



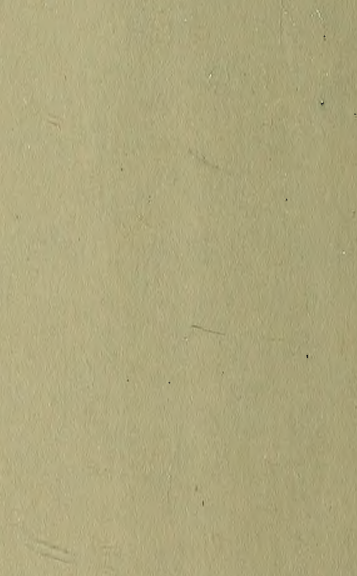






\section{OR NITHOLOGIA}

S V E C I C A.

QL

677

$\sin 2$

MU UVAKOMGST

817

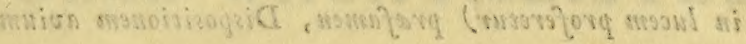

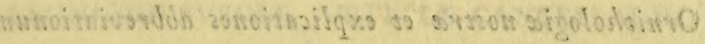

A UCTORE

\section{SV. NILSSON,}

PHILOSOPHIEE DOCTORE?

IN ACADEM. IUNDENSI ADJUNCTO ET SOCIET. PHYSIOGR。 MEMBRO.

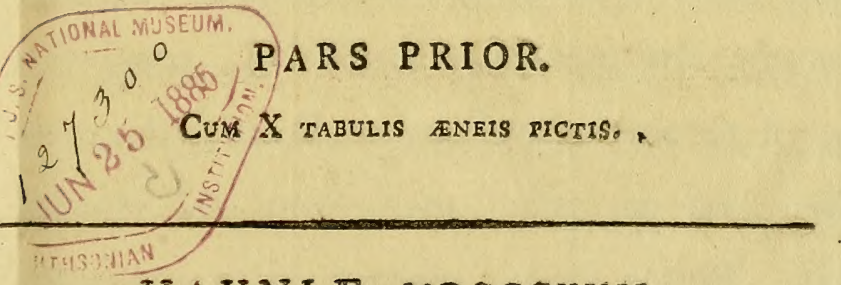

HAVNI㤅, MDCCCXVII.

APUD $7 . H$. SCHUBOTHIUM, AUTA REGTA BXBLOPORAM. 
PREMONENDUM.

Parti Operis hujus posteriori (que proxime anno D. v. in lucem proferetur) prafamen, Dispositionem avium, fata Ornithologia nostra et explicationes abbreviationum complex. urum, praponetur. 


\section{gатет}

Won facile scriptor invenietur, qui Patrono justius vel devotius memoriam laboris et industrix sua consecraret, quam nos Tibi, Vir illustrissime! Quo nihil Patria in Ornithologia, nec mihimet ipsi in beneficis majus et eminentius obtigerit. Tua virtus erat, Vir generose! qua atate apud plerosque ludis tantum obnoxia, haud fallacem natura interpretem se exhibuit, nomenque apud rerum naturalium studiosos, dum natura ipsa perennat, mansurum sibi vindicavit. Postea vero, summos in republica ho- 
nores jamdudum adeptus, quantum Tibi æquata Themidis libra tribuit otii, potissimum Faunorum dedicas choro. Tu idem eras, qui prima eademque leviora tentan. tem, me hortabaris ut pergerem; meditanti vero majora mihi et robur et animum addebas, cum ea conferres auxilia, quibus sublatis sublata mihi fuisset copia hoc edendi specimen. Nihil igitur aquius habeatur, quam ut Tuum ipsius Opus Tibi porrigam; quod quidem, quo minus pretii in manibus meis perdiderit, eo melius mihi successisse 
putabo. Sed quomodocunque vulgo judicabitur, equidem tum habeo quod mihi in perpecuurm gratulabor, si eodem, quo antea, favore et gratia, me semper dignum censueris.

Mansurus, dum vixero,

Nominis Tui illustrissimi

cultor devotissimus

Sv. Nilsson. 
O R N I T H O L O G I A $S V E$ C I C A. 


\section{SYNONYMA.}

Avis senior:

Falco chrysaétos Lin. Faun. Sv. p. I9 - n. 54. Syst. snat. edit. XII. I. p. 125. n. 5. Retzii Faun. Svec. p. 59. n. I. Gmel. Syst. I. p. 256. Lath. Ind. Orn. I. p. 12. 1. 8. Wachtmeister, Wet. Acad. N. Handl. I80I. p. I85. (fem. var.) p. I87. (mas var).

'Aquila auren, Briss. Orn. I. p. 43 I.

Le grand Aigle, Buff. Hist. Ois. I. p. 76. ver's. Germ.

Martini I. p. 94. tab. I. Planches enlum. 410.

Golden Eagle, Pen. Brit. Zool. I. p. I61. Lath. Syn. I.

p. 3 I. vers. Germ. Bechst. I. p. 3I. n. 5. Arct.

Zool. II. P. 214. A.

Der Goldadler Bechst. Naturg. Deutschl. ") 2 B. p. 205.

Suis junior:

Falco fulvus, Lin. Syst. Nat. XII. I. P. I25. n. 5.

Gmel. Syst. I. p. 256. Lath. Ind. Onn. I. p. 10.

n. 4. Wachtm. Wet. Ac. Hand. I80I. p. I8I.

Falco Melanatus et fulvus, Retz. Faun. Sv: 60.11. 2 s

Falco niger; Gmel. I. p. 259.

Falco Melanonotus Lath. Ind. I. p. I6. n. 26.

Aquila, Briss. On. I. p. 419.

Melanaètus sen Aquila nigra, Briss. Orn. I. p. 434.

Aigle commun, Buff. Ois. I. p. 86. vers. Germ. Martini

I. p. IOS. tab. I2. Pl. enl. 409.

Black Engle, Pen. Brit. Zool. I. p. I65. Aret. Zool. II.

p. I95. Lath. Syn. I. p. 28. 12. 2. vers. Germ. Bechst. I. p. 25. n. 2.

Ring-tailed Engle, Lath. Syn. I. p. 32. 11. 6. ver's. Germ.

I. p. 28. n. 6 . et var. A. p. 29. Edw. aves T. I. tab. I.

2) Editio prima citatur, ubi non expresse dicitur: ate Auft. 
Black backed Eagle, Lath. Syn. I. p. 49. vers. Germ. I. p. 39. n. 22.

Der gemeine Adler, Bechst. Nat. Deutschl. 2. B. p. 2 L. Svecis: Örn, Landörn.

Museum Paykullianum - Museum Lundense.

\section{OBSER V.}

Omnes fere ornithologi $F$. chrysätum et fulowm ut distinceas Species enumerant. Illustrissimus Comes Wachtmeister primus erat qui hoc non solum in dubium vocabat sed etiam contrariuin demonstrabat ); (vide Försök till de Sverske Falk-Arters beskrifning och bestämmande. Wet. Ac. Handl. 1801. p. I7r. \&c.) Quem ego quoque eo lubentius sequor, quod utramque avem et chrysaëtum et fulvum Auctor. sæpenumero unâ vidi et diligenter comparavi, nec vero miniman partium constantiorum vel proportionum differentiam indagere potui. Sola fere difea rentia in colore caudze semper consistit. Sed hajus sunt innumerabiles varietates et transitus a fulvo in chrysaëtnm. Ne vero quis dubitet, an verum viderim chrysä̈thm; Auctores prastantiores, qui avis mentionem fecerunt, breviter recensere volumus: $\boldsymbol{F}$. chrysätos, Lin. Frun. Svec. cun mea ave annosa optio me convenit, proter: Caput collsmque supra magis nigricantia. Hoc vero loco aliquid omissum esse, absque dubio est. Leche enim, maximus tunc temporis olnithologus, a quo Descriptiones plurimas avium recepir Linné, ita in adversariis de $F$. chrysaëto haber: Caput tegitur penris flavo-fulvis longisque, instar penua, rum colli galli-gallinacei; et Willoughby, quam citavit Linné, disit: The Feathers of the neck are rigil and ferrugineous: Will. Orn. Ang. p. 58. (Rajum, quen etiam citavic Linné, ad manum non habeo). İa ígitur, ni fallor, dicere voluit Linné: corpus.... ferrugineo fuscoque uebrlosum, Caput collamque supra magis ferrugines, subtus nigricantic.

The Golden Eagle, Pen, 1. c. p. 162, in nostrum maxime qunàiat, estque eâdem magnitudine ac The blaçk Eagle 1. c. p. 866. 2 qุuo โุantumıodo cauda colore differt.

-) Hoc posta cum Illustr. Wachtmeister assumsit Bechstein in 2 da edit. Nat. Deuk, Bs a. p. 521 et 522. 
Le grond Aigle et Aigle commun, Buff. P1. enl. 409. et 4:0. Utraque figura, ab Auctore ipso citara, absque ullo dubio ad meam pertinet avem, scil. fig. 410 ad seniorem, et 409 ad juniorem. Si vero $c$, que fusa et elegantia de generoso suo Grand Aigle on Aigle royal scripsit cloguens Auctor, cum is conferimus, quæ de ignobili Aigle commun dixit; facile pro diversis avibus eas habeamus. Illa vero omnia Auctor, stilo migis splendido et magnifico quam vero studens, ab antiquis Scriptoribus, mirabilibus rebus addicis, desumsic, propriaque forte imaginatione auxit, ut Reginam avium totâ majestate et præxstantia ostenderet. Non igitur quas descripsit, sed quas depinxit aves consulendas snnt.

Plurina qua Bechstein de suo Goldadler 1. C. dixit a Bufton desumta sunt, eidemque fonti originem debent.

\section{DESCRIPTIO.}

A. Femina senior:

Longirudo avis ab apice rostri, ad apicem cauda 3. ped. 3 poll. Rostrum ab angulis oris ad apicem $2 \frac{1}{2}$ poll. A flexura alr ad ejusdem epicem 2 ped. 2. poll. Cauda extra alas extenditur 3 poll. Cauda I. ped. 3. poll. Tibie $4 \frac{\pi}{2}$ poll. Digitus medius cum ungue $3 \frac{\pi}{2}$ poll. Digit. post. $3 \frac{2}{5} ;$ Unguis medius ant. $I_{\frac{3}{8}} ;$ ung. post. $2 \frac{2}{8}$ poll.

Rostrum coeruleo-nigricans, apice nig"o, basi rec. sum, mox ante ceram in uncum validum, acutum, de. pendentem exiens. Cera et Iris flava. Os frontis supra oculos margine rotundato eninens. Tibix robu. stæ, usque ad digitos teftx, antice pennis plumosis luteo-rufescentibus, postice plumis albis. Digiti lutei unguibus nigris, validis, acutis.

Caput et collum teguntur supra pennis longis, acu. tis, splendentibus, fulvoferrugineis, brunneo immixto; frons saturatior. Regio inter nares et oculos albida, setis nigris adspersa. Gula er jugulum saturate fusca, vel nigricantia. Doxsum, pectis, abdomen et tectrices 
alarum fusca, nigro, ferrugineo et albido immixtis. Crissum et femora intus rufescentia, extus rufo-fusca. Remiges nigricantes; primores versus basin cinerascentes, albo variegatx, qux albedo in sequentibus angetur. Rectrices basi albr, apice nigricantes, in medio (ubi hi colores confuunt) fusco, cinereo et albo, irres gulariter variegatæ.

B. Mas senior:

Non modo co pore, sed etiam rostro, unguibus \& minor præcedente, cui prxterea subsimilis.

Varietas Maris senioris:

Magnitudo corporis, rostri, unguitum \&c. minor quam feminæ. Caput et collum supra fulvo - ferruginea, subtus saturate fusca. Dorsum nigricans, pennis di。 lutioribus insertis. Alx supra fusex marginibus pen。 narum grisescentibus. Pectus et abdomen diute ferruo ginea, maculis oblongis nigro-fuscis, in abdomine fere confuentibus. Remiges primores nigrx, secundarix intimx fuscx cinereo et albido variegatx. Cauda supra $a b$ apice ad tertiam partem nigra, immaculata, dehine cinereo parum variegata; subtus fasciis 2 vel 3 . angustis, irregularibus, cinereo-albis. Tibix pennis griseo-ferruginescentibus vestitæ. Mus. Payk.

C. Avis jurior:

Longitudo circa $2 \frac{\pi}{2}$ ped. Rostr. $2 \frac{8}{8}$ poll. A fexura alx ad apicem 2. ped. I. poll. Cauda I. ped. I. poll.

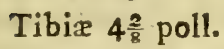

Rostri forma et color ut in procedentibus. Cera lutea. Iris fusco-lutea. Tibir antice pennis plumosis sordide albis; postice plumis albis vestitx.

Caput et collum supra ferruginea, apicibus pennarum dilutioribus; fronte et vertice magis fuscescentibus. Gula et jugulum fusco-nigricantia. Dorsum, pectus et abdomen fusca, pennis nigricantibus immix- 
tis. Teetrices alarum in ferrugineum vel griseum in. clinantes. Crissum rufescenti-album. Remiges apice nigræ, primores juxta basin latere interiore cinerea, qui color in sequentibus magis albescit, ita ut secun. darix sint apice nigrx, basi nivex, in medio ex albo et fusco varix. Cauda alba, vel sordide alba, ab apice ad tertiam partem nigricans.

\section{OBSERV.}

Plurimas hujus speciei varietates in variis Patriæ Museis et in natura vidimus. Illas vero oinnes adferre superfluum duximus, cuin plerumque in eo consistunt quod alis specimina ma. gis, alia minus fulvi coloris supra caput et collum habent; alia parcius, alia uberius colore ferrugineo sunt adspersa. Cum enim diu vivit hac avis; diu queçue crescit, et per totum hoc tempus varias colorum mutationes subit. Caudx autem coJore maxime variat. Cauda enim, ut jan diximus, in ave juniori, alba est et ab apice ad tertian partem nigra; tempore vero, hi duo colores, modo irregulari, nee in duobus speciminibus exacte simili, miscentur, fuscumque colorem, cinereo variegatum confluentes effiunt. Qua vero ærate hac mutatio plane peracta sit; nescio.

\section{HISTORIA AVIS.}

Septentrionalium Patria provinciarum loca solitaria, nemorosa et petrosa prasertim incolit, ibique in rupibus et excelsis arboribus nidum ponit. Altissime vo. lat. Lepores, Capreolos, Tarandorum hidos, aliaque Mammalia minora et aves prædatur. Alio victu deficiente, eadavera petit. Hieme instante montuosas interdum regiones relinquit, et in Scaniz usque campos descendit, ubi Leporibus præsertim infestus. Cautus fixuque difficilis. Terra nive obrutâ, in pedicis vul. pinis interdum capitur. 


\section{FALCO NEVIUS:}

DIAGN: Rufo-fuscus, Remigibus nigricantibus Rectricibus saturare fuscis apicibus pallidis.

\section{SYNONYMA.}

Falco navius, Gmel. Syst. I. p. 258. Lath. Ind. I. pi I4. n. I8.

Aquila nevia, Briss. Orn. I. p. 425.

Aquila Mogilnik, Nov. Comm. Acad. Petrop. XV. p. 445 tab. XI. b.

Falco Mogilnik, Lath. Ind. I. p. I7.

Rough-footed Engle, Lath. Syn. I. p. 37.' n. I4. vers.

Germ. Bechst. I. p. 35. n. 14.

Russian Engle, Lath. Syn. I. p. 43. 11. 24.

Funior Avis:

Falco muculatus, Gmel. Syst. I. p. 258. Lath. Ind. I. p,

15. n. I9.

Spotted Engle, Lath. Syn. I. p. 38. n. I5. vers. Germ, Bechst. I. p. 35. n. I5.

Der Schreier (F. nævius) Bechst. Nat. Deut. 2. B. p. 226. Mus. Payk,

\section{OBSER V.}

Cel. Bechstein 1. c. sub nomine f. nevii Lin. (i. e. Gmel.) avem describit, quan Gmelin, Lathain \&e f. maculatums appelJant. Avis quan ego infra describam, cum f. navio Gmel. Lath. probe convenit. Cum vero etian in $f_{0}$ maculatum quadrat, prerer maculas schpularum et alarum tectricum albas, et cum constat quam varient falcones pro varia etate; haud temere has aves conferendas putavi. Judieet vero is, cui avem per omnes attes observare contingat, an recte contulerim. Figura Frischii 71 . plerumigue ad hanc avem citatur, tnea autern senter. tia potius ad $F$. Buteonein pertinet. 


\section{DESCRIPTIO.}

Longitudo avis circa 2, ped. Rostr. 2. poll. A flexura alx ad apicem I. ped. 6. poll. Cauda circa 9. poll. Tỉbix 2. poll. Digit. med. $2 \frac{2}{8}$ poll. Digit. post. I $\frac{7}{3}$ poll.

Rostrum coeruleo - nigricans, dente obtusissimo, basi elongeto-reßtum, apice valde aduncur, cera lutea. Regio inter nares et oculos setulis nigris adspersa; Tibiæ, usque ad digitorum exortum, pennis cinereo-rufis, postice plumis albis, tectx. Digiti lutei.

Corpus totum rufo-fuscum, capite et femoribus aliquantum dilutioribus. Teß̂rices alarum minores pallide xufo-fuscæ, marginibus pallidioribus. Remiges primores nigrx, basi alba; secundarix nigricantes, basi cinerascentes, apice dilutiore. Tectrices alarum inferiores rufo - fuscr maculis albis. Cauda alis parum longior, nigro-fusca lineis transversis, obsoletis, cinerascentibus, apice albido. Tectrices caudæ inferiores albæ. Crissum albo-rufescens.

\section{LOCUS.}

Specimer, in Lapponia occisum, in Museo Paykulliano esservatur:

\section{FALCO LAGOPUS.}

Diagn: Corpus albo-fuscoque varium. Caput et collum alba striis fuscis. Cauda alba, versus apisem saturate fusca, apice albo.

\section{SYNONYMA.}

F. Lagopus, Brün. Orn. bor. p. 4. 11. I5. Gmel. Syst. Nat. I. p. 260. Lath. I. p. 19. 11. 33. Retz。 Faun. Svec. p. 62. ^. 6. Wachtmeister, Wet. Acad. H. I80I. p. I9I. n. 5. 
Falco communis leucocephalus, Gmel. Syst. 1. p. 270. 8. Lath. Ind. Orn. I. p. 30. $\delta$.

Fautcon is tête blanche, Briss. Orn. I. p. 325.

White-hended Falcon, Lath. Syn. I. p. 66. 11. 49. C.

Rough-legged Falcon, Pen. Brit. Zool. 2. p. 200. n. 92.

Lath. Syn. I. p. 75. n. 54. vers. Germ. Bechst. I. p.

64. 11.54.

Der rauhleinige Falke, Bechst. Nat. Deut. 2. B. p. 228. Der rauhfussige Falke, Teutsche Ornith. Heft. II. cum figuris coloratis maris et feminx egregiis.

Funior avis:

Falco Sclavoricus, Lath. Ind. I. p. 26. 11. 54.

Falco pennatus. Ginel. Syst. I. p. 272. Lath. Ind. O'n,

I. p. 19.

Faucont patu, Briss. Orn. VI. app. I. p. 22. t. I.

Booted Falcon, Lath. Syn. I. p. 75. 11. 55.

F. S. Fohannis, Lath. Ind. I. p. 34. 11. 74?

St. Fohn's Falcon, Arett. Zool. II. p. 93. tab. 9?.

Mus. Lund. Mus. Payk.

\section{DESCRIPTIO.}

Longit. avis circa 2 ped. A flexura alr ad apicem

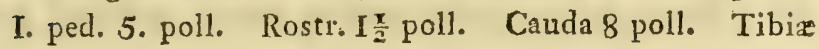
3 poll. Digit. medius 2 poll. Digit. postic. Ir poll. Rostrum nigro-fuscum, breve, parum aduncum, dente marginis obtusissimo vix notabili. Cera lutea. Iris flava. Tibix ad digitos usque tetze pennis sordide-albis, fusco longitudinaliter maculatis.

Caput, collum et peetus lutescenti-alba, striis ad ra. ches pennarum fuscis. Regio inter nares et oculos alba, setulis nigris. Dorsum et alre supra fusca marginibus pennarum ferrugineis et albidis. Abdomen saturate fuscum macnlis (interdum) albis. In aliis (maribus) abdomen lutescenti album, maculis saturate cuscis fere 
confuentibus. Remiges primores ab apice fere ad me. dium nigricantes, intus albo varix; secundaria fusca, basi alba maculis fuscis. Cauda alis complicatis parum longior, supra saturate fusca, interiore medietate et apice extimo albis; subtus alba apice griseo. Teetrices superiores albæ maculis fuscis, inferiores sordide albæ. Femore sordide alba, maculis fuscis, in maribus, transversis; in feminis, longitudinalibus.

Funior avis:

Caput, collum, totumque corpus supre fusca marginibus pennsum albis, vel cinereo-albis; pectus, abdomen et fernora alba maculis transversis fuscis. Tibix saturate furcr undulis albis. Cauda alba fasciis nigris, quarum extima ceteris latior.

Mus. Rayk. L Lapponin.

\section{LOCUS.}

Habitat in Lapponia et Nordlandia. Rarissine in Sveciam australiorem descendit. Mores ejus nondum mili bene noti.

\section{DISSECT. II.}

Tibice antice quoad parten plumis vestite; postice nude.

\section{A.}

Margo maxilla superioris dente obtusissimo aut quillo.

\section{FALCO ALBICILLA.}

Diagn: Corpus fuscum griseo immixto, capite et collo pallidioribus (sordide albidis); Cauda ro. tundata, brevis, alba, 


\section{SYNONYMA.}

Falco albicilla, Lin. Faln. Sv. p. I0. 13, 55. Gmel. Syst. 1. p. 253. Lath. Ind. Om. I. p. 9. n. 2. Wachtm. Wet. Ac. Handl. I80I. p. I75. I. Retz. Faun. Sr. p. 61. n. 3. Ödman, Alta Upsal. Vol. IV. p. 225.

Valtur Albicilla, Lin. Syst. nat. XII. I. p. I23. n. 8. Fabric. Faun. Groenl. p. 53.

Falco leucocephalus, Lin. Syst. Nat. XII. T. I. p. 124. n. 3. Gmel. Syst. I. p. 255. Lath. Ind. Orn. I. p. II. 11.5 .

Falco albicaudus, Gmel. Syst, nat. I. p. 258.

Falco hinnularius, Lath. Ind. Orn. I. p. 15. n. 20.

L'Aigle ì queue blanche, Briss. Orn. I. p. 427.

L'Aigle à tête blanche, Briss. Orn. I. p. 422.

Le petit Aigle à queue blanche, Briss. 1. c. p. 429.

Lo Pygargue, Buff. Ois. I. p. 99. vers. Germ. Martini

I. p. I25. Pl. enl. $4 \mathrm{II}$.

Cinereous Eagle, Pen. Brit. Zool. I. p. I70. tab. XVIII.

Lath.Syn. I. p. 33. n. 8 Vers. Germ. I. p.32. n. 8. Lesser zohite tailed Eagle, Lath. Syn. I. p. 39.' n. I6.

Vers. Germ. I. p. 36. n. I6. Anh. p. 658. n. I6. Bald Engle, Lath. Syn. I. p. 29. vers. Germ. I. p. 25. 11. 3.

Der Fischadler, Bechst. N. D. 2. p. 222. Funior avis:

F. Melnnatus, Lin. Syst. Nat. XII. I. p. 124. n. 2. Lath. Ind. Orn. I. p. 10. n. 3. (Diagn.) Gnel. Syst. Nat.

I. p. 254. (Diagn.)

Black Engle, Will. Orn. (Angl.) p. 6I. t. 2. fig. inf. Svecis: Hafsörn.

OBSER V.

Me contulisse F. Albicillnm, lessocephalum, Albicaudum \&c. mirum videbitur nemini, qui avem sub varia ætate' examinavit. 
Cur vero huic speciei $F$. Melanctun quoçue adjunxerim; explicationem fortasse postulare videbitur. Caussam breviter expo. nan: Willoughby 1. c. (quen citarunt omnes Ornithologi F. nedonatum commemorantes), describit Aquilam sub nomine: the Black Engle, called Melanä̌nus or Aquila valeria, de qqua inter alis dicit: The jaws and Eye-lids were bare of Feathers - The herh, neck and brest black-D- The Beak- - black at the sud, then yellow \&C- - The Leggsueve feathered down but a little bolow the krees bro. qua quidem omnia in $F$. albicillam junicrew, non vero in $F$. fulum (i. e, chrysäerum jun.) quadrant. Gmelin autem et secundun illum Latham, Diagnosi bajus avis descriptionem breven a $F$. fulwo desumtam addiderunt.

\section{DESCRIPTIO.}

A. Feminn senior:

Longit. avis 3 ped. 3 poll. Rostr. $3 \frac{2}{3}$ poll. A fex. ura ale ad apicem 2. ped. 3. poll. Cauda II. poll. Tibia $4 \frac{2}{8}$; Digit. intermed. 4. poll. Digit. post. 3. poll. Unguis post. $I_{8}^{7} ;$ unguis antic. intermed. $I \frac{7}{2}$.

Rostrum cerino-Iuteum, elongatum, reetum; maxil. Ia superior margine sinuosa, apice unco dependente incurvato. Cera lutea in qua nares oblongo-ovales, oblique-transversa. Iris flava. Tibir plumis fuscis ad medium antice teEtx, indeque cum digitis lutex. Femora extus pennis longis fuscis. Regio inter nares et oculos plumis destituta, setulis albis adspersa.

Caput et collum, pennis acutis, longis, supra sub. tusøูue, pectus et alæ complicatæ supra pallide grisea, dilute fusco immixto. Gula sordide albida, Dorsum et Uropygium saturate fusca, Abdomen fuscum pallide griseo immixto, Crissum nigricans. Remiges prino. res nigræ, basi cinerascentes; secundariæ fuscr. Cauda rotundata, cum alis complicatis æqualiter extenditur, Reenuces albx, versus basin fusco variegatx. Tectrices supenores quatuor elongatæ, albæ apice (in. terdum) fusca. 
B. Meres minores sunt, plusque fusci coloris in capite et collo habent. Quo senior avis, eo albidior, quo jwo nior eo obscurior; ita ut pulli prino atatis anno fncilo lime pro diversa specie haberi possint.

C. Pullus, primo atatis anno:

Feminâ amnosâ tzulto minor. Longit 2. ped. 6. poll. Rostr. 27 poll. A flexura ala ad apicem 2. ped. I. poll. Cauda IO poll.

Rostrum et cera nigra; regio inter nares et oculum setulis nigris adspersa. Iris avellana. Tibix plumis fuscis antice semitectx, dehinc cum digitis lutex. Ungues nigri.

Caput et collum nigricantia, gula pallidiore. Dor. sum et alarum teetrices fusco ferruginea maculis nig: 0 . fuscis (singula penna apice nigrofusca). Pectus et ab. domen ferruginescentia maculis oblongis nigris. Remiges primores nigræ, basi cinerea; secundariæ griseofuscr. Reetrices nigricantes, rachi et latere interiore fere ad apicem albæ, fusco punetatr. Tectrices Cau* d:x superiores albo et fusco variegati; inferiores alba, apice nigricantes.

Mus. Lunc.

D. Avis biennis:

Rostrum dilute fuscum, versus basin pallidius (Initga scens). Iris quan pracedentis pallidior. Caput eq collum supra fusca apicibus pennarum pallidioribus; subtus dilute griseo-fuscescentia. Dorsum et alä su. pra fusce, ferrugineo et griseo immixtis. Pectus et abdomen pennis albicantibus, apicibus fuscis. Femo. sa pennis longis saturate fuscis, Cauda ut prioris, sed albedine anctiori.

Mus. Iund, 


\section{O BSER V.}

Illustriss. Comes Wacktmeister, pullos ex nido desumtos. per biennium et quod excurrit, educavit, mutationesque colorum observavit. Hrec eadem Specimina, unde eriam Descriptiones datas ego formavi, in Museo Academix Lundensis adhuc asservantur. Ex his vero patet, pullam Albicille in omnibus fere cum F. Ossifrago Auctor. congruere. Rev. jgiturödman et Ill. Wachtmeister non sine jure suspicantur, juniorem Albicillam nomine Ossifragi sape esse descriptam. Caussas vero, cur nihilo tamen minus ego Ossifragum ut distinctan Speciem enumerem, hac est: Avem a nob. Wormekiold e Groenlendia allatam accepi, que quoad colores cum Albicilla bienni probe conrenit, sed magnitudine rostri, unguiam \&c Albicillam vel seniorem facile superat, Rostrun prxterea habet aterrimum, sed ceram $\mathbf{8 2}$ vam. Cauda quæ in Albicilla rotundata, in hac xqualis est. Hanc avem, quam et postea in Mus. Payk. vidi, quæque in Teutw sthe Ornith. Heft, XVII, nomine Seeadler egregie depicta est, pro genuino Ossifrago habco.

\section{HISTORIA ALBICILL屟.}

Nemorosa et rupestria loca juxta maria et lacus progo sertim amat. Superioris tamen quam inferioris Svecia frequentior incola. Nidum vel in excelsis arboribus, vel in altis rupibus construit. Tria ova ponit, quoxum plerumque duo tantum prolifera. Vivam vel recentem prædam pullis adportat. Mammalia mediocris magnitudinis, aves et pisces prædatur. Etian infantes aliquando subripuit. Putrescentibus cadaveribus vescitur. Phocam vitulinam \&c. adgressus, interdum in fundum sequi cogitur. Vox acutissime sibilans. Pulli facillime mansuescunt. (Vide nova ACt, Upsal. IV. p. 234 et sequ. ubi Rev. Ödman Historiam avis ex atopsia fuse, concinne et ingeniose dedit.)

$$
\text { V. FALCO OSSIFRAGUS. }
$$

Dragn: Corpus ferrugineum maculis fuscis. Capur et collum supra saturare fusca apicibus pennarum 
pallidis. Retrices nigricantes, rachibus et interiore latere albidis. Cauda aqualis. Rosirum nigrum, Cera lutea.

\section{SYNONYMA.}

Falco Ossifragus, Lath. Ind. Orn. I. p. 12, 11. 7. Gmel. Syst. Nat. I. p. 255.

The Sea-Eagle or Osprey, Will. Orn. (Angl.) p. 59. to 1. fig. infer. Pen. Brit. Zool. I. p. I67. n. 44. f. I7. Aret. Zool. II. 12. 86. A. Lath. Syn. I. p. 30. n. 4. vers. Germ. I. p. 26. n. 4.

L'Orfraie, Buff. Ois. I. p. II3. tab. 3. Pl. enl. 4 I5.

Le grand Aigle de mer, Briss. Onn. I. p. 437.

Der Seendler, Borkhaus. Teutsche Ornith. H. XVII. (Figura maris et femina egregia).

Mus. Payk, e. Lapponia.

OBSERV.

Cur hanc avem, juniori Albicilla subsimilen, it distinctan Speciem exposuerim, caussam supra dixi. Forte tamen mera pro slimate varietas Albicilla. Junior salcem non est.

\section{DESCRIPTIO:}

Longit. avis 3 pedes et aliquot pollices; Rostrum $3 \frac{\pi}{2}$ poll. A flexura alæ ad apicem 2 ped. 4 . poll. Canda I. ped. 3 poll. Tibir $4 \frac{\pi}{2}$ poll. Digit. medius $4 \frac{\pi}{2}$ poll. postic. $3 \frac{T}{2}$ poll. unguis medius $I \frac{5}{8}$ poll. ung. postic. 2 poll.

Rostrum nigrum forma præcedentis at aliquantum majus et robustius. Cera flava. Regio inter nares et oculos setis nigris adspera, Pedes frovi unguibus nigris.

Caput et collum supra pennis acutis, longis, saturate fuscis apice extimo pallidis; subtus dilute griseo. 
gufis, apice nigris. Dorsum et alarum tectrices ferrnginea maculis nigricantibus, Remiges primores nigrx basi cinerex; secundarix nigricantes, latere interiore cinereo variegate, apice pallidiores. Femora teguntur peunis longis cinereo-fuscis. Tibix antice pennis fuscis semivestita, inde cum unguibus lutez. Cauda subxqualis nigra, interiore Rectricum latere et ad raches aibo variegata. Tectrices superiores et inferiores ex fusco, fermgineo et albo variegatx.

\section{LOCUS:}

Rupes septentrionis, mari adjacentes, habitat. Animalia, qua in undis degunt, prasertim devastat.

\section{FALCO HALIETUS.}

Diagn: Corpus subrus, caput et collum supra sub. tusque alba; latera colli et dorsum fusca; pennæ femorum brevissimæ. Cera pedesque cærulei, unguibus teretibus, subæqualibus.

\section{SYNONYMA.}

Falco Haliatus, Lin. Faun. Sv. p. 22. n. 63. Syst. Nat. XII. T. I. p. I29. Gmel. Syst. I. p. 263, Lath. Ind. I. p. I7. Retz. Faun. 62, 11. 5. Wachtmeister Wet. Ac. H. I80I. p. 188.

LiAigle de Mcr. Briss. Orn. I. p. 440 tab. 34. Le Balbuzard, Buff. Hist. Ois. I. p. I03. t. 2. vers.

Germ. Martini p. I82. Pl, enl, 4 I4.

Osprey, Pen. Brit. Zool, I. p. I74, n, 46. Aret. Zool.

II. 11. 9I. Lath. Syn. I. p. 45. n. 26-Suppl. p. I3.

Der Fischanr, Bechst. Nat. Deutschi. 2. p, 229.

Der' Flufsaller, Borkh. Teutsche Om. II. IX. (Mias et fem. egregie depicti.)

Suecis: Fisk-kjuse, Sjöhök.

Mus, Lund. Upsal, Paykull. 


\section{DESCRIPTIO.}

Longit. avis circa 2 pedes. Rostr. $I \frac{g}{8}$ poll. alx compositz I.ped. 8 poll. Cauda $8 \frac{1}{2}$ poll. Tibia $2 \frac{2}{8} ;$ Digit. med. fere 3. poll. Digit. post. $2 \frac{\frac{1}{8}}{8}$ poll.

Rostrum coeruleo-nigrum, longiusculum, basi rec. tum, maxilla superior in médio dente obtusissimo, apice unco valido, Cera coerulea. Iris pallide flava. Regio inter oculos et nares setulis nigris adspersa. Pedes coerulei, nudi, scutellis parvis, imbricatis scabri. Digiti, quorum extimus versatilis, subtus rugosissimi. Femora pemis plumosis, brevibus, albis tecta. Ungues nigri, magni, in semicirculum curvati, teretes, aliquantum compressi, fere æquales, exteriore tamen et postico paulo majoribus. Caput, collum, pectus et abdomen alba, vertice et pectore striis et maculis fuscis adspersis. Ab oculo ad latera colli utrinque de. scendit fascia fusca. Dorsum et tectrices alarum saturate fusca. Remiges nigro-fuscx, basi et (ad partem) latere interiore albx, fusco transversim fasciatx. Cauda alis complicatis brevior, æqualis, supra obscure - cinereo fuscoque fasciata, subtus albida fasciis fuscis. Par intermedium Rectricum immaculatum, fuscum. A. nvis junior:

Caput supra et pectus magis maculata. Dorsum et alæ supra fusca, marginibus pennarum albidis. Cauda fasciis supra magis conspicuis. Crissum rufescens.

B. variet:

Mus. Payk.

Primo descriptr similis, pectore antem anteriore fusco marginibus pennarum albescentibus.

Mus. Paýk.

\section{HISTORIA.}

In Scania rarior, in ceteris Patrix provinciis frequens. Iam maria jam lacus visitat. Supra aquas voliNilsson Oruith. 
tans, in pisces majores se præcipitat. Neque semper latrocinlum ejus inpunitum; unguibus enim aduncis in piscem nimis magnum infixis; gitatis alis ejulans avis in undas derrahitur. Lacubus glacie obductis, ora nostras relinquit; tempore vero, quo dissolvitur glacies, redit. Nidum in praltis arboribus construit. Ova'3. alba, brunneo-marmorata. (Historißm avis cet. vide Nov. Act. R. Ac. Scient. Holm. Tom. V. p. 30I. et Act. Upsal. T. V. p. 54.)

\section{FALCO LONGIPES, miki.}

\section{(Tab. I.)}

Diagn: Supra nigro-fuscus maculis colli testaceo albis; subtus testaceo albus maculis nigricantibus. Femora fusco undulata. Cauda fasciis fusciis et cinereis. Rostrum validum. Tibiæ prælongæ: digitis brevibus.

\section{DESCRIPTIO.}

Habitu distinctissima avis, inter majores est. I. ped. II. poll. longa. Rostrum $I \frac{6}{8}$ poll. A flexura alx ad apicem 15 poll. Cauda circa 9 poll. extra alas extenditur 4 poll. Tibiz fere 5 poll. Digitus posticus $I_{2} \frac{x}{2}$ poll. intermed. $I \frac{\sigma}{8}$.

Rostrum nigro - fuscum, validum, aliquantum elongatum, fere edentulum, maxilla superiore unco dependente, longo, terminata. Cera lutea? Iris - Regio inter nares et oculos pilis adspersa. Pedes lutei, tibiis elongatis, digitis brevibus, unguibus nigris.

Caput supra nigricans. Occiput, cervix et colli la. tera variegata pennis basi albo-testaceis, apice nigricantibus. Circa oculos circulus nigricans e quo macula nigra versus nucham ducta. Dorsum nigro-fuscum 
pennis hine inde margine tenui fuscis. Tectrices ala. rum minores colore dorsi, at magis in fuscum inclinantes, marginibus tenuibus ferruginescentibus. Remiges extimæ nigrx, basi alba fasciis fuscis; ceteræ cum tectricibus majoribus cinereo- fuscr, fasciis transversis, angustis, obsoletis, nigricantibus, basi et la. tere interiore a basi albre vel testaceo albr. Corpus subtus testaceo vel flavo-album; gula et genis striis nigris, pectore et abdomine maculis oblongis vel sub. cordatis nigricantibus, in infimo ventre confluentibus. Tectrices alarum inferiores testaceo-albx maculis nigricantibus. Crissum maculis transversis parvis et rarioribus. Femora teguntur pennis brevibus albo-testa. ceis, undulis numerosis nigro-fuscis. Rectrices latere exteriore cinerea fasciis angustis (6.) fuscis; basi at latere interiore testaceo-albx fascids angustioribus fuscis; pone apicem albidum fascia lata nigricante Rectrices intermedix binx, prxter fasciam pone apicem latam nigram, cinereo, fusco et albido irregulariter maculatx et litturatx.

OBSERV.

Pulchram hanc et distinctissimam avem nullibi descriptam invenio, nisi forte Falco leucopsis Bechsteinii (Naturg. Deutschto 2re Aufl. 2. B. p. 572) huc pertinet. Nomen F. longipes, quod Illustr. comes Wachtmeister ei addidit, ut maxime characteristicum retinui. Figura data Benevolentis nobiliss. Payknll dę betur.

\section{LOCUS.}

E Lapponia allata in Museo egregio Paykulliano oso servatur.

VIII. FALCO ERUGINOSUS.

Diagn: Corpus saturate fuscum, gula et vertice sua fescenti-albis, Pedes longi, graciles. 


\section{SYNONYMA.}

Falco aruginosus, Lin. Syst. Nat. I. p. I30. Faun. Svec.

p. 66. Retz. Faun Sv. p. 74. n. 2I. Gmel. Syst. Nat. I. p. 267. Lath. Ind. Orn. I. p. 25. Wachtm. Wet. Ac. H. I80I. p. 203.

Ie Busard de Marais, Briss. Orn. I. p. 40I.

Le Busard, Buff. Ois. I. p. 218 . tab. IO. Pl. enl. 424.

vers. Germ. Martini II. p. 22. tab. 27.

Moor Buzzard, Pen. Brit. Zool. I. p. 192. n. 57. tab. XXVII. Arct. Zool. II. p. 225. L - Lath. Syn. I. p. 53. Sup. p. I5. vers. Germ. Bechst. I. p. 48. 11. 34.

Accipiter korschun: Nov. Comm. Acad. Petrop. T. XV. p. 444. tab. XI. a. (ad hunc pertinet, minime ad $f$. inilvum, ut Lath. vult.)

Rostzpeihe, Bechst. Nat. Deutsch. 2. p. 250.

Funior avis:

Falco rufus, Gmel. Syst. Nat. I. p. 266. Lath. Ind.

Orn. I. p. 25. 11. 5I. Retz. Faun. p. 65. 11. 10.

Wachtm. Wet. Ac. H. I80I. p. 205.

Le Busard roux, Briss. Orn. I. p. 404.

La Harpaye, Buff. Ois. I. p. 217. Pl. enl. n. 460. vers.

Germ. Martini II. p. 20. tab. 26.

Harpy - Falcon, Lath. Syn. I. p. SI. n. 32. vers. Germ.

I. p. 46 . n. 32 .

Brandfalke, Bechst. Nat. D. 2. p. 26 I.

Svecis: Hönstjuf.

Mus. Payk: \&c.

\section{DESCRIPTIO.}

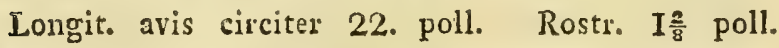
A flexura alx ad apicem I. ped.4. poll. Cauda 7. poll. Tibia 3. poll. Digit. antic. med. $2 \frac{3}{8}$; post. $I \frac{3}{8}$

Rostrum nigrum dente obtusissimo, vix notabili. Cera virescenti-flava. Iris lutea. Regio inter nares 
et oculos setis nigris, nares quoad partem tegentibus, vestita. Pedes longi, graciles, lutei, unguibus nigris.

Caput et collum supra rufescenti-1. testaceo-alba lineis longitudinalibus fuscis. Gula et superior juguli pars alba, rufescenti tincta. Ceterum corpus supra saturate fuscum, rufo tinctum; subtus fuscorufum. Remiges primores nigricantes latere interiore basi cinerex, secundariæ fuscæ apice pallido, latere interiore cinerex. Cauda supra fusco-rufa, subtus grisea.

A. variet. Antecedenti similis, sed humeri macula mag. na, ovali, rufescenti-alba.

Mus. Upsal.

B. Funior; F. rufus Auctor.

Magnitudine, forma et proportione partium primo descriptæ similis. Color partium nudarum idem. Caput supra :pallide ferrugineum striis fuscis. Gula albo ferruginescens, immaculata. Dorsum et tectrices alarum superiores saturate fusca pennis ferruginescenti marginatis. Superior pars pectoris pallide ferruginea maculis longitudinalibus fuscis. Abdomen, crissum et femora saturate ferruginea. Remiges primores nigræx; secundariæ cinerex. Cauda supra cinerea, lateribus ferruginea, subtus alba.

Mus. Payk

\section{HISTORIA AVIS.}

Amat loca uliginosa, flumina et paludes. Nidum in arboribus depressis vel in ipsa terra, locis paludosis ponit. Ranas, Lacertas. pisces et aves aquaticas pre. datur. Rarior. Migratoria.

\section{FALCO STRIGICEPS*), mihi}

DIAGN : Caput torque e pennis rigidis cinctum, Ro. strum breve. Tibiæ longæ, graciles. Cauda fasciata.

* Variat admodum, unde varia ei data sunt nomina, e quibus tamen nullum charąteristicum. Fo cyanus colorem maris 


\section{MAS.}

Supra canus; subtus albus.

\section{FEMINA.}

Supra fusca, subtus luteo rufa maculis fuscis.

\section{$S$ YNONYMA.}

Falco cynums, Lin. Syst. nat. I. p. 126 (mas.) Lath。 Ind. Orn. I. p. 39. 11. 94. Gmel. Syst. nat. I. p. 277. (mas).

Filco pygargus, Lin. Syst. I. p. I26. Gmel. Syst. I. p. 277.

Falco torquatus, Briss. Orn. I. p. 345.

Lnarius cinereus, Briss. Orn. I. p. 365. (mas.)

L' Oiseau St. Martin, Buff. Hist. Ois. I. p. 2İ2. vêts.

Germ. Martini II. p. I2. tab. 24. Pl. enl. 459. (mas).

In Soubuse, Buff. Ois. I. p. 215. tab. 9. P1. enl. 443. (fem.) vers. Germ. Martini II. p. I7. tab. 25. (fem).

Henharvies, Pen. Brit. Zool. I. p. I93. n. 58. Lath.

Syn. I. p. 88. Sup. p. 22. (mas) vers. Germ.

Bechst. I. p. 78. n. 74.

Ring-tnil, Peln. Br. Zool. I. p. I94. 11. 59. Lath. Syn. I. p. 89. - Suppl. p. 22. (fem.) vers. Germ. I. p. 79. n. 75 .

Accipiter macrourus, Nov. Com. Acad. Petrop. T. XV. p. 439. tab. VIII. et IX.

Falco macrourus, Gmel. Syst. I. p. 269. Lath. Ind. I. p. 29. n. 64 .

tantum indicat. $F$. pygargus femina appellatur; hoc tamen nomine $F$. albieilla, $a b$ antiquis ornithologis nominabarus. Cum vero ab omnibus mihi cognitis Falconibus facillime distinguitur pennis illis rigidis, erectis, qua more Striguru coptut cingunt, nomen Strigiceps sptissimum mihi visum est. 
F. hud'soniuls, Gmel. Syst. Nat. I. p. 277. Edw. aves I07. t. 107. (fem. var. f.).

Der blaue Habicht, Bechst. Nat. D. 2. B. p. 256 (mas). Die Halbrocyhe, Bechst. N. D. 2. p. 253. (fem.)

\section{DESCRIPTIO.}

A. mans senior:

Longit. avis I. ped. 5. poll. Rostrum I. poll. A flexura alx ad apicem $13 \frac{\mathrm{r}}{2}$ poll. Cauda 8 poll. Ti-

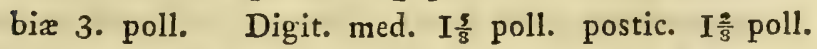

Rostrum breve, fere edentulum, nigrum, basi undique setulis nigris nares subtegentibus instructum. Cera viridi-lutea. Iris et pedes lutei. A gula circa aures versus nucham series pennarum brevium rigidarum; unde Caput Strigem parvam refert. Tibix lon$g \mathfrak{x}$, tenties. Ungues fusci.

Caput, Dorsum et Alæ supra coerulescenti-cana, pennis scapularum fuscescentibus. Occiput macula fuscescenti, albo immixto. Gula, jugulum et anterius pectus pallide coerulescenti-cinerea. Ceterum subtus tota alba, lineis rarioribus fuscis ad raches pennarum. (Sæpius tamen immaculata.) Remiges primores nigrx, basi albx, secundarix fusco-cinerex apice pallidx, latere interiore albx. Cauda cinereo-canescens fasciis fuscis. Rectricibus intermediis sæpe immaculatis. Tectrices superiores et inferiores alba.

B. femina:

Mare major, rostro pedibusque aliquantum robustioria bus.

Caput supra fuscum marginibus pennarum rufescentibras. Fascia per oculos fusca, supra et infra quam fascia albida. A sinu oris ad aures macula semilunaris fusca, sub qua, a jugulo ad nucham, collare e pen. nis rigidis, nigris, albo-marginatis. Dorsum fu- 
sco-fuliginosum. Subtus avis Inteo-rufa maculis longitudinalibus fuscis. Femora albida maculis et striis ad raches ferrugineis. Remiges primores supra fuscr, subtus albæ fasciis transversis nigris. Rectrices fasciis supra cinereis et fuscis, subtus albis et fuscis, fere pollicaribus.

C. Mas junior:

Caput supia fuscum marginibus pennarum ferrugineis, suprà et infra oculum fascia albida. Macula auris semilunaris fuscescens. Dorsum et Alæ supra cinerea, fusco tincta. Remiges primores nigrx. Cauda cinerea fasciis fuscis. Pectus cinereo album striis fuscis. Abdomen et crissum alba.

D. variet.

Supra fusca marginibus pennarum pallide rufescentibus. Fascia per oculos fusca, supra et infra quam, fascia alba. Macula auris rufo-fusca. Collare album punctis fuscis. Subtus avis alba, rachibus pennarum rufo-fuscis. Remiges et Rectrices ut antecedens.

Mus. Payk.

E. variet. Latera capitis, caput supra et dorsum fusca. Stria alba supra oculos et macula alba infra. Avis subtus testacea immaculata. Remiges fuscr fasciis saturatioribus, latere exteriore cinereo. Rectrices I. I. pallide testacex, basi albx, apice fascia fusca. 2. 3.4.5. fasciis latis fuscis, et testaceis; 6. 6. cinereo-fusc fasciis latis saturatioribus.

E Gottlandis. Mus. Holm.

F. variet. fem.

Caput suprą, orbita et macula auris nigra. Dorsum obscure fuliginosum pennis nigris insertis. Supercilia albida. Collare e pennis nigris albo marginatis. Iugulum sordide album maculis fuscis fere confluentibus. Pectus et abdomen alba maculis et striis minutis 
raris, fuscis. Remiges nigricantes tęricibas penultimis nitide cinereo-coeruleis apicibus et fascia per medium nigris. Reetrices rufo-cinerex, intermedix nitide cinerex, omites fasciis quatuor distantibus nigris, quarum ultima latior. F. Hudsonius Gmel.

Mus. Payk.

\section{HIS T O R I A.}

Rarior. Migratoria. Autumno abiens, vereque redux in Scania obvenit. Mures et aviculas prædatura supra agros et campos haud multum a terra elevata volitat.

\section{F A LCO MILVUS.}

DIAGN: Corpus ferrugineum maculis fuscis, capite albicante. Cauda forficata. Pedes breves, cum cera, lutei.

\section{SYNONYMA.}

Falco Milvus, Lin. Syst. Nat. I. p. I26 11, I2. Gmel. Syst. Nat. I. p. 26I. Lin. Faun. Sv. 11. 57. Retz. Faun. p. 66. n. II. Lath. Ind. Orn. p. 20. 11. 37. Wachtm. Wet. Ac. H. I8OI. p. 197. n. 8.

Milan royal, Briss. Orn. I. p. 414. t. 32. Buff Ois. I. p. 197. t. 7. vers. Germ. I. p. 267. tab. 2I. Pl. cnl. 422.

Kite, Pen. Brit. Zool. I. p. I85. n. 53. Ar\&t. Zool. Ir. p. 223. H Lath. Syn. I. p. 6 I. 11. 43. Suppl.

p. I7. vers. Germ. Bechst. I. p. 56. n. 43. Anh. p. 662 . n. 43.

Gabelweihe, Bechst. Nat. Deutsch. 2.' p. 243. Teuto sche Ornithol. H. V. tab. I. 2. mas et tem. Avis junior:

Falco ater, Ginel. Syst. I. p. 262. Lath, Ind, 1. p. 2I. n. 39. Retz. Faun, p. 6б. 11. I2. 
Milan noir, Briss. Onn. I. p. 413. Buff. Ois. I. p. 203. P1. enl. 472. vers. Germ. Martini I. p. 274. Black kite, Lath. Syn. I. p. 62. 1. 44. vers. Germ. I. p. 57. 11. 44. Anh. p. 663.

Svec. Glada.

\section{OBSERV.}

Falconte atro, Auctor. diligenter cum Milvo collato; non possum non ad partes cel. Le Vaillant accedere, qui utramque avem pro eadem specie habet. Pullos scil, e nido Milvi exceptas educavit, qui ad secundam usque deplumationem $F$. atrun aut tor. perfecte referebant. (vide Hist. nat. Ois. d'Afr. par $L e$ aillant, ed. min. T. I. p. 157 .

\section{DESCRIPTIO.}

A. senioris avis:

Longit. 2. ped. 2. poll. Rostr. I $\frac{6}{6}$. A flexura alæ ad apicem I. ped. $8 \frac{\pi}{2}$ poll. Cauda fere 13 poll. Ti. bix $2 \frac{5}{8}$ poll. Digit. med. $2 \frac{1}{8}$; Digit. post. $I \frac{\kappa}{8}$.

Rostrum nigricans, elongatum, superne compressum; maxillx superioris margo dente obtusissimo; apex unco magno instructus. Cera et Iris flava. Regio inter nares et oculos, mentum et orbitx, setulis nigris. Tibir breves, antice ad dimidiam partem pen. nis plumosis lutescenti-albis vestitx, dehine cum digitis, lutex. Ungues nigri, acutissimi.

Caput et colluin alba, fusco striata, ferrugineo immixto. Dorsum et alarum tectrices pennis saturate fuscis, marginibus late ferrugineis vel albicantibus. Iugulum, pectus et abdomen ferruginea striis longitudinalibus fuscis. Crissum pallide ferrugineum. Remiges primores nigrx, latere interiore a basi ad medium albx; intermedix $(6,7.8$.$) extus ferruginex, intus$ albæ fasciis fuscis; secundariæ nigricantes apice albicantes, latere interiore pallidiores, obscuro fasciata. 
Cauda ferruginea forficata. Reetrices intermedix cum alis compositis aqualiter extenduntur; laterales 2. poll. longiores sunt ef fuscr.

B. juniorís a ais:

Rostrum, pedes \&c. formâ ct proportione exą̧ præcedentis, quâ tamen, ut junior, aliquantum minor. Caput et collum albida striis longitudinalibus numero. sis fuscis. Dorsum et tectrices alarum saturate rufofusca rachibus pemurum nigris. Pectus, abdomen, femora et crissum saturate ruto-ferruginea striis longitudinalibus nigris. Remiges nigricantes. Cauda alis parum longior, aliquantum forficata. Reetrices fuscx fasciis obsoletis saturatioribus, apice albidx.

\section{HISTORIA MILVI.}

Migratoria avis, lati nuncia veris. Inter XX. et XXVtum diem Mens. Martis Milvos e meridie in Scaniam primos redeuntes, alteque per paria volantes, per plures annos observavi. In Scania, quam in ceteris Patrix provinciis frequentior avis. Volatus pulcherrimus. Immotis enim alis, caudre tantum flexibus cursum dirigens, jam sublimis cum conjuge ludens, coelo sereno, gyros per ära ducit; jam humilis supra campos et agros volitans, rapinam meditatur. Interdiu in arvis et pratis interdum sedit. Noetu in arboribus quiescit. Timida admodum et ignava, nullas nisi inermes pradas petit. In Mures, Lacertas, Ranas, aviumque pullos rostro impetum facit. Nidum in sum. mo arboris excelsa cacumine et ramulis construit. Ova 3. ponit. Iam medio Septembri provincies Svecire superiores relinquit. In Scania ad initiun Octabris obvenit. 


\section{FALCO BUTEO.}

Dragn: Cera pedesque seminudi lutei. Cauda brevis, ciscreo fuscoque transversim lineata. Rostrum dente obtuso. Lorz setulis adspersa.

\section{SYNONYMA.}

Falco Buteo, Lin. Syst. Nat. I. p. 127. Faun. Sv. F. 2 I. n. 60. Retz. Faun. p. 69. 11. I6. Gmel. I. p. 265. Lath. Ind. I. p. 23. n. 47. Wachtm. Wet. Ac. H. 180I. p. 199. 11. 9.

La Buse, Briss. Orn. I. p. 406. Buff. Ois. I. p. 206. tab. 8. Pl. enl. n. 4I9. vers. Germ. Martini II. p. 3. tab. 22.

Common Buzzard, Pen. Brit. Zool. I. p. I88. n. 54. tab. 25. Aret. Zool. I I. p. 224. J. Lath. Syn. I. p. 48. Suppl. P. I4. vers. Germ. Bechst. I. p. 44. n. 28. Anh. p. 659 .

Bussard, Bechst. Nat. Deutschl. 2. p. 238.

Varietates:

Falco versicolor, Gmel. Syst. I. p. 272. Lath. Ind. I. p. 33. n. 73.

Spottêd Falcon, Pen. Brit. Zool. I. p. 189. 11. 55 . tab. 26.

Falco nlibidus, Gmel. Syst. I. p. 267.

Der weissliche Bussard, Teutsche Ornith. H. XI.

Falco variegatus. Lath. Ind. Orn. p. 24. 11. 48. Speckled Buzzard, Lath. Syn. I. p. 97. vers. Germ. Svecis: Qvidfogel, Musväg. I. p. 88.

\section{DESCRIPTIO.}

Longit: avis I. ped. 8. poll. Alx extensæ 4. ped. A fexura alx ad apicem I. ped. 3 poll. Rostr. $I \frac{x}{2}$ poll.

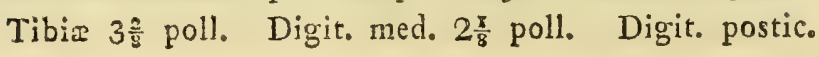


$I_{\frac{\pi}{2}} ;$ Cauda $8 \frac{r}{2}$ poll. - extra alas compositas extenclitur ad I. poll.

Rostrum nigricans, dente marginis obtusissimo. Cera et anguli oris flava. Iris pro ætate variat, fuscogrisea, rufa, et pallide grisea. Regio inter nares et oculos setulis nigris, qux quoad partem nares rotundato-triangulares tegunt, adspersa. Pedes lutei unguibus nigris. Tibix quoad partem antice teguntur pennis plumosis.

Caput et collum supra, dorsum et alarum tectrices fusca marginibus pennarum in aliis magis, in aliis minus ferrugineis et albidis. Subtus corpus album, luteo leviter tinctum maculis fuscis in pectore majoribus et fere confluentibus, in abdomine distinctis, cordatis. Gula striis fuscis. Crissum maculis raris coldato-fasciatis. Remiges primores nigricantes, latere interiore a basi albx; secundarix fuscx, apice albicantes, latere interiore albx, fusco fasciatx. Canda sub. xqualis. Rectrices obscuro-cinerex fasciis, angustis, numerosis (IO-I2) fuscis, apice et latere interiore fer. rugineo-albz. Femora fusca, marginibus pennarum ferrugineis.

\section{OBSERV.}

Variat ad modum pro vario sexu et varia atate totum Accipitrinum Genus, nec ulla Species quam hac magis. Causs vero adhuc incognitx. Neque cnim clima, sexus, ætas sole suns sationes. Non igitur mirum ornithologos, qui in Specieburs determinandis sihili fere nisicoloris rationem habuerint; his varietatibus noinenclnturam scudiose auxisse. Sunt vero pracipux varietates:

Var. A. Fuliginoso-fusca, slarum tectricibus dilutins marginatis. Crissum albo fuscoque transversim fasciatum. Abdomen undulis rarioribus albis. Femora fuliginoso-fusca immaculata. Cauda fusco cinerenque transversim lineasa. fascia latiore fusca pone apicen alticantem.

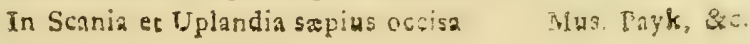


Var. B. Fuliginoso-fusca, gula et jugulo albis, fusco maculatis. Abdomen albo fuscoque transversim fasciatum. Crissum rufescenti album maculis raris transversis fuscis. Cauda ut in var. A. Mus. Payk.

Var. C. Caput et collum ad siernum usque, Dorsum et alarum tectrices fusca, pennis prasertim colli anterioris et alarum! tectricum ferruginescenti-albo marginatis. Macula major oblonga, albida, sub oculis. Pectus, abdomen, crissum alba immaculata, ferrugineo leviter tincta. Uropygium lutescenti-album, macula magna fusca in medio. Rectrices basi ct ad raches ferruginescenti albæ, lineis transversis, numerosis, cincreis et fuscis.

E Hallandia.

Mus. Payk:

$\boldsymbol{V}$ ar. $D$. Caput et collum alba maculis rarioribus fuscis. Dorsum et alarum tectrices fusca, pennis albo lata fimbriatis. rectus, abdomen et femora extus alba immaculata. Femora intus et crissum albo rufescentia. Reetrices medizalba fasciis transversis fuscis; ceter fusco et cinereo transversim fasciata: cet. ur anteced.

Obs. Huc pertinet The spotted Falcon, Pen. 1. c.

Var. E. Caput et tota avis subus alba, maculis fuscis in vertice raro sparsis. Ab angulis oris ad latera colli macula fusca in serie dispositz. Superior pars pectoris maculis fuscis fere confuentibus. Abdomen maculis nomnullis parvis fuscis. Tectrices alarum minores alba maculis fuscis. Cet. ut anteced.

E Nordiandia ine Mus. Payk.

Var. F. Caput et tota avis subtus staceo-alba maculis raris fuscis ad latere peftoris. Dorsun et alarum tefrices minores testacen alba macnlis fuscis. Remiges fuscz, omnes, preter primores tres apicibus alba. Refrices medietate interiore et apice alba, exteriore cinerex lineis transversis fuscis. Junior ost. E Scania.

Miss. Payk.

Var. G. Colore prina descriptæ, sed magnitudine minore. E Lapponia Mus. Payk.

In Mus. Paykuliano tota hæc series varietatum invenitur, quas unâ vidi et diligenter comparavi. In omnibus, prater colores, similes sunt. Rostri eadem forma et magritudo. Pedes eadem maguitudine, iisdem scutellis tegurtur. Ungues similcs. Proportio demum partiun eadem. 
Idem habitus; unde, me judice, eadem Specics. Vorietna tem $G$ in Hallandia nidificantem ipsi sclopeto occidimus. Nidus, pulli, conjux, vox, volatus \&c exacre Butconis.

\section{HIST ORIA.}

Segnis et ignava avis, hyeme instante abit, verno tempore redux. In sylvis, prata adjacentibus, frequens. Nidum planum ex ramulis aridis in arbore quadam struit. Ova 3. 1.4. Mammalia minora ut Erinaceos, Talpas, mures et aves ex. gr. Perdices, Coturnices \&c. prrsertim vero amphibia: Ranas, Natrices ot Lacertas prædatur. Tetraonis urogalli feminam etiam in nido olim inveni. Autumni tempore, prasertim in Scania frequens. Jam supra silvas tota familia gyros per aërem ducit, jam sæpibus insidit, jam in pratis sylvestribus, depressis, amphibia quxritura ambulat. Vel sedens vel volans sonum ejulantem Gaah! edit.

\section{FALCO APIVORUS.}

DiAGN: Rostrum plane edentulum. Lora pennis rekta. Ungues rectiusculi, Cauda cinereo fl. scoque fasciata.

\section{SYNONYMA.}

Falco Api vorus, Lin. Syst. Nat. I. p. 130. 11. 28. Lin。 Faụu. Svec. 23. 1. 65. Gmel. Syst. I. p. 267. Lath. Jnd. I. p. 25. n. 52. Wachtm. Wet. Ac. Hi. I80I. p. 201.

Ln Bondrée, Briss. Orn. I. p. 4IO. Buff. Ois. I. p. 208, Pl. enl. 420.

Honey - Buzzard, Pen. Brit. Zool. 1. p. 190. n. 56. Latho Syn. I. p. 52. vers. Germ. I. p. 47. Suppl. p. I4. Wespenfalke, Bechst. Nat. Deutschl. 2. p. 264. Wespen-Bussard, Teutsche Ornith. Borkh. \&r. H. XIV 


\section{DESCRIPTIO.}

Longit. avis I. ped. IO poll. Alæ extensæ 3. ped. 9 poll. Rostrum $I \frac{x}{2}$ poll. A flexura alx ad apicem I. ped, $2 \frac{x}{2}$ poll. Tibix $2 \frac{x}{8}$ poll. Digitus med. $2 \frac{3}{8}$ poll. Digit. postic. I $\frac{x}{2}$; Cauda 9 poll.

Rostrum nigrum, plane edentulum, modice curva. tum, supra compressum, fere carinatum. Cera flava. Iris lutea. Nares oblongx, obliqux nudx. Tibix antice pennis fere semitect: dehinc lutex, unguibus rectiusculis nigricantibus. Regio inter nares et oculos pennis (non setulis) tecta, unde tumida apparet.

A. Mas:

Caput supra et cervix fusco alboque varia. Latera capitis cinerea maculis minutis albis. Dorsum et alæ supra saturate fusca. Remiges primores nigricantes; secundarix fuscx latere interiore a basi albido maculatx. Subtus tota avis alba maculis distantibus rotundato-cordatis, fuscis. Gula striis fuscis. Crissum maculis transversis fuscis. Cauda supra cinerea fusco nebulosa, fasciis quatuor distantibus, pollicaribus (extima tamen latiore) saturate fuscis, apice albo; subtus cinereo - alba obsolete fásciata.

\section{B. Femina :}

Mus. Payk.

Latera capitis cinerea albo immixto. Caput supra, Dorsum et tectrices alarum fuliginoso-fusca, marginibus pennarum dilutioribus. Subtus cinereo-fusca rachibus fuscis, in abdomine et crisso maculis transversis albis, distantibus. cet. ut anteced.

Mus; Payk.

C. Funior:

Supra nigro-fusca, apitis et colli singula pema apice maculâ albâ. Remiges nigrícantes apice macula alba. Subtus albida; gula strits, pectore et abdomine 
maculis latis nigro-fuscis. Rectrices nigro-fuscr, cinereo et albo transversim lineatx, fascia lata nigricante pone apicem album. Femora et crissum lutescentialba, maculis transversis fuscis.

Mense Augusti in Smolandia occisa.

D. varict:

Caput et collum supra ferruginea punctis albis et nigris adspersa. Frons albida. Macula per oculos fu. sca. Dorsum et alarum teetrices nigro - fusca marginibus ferrugineis. Avis tota subtus ferruginea lineis ad raches fuscis. Cauda nigricans lineis transversis cinereo-rufis, pone apicem album fascia lata nigra.

Mus. Payk. An, fem. junior?

OBSERV.

Variat admodum, sed hoe uno charątere ab omnibus, quos ega saltem vidi, Falconibus, primo in aitu distinguitur, quod regio inter rostrum et oculos pennis, non setulis dense et tumide sit vestita

\section{HISTORIA.}

Rarior. Migratoria. Multa et quoad habitum et quoad mores cum Buteone habet communia. Imbellia tantum Animalcala prædatur. Mammaliis parvis, amphibiis et Insectis vescitur. In arboribus exselsis nidulatus. Ova ponit $3-4$.

\section{FALCO PALUMBARIUS.}

DiAgn: Supra fuscus, subtus albidus undulis vel maculis fuscis. Cauda longa, rotundara, fasciis latis (5.) fuseis et cinereis.

a. senzior:

Tota avis subtus alba, undulis fuscis numerosissimis pulcherrime ornata. F. palumbarins, Lin. Nilsson Ornith. 


\section{B. junior :}

Subtus rufescenti alba, maculis longitudinalibus fuscis. F. Gallinarius, Gmel, F. Gentilis, Iin. Faun.

\section{SYNONYMA.}

Q. Senior:

Falco palumbarius, Lin. Faun. Sv. 23. 11. 67. Syst, Nat. I. p. I30. 1. 30. Lath. Ind. On. I. p. 29. Retz. Faun. Sv. 74. n. 22. Gmel. Syst. Nat. I. p. 269. Wachtm. Wet. Ac. H. 1802. p. 249.

Astur, Briss. Orn. I. p. 314.

L'Astour, Buff. Ois. I. p. 230. tab. I2. Pl. enl. n. 418. vers. Germ. Martini II. p. 46. tab. 32.

Gooshnwk, Pen. Brit. Zool. I. p. I84. tab. 24. Lath. Syn. I. p. 58. Suppl. p. I6. vers. Germ. I. p. 53. 11. 39.

Der Stockfalke, Bechst. Nat. Deutschl. 2. p. 268. Teutsche Ornith. H. VI. tab. I. 2. mas et fern.

B. junior:

Falco gentilis, Lin. Faun. Sv. p. 20. n. 58. Retz. Faun. 66. n. I3. Gmel. Syst. I. p. 270.

Genzil Falcon, Pen. Brit. Zool. I. p. 18I. n. 50. tab. XXI - XXII.

Falco Gallianrius, Ginel.Syst. 1. p. 266. Wachtm. Wet. Ac. H. I802. p. 25 I.

Falco Buteo, var. $\gamma$. Lath. Ind. Orn. I. p. 24.

Falco marginatus, Lath. Ind. Orn. I. p. 26. n. 55. (Descriptio optima).

Autour sors, Buff. Pl. enl. $46 \mathrm{I}$.

Hiihnerfalke, Bechst. N. D.2. p. 263.

Svecis: Slaghök. 


\section{DESCRIPTIO.}

Longit. avis circa 2. ped. Rostr. I $\frac{3}{3}$ poll. A flexura ala ad apicem I. ped, 3. poll. Cauda $10 \frac{\mathrm{T}}{2}$ poll. extrá alas ad 5. poll. et magis extenditur. Tibix $3 \frac{x}{2}$ poll. Digitus med. $2 \frac{5}{8}$ poll. Digit, postic $2 \frac{3}{8}$ poll. unguis postic, $I \frac{3}{8}$ poll. anter, med. I $\frac{\frac{2}{8}}{8}$ poll.

Rostrum nigrum, basi coerulescens; margo maxillx superioris dente obtusissimo. Cera virescenti lutea. Nares sinnoso-rotundatx. Iris flava. Regio inter oculos et nares setulis nigris adspersa. Anguli eris et palpebrarun margo, flavi. Tibix, antice fere ad medium tectx pennis mollioribus, dehinc cum digitis, lutex. Ungues nigro-fusci, validi.

Caput supra saturate fuscum, fascia alba, striis par-vulis fuscis adspersa, a fronte supra oculos ad nucham. Cervix, dorsum et alæ supra fusca. Tota avis subtus alba; in gula lineis longitudinalibus; in jugulo, pectore, abdomine, femoribus et alis subtus lineis transversis, seu undulis numerosissimis fuscis pulcherrime ornata. Remiges fuscx, saturatius fasciatx, latere interiore a basi maculis albis. Cauda longa, rotundata. Rectrices cinerex, fasciis 5.1.6. fuscis, latis, (fere pollicaribus) extima latiore; apice albo.

\section{Avis junior:}

var. a. Supra saturate fusca, marginibus pennarum ferruginescentibus. Supercilia, latera capitis et colli ferrugineo-albida striis longitudinalibus fuscis. Subtus alba, rufo leviter tincta, maculis longitudinalibus fuscis, postice obtusis; in femoribus et crisso angustioribus; in hypochondriis latioribus fere cordatis. Remiges et Rectrices ut in anteced. sed fasciis caudx fusçis saturatioribus linea albida marginatis. ynr. $\beta$. Prracedenti similis, at subtus testacea maculis rufo-fusçis. Mus. Payk. 
car. \%. Similis varietati a, sed pennæ Dorsi, teetricesque alarum albo marginatx.

Mus. Payk. yar. $\delta$. Alba, maculis longitudinalibus rufescentibus in capite, collo et alarum tectricibus minoribus; lineis longitudinalibus rufescentibus in peetore, abdomine et femoribus. Præterea tota alba.

E Norrlandia. Mus. Payk.

\section{HIST ORIA.}

In nemorosis Svecix haud rara. Hiemes nostras edurat. Plures vero, præsertim juniores, hieme quam xstate, Scanix campos visitant. Audax avis et sanguinem sitiens, Tetraones, Perdices, Columbas, Anates et juniores Lepores prædatur. Sub ipsis fenestris ædium Gallinis cædem parat. In sylvis densis nidum ponit in arbore excelsa. Cum Corace supra sylvas in aère pugnantem, illumque fugantem vidi. Modus aggrediendi singularis. Volvens enim sese in aëre, pedibus sursum protractis, in pectus et abdomen hostis sui impetum faciebat.

Pullus, medio mens. Julii, juxta nidum occisus, hoc gaudebat colore: Anguli oris et cera viridi lutea. Iris albida. Corpus supra obscure-fuscum pennis ferxugineo marginatis; subtus albide-rufescens maculis ovalibus et cordatis fuscis. Canda ut in ceteris.

\section{FALCO NISUS.}

Diagr: Supra coeruleo-cinereus; subtus albus, transversim undulatus. Cauda longa aqualis coe. zuleo - cinerea fasciis (5) nigricantibus apice albo. Tibix tenues, longæ. Digitus medius pralongus. 


\section{SYNONYMA.}

Falco nisus, Lin. Faun Sv. 23. 11. 68. (fem.) Syst. nat. I. p. 130. Gmel. Syst. Nat. I. p. 280. Lath. Ind. I. p. 44. n. 107. Wachtm. Wet. Acad. H. I802. p. 272 .

Sparrow Hawk, Pen. Brit. Zool. I. p. 198. n. 62. Lath. Syn. I. p. 99. 11. 85. Vers. Germ. I. p. 89. (junior fem.)

L'Epervier, Briss. Orn. I. p. 3 IO. Buff. hist. Ois. I. p. 225. tab. II. Germ. vers. Martini II. p. 35. tab, 3I. Pl. enl. 4I2. 467.

Der Sperber, Bechst. Nat. Deutsch, 2. p. 320.

Svecis: Sparfhök.

\section{DESCRIPTIO.}

Longit. avis circa 12 poll. Rostr. $\frac{8}{8}$ poll. A flexur' alæ ad apicem 8 poll. Cauda $5 \frac{x}{2}$ poll. Extra alas cau. da extenditur 2 poll. Tibia $2 \frac{2}{8}$ poll. Digit. med. $I \frac{T}{z^{2}}$ poll. Digit. postic. I. poll.

Rostrum coerulescens apice nigro. Margo maxillx superioris dente obtusissimo. Cera viridi-lutea. Iris flava. Pedes longi, tenues, flavi. Digitus medius prælongus, gracilis, sub articulis nodulis verrucosis, Ungues nigri. Cauda rqualis, longa.

Caput supra, dorsum et alarum tectrices coeruleocinerea. Occiput albo máculatum. Supercilia alba. Gula et jugulum alba lineis longitudinalibuis ferrugineis. Latera juguli in ferrugineum tendentia. Pectus et abdomen alba undulis ferrugineis in peetore crebris, in abdomine rarioribus. Crissum album. Remiges primores cinereo-fuscre, saturatius fásciatre; intus maculis albis; sccundarice colore dorsi, intus albo maculatæ. Rectrices fusco-cinerex fasciis 4 1. 5. nigrricantibus, apice albido. 
Femina:

Mare major. Longit. 14. 1. I5. poll. Supra fusco cinerea subtus albo undulis fuscis.

Avis junior:

Supra saturate fusca, capite, collo et alarum tectricibus ferruginco marginatis. Frons et supercilia albida striis fuscis. Subtus albida ferrugineo tincta; jugulo striis, pectore maculis cordatis, abdomine et hy. pochondriis fasciis transversis fuscis vel saturate ferrugineis, fusco marginatis. Femora et crissum albida, ferrugineo-tincta. Cetera ut anteced.

\section{HISTOR I A.}

In sylvis et sylvarum vicinitate frequens. Rapax, audax, gulæ satisfaciendi semper cupida. Columbis, Turdis, Alaudis, ceterısque aviculis infesta. Muribus, Lacertis et Insectis quoque vescitur. In arboribus excelsis nidulatur. Ova $3 \cdot 5$. sordide albida maculis ferrugineis.

B.

Maxilla superioì, pone apicent, dente exscrto, acuto. Farcones, proprie.

\section{FALCO GYRFALCO.}

DIAGN: Rostrum breve, validum (quasi tumidum) dente acutissimo. Cera et pedes robusti, coeruJei. Cauda alis longior.

\section{MAS.}

Albus, supra transversim fusco maculatus.

\section{FEMINA ET JUNIOR.}

Sup ra fusca, subtus albida maculis fuscis. 


\section{SYNONYMA.}

Falco Islandicus, Lath. Ind. On. I. p. 32. Wachtm: Wet. Ac. H. I80I. p. I93. 11. 6. Fabric. Faun. Groen. p. 58. n. 35. vâ $\beta$. Brün. Orn. bor. p. 2. ii. $7-8$.

Falco candicans, Gmel. Syst. Nat. I. p. 275.

Le Gerfrult, Briss. Onn. I. p. 380. tab. 30. fig. 2.

Le Gerfaul blanc, Buff, Ois, I. p. 24I. P1. enl. 446.

Gyvfalcon, Pen. Brit. Zool. I. p. I77. tab. 19.

Fceland Falcon, Lath. Syn. I. p. 70. 11. 50. vers. Germ. I. p. 63. n. 50 .

Geyerfalke, Bechst. Nat. D. 2. p. 308.

Yunior:

Falco rusticolus, Lin. Faun. Sv. p. 19. n. 56. Fabric, Faun. Groenl. p. 55. 11. 34. (obs. Collared Falcon, Pen. Arct. Zool. II. p. 222. G. est mea varietas B.)

F. Gyrfalco, Lin. Faun. Sv. p. 22. n. 64. Gmel. Syst. I. p. 275. Wachtm. 1. c. p. I95. Lath. Ind. I. p. 32. n. 68. Retz. Faun. Sv. p. 73. n. 20.

Falco Islandus, Brïn. Orn. bor. p. 2. 11. 9.

Gerfault d'Island, Buff. pl, enl. 210. Briss. Onn. I. p. 733. tab. 31 .

Adhue junior:

Fnlco fuscus, Fabric. Faun. Groenl..p. 56.

Falco Umbrinus, Billb. Wett. Acad. H. 1809. p. 202. tab. VI. (Specimen in Museo Holm. asservatum, vidimus.)

Gerfault de Norvege, Buff. P1. en1. 462.

Svecis : Blöfot.

In Museo Payk. series varictatum asservatur. 


\section{DESCRIPTIO.}

Mas senion:

Longit. avis circa I ped. IO poll. Rostr. I $\frac{x}{2}$ poll. A flexura alx ad apicem I. ped. 3. poll. Cauda $8 \frac{1}{2}$ poll. extra alas extenditur 2 poll. Tibir $2 \frac{5}{8}$ poll. Digit. med. $2 \frac{6}{8}$ poll. Digit, post. $1 \frac{6}{8}$ poll.

Rostrum valietum, crassum, breve, coerulescens; maxilla superior supra tumide convexa, a basi usque curvata, apice nigricante, pone apicem dente acutissimo; maxilla inferior apice truncata, pone apicem incisurâ, denti maxillæ superioris respondente. Cera coerulea. Iris - - Regio inter nares et oculos palpebraque inferior setulis nigris adspersis. Tibix antice pennis plumosis semitectx, dehinc coerulex, scutellis parvis rotundatis, undique teetx. Ungues fusci.

Caput et collum supra alba, lineis ad raches penna. rum fuscis. Dorsum et alarum tectrices alba, maculis vel fasciis transversis (interdum subcordatis) fuscis. Avis tota subtus alba, sæpius inmaculata. Remiges albx, tænia ad rachin dentata, fusca, versus apicern fuscx, apice ipso albæ. Cauda aliquantum rotundata. Rectrices albr, maculis vel fasciis angustis, transversis, fuscis. Femora alba, extus maculis ntinutis, raris, fuscis.

Femina:

Mare major, circa 2 ped. longa. A flesurą alæ ad apicem I6 poll. Cauda IO poll; extra alas 3. poll. extenditur.

Supra fuscamarginibus pennarum pallidioribus; superciliis et maculis nachæ albidis. In alarum tectricibus et in dorso postico macula rariores, parva, rotundatx, albidx. Subtus albida maculis fuscis in gula, jugulo et crisso rarioribus, longitudinalibus; in pectove et abdomine numerosis, ovalibus et subcordatis. 
femora pennis longis fuscis, albo maculatis teguntur. Rerniges primores fuscer ad apicem saturatiores, latere interiore maculis transversis (I4-I2) luteo-albis; secundirice fusca apice palidiores intus mnculis obsoletis albialis. Rectrices fuscr, lineis luteo-albis, transversis, rachi interruptis (circiter 12.)

O BSER V.

Femin etiam, pro ztate variant. Junior forte est, quam descripsi.

Avis primo ctatis anno, feminx descriptx similis, cera et pedibus aliquando luteis.

\section{O BSER V.}

Inter has duas, qua tanquam extremitates seriei consideran. $d x$, multæ sunt varietates et $a b$ una in alteram transitus. 'In quibus ut in omnibus fere congencribus observandum, quod, quo junior avis eo obscurior, quo senior eo albidior.

variet. a Caput et collum supra fusca marginibus pennarum sordidela 2 bis; ab angulis oris macula fusca interrupta de. seendit. Dorsum et Remiges intimx cinereo-fusca, lineis eransversis ot maculis parvis albicantibus, Teqriees alarum fusca maculis parvis raris, albescentibus. Avis subtus albicans maculis in pectore et abdomine longitudinalibus, postice dilatatis; in femoribus et crisso transversis. Gula immaculata. Reetrices cinereo-fusca lineis transversis albidis, apice albz.

var. $\beta$. Caput et collum snpra fusca marginibus pennarum albis. Dorsum et ale supra cinereo-fusca albo transversim fasciata (undulata). Avis subtus alba striis in jugulo fuscis; latera pectoris et abdominis maculis rotundatis, subcordatis vel transversis fuscis. Femora et crissum alba far sciis transversis foscis. Rectrices cinere fasciis angustis fiiscis:

war. $\gamma$. Caput et collum alba striis longitudinalibus fuscis. Dorsum et tectrices alarum fusca marginibus et imaculis 
transversis albis. Subrus alba testacen tincta. Gula, jugulum et pe@tus striis rarioribus. Abdomen: maculis parvis subcordatis vel rotundatis; femoract crissum maculis trans. versis, fuscis. Remiges primores nigro-fuscx extus albido transversin maculate apicibus albis. Cauda supra alba parum cinerascens fasciis numerosis angustis fuscis.

\section{HISTORIA.}

Rigidissimas Septentrionis terras, Islandiam, Groenlandiam, Norvegiam et Lapponiam precipue incolit. Raro in australcs Svecix plagas descendit. Occisus tamen in Ostrogothia, in Gottlandia, et ab Ill. comite Wachtmeister in Scania, juxta litus ad Aohus, mens. Novernbl.

Princeps inter Falcones venatui aptos, maximique a Falconariis xstimatus. Pulchritudine formx, audaciâ animi et celeritate volatus insignis. Nemo tamen nostratum jam institutionem falconum curat.

\section{FALCO PEREGRINUS.}

DIAGN: Supra coeruleo-cinereus, nigricante transversim maculatus; subtus albus abdomine et femoribus nigricante undulatis. Macula fusca sub oculis descendens. Cauda brevis. Digiti prælongi.

\section{SYNONYMA.}

Falco peregrinus, Briss. Orn. I. p. 34I. (mas senior) Fulco gentilis, Briss. Orn. I. p. 33\%. (junior)

Falco peregrinus, Gmel. Syst. I. p. 272. Lath. Ind. Orn. I. p. 33. 11. 72. (mas senior.); Wachtm. Wet. Ac. H. 1802 . p. 256.

Falco, Briss. Orn. I. p. 32 I. 
Faucon noir et passager, Buff. Pl. enl. 469.

Frucon sors, Pl. enl. 470 .

Falco comnunis, Lath. Ind. Om. I. p. 30. n. 67. (junk. or) Gmel. Syst. I. p. 270.

F. communis, ater, Gmel. 1. c.

Common Falcon, Lath. Syn. I. p. 65. vers. Germ. I. p. 59. Peregrine Falcon, Pen. Brit. Zool. I. p. I78. n. 48, tab. 20. Lath. Syn. I. p. 73 Suppl. p. 18 vers. Germ. I. p. $65,11.52$.

Der Wanaerfalke, Bechst. Nat. D. 2. p. 300.

\section{DESCRIPTIO.}

(Mis:)

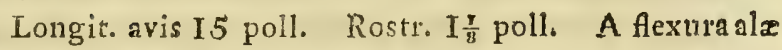

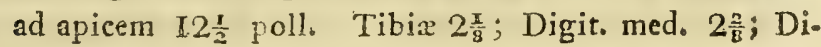
git. post. $I_{\frac{\pi}{2}}$. Cauda $5 \frac{1}{2}$ poll.

Rostrum cocrulescens, basi pallidius, dente prope apicem acuto. Iris fusca. Cera et orbitæ nudæe, lutex. Pedes lutei, digitis prælongis, sub articulis verrucosis. Nares rotundatr puncto centri flavo.

Caput, collum supra et macula sub oculo longitudinalis fusco-nigricantia. Dorsum et alarum tectrices cinereo-coerulescentia, fusco transversim lineata, rachibus pennarum nigris. Subtus tota alba, pectore inferiore, abdomine, femoribus et crisso transversim nigricante undulatis. Teetrices inferiores alarum albo maculis fuscis. Remiges primores nigre, latere interiore maculis oblongis transversis (circiter 10) slbidis. Cauda alis complicatis vix longior, saturate cinerea, nigricante transversim lineata (lineis $8-10$ ) versus apicem saturatior apice albo.

\section{Femina:}

Mare major; 17. poll. longa. Supra saturate cine. rea maculis transver is fuscis. Subtus alba macula sub 
oculo fusca, pectore, abdomine et femoribus nigro maculatis.

Funior:

Supra saturace fusca marginibus pennarum ferruginescentibus. Macula nigra sub oculo descendens. Subtus testaceo - albida gulâ immaculata, pectore et abdo. mine maculis longitudinalibus fuscis, fere confluentibus. Crissum pallide testaceum lineis transversis fuscis. Cauda fasciis angustis fuscis et cinereis apice te. staceo.

varietas:

Supra cinereo-fusca marginibus pennarum obsolete ferruginescentibus. Macula sub oculis nigro-fusca. Subtus testaceo-alba, pectore, abdomine, femoribus et crisso fusco-undulatis. (Obs. Est mas biennis vel triennis, ni fallor.)

\section{HIST ORIA.}

Rario:. In rupibus nidulari refertur. Pro venatí proxime ad Gyrfalconem, accedit.

\section{(?) $1 \%$ FALCO LANARIUS.}

Dragn: Supra fuscus superciliis albidis; subtus albidus maculis longitudinalibus nigro - fuscis. Cauda fusca, maculis transversis vel rotundatis albidis. Tectrices alarum inferiores et hypo. chondria nigro - fusca maculis rotundatis albis。

\section{SYNONYMA.}

Falco Lanarius, Lin. Faun. Sv. p. 22. n. 62. Syst. nat. I. p. I29. 11. 24. Gmel. Syst. I. p. 276. Lath. Ind. On. I. p. 38. 11. 92.

Lntarizs, Briss. Orn. I. p. 363 . 
Le Lanier, Buff. Ois I. p. 243.

Lanner, Pen. Brit. Zool. I. p. I82. tab. 23 ? - Lnth. Syn. I. p. 86. 11. 72.

Die Lanette, Bechst. N. D. 2. p. 296.

Mus. Payk.

\section{DESCRIPTIO.}

Longit. 17. poll. A flexura alix ad apicem 13. poll. Tibix $2 \frac{3}{8}$ poll. Digit med. 2 poll. Digit. po. stic. I $\frac{T}{2}$; Rostr. T. poll. Cauda 7. poll.

Rostrum brevel, crassum, tumide convexum coernle. um apice nigricans, dente acuto pone apicem. Nares rotundatæ puncto centri. Tibix antice, quoad partem plumis albis tectx, scutellis rotundatis ubique instructr. Ungues fusci.

Caput supra saturate fuscum marginibus pennarum hinc inde rufescentibus. Frons, fascis supra oculos et nucha albida striis et lineis longitudinalibus fuscis. Gula alba immaculata. A sinu oris ad latera gula de. scendit macula oblonga nigricans. Dorsum et alie su. pra cinereo - fusca, marginibus pennarum obsolete rufe. scentibus. Pennx scapulares postice una vel altera ma. cula rotundata, sordide alba. Pectus et abdomen albida maculis longitudinalibus nigro-fuscis. Crissum sordide album rachibus fuscis. Remiges primores nigro-fuscx, prima margine exteriori-tenui albido, ceterr latere exteriore immaculatx, interiore maculis transversis ovalibus (I2.-8.) flavescenti-albis; secundaria fusca margine et apice pallidx, latere interiore ma. culis transversis albidis. Cauda alis longion cinereo. fusca apice late alba. Rectrices. latere interiore maculis (9-I0) transversis, exteriore juxta apicem maculis roiundatis ferruginescenti-albis. Remiges et Rectrices subtus cinerea, fasciis transversia numerosis albis. 
Alarum tectrices inferiores antice (sub fiexurâ alæx) albæ maculis fuscis; postice et hypochondria nigro-fusca anaculis rotundatis albis, magnitudine pisi majoris.

OBSERV.

Hunc esse Lanarim Au Ror. cuique Descriptiones conferen. ti perspicuum erit. Alia est quastio an re ve:a sit Species a 25. peregrino distincta, quod obstinate urgere nee possum nec volo. Multa enim haber cum $\mathbb{F}$. peregrino, jnriore, communia, sed color caudx, hypochondriorum ec testricum alarnm inferiorum diversus, et, quantum ex Specimine farcto videri licer, gracilior est, caudą̧ue longius extensa. Judicent præterea peritiores.

\section{LOCUS:}

Specimen e Scania, in Museo Paykullino assarvatur.

\section{FALCO SUBBUTCO.}

Dragn: Supra coeruleo-fuscus capire nigricante, - subtus albidus maculis longitudinalibus nigris. - Lunula sub oculis nigra. Femora et crissum fer. ruginea. Cauda brevis.

\section{SYNONYMA.}

Frlco Sulbuteo, Lin. Faun. Sv. p. 20. n. 59. Syst. nat. I. p. 127. Gmel. Syst. Nat. I. p. 283. Lath. Ind. Orn. I. p. 47. Retz. Faun. Sv. p. 68. Wachtm. Wet. Ac. H. I802. p. 263.

Dendrofalco, Briss. Orn. I. p. 375.

Hobreau, Buff. Ois. I. p. 277. tab. I7. Pl. "enl. 432. 431 .

Hobby, Pen. Brit. Zool. I. p. I9\%. n. 6I. Lath. Syn. I.

p. I03. n. 90. - Supl. p. 28.

Bammfalke, Bechst. Nat. Deutsch!. 2. p. $30 \%$

:Susçis: Lärkfalk. 


\section{DESCRIPTIO.}

Longit. avis circa I3 $\frac{\pi}{2}$ poll. Rostr. $\frac{6}{3}$ poll. A Alex-

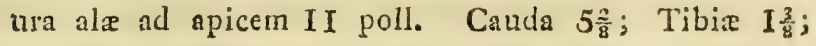

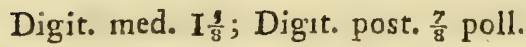

Rostrum coerulescens breve, maxilla superior pone apicem dente acuto, cui incisura maxillæ inferioris respondet. Cera et orbite nudre, lutex. Iris fusca. Pedes pallide favi, unguibus nigris. Canda xqualis, cum alis compositis æqualiter fere extenditur.

Caput et collum supra nigro-fusca cinereo et rufo tineta. Nucha maculis binis albo-rufescentibus. Dorsum et alarum teetrices saturate coeruleo-fusca rachibus nigris. Sub utroque oculo macula nigra decurrit. Gula, jugulum et, quoad partem, latera colli, alba. Pectus et abdomen albida maculis longitudinalibus fuscis. Crissum et femora ferruginea. Remiges primores nigra, latere interiore maculis (circa 8.) trans. versim ovalibus rufo-albis, secundarix fusco cinerex apicis margine pallido, latere interiore, ut priores, maculatx. Rectrices cinerea fuscx, latere interiore fasciis $-8 \mathrm{IO}$, rufo-albis. Par intermedium fusco cines 'reum, unicolor, acutius.

Avis junior:

Supra saturate fusca marginibus pennarum ferrugi, nescentibus; subtus testaceo-alba maculis longitudinalibus fuscis. Cet. ut anteced.

\section{HISTORIA.}

In sylvis hand rara. In arboribus nidulatur. Admiranda celeritate volans, aviculas, præsertim Alaudas pradatur. Migratoria, hyeme instante sbit, vere redit. 


\section{FALCO LITHOFALCO.}

Diagn: supra coeruleo-cinereus rachibus nigris; subtus albidus maculis longirudinalibus fuscis. Cauda longa, fascia nigra pone apicem album.

\section{SYNONYMA.}

Falco Lithofalco, Gmel. Syst. Nat. I. p. 278 (mas) Lath. Ind. On. I. p. 47. (mas.) Wachtm. Wet. Ac. H. 1802. p. 269.

Falco asalon, Gmel. Syst. I. p. 284. Lath. Ind. Om. I. p. 49. (fem. et junior avis.)

Litho -falco, Briss. Orn. I. p. 349. (mas.)

Le Rochier, Buff. Ois. I. p. 286. P1. enl. 447. (mas.) Emerillon, Buff. Ois. 1. p. 288. P1. enl. 468. (fem.) Merlin, Pen. Brit. Zool. I. p. 200. 17. 63. (fem.) Lath. Syn. I. p. 106. n. 93. Suppl. p. 29.

Der Merlin, Bechst. Nat. D. 2. p. 328. (fem.) Mus. Lund. Payk. \&c.

\section{DESCRIPTIO.}

Mas:

Longitudo circa $I_{2}^{\frac{\gamma}{2}}$ poll. Caput et dorsum supra coeruleo-cineren rachibus nigris. Latera capitis et cervix ferrugineo alba striis et maculis fuscis. Gula sordide alba immaculata. Pectus et abdomen albicanria maculis longitudinalibus fuscis. Femora et crissum ferruginescentia striis nigris. Remiges nigricantes latere interiore maculis albis. Cauda extra alas $I \frac{r}{2}$ poll. extenditur, supra cinereo-coerulea lineis 4 vel 5. obsoletis, transversis, fuscis, fascia lata nigra pone apicem album.

Femina in Scania, Mense Aprili, occisa.

Longit. I2 $\frac{x}{2}$ poll. Alx extens 2 ped. $2 \frac{6}{8}$ pall. A flexura alxad apicem $8 \frac{x}{2}$ poll. Rostr. $\frac{5}{8}$; Tibiz I $\frac{5}{2}$ poll. 
Digit. med, I $\frac{\mathrm{r}}{2}$ poll. Cauda 5 poll.; extra alas extensa, fere 2 poll.

Rostrum coerulescens, basi pallidius. Cera et anguli oris lutea. Iris fusca. Nares rotundatr. Pedes pulchre flavi, digitis, sub articulis, nodis verrucosis. Ungues nigri.

Caput et collum supra fusca ferrugineo immixto, striis longitudinalibus nigris. Latera capitis, Supercilia et cervix inferior ferrugineo - alba maculis et striis fuscis. Dorsum et alarum tectrices saturate coeruleocinerea rachibus nigris, apicibus et maculis transversis obsoletis ferrugineis. Subtus avis alba, flavo tineta; Gula immaculata; pectore et abdomine maculis longitudinalibns, ad latera subcordatis rufo-fuscis rachibus nigris. Crissum et femora albida striis raris fu. scis. Remiges nigro-fusca, maculis numerosis, transversis, ferrugineo albis. Cauda saturate fusca fasciis 7. angustis albo-lutescentibus; fascia lata nigricans pone apicem album.

\section{HIS TORIA.}

Raxa. Migratoria. In sylvis nidulatur, in arboribus excelsis. Ova 5-6. ponit. Aviculas pradatur.

\section{FALCO TINNUNCULUS.}

Diagn: Corpus supra rufum maculis nigris. Cauda longa rotundata fascia lata nigra pone apicem al. bum.

\section{SYNONYMA.}

Falco Tinnunculus, Lin. Faun. Sv. p. 2I. Ir. 6I. Retz. Faun, Sv. p. 7 I. 11. I8. Lin, Syst. Nat, I. p. I27. Gmel. Syyst. I. p. 278. Lath. Ind. Orn. I. p. 4I. Wachtm. Wet. Ac. H. I802. p. 265.

Nilsson Ornith. 
Tisnusuculus, Briss. Orm. I. p. 399. (mas.)

Accipitev alaudarius, Briss. Orn. I. p. 379. (fem.)

La cresserelle, Buff. Ois. 1.p. 280. t. I8. P1. enl. 401 - 47 I.

Kestrel, Pen. Brit. Zool. I. n. 60. Lath, Syn. I. p 94.

79. Supl. p. 25.

Tharmfalke, Bechst. Nat. D. 2. p. 3 II.

Avis primo atatis anno:

Falco fasciatus, Retr. Faun. Sv. p. 70. 11. 17.

Falco brunneus, Bechst. Nat. D. 3. p. 748. Item An. hang zu Lath. p. 679. tab. II.

Svecis: Tornfalk.

\section{DESCRIPTIO.}

(Mns)

Longit. I4. poll. Rostr. $\frac{\delta}{8}$ poll. A flexura alr ad

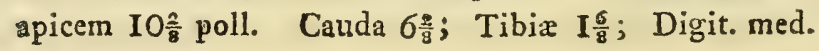
I $\frac{\mathbf{T}}{8}$ poll. postic. $\frac{7}{8}$.

Rostrum coerulescens basi flavicans. Maxilla supe. rior pone apicem dente acutissimo. Cera, orbitæ nudæ et pedes lutei. Nares rotundatæ, in medio puncto flavo. Iris fusca.

Caput et collum supra cinerea rufo tincta, lineis lon. gitudinalibus fuscis. Dorsum et alarum tectrices rufa maculâ transversali nigra prope apicem singulæ pennæ. Uropygium cinereum. Cauda cinerea, pone apicem album fascia lata nigra, Subtus avis pallidius rufa. A sinu oris ad latera juguli stria nigricans decurrit. Gula et crissum immaculata. Pectus et abdomen macu. lis parvis, ovalibus nigris. Remiges nigricantes latere interiore maculis transversis albis. Tectrices alarum inferiores albe maculis subcordatis nigris.

(Femina) Longit. I6 poll. Supra tota rufa capite et collo striis longitudinalibus; dorso, teetricibus alarum et mopygio maculis transversis fuscis. Cauda rufa fa- 
sciis fuscis, quarum ultima latissina, apice albo. Subtus avis albida, pectore et abdomine testaceo tinetis, maculis longitudinalibus fuscis.

Funior: Feminx similis præter maculas dorsi fuscas, qux in ave juniori fascias formant numerosas, saturate fuscas. Subtus pallide testacea maculis longitudinalibus fuscis fere confluentibus.

\section{H ISTOR I A.}

Frequens. Migratoria, In turribus, templis, ruinis, in arboribus cavis, in arborum quoque ramis nidulatur. Ova ponit 3 vel 4. Mammalia parva, aviculas, Amphibia et Insecta prædatur. In aëre sese librans, oculis pradain quzrit, in quam mira celeritate se pracipitat. 


\section{GENU.S Ir. \\ $S$ T R I X.}

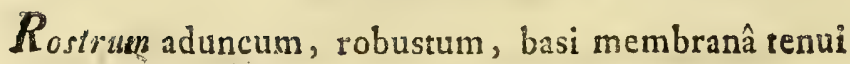
tęum. Mandibulis utrisque mobilibus.

Capat grande, oculis antice positis, auribusque magnis.

Nares pennis setace is recumbentibus obtectx Faciem cingunt pennæ rigidæ.

Remiges molliores, exterioribus margine extus serratis.

Digitus extimus versatilis.

DISSECTIO I.

Capite aurito.

\section{STRIX BUBO.}

Diagn: Corpus restaceo, nigro et albido varium. Irides fulva.

\section{SYNONYMA.}

Strix Bubo, Lin. Faun. Svec. p. 24. n. 69. Retz. Fauno Sv. p. 76. n. 24. Tengmalm Wet. Acad. H. I793. p. 236. Gmel. Syst. Nat. 1. p. 286. Lath. Ind. Orn. I. P. 5 I. 
Le grand Duc, Buff. Ois. I. p. 332. tab. 22. P1. enl. 435. Briss. Orn. I. p. 477. n. I.

Engle Owl, Pen. Brit. Zool. I. p. 202. n. 64. tab. 29. Are. Zool. II. p. 288.|n. II4.

Great-eilred Owol, Lath. Syn. I. p. II6. vers. Germ.

Bechst. I. p. 109. Suppl. n. 40.

Der. Uhu, Bechst. Nat. D. 2. p. 333.

Svecis: Uf. Hornuf. Berguf.

\section{DESCRIPTIO.}

Longit. ferninx 2. ped. 4. poll. Rostr. 2. poll. A flexura alx ad apicem I. ped. 8. poll. Cauda II poll. Tibix 3. poll. Digit. med. cum ungue $3 \frac{\pi}{2}$; Digit. postic. 2. poll. Ungues antici subtus planiusculi, po. sticus teres.

Rostrum coeruleo-nigricans, validum, valde aduncum, basi membranâ tectum. Pennæ setaceæ, basi albre apice nigre, ad dimidiam partem, et magis, ro. strum ohtegunt. Nares obovatx. Oculi magni eminentes, nigri, Iridibus fulvis. Apertura aurium, pro magnitudine capitis minor quam in congeneribus. $\mathrm{Ca}$ put magnum, felinum caput fere referens. Pedes ad ungues usque pennis plumosis densis, teeti. Ungues coeruleo-nigri, magni, acuti.

Corpus supra nigricans marginibus pennarum testaceis et albidis, nigricante variegatis. Aurium pennx numero sex, longitudine inxquales circa 3. poll. nigrx, latere interiore testaceo variegatx. Facies griseo et fusco variegata. Pectus et abdomen testacea maculis longitudinalibus nigricantibus, margine variegatis. Pedes, crissum et tectrices caudæ inferiores sordide testa* ceo albida undulis transversis fuscis. Remiges fuscofasciatx, latere interiore testacex, exteriore sordidx fu- 
sco tenuissime variegatæ et undulatx. Cauda testacea fusco fasciata et variegata, Rectricibus binis intermediis saturate fuscis maculis et punctis albidis variegatis.

\section{H IS T OR I A.}

In praruptis Svecix superioris haud infrequens nidulatur. Ova 2. 1.3. Non nisi hieme in campos Scaniæ descendit. Mures, Lepores aliaque Mammalia minora, Tetraones et Anates \&c. prædatur. Femina quam descripsi, occisa est prope Ystad, media hyeme, cum in eo esset ut Vulpi cxden pararet. Vox Maris, noĉu audita, lugubris, ululans, abrupta et gravis; feminæ paullo debilior.

\section{XXII, STRIX OTUS.}

DiAGn: Aurium pennæ longæ. Corpus fulvo, fusco et albido varium. Iris flava. Orbiræ nigræ. Pedes albo-fulvescentes, immaculati.

\section{SYNONYMA.}

Strix Otus, Lin. Faun. Sv. p. 24. 1n. 7 I. Retz. faun. Svec. p. 77. 11. 26. Lin. Syst. Nat. I. p. I332. n. 4. Gmel. Syst. I. p. 288. Lath. Ind. Orn. I. p. 53. n. 7. Tengm. Wet. Ac. H. I793. p. 261.

Moyen Duc ou Hibou, Briss. Onn. I. p. 486. Buff. Ois.

I. p. 348. tab. 22. P1. enl. 29. (fig. mala.) Long-Eared Owl, Pen. Brit.Zool. I. p. 203. n. 65. tab. 30. Arct. Zool. 2. p. 229. Lath. Syn. I. p. I2I. n. 5. vers. Germ. I. p. II 4 .

Die Mittlere Ohreule, Bechst. Nat. D. 2. p. 340. Svecis: Hornuggla. 


\section{DESCRIPTIO.}

Longit. avis I4 poll. Alæ extens 3 ped. Rostr. I. poll. A flexura alx ad apicem II $\frac{1}{2}$ poll. Cauda 6 poll.

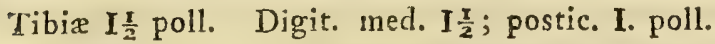

Rostrum nigrum basi membrana tectum. Nares ovales. Pennæ setacex albr, apicibus nigris rostrum circumdant et ad partem obtegunt. Iris aurantia. Orbitr, prasertim supra et subtus nigræ. Facies præterea rufescens, circulo e pennis nigris apice luteo et albo variegatis, ab auriculis pone aures ad gulam cincta.

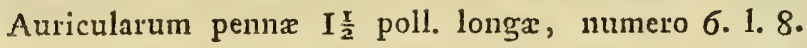
nigricantes, margine extcriore testaceo, interiore albo.

Corpus supra e ferrugineo, luteo et saturate - fusco longitudinaliter maculatum, fuscoque et albo tenuissime variegatum; maculis albis in Alarum tectricibus. Pectus et abdomen pallide fulva et albida maculis longitudinalibus fuscis, quarum margines albi, fusco te. nuissime transversim striati sunt. Remiges latere interiore a basi albx, exteriore ferrugineo-lute fasciis fuscis, versus apicem fasciis griseis et fuscis. Cauda xqualis, alis brevior, pallide fulva fasciis angustis, transversis fuscis, apice albida. Crissum et pedes ad ungues tecti pennis lanatis pallide ferrugineo-luteis, immaculatis.

\section{HISTORIA.}

Parum frequens. Prærupta, nemora, ruinas ædium incolit. In hortis ad Lund, non raro hyeme obvenit. In cavis arboribus vel in nido, quem alia avis deseruit, ova 4. 1. 5. alba ovato-globosa, ponit. Etiam in quercetis Seanix nidulantem inveni. Mammaliis minoribus et aviculis vescitur. Pulli facile mansuescunt. 


\section{OBSERV.}

Strix Scops, que in Retzii faun. Sv. p. 77. adfertur ut incola Svecix septentrionalis, nunquan, quant tim ego scio, in Svecia inventa est. In Mus. Grill. mentio ejus non fit. Neque in Museo quodam Patriz hane avem nisi ab exteris allatan vidi, neque venatori cuidam occurrisse audivi.

\section{DISSECTIO. II.}

Capite lavi.

XXIII. S'TRIX NYCTEA, Lin. Faun. Sv.

DIAGN: Corpus album, vel immaculatum vel fusco transversim maculatum fasciatumve, facie alba.

\section{SYNONYMA.}

Strix Nyctea, Lin. Faun. Sv. p. 25. n. 76. Retz. Faun. Sv. p. 78. n. 28. Lin. Syst. Nat. I. p. I32. Fabric。

Faun. Groenl. p. 60. n. 36. Lath. Ind. Orn. I. p. 57. Tengm. Wet. Ac. H. I793. p. 264. Harfang, Buff. Ois. I. p. 387. P1. enl. 458. Sirowy Orol, Pen. Arct. Zool. 2. p. 233. Lath. Syn. I. p. I32. 12. I7. vers. Germ. I. p. I24. - Suppl. p. 45.

Die Schneenle, Bechst. Nat. Deutsch1. 2. p. 350.

Strix nivea, Thunb. Wet. Ac. Handl. 1798. p. 184. Strix Scandiacin, Lin. Faun. Sv. p. 24. 11. 70.?

Svecis: Harfång.

\section{OBSER V.}

Br:bo Scandianus, Rudb. unde Strix Scandiaca Lin. desumta est, inter aves Rudbeckii piałas, quas Holmise vidi, non invenitur Ad quam igitur Speciem optimo jure referatur hæc avis; certe dicere non possum. Ab Ornithologo vero summe fidei, 
qui iconem Bibliothece Grilliana Söderforssenssi vidit, audivi figuram Rudbeckii ad Strigem nyctenm pertinere.

\section{DESCRIPTIO.}

Longit. avis 2 pedes. A flexura alæ ad apicem 16

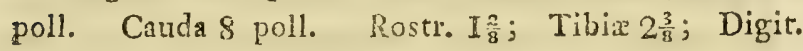
medius cum ungtie $2 \frac{2}{5}$ poll.

Rostrum nigricains, in pennis setaceis fere reconditum. Iris flava. Caput, pro magnitudine corporis cum congeneribus comparati, parvum. Pedes ad uurgues usque pennis densissime tecti. Ungues arcuati; acutissimi, basi pallidi, apicem versus nigri. Cauda alis aliquantum longior, subrotundata.

vnriet. a. Alba, capite supra maculis, corpore superne fasciis, subtus undulis fuscis. Cauda versus apicem fasciis fuscis. Facies et pedes lanati, albi immaculati.

Mus. Payk. Ex Uplandia.

variet. $\beta$. Alba, capite supra maculis parvis fuscis; dorso undulis rarioribus fuscis. Cauda et Remiges apice fusco maculatæ. Pectus undulis paucis, obsoletis. Ceterum tota alba. Ex Enara.

Mus. Payk. var. $\gamma$. Tota alba apicibus Remigum Imx et IIdæ maculâa transversali fusca.

Mus. Payk. E Lappania,

var. $\delta$. Tota alba, immaculata. Mus. Thunb.

\section{OBSERV:}

Pro atate et sexu magis fortasse quam pro vario coelo variat hac Species, in qua eadem regula videtur esse observanda ac in. ter Falcones, scilicet quo junior avis eo obscurior, quo senior eo albidior. Tori albi Mares sunt seniores. Talis est Str. niven, Thunb. quam in Mus, Upsal, vidimus. 


\section{HISTORIA AVIS.}

Regiones Sveciæ Septentrionales, ubi in præruptis nidulatur, hand rara incolit. Hyeme in Scania quoque, quamvis rarius obvenit. Tetraones, aliasque aves et Lepores (unde Harfang dicta) pradatur. Etiam interdiu venatur, volatusque ut Falconis caler; unde "rapinam suam raptim et fere perpcsadiculariter involat. Sonus ejus clamorem hominis periclitantis amalatur." Fabl.

\section{STRIX LAPPONICA.}

Diagn: Supra ex fusco et albo variegata, subtus albida maculis longitudinalibus fuscis. Facies cinerea lineis concentricis fuscis circa oculos. Cauda longa, griseo variegata, apice fusco.

\section{SYNONYMA.}

Strix Lapponiea, Retz. Faun. Sv. p. 79. n. 30. Mus. Carlson. Fasc. V. tab. (fig. mala.)

Mus. Payk. Mus. Upsal.

\section{DESCRIP TIO.}

Magnitudo fere Bubonis. Caput grande. Longit. avis circa 2 ped. 2 poll. Rostrum luteo-albescens. Iris flava. Pedes usque ad ungues plumis sordide albis, fusco undulatis vestiti.

Caput et collum supra maculis seu undulis parvis albidis et fuscis, numerosis adspersa. Dorsum et tectrices slarum fusca albo variegat et undulata. Oculi macula semilunari nigra antice semicircumdati, ante quam macula major semilunaris alba. Cetera facies cinerea circulis concentricis fuscis. Superior pectoris pars fusca macnlis parvis numerosis albidis, unde albi- 
da apparet striis longitudinalibus fuscis, transversim lineatis. Abdomen album maculis longitudinalibus fu. scis. Remiges primores albido fuscoque fasciatæ. Cauda alis longior, supro cinereo fuscoque tenuiter variegata (undulata) versus apicem fusca; subtus basialbida, apice fuisca.

\section{LOCUS.}

Lapponia. Mores ignoti.

\section{STRIXLITURATA.}

Dracn: Supra maculis longitudinalibus albis et fuscis; subtus albida maculis longirudinalibus fuscis. Area oculorum sordide cinerea, pennis rigidis albo nigroque maculatis cincka.

\section{SYNONYMA.}

Strix litulratn, Mus. Grill. p. 4. Tengm. Wet. Acad. H. 1793. p. 267. Retz. Faun. Sv. p. 79. n. 29. Mus. Payk.

\section{DESCRI P TIO.}

Magnitudo fere Strigis vayctea, capite aliquantum majore. In ordine naturali proxime ad St. Aluconem accedit. Longitudo avis circa I. ped. 8 poll. Rostrum flavescens. Iris - Arex oculorum sordide cinerex cinguntur pemnis rigidis, albis, nigro striatis et maculatis. Caput supra et collum maculis longitudinalibus fuscis et sordide albidis. Dorsum et alarum tectrices fuscx maculis albidis. Tota subtus sordide alba maculis longitudinalibus fuscis, in crisso sagittatis. Remiges areis fuscis et griseo - albis alternatis. 
Cauda alis longior, albido fuscoque transversim fasciata. Pedes pennis sordide albis vestiti.

\section{LOCU.S.}

Specimen unicum juxta Elfkarleby Uplandix oecisum. Mores ignoti.

\section{STRIX ALUCO.}

Strix Aluco et Stridula Lin. Faun. Sv. *).

Dragn: Caput prægrande. Irides nigræ. Corpus griseo, ferrugineo, fusco nigroque varium. Alæ supra maculis albis.

\section{S YNONYMA.}

Strix aluco, Lin. Faun. Svec. p. 25. 11. 72. (mas.) Lin. Syst. Nat. I. p. 292. Lath. Ind. Orn. I. p. 59. n. 26. Tengm. Wet. Ac. H. I793. p. 270. Retz. Faun. p. 80.

Ulula Gesneri, Grå svartögda Uglan, Rudb. pict. fol.

I7I. (Optina maris figura.)

Brown O2vl, Pell. Brit. Zool. I. p. 2IO. 11. 69. tab. 32.

(figura mala) mas.

Skrikugla, Rudb. pict. fol. I69. (fem.) unde, Strix Stridula, Lin. Faun. Sv. p. 26. 11. 77. Lath. Ind. Onin. I. p. 58. 11. 25. Gmel. Syst. I. p. 294. (fem.) Tnwny ovvl, Pen. Brit. Zool. I. p. 208. n。 68.

*) Per totum hoc Genus eadem retinui nomina, quantum potui quæ in Faunâ sua Svecica habet Ill. Linné; quænam zutem sine aves, clarius interdum, ex iconibus Rudberkianjs quam ex Descriptionibus Linnei liquet. 
Chat-hunt, Briss. Orn. I. p. 500. Buff. Ois. I. p. 362. t. 25. Pl. enl. 437.

Die Nachteule, Bechst. Nat. D. 2. p. 353.

Svecies: Haruggla; Kattuggla.

\section{OBSERV.}

Linné multas aves in Fauna Svecica ex figuris Rudbeckiii piatis descripsit, inter qaas aves Stridula n. 77. numeranda. Eqdem enim adfert Synonyma, idem Svecanum nomen ac Rudbeck. Hac vero figura Rudbeckii Aluconis feminam bene refert. Striduzla igitur Linnéi ex numero Specierum delenda. Alii aliam hoc nomine descripserunt.

\section{DESCRIPTIO.}

Mas:

Longit. 15. poll. Alx extensæ 2. ped. IO. poll. A sinu ori, ad apicem Rostri $I_{\frac{2}{3}}$ poll. A flexura ala ad apicem $1 O_{\frac{1}{2}}^{\frac{x}{2}}$ poll. Cauda $6 \frac{\mathrm{T}}{2}$ poll. extra alas fere 2. poll. extensa. Tibiz 2. poll. Digit, med. cum ungue I $\frac{i}{2}$ poll, Digit. postic. I. poll.

Rostrum apice flavescens, basi coerulescens, oculi magni eminentes coeruleo-nigri. Pedes ad ungues us: que tecti pennis plumosis sordide albis, extus punctis fuscis. Ungues coeruleo nigricantes. Area oculorum cinerascens rufo immixto. A fronte, circa aures ad gulam, circulus e pennis basi nigris, apice ex rufo, albo et nigro variegatis.

Corpus torosum. Caput et collum supra ex nigro, rufo et albo pulcherrime variegata. Dorsum et alx supra sordide griseo-rufa, fusco longitudinaliter maculata et transversim vaziegata. Pennæ scapulares margine cxteriore albx, et tectrices alarum majores margine exteriore maculis albis. Pectus et abdomen albida, fer. ruginescente immix to maculis longitudinalibus fuscis, 
undulis fuscis interdum marginatis, Crissum album maculis sagittatis fuscis. Remiges (quarum prima brevissima, 3tia, 4 ta et 5 ta fere rquales, ceteris longiores) fasciis fuscis et ferrugineo-albidis, apice grisea punctis fuscis. Cauda fusca fasciis angustis ferruginescentibus, apice albida.

\section{Femina:}

Longit. 14. poll. Eaden colorum dispersio ac in mare; magis tamen rufa est ubi ille griseus vel albidus.

\section{HISTORIA.}

Inter Striges Svecia maxime vulgaris. Interdiu in sylvis inter ramulos arborum aut in cavis truncis irrin sui et clamoribus ceterarum avium se abscondit. Noatu venatum evolans, domos etiam frequentat Felibus et $\mathrm{Co}$ lumlis imprimis infesta. Mures etiam et aviculas prx. datur. In cavis arboribus, vel in nido quem struxit connix \&c. ova 3 - 5. ovato-globosa ponit. Vox maris ululans, femine stridens.

XXVII. STRIX BRACHYURA, mihi.

Diagn: Rostrum et orbita nigra. Corpus supra fuscum maculis luteis striis longitudinalibus fuscis, Cauda alis bre. vior.

\section{SYNONYMA.}

Strix tota flammea, Rudb. pict. unde, Strix capite lavi corpore luteo, Lin. Faun. Sv. 73. Ulula flammeata, Frisch, tab. 98. Strix flammen, Pontopp. Alt. Dan. I. t. 25. fig. 5. Strix aluco, Brüun. Bor. 1. I7. (optime descripta.) 
Strix ulula, Tengm. Wet. Ac. H. I793. p. 279. Retz. Faun. p. 82. n. 33. Lath. Ind. On. I. p. 60. n. 27. Strix brachyotos, Ginel. Syst. Nat. I. p. 289. Lath. Ind. Orn. I. p. 55. n. I I.

Strix accipitrina, Gmel. Syst. Nat. I. p. 295. Strix Arćticn, Mus. Carlson. fasc III. n. 5 I.

? Noctua minor, Nov. Comm. Acad. Petr. XV. p. 447. tab. XII.

Short-Eared Owol, Pen. Brit. Zool. I. p. 204. n. 66. tab. 3 I.

Die Brandeule, (Str. striduln) Bechst. N. D. 2. p. 356. Svecis: Branduggia.

\section{O BSER V.}

Mira in Synonymia hujus avis confusio, cujus ansami ipss Linné primus prebuit. In Fanna enim Svecica, ad n. 73. Strigen totam fammineam, Rudbeckii, citavit. Hæc vero icon Rudbeckii, weam exąte refert avem. In Systemate Natura, XIIm edit. hunc eunden numerum 73. Fanine, ad suain Str. fammeams (ad quam minime pertinet figura Rudibeckiana), murata Dingnosi, adfert. Hunc errorem non observavit cel. Tengmalm, qui in Actis Scient. Holn. monographian Serigutn seripsit. Ad hanc nostram avem, quan Ululum vocat, adfert Str. uluiem L in. Syst, nat. sed falso. Præmonet autem ne Ulula Fauna, pro eâdem atque Ulula Syst. Nat. habenda sit. Sed quare? Nihil de Ulala, in Syst. Nat. dixit Linné quod non anten in Fauna Sv. do Ulula dixerit; nisi Synonymism respicias, que falsa est. Ululs vero et Fannce et Systematis ad sequentem Speciem, quam Tengmalm Funeream temere dixit, pertinet. Hxc mea brachyura, in Syst. Lin. nullibi descripsa est. Strigem brachyotum, a celebri orתithologo Germanico missam, apud. cel. Thunberg Upsalix vidi. Hac vero nostra est brachyura.

\section{DESCRIPTIO.}

Longit. avis I3. poll. Rostr. I $\frac{3}{y}$ poll. A flexura alx ad apicem 13 poll. Cauda $4 \frac{r}{2}$ poll. Tibia $2 \frac{2}{\frac{2}{3}}$ poll. Digit. med. $I \frac{T}{2}$ poll. - postic. I. poll. 
Corpus gracilius et caput minus quam antecedentis. Forma, magnitudine et quadammode colore, ad Str. Ormm proxime accedit.

Rostrum nigricans apice pallido. Iris fiava. Circa oculos circulus niger, extus latior. Ungues magni, acuti, pallide fusci. E pennis capitis una vel plures ceteris longiores, quas viva erigere potest avis. In mortua haud observantur.

Caput et collum supra fusca striis longitudinalibus luteis. Dorsum et alæ supra fusca maculis luteis. (In aliis testacea 1. ferruginea, maculis longitudimalibus fuscis.) Pectus et abdomen pallide lutea. Striis longitudinalibus fuscis. Crissum et pedes pallide lutei, immaculati. Remiges primores ferrugineo. lutex, versus apicem fasciis fuscis; secundarice fusco et testaceo fasciatr. Cauda alis I. poll, brevior, testacea, fasciis $5-6$ fuscis, apice alba.

\section{HISTORIA.}

In superioribus Patrix provinciis xstatem degens, verno et autumali tempore tantum in Scania obvenit; unde migratoriam existimo. Mures prasertion venatu. Nidificat - -

\section{OBSERV.}

Strix fanumes, qualis a Tergmalm ct Retzio describitur, Australis Europe est incola, nec in Svecia unquam inventa.

XXVIII. STRIX ULULA, Lin. Faun. Sv.

Diagn: Supra fusca maculis albis; subtus alba fasciis transversis fuscis. Cauda longa, cuneifar. mis, fusca, lineis transversis albis. 


\section{SYNONYMA.}

Strix" Ulula, Lin. Faun. Sv. p. 26. 11, 78. Lin. Syst. Nat. I. p. I33. n. 10.

Strix funeran, Tengm. Wet. Aead. H. 1793. p. 284. Lin. Syst. Nat. I. p. 133. n. II. (Descriptio.) Lath. Ind. Orn. I. p. 62, 11. 35. Ginel, Syst. Nat, I. p. 294. (Descriptio.)

Strix Huidsonia, Gmel. Syst. Nat, I. p. 295.

Strix Uralensis, Gmel. Syst. I. p. 295.

Chouette à longue quelie de Sibirie, Buff. Pl. enl, $2,463$. Die Habichtseule, Bechst. Nat, D. 2. R. 372.

\section{DESCRIPTIO.}

Longit. avis $I 6 \frac{x}{2}-I 7$ poll, Rostr. $T \frac{T}{8}$ poll. A flexura alxad apicem $9 \frac{r}{2}$ poll. Cauda 7. poll; extra alas extensa ad $3 \frac{x}{3}$. poll. Tibix brevissima $\frac{7}{8}$ poll. Digit. med. I $\frac{3}{8}$ poll.

Rostri maxilla suparior lutea, inferior nigricans. Nares rotundatæ plumis rigidis tectr. Iris - Tibir usque ad ungues vestitz plumis sordide albis, undulis obsolete fuscis. Cauda longa, cuneiformis; angues fusci acutissimi.

Corpus gracile; caput, pro magnitudine corporis, minus quam in plurimis congeneribus.

Caput supra saturate fuscum maculis parvis rotunda. ris, albis, Fascia nigra semicirctalaris ab exteriore oculf cantho, circa tempora ad gulam ducta. Tempo. ra et genæ cinereo-alba. Cervix et anterior pars Dorsi fusca maculis albis. Medinm Dorsum fuscum fere inmaculatum. Uropygium fuscum fasciis transversis albis. Pennx scapulares fuscr maculis albis in unam maculam magnam longitudinalem; fere confluentibus. Subtus tota sordide alba, lineis in pectore et abdomine Wilssons Ornith. 
transversis, fuscis. Remiges fuscr margine exteriore maculis albis, latere interiore maculis transversim-ovalibus (5-7) albis; omnes prater exteriores duas apice albo marginatx. Reetrices fuscx, apice albx, lineis transversis $(7-8)$ albis, latere exteriore in fascias extensis.

Femina aliquantum minor.

\section{HISTORIA.}

Rarior. Arctoas patrix plagas incolit. Rarissime in sylvis Scanix instante hyeme, inventa. Interdiu venatur. Alte, modo Falconum, volat. Tetraonibus muribusque infesta.

\section{XXIX, STRIX FUNEREA, Lin. Faun. Sv.}

Diagn: Supra castaneo-fusca, maculis et punctis albis; subtus alba maculis fuscis nebulosa. Area oculorum albida. Inter oculos macula nigra. Cauda subæqualis maculis albis per paria positis. Pedes ad̉ ungues plumis dense vestiti.

\section{SYNONYMA.}

Noßึua major oculis majoribus, iridibus pallide luteis, Rüdb. pict. fol. I70, et hinc.

Strix funerea, Lin. Faun. Sv. p. 25. 11. 75.

Noctua major, oculorum iridibus croceis, Rudb. piat.

fol. 165. et hinc.

Strix capire lavi, corpore fusco, iridibus oculorum fulvis,

Lin. Faun. Sv. p. 25. in. 74.

Strix noctur, Tengm. Wet. Acad. H. I793. p. 289.

Strix Tengmalmi, Gmel. Syst. I. p. 29I. Lath. Ind. Orn.

I. p. 64. n. 42. 
Little Owol, Pen Brit. Zool. I. p. 2II. 11. 70, fol. tab. B. 5 .

Rawfiissiger Kantz, Strix Dasypuis, Meyer Vögel Esthl, p. 37 .

\section{O BSERV.}

Omnes fere Onithologi in hac ave apellanda hallucinan. tur. Eam esse funeream Lin. Fạun. Sv, bene ostendunt figurz Rudbeckianx, ex quibus avem descripsir Linué. Bechstein in Nat. Deut. 3. p. 665 , nočnam Tengmalmi cum passerina Linnéi conjungit, sed falso.

\section{DESCR IP TIO.}

Longit. svis $9 \frac{\pi}{2}-10$ poll. Alre extensx 22. poll。 Rostr. ab angulo oris ad apicem $\frac{6}{8}$ poli. A flexurn ala ad apicem $\sigma_{\frac{\pi}{2}}$ poll. Canda $3 \frac{5}{8}$ poll.; extra alas ad $I$. poll. extensa. Tibia breves vix I. poll.

Rostrum flavescens, lateribus in ave mortua fuscescentibus. Nares rotundatx postice membranâ fornicatâ. Areq oculorum magna e pennis divaricatis, rio gridis, albis nigro immixto Inter oculos et basin ro. stri macula atra oculos semicircundat. Circa aures ad jugulum circulus c pennis fuscis albo pulcherrime variegatis. Aures permagne. Iris pulcherrime flava. Pedes pennis plumosis albis, extus fusco immixto, ad ungues densissime vestiti. Ungues fusci, acutissimi. Cauda alis longior, expansa angulum obtusissinum format.

Avis tota supra rufescenti-fusca, capite maculis minutis numerosissimis, dorso et alarum tectricibus maculis aliquantum majoribus albis. Gula et crissum alba immaculata. Peetus et abdomen alba maculis fu. scescentibus irregularibus nebulosa. Remiges colore dorsi maculis (circiter 5.) margine exteriore et latere 
interiore pisiformibus albis. Reetrices colore dorsi maculis utrinque 4-5. pisiformibus aut transversis albis, per paria positis.

\section{HIST ORIA.}

Habitat in sylvis Sveciz superioris. Hyeme instante in sylvis et hortis Scanix, quamvis rarius, occurrit. Vescitur aviculis \&c. nidulatur -

\section{STRIX NUDIPES, mihi.}

\section{(Tab. II.)}

Diagn: Supra griseo-fusca maculis et punetis albis. Cauda tæniis albis rachi interruptis. Digiti subnudi, pilis rarioribus, adpressis instructi.

\section{SYNONYMA.}

Strix noctur, Retz. Faun. Sv. p. 85. Descriptio Retzii。

(Descriptio Tengmalmi ad antecedentem pertinet.)

Der kleine Kautz, Strix passerina, Bechst. Nat. Deutschl.

2. p. 367 .

Sperlingskautz, Meyer Vögel Esthl. p. 36.

\section{O B S ER V.}

Hane avem non novit Linné. Quin vero sit distinda species et $a b$ antecedente et $a b$ insequente; non dubitare potest is, qui omnes tres unâ viderit. Non enim tam colores quam forma partiun constantiorum ab Ornithologo consulenda sunt. Specimen quad heic describatn, in Museo Academix Lundensis asservatum, idem est cujus descriptionem dedit cel. Retzius 1. c. Hæc est passerina Germanorum, minime vero tinnéi; id quod satis docent icones Rudbeckianx. Nomine igitur passerine illam ap. pellare ron licuit. Aliud quarens, id mihimaxime charafteristicum visum est, quod avem ab omnibus europæis Strigibus inauritis, quantum ego scio, distinguit. 


\section{DESCRIPTIO.}

Longit. avis 8 poll. Rostr. $\frac{6}{8}$ poll. A Aexura alx ad apicem a poll. Cauda $2^{6}$ poll. Tibiæ longæ $\mathrm{I} \frac{\mathrm{T}}{2}$ poll.

Rostrum Havescens, robustius quam in antecedente, quamvis avis minor. Area oculorum parva, pennis griseo fuscis albo immixto. Iris flava. Tibix pennis plumosis albis parcius tectr. Digiti fere nudi, pilis rarioribus adpressis. Ungues quam præcedentis erassiores, fusci, basi pallidi. Cauda alis parum longior, $x$ qalis.

Avis tota supra griseo-fusca maculis albis pisiformibus, in capite minoribus et frequentioribus. Gula jugulum et latera colli anteriora aiba, immaculata. Pectus et abdomen alba, maculis longitudinalibus fuscescentibus, fere confuentibus. Remiges colore dorsi, latere interiore maculis transversim ovalibus, margine exteriore maculis triangularibus (circa 5-6.) albis, unde alx complicatx fere tessellatx videntur. Rectrices colore dorsi, trniis 5. albis, ad rachin interruptis, apice albo.*)

\section{LOCUS:}

Specimen descriptum e Scania est. Rara, ni fallor avis, sed forte cum funerea vel passerina a venatoribus confusa.

\section{XXXI.STRIX PASSERINA, Lin. Faun.Sv.} (Tab III)

Diagn. Supra fusco-cinerea punctis et maculis minuris albis, subtus alba maculis longirudinalibus fuscis. Area oculorum corpori concolor. Cauda lineis quatuor et apice albis. Pedes ad ungues plumis dense vestiti.

\#) A passerina, dignoscitur : Tibiis longinribus, digitis nudiusculis, oculis, rostro et unguibus majoribus. 


\section{SYNONYMA.}

Strix passeriua, Lin, Faun. Svec. ip. 26. n. 79. (femina.) Rudb. pict. fol. 163. Tengm. Wet. Acad. Handl. 1793. p. 294. Retz. Faun. Sv. p. 86.

Ziverg Inutz, Strix pygzzen, Meyer vögel Esthl. p. 38 Svecis: Sparfriggia.

\section{OB SER V。}

Hallucinantur Ornithologi plurimi passerinam Linnéi citaturi. Ab ornithologis Britannicis Strix funeren Lin, pro passerita Lin. habetur, testante cel. Tengmalm, (1. c. p. 288.) qui Musea eo. aum pervestigevit. A Germanis mea nudipes, passerina vocatur, et cum tandem verain passerinam Lin. invenerunt, pygixeam appellarunc. Inter aves pictas Rudbeckii, figura hujus avis optima invenitur, quam in passerina describenda ante oculos habuit Linné. Ut igitur quantum exinde sumserit, quisque videat: schedam illam adferre placet, qua, Rudbeckii manu scripta, juxta figuram adhuc exstai: Noctua Gesn. p. 620. Aldr. z. I.p 54. Noctua minor Will. p. 69. Raji. Syn. 26. Lilla eller minsta Stenrglau Sved. In sylva hand longe Uspalis Norlen (?) dicta, a the occisa et in nido cjus 4. ova exigna, maculosa.

\section{DESCRIPTIO.}

(Mas.) Longit. $6 \frac{2}{8}$ poll. Alæ expansæ II. poll. Rostr. $\frac{x}{2}$ poll. A flexura alx ad apicem 4. poll. Cauda $2 \frac{x}{8}$ poll.; extra alas extenditur $\frac{6}{8}$ poll. Tibix breves vix $\frac{x}{2}$ poll. superant. Digit. med. $\frac{7}{8}$ poll.. postic. $\frac{3}{8}$ poll.

Rostrum viridi-flavescens, apice pallidiore. Nares rotundatæ, postice membranâ fornicatâ. Iris pulcherrime crocea. In regione periophthalnica, rigidx illæ pennæ divaricatæ, qux in congeneribus conspiciuntur, in hac desiderantur. Pedes usque ad ungues pennis plumosis albis, extus fuscescentibus, densissime vestiti. Ungues nigro - fusci, acutissimi.

Tota avis supra fusco-cinerea. Caput punctis subrotundis albis, antice frequentius sparsis. Collum su. 
pra maculis confertioribus, magnitudine pisi minoris. Dorsum et alarum tectrices maculis minoribus, minusque confertis. Latera capitis pone oculos fasciis minoribus transversis fuscis et albis. Subtus avis alba. Gula et crissum immaculata. Pectus maculis fuscis irregularibus, fere confuntibus, ad latera transversis. Abdomen striis longitudinalibus distinctis. Remiges cincreo - fusca margine exteriore et latere interiore maculis albis. Rectrices æquales, cinereo fuscæ lineis transversis quatuor saturatius marginatis et apice albis. Femin? $7 \frac{1}{7}$ poll. longa. Alx extens: $13 \frac{\mathrm{r}}{2}$ poll. Color exakte Maris sed maculæ fuscx pectoris et abdominis longitudinales sunt, et magis distinctx.

\section{HISTORIA.}

In sylvis rarius. Par quod descripsi, in Hallandia, media hyemecepi. Feminam undique vel insectabantur vel comitabantur avicula garrientes et clamantes, qux tamen omnes in certa quadam distantia se tenere tutius ducebant. Nec incallide. Celeriter enim volabat, nec incommodo illi fuit lux. Mas, quem in alâ altera plumbo fixi, unguibus tenebat Parum coeruleun, quem media die necaverat. Vivum in cavea per quosdam dies tenebam. Aviculis vescebatur, quas tamen interdiu non tangebat, nec vesperâ quidem, quamdiu in cubiculo accensa erat candela. Hac vero exstincta, coenare statim adgressus est. Prxdam ante coenam curate deplumabat more Falconum. In cavea excellenter scandebat, et de summa pendebat. Nullum edebat sonum nisi garritum quendam, (fere: kirr! kirr!) Emberiz. citrinellam, in caveam iminissam, mox necavit. In cavis arboribus nidulatur. ova 4. 


\section{ORDO II. \\ P 1 C E, Lath。}

DISSECT. I.

Pedibus ambulatorits.

\section{GENUS III. \\ L A N I U S}

$\boldsymbol{R}_{\text {ostrum, validum, compressum, rekum, maxil- }}$ lis apice ineurvatis, superiore longiore, pone apicem incisurâ (unde denticulus interdum oritur) utrinque.

Nores pennis setactis te Ztr.

$P$ edes validiusculi, ambulatorii.

Carda longa, plerumque cuneata

XXXII. LANIUS COLLURIO, Lin. Faun. Sv,

Diagn: Capur supra cinereum, Dorsum rufo-brune neuim. Fascia per oculos nigra. Subtus roseoalbus. Cauda nigra, lateribus versus basin albis. $\left(\mathrm{Mas}_{\diamond}\right)$

\section{SYNONYMA.}

Lanius collario, Lin. Faun. Sv. p. 27. n. 8 I. Lin. Syst. Nat. I. p. 136. Retz. Faun. Sv. p. 88. 11, 38. Lath. Ind. I. p. 69. n. II. 
Ecorcheur, Buff. Ois. I. p. 304. t. 2I. Pl. enl. n. 31. fig. 2 .

Rellbacked Shrike, Pen. Brit. Zool. I. p. 215. n. 72 Lath. Syn. I. p. 167. 11. 25. Suppl. p. 52.

Der Dorndreher, L. Spinitorquus, Bechst. Nat. D. 2. p. 392.

Svec. Törnskata.

\section{DESCRIPTIO.}

Longit. avis $6-7$. poll. Rostr. $\frac{6}{8}$ poll. A flexurn alix ad apicem fere 4. poll. Canda $3 \frac{2}{5}$; extra alas $I \frac{x}{2}$ poll. Tibix fere I. poll. Digit, med. $\frac{7}{3}$ poll., postic. $\frac{5}{8}$ poll.

Rostrum nigrum, basi subtus pallidum. Nares ova. les, supra quas penne setacex. Anguli oris vibrissis 4-6 nigris. Iris fusca. Pedes nigri.

Caput, collum supra et uropygium cinerea, fronte pallidiore. Basis rostri supra nigra. Fascia nigra a neribus per oculos ad laterà colli. Dorsum et teerrices alarum pulcherrime rufo-brunnea. Avis subtus alba pectore roseo colore tincta. Remiges fuscr nurginibus presertim interiorum rufo-brtmneis. Cauda aliquantum cuneiformis, longa, Rectricibus mediis binis nigris inmaculatis, ceteris medietate interiore albis, exteriore nigris.

Femina: Mari valde dissimilis. Supra fusco-ferruginea undulis obsoletis nigris et albidis. Per oculos ad tempora fascia rufo-fusca. Subtus alba, luteo tincta, undulis præsertim in pectore et hypochondriis fuscis. Remiges fuscx. Rectrices rufo-fuscx, extima albo fimbriata, ceteræ, exceptis quatuor intermediis, apice albo marginatis. 


\section{HISTORIA.}

Frequens; migratoria. Post medium mens. Maji, in hortis et fruticetis Scanix jam redux occurrit. In fruticibus, præsertion Pruni spinose vel Crategi, nidum, haud multum a terra elevatum, ponit. Ova 6. albida, rufo maculata. Vescitur aviculis, amphibiis, coleop. teris, qua spinis transfixa in futicibus circa nidum sæpe pendent. Egregie cantillat Mas, alias aves optime imitans. Pullis exclusis parentes e summo fruticis cacumine, voce Gäck! Gäck! Gäck! caudâque huc et illuc motâ, queruntur, hoste adveniente. Aviculas, qux ad nidum se adpropinquant, magno clamore statim fugat. Initio mens. Septembris abit.

\section{LANIUS EXCUBITOR.}

Diagn: Corpus supra canum, subrus album. Alre nigræ maculis binis albis. Cauda nigra lateribus albis.

\section{SYNONYMA.}

Lanius Excubitor, Lin. Faun. Sv. p. 27. n. 80. Lin. Syst. Nat. I. p. I35. n. I I. Gmel. Syst. I. p. 300. Rètz. Faun. Sv. p. 8\%. 11. 37. Lath. Ind. I. p. 67. n. 6 .

Pie-gricsche grise, Buff. Ois. I. p. 296. t.20. Pl. enl. 445.

Grea Shrike, Pen. Brit. Zool. I. p. 2I3. n. 7 I. tab. 33.

Lath. Syn. I. p. I60. n. 4.

Der gresse graue Wirger, Bechst. Nat. D. 2. p. 376. Suecis: Warfogel. 


\section{DESCRIPTIO.}

Longit. evis 9. poll. Ab angulo oris ad apicen Rostri I. poll. A flexura alx ad apicem $4 \frac{6}{8}$ poll. Cauda

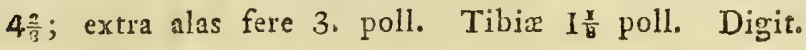
med. $\frac{7}{3}$ poll. , postic. $\frac{5}{8}$ poll.

Rostrum nigrum basi subtus flavescens. Nares ova. les setis tectx. Anguli oris vibrissis sex nigris. Iris fusca. Pedes plumbeo-nigri, unguibus nigris.

Totum corpus supra pallide cinereum, superciliis, fronte et pennis scapularibus in album inclinantibus. A rostro per ocules ad latera occipitis fascia nigra. Totum corpus subtus album, peftore in purpureum leviter inclinante undulis obsoletis, vix conspicuis. Remiges nigrx a basi ad medium fere albx, secundarix 2pice albx. Cauda longa, cuneiformis, Rectricibus intermediis basi et apice tenuiter albis, in ceteris albedo apicis et basis magis magisque augetur.

Feminn differt coloribus magis sordidis, undulisque pectoris fuscescentibus, magis distinetis.

\section{HISTORIA:}

In sylvis rarius. In Scania, instante hyeme, rarissime tamen visa. Vescitur aviculis, muribus, insectis. Nidulatur in arboribus. Ova 4- 6 .

\section{XXXIV* LANIUS GLANDARIUS, *) mihi.}

DIAGN: Corpus rufescenti-cinereum, tectricibus alarum coeruleis, lineis transversis albis et nigris.

*) Tum propter furman rostri \& 2 . tum propter mores a Corwis separandus et in ter $L$ suios collocandus est. 


\section{SYNONYMA.}

Corvus glindarius, Lin. Faun. Sv. p. 30. n. 90.

Coruus glandarizs, Retz. Faun. Sv. p. 93. n. 44. Lin。

Syst. nat. I. p. I56. n. 7. Gmel. Syst. I. p. 368.

Lath. Ind. Orn. I. p. I57.

Grrulus, Briss. Om. II. p. 47. I.

Gery, Buff. Ois. II p. I07. t. 8. Pl. enl. 48 I.

Jay, Pen. Brit. Zool. I. n. 79. Lath. Syn. L. p. 384. n.

19. Aret. Zool. II. p. 252. E.

Der Holzheher, Bechst. Nat. D. 2. p. 449.

Svec: Skogskata; Nörskrika. Ostrogothis: Kornskrika。

\section{DESCRIPTIO.}

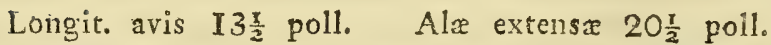

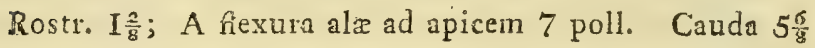

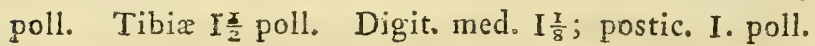

Rostrum nigrum, reeum, compressum, supra subtusque convexum, maxilla superiore apice decurvata, utrinque denticulo, vel excisura, instructa. Supra angulum oris vibrissa nigra sex. Nares pennis setaceis teetr. Iris griseo-albida. Pennæ Pilei longx, in cristam erigi possunt. Pedes fusco - incarnati. Cauda fere rqualis, pare intermedio et extimo ceteris Rectricibus parum breviore.

Corpus fere totum rufo-cinereum; latera tamen colli magis in rufum, dorsum in cinereum tendentia. Frons albida vel pallide rufescens, maculis longitudinalibus nigris. Ab angulo oris ad latera gula descendit utrinque macula nigra. Gula albida. Crissum et Uropy. gium alba. Reniges primores nigro-fuscr, latere exteriore sordide alba; sequentes quatuor nigrx latere exteriore medio albr, unde in ala composita macula magua alba oritur. Teetrices remigum primorum la- 
tere exteriore lineis transversis nitentibus nigris, pallide coeruleis et albidis pulcherrime ornata. Rectrices nigre basi cinerex lineis transversis nigris; par laterale ifuscum subtus cinerascens.

\section{HISTORIA.}

Frequens. Pro anni temporibus locum mutat. Estatem in regionibus superioris Svecix montuosis et nemorosis degit; autumno et hyeme in Seania aut per familias, aut gregatim occurrit. Vescitur glandibus, nucibus, frugibus, aviculis in laqueis (Donor Sv.) pendentibus \&c. In arboribus nidulatur, Ova 5-7. 


\section{GENUS IV. \\ $C O R \quad V \quad S$.}

$\boldsymbol{R}_{\text {ostrum validum, compressum, secundum rotam }}$ suan longitudinem aliquantulum decurvatum, marginibus (plerumque) integris.

Nares setis rigidissimis tectæ.

$p_{e d e s}$ validiusculi, ambulatorii.

Caudo varia in variis.

\section{XXXV, CORVUS CORAX.}

Diagn: Ater, purpureo nitens. Cauda cuneato-rotundata. Tibiæ cum rostro æquales, digito midio longiores.

\section{SYNONYMA。}

Corvus Corax, Tuin. Faun. Sv. p. 29. n. 85. Retz. Faun. p. 90. 11. 40. Lin. Syst. I. p. 155. n. 2. Gmel. Syst. I. p. 364.

Corbeau, Buff. Ois. III. p. 13. t. 2. P1. enl. 495. Rnven, Brit. Zool. I. p. 2I8. n. 74. Art. Zool. II. p. I34. Lath. Syn. I. p. 367. n. I. Suppl. p. 74. Der gemeine Rabe, Bechst. Nat. D. 2. P. 402. Svecis: Korp. Scanis rusticis: Ravn.

\section{DESCRIPTIO.}

Longit, avis 2. ped. Rostr. 3 poll. A flexura alz ad apicem I7 poll. Cauda 9 poll. Tibix 3 poll. Digit. med. $2 \frac{2}{3}$ poll.; postic, $I \frac{7}{5}$ poll. 
Rostrum nigrum, validum, supra convexum, compressum, versus apicem aliquantum decurvatum, denticulo utrinque instructum. Nares ovales, pennis setaceis rigidissimis, densissime tectr. Iris extus albi$\mathrm{da}$, intus griseo-fusca. Pedes validi nigri. Cauda cum alis aqualite: extenditur. Alæ acutx.

Tota avis atra, nitore supra purpureo, subtus atroviridi fulgens.

Femina magnitudine parum inferior, colore similis.

\section{HISTORIA.}

Vulgaris. Manens. Esstatem in sylvis degit. In arboribus excelsis et in prxruptis montium, jam sub finem hyemis, nidulatur. Ova 4-5. Vescitur Mammalibus minoribus, aviculis, amphibiis, insectis et frugibus, præsertim vero cadaveribus. Olfactu valet. Vox solita Krack! Krack! vel Kruck! Kruck! Tempore vero propagationis mas et femina, coelo sereno, pulcherrimos gyros supra sylvas per aërem ducentes, clangorem Clong! Clong! remotissime sonantem edunt. Longævum esse etiam rustici norunt; et quia clong illud raro exauditur, dicunt avem ante annum centesimum illud producere non posse. Sancta praterea habetur, nec facile a quodam occiditur.

\section{CORVUS CORONE.}

DIAGN: Ater, supra violaceo niters. Cauda rotun. data. Rostrum validum Tibiis brevius, Nares setis tektx.

\section{SYNONYMA.}

Corvus Corone, Lath. Ind. Orn. I. p. I5I. Emel. Syst,

I. p. 365 . 
Comix Gosneri, Rudb. pict. fol. I56.

Corbine ou Corneille noire, Buff. Ois. V. p. 56. t. T. f. 3. vers. Germ. Otro. VII. p. 77. cum, fig. Pl. enl. 483.

Carrion Craw, Pen. Brit. Zool. 1. p. 75. t. 34. Lath. Syn. I. p. 370. n. 3. vers. Germ. I. p. 304. n. 3. Die Rabenkrähe, Bechst. Nat. Deutsch. 2te Aufl. 2 B. p. 1167.

Gottlandis: Rauk. Mas. Upsal. Mus. Payk.

O BSER V.

Corvus corone $\mathrm{Iin}$. in Faun. Sv. p. 29. n. 86. planis ad roo strum detritis, vix alius est quam Corvus frugilegus.

\section{DESCRIPTIO.}

C. Cornce minor. Circa 20. poll. longus. Rostr. $2 \frac{2}{3}$ poll. A fiexura alæ ad apicem 13. poll. Cauda $6 \frac{6}{8}$ poll. Tibix $2 \frac{5}{8}$ poll. Digit. med. $I_{\frac{6}{3}}$ poll. Digit. postic. $I \frac{3}{8}$ poll.

Rostrum nigrum. validum, supra convexum, compressum, aliquantum decurratum præsertim juxta apin cem- Nares rotundatr, setis rigidissimis, numerosis, nigris, tectx. Supra sinus oris et ad oculos pili nigri. Cauda alis longior, aliquantum rotundata, Rectricis bus apice obtuse-rotundatis, rachibus tamen aliquan. tum elongatis (an semper?).

Totus ater, supra purpureo vel violaceo colore nitens.

var. a: Corvus Clericus, macula gulari alba. Mus. Carlson. I. tab. 2. Specimen in Museo Payk. adhue asservatum vidi. Junior est avis.

\section{OCUS.}

In Svecia passim. Rara sine dubio est, nam vivam arem videre nondum milai contigit. In Gottiandix pa- 
roeciis Grbttlinglo et $V a m b l i n g h o$ habitare refertur. (Wet. Ac. Hand. 1809. p. 193) ibique laud rara esse debet, cum a rusticis proprio nomine Rauk appelletur. Spe. cimen descriptum e Gottlandia est. Varietas, quam Corv. Clericum appellavit Sparrmann 1, c. in Wermlandia occisa dicitur.

\section{CORVUS FRUGILEGUS.}

Diagn: Ater, purpureo nitens. Cauda rotundata, Rostrum subrectum, acutum. Pennx circa basin rostri detritie.

\section{S YNONYMA.}

Corvus frugilegus, Lin. Faun. Sv. p. 29. 11. 87. Retz, Faun. p. 91. n. 42. 8. Lin. Syst. Nat. I. p. I56. n. 4. Gmel. Syst. I. p, 366. Lath. Ind, Orn. I. p. I52. $\mathrm{n}, 5$.

Le Freux ou la Frayonne, Buff. Ois. III. p. 55. vers. Germ. Otto. VII. p, 98. cum. fig. Pl. enl. 484. Rook, Pen. Brit. Zool. I. p. 22I, n. 76. Lath. Syn. I. p. 372. vers. Germ. I. p. 307. n. 4. Suppl. p. 76 . Aret. Zool. II. p. 250. A.

Die Saatkrähe, Bechst. Nat. D. 2te Auf. 2 B. p. IIg9. Svecis: Roka, Råka.

\section{DESCRIPTIO.}

Longit. avis I ped. 6. poll. Alz extensz 3. ped.

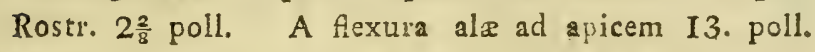
Cauda \%. poll. Tibiz $2 \frac{3}{3}$ poll.; Digit, med. 2. poll. Digit, postic. II $\frac{T}{2}$ poll.

Rostrum nigrum, magis rectum, minus validum et minus compressum, magis vero acutum quam antecedentis. Nares ovata, pennis setaceis fere detritis ob. Nibson Ornith, 
tectx. Basis rostri (per oculos ad angulos oris et gulam) cute calva, rugosa, albida instructa. (In pullis hac calvities non observatur; sunt enim capistro instructi, sicut C. Corone, a quo vix nisi formâ rostri di. stinguuntur.) Oculi fusci. Cauda alis vix longior rotundata, reetricibus apice obtuse rotundatis.

Totus ater corpore supra et subtus purpureo nitente, rectricibus et primoribus remigibus atro-viridi splendentibus.

Femina mari similis.

\section{HISTORIA.}

Migratoria. In Scania campestri et in Oelandia fre* quens. Sero abit (ad medium fere Septembrem); ma. ture redit. Anno 1813. jam circa XX. d. Mens. Februarii in Scaniarn revertebatur. In arboribus, pras sertim Ulmis et Fraxinis excelsis, circa vicos et domos gusticorum satis gregatim nidulatu, ubi clamorem et garritum perpetuo instituit. Rustici et nidum et aves suentur, præsentiam earum inter bona omina numeran. tes. Verno tempore aratrum sequitur, vermiculos et forte sate lecturus. Ova 3-5. Pulli primoxtatis anno capistro reverso sunt instructi, quod teram frequenter fodientes deterunt.

\section{XXXVIII, CORVUS CORNIX.}

Dragn: Corpus cinereum, capite, jugulo, alis et cauda nigris.

\section{SYNONYMA。}

Corvus Cosrix, Lin. Faun. Sv. p. 30. 11.88. Retz. Faun. Sv. p. $92,12,42$. 
Corvus Cornix, Lin. Syst. Nat. I. p. I56. 17. 5. Gmel, Syst. I. p. 366. Lath. Ind. Orn. I. p. 153.

Cornix cineren, Briss. Orn. II. p. I9. 11. 4.

Corneille mantelée, Buff. Ois. III. p. бI. tab.4. Pl. enl. 76.

Hooded Crow, Pen. Brit. Zool. I. p. 223. n. 77. Aret. Zool. II. p. 25I. D. Lath. Syn. I. p. 374. 5. Suppl. p. 77 .

Nebelkrähe, Bechst. Nat. D. 2. p. 425.

Svecis: Kråka.

\section{DESCRIPTIO.}

Longit. avis $I \frac{x}{2}$ ped. Alæ extens $x$ fere 3. ped. Rostrum et pedes nigra. Nares rotundatx, pennis setaceis dense tecix. Iris pallide grisea. Cauda rotundata cum alis æqualiter extenditur.

Caput, gula et jugulum, alx et cauda nigra violaceo splendentia; ceterum pallide cinerea.

var. a. Tota alba. Cornix alha Scandiana. Rudb picto fol. I57. Talis in Museo Payk. e Wik Uplandia as. servatur.

\section{H IS T OR I A.}

Vulgaris. Manens; ab una tantum in alteram pao tria plagam pro anni tempestatibus demigrans.*) \#sta. tem in sylvis degens in arboribus nidulatur. Ova 46 pallide viridia maculis fuscis, Mens. Aprili et Majo ponit. Vorax. Mures, lacertas, ranas, vermes, pisces ad litus ejectos aliaque eadavera edit. Cerasos aliasque fruges devastat, unde hortis noxia. Aves cubantes infestat, ovaque ebibit, fugata matre. Etiam

*) Per totam hiemem $18 \times 4$. nullam in Hallandia sylvestri coro nicens videbam. 
aves juniores consumit. Autumno gregatim volat. Erucas depescitur, hinc purgat pascua et prata a ver. mibus, qui foenum consumunt. (Lin.) - Hieme in viis plateisque urbium et inter stercus nutrimentum quarit:

\section{XXXIX, CORVUS MONEDULA.}

Diagn: Nigra, subtus nigro-cinerea. Occiput, Cervix, latera capitis et colli incana.

\section{SYNONYMA.}

Corvus Monedula, Lin. Faun. Sv. p. 30. n. 89. Retz. Faun. Sv. p. 93. 11. 43. Lin. Syst. Nat. I. p. I56. n. 6. Gmel. Syst. I. p. 367. Lath. Ind. Onn. I. p. I54. n. II. Briss. Orn. I I. p. 24. n. 6.

Choucas, Buff. Ois. III. p. 69. Pl. enl. 523.

Fackdarv, Pen. Brit. Zool. I. p. 234. n. 8I. Arct. Zool.

II. p. 25 I. C. Lath. Syn. I. p. 378. 9. Suppl. p. 78.

Die Dohle, Bcchst. Nat. D. 2. p. 439.

Svecis: Kaja. Scanis: Allika.

\section{DESCRIPTIO.}

Longit. avis I3: poll. Als extensx 2. ped, 2. poll. Rostrun nigrum, naribus setis rigidis dense tectis. Iris albida, Cauda rotundata, circa I. poll. extre alas extensa, Pedes nigri.

Frons, vertex, dorsum, alx, cauda et gula nigra. Occiput, cervix, latera colli et capitis pallide incono quasi irrorata. Peetus, abdomen et tectrices alarum inferiores nigra, cinereo tincta. var. a. Tota alba, levissime testaceo tincta.

E. Svecia. Mus. Payk. 


\section{HISTOR I A.}

Frequens. Alix manent, aliæ demigrant. Habitat gregatim in arcibus, turribus urbium, templis et xdibus, ibique hibernat. Nidulatur Mens. Aprili, in muris, caminorum spiramentis, et sub tectis. Etiam in cavis arboribus in sylvis ex. gr. ad Roslätt Scanix. Ova 4-6. virescentia maculis striisque fuscis ponit. Vescitur insectis, vermibus, ovis avium, frugibus et cadaveribus. Avis docilis, loquax, furax.

\section{CORVUS PICA.}

Diagn: Albo-nigroque varius, cauda longa cuneiformi.

\section{SYNONYMA.}

Corvus Pica, Lin. Faun. Sv. p. 3I. n. 92. Retz. Faun.

Sv. p. 95. n. 46. Lin. Syst. Nat. I. p. I57. n. 13.

Gmel. Syst. Nat. I. p. 373. Lath. Ind. Orn. I. p.

I62. n. 3 I. Briss. Orn. II. p. 35. I.

La Pie, Buff. Ois. III. p. 85. n. 7. Pl. enl. 488.

Mngpie, Pen. Brit. Zool. I. p. 225. n. 78. Ark. Zool.

II. n. I36. Lath. Syn. I. p. 392. 11. 29. Suppl。

p. 80.

Elster, Bechst. Nat. Deut. 2. p. 462.

Svecis: Skata.

\section{DESCR IP TIO.}

Longit. avis I8 poll. Rostr. I $\frac{T}{2}$ poll. A flexura alx ad picem 8 poll. Cauda IO $\frac{x}{2}$ poll. Tibix $2 \frac{2}{\frac{1}{4}}$ poll. Digit. med. I $\frac{x}{2}$ poll. Digit. post. I. poll.

Rostrum nigrum supra convexum, subcompressum, maxilla superiore, apice aliquantum decurvara, Na- 
res tectz pennis setaceis, rigidis. Supra angulos oris vibrissæ nigræ. Iris pallide brunnea. Pedes nigri.

Inter pulcherrimas Europa aves jure numeranda. Caput, collum, dorsum, et pectus ad medium, crissum et femora, nigra, rachibus pennarum in gula cinereis. Ceterum pe\{rus, abdomen et pennæ scapulares nivea. Remiges primores albx, latere exteriore et apice nigræ, secundaria cum tectricibus nigra, atro-coeruleo nitentes. Cauda nigra, nitore atro-viridi pulcherrime fulgrens.

var. w. Rostrum et pedes nigra. Ceterum ubi antecedens nigra est, hre sordide cinerea vel sordide alba; ubi illa alba, hre quoque alba. Ita ut caput, collum, pectus anterius, dorsum, femora et crissum sordide cinerea sint. Pectus posterius, abdomen et pen"1æ scapulares alba. Basis alarum sordide cinerascens. Remiges primores albæ, apice et latere exteriore sordide albidx. Cauda albida. Corvus Lapponicus Thunb. Wer. Ac. H. I798. p. I78. Corv. Tornensis, Sparm. Svensk Ornithologie p. 38 , cum fig.

\section{OBSER V.}

Specimen in Mus, Thunberg. vidi. Magnitudine et partium proportione exacte eum $C$. Pica convenit, præter caudam aliquananm breviorem in ave morbo laborante, aut certe juniore. In plagis Patrix septentrionalibus haud rara est. Ex Uplandia quoque haber cel. Swartz. Mus. Holm. war. $\beta$. cinereo-fuliginoso-alboque varia, oculis rubicundis, rostro pedibusque nigris. Mus. Carls, III. n. 53 .

Mus. Holm.

*a. $\vartheta$. Ferrugineo-alboque varia。 Mus. Carls. IV. n. 87. 


\section{HISTORIA.}

Manens. Vulgaris.*) Loca ab hominibus habitata incolit, raro sylvas quærit. Nidum plerumque in summo arboris cacumine juxta domos ponit, e ramusculis constructum, radiculis parvulis stratum et ramulis spinosis supra tectum, foramine laterali. Ova 4. 6. Vescitur insectis, frugibus, ovis et pullis avium, cadavere et stercore \&c. Sagax, loquax, furax.

\section{CORVUS INFAUSTUS, Lin.} Faun. Sv.

Diagn: Corpus pallide rufescenti-cinereum, Capite fusco. Cauda longa cuneato - rorundata, Rectricibus fulvo - ferrugineis, intermediis binis cinereis.

\section{SYNONYMA.}

Corvus infaustus, Lin. Faun. Sv. p. 32. n. 9.3. Mus. Carls. IV. tab. 76 (Fig. bona.) Retz. Faun. Sv. p. 95. n. 47. Lath. Ind. Orn. I. p. I50.

Lanius infanstus, Lin. Syst. Nat. I. p. I38. n. 2.5.

Gmel. Syst. Nat. I. p. 310.

Corvus Sibericus, Gmel. Syst. I. p. 373.

Siberian Fag, Lath. Syn. I. p. 39I. n. 26.

Geni de Siberie, Buff. Ois. III. p. I18. Pl. enl. 608. (figura nimis colorata.)

Svecis: Lappskata, Olycksfogel. Femtlandis: Rödrjuxa. Dalekarlis: Lafokrika.

*) Singulare tamen, quod in oppidulo Scania Sknnör et in insula Hven nunquam habitat, nec, nisi instante procella, ibj visa est. 


\section{DESCRIP TIO.}

Longit. avis II. poll. Rostr. I. poll. A flexure alx ad apicem $5 \frac{1}{2}$ poll. Cauda $5 \frac{1}{2}$ poll. Tibix $1 \frac{3}{8} ;$ Digit. medius I. poll. Digitus postic. $\frac{6}{8}$ poll.

Rostrum nigium, crassum, subtrigonum, (haud compressum* ${ }^{*}$, rectum, mandibula superiore longiore, apice aliquantum decurvata; basi setis nigris antice rergentibus. Nares tect $x$ pennis rigidis albo rufescen. tibus. Pedes fusci? - Corpus totum tegitur pennis tumidis, mollibus, pinnatis, pinnis raris, (nec contiguis) tanquam in Lanio Glandario, cum quo multa quoad habitum videtur habere communia. Etiam hic, ni fallor, pennas capitis longioies in cristam erigere potest. Pennæ Gula pilis, antice vergentibus, terminata.

Caput supra et ad latera usque sub oculis saturate fuscum rufo tinctum, fronte pallidiore in testaceum yergente. Collum, dorsum et pętus pallide cinerea rufo leviter tincta. Abdomen, hypochondria et crissum magis iufescentia. Caudx tectrices inferiores pallide rufæ. Remiges fuscr, basi ferruginex, latere exteriore cinerex. Teetrices Remigum primorum proxi$m x$ ferruginex, apice fuscr. Rectrices fulvo-ferruginex intermediis duabus saturate cinereis.

*) Jam ad hoc jam ad aliud genus ab ornithologis allata est hec avis. Neque vero Corvis neque Laniis propter formam rostri bene jungitur. Cum vero inter Corvos plerique illam sumerane, hoc ad interim retinui. Alio tempore, cum congeneres exoticos sat bene examinavero, illi in serie avium naturali locum suum accuratius designare conabor. Lanii enim Linnéi, natura duce, in plura genera abire debent, quorum uni hic adnumerandus est. 


\section{HISTORIA.}

Habitat in nemorosis Lapponix, Jemtlandia et Dale. karlix. Juxta Garpenberg in pinetis densis vulgaris est ibique Lafskrika appellatur; (Dr. Lindroth in litt.) Etiam in Uplandiam descendit sed rarissime. Illustr. Comes Wachtmeister Holnix emit, et, quo semper est erga scientiam nostram favore, ad nos misit specimen juxta Holmiam captum initio Mens. Novembris, aurd adhuc leni.

Proterva avis commessantes peregrinatores sxpe adiens, carnesque disjettas rapiens.(Lin.) Victus varius, forte pro varia amni tempestate. Cel. Leche in ventriculo ejus invenit baccas juniperi, pilos quosdam breves et caudam Muris. (Alvers. Lechei.) Ille vero, quem ab IIl. Wachweister recepi, ventriculum habebat insectis, presertim Coleopseris fartum. 


\section{GENUS $V$. \\ CARYOCATACTES.}

Rostram conico-subulatum, subrectum, teretiuseulum, aliquantulum conpressum, apice depressorruncatum. Mandibula inferior intus gibbo longitudinali.

Nores pennis setaceis tedz.

Lingau bifurca.

Pedes validiusculi, ambulatorii.

\section{CARYOCATACTES NUCI- FRAGA, wihi.}

DiAGN: Corpus fuscum albo guttatum, alis caudaque nigris, rectricibus apice albis.

\section{SYNONYMA.}

Corvus Caryocatactes, Lin. Faun. Sv. p. 3I. n. 9I. Retz.

Faun. Sv. p. 94. 1. 45. Lin. Syst. p. 157. n. IO.

Gmel. Syst. I. p. 370.

Nucifraga, Briss. Om. II. p. 50. I. tab. 5. fig. I.

Casse-noix, Buff. Ois. III. p. 122. t. 9. PI. enl. 50.

Nutcracker, Pen. Brit. Zool. 2. p. 62.5. app. tab. III.

Ark. Zool. II. p. 252. Do Lath. Syn. I. p.

400. n. 38. Suppl. p. 82 .

Der Tannenheher, Bechse. Nat. D. 2. p. 457.

Svecis: Nötkraka. 


\section{DES CRIPTIO.}

Longit. avis 13 poll. Rostr. 2. poll. A flexura alx ad apicem $7 \frac{x}{3}$ poll. Canda $5 \frac{t}{2}$ poll. Tibia $I_{\frac{\pi}{2}}$ foll. Digit med. $I_{8}^{3}$; postic. $I_{8}^{\frac{T}{8}}$ poll.

Rostrum nigrum, conico-subulatum, strikum, sine angulis; mandibulis fere zqualibus, apice depres. sis, obtusis. Mandibula inferior intus gibbo instruc. ta. Nares rotundatr pennis setaceis albidis teetz. Pedes nigri. Cauda alis longior, rotundata.

Corpus brunneum pileo et uropygio immaculatis. Inter oculos et rostrum macula alba. Gula striis albis. Ceterum corpus maculis parviis, ovatis, albis, subtus majoribus, supra minoribus numerosis adspersum. Alx nigrx, supra nitentes, brsi maculis parvis triangularibus albis. Remiges medix (6-8) latere interiore maculâ alba, apice albo marginatx. Cauda nigra. Rectricibus intermediis apice albis, qux albedo in cetesis magis augetur. Teetrices caudx superiores nigræx, inferiores alba.

\section{HISTORYA.}

Manens, ab una in alteram plagam tantum demigrans. In superioris Svecix pinetis astate passim habitat. Au. tumno coryleta et querceta frequentat. In Scaniam non nisi hyeme, et quidem rarissime advenit. Vietus: insecta, glandes, nuces, avium ova et pulli. Nidulatur in cavis arboribus. Ova $5-6$. 


\section{GENUS VI. \\ C O R A C I A S.}

Rosirum validum, fere rectum, compressum, supra convexum, apice deflexo.

Nares nudæ, oblongæ.

Vibrissx rigidx.

$p_{\text {edes ambulatorii, breves. }}$

\section{CORACIAS GARRULA.}

Diagn: Coerulea dorso rubro, remigibus nigris.

\section{SYNONYMA.}

Coracias Garruln, Lin. Faun. Sv. p. 32. 11. 94. Retz. Faun. Sv. p. 97. 11. 48. Lin. Syst. nat. I. p. I59. i. I. Gmel. Syst. I. p. 378. Lath. Ind. Orn. I. p. 168.

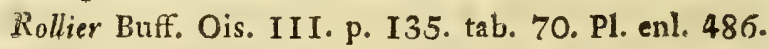

Roller, Pen. Brit. Zool. 2. p. 624. tab. append. II. Are?. Zool. II. p. 235. G.

Mandelkrähe, Bechst. Nat. D. 2. p. 472 .

Svecis: Bläkråka; Spanskkråka.

\section{DESCR I P TIO.}

Longit. avis $12 \frac{1}{2}$ poll. Rostr. I $\frac{6}{3}$ poll. A flexure alte ad apicem 8. poll. Canda fere 5. poll. Tibix fere

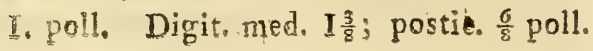


Rostrum nigrum basi subtus fuscum, compressum, secundum totam longitudinem aliquantum decurvatum, basi supra pennis denudatum; maxilla superior apice dependente, pone quem incisura lata utrinque. Nares nudx, oblongx, oblique positx. Supra angulos oris vibrissæ 6. rigidæ, nigræ. Iris intus brunnea, extus grisea. Regio circa oculos nuda. Pone oculos macula nuda, tempore propagationis verrucosa. Pedes lute. scentes unguibus nigris. Cauda alis longior, æqualis, Reetricibus obtusis, extima tamen utrinque longior: acuminata.

Caput, nucha, pectus et tectrices alarum majores coerulescenti-viridia; abdomen, crissun et alarum tectrices inferiores eodem colore, sed pallidiore. Cer. vix, dorsum et remiges intimx ferrugineo-hepatica. Uropygium et tectrices alarum minores (basis alarum) pulcherrime coerulea, nitidissima. Remiges supra nigrx, basi pallide coerulescenti-virides, subtus latere interiore splendidissime cocrulex. Rectrices basi fu. sco-coerulex versus apicem albo-virides, pari intermedio viridi-nigro, unicolore; extimo apice nigro.

Femina differt colore capitis, colli et pectoris sordidioribus; reetricibus xqualibus, neque extimis apice ni. gris.

\section{HIS T ORIA.}

Migratoria. In plerisque sylvis haud infrequents. Nidulatur in cavis a:boribus. Ova 3-7. alba, immaculata. Vescitur Insectis, Lumbricis, Ranis, Limaci. bus. Avis timida, nunquan mansuescit. Vox garrt. la. Abit circa medium Septembrem. (vide præterea Historiam avis fuse expositam in Wet. Ac. 11. Hiandl。 I787. p. 308.) 


\section{CORACIAS GALBULA,}

\section{Bechst.}

Diagn: Wis: Flavus, loris etremigibus nigris, rectricibus nigris apice Aavo. Femina: supra luteo-viridis, subtus sordide virescens striis fuscis; Remiges fuscx; Cauda olivacea apice flavo.

\section{SYNONYMA.}

Oriolus Galbula, Lin. Syst. nat. I. p. I60. I. Gmel. Syst. I. p. 382. Retz. Faun. p. 98 n. 49. Lath. Ind. I. p. I86. n. 45. Sparm. Svensk Ornith. cum fig. maris et fem.

Coracias Oriolus, Lin. Faun. Sv. p. 33. 11. 95.

Oriolus, Briss, Onn. II. p. 320. n. 58.

Loriot, Buff. Orn. III. p. 254. tab. I7. P1. enl. 26. (mas.)

Die gelhs Racke, Bechst. Nat. D. 2te Aufl. 2. p. I292. The Oriole, Pen. Brit. Zool. II. p. 626. Append. tab. IV. Lath. Syn. II. p. 449. n. 43. Sup. p. 89.

Svecis: Sommarguling; Gultrast, Gylling.

\section{DESCRIPTIO.}

Iongit. avis 9 poll. Rostrum fusco-rubrum, conico-subulatum, supra convexum, aliquantulum decurvatum, (fere reetum); maxilla superiore longiore apice deflexo, pone quem incisura. Nares patula nudr, ob. longæ. Lingua apice membranacea, lacera. Iris rubra. Pedes plumbei.

Caput, collum et corpus totum pulcherrime flavum loris nigris. Alæ, cum teetricibus, nigre macula flava. Cauda nigra reetricibus lateralibus apice flavis. 


\section{H IS T O R I A.}

In quibusdam Finlandiz provinciis ex. gr. in Carelia et Savolaxia frequens est ibique Kuhankeittiajü appella. tur (Faun. Fenn. p. I9.) In Westrobothnia quoque visa refertur. Migraroria. Vescirur cerasis aliisque baccis, insectis et larvis. In ramss arboris aut arbuscule cujusdam nidum suspendit. Ova $4-6$ alba, extre mitate obtusiore fusco maculata. 


\section{GENUS VII.

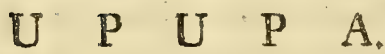

Rostram longum, tenue, arcuatum, teres, basi trigonum, obrusciusculum.

Nares parvæ, ovales, nudæ.

Lingua integra, obtusa, triquetra, brevissima.

Pedes ambulatorii.

\section{VL. UPUPA EPOPS.}

Diags: Caput, collum et peetus ferrugineo-lurea, crista apice nigra. Corpus supra nigro et albo varium; abdomen album; Cauda nigra fascia alba.

\section{SYNONYMA.}

Upupa Epops, Lin. Faun. Sv. p. 3\%. n。 I05. Retz. faun. Svec. p. 107. 11. 60. Lin. Syst. Nat. I. p. 183. Gmel. Syst. I. p. 466. Briss. Orn. II. p. 455. $t$, 43. fig. I.

In Huppe, Buff. Ois. VI. p. 439. t. 2I. P1. enl. 52.

Hoopoe, Pen. Brit. Zool. I. n. 90. t. 39. Aret. Zool. II.

p. 283. A. Edw. tab. 345- Lath. Syn. II. p. 687. Sup. p. I22.

Gemeiner Wiedehopf, Bechst. Nat. D. 2te Aufl. 2. p. IO7I.

Sivecis: Härfogel; Scnnis Popp: Hallandis Oäringsfogel. 


\section{DESCRIPTIO.}

Longit, avis I2 poll. Alx extensæ I9, poll. Rosir. I $\frac{\pi}{2}$ poll. Tibix $\frac{6}{8}$ poll. Cauda zqualis, alis longior, Rectricibus decem.

Rostrum coeruleo-nigricans basi incarnatum. Iris fusca. Pedes nigricantes. Crista duplex plicatilis e pennis circa 2 poll. longis ferruginescenti-luteis, apice nigris.

Caput, collum, interscapulium et basis alarum, peceus et inferiores alarum tectrices sordide aurantia. Dorsum et alx complicatæ nigræ fasciis albis. Abdomen et crissum alba. Cauda nigra, fascia in media alba.

\section{HISTORIA.}

In sylvis passim. Migratoria. Timida, Irata vel ferrefacta cristam erigit, Vocem oppoppopp! oppoppopp! subsistens recitat. Propter singularem er insolitum sonum, jam hoc jam illo loco in sylva auditum, nulio tamen illius auctore invento, inter mala omina a vulgo numeratur, In aliis provinciis bellum, (unde Härfo. gel.) in aliis annonx caritatem (unde Oäringsfogel) pra. dicere putatur innocens avicula. Vescitur insectis. Nidulatur in cavis arboribus sordida et foetida avis. Qva ponit 4.6 sordide cinerascentia: 


\section{GENUS VIII. \\ C E R T H I .}

Rostrum tenue, arcuatum, compressum, acutum. Nares oblongæ, membrana semiredæ.

Lingua longa, tenuis, acuta.

Pedes ambulatorii, ungue postico prxlongo.

\section{CERTHIA FAMILIARIS.}

Diagn: Supra ex fusco, albo et ferrugineo variegata, subrus alba. Remiges fuscr fascia albida. Cauda cuneata, rigida.

\section{SYNONYMA.}

Certhia familinris, Lin. Faun. Sv. p. 38. 11. 106. Retz. Faun. P. 108. n. 6I. Lin. Syst. Nat. I. p. I84. n. I. Gmel. Syst. I. p. 469. Lath. Ind. Om. I. p. 280. n. I.

Certhia Briss. Onn III. p. 603. n. I.

Grimperenu, Buff. Ois. V. p. 48 I. tab. 2I. f. I. Pl. enl. $68 \mathrm{I}$. f. I.

Common Creeper, Pen. Brit.Zool. 1. 13. 92. tab. 39. Arct. Zool. II. n. I74. Lath. Syn. II. p. 701 . Suppl. p. 126.

Der gemeine Baumlaiifer, Bechst. N. D. 2. p. 550. Motacilla scolopacina, Norske Vidensk. Selsk. nye Skruift.

2. B. p. 367 , cum fig.

Svecis: Trädkrypare. 


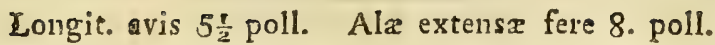
Rostrum supra nigricans, subtus albidum apice fuæco. Nares oblique ovatz. Iris fusca. Cauda ex pennis XII. rigidis, decurvatis constans, extensa cuneiformis est, composita in duas euspides divisa. Pedes pallide fusci; digiti ad primun articulum connati; ungues griseo-albi, quorum præsertim posterior pralongus.

Caput et collum supra fusca, maculâ ovali alba in singula penna. Ab angulo oris per oculum fascia fusca; supra oculum alba. Dorsum et penne scapulares ferrugineo-fusca maculis albidis. Uropygium ferugineum. Subtus tota avis pulcherrime alba. Remiges nigro-fuscr, versus apicem margine exteriore luteorufæ; versus basin margine exteriore virescenti-grisex; prima maculâ alba latere interiore ad basin notata; secunda et tertia unicolores; Ceteræ fasciâ per medium albida. Tectrices nigro-fusca maculis albis et ferrugineis. Rectrices griseo-fuscr, extimx margine albr,

\section{HISTORTA.}

In sylvis haud infrequens. Hyemes nostras perfert. Truncos arborum scandens, insecta eorumque ova et larvas legit. Nidulatur in cavis arboribus. Ova 6-9 alba puntis fuscis et ferrugineis ponit. Homines pa. rum metuit, unde nomen faniliaris. 


\section{GENUS IX. \\ $S I T T A$ 。}

Rostrum subulatum, rectum, porrećtum, ieretiusculum, acutum.

Nares rotundatx, pennis setaceis obteetr.

Lingara bifido-lacera.

Pedes ambulatorii, digito postico elongato valido.

\section{SITTA EUROP㞋A.}

Diagn: Supra cano-coerulea, subrus albida, fascia per oculos nigra. Reetrices nigræ, laterales quatuor pone apicem albæ.

\section{SYNONYMA.}

Sitti Europer, Lin. Faun. Sv. p. 37. n. I04. Retz。 Faun. p. 106. n. 59. Lin. Syst. Nat. I. p. 177. Gmel。 Syst. Net. I. p. 440. Lath. Ind. Orn. I. P. 26 I. Briss. Orn. III. p. 588. I. t. 29.

La Sitelle ou Torchepot, Buff. Ois. V. p. 460. t. 20. PI. enl. 623. f. I.

Nuthatch, Pen. Br. Zool. I. p. 255. n. 89. Arct. Zool. II. 11. 170. Lath. Syn. II. p. 648. Suppl. p. II7.

Gemeine Spechtmeise, Bechst. Nat. D. 2. p. 523. Svecis: Nötväcka, Nötpacka. 


\section{DESCRIPTIO.}

Longit. avis $5 \frac{x}{2}$ poll. Rostr. $\frac{6}{8}$ poll. Alx extensæ 9 poll. Cauda 2 poll. Tibix $\frac{6}{8}$ poll. Digit. med. $\frac{5}{8}$ poll.; postic. $\frac{6}{8}$ poll.

Rostrum coeruleo-fuscum, subtus albidum. Iris griseo-fusca. Pedes cinereo-lutescentes. Cauda alis aliquantum longior, æqualis, Rectricibus XII.

Frons coerulea, caput supra, cervix, dorsum et alarum teetrices cano-coerulescentia. A naribus per oculos ad latera colli (usque ad scapulas) utrinque fascin nigra. Avis subtus alba pectore et abdomine rufo levissime tincta. Hypochondria rufa. Crissum album marginibus pennarum rufis. Remiges fuscescenti-cinerex, primx quatuor basi albx, ceterx margine interiore albidx. Rectrices nigrx, versus apicem albx, apice ipso cinerex, intermedix binæ cinerex immacula. tx.

Fennine colores aliquantum pallidiores.

\section{HISTORIA.}

In sylvis ubique. Manens. Parum timida. Truncos arborum scandit insecta lectura. Vescitur quoque nucibus et glandibus, quas in rima corticis trunci cujusdam positas rostro findit acutissimo. Vox sibilans Tjutt-Tjutt! - Tjutt - Tjutt! Nidulatur in cavis arboribus. Ova $5-7$. sordide alba maculis rufis. 
DISSECTIO. II.

Pedibus scansoriis :

\section{GENƯ⿱ $\ddot{X}$. \\ P I C U S.}

Rostrum subulatum, polyedrum, reetum, apice cuneato.

Nares ovatæ pennis setaceis tectæ.

Lingua longissima, lumbriciformis, mucronata, apice retrorsum seris aculeata.

Pedes scansorii.

* Pedibas telradadoylis:

XLVIII. PICUS MARTIUS.

Diagn; Niger, pileo coccineo.

\section{SYNONYMA.}

Picus Martius, Lin. Faun. Sv. p. 34. n. 98. Retz. Faun.

Sv. p. IOI. 11. 53. Lin. Syst. Nat. I. p. I73. n.I.

Gmel. Syst. Nat. I. p. 424. Lath. Ind. I. p. 224. I.

Piculs niger, Briss. Orn. IV. p. 2 I. n. 6 .

Le Pic noir, Buff. Ois. VII. p. 4I. t. 2. Pl. enl. 596.

Great black Woodpecker, Arct. Zool. II. p. 276. A. Lath.

Syn. II. p. 552. I. Suppl. p. I04.

Der Schroartzspecht, Bechst. Nat. D. 2. p. 499.

Svecis: Spillkräka, Tillkräka. 


\section{DESCR I P T IO.}

Longit. avis I. ped. 5 poll. Rostr. basi albidum, versus apicem coeruleo-fuscum. Iris pallide flava. Palpebrx nudx. Pedes plumbei.

Tota avis atra capite supra a fronte ad occiput coc. cineo.

Femina color niger aliquantum in fuscum inclinans, occipite tantum coccineo.

\section{HIST ORIA.}

In sylvis, prisertim in pinetis. Vix in Scania obvenit. Manens. Coleoptera eorumque larvas lectura, truncos arborum putridarum scanditet terebrat. Hyeme silens, vere clamitare incipit. Imitatione vocis facillime allicitur. In cavis arboribus ova ponit IV. alba, nitida.

\section{PICUS VIRIDIS.}

Diagn: Viridis pileo coccineo, regione oculorum nigra.

\section{S YNONYMA.}

Picus vividis, Lin. Faun. Sv. p. 35. 11. 99. Retz. Faun. p. I02. n. 54. Lin. Syst. Nat. I. p. I75. n. I2. Gmel. Syst. I. p. 433. Briss. IV. p. 9. I. Lath. Ind. orn. I. p. 234.

Pic verd, Buff. Ois. VII. p. 7. t. I. Pl. enl. 37 I. 879. Green Woodpecker, Pen. Brit. Zool. I. 11. 84. Arct. Zool. II. p. 277. B. Lath. Syn. II. p. 577. 1. 25. Suppl. p. IIO.

Der Griuzspecht, Bechst. Nat. D. 2. p. 505.

Svecis: Grönspetr; Gröngöling. Scnnis: Gyllenrenn. 


\section{DESCRIPTIO.}

Longit. avis I3-14 poll. Rosir. a fronte ad apicem I $\frac{\sigma}{8}$ poll. A flexura alæ ad apicem 7. poll. Cauda $4 \frac{x}{2}$ poll. Tibix I $\frac{2}{3}$; Digit. antic. exter. $I \frac{3}{8}$ poll.; postic。 inter. $\frac{5}{8}$ poll.

Rostrum validum, coeruleo-nigrum, basi subtus flavescens, maxilla superior trigona, versus apicem cuneata, acuminata. Nares ovato-oblongx setis tectæ. Lingua 5 poll. longa, teres, retractilis, apice mucrone rigido ad latera setis retrorsum positis instructo. Iris alba, Pedes virescenti-plumbei.

Caput supra a fronte ad nucham splendide coccine: um, basi tamen pennirum glauco interlucente. Regio circa oculos nigra, maculis interdum albis, minutis. A basi maxilla inferioris ad latera gulæ fasciâ utrinque nigra, coccineo immixto. Sic latera capitis nigra, excepta regione aurium, quæ cum jugulo, pectore et abdomine, pallide cinereo-virescens, luteo tincta. Gula magis albescit. Collum supra, dorsum et tectrices alarum olivacea uropygio splendide virıdi-flavo. Crissum, latera abdominis et tectrices caudx inferiores sordide albida, luteo - viridi tincta, undulis fuscis. Remiges nigrescentes latere interiore maculis transversis albis; primores latere exteriore maculis luteo-albidis, cetera exteriore olivacea. Tectrices superiores remigum primorum nigricantes maculis alternis albidis, latere exteriore et interiore. Tectrices inferiores luteoalbx undulis nigricantibus. Reetrices XII. quarum I. 1. mollior, brevissima, fusca, margine et apice obtuso olivacea. Ceteræ rigidx, acutr; nigricantes, lineis transversis virescentibus, obsoletis.

Avis junior: Pilei singulx penne apice coccinex, pone quem macula ad rachin nigra, basi cinerea. Dorsum sa- 
turợe virì̉e, undulâ singularum pennarum albâ. Ưro. pygium viridi-favum, undulis obsoletis albis et fuscis. Latera capiris nigra apicibus pennarm albidis. Avis subtus sordide albe naculis nigris, in gyla angustis. simis, in jugulo et colli lateribus, in pectore et abdomine fascis binis in singulis pennis; in crisso ternis.

\section{ORSERV.}

Hoc colore gaudent pulid utriusque sexus ad priman usque pennaruar mutationem. A medio M. Julio ad frnem Augusti in sylvis frequontes.

\section{HISTORIA.}

Manens. In sylvis ubique haud infrequens. Trun. cos arborum erregie scandit. Coleopteris et formicis vescirur. Hycme formiceta fodit fornicas quærens, tuncque a pueris laqueis capitur. In cavis arboribus ova ponit $5-8$ alba. Nullos Inisi jam putrescentium arborum truncos terebrat, unde innoxia habenda avis, et utilis; inseta enim noxia consumit.

\section{PICUS CANICEPS, mihi.}

Dragn: Viridis, capite, collo corporeque subtus cinereis; Fascia nigra inter nares ct oculos, alia* que tenuiore ad latera juguli.

\section{SYNONYMA.}

Picus Norvegicus, Lath. Ind. Orn. I. p. 236. 1. 33.

Picus canus, Gmel. Syst. Nat. I. p. 434.

Picus viridis Norvegicus, Briss. Orn. IV. p. I8. 11. 4. Grey-headed green Woodpecker, Pen. Arĉ. Zool. II. n. 277. Lath. Syn. II. p. 583. n. 30. Edw. tab. 65. Gringraner Specht, P. virili - cnntis, Meyer Vögel Livund Esthl, p. 59. 
OB SIERV.

Thter aves Rudbeckii pictas optima hujus avis icon invenikur foil. 12j. Avis itaque jam cunc temporis cognita, sed a Linaè cum $P$. viridi confusa.

\section{DES CRIPTIO.}

Longir. avis I2. poll. Alæ extensæ I7 $\frac{r}{2}$ poll. Rostr. a fronte ad apicem I $\frac{2}{3}$ poll. A flexura alæ ad apicem

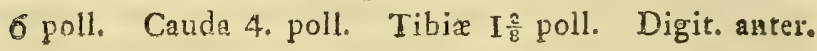

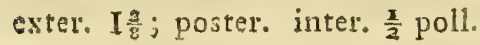

Rostrum debile coeruleo-fuscum, subrus versus ba. vin viridi-flavum. Nares oblongo-ovales pennis setaceis rectx. Iris rubenti-albs. Pedes cinereo-virides, unguibus griseo - fuscis.

Caput et collum cinerea, maculis minutis fuscis ver: sus frontem. A naribus ad oculos stria nigra, postice latior. A maxilla inferiore ad latera colli utrinque stria angustior nigra, interrupta. Dorsum et alarum tentices olivacea in coeruleum levissime inclinantia. Uropygium viridi-flavum, splendens. Subtus tota avis pallide cinerea olivaceo tinta, gula pallidiore. Crissum et tectrices caudx inferiores sordide olivacea undulis obsoletis fuscis. Remiges fuscx latere interiore maculis transversis albis, margine exteriore macuIis minoribus luteo-albis. Rectrices nigro-virides $s u$ pro unicolores intermediis binis obsolete fasciatis; $s u b$. : $2.2-3.3$. apice extus sordide incanæ; 6. 6. lute. scenti-albx apice et tæniâ ad rachin dentatâ nigris.

\section{HISTORIA.}

In provinciis Sveciz austratioribus rarius, nec unguam nisi hyeme hanc speciem inveni. In septentrio. nolibus minus rara. Mores pracedentis. Vescitur insectis. Nidulatur in cavis arboribus. Ova alba. 


\section{PICUS LEUCONOTUS.}

DIAGN: Alb, nigroque varius, dorso postico albo. Pileus maris ruber, fomince niger. Pektus et abdomen albida striis nigris. Crissum rubrum.

\section{SYNONYMA.}

Pičs lenconotus, Meyer Vögel Liv - und Esthl. p. 60. Ricus varius mojor, Rnib. pict. fol. I2I. fem. 122. mas.

Picus major vuriet. medius. Större Hack-eller Brolsplet, Sparrm. Sr. orn. (fig. bona.)

Mus. Lund. Mus. Upsel.

O B SER V.

III. Rudbeck 1. c. marem et femina in optine depinxit, quas pieturas in are incidendas curavit cel. Sparrmann. Cognita igitur jam dudum in Svecia avis, sed pro varietate sequentis habiza.

\section{DESCRIPTIO.}

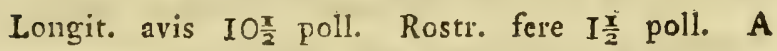
fexura alx ad apicem 6 poll. Cauda 4. poll. Tibix $I_{\frac{1}{8}}$; Digit. ant. exter. I $\frac{x}{3}$; postic. inter. $\frac{x}{2}$ poll.

Rostrum validum, pallide coeruleum. Nares teetx pilis albidis apice nigris. Iris purpurea. Pedes coeruleo-cinerei.

Frons albida. Caput maris supra a fronte ad nucham miniatum; femina nigrum. Latera capitis et colli alba. Amaxilla inferiore decurrit fascia nigra, qux in latere colli extenditur. Cervix et dorsum anticum nigra. Dorsum posticum et Uropygium alba apicibus pennarum obsolete nigris. Gula alba. Pectus et abdomen aibida, illud luteo, hoc rubro tincta, striis ni- 
gris longitudinalibus. Crissum carmineury. Ala composita nigra maculis albis fascias transversas formantibus. Rerniges nigrex maculis subrotundatis albis latere interiore et exteriore. Tectrices proximæ nigræx, versus apicem maeula rotundata alba; teetrices penultimre medietate interiore nigrx, exteriore albx. Cauda rigida cuneiformis. Rectrices basi nigra, versus spicem albidx maculis nigris per paria positis; par intermedium nigrum immaculatum. Teetrices superio. res caudr nigræ.

\section{HISTORIA.}

Habitat in sylvis cum congeneribus. Vescitur insectis. Ova $4-5$ alba in cava arbore ponit. Instante hyeme hortos Scanix interdum frequentat.

\section{PICUS MAJOR.}

Dragn: Albo nigroque varius. Dorsum nigrum. Scapula macula magna alba. Occiput maris coccineum femine nigrum. Crissum miniaceum. Pectus et abdomen alba immaculata.

\section{SYNONYMA.}

Picus mnjor, Lin. Faun. Sv. p. 35. n. I00. Retz. Faun. Sv. p. I03. n. 55. Lin. Syst. Nat. I. p. I76. Gmel。 Syst. Nat. I. p. $435 . \mathrm{n}$. I7. Lath. Ind. On. I. p. 228. 11. I3.

Picus varius major, Briss. Orn. IV. p. 34. n. I3. L'Epeiche ou Pic variê, Buff. Ois. VII. p. 57. Pl. enl. I96. 595.

Grent spotted Woodpecker, Pen. Brit. Zool. I. p. 243. 11. 85. Arct. Zool. II. n. I62, Lath. Syn. II. p. 
564.' 11. 12. Suppl. p. I07.

Der grosse Buntspecht, Bechst. Nat. D. 2. p. $5 I I$.

Svecis: Hackspett; Wedknarr.

\section{DESCRIPTIO.}

Longit. avis IO. poll. Rostr. I. poll. A fexura alm ad apicem $5 \frac{6}{8}$ poll. Cauda $3 \frac{\sigma}{3}$ poll. Tibix I. poll. Digit. ant. exter. $\frac{6}{8}$ poll. Digit. post. inter. $\frac{3}{8}$ poll.

Rostrum crassum, validum, coeruleo-fuscum, suhm tus pallidius. Nares teetz pennis setaceis, nigris. Iris albida. Pedes coertulescenti - virides.

Frons sordide rufescenti albida. Vertex niger. Fascia occipitalis transversa pallide coccinea. Ab angulo oris descendit fascia nigra et in latere colli se extendit. A fronte per oculos fascia alba, qua in temporibus ex. tenditur er ad latera cervicis descendit. Cervix, Dorsum, Uropygium nigra. Pennz seapulares et teetrices Remigum interiorum niver, basi tantum nigri, unde macula magna longitudinalis alba in ala clausa oritur. Remiges nigra maculis rotundatis albis per paria positis. Gula alba. Jugulum, pectus, abdomen alba, sordida rubigine tincta. Crissum miniacenm. Cauda cuneata Reetricibus rigidis, 2 vel 4 . intermediis nigris, immaculatis; laterales versus apicem sordida alba fasciis nigris.

Fenina caret fasciâ occipitis cocciner; Dorstum non tarn splendide atrum, sed magis nigro-fuscescens.

\section{HIS TORIA.}

Habitat in sylvis ubique. Manens. Ictibus ciretis. simis, quos truncis putridis infligit, etian eminus se prodit Ova in cavis axboribus $4-6$ alba ponit. Vescitur insektis. 


\section{PICUS MEDIUS.}

Diagn: Albo nigraque varius. Dorsum nigrum. Scapula macula magna alba. Vertex maris et femince coccineus. Crissum roseum. Peltus et abdominis latera striis nigris.

\section{SYNONYMA.}

Picus medius, Lin. Faun, Sv. F. 35. n. IOT. Lin. Syst. nat. I. p. I76. n. I8. Gmel. Syst. I. p. 436. Lath. Ind. orn. I. p. 229. 11. I4.

Pic varié à tête ronge, Buff. Pl. enl. $6 I$ I.

Middle Woodpecker, Pen. Brit. Zool. I. p. 244. n. 86. tab. 37. fig. sup. Arê. Zøol. I I. p. 278. D. Lath. Syn. II. p. 565. n. I3. Suppl. p. 107. Mittlere Buntspecht, Bechst, Nat. D. 2. p. 5 IG. (fem, ad $P$. majorem pertinet.)

\section{DESCRIPTIO.}

Longit. avis $8 \frac{\pi}{2}$ poil. Alæ extensæ 14 poil. Rosit. a fronte $\frac{7}{8}$ poll. A flexura alx ad apicen 5 poll. Cauda $3 \frac{1}{8}$ poll. Tibia $\frac{7}{3}$ poll. Digit. ant. exter. $\frac{7}{3} ; \mathrm{Di}$ git. postic. inter. $\frac{3}{8}$ poll.

Rostrum brevius et debilius, magisque compressum quam antecedentis, basi plumbeum, versus apicem coeruleo nigricans. Iris griseo-alba. Pedes griseoolivacei.

Frons sordide ferruginescens; vertex coccineus. Gula, jugulum, latera colli et capitis alba fasciâ nigra a sinu oris plerumque orta, in lateribus colli in maculam fere triangularem extensa. Cervix et Dorsum nigra. Scapulæ albx. Pectus album luteo tinctun et abdomen album rubro tinctum, striis ad latera longitudi- 
nalibus nigris. Crissum saturate roseum. Remiges nigre maculis utrinque albis. Rectrices quatnor in. term dix nigræ immaculatx, ceteræ versus apicem soldid: albx, nigro maculatx vel fasciatx.

\section{HIS TORIA.}

In sylvis eum præcedente passim. Vescitur insecris. Truncos arborum putridarum scandit et terebrat. Ova in cavis arborum ponit.

\section{PICUS MINOR.}

Diagn: Albo nigroque varius, dorso albo fasciis nigris. Vertex maris ruber, femince albus, Cris= sum sordide albidum fusco maculatum.

\section{SYNONYMA.}

Picus minor, Lin. Faun. Sv. p. 36. n. I02. Retz. Faun, Sv. p. 104. 11. 57. Lin. Syst. nat. I. p. 177. Lath, Ind. orn. I. p. 229. 11. I5. Gmel. Syst. I. p. 437. Picus varius minor, Briss. On. IV. p.4I. n. 15. La petite Epeiche, Buff. Ois. VII. p. 62. P1. enl. 598. Lesser spotted IVoodpecker, Pen. Brit. Zool. 1. n. $8 \%$ tab. 37. Arct. Zool. II. p. 278. E, Lath. Syn. II. p. 566. 11. 14. Suppl. p. 107.

Kleiner Buntspecht, Bechst. Nat. D. 2, p. 5 I8.

Picus minor, Lilla Hackspeten, Sparrm. Sv. Omith. (fig. bona.)

\section{DESCRIPTIO.}

Longit. avis 6. poll. Ala extensa II. poll, Posis, $\frac{1}{2}$ poil. A flexura alæ ad apicem fere 4 poll. Tibiz $\frac{3}{3}$ poll. Digit, enter. exter, $\frac{5}{8}$ poll. Digit. post, inter. vix $\frac{3}{8}$ pol! 
Rostrum virescenti nigricans. Iris fusco- Iufa. Pedes plumbei. Pennæ setacea sordide fuscescentes nares regunt. Frons alba. Vertex carmineus. Occiput, cervix et interscapulium nigra. Ceterum Dorsum album, fasciis angustis nigris. Tempora rufescentia. Later colli alba fesclâ nigra a sinu oris orta. Subtus avis sordide albo-rubescens. In lateribus striis longitudinalibus, in crisso maculis minutis cordatis fuscis. Alr compositz nigræ fasciis albis (circa 6.). Rectrices quatuor intermedia nigræ, immaculata; ceteræ introrsum nigræ, extrorsum oblique-albx, versus apicem ferru ginescentes fasciis nigris. Tectrices caudæ superiores nigrx.

Femina dignoscitur pennis supra basin rostrifuscis, fron: te et vertice sordide albis.

\section{HISTORIA.}

Manens; ab una tantum in alteram potrix plagam de migrans. In sylvis frondosis et in fruticetis rarius. Non nisi hyeme in Scania eam vidimus. Ora 7. alba immaculata, fere globosa in cava arbore ponit. Vescirur insectis.

* Pedibas tridaciglis.

\section{PICUS TRIDACTYLUS.}

Diagn: Albo nigroque varius. Vertex maris flavus, feminu albus. Pedes tridactyli.

\section{SYNONYMA.}

Picus tridactylus, Lin. Fann. Sv. p. 36. n. I03. Retz. Faun. Sv. I05. n. 58. Lin. Syst. nat. I, p. 177. n. 21. Gmel. Syst. I, p. 439. Lath. Ind. I. p. 243. 22. 56 . 
Epeiche ouil Pic varié ondé, Buff. Ois. VIr. p. 78. Pl. enl. 11. 553. (quartus digitus falso appos tis.)

Three-toed Woodpecker, Edw. t. II4. Pen. Aret. Zool. II. p. I68. Lath. Syn. II. p. 600 . 11. 5 I. Suppl. p. II 2 .

Der dreyzehige Specht, Bechst. N. D. 2. p. 52I.

\section{DESCRIPTIO.}

Longit. avis $8 \frac{\pi}{2}-9$ poll. Rostr. I. poll. A flexurs slx ad apicem fere 5 poll. Canda 3 poll. Tibia $\frac{7}{8}$ poll. Digit. anter. exter. $\frac{6}{5}$; Digit. post. $\frac{5}{2}$ poll.

Rostrum coeruleo-nigricans, basi albidum, validum. Iris fusco-rufa Pedes grisei. Digit. postic. solus, major binis anterioribus.

Frons et vertex flava; occiput nigrum. Latera colli et capitis nigra faseiis duabus albis, quarum una supra, altera infra oculum. Cervix alba. Dorsum nigrum, antice albo immixto. Subtus avis alba lateribus pectoris et abdominis maculis et striis nigris. Crissum sordide albidum lineis transversis nigris. Re. miges nigrra margine exteriore et interiore maculis albis, apice albæ. Teetrices nigræ immaculata. Rec. trices sex medix nigra iminaculati, sequentes dux utrinque nigr:x, versus apicem albæ fasciis nigris. Femince vertex argenteus, nigro longitudinaliter striatus.

\section{HISTORIA.}

Hebitat in pinetis rara avis. In Scania nunquam visa. In Smolandia accisam esse certe scio. In UpIandia preterea, Sudermannia et Westrogothia occurs rit, Mores mihi nondum bene noti. 


\section{GENUS XI. \\ J $\mathrm{Y} N \mathrm{~N}$.}

$\boldsymbol{R}_{\text {ostram rectum, teretiusculum, acuminatum. }}$ Nares nudæ, oblongæ, prope se invicem positx. Lingura teres, lumbriciformis, longissima, apice mucronata.

$p_{\text {edes scansorii. }}$

\section{JYNX TORQUILLA.}

DiAGs: Supra grisea maculis punctisque nigris, albis et rufescentibus. Subrus flavescenri-albida lineis transversis et maculis triangularibus nigris.

\section{SYNONYMA.}

Fynux Torquilla, Lin. Faun. Sv. p. 34. n. 97. Retz, Faun. Sv. p. 100. n. 52. Lin. Syst. nat. I. p. I72. Gmel. Syst. I. p. 423. Briss. Orn. IV. p. 4. t. I. f. I. Lath. Ind. Orn. I. p. 223.

Torcol, Buff. Ois. VII. p. 84. t. 3. Pl. enl. 698. Wryseck, Pen. Brit. Zool. I. p. 237. n. 83. tab. 36. fig. inf. Lath. Syn. If. p. 548. t. 24. Suppl, p. 103. Der Wendehals, Bechst. Nat D. 2. p. 527.

Svecis: Göktyta. 


\section{DESCRIPTIO.}

Longit, avis 7. poll. Als extensx II. poll. Rostr. A fronte ad apicem $\frac{x}{2}$ poll. A flexura alr ad apicem $3 \frac{r}{2}$ poll. Canda $2 \frac{x}{2}$ poll. Tibia $\frac{6}{8}$ poll. Digit. anter. exter. $\frac{7}{8}$ poll. poster. inter. fere. $\frac{x}{2}$ poll.

Rostrum griseo-fuscum. Iris luteo-fusca. Pedes plumbeo-virescentes. Cauda longa rotundata reetricibus duodecim, obtusis, quarum extima utrinque brevissima, vix pollicaris.

Caput cinereum maculis minutis nigris et albis ferrugineo inmixto. A vertice ad medium dorsum fascia longitndinalis nigra, ferrugineo varia. Ceterum supra grisea, nigro, albo et ferrugineo pulcherrime ma. culata et variegata. Subtus a gula ad stemum ferruginescente-lutea lineis transversis nigris. Pectus et abdomen alba, luteo tincta, maculis minutissimis, triquetris, nigris adspersa. Remiges saturate fuscr latere exteriore fasciis ferrugineis et nigricantibus, margine interiore maculis ferrugineo-albis; intima dorso concolores. Cauda cinereo et nigro tenuissime varie. gata lineis 5. nigris transversis, quarum interiores la. riores sunt.

\section{HISTORIA.}

Migratoria. Advenit initio m. Maji. Habitat in syl. vestribus, minus tamen frequens. Verno tempore vox acuta: $G i, g i, g i !$ sape auditur. In truncis arborum purridis ova ponit 6.8 alba, splendida. Vescitur insectis et larvis. Caput utrinque contorquet; cristam irata erigit. 


\section{GENUS XII. \\ CUC U L U S.}

Rostram subgracile, terefiusculum, aliquantum incurvatum, versus apicem compressum.

Nares parvæ, rotundatæ, nudæ, margine prominu$1 x$.

Lingua plana, integra,

Carda longa, rotundata.

Pedes scansorii.

\section{CUCULUS CANORUS.}

DIAGN : Supra saturate cinereus, peetore ei abdomine albis, undulis nigricantibus. Cauda nigra maculis albis。

\section{SYNONYMA.}

Cuculus canorus, Lin. Faun. Sv. p. 33. n. 96. Retz. Faun. Sv. p. 99. n. 50. Lin. Syst. Nat. I. p. I68. n. I. Gmel. Syst. I. p. 409. Briss. orn. IV. p. I05. n. I.

Coucou, Buff. Ois. VI. p. 305. Pl, enl. 81 I.

Cuckoo, Pen. Brit. Zool. I. p. 232. n. 82. (tab. 36. juniorem refert.) Lath. Syn. II. p. 509. n. I. Sup. p. 98.

Gemciner Kuckuk, Eechst. Nat. D. 2. p. 484. 


\section{DESCRIPTIO.}

Longit. avis I4. poll. 'Alæ extensæ 2 ped. Rostrum a fronte ad apicem $\frac{\sigma}{2}$ poll. A flexura alx ad apicem 9. poll. Tibia $\frac{5}{8}$ poll. Digit. anter. exter. I $\frac{2}{8}$ poll.; postic. inter. $\frac{x}{2}$ poll. Cauda $6 \frac{x}{2}$ poll. extra alas $2 \frac{x}{2}$ poll. extensa.

Rostrum nigrum, suhtas versus basin virescens. Anguli oris, palpebrarum margo, Iris et pedes pulcherrime flava. Os intus incarnatum. Cauda rotundata rectricibus decem, quarum extims utrinque ceteris multo brevior.

Caput et collum supre, dorsum et alarum tectrices saturate cinerea, Dorso et tectricibus alarum saturatioribus. Subtus a gula ad sternum pallide cinerea. Pectus, abdomen, hypochondria, tectrices alarum inferiores et femora alba lineis transversis nigricantibus. Remiges saturate fuscx, margine interiore maculis transversis albis. Rectrices nigrx apice albo, intermedix binx maculis albis juxta rachin; ceterz quoque margine interiore maculis minutis albis. Tectrices cauda longx, superiores cinerex, inferiores albæ, luteo tinc. $\mathrm{t} x$ maculis raris nigricantibus.

Femina: minor, collo subtus cinereo, luteo immixto, undulis fuscis. Abdomen sordide album, undulis fuscis.

\section{Variat admodum pro atate avis.}

Funior, ad finem mens. Augusti occisa: Longit. fere I3 poll. Rostr. supra fuscum, subtus virescens, angulis oris aurantiis. Iris griseo-brumnea. Pedes cum unguibus cerini.

Supra tota saturate fusca Uropygio in cinereum inclinante. Singulx pennx apice albo marginatx. Supra collum et tectrices alarum prosertim rufo transver- 
sim variegata. Maculæ albæ supra frontem et in nu. châ. Regio circa oculos fusco alboque tenuiter maculata. Tota subtus sordide alba undulis fuscis, in collo crebrioribus, in pefore et abdomine tenuioribus. Remiges fuscr margine exteriore maculis rufis, latere interiore maculis transversis intus albis, extus rufis. Cauda saturate fusca maculis ad rachin et in marginibus albis, fasciis præterea obsoletis rufis; pone apicem album fascia lata nigricans. Tectrices caudx inferio. res sordide albx, inmaculata.

\section{OBSERV. ANATOMIA.}

Oesophagus habet dilatationem musculosam supra cardiam. Neque oesophagus neque ventriculus magis quam in ceteris avibus ruber; (ut dicit Bechst. 1. c.) Ventriculus vero intus pilis nigris, adpressis, vestitus; (Larvis plenus.) Pilos hos in adultis nunquam vidinus.

\section{HIS TOR IA.}

Migratoria. In sylvis ubique. Circa medium $\mathrm{m}$. Maji, cum frondescit Sorbus aucuparin, in Scaniam redit. Sono læto, æstatis nuntio, statim se prodit. Circa medium Julium silescit. Vescitur insectis et larwis. Nidum nunquam ipsa sibi construit. ovun ponit in nidulo Motacilla vel Sylvice cujusdam, quæilIud diligenter fovet, pullumque fideliter cibat.

\section{? LVIII. CUCULUS RUFUS. Bechst.}

DiaGN : Supra rufus fasciis nigricantibus. Cauda rufa, fasciata, pone apicem album fascia lata nigra. Subtus albidus undulis nigricantibus. 


\section{SYNONYMA.}

Cuculus rufus, Bechst. Nat. D. 2. p. 495. Meyer Vögel Liv - u. Esthl. p. 54.

Cuculus hepaticus, Sparrm. Mns. Carls. III. tab. 55. (fig. mediocris, cauda nimis longa. Specimen de-

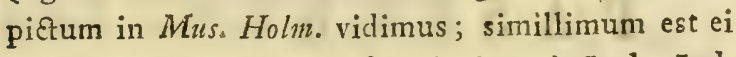
quod ex Museo. Lund. descripsimus.) Lath. Int. On. I. p. 215. n. 25. Retz. Faun. Sv. p. I0O. n. 5 I.

\section{DESCR I P TIO.}

Magnitudine, formâ et proportione partium exacte cum præcedente convenit, colore tantum pennarum differt.

Caput, collum, dorsum et tectrices alarin superiores rufo-brunnea fasciis nigricantibus, angustioribus. Uropygium et tectrices caudx superiores rufa, fere immaculata. Subtus tota avis albida undulis nigricantibus, collo subtus rufo tincto. Remiges exacte ut in Canoro juniore, descripto. Reetrices rufæe fasciis nigris, angulum ad rachin formantibus. Maculx, ad rachin inter fascias, albx. Pone apicem album fascia lata nigra. Tectrices caudx inferiores albæ, rufo tinctæ immaculatx.

\section{OBSERV.}

Vix nisi varietas $C$. canori. In omnibus enim preter pennarum colores cum illo convenit, neque tamen in his tam vehementer a juniore Canoro discedit. Sunt qui hanc pro femina canori habeant, sed perperam. Alii hunc a canoro dignoscen。 dun dicunt sentriculo intus piloso; sed ventriculum intus pilo. sum C. Canorus jwaior quoque habet. Vide supra.

\section{LOCUS.}

Habitat passim cum pracedents. 


\section{ORDO III. \\ PAS SER ES, Lath。 \\ FAMILIA I. \\ C O N I R O S T R E S.}

$\boldsymbol{R}_{\text {ostrum robustius conicum, acutum mandibulis in- }}$ tegris, superiore etiam mobili. Nares plumis setosis reeta. Pedes tenues ambulatorii. viçirs: semina, nuclei, rarius insecta.

\section{GENUS XIIT. \\ CRUCIROSTRA, Meyeri.}

Rostrum robustum, compressum, supra subtusque convexum mandibulis cruciatim curvatis.

Nares rotundatx, plumis setosis tectr.

Pedes ambulatorii.

LIX. CRUCIROSTRA PINETORUM. Mey.

DIAGN : Rostrum valde robustum digico medio brevius. Maxilla magis adunca apicibus obtusioribus; inferiore subtus gibbo-convexa, apice supra dorsum superioris haud adscendente. Color corporis olivaceus vel miniarus. 


\section{SYNONYMA.}

Cracirostrn pinetorum, Meyer Vögel. Liv-und Esthl. p. $7 \mathrm{I}$.

Der Kiefern-Krentzichnabel, Bechst. Nas. D. 2. Auf. 3. B. p. 20. tab. 32. fig. 2. 3.

Loxin curvirostre major, Gmel. Syst. I. 2. p. 843. $\%$. Der grosse Kinumischnahel, Otto in Buff. Naturgesch. der Vögel, X. B. p. 48. cúm fig.

Loxia alia species, Rudb. piet. fol. IOO. (cognita igitur jam Rudbeckio nostro ut Species distin Eta.)

Svecis: Större korsnäbb.

\section{DESCRIPTIO.}

Longit. avis fere 7. poll. Maxilla superior $\frac{6}{8}$ poll; inferior $\frac{5}{3}$ poll. A flexura ale ad apicem 4. poll. Cau. da $2 \frac{1}{2}$ poll. Tibia $\frac{6}{8}$ poll. Digit. med. I. poll. Digit. postic. $\frac{6}{8}$ poll.

Rostrum corneo-fuscum, crassius et robustius quam sequentis; maxilla inferior prasertim lata et robusta marginibus inflexis, subtus gibbo-convexa, obtusior, apice parum incurvato, dorsum maxille superioris haud attingente. Nares rotundatix ad basin rostri sitx, pennis setosis pallide rufescentibus tectx. Oculi magni, eminentes, Iridibus fuscis. Caput pro magnitudine avis magnum. Pedes incarnato fusci. Cauda bifurca.

Caput, collum et totum corpus sordide miniata. Tectrices caudx inferiores griseo-fuscx marginibus lap tis albis. Remiges et Rectrices subtus pallide grisex, supra fusca mnrginibus pallidioribus, vel olivaceis vel albidis. Tectrices alarum superiores fuser minia. to tinetx.

Mas semior: Sordide olivaceus, vertice, uropygio et gula luteo-olivaceis. 
Fenins: Saturate grisen cepite fuscomaculato, dorso oli vaceo tincto, uropygio luteo-viridi; abdomine et cris. so albidis.

OBSERV.

pro mtate cs sexu valde variat.

\section{LOCUS.}

Vivam nondum vidi hanc avem. In Museis vero Holm. Upsal. \&e specimina asservantur. Etiam inter aves Rudbeckii pictas occurrit. E Scania et Siellandia receptam sxpe vidi. Unde liquet aven non esse apud nos tam raram, quamvis, cum sequente confusa, nondum diligenter observata fuerit. Est vero distincta Species in Pinetis quxrenda, ubi semina e strobilis deligit. Etiam in eo a sequente Specie differt, quod mense Majo ova ponit, testante Otto. 1. e.

LX. CRUCIROSTRA ABIETINA, Mey.

Dragn: Rostrum minus validum, longirudine digiti medii. Utraque maxilla acuminata, cruciatim exserta; inferioris apice supra dorsum superioris adscendente. Color corporis olivaceus vel lu:co-miniatus.

\section{SYNONYMA.}

Loxin curvirostra, Lin. Faun. Sv. p. 8I. 11. 224. Retz. Faun. Sv. p. 232. 1. 209. Lin. Syst. nat I. p. 299. I. Gmel. Syst. Nat. I. p. 843. Lath. Ind. Orn. I. p. 370 . 
Bec croisé, Buff. Ois. III. p. 449. t. 2\% fíg. 2. vers.

Germ. Otto X. p. 2I. cum fig. Pl. enl. 218.

Crossbill or Sheld apple, Pen. Brit. Zool. I. p. 319. n. 115. tab. 49. fig. sup. Aret. Zool. II. n. 208. Lath. Syn. III. p. 106. I.

Der Fichten-Krentschmbel, Bechst. Nat. D. 2 Auf. 3. B. p. 4. tab. 32. fig. I.

Crucirostra abictina, Meyer Vügel Liv-und Esth. p. 72 .

Svecis: Mindre Korsnäbb. Kägelrifvarc. Korsskärare.

\section{DESCRIPTIO.}

Longit, avis $6 \frac{x}{2}$ poll. Rostr. $\frac{6}{3}$ poll. A flexura alæ ad apicem $3 \frac{5}{6}$ poll. Cauda $2 \frac{2}{2}$ poll. Tibix $\frac{5}{8}$ poll. Digit. med. $\frac{6}{8}$ poll. Digit. postic. $\frac{5}{8}$ poll.

Rostrum corneo-fuscum, validum, maxillis longioribus, acuminatis, vel dextrorsum vel sinistrorsum ita cruciatis, ut apex maxillx inferioris emineat supra dorsum maxillæ superioris. Nares rotundatx pennis setosis rectx. Iris avellana. Pedes corneo-fusci uncuibus acuminats, nigricantibus. Cauda bifurca. Mns senior: Dorsum viride, Iuteo tinctum; Latere capitis stperius ad nucham fusca striis albis; vertex viridi-flavus. Uropygium favum. Avis subrus viridi-flava. Crissum album maculis fuscis. Remiges et Reftrices subtus pallide grisex, supra saturate fusce marginibus tcnuiter olivaceis. Teßtrices alarum sattt. rate fuscr olivaceo tincrx, majores apice pallide marginatæ.

Mas post priman pennnum mutationem: Dorsum sordide virescens, capite et coppore subtus pallide miniatis. Uropygium miniato-flavum. Crissum griseo-fuscum marginibus pennarum latioribus albis. Remiges et Rectrices ut antecedentis. 
Avis junior ante priman dep? umationem: Supra fusca marginibus pennarum virescentibus. Uropygium pallide lutescens maculis fuscis; subtus sordide albida striis longitudinalibus fuscis. Remiges et Rectrices ut anecedentes.

\section{HISTORIA.}

In sylvis abietinis Svecix. In sylvas frondosas et hortos Scanix, instante hyeme, (non tamen omnibus amnis), gregatim advenit. De hac ave ita habet Linnein Faun. Sv: "Habitat in sylvis abietinis, viktitans "strobilis Abietis, ubi conos primo dejicit rostro ab ar"bore liberatos, quotquot unquam valet, dein singu"Ium per se arripit, pedibus tenet uti Psittacus vel "Sciurus, rostri forfice per singulas squamas frangen"cio semina investigat; et quod singulare, magnitudi。 "ne et colore omnino abietis conum refert, et structu"ra rostri ad semina hujus sola edenda ce eata videtur; "licet et semina Cannabis et baccas Juniperi, Sorbi, "nuces Coryli edere queat. Mira fortitudo parvx avis, "utpote qux bacillos ligneos crassitie digiti, brevi mo"ra levique negotio detruncat."

Supra ramulos superiores pinorum nidum construit. Mensibus hyemalibus ova ponit et pullos exeludit. 


\section{GENUS XIV. \\ L $\quad \mathrm{O} \quad \mathrm{X} \quad \mathrm{I}$ A.}

$R_{\text {ostrum crassum, conicum, haud compressum, su- }}$ pra subrusque convexum: mandibula inferior margine laterali inflexa.

Nares parvæ, rotundatx, ad basin rostri sitx, plumis serosis, brevibus, tectz.

Pedes ambulatorii.

\section{LOXTA ENUCLEATOR.}

DiagN: Maxilla superior longior, apice adunso. Linea alarum duplex aiba. Cáda nigricans.

\section{SYNONYMA.}

Loxin Enuclentor, Lin. Faun. Sv. p. 8I. n 223. Retz. Faun. p. 234. n. 2II. Lin. Syst. Nat. I. p. 299.

3. Lath. Ind. On. I. p. 372. Gmel. Syst. I. p 845.

Gros-bec de Canada, Buff. Ois. IIl. p. 45\%. Pl. enl, I35. f. I.

Greatest Bulfinch, Edw. tab. I23. I24. (fig. med.)

Pine Grosbenk, Pen. Brit. Zool. I. p. 317. n. II4. tab. 49. fig. inter. Aret. Zool. II. n. 209. Sup. p. 64.

Lath. Syn. II I. p. III. n. 5. Suppl. p. 148.

Hanken - Kreusschmabl, Bechst. Nat, D. 2 Auft. 3. B p. 28. 
Svecis: Svenska; Svensk Papgoja; Nottraka; Smolandis perperom: Waktel.

DESCRTPTIO.

Lonngit. aris $7 \frac{x}{2}-8$ poll. Rosti. $\frac{5}{8}$ poll. A flexura alr ad apicem $4 \frac{\pi}{2}$ poll. Canda 3 poll. Tibiz $\frac{7}{3}$ poll. Digit. med. $\frac{7}{8}$ poll.; postic. $\frac{5}{8}$ poll.

Rostrum saturate fuscum, subtus basi pallide incare natum, validum, maxilla superioris dorso compresso, apice longo dependente, eurvato. Nares rotundatæ, pennis setosis nigricantibus tectr. Lora fusca. Iris Pedes fusco nigricantes, cauda subbifurca.

Caput et corpus supra viridia marginibus pennarum Ravis, prasertim in capite et collo; Uropygium et reetrices alarun minores saturate cinerea viridi tincta. Collum subtus et pectus pallide viridia favo tincta. Abdomen cinereum. Crissum albidum. Remiges nigricantes margine exteriore tenui albida. Utraque series tecricum majorum nigricantes apice albo marginatæ. Recrices nigricantes margine tenuiter pallidx.

\section{OBSERV.}

Variat ad nodum avis pro varia atate et sexi. Sunc igitur qua rubro sint, ubi descripta flava.

wariet. a : alta, capire et collo supra, et uropygio lurescentibus, abcomine maculis confertis fuscis. Ala nigricantes albo maculate et striace,... Cauda nigricans.

Mus. Payk.

\section{HISTORIA.}

Habitat in Pinetis superioris Svecix; in Scaniam 1'a. rissime et nunquam nisi instance frigore, advenit. In quibusdam Smolandix plagis interdum hyeme gregatim er copiosa. Vescitur seminibus Pini syllestris et Abie. 
tis, baccarmque Juniperi, Sorbi aucuparix, Crat:egi arix \&.c. Laqueo, baculo alligato, facili negotio a pueris capitur stolida et hebes avis. Mor mansuescit. Bene cantat. In arctois patrir plagis nidulatur.

\section{LOXIA COCCOTHRAUS'TES.}

DiAGn, Rostrum validissimum. Dorsum castane. um, maculâ alarum transversa alba. Remiges mediæ apice rhomber. Gula nigra.

\section{SYNONYMA.}

Loxia Coccothraustes, Lin. Faun. Sv. p. 80. 13. 222. Syst. Nat. I. p. 299. Gmel. Syst. I. p. 844. Briss. Om. III. p. 219. I. Lath. Ind. Orn. I. p. 37 I. n. 4.

Le Gros-bec, Buff. Ois. III. p. 444. tab. 27. f. I. P1. enl. 99. 10. vers. Germ. Otto X. P. 5. cum 2. figg.

Harv-Groshenk, Pe1. Brit. Zool. I. p. 31 6. n. II3. Ark. Zool. II. p. 354. C. Lath. Syn. III. p. 103. 11. 4. Suppl. p. I48. vers. Germ. Bechsr. III. p. 104. 11. 4. Edw. 188. (fig. med.)

Der Kirsch-Rernbeisser, Bechst. Nat. D. 2 Auf. 3. B. p. 35 .

Svecis: Stenknäck.

\section{DESCRIPTIO.}

Iongit. avis 7. poll. A.le extens: I2 poll. Roses. $\frac{6}{2}$ poll. A fexura alæ ad apicen 4. poll. Cauda $2 \frac{2}{3}$ poll. extra alas $\frac{7}{8}$ poll. extensa. Tibiz $\frac{7}{8}$ poll. Dir git. med. $\frac{7}{8}$; Digit, postic. $\frac{7}{4} \frac{5}{6}$ poll. 
Rostrum crassissinum, conicum, mandibulis subr. qualibus, inargine aliguantum infexis; vere et xstate nitide plumbeum, subtus versus basin album; hyeme pallide incamatum apice nigricante. Nares parvæ ro. tunàata ad basin rostri sita, plumulis setosis nigris teeta. Iris albida. Pedes pallide incamati unguibus griseo-fuscis.

Caput et rectrices superiores caudr luteo-brunnea, versus frontem pallidius versus occiput ohscurius castaneum. Collum supra et ad latera pallide cinereum. Dorsum et pennip scapulares saturatc castanea. Gula, lora et linea circa basin rostri nigra. Ceterum avis subtus pallide incarnato-cinerea, versus crissum in album transiens. Remiges nigra versus apicem atro. coeruleo nitentes, latere interiore in niedio maculâ magna alba; I- 4 apice acutx; 5 - I 6 apice rhombeo truncatx. Teetrices minores nigrx; tedrices majores albx, basi tantum nigra, intim: cum intimis Remigibus castancx. Reetrices outusr, niytr, latere interiore (latiore) juxta apicen alba; intermedia binælu. teo-brunnex.

Avis junior versus hyemen copen: Rostrum incarnatum apice nigro-fuscum. Caput, latera colli, pectus et hypochondria incarnato-cinerea, capite tamen saturatiore. Gula fusco-nigricans. Abdomen te crissum alba. Remiges nigro-fusce margine exteriore cinereo. Fascia alarum sordide alba, Dorsun castaneum.

\section{OBSERV.}

Hoc fere colote stiam Femina gaudet. 


\section{HIST ORIA.}

Rara. Migratoria advenit in Scaniam cirea vigesimum M. Aprilis, florescente Anemone nemorosa *). Etiam in Hallandia eam vidi. Estate in sylvis frondosis habitat. Vescitur seminibus et nucleis vairarum arborum et fruticum. Cerasos quoque devastat, unde hortis noxia. Nidum in arboribus et fruticibus ponit. Ova 3.5 viridi-cinerea maculis fuscis striis. que nigro-coeruleis,

\section{LOXIA PYRRHULA.}

Diagn: Caput alæe et cauda nigra. Uropygium album. Dorsum cinereum. Mas: subtus ruber Fenina: subtus rufescenti-cinerea.

\section{SY NONYMA.}

Loxin Pyrrhula, Lin. Faun. Sv. p. 8I. 10. 225. Retz. Faun. Sv. p. 235. n. 212. Lin. Syst. I. p. 300. 4. Gmel, Syst. I. p. 846. Briss. Orn. III, p. 308. n。 I. Lath. Ind. Orn. I p. 387.

Bouvreuil, Buff. Ois. IV. p. 372. t. I7. Pl. enl. I45. Bulfuch, Pen. Brit.Zool.I.p. 320 n II 6 . Aret. Zool.

II. p. 353. A. Lath. Syn, III. p. I45. n. 5 I. Suppl. p. I52.

Der rothbritstige Gimpel, Bechst, Nat, D. 2 Auf. 3. B. p. 55.

Svecis: Domherre. var. a: Loxia Flanengo, Gmel, Syst. I. p. 864. n. 92,

*) Neque tamen omnes e Seaniz demigrant. Hoc ipso anno ad finem $M$. Jariarii avis hujus speciei in horto juxta Lund oćcisa erat et ad me delata,

Nilison Ornith. 
Flamengo Grosbeak, Lath. Syn. Suppl. p. 155. Suecis: Domherre.

\section{DESCRIPTIO.}

Longit. avis $6 \frac{\pi}{2}$ poll. Rostr. $\frac{x}{2}$ poll. A flexura ala ad apicem $3 \frac{6}{8}$ poll. Cauda $2 \frac{6}{8}$ poll. Tibix $\frac{6}{8}$ poll. Digit. med. $\frac{\sigma}{3}$ poll.; postic $\frac{\pi}{2}$ poll.

Rostrum breve, crassum, convexo-conieum, nigrum, maxilla inferior marginibus inflexis, superior aliquantum longior apice acuto. Nares rotundatx, parvæ, ad basin rostri sitæ, plumis setosis nigris tectæ. Iris brunnea. Pedes nigri.

Pileus, genx et mentum nitide atra. Cervix, Dorsum et scapulx coeruleo-cinerea. Uropygium album. Collum antice et ad latera, Pectus et pars posterior ab. dominis pulcherrime carminea. Pars posterior abdominis, crissum et tectrices inferiores alarum et caudæ, albæ. Remiges nigricantes, intericres atro-coeruleo nitentes; intima latere exteriore rubra. Tectrices majores nigra atro-coeruleo nitentes, apicibus cinereoalbis. Cauda cum tectricibus superioribus nigra, atrocoeruleo nitens.

Fennina: distinguitur Dorso cinereo, fusco tinkto; Corpore subtus rufescenti - cinereo; pedibus fuscis. yar. a: Dorsum album postice maculis raris fuscescentibus. Cauda alba lateribus longitudinaliter fuscescenribus. Remiges $4-5$ extus nigræ, ceteræ albx. Caput supra album rubro tinctum. Collum, peetus, $a b$ domen pallide-miniata, albo tincta. L. Flnmengo Mus. Carls. I. t. I7.

\section{OBSERV.}

Hoc idem Specimen, quod are incidendum euravit Ill. Carison 1. $c_{\text {, }}$, in. Museo Paylsullianoadhuc asservatur. Per litte- 
ras a Beat. Dr, Lindroth datas, scio, hanc arem fuisse in caven inclusam, ibique pennas mutasse.

variet. $\beta$. Tota nigl'a, linea alarum longitudinali et tectricibus candz inferioribus albis.

Mus. Payk.

\section{H IS T O R I A.}

Habitat xstate in Svecia superiore; hyeme Scanix sylvas et hortos visitat, tuncque baccis Sorbi aucuparix præcipue delectatur. Avis familiaris et maxime docilis. Cicurata varias melodias cantare discit. Amoris erga conjugem multa exstant exempla. Nidulatur in sylvis acerosis. Ova $3-6$ coerulescenti-alba, fusco maculata.

\section{LOXIA CHLORIS.}

DIAGN: Flavicanti-virescens, remigibus primoribus antice luteis; rectricibus quatuor lateralibus basi luteis.

\section{SYNONYMA.}

Loxia chloris, Lin. Faun. Svec. p. 82. 11. 226. Retz. Faun. Sv. p. 236. n. 2I3. Lin. Syst. I. p. 304. n. 27. Gmel. Syst Nat. I. p. 854 . Briss. Orn. III.

p. I90. n. 54. Lath. Ind. I. p. 382. n. 39.

Le verdier, Buff. Ois. IV. p. I72. t. I5 P1. enl.267. f. 2. Grecnfuch, Pen. Brit. Zool. n. II7. Axct. Zool. II. p. 353. B. - Lath. Syn. III. p. I34. n. 36. Suppi. p. 152. Der grïne Rernbeisser oder Grinling, Bechst. N. D. 2 Auf. 3. B. p. 45.

Svecis: Gröning.

\section{DESCRIPTIO.}

Longit. avis 6 poll. Alx extense IO poll. A flexura alx ad apicem $3 \frac{x}{2}$ poll. Cauda $2 \frac{\frac{2}{8}}{8}$ poll. Tibia $\frac{5}{8}$ poll. Digit. med. $\frac{5}{8}$ poll. Digit. postic. $\frac{x}{2}$ poll. 
Rostrum supra incarnatum subtus pallidum; Nares sotundatæ plumulis setosis, parvulis tectx. Iris fus sca. Pedes fusco-incarnati, unguibus fuscis.

Supra olivac $\in$ a cinereo immixto, fronte, superciliis et uropygio viridi-flavis. Subtus viridi-flava, ita tamen ut collum subtus et pectus magis sint viridia, $a b$. domen magis flavum. Crissum et inferrores caudx tectrices alba, flavo tincta. Remiges nigricantes, margine interiore, presertim versus basin albx; primores latere exteriore a basi rersus picem dilute flava, secundariz extus cinerex, basi virides. Teetrices alarum minores olivacez, margine alæ, et tectricibus inferioribus pallide Aavis. Cauda bifurca, nigricans reftricibus qquatuor lateralibus utrinque a basi ultra medium dilute favis, apice cinereo marginatis, ceteris versus basin olivaceo-, versus apicem cinereo-marginatis. Tectrices superiores longæ cinereæ, versus basin olivaceæ. Femina: Supra griseo-fusca uropygio luteo-viridi; subtus fusco-cinerea pectore et abdomine viridi-flavo inmixto. Crisso sordide albo.

yar. $\alpha$ : Tota alba.

Mus. Lund.

\section{HISTORIA.}

Fistate avem in Scania non vidi, auturnno autem et hyeme ${ }^{*}$ ) solitariam et per familias inveni. Nidum

F) Illam quoque in Hallandia, sub $57^{\circ}$ lar. mense Januarii, frigore rigidissimo et nive altissima 1814 , inter frutices Juniperi et Carduorum semina legentem vidi. Hoc eam ob caussam adfero, quia cel. Bechst. 1. x. p. 50. observarit hane avem, frigore duro, niveque alta, Germaniam relinquere australiores terras petituram. 
in arboribus et fruticibus construere dicitur. Ova 4. 6 pallide argentea, puntis fuscis et violaceis. Vescitur seminibus Cardui, baccarum Juniperi, Lini, Canabis \&c. Mox cicuratur. Docilis avis. Cantus maris haud insuavis. 


\section{GENUS XV. \\ FR I N G I L L A.}

$\boldsymbol{R}_{\text {ostrum conicum, ręurn, acuminatum, (non tam }}$ crassum aut convexum ac antecedentis generis.) maxillis subæqualibus, integris.

Nares parvæ, subovatx, plumulis setosis teetx. Pedes ambulatorii.

\section{FRINGILLA NIVALIS.}

DIAGN: Supra fusca capite et collo cinereis, subtus alba. Remiges secundariæ tęricesque albr. Ręrices laterales nivex, intermedix fuscx, albo marginatæ.

\section{SYNONYMA.}

Fringilla nivalis, Lin. Syst. Nat. I. p. 32I. n. 2 I. Gmel. Syst. I. p. 9 I I. Briss. Om. III. p. I62. t. 15. fig. I. Lath. Ind. Orn. I. p. 440. no I9.

Sizow Finch, Lath. Syn. III. p. 264. 11. I5. vers. Germ. Bechst. III. p. 259. n. I5.

Pinçon de neige ou Niverolle, Buff. Ois. IV. p. I36. vers. Germ. Otto XI. p. I42.

Der Schreefink, Bechst. Nat. D. 2 Auf. 3, B. p. 136. tab. XI. Mus. Payk. 
DESCRIPTIO.

Longit. avis circa $7 \frac{\mathrm{r}}{2}$ poll. . Rostr. $\frac{x}{2}$ poll A. flexura alx ad apicem fere 5. poll. Cauda $2 \frac{\pi}{2}$ poll. Tibix I. poll. Digit. med. $\frac{7}{8}$ poll. postic. $\frac{6}{3}$ poll. Unguis posticus $\frac{7}{76}$ poll. modice curvatus.

Rostrum luteum, maxilla superiore apice supra longitudinaliter nigra. Pedes fusci.

Caput et collum cinerea, fusco tincta, capite superne et auris regione saturatioribus. Gula et jugulum albida. Dorsum et alarum tectrices minores fusca, cinereo marginata. Pectus et abdomen cinereo - alba, luteo levissime tincta. Remiges nigricantes, latere exteriore tenuissime pallidx; I-3 immaculatx; 4- 7 apice albx; 8 intus dimidiato oblique alba; 9 -I5 totæ nivex; intimx colore dorsi. Tectrices proximx Remigum primorum nivex apicibus nigris. Cauda subbifurca, Rectricibus apice obtusis. Laterales I-I. totæ nivex; $2-2 ; 3-3 ; 4-4$. nivex apicibus fuscis; 5.5 . 6. 6. fuscx marginibus et apice pallidioribus. Teetrices caudx inferiores albæ, apice fuscescentes.

O BS.ERV.

Cel. Bechstein 1. co dicit gulam maris esse nigram, albo mao sulatam. Descriptun itaque specimen forte est femina.

\section{LOCUS.}

E Norrlandia recepit avem Nobiliss. Paykull.

\section{LXVI, FRINGILLA MONTIFRIN. GILLA.}

Diagn: Alarum basis subtus flava. Peetus et Scapulæe fulvo-ferruginea. Rectrices nigricantes, extima versus basin extus alba. 


\section{SYNONYMA.}

Fringilla Montifringilla, Lin. Faun. Sv. p. 85. 1. 233. Retz. Faun. 244. n. 221 . Lin. Syst. Nat. I. p. 318. n. 4. Gmel. Syst. I. p. 902 . Eriss. Or11. III. p. 155. Lath. Ind. Or1n. I. p. 439.

Le Pingont d,Ardenne, Euff. Ois. IV. p. I23. vers. Germ. Otto X. p. II5، 127. Pl. enl. 54, fo 2.

The Brambling, Lath. Syn. III. p. 261. 11. I3. vers. Germ. Bechst. III. p. 254. 11. I3. Pen。 Brit. Zool. I. p. 337. n. I26. Aret. Zøol. II. p. 38 I. E.

Der Bergfnk, Bechst. Nat. D. 2te Auf. 3. B. p. 97.

Carduelis Lulensis, Rudb. pict. fol. 88. fig. sup. unde Fringilia Lulensis, Lin. Faun. Sv. p. 86. 11. 234. Retz. Faun. Sv. 245. Icon. fig. 2. Lin. Syst. I. p. 318. Gmel. Syst. I. P. 902 .

Svec: Bergfink. Norrquint.

OBSERV。

Rudbeckii Carduelis Lulonsis, unde Fring. Lulensis Lin. desumea, nulla alia est quạm Fr. montifringillo; ne varietas quidern. Sed figura in Retzii Fauna minus fideliter reddita.

\section{DESCRIPTIO.}

Longit. avis. $6 \frac{\mathrm{r}}{\mathrm{e}}$ poll. Rostr. fere $\frac{\mathrm{r}}{2}$ poll. A fexura alix ad apicem $3 \frac{5}{8}$ poll. Cauda $2 \frac{5}{3}$ poll. Tibix $\frac{5}{8}$ poll. Digit. med. $\frac{\sigma}{8}$; postic. $\frac{x}{2}$ poll.

Rostrum basi favum, apice fuscum. In juniori ave supra corneo-fuscum, versus basin fusco lutescens, subtus flavium apice fuscum. In senioribus supra albidum, subtus pallide luteum, apice tantum fuscum. Iris fusca. Pedes fusco-incarnati.

Variat admodum pro varia atate et sexu:

Mres adultzus: Caput supra et ad latera nigrum, marginibus pennarum rufis. Collum supra etad latera palli- 
de cinerenm. Supra utrumque oculum et ad latera cervicis utringue usque ad Dorsum decurrit fascia nigra. Dorsi pennax versus basin cinerex, versus apicem nigrx marginibus latioribus rufis, unde Dorsum rufum maculis nigris. Uropygium album ad latera nigtum. Penne scapulares, Gula, jugulun et superior pars pectoris rufo-feruginex. Ceterum pectus, abdomen et crissum alba. Ala supra nigre tectricibus minoribus fulvis. Te êrices majores utrjusq̨ue ordinis nigræa apicibus fulvescenti-albis. Remiges nigrx margine interiore a basi albre, margine exteriore tenuius viridi-flavx; intime quntuor rufo extus latius marginatx; 4-15. ma culâ alba latere extcriore infra medium. Tectrices inferiores pulcherrime flavx. Cauda bifurca nigra, rec. trice extima utrinque versus basin extus alba, qua albedo usque ad apicem oblique per rectricem continua. tur. Intermedix binx latius cinereo-Iuteo marginate (sæpius) ceteræ tenuissime luteo - viridi marginate. Fenina forte junior: Caput cinereum rufo tinetum, stria nigricans supra utrumque oculum ad latera cervicis ducta. Corvix et latera colli cinerea. Dorsum nigricans marginibus pennarum latioribus saturate rufis. Gula Pectusque superius pallide cinereo - rufa. Scapula rufx. Strix binz alarm obliqux, pallide rufx (nor albz ut in Mare) cet. anteced.

Mas annosus: Caput et collum superne lateribusque nitide nigra. Dorsum nigrum marginibus pennarum tenuibus rufis. Gula, jugulum, superius pectus et scapulæ luteo-rufa. Tectrices alarum minores, superiores, pulcherrime fulvæ, inferiores flavi: Peetus infe. rius et abdomen alba. Crissum albo-rufescens. var. a: In ceteris Montifringille juniori similis, sed habet maculam pectoris transversam, semilunarem, nigram. Mus. Payk, e Lapponia: 


\section{HISTORIA.}

Habitat astate in Norrlandix et Lapponix sylvis. Autumnali et verno tempore transmigrans, in agris Scanix inter $F r$. coelibes gregaria obvenit. Abit itaque post medium Octobrem; redit initio M. Aprilis. Hieme non occurrit. Vescirur seminibus. Facile cicuratur, nee tamen bene cantat.

\section{FRINGILLA COELEBS.}

Diagn: Dorsum castaneum uropygio luteo-viridi. Alæ nigræ fasciis versus basin binis obliquis albis. Rectrices nigra extimis duabus oblique albis.

\section{SYNONYMA.}

Fringilla coelebs, Lin. Faun. Sv. p. 85. n. 232. edit. Ina p. 76. n. 200. fem. Retz. Faun. Sv. 243. n. 220. Lin. Syst- I. p. 3 I 8. n. 3. Gmel. Syst. Nat. I. p. 90I. Briss. Orn. III. p. I48. 36. Lath. Ind. Om. I. p. 437. 11. I2.

Le Pincort, Buff. Ois. IV. p. I09. fo 4. P1. enl. 54. fig. I.

Chaffinch, Pen. Brit. Zool. I. P. 335. n. I25. Aret. Zool. II. p. 38 I. F. Lath. Syn. III. p. 257. IO. vers. Germ. III. p. 250. n. IO. Suppl. p. I65.

Gemeiner Fink, Bechst. Nat. D. 2 Auf. 3. B. p. 75. Svecis Bofink.

\section{DESCRIPTIO.}

Longit. avis fere $6 \frac{\mathrm{r}}{2}$ poll. Alæ extensæ $10 \frac{\mathrm{r}}{2}$ poll. Rostr. fere $\frac{T}{2}$ poll. A flexura alæ ad apicem $3 \frac{2}{8}$; Cau-

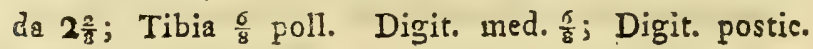
poll. 
Rostrum hyeme pallidum, fere album, apice corneofusco; verno autem et æstivo tempore obscure coeruleum. Nares rotundatæ plumulis setosis teetæ. Iris castanea. Pedes incarnati, digitis obscurioribus. Cauda bifurca.

Caput supra cinereo- coeruleum fronte nigra. Latera capitis ferruginea. Gula, jugulum, pectus et hypochondria incarnato-brumen, colore versus crissum in album tendente. Dorsum et Scapulx castanea, oliva. ceo tincta; uropygium viridi-luteum. Remiges nigrre. margine interiore a basi albo, exteriore tenuiter viridi-flavo, tribus primis exceptis omnes versus basin latere exteriore maculî alba notatx. Tectrices breviores albr, basi tantum nigrr; longiores nigrx apice albo, unde striæ binæ obliqux in ala clausa. Tećtrices infe. riores albæ. Rectrices nigra; I. I. extus dimidiato oblique albx, latere exteriore versus apicem fusco; 2.2. maculâ apicis mangnâ, cuneiformi, albida. Interme. dix binx griseo - fuscx.

Femina: Caput et collum supra griseo-fusca. Latera capitis, collum antice et pars superior pectoris rufo-cinerea; cetera pars peêtoris, abdomen et crissum albida, Rostrum vere cinereo-fuscum, hyeme albidum.

\section{HIST ORIA.}

In Sylvis ubique frequens. Plurimæ dinigrant in. stante frigore. Medio M. Martis peracto redeunt et $a b$ uno in alterum hortum per familias volitant. Ineunte M. Aprili, coelo sereno cantum lætum, verisque nun. tium, ex arboris cacumine modulari incipiunt. Mensibus vero insequentibus horti et luci et sylvæ sonos lones et duices undique resonant. Nidum pulcherri. mum, semiglobosum in ramo arboris ponunt. Ova 4-5. pallide virescenti-coerulea, punctis striisque fu- 
scis. Prolem reneram Insefis alunt. Vescuntur praterea granis varia speciei, seminibus berularum, alno. rum \&c. glandibus fageis \&c. Collecta messe, agros Scania innumerabilibus fere gregibus visitant. Hyeme instante, australiores terras sensim petunt. Paucx tantum, nec mares soli, nobiscum hibernant.

\section{FRINGILLA DOMESTICA.}

DIAGN: Caput supra griseum. Dorsum rufo - brunneum maculis nigris. Remiges et Reetrices saiurate fuscx. Fascia alarum alba, solitaria.

\section{SYNONYMA.}

Fringilla domestica, Lin. Faun. Sv. p. 88. n. 242. Retzo Faum. Sv. p. 249. n. 228. Lin. Syst. Nat. I. p. 323. 11. 36. Gmel. Syst. I. p. 925. Lath. Ind. Orn. I. p. 434. n. I.

Passer domesticus, Briss. Orn. III. p. 72. n. I. Le Moinenu franc, Buffo Ois. III. p. 474. t. 29. f. I. vers. Germ. Otto X. I24. cum. fig 2.

House Sparrow, Pen. Brit. Zool. I. p. 338. n. 127. Aret. Zool. II. p. 382. G. Lath. Syn. III. p. 248. in. I. vers. Germ. Bechst. III. p. 242. n. I. Suppl. p. I63.

Houssperling, Bechst. Nat. D. 2 Auf. 3. B. p. IOZ. Svecis: Gråsparf, Gräspink。 Husfrnk.

\section{DESCRIPTIO.}

Iongit. avis fere 6. poll. Alx extensæ $8 \frac{x}{2}$ poll. Rostr. $\frac{\mathrm{r}}{2}$ poll. A. flexura alx ad apicem 3 poll. Cauda $2 \frac{r}{8}$ poll. Tibiz $\frac{6}{2}$ poll. Digit. med. $\frac{5}{8}$ poll.; postic. 焉 poll. 
Rostrum crassum, supra fuscum, subtus basi favum; hyeme supra fusco incarnatum. Nares rotundate, plumulis setosis tecte. Iris griseo-lutes. Pedes pallide griseo-rusci, digitis subrus sordide luteis. Cauda aliquantum bifurca.

Caput supra cinereun rufo tinctum; pone oculum fascia rufo-fusca, postice, ad latern cervicis, latior. Macula circa oculos nigra. Supra oculos lineola alba. Dorsum et Scapula rufo-brunnea maculis longitudinalibus nigris. Uropygium et tectrices caudæ gुrisea, rufescenti tincta. Gula et jugulum usque ad sternum nigra pennis cinereo marginatis. A maxilla inferiore ad latera juguli stria albida. Pectus et Hypochondria pallide grisea, rufo-tincta. Abdomen albidun. Crissum griseum marginibus pennarum albidis. Remiges saturate fuscr, primores tenuius, secundariz latius rufo - brunneo extus marginata. Tectrices minores alarum saturate rufx. penultime nigrx apicibus albis; proxima nigre rufo-brumneo latius marginata. Rectrices saturate fusce rufescenti tenuiter marginatx.

Femina: Caput rufo griseum. Supra oculos linea albida postice latior. Fascia alarum rufescenti-alba. Tota subtus sordide griseo - albida.

war a. Fringilla candida plumis ac pennis omnibus, rostre, pedibus digitisque albis. Mus. Carls. I. ז. 20.

ver. $\beta$. Tota sordide alba. Mus. Fayk.

war. $\mathscr{\gamma}$. varia, pennis albis ubique immixtis. Capta ad Lund, initio Mens. Maji.

var. 8. Supra pallide fusco ferruginescens, subtus et canda albida, sordide lutescenti tincta. Rostr. et pedes albi. Mus. Payk.

var. $\varepsilon$. Caput, colium et tota avis subtus sordide aiba luteo tincta. Remiges fasciâ superiore alba, altera In- 
tea. Cauda fusca rectricibus laterilibus dimidiato ob. lique albis.

var. $\zeta$. Alt fasciis binis albis, obliquis, quarum superior major. Remiges et Rectrices tenuiter albo marginatix. Fenr. Mus. Upjsal.

\section{HISTORIA.}

Juxta domicilia hominum ubique. Permanens. Cantus haud quidem amoenus est; pendentibus tamen alis, expansâque et erecta caudâ, circa thori sociam saliens, garritu læto: Dip, dack, dack! Zitschischi! ver instans celebrat. Coitus cupidissima avis. Nidulatur jam ad finem Mens. Martis; sub tectis, in murorum rimis, in arborum cavis. Etian nido Hirundinis interdum potitur. Ova 5-6, rarius $7-8$, virescenti alba, punctis fuscis. Vescitur insectis et seminibus.

\section{FRINGILLA MONTANA。}

Diagn: Caput et collum sipra rufo-fusca. Dorsum maculis ferrugineis et nigris. Remiges er Rectrices saturate fuscx. Linex alarum bina, albæ.

\section{SYNONYMA.}

Fringilla montann, Lin. Faun. Sv. p. 88. x. 243. Retz. Faun. p. 250. n. 229. Lin. Syst. 1. p. 234. 11. 37.

Gmel. Syst. I. p. 925. Lath. Ind. I. p. 433. 11. 2. Passer montanus, Briss. Orrz. III. p. 79. 2.

Le Friquet, Buff. Ois. III. p. 489. t. 29. fig. 2. Vers.

Germ. Otto X. p. I63. cum fig. PI. enl. 267. f. I. Tree or monninin Finch, Pen. Brit. Zool. I. p. 339. n. I28. tab. 52. fig. sup. Arg. Zool. II. p. 246. Lath. Syn. III. p. 252. n. 2. Suppl. p. 163. 
Feldsperling, Bechst. Nat. D. 2 Auf. 3. B. p. 124.

Svecis: Fältsparf. Trädsparf.

\section{DESCR IP TIO.}

Longit. avis $5 \frac{\pi}{2}$ poll. Alæ exrens $x$ 8 $\frac{r}{2}$ poll. Rostr. $\frac{3}{8}$ poll. A flexurn alix ad apicen $2 \frac{6}{5}$ poll. Canda $2 \frac{\pi}{8}$ poll. Tibia $\frac{6}{8}$ poll. Digit. med. $\frac{5}{8}$ poll. Digit. postic. $\frac{x}{2}$ poll.

Rostrum nigro-fuscum, basi inprimis subtus luteum. Iris castanea. Pedes sordide incarnati. Cauda xqualis.

Caput supra et cervix pulchre rufo-fusca (casta. nea). Latera capitis alba macula nigra. Circa collum torques albus, in cervice interruptus. Dorsum anticum et Scapulx griseo - ferruginex maculis nigris. Dor. sum posticum fusco-griseum, immaculatum. A gula nigra decurrit stria concolor. Latera gula et juguli alba. Pectus pallide cinereum (argenteum). Abdomen album. Crissum sordide album, griseo-fusco maculatum. Hypochondria fusco grisea. Remiges saturate fusce, primores interrupte-, secundariz continuoextus ferrugineo marginatz. Teßrices minores ald. rum basi rufe; penultime nigra apicibus albis; proxime nigre marginibus ferruginets apicibus albis. Rectrices saturate fuscæ grisescenti-luteo marginata.

Femina: Capite supra pallidiore, maculâ gule et laterum aapitis nigra, minor.

\section{HISTORIA.}

In hortis, senticetis et fruticetis juxia agros. Nhe merosior, in Scania saltem, quam antecedens. Sem. per gregaria excepto propagationis tempore. Hibernat nobiscum. Nidulatur in cevis arboribus ex. gr. Sa- 
licibus, Malis \&c. Ova 5-\%. albo grisea, maculis rufis et fuscis. Vescitur insectis et seminibus.

\section{FRINGILLA CANNABINA.}

Dracin. Remiges primores Rediricesque nigra urroque nargine albæ. Pectus maris senioris sanguineum; fenince sordide flavescens striis fuscis.

\section{SYNONYMA。}

Fringilla camabina, Lin, Faun. Sv. 87. n. 240. Retz. Faun. Sv. p. 247. n. 226. Lin. Syst. Nat I. p. 322. i. 28. Gmel. Syst. I. p. 9 I6. Lath. Ind. I. p. 458. n. 82 .

Linaria vubra major, Briss. Om. III. p. 135. 30. (senior).

Linnin, Briss. Orn. III. p. I3I. n. 29. (mas senior.)

Fringilla Linota, Gmel. Syst. I. p. 916 . Lath. Ind. I. P. 457. 11. 8I.

La grande Linotte des Vignes, Buff. Ois. IV. p. 58. vers.

Germ. Otto XI. p. 5. Pl. enl. 485. f. I.

In Linotte, Buff. Ois. IV. p. 58. t. I. Pl. enl. I5I. f. I. Greater Redhended Limet or Redpole, Pen Brit. Zoole I. p. 343. n. I3I. tab. 54. Aret, Zool. II. 11. 26 I. Lath. Syn. III. p. 304. n. 74. vers. Germ. Bech. II I. p. 294. n. 74. Sup. p. I67.

Limet, Pen. Brit. Zool. I. p. 342. n. I30. Lath. Syn. III. p. 302. n. 73. vers. Germ. Bechst. III. p. 29 I. n. 73.

Gemeiner Hürifing, Bechst. Nat. D. 2 Auf. 3. B. p. I4I.

Svecis: Hämpling. 


\section{DESCRIPTIO.}

Longit, avis $5 \frac{1}{2}-6$ poll. Alæ extensix $9 \frac{r}{2}$ poll. Ro. str. $\frac{3}{3}$ poll. A flexura alx ad apicem $3 \frac{x}{8}$; Cauda $2 \frac{2}{8}$ poll. Tibia $\frac{5}{3} ;$ Digit. med. $\frac{6}{8}$ poll. Digit. post. $\frac{1}{2}$ poll. Canda extra alas extensa I. poll.

Rostrum basi crassum, teres, apice acutum maxillis subæqualibus, supra coeruleo-nigrum, subtus griseoincarnatum apice nigricante. Nares parvæ rotundatæ, plumulis tekx. Iris saturate castanea. Pedes incarnato - fusci, unguibus nigris.

Pro xtare et anni tempestatibus variant admodum mares:

Mas sentior verno tempore:

Frons sanguinen marginibus pennarum (interdum) pallidis. Ceterum caput cinereum, circa oculos et ad latera colli maculs albida. Dorsum, scapulæ et alarum tectrices minores fusco-rufa. Gula et collum antice alba luteo tincta striis longitudinalibus fuscescentibus. Pectus sanguineum pennis tenuissime albido marginatis. Ceterum tota subtus alba, pone pectus sanguineum et in hypochondriis luteo-tincta. Remiges nigræ margine interiore albx; primores I-4. tenuius, 5-9 latius margine exteriore fere ad apicem albx; secundarix apice albo marginata, latere exteriore griseo - rufescentes. Teetrices proximx latere interiore nigr:e, exteriore pallide-rufx. Cauda bifurca, Reetricibus nigris utroque margine albis. Tectrices superiores nigrt marginibus latius albis.

Mns autumni empore:

Frontis et petoris sanguineus color vix conspicitur nisi sejunctis pennis. Margines enim sordide albidz latiores sunt. Cervix et colli latera cinerea, capite supra maculis nigricantibus. Dorsum saturate fuscum marginibus pennarun rufescentibus. Tectrices caudz Nilsson Oruith.

$\mathrm{K}$ 
superiores albo - rufescentes striâ ad rachin nigra. Reetrices dur intermedia rufescenti-marginatio. Ceterum ut antecedens.

Fernina:

Caput cinereum supra striis longitudinalibus saturate fuscis. Dorsum fusco-rufum marginibus pennarum pallidioribus, rachibus saturatioribus. Subtus sordide lutescenti - alba striis in pectore et hypochondriis griseo-fuscis. Remiges et rectrices ut in mare.

\section{HISTORIA.}

Haud rara. Aestate in firticetis habitat; hyeme arva frequentat. Aliz manent, alix demigrant. $\mathrm{Hx}$ per familias redeuntes, jam medio M. Martis de arboribus hortorum cantant; Cantus egregius. Nidificant in fruticetis et inter arborum minorum ramulos. Ova

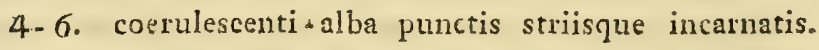
Victitant seminibus. Facile cicurantur.

\section{FRINGILLA FLAVIRO- STRIS, Lin. Faun. Sv.}

(Tab:IV)

Diagn: Supra nigricans marginibus pennarum rufescenti-lureis; subrus rufescenti-lutea pecto. re maculis fuscis. Abdomen album. Remiges et rectrices nigricantes. Reetrices et intermedix Remiges albo marginatæ.

\section{SYNONYMA.}

Fringilla fuscarostro flavicante. Sved. Riska. Rudb.av. pict. fol. 58. unde. 
Fringilla flavirostris, Lin. Faun. Sv. p. 87. 11. 239. Pallas. Iter II. p. 710.

The Tvite, Pen. Brit. Zool. I. p. 346. 11. 133. tab. 53. Arct. Zool. II. p. 380. C; vers. Germ. II. p. 354. C. Lath. Syn. III. p. 307. n. 76. var. A. vers. Germ. III. p. 297. n. 76. var. A.

Gran Irisk, Leems Finn. p. 256.

\section{OBSERV.}

Hallucinantn plerique Ornithologi de Synonymia hujus avis. Brisson et Bufton varietatem purant Fring. coelibis, quo nihil falsius. Cum scriberet cal. Retzius Faunam Svecicam, rondum nec. F. flavirostrem nec Iconem Rudbeckinuan viderat. Ab initatione vero hujus iconis, ab Assess. Lindroth male factà, et a Descriptione Linnei mancâ seductus, aven pro femina $F r$. Linaric habuit. Cum vero avem ipsam postea vidit, illam eșse distinctam specien, ipse monuit. Figura autem Lindrothiana nec flavirostrem, nec Linariam bene refert. Icon Rudbeckii, q̨uam ipse vidi, melior est. Frischs Vögel Deutschl. Hafniae quondain vidi, er ni memoria vehementer fallit, Linaria ejus peczore subluteo, Tab. X. f. I., quam ad cannabinam refert Bechst. hac ipsa est flavirostris. Rostrum enim parvum, luteum, huic bene, minime vero canzanbina competit. A Pennarit bene descripta est 1.1. cc. nomine Tvite, quatn tamen avem Fringilla montizm varietatem facit Lathnm. Quznain sit avis Fr. montizizm Lath. (montana Briss.) nescio; in omnibus tamen cum Bechsteinie consentire non possum. Cel. Pallas 1. c. dicit: in pecírore summitatibus penzaram sape puniceo variantibus \&c. Hoc sine dubio non nisi rstate fit; quod ego tamen non observavi, quamvis avern per totum annum in cavea habui.

\section{DESCRIPTIO.}

Longit, avis $5 \frac{3}{8}$ poll. Alx extensa $9 \frac{\mathrm{T}}{2}$ poll. Rostr. $\frac{2}{8}$ poll. A flexura als ad apicem 3. poll. Cauda $2 \frac{\pi}{3}$ poll; extra alas extensa $\mathbf{I} \frac{2}{3}$ poll. Tibix $\frac{5}{8}$ poll. Digit. med. $\frac{5}{8}$ poll.; postic. $\frac{x}{2}$ poll: 
Rostrum breve teretiusculum acutum, mandibulis aqualibus, flavum apice nigricante. (In feminae rostro stria longitudinalis nigricans a basi ad apicem superne ducta.) Nares rotundatx plumis setosis testaceis, tectr. Iris fusca. Pedes maris nigri, feminiz fusci. Cauda bifurca.

Caput, collum, dorsum, scapulix, uropygium supra nigro-fusca pennis rufescenti-luteo (sordide fulvo) marginatis. Uropygium maris praterea rubicundo colore tinftum. Gula, jugulum, peetus et Hypochon. dria rufescenti-lutea (sordide fulva) pectore superiore et hypochondriis fusco maculatis. Abdomen et cris. sum alba, immaculata. Remiges nigræ margine interiore a basi albidx, ( -4 fere aquales, ceteris longiores; 8 -I5 apice emarginatæ.) prima quatuor margine exteriore tenuissime pallida; sequentes quinque ( 5 9) margine exteriore latius albr; secundaria apice albido marginatx. Remiges subtus pallide cinerea, Tectrices superiores minores suturate fusca rufescenti obsolete marginatix; Tectrices longiores (proximx) saturate fuscr apicibus rufescenti-albis. Rearices nigro-fusca margine exteriore tenuius, interiore latius albx; par intermedium utroque margine rufescenti-luteo. Teetrices caudz inferiores griseo-fusce, albo latius marginatz.

\section{HISTORIA.}

Arctoarum regionum incola, frigore intenso austra liores interdum Patriz plages petit. Omni fere hyeme gregaria obvenit in Scania prope Lund, ibique capitur cun congeneribus. In Siælandiâ captam quoque vidimus. In Mus. Upsal. specimen ex Uplandia asservatur. Stupida et mansueta avis, captu facillima. Se. minibus Brassica et Sinapis arvensis, aliisque quz hye- 
me supersunt, delectatur. Facili negotio cicuraturCantus vero haud gratus, nimis enim garriens et stri. dul..s.

\section{FRINGILLA LINARIA.}

DIAGN : Vertex coccineus, gula nigra; Remiges et Reatrices nigro-fusca, fasciis alarum binis albidis.

\section{SYNONYMA.}

Fringilla Linaria, Lin. Faun. Sv. p. 87. n. 241. Retz. Faun. Sv. p. 248. n. 227. Lin. Syst. Nat. I. p. 322. n. 29. Gmel. Syst. I. p. 9 I7. Lath. Ind. I. p. 82. n. 83 .

Linaria rubra minor, Briss. Orn. III. p. I38. n. 3 I. Sizerin ou petite Linotte des vignes Buff. Ois. IV. p. 216.

vers. Germ. Otto XI. p. 309. cum. fig. Pl. enl. I5 I. fig. 2.

Lesser red-headed Linnet or Redpole, Pen. Brit. Zool. I. p. 344. n. 132. t. 54. Art. Zool. II. n. 262. Lath. Syn. III. p. 305. 75. vers. Germ. Bechst. III. p. 295. 11. 75.

Bergzeisig, Bechst. Nat. D. 2te Auf. 3. B. p. 23 I. Svecis: Gråsiska. Iris. Winterhämpling.

\section{DESCRIPTIO.}

Longit. avis $5 \frac{3}{8}$ poll. Rostr. $\frac{5}{6}$ poll. A flexura alæ ad apicem fere 3. poll. Cauda $2 \frac{1}{8}$ poll. Tibia $\frac{5}{3}$ poll. Digit. med. $\frac{1}{2}$ poll. Digit. post. $\frac{x}{2}$ poll.

Rostrum breve, acutum valde compressum, flavum, supra et subtus stria longitudinali nigra. Nares totundatz, pennis setosis, longioribus, numerosis, ru- 
fescentibus plane tectx. Iris castanea. Tibix nigro. fuscr digitis et unguibus nigris.

Vertex coccineus fronte grisescente, albido irrorata. Occiput, cervix, dorsum, scapulæ, uropyginim albida maculis longitudinalibus nigro-fuscis, i. e. singula penna nigro - fusca lutescenti-albo marginata. Latera capitis, collique et pefus albida luteo tineta maculis parvis fuscis. (Pectus maris verno tempore sanguineum). Gula nigra. Inter oculum et rostrum macula parva nigra. Abdomen et crissum alba; Hypochondriis et caudre tectricibus inferioribus fusco-striatis. Remiges nigro-fuscx margine interiore a basi alba; secundaria apice albo marginate, intimx latius albo marginatæ. Teetrices utriusque ordinis nigro-fuscæ apicibus albis, unde fasciæ alarum transversæ dux, angustx, quarum superior a pennis scapularibus fere obtegitur (unde alii auctores nnam, alii duas fascias dicunt). Cauda valde bifurca. Rectrices nigro-fusca albo tenuiter marginatx.

Fenina: distinguitur colore supra obscuriore, scil. nucha, cervix et dorsum anticum nigro - fusca marginibus pennarum lutescentibus, dorsum posticum pennarum marginibus albis. Lineæ alarun lutescenti - albæ. Gulx macula nigra minor marginibus pennarum albidis. Pectoris et hypochondriorum maculæ nigro - fuscæe, magis conspicua.

anar. «. Rostrum et pedes albidi. Vertex et pectus coccineo tincta. Corpus præterea album pennis fuscis in scapulis et superiore dorso immixtis. Alæ et cauda nigro-fuscæ pennis albis insertis. Mus. Lund. IIl. Wachtm. vivam coluit aviculam.

vnr. $\beta$. Albida maculis parvis longitudinalibus fuscis supra numerosis subtus raris adspersa. Vertex pallide coccineus.

Mus. Payk. 


\section{H IS T ORIA.}

In septentrionalibus Sveciæ reg:onibus æstatem degens, hyeme instante in Scaniam gr:garia advenit, et in alnetis pracipue occurrit. Vescitur seminibus alni, cardui, arte risie, lini, camnabis \&rc. Instante vere plagas nostras relinquit arctoas petitura. Nidum in arbusculis minoribus ponit. Ova 4- 6 coerulescenti - viridia, extremitate crassiore rubicundo maculata. Virgulis visco illitis, a pueris capitur. Facile cicuratus. Docilis nec cantus suavis.

\section{FRINGILLA CARDUELIS.}

Diagn: Facies coccinea. Remiges latere exteriore ulra medium lutex. Reetrices duæ extimæ introrsum medio, reliqua apice albx.

\section{SYNONYMA.}

Fringilla corduelis, Lin. Faun. Sv. p. 86. 11. 236. Retz. Faun. Sv. p. 245. n. 223. Lin. Syst. Nat. I. p. 31 8. n. 7. Gmel. Syst. Nat. I. p. 903. Briss. Orn. III. p. 53. n. I. Lath. Ind. Orn. I. p. 449, n. 58. Ie chardonneret, Buff. Ois. IV.p. 187. t. IO. vers. Germ.

Otto XI. p. 260. 264, cum. fig. Pl. enl, n. 4. f. 1. Goldfink, Pen. Brit. Zool. I. p. 332. n. I24. Aret. Zool. II. p. 283. H. Lath. Syn. III. p. 28 I. n. 5I. vers.

Germ. Beclist. III. p: 274. n. 5 I.

Distelzeisig, Bechst. Nat. D. 2 te AuA. 3. B. p. 200. Svecis: Steglitsa.

\section{DESCRIPTIO.}

Longit. avis fere $5 \frac{\pi}{2}$ poll. Alx extensx $8 \frac{\pi}{3}$ poll. Rostr. $\frac{x}{2}$ poll. A flexura alæ ad apicem. $3 \frac{T}{2}$ poll. Caun. 
da $2 \frac{\mathrm{T}}{3}$ poll. Tibix $\frac{5}{8}$ poll. Digit. med. $\frac{3}{8}$ poll; postic. 굴 poll.

Rostrum acutissimum, versus apicem aliquantum decurvatum et compressum, albidum apice fusco. Iris castanea. Pedes pallide fusci. Cauda aliquantum bifurca.

Frons, genæx et guia coccinea loris et lineâ circa basin rostri nigris. Vertex niger demittit fasciam nigram in latera colli utrinque. Occiput macula albida. Pone oculos fascia alba descendit et cum jugulo albo se conjungit. Cervix, scapulx et dorsum anticum castanea. Uropygium albo fuscoque mixtum. Tectrices caudx superiores albx. Latera pectoris et abdominis pallide castanea. Medium pectus, abdomen et crissum alba. Remiges nigræx (atræ) maculâ apicis alba; extima inmaculata; ceteræ latere exteriore ultra medium flave. Tectrices nigræ apicibus longiorum flavis. Rectrices nigrx, laterales dux vel tres latere interiore pone apicem macula majori alba, reliqux apicibus albis.

Femina dignoscitur lineâcirca basin rostri rufescente; albedine laterum capitis fuseescente; Tętricibus alarum minoribus fuscis.

\section{HISTORLA.}

Passim. Hyeme quam xstate in Scania frequentior. In hortis et fruticetis agros adjacentibus inque sylvis nidulatus. Ova 4.6 pallide glauca, rufo maculata. Vescitur seminibus. Facile cicuratur avis pulcherrima. Egregie cantat. In cavea cum Fr. cannaria coit, unde Fr. cardueles hybrida, jam patri jam matri magis similes oriuntur. Avis lxta, docilis, agilis. 


\section{FRINGILLA SPINUS,}

Diagn: Supra viridis subrus lutea. Remiges versus basin extus lutex, primis quatuor immaculatis. Rectrices flavæ apicibus nigris.

\section{SYNONYMA.}

Fringilln Spinus, Lin. Faun. Sv. p. 87. n. 237. Retz. Faun. Sv. p. 246. n. 224. Lin. Syst. Nat. I. p. 322. 11. 25. Gmel. Syst. Nat. I. p. 914. Lath. Ind. I. p. 452.17 .6 .5 .

Ligkrinus, Briss. Orn. III. p. 65.

Le Tarin, Buff. Ois. IV. p. 22I. vers. Germ. Otto XI. p. 3 II. cum. fig. Pl. enl. 485 . f. 3.

Siskin, Pen. Brit. Zool. I. p. 340. n. 129. tab. 53. Arct. Zool. II. n. 243.id. p. 383. I. Lath. Syn. III. p. 289. n. 58. vers. Germ. Bechst. IIL.p. 28 I. n. 58. Sup. p. 166.

Erlcnzeisig, Bechst. Nat. D. 2te AuR. 3. B. p. 220. Svecis: Grönsiska.

\section{DESCRIPTIO.}

Longit. avis $4 \frac{6}{5}$ poll. Rostr. $\frac{3}{8}$ poll. A flexurâ alæ ad apicem fere 3 poll. Cauda $I \frac{6}{8}$ poll. Tibix $\frac{2}{16}$ poll. Digit, med. $\frac{9}{16}$ poll. Digit. post. $\frac{3}{8}$ poll.

Rostrum acutissinum griseum versus apicem compressum, fuscum. Iris fusca. Pedes fusci. Cauda bifurca.

Frons, vertex et gula nigra marginibus pennarum verticis cinerascentibus, gulx luteis. Cervix, dorsum, scapulæ et longiores caudæ tectrices olivacex, dorso striis obsoletis nigricantibus. Teetrices alarum minores, uropygium, linea per oculos, jugulun 
et peđus virescenti - luted. Abdomen albun. Crissum et bypochondria alba luteo tincta striis nigris. Remiges nigra, primores extus viridi-luteo tenuiter, intima albido late marginata; omnes, quatuor extimis exceptis, latere exteriore juxta basin pallide flava. Tectrices majores nigræ, apice lutex. Rectrices pulcherrime favæ apicibus nigris virescenti - favo tenuiter marginatis, extimæ latere exteriore fere ad basin et in termedix totæ nigræ.

Fenina: Dorsum griseo-viride maculis nigris. Gula et latera albida. Pectus album viridi tinctun maculis nigricantibus. Pedes griseo-fusci.

varietas! a. Nigricans maculis supra rarioribus, subtus confuentibus luteo-viridibus, splendidis, puliherrime ornata. Culta fuit.

Mius. Payk. Mus. Upsal.

\section{H IS T OR I A.}

Passim. Autumnali tempore et hyeme turmatim in sylvis, præsertim inter alnos et betulas, quarum seminibus delectatur, obvenit. Fstate avem in Scania nondum vidi. In Hallandia vero et aliis provinciis superioris Svecix in pinetis, locis montmosis nidulatur. Ova 5-6 griseo-alba, punctis rufo-purpureis. Facile cicuratur avis gilis, docilis, mansueta. Bene cantat.

LXXV. FRINGILLA FLAMMEA, Lin, Faun.

Disçs: Fusca, crista fammea. Rud. pict. Habitat in Norrlandia. Lin. faun. Sv. p. $87 \cdot$ n. 238. 
OBSER V.

Inter aves Rudbeckii piatas, quas Holmix vidinus, hæc non reperitur. Inter icones autem ejusdem auctoris, quas possider Bibliotheca Grilliana Söderforsensis, exstare refertur. Sola igitur est inter aves, quarum mentio fit in Fauna Linneana, quam nonduin vidi. Cel, autem Ifeyeri (in Beschreibung der Vögel Livuxd Esthlauds p. 77) ni fallor, huc pertinet, quam vero descriptionem, eam ob caussam adferendam putavi, ut Ornithologis, quibus Arctoas aves investigare et iconem Rudbeckii allatam examinare contigerit, ansain praberem avem Rudbeckii et Meyeri conferendi.

FRINGILLA ERYTHRINA, Meyeri.

Mas: Rostrum pallide corneum; Iris brunnea; pedes fusco-incarnati. Vertex, gula, superior pars pectoris et uropygium pulchre carminea; Dorsum et alæ grisea, roseo colore tincta; $\mathrm{Ab}$ domen et crissum albida. Longit. $5 \frac{\pi}{2}$ poll.

Femina: Supra grisea, fuscescenti tincta; Subtus sordide alba; Gula, collum et pectus maculis longitudinalibus pallide griseo-fuscis; Remiges et Rectrices flavo-virescenti tenuiter marginatr. Longit. $5^{\frac{T}{4}}$ poll.

\section{SYNONYMA.}

Loxin erythrina, Pallas nov. comment. Petrop. XIV. p. 587. t. 23. f. I. mas et femina. Gmel. Syst. I. p. 864. n. 91 .

Loxin erythran. Endler et Scholz, Beitr. zur Schlesischen Naturgesch. Jahrg. I. p. I7. t. 5. mas. Jahrg. 2. p. 185 t. 47. femina. Naumanns Vögel Nachtr. 3 tes Heft. p. 25. t. 20. f. 40.

Loxin cardinalis, Beseke p. 77. n. I66. 
Fringill a fammen, Gmel. Lin. Syst. I. p. 9I5.n. 26.

Fringilla rosca, Gmel. Syst. I. p. 923. n. 9 I.

Fringili purpurea, Gmel. 1. c. p. 923. n. 90.

Fringilla obscisa, Gmel. 1. c. p. 862. n. 88. femina. (vide cel. Meyer. l. c.)

\section{OBSERV.}

Si Fringills fammea Lin. hæc est (quod pro certo dicere non possum, cum Iconem Rudbeclianam a Linné citatam nondun viderim) et si figura Meyeri fideliter reddita est; potius ad Loxias quas ad Fringillas Linneanas referenda mihi videtur hac species. 
GENUS XVI.

\section{E M B E R I Z A.}

$\boldsymbol{R}_{\text {ostram subconicum, mandibula superiore angu- }}$ stiore versus apicem compressa; inferiore latiore lateribus inflexo-coarctara; utraque basi deorsum a se invicem discedente.

Palatum tuberculo osseo.

Nares rotundatx, juxta basin rostri, prope se invicem positæ, plumis setosis teetr.

pedes ambulatorii.

DISSECTIO. I.

Unguis posticus rectior, digito longior.

\section{IXXVI. EMBERIZA LAPPONICA.*)}

DiAgN: Dorsum nigricans marginibus pennarum ferrugineis. Supercilia alba. Reetrices duæ

* Caussam cur inter Fringillas ab Ornithologis nunerate sit. hac avis, ego salecm videre non possum. Forma enim rostri, a quo character genericus imprimis sumendus, cum ceteris Emberizis exacte convenit. Etiam tuberculum palari adest non minus conspicuum quam in Ember. nivali, Schoericlo et aliis, quas ad hoc Genus numerandas nemo dubieavit. 
extimæ maculâ apicis cuneiformi alba. Vertex: Maris nizer. Cervix rufo-ferrugines.

\section{SYNONYMA.}

Fringilla Lapponica, Lin. Faun. Sr. p. 86. 17. 235. Retz. Faun. p. 242. 1. 219. Fabric. Fann. Groen. p. I19. 11. 82. Lin. Syst. I. p. 3I7. 13. I. Gmel. Syst.

I. p. 900. Lath. Ind. Orn. I. p. 440. n. I8. Mus.

Carls. fase... (Figura præsertim feminx mala). Fringilla cnlcarata, Pallas Iter II. p. 710. n. 20. t. E. Fringilla montana, Briss. Om. II. p. 160.

Le grand montain, Buff. Ois. IV. p. 134. vers. Germ. Otto XI. p. I33. I36. I39.

Lappland Finch, Arct. Zool. II. n. 259. Lath. Syn. III. p. 263. n. I4. Vers. Germ. Bechst. III. p. 256. n. I4.

Grauer Sporner, Bechst. Nat. D. 2 Auf. 3 B. p. 246. Svecis: Lappsparf. Mus. Lund. Payk. Ups.

\section{DECRIPTIO.}

Longit. avis $6 \frac{\pi}{2}$ poll. Rostr. $\frac{3}{8}$ poll. A flexura al ad apicem fere 4. poll. Cauda $2 \frac{1}{2}$ poll. Tibia $\frac{7}{3}$ poll. Digit. ant. intermed. $\frac{5}{8}$ poll. postic. $\frac{\frac{T}{x} \frac{T}{8}}{8}$ poll. unguis postic. $\frac{3}{8}$ poll. fere reetus.

Rostrum luteum apice nigro, formî ex乞̨̧ similis rostro Emberiza. Iris subbrunnea. Pedes nigricanres. Cauda bifurca.

Mas: Caput totum, guia, jugulum usque ad sternum, et latera pectoris nitide atra. Cervix rufo-ferruginea. A naribus peroculos stria alba, pone oculos latior, in. ter jugulum atrum et cervicem ferrugineum continuats usq̨ue ad flexuram alı. Dorsum, scapula et alarum 
minores teenrices nigra marginibus ferruginescentibus vel albidis. Peelus medium, abdomen et crissum nlba. Remiges saturate fusce, primores (præsertim prima) margine exteriore renuiter albidx, secundarice apice emarginata, pallida, marginibus fernginescentibus. Teçrices proximce nigricantes margine exteriore ferruginescentes apice albida; pemitivince albo marginata. Rectrices niyro-fusca, extima macula apicis cuncifor. mi albâ et latere exteriore fere tota alba, secunda macu. lâ apicis cuneiformi alba.

Femina: Mari dissimilis. Caput supra, dorsum et scapula nigricantia marginibus pemarum ferrugineis. Cervix et uro ygium ferruginea maculis minoribus nigricantibus. Latera capitis nigricantia ferrugineo immixto striâ ferrugineo-alba a rostro, per oculos et pone aures crrcumdata. Subtus alba jugulo, pectore et hypochondriis ferruginescenti tmetis, nigricante maculatis. Cetera ut in mare.

Mas janior: Capur supra nigrum marginibus pennarum tenuiter ferruginescentibus. Collum antice nigrum marginibus pennarum cinerascentibus. In ceteris feminæ subsimilis.

\section{HISTORIA.}

ArEtor regiones patria. Hyeme versus australiores plagas demigrare refertur, neque illam in Scania unquam vidi, nec visam audivi. Vißitat seminibus graminum et plantazum arvensium varii generis. In arvo ad tumulos paludosos ova 5- 6 ponit. Nidus sine arte ex gramine muscoque confectus, plumis raris in disco. Pra ceteris egregie cantillat, non tamen terr insidens, sed in aëre vibrans ut Alaudx. Frabr. l. c. 


\section{EMBERIZA NIVALIS.}

Diggn: Remiges secundariæ et Reetrices dure vel tres exrimx albx, apice nigre. Dorsum pennis nigris rufescenti marginatis, Subtus alba peltore rufescente.

\section{SYNONYMA.}

Emberiza nivalis, Lin. Faun. Sv. p. 82. n. 22\%. Retz. Faun. p. 237. n. 214. Fabric. Faun. Groenl. p. II7. n. 81. Lin. Syst. Nat. I. p. 308. n. I. Gmel. Syst. I. p. 866. Lath. Ind. I. p. 397. n. I.

Hortulanus nivalis, Briss. Orn. III. p. 385. n. 9. I'Ortolın de neige Buff. Ois. IV. p. 329. vers. Germ. Otto XII. p. I73 cum fig. P1. enl. 497. f. I.

Snow Bunting; Pen. Brit. Zool. I. p. 329. n. I22. t. 50. Aret, Zool. 1I. n. 222. Lath. Syn. III. n. 16I. vers. Germ. Bechst. III. p. I59. 11. I.

Der Schneenmmer Bechst. Nat. D. 2 Auf. 3. B. p. 305. Svecis: Snösparf.

\section{OBSERV.}

Ember. giacialis, Lath. Ind. I. po 398 ; Emb. musteiitu Gmel. 2. c.! I. p. $86 \%$ montana Lath. 1. c. p. 398 . huc pertinent, nec, ine judice, aliz sunt quan feminæe et juniores mares. Cel. quidem Bechstein, 1. c. p. 314. \&rc. E. mustelinam et montauam Ginel. pro specie a nivali diversan assumit; sed tum descriptio, tum figura data contrariun probare videntur. Vidi saltem specimina Emb. nivalis, qux in omnibus cum descriptione et figura jain allara convenirent. Etiam mores iidem.

\section{DESCRIPTIO.}

I.ongit. avis $6 \frac{x}{2}$ poll. Alx extens $x$ I2 poll. Rostr. $\therefore$ poll. A flexurs alz ad apicem $4 \frac{r}{2}$ poll. Cauda fere 
3 poll. Tibir $\frac{7}{2}$ poll. Digit. med. $\frac{7}{8}$ postic. $\frac{6}{8} ;$ unguis postic. $\frac{I}{2}$ poll.

Rostrum flavicans apice nigrum. Nares rotundatr, plumis setosis obtectx. Iris fusca. Pedes cum unguibus nigri. Cauda aliquantum bifurca.

Mas: Caput, collum, uropygium et tota avis subtus alba; capite et collo supra, maculà aurium et pectore (inter. dum) sordide rufo tinctis. Femora fusca. Dorsum et scapulæ nigræx marginibus latioribus albo rufescentibus. Remiges primores nigræ albo tenuiter marginatæ, a basi ad tertiam partem albx, qux albedo magis magisque augetur, ita ut IO-I3 sint nivex macula parva nigra in latere exteriore pone apicem; I4 - I5 niver immaculatr; 16-18 cum suis tectricibus nigra, margibus rufescentibus. Tectrices proximæremigum primorum albæ, apice nigræ; ceteræ tectrices albæ. Tectrices inferiores Remigum nivex. Rectrices dux intermedix nigra, versus apicem pallide rufescenti marginatw, sequens nigra, basi, præsertim latere exteriore albo, qux albedo ita crescit in sequentibus, ut ultimæ tres vel dux plane sint niver summis apicibus tantum nigris. Tectrices superiores nigræ rufescenti latius marginatæ. Femina distinguitur coloribus magis rufis minusque albis. Supra sordide rufa maculis dorsi et scapularum nigris, macula aurium saturate rufa; collo supra magis cinerascente. Collum,antice cinerascenti, pectore superiore et hypochondriis rufescenti colore tinctis. Tectrices alarum nigr cinereo-albo marginatx. Tectrices majores proxime nigre margine exteriore rufæ, apice albæ. Remiges nigrx, primores/margine exteriore tenuius cinereo - albx; secundarix apice albo marginatæ, a basi versus vel supra medium albæ. Intima tres nigre marginibus rufis. Cauda fere prioris, nec tamen color albus tam niveus. 


\section{HIS T ORIA.}

In sumnis Lapponix alpibus xstatem degit. Instante hyeme in provincias australiores turmatin advenit. Jam ante medium mens. Novembris instante nive, in Scania occurrit. Tota hyeme in agris et viis Scanix gregaria obvenit, semina in stercore et in herbis arvensibus quxsitura. Etim arboribus et tectis interdum insidit. Instante vere in patriam revertitur. Marem hyeme captum, per astatem in eubiculo vivum retinui. Timidus admodum et inquictus, tam noctu quam interdiu agilis. Ubi se solum putabat, egregie sed submissa voce, cantabat. Tam pavidus erat ut ab aviculis, illo longe minoribus de pastu fugaretur. Mutationem coloris sub æstate nullam observavi.

\section{DISSECTIO. II.}

Unguis posticus curvatus, longitudine fere digiti.

\section{EMBERIZA MILIARIA. Lin. Syst.}

DIAGN : Supra rufescenti-grisea maculis longitudinalibus nigro-fuscis; subrus flavescenti-alba maculis nigro-fuscis.

\section{SYNONYMA.}

Emberiza calandra, Lin. Faun. Sr. p. 83. n. 228.

Enberiza Miliaria, Lin. Syst. Nat. I. p. 308. n. 3.

Gmel. Syst. I. p. 868. Lath. Ind, I. p. 402. nI I2. Retz. Faun. Sv. p. 239.11. 215.

Cynchramus, Briss. Orn. III. p. 292. IO.

Le Proyer, Buff. Ois. IV. p. 355. t. Іб. vers. Germ. Otto XII. p. 237. cum. fig. Pl. enl. 233. 
Common Bunting, Pen. Brit. Zool. I. p. 324. n. I8. Aret. Zool. II. 1p, 366. B. Lath. Syn. III. p. I7I. n. 8. vers. Germ. Bechst. III. p. I69.

Der Graummer, Bechst. Nat. D. 2te. Auf. 3. B. p. 262.

Svecis: Kornlarka.

\section{DESCRIPTIO.}

Longit, avis $7 \frac{x}{2}$ poll. Alx extensx I2 poil. Rostr. fere $\frac{1}{2}$ poll. A flexura alæ ad apicem $3 \frac{\pi}{2}$ poll. Cauda $2 \frac{5}{8}$ poll. Tibix I. poll. Digit. med. fere I, poll. Digit. postic. $\frac{5}{8}$ poll.

Rostrum robustum, tuberculo palati exserto, supra nigro - fuscum, subtus luteum. Iris castanea. Pedes pallide griseo-lutescentes, articulis digitorum fuscescentibus.

Quoad colores alaude valde similis. Supra tota rufogrisea maculis longitudinalibus nigro-fuscis. Latere capitis luteo-alba striis rufo-fuscis in temporibus magis confertis. Subtus tota albida sordide luteo tincta. Mentun immaculatum. Ingulun et pectoris superio: pars maculis nigricantibus fere triquetris; ceterum pectus, hypochondrix et caudx tectrices inferiores striis longitudinalibus fusco-nigris. Remiges nigro-fuscx, latere exteriore rufescenti-griseo marginatx, secunda. xix apice albido marginatæ; intimæ tres apice latius albx. Tecrrices minores rufescenti-grisex, majores utriusque ordinis fusco-nigrx, margine rufescenti, apice rufo-albidx. Tectrices inferiores sordide albz striis rarjoribus fuscis. Cauda aliquantum bifurca, satmrate flisen; Rectrices rufescenti-albo, apice latius; marginata; extima versus apicen maculâ obsoleta al. bida, cuneiformi. 
wariet. a: Ember. nilieria varia: Dorsum et alæ pennis albis insertis.

Prope Lund visa.

\section{HISTORIA.}

Habitat in Scania campestri frequentissima. Manens. Estate in pratis, agris, arvis per paria occurrit. Mas in lapide sape, carduo, salice, vel alio quodam loco elevato, sedens, abrupta et stridula voce cantat. Hoc anni tempore pendentibus volitat pedibus. Victitat se. minibus, frumentis, insectis. Nidulatm in terra inrer sata agrorum vel gramina pratorum. Ova 4-6 cinerea punctis rufis striisque nigris. Collecta messe gregatim volitat. Per totam hyemem in agris, viis, plateis et fimetis semina hordei, avena \&c. turmatim quirit*).

\section{EMBERIZA HOR'TULANA}

DiAGN: Caput et collum supra olivaceo-cinerea, gula flava; Abdomen rubro-testaceum; Rectrices nigro-fuscr lateralibus duabus latere interiore versus apicem albæ.

\section{SYNONYMA.}

Emberian Hortulnza, Lin. Faun. Sv. p. 84.11. 229. Retz. Faun. p. 240. 11. 216. Lin. Syst. Nat. I. p. 309. 4. Gmel. Syst. I. p. 869. Lath. Ind. I. p. 399. n, 5.

*) Notact dignum videtur quod hac, ram frigoris patiens avis, ut non modo rigidissimas apud nos perferat hyemes, sed etiam frigore maxime intenso, coelo sereno, voce larissima canter; non nisi in provincia Patrix maxime australi, Scania nempe, occurrit. In Hallandia illam nunquam vidi, nec in cetera Svecia superiore visan audivi. Neque inter aves Gottlandicas a cel. Billberg enumeratas occurrit. Numeratur tamen inter aves Norvegia a cel. Brïnniche es Ström. In Finlandia tara est. (Faun. Fenn. p. 29.) 
Horulanus, Briss. Orn. III. p. 269. 4.

Orto/an, Buff. Ois. IV. p. 395. t. I4. vers. Germ. Otto. XII. p. I28-144. cum fig. Pl. enl. 247. f. 1.

Ortolan Bunting, Aret. Zool. IL. p. 367. D. Lath. Syn.

III. p. I66. 5. vers. Germ. Bechst. III. p. I64. 11.

5. Suppl. p. I5\%.

Emberiza Malbyensis, Mus. Carls. tab. 2I.

Gartenanmer, Bechst. Nat. D. 2te Auf. 3. B. p. 283.

\section{DES CRIPTIO.}

Longit. avis $6 \frac{x}{2}$ poll. Alx extens $x$ I I poll. Rostr. 7. poll. A flexura alx ad apicem $3 \frac{x}{2}$ poll. Cauda $2 \frac{r}{2}$ poll.; extra alas $I_{8}^{3}$ poll. extenditur. Tibix $\frac{6}{8}$ poll. Digit. med. fere $\frac{\kappa}{2}$ poll. Digit. postic. $\frac{\tau}{2}$ poll.

Rostrum supra incarnatum, subtus luteo-inearnatum. Nares ovatx, membrana fornicata semitectx, nudx. Tuberculum palati minus perspicuum. Iris fusca. Palpebra flavæ. Pedes incarnati, unguibus, presertim postico, pallidis. Canda æqualis.

Caput et collum supra cinerea olivaceo leviter tincta. Latera colli et anterior pars pectoris pallide cinerea, olivaceo tinła, gulâ, jugulo et stria ab utroque angu1o oris decurrente pallide flava. Dorsum et Seapulie griseo - rufa maculis longitudinalibus nigris, uropygio immaculato. Pectus, abdomen et crissum testaceoferruginea, marginibus pennarum dilutioribus, versus caudam magis in testaceum inclinantia, Remiges nigrofusce, primores margine exteriore tenuiter albidx; secundaria præsertim intimæ latius griseo-rufo extus marginatx. Tectrices minores nigro-fuscx, olivaceo obsolete marginatx; majores utriusque ordinis rufescentialbido latius marginatx. TeQtrices inferiores pallide lutex. Cauda saturate fusca, Rectricibus lutescenti rufo matginatis, extimis duabus late in interiore ab api- 
ce fere ad medium albis; extimâ quoque margine exteriore medio alba.

Femina: dignoscitur prasertim cinereo capitis colore magis sordido lineis ad raches pemnarm nigricantibus; pełore quam maris pallidiore.

\section{HISTORIA.}

Migratoria avis advenit initio M. Maji et abit M. Ar. gusto et Septembri. In Seania sylvestri passim, in Hallandia frequens. In frutice vel arbore sedens cantum jucundum, E. citrinella voci quodammodo similem: Tinktinktinktink tjöhrrr! vel interdiu vel noctu modulatur. Nidum straminibus bene constructum, mense Junio sub frutice in ipsa terra inveni. Ova 4-5 pallide grisea maculis striisque nigris, Pullis exclusis cantus silescit. Juxt finem Augusti, pullis educatis iterum auditur cantus. Victitat insectis et seminibus. Abit ante medium Septembrem.

LXXX. EMBERIZA CITRINELLA.

DiaGn: Subtus citrina. Reetrices extimæ duæ, latere interiore apicis macula cuneiformi alba. Caput maris fere citrinum; femince olivaceum maculis nigris.

\section{SYNONYMA.}

Embiriza citrinella, Lin. Faun. Sv. p. 48. n. 230. Retz. Faun. p. 240. n. 217. Lin. Syst. I. p. 309. 11. 5.

Gmel. Syst. I. p. 870 . Lath. Ind. I. p. 400. n. 7. Emberiza flava, Briss. Orn. III. p. 258. n. I.

Ie Bruant, Buff. Ois. IV. p. 342. t. 8. vers. Germb

Otto XII. p. 206. cum fig. Pl. enl. 30. f. I. 
Yellow Bunting, Pen. Brit. Zool. I. p. 325. n. II9. tab. 50. Aret. Zool. II. p. 367. C. Lath. Syn. III. p. I70. vers. Germ. Bechst. III. p. 167. 11. 7. Suppl. p. 157.

Der Goldammer, Bechst. Nat. D. 2 te Aun. 3. B. p. 252. Svecis: Gulsparf.

\section{DESCRIPTIO.}

Longit. avis 7. poll. Alæ extens: II. poll. Rostr. $\frac{3}{3}$ poll. A flexura al $x$ ad apicem $3 \frac{1}{2}$ poll. Cauda fexe 3 poll. Tibix $\frac{7}{8}$ poll. Digit. med. $\frac{7}{8}$; Digit. postic. $\frac{4}{2}$ poll.

Rostrum supra fuscum, subtus pallide griseum. Tuberculum palati exsertum. Nares plumulis fere tectx. Iris fusca; veitex depressus, rostro vix elatior. Pedes pallide incarnati digitis saturatioribus. Cauda bifurca.

Caput et collum pallide flava striis rarioribus oliva. ceis. Cervix olivacea. Dorsum et scapulæ griseo-rufescentia maculis longitudinalibus nigris. Uropygium aurantiun. Gula, jugulum, medium pectus, abdomen et tectrices alarum inferiores pallide flava. Superior pars pectoris et hypochondria olivaceo tincta, striis fuscescentibus. Remiges nigricantes margine exteriore prinarum viridi-flavo, secundariarum rutescente. Tectrices majores nigræx, rufescenti margina. tæa apicibus pallidioribus, minores olivacex. Rectrices nigricantes, extimæ dux macula longa cuneiformi alba in interiore apicis latere, medix binx rufescenti latius marginatx.

Femina: Mari valde dissimilis. Caput supra olivaceum maculis longitudinalibus nigris. Uropygium fusco-rufum. Gula flava. Collum antice griseo-olivaceum. Pectus medium et abdomen pallide fava. Crisstum al- 
bum flavo tinctum striis longitudinalibus nigris. Cetera ut in Mare.

variet: Tota flavescenti-alba, Fringilla canarice eminus simillima. In Hallandia initio Mens. Decembris sæpius visa.

\section{HISTORIA.}

Frequens. Manens. In pratis et juniperetis astivat. Nidulatur sub fruticibus. Ova $3-5$ sordide albida maculis striisque fuscis. Cantus maris haud ingratus, his fere syllabis: Tittitiť tȳy ! exprimi potest. Victitat astate inprimis insectis; hyeme vero domos, plateas, stabula et fimeta adit, semina præsertim hordei et avenæ lectura*).

\section{EMBERIZA SCHOENICLUS.}

Diagn: Supra nigra marginibus pennarum rufis; subtus alba striis pedtoris et hypochondriorum fuscis. Rectrices nigricantes, laterales duæ maculâ cuneiformi alba. Caput et jugulum no $r$ is nigra.

\section{SYNONYMA.}

Enheriza Schoeniclus, Lin. Faun. Sv. p. 84. n. 23 I. Retz. Faun. Sv. p. 24I. n. 2 I8. Lin. Syst. Nat. I. p. 3 I I. n. I7. Gmel. Syst. I. p. 88 I. Lath. Ind. I. p. 402. 11. 13 .

Passer torquatus s. arundinaceus, Briss. Orn. III. p. 274. 5.

*) Inter superstitiosas rusticorum opiniones, etiam hac quibusdam locis obvenit, quod hac ave edenda, morbo quem Idterum appellant, mederi putent. 
Ortolan de vosenux, Buff. Ois. IV. p. 315. vers. Germ. Otto XII. p. 149. cum 2. fig. Pl. enl. 247. f. 2. (mas ni fallor, junior.) et 497. f. 2. (fent.)

Rced Bunting, Pen. Brit. Zool. I. p. 326. n. I20. Arct. Zool. II. p. 368. E. Lath. Syn. III. p. I73. 11. 9. vers. Germ. Bechst. III. p. I70.9. Sup. p. 157. Rohrammer, Bechst. Nat. D. 2 Auf. 3. B. p. 269. Szecis: Säfsparf.

\section{DESCR IP TIO.}

Longit. avis 6. poll. Alx extens $x$ 9 $\frac{x}{2}$ poll. Rostr. fere 3 poll. A Alexura alix ad apicem $3 \frac{2}{8}$ poll. Canda $2 \frac{1}{2}$ poll. extra alas $I \frac{T}{2}$ poll. Tibiæ $\frac{7}{8}$ poll. Digit. med. $\frac{6}{8}$ poll.; postic. $\frac{r}{2}$ poll.

Rostrum supra nigricans, subtus flavescens. Tuberculum palati minus conspicumm; Nares membrana subfornicata, setulis semitectx. Iris fusca. Pedes fuscocarnei digitis et unguibus nigro-fuscis. Cauda bifurca.

Caput, gula et collum antice nigra. Ab angulo oris utrinque decurrit fascia alba, quæ tanquam torques cervicem cingit. Dorsurn et scapulæ peninis nigris ferrugineo marginatis. Uropygium et caudx superiores tectrices cinerea rufo immixto. Pectus et abdomen al$\mathrm{ba}$, illo et hypochondriis striis longitudinalibus fuscis. Remiges nigro-fuscx, primores margine exteriore tenuius, secundarice latius ferrugineo marginatx. Tectrices majores nigricantes dilute ferrugineo latius marginatr. Tectrices breviores saturate ferruginex. Rectrices nigricantes extima extus dimidiato oblique alba, latere tamen exteriore versus apicem fusco; secunda 'maculâ cuneiformi alba; par intermedium gri. seo - marginatum. 
Femina mari valde dissimilis: Caput supra rufo-brunneum maculis nigris. Collum supra in cinereum inclinans. Latera capitis rufo-brunnea. Supra oculos fascia rufo-albida. Collum antice sordide album, stria nigro-fusca utrinque decurrente juxta gulam et jugulum. Ceterum subtus sordide alba rufescenti tincta, pectore et hypochondriis, striis fuscis. Color dorsi et alarum dilutior quam in mare.

Avis junior: Tota supra saturate rufa maculis nigricantibus. Collum antice et pectus superius sordide rufescens maculis obsoletis fuscis.

Varietas: minor pracedente. Rostri et pedum eadem forma. Caput supra nigrum tæniâ longitudinali ferruginea a fronte ad nucham ducta. Latera capitis ferruginea, fascia circa aures nigra. Gula rufescenti-alba. Jugulum album fascia alba versus nucham ducta. Dorsum nigricans pennis griseo-rufescenti marginatis. Remiges nigricantes rufo marginatis tectricibus majoribus albido terminatis. Subtus (Antho pratensi exacte similis) alba maculis nigris in pectore et hypochondriis. Cauda nigro-fusca Rectrice tantum extima*) ob. lique transversim alba. Femina est verno tempore oc. cisa. An femina annosa? an distincta species?

\section{H IS TORIA.}

Rara. Migratoria redit circa XX. M. Aprilis tunc. que in salicetis Scanix interdum obvenit. Estate locis paludosis inter arundines habitat, nidumque in terra inter gramina vel radices fruticis cujusdam ponit. Ors $4-5$.

* Adsunt vero duodecim, ita ut nulla sit perdita. 
FAMILIA II.

\section{SUBULATIROSTRES.}

Rostrun tenuius, cultrato-subulatum, maxilla superiore immobili. Vicfqu: Baccæ, Vermes, Insecta, Semina.

\section{SUBDIVISIO I.}

Maxilla superiore pone apicem excisa.

\section{GENUS XVII. \\ T U R D U S.}

Rostrum rectiusculum, vix decurvatum, tereti-cultratum, versus apicem compressum. Mandibu. la superior pone apicem deflexum excisit. Narcs ovales, nudx, superne membranâ semireetx. Anguli oris vibrissis antrorsum vergentibus ciliati. Lingua lacero - emarginata.

Pedes ambulatorii.

\section{LXXXII, 'TURDUS VISCIVORUS.}

Diagn: Supra olivaceo-fuscus ReEtricibus tribus extimis apice albis. Subrus albo-Aavicans maculis nigris in pectore triquetris, in abdomine trans- 
versim ovalibus. Te\&rices alarum inferiores albx, superiores majores apice albæ.

\section{SYNONYMA.}

Turdus viscivorus, Lin. Faun. Sv. p. 79. 11. 216. (male descriptus) Retz. Faun. Sv. p. 226. 11. 20I. Lin. Syst. Nat. I. p. 29I. Gmel. Syst. I. p. 806. Lath. Ind. Orn. I. p. 326. n. I.

Turdus.najor, Briss. Orn. II. p. 200. I.

La Draine, Buff. Ois. III. p. 295. t. 19. f. I. vers.

Germ. Otto VIII. p. 26I. cum fig. bona. Pl. enl. 489.

Missel Thrush, Pen. Brit. Zool. I. p. 30I. 1. 105. Arê.

Zool. II. p. 34I. B. Lath. Syn. III. p. I6. I. vers.

Germ. Bechst. III. p. I3. n. I.

Die Misteldrossel, Bechst. Nat. D. 2 Auf. 3. B. p. 325 Svecis: Dubbel Kramsfogel, Björktrast.

\section{DESCRIP TIO.}

Longit. avis fere 12 poll. Alx extensx I9. poll. Rostr. a fronte ad apicem $\frac{\sigma}{8}$ poll. A flexura alæ ad apicem fere $6 \frac{r}{2}$ poll. Cauda $4 \frac{x}{2}$, extra alas 2 poll. extenditur. Tibia $I_{\frac{3}{8}}^{3}$ poll. Digit. med. $I_{\frac{2}{8}}^{\frac{2}{5}}$ poll. Digit. postic. $\frac{7}{8}$ poll.

Rostrum saturate fuscum basi subtus flavescens. Nares ovales. Os intus et lingua flava. Supra angulos oris vibrissie nigrx. Iris fusco-castanea. Pedes sordide lutei, articulis digitorum fuscescentibus, unguibus fuscis.

Caput, Cervix, Dorsum, Uropygium, Scapulx et alarum minores tectrices griseo-fusca, olivaceo tincta; Dorso postico in luteo-viride tendente. Latera capitis et colli luteo-alba fusco maculata. Palpebrae albæ. 
Avis subtus alba luteo tincta, maculis nigricantibus, in collo et superiore pectore triangularibus, in inferiore pectore, hypochondriis et abdomine transversin ovalibus; in gula minoribus, in pe¿tore et hypochondriis majoribus. Teetrices caudx inferiores griseo-fusce striâ juxta rachin latâ, alba marginibus lutescentibus. Remiges saturate fuscx, latere interiore versus basin albx, latere exteriore olivaceo, apice tenuiter albo marginatx; Tectrices majores utriusque ordinis saturate fuscer apicibus albis. Tectrices inferiores niver. Cauda æqualis saturate griseo-fusea, Rectricibus tribus extimis apice albis, ceteris albido tenuiter marginatis. Femina mari similis, corpore tamen subtus magis albo, minus luteo.

\section{HIST OR I A.}

Alii migrant, alii nobiscum hybernant. Sic in Hallandia per totam rigidissimam hyemem anni I8I4 illos inter T. Pilares junipereta visitantes, pluries vidi*). Demigrantes in Scania interdum, sed rarius autumno et vere occurrunt. Estivant in sylvis, præsertim pinetis montuosis superioris Sueciz. Inter ramos arborum nidulantur. Ova $3-5$ virescenti-aiba maculis punctisque rufis. Vietitant æstate Insectis et Lumbricis, qui. buscum teneram quoque alunt prolem; autumno baccis sorbi; hyeme juniperi. Verno tempore inter primas aves, e cacumine arboris summe, jucunde cantant.

\section{TURDUS MUSICUS.}

Diagri: Supra olivaceo-fuscus Rectricibus unicoloribus. Subtus albus, collo antice ferruginescen.

*) Hoc eam ob caussain notandum putavi, quia Bechst. 1. c. p. 330 dicit hanc aven in Germania rarius nisi leniori frigore. hibernars. 
ti-luteo tincto, maculis obcordatis fuscis. Tecfrices alarum inferiores ferrugineo-favæ. Superior majores apice ferrugineo-luteæ.

\section{SYNONYMA.}

Turdus Musicus Lin. Faun. Sv. p. 79. n. 2I7. Retz.

Faun. Sv. p. 227. 11. 202. Lin. Syst. Nat. I. p. 292. 11. 2. Gmel. Syst. I. p. S09. Lath. Ind. I. p. 327. n. 2.

Turdus Minor Briss. Orn. II. p. 205. 2.

La Grive Buff. Ois. III. p. 280. Vers. Germ. Otto VIII. p. 227 cuin figura. Pl. enl. 406.

Throstle Pen. Brit. Zool. I. p. 306. 11. 107. Arê. Zool.

II. p. 342. C. Lath. Syn. III. p. I8. 2. Vers. Germ.

Bechst. III. p. I5. n. 2.

Singdrossel Bechst. N. D. 2 Auf. 3 B. p. 340.

Svecis: Kramsfogel, Talltrast, Fjälstar, Klädra. Smol. Klera.

\section{DESCRIPTIO.}

Longit. aris $8 \frac{5}{2}$ poll. Alir extens $13 \frac{T}{2}$ poll. Rostr. $\frac{5}{8}$ poll. A flexura alx ad apicem $4 \frac{1}{2}$ poll. Cauda $3 \frac{x}{8}$; Tibia I $\frac{2}{8}$ poll. Digit. med. I. poll. Digit. post. $\frac{6}{8}$ poll.

Rostrum nigro-fuscum, subtus versus basin lutescens. Supra sinum oris vibrissx plurima nigre. Os intus flavum. Nares ovales. Iris brumnea. Pedes margaritacei. Cauda æqualis Reetricibus acuminatis.

Pracedenti fere similis sed minor. Caput, Collum, Scapulæ, Dorsum, Uropyginm et Tectrices caudre superiores grisescenti-fusca olivaceo tineta. A naribus ad oculos stria luteo-alba. Latera capitis olivaceo-fusca striis et punctis lutescentibus. Tota subtus alba, collo antice et pectore pallide rufescenti-luteo tinctis. 
Ad latera gula stria utrinque nigra decurrit. Collum antice, pectore et hypochondriis, maculis obcordatis nigro-fuscis. Abdomen album maculis raris minoribus ovalibus. Crissum album maculis longitudinalibus fuscescentibus. Remiges fusca basi intus pallide ferrugineo-lutea, extus olivaceo tenuiter marginatio. Tectrices majores utriusque ordinis colore dorsi, apice macula triangulari ferrugineo-lutea. Tectrices inferiores ferrugineo-flavx. Rectrices fuscz olivaceo tinc. tæe, unicolores.

\section{HISTORIA.}

Frequens. Migratoria. Autumni tempore per Scaniam in terras meridionales demigrantes, sylvas, ubi Sor* bos inveniunt, quorm! baccis præcipue delectantur, frequentant. Laqueis (Donor dictis.) hoc anni tempo. re capiuntur. Ad finem M. Martis vel initium M. Aprio lis, nive nondum, nisi locis palustribus soluta, revertuntur. Vere et per totam æstatem e cacumine summarum arborum cantus gratus, modulatione plenus, prasertim mane et vesperi, exauditur. Etiam in Scania nidulatur. Nidum forma semiglobosum, intus muscis, luto et argilla solide conglutinatis, vestitum, supra infimos arborum ramos vel in frutices ponit. Ova $3-6$ cocruleo-virescentia punctis fuscis. Victitat xstate insętis et lumbricis, antumno baccis.

\section{TURDUS ILIACUS.}

Diagn: Supra olivaceo-fuscus Rectricibus unicolo. ribus. Stria a naribus ad nucham luteo-albz. Latera colli maculâ lureâ. Ala subcus ferrugineo - rufr. 


\section{SYNONYMA.}

Turdus Iliacus Lin. Faun. Sr. p. 79. 11. 218. Retz. Faun. Sv. p. 228. n. 204. Lin. Syst. Nat. 1. p. 292. n. 3. Gmel. Syst. Nat. I. p. 208. Briss. Om. II. p. 208. 3. t. 20. f. I. Lath. Ind. On. I. p. 329. n. 7. Le Mnuvis Buff. Ois. III. p. 309. Vers. Germ. Otto VIII. p. 296, cum figura.

Redwoing Pen, Brit. Zool. I. p. 307. n. I08. Arct. Zool.

II. p. 342. D. Lath. Syn. III. p. 22. n. 7. Vers。

Germ. Bechst. III. p. I9. n. 7 .

Rothdrorsel Bechst. N. D. 2 Auf. 3. B. p. 360.

Aucupibus Scanensibus: Rödwinge.

\section{DESCRIPTIO.}

T. Musico aliquantum miror. Jongit. aris 8 poil. Alr extensx fere I 4 poll. Rostr. $\frac{5}{3}$ poll. A flexura alx ad apicem $4 \frac{6}{8}$ poll. Cauda $3 \frac{2}{3}$ poll. Tibia $I \frac{2}{3}$ poll. Digit. med. I. poll. postic $\frac{6}{8}$ poll.

Rostrum nigrum basi subtus et angulis oris luteis. Nares ovales. Iris brumea. Pedes griseo-lutescentes, ungvibus saturate fuscis.

Caput supra, cervix, dorsum, scapulix, tectrices minores et uropygium fusca, olivaceo tincta. Tem. pora fusca lineis luteis. A naribus supra oculos versus nucham stria luteo-alba. A maxilla inferiore sub temporibus decurrit utrinque stria lutea, qua in latere colli in maculam majorem luteam extenditur. Gula, jugulum, pectus et abdomen alba rufescenti-iuteo tincta, gulx et juguli lateribus maculis fere confuentibus saturate fuscis. Pectus et latera abdominis maculis olivaceo - fuscis fere ovalibus. Abdomen medium album. Hypochondria et tectrices alarum inferiores ferrugineo-rufa. Remiges saturate fuscr latere inte- 
riore a basi cincrascentes, margine exteriore olivacex, tectrices majores extus griseo-olivacez. Reetrices fu. scx olivaceo tinctx, subtus cinerex.

Variet. a. Totn alba rufescenti-nebulosa, hypochondriis et alis subtus rufis. Mus. Holm.

\section{HISTORIA.}

Rarior. Migratoria. Estivat in sylvis Suecîx supe. rioris. Per Scaniâm demigrans, versus hyemem ab aucupibus, laqueis recipitur, parcius tamen et serius quam antecedens. Cantus ex arboribus astate andiendus parum jucundus, his fere syllabis: try-try-try. $-t r y$ - try - try - $t i$ ! exprimi potest (cel. Auselius in litt.)*) Vescitur insectis, lumbricis, et autumno baecis.

\section{TURDUS PILARIS.}

Diagn: Caput et Uropygium cinerea. Dorsum castaneum. TeEtrices alarum inferiores alba. Rectrices nigræ, extimæ margine interiore apice albicantes.

\section{SYNONYMA.}

Turdus 'Pilaris, Lin. Faun. Sv. p. 78. n. 215. Retz. Faun. Sv. p. 227. 11. 203. Lin. Syst. Nat. I. p. 29 I. 11. 2. Gmel. Syst. I. F. 807. Briss. Orn. II. p. 214. n. 5. Lath. Ind. I. p. 330. n. Ir.

*) Temore igitur in Faun. Sv. dicitur avem egregie cantillare: Ve vero omnia qua hoc loco a limné de $T$. Iliaco affertuntur, ctian nomina Svecana, (descriptione ramen excepta) non huic sed T'. Musico competunt.

Nilsson Ornith. 
La I itorme, Buff. Ois. III. p. 301.t. I9. Vers. Germ.

Otto VIII. p. 276. cum figura.

Ficldfare, Pen. Brit. Zool. I. p. 304. 11. IO6. Arct. Zool. II. p. 340 . A. Lath. Syn. III. p. 24. n. II. Vers.

Germ. Bechst. III. p. 2 I. n. II.

Wachholderdrossel, Bechst. Nat, Deutschl. 2 Auf. 3 B. p. 336 .

Suecis: Björkdrossel. Snöskata.

\section{DESCRIPTIO.}

Longit. avis 10 poll. Rostr. $\frac{5}{8}$ poll. A flexura ala ad apicem $5 \frac{\mathrm{T}}{2}$ poll. Cauda 4 poll. Tibia $\mathbf{I} \frac{3}{8}$ poll. Digit. med. I $\frac{x}{8}$ poll. Digit. post. $\frac{6}{8}$ poll.

Rostrum Aavescens apice fuscum Os intus et lingua flava. Nares ovales. Iris fusca. Pedes nigrofusci. Cauda xqualis.

Caput, collum strpra et uropytrium cinerea, vertice lineis longitudinalibus nigris. A naribus, supra ocu. los linea rufescenti-alba. Lora nigra. 'Tempora cinerea lineis lutescentibus. Dorsum et Scapulæ saturate fusco-rufa (castanea) A gula ad medium pectus ferruginescenti-lutea, jugulo et superiore pectore maculis longitudinalibus nigro-fuscis. Latera pectoris et hypochondria pennis versus apicem nigro-fuscis, albia do latius marginatis. Medium pectus et abdomen alba. Crissum pennis griseo-fuscis, fascia juxta rachin longitudinali, apice et marginibus albis. Remiges nigrofusca margine exteriore pallide cinerascentes. Tectriees alarum extuscinerascentes, Tectrices inferiores albæ. Rectrices nigricantes extimis tribus margine interiore apicis albidis.

Yav. Caput et collum alba pennis quibusdam fuscis insertis. Tecrices alarum pennis dnabus albis. Cetera exacte ut in T. Pilari. T. Visciqorus, Var. Wet. 
Ac. H. I808. p. 197. Tab. V. (Specimen depictum in Mus. Upsal. vidi.) Briss. Om. II. p. 217. A.

\section{HIST ORIA.}

Frequens. Aliae demigrant, aliæ manent, hæque in sylvis et juniperetis, ubi baccas devastant, maximis gregibus associatr, per totam hyemem obveniunt. Timida admodum avis fixuque difficillina, autumnali rempore baccis sorbi allecta laqueis (Donor) capitur. Nidulatur: in sylvis præcipue in Pinetis Smolardix aliarumque provinciarum superjoris Suecir. Nidus in ramo positus continet ova 4.5 pallide grisea maculis obsoletis tuscescentibus. Vescitur insectis, lumbricis, baccis.

\section{TURDUS MERULA.}

Diagn: Mas ater rostro palpebrisque fulvis. Femina nigricans rostro intus flavo.

\section{SYNONYMA.}

Turdws Merula, Lin. Faun. Sv. p. 80. n. 220. Retz. Faun. Sv. p. 230. 11. 206. Lin. Syst. I. p. 295. 11. 22. Gmel. Syst. I. p. 83 I. Briss. II. p. 227. n. IO. Lath. Ind. I. p. 340.

Merle Buff. Ois III. p. 330. t. 20. Vers. Germ. Otto IX. p. 5. cum 2 figuris. Pl. Enl. 2. mas. 555. fem. Blackbird, Pen. Brit. Zool. I. p. 308. n. 109. tab. 47. Arct. Zool, II. p. 345. I. Lath. Syn. III. p. 43. n. 46. Vers. Germ. Bechst. III. p. 39. n. 46. Id. Supl. p. I4I.

Schwarzdrossel, Bechst. N. D. 2 Auf. 3 B. p. 376. Svecis: Koltrast. Scanis: Solswärta. 


\section{DESCRIPTIO.}

Longit. avis 10 poll. Rostr. $\frac{\sigma}{8}$ poll. A flexura alre ad apicem $5 \frac{2}{2}$ poll. Cauda $4 \frac{x}{2}$ poll. Tibia $1 \frac{3}{8}$ poll. Digit. med. I $\frac{2}{8}$ poll. Digit. post. $\frac{7}{8}$ poll.

Rostrum extus er intus fulvum. Supra sinus oris Vibrissx nigræ. Iris fusca. Margo palpebrarum fulvus. Pedes nigri.

Mas toto corpore atro.

Femina. Rostrum fuscum intus flavum. Pedes saturate fusci. Supra tota nigro-fusca. Gula albida maculis fuscis. Peetus superius sordide ferruginescens maculis fuscis. Abdomen fuscum cinereo tinctum.

Avis $\mathfrak{z}$ unior, ante prinan deplanationem: Supra saturate fusca, capite, collo, dorso antico et scapulis rachibus ferrugineis. Gula et jugulo maculisque in pecto. re et abdomine sordide ferrugineis.

frar. a : Capite albo maculis rarioribus fuscis; corpore nigro maculis majoribus albis.

Mus. Payk.

Var. ค: Griseo - fusca.

\section{HISTORIA.}

Ubique in sylvis. Solitaria. Manens. In nemoribus densissimis æstivat, hyeme hortos quogue visitat. Sub finem hyemis nidulatur. Nidus in fruticibus ex muscis et ramulis, argilla et luto conglutinatis con. struetus, Ova 4.5 griseo-viridia maculis striisque brunneis. Terrefaßta sono Tack! Tack! avolat. Cantus Maris triste suavis, præsertim vesperi audiendus. Victitat insectis, lumbricis, baccis. Laqueis capitur, baccis Sorbi allecta. 


\section{TURDUS TORQUATUS.}

DIAGN : Nigricans alis et corpore subtus albido mar. ginatis, macula semilunari alba circa partem pectoris superiorem.

\section{SYNONYMA.}

Turdus Torquatus, Lin. Faun. Sv. p. 80. n. 22I. Retz. Faun. Sv. 230. 11. 207. Lin. Syst. Nat. I. p. 296. n. 23. Gmel. Sy,st. I. p. 832. Lath. Ind. I. p. 343. Merula Torquata, Briss. II. p. 235. n. I2.

Merle à plastron blanc, Buff. Ois. III. p. 340. t. 3I. Vers. Germ. Otto IX. p. 36. cum figura. Pl. Enl. 5 I6 (Mas) I82. (junior avis).

Ring-Ouzel, Pen. Brit. Zool. I. p. 3IO. n. IIO. t. 46. Aret. Zool. II. p. 344. H. Lath. Syn. III. p. 46. n. 49. Germ. Vers. Bechst. III. p. 43. n. 49. Sup. p. I4I.

Ringdrossel, Bechst. N. D. 2 Auf. 3 B. p. 369.

\section{DESCRIPTIO.}

Longit. avis II. poll. Alx extens $x$ I $7 \frac{\pi}{2}$ poll. Ro. strum fere $\frac{6}{8}$ poll. A flexura alix ad apicem $5 \frac{6}{8}$ poll. Cauda $4 \frac{T}{2}$ poll. Tibia $I_{\frac{3}{8}}^{\frac{3}{8}}$ poll. Digit. med. $I_{\frac{T}{B}}^{\frac{T}{B}}$ poll. Digit. post. $\frac{7}{8}$ poll.

Rostrum adulti maris luteum apice et basi supra nigricans. Os intus flavum. Sinus oris flavi, vibris. sis nigris supraciliatis. Iris castanea. Pedes nigro-fusci.

Supra niger pennis dorsi et uropygio tenuissime pallido marginatis; pennis pectoris, tectricibus alarum et caudx inferioribus albo marginatis. Supra pectus in infima juguli parte macula transversalis magna, semilunaris sordide alba. Remiges et majores tectrices ni- 
gro-fusca griseo-albo extus marginatx. Rectrices obtuse-acuminatie, extima ceteris aliquantum brevior apice tenuiter albo marginata.

Femina dignoscitur colore magis sordido. Rostrum fu. scum basi subtus sordide flavum, intus flavum. Pedes fusci.

Fusco-nigra pennis supra griseo, subtus albo marginatis. Lunula juguli sordide cinerascenti-albida, undulis obsoletis fuscescentibus.

\section{HISTORIA.}

Rara avis. Migratoria. Demigrantem mense Octobri et revertentem M. Aprili in Scanix hortis et sæpibus vivis, rarius tamen, inveni. In superioris Svecia sylvis montuosis nidulatur. Cel. Leche sclopeto occidit seniorem avem hujus speciei, ad Mariestad Mense Majo, et juniorem ad Gothoburgum medio Julii, "quce cerasos jam maturos cun socüs depascebatur. "Leche in Advers.

\section{TURDUS ROSEUS.}

Diagn: Corpus roseum capite, alis et cauda nigris, occipite cristato.

\section{SYNONYMA.}

Turdus Roseus, Lin. Faun. Sv. p. 79. 11. 219. Retz. Faun. Sv. p. 229. n. 205. Lin. Syst. Nat. I. p. 294. n. I5. Gmel. Syst. 1. p. 8I9. Lath. Ind. I. p. 344. n. 59.

Merula Rosea, Briss. II. p. 250. n. 20. Merle couleur de rose, Buff. Ois. III. p. 348. t. 22 .

Vers. Germ. Otto IX. p. 55. Pl. Enl. 25 I. 
Rose-coloured Ousel or Thrush, Pen. Brit. Zool. 2. p. 6. 7. tab. append. V. Are?. Zool. II. p. 344. G. Lath. Syn. III. p. 50. Vers. Germ. Bechst. III. p. 46. n. 52. Sup. p. 142.

Rosenfurbige Drosscl, Bechst. N. D. 2 Auf. 3 B. p. 393. Mus, Payk. M. Ups.

\section{OBSER V.}

Specimina, quæ in variis Potriæ Museis vidi e Lapponia sunt. Etiam in Dalekarlia visa refertur avis. Cum vero migratoria est, nec unquam in Svecia meridionali inventa; demigrariones e Lapponia per Finlandian et Russiam instituere videtur.

\section{DESCRIPTIO.}

Longit. avis fere $8 \frac{1}{2}$ poll. Rostr. $\frac{6}{8}$ poll. A flexura alx ad apicem $5 \frac{2}{8}$ poll. Cauda $2 \frac{5}{3}$; Tibia $I \frac{2}{8}$ poll. Digit. med. $I \frac{1}{8}$; Digit. post. fere I poll.

Rostrum roseum basi nigricans (in ave mortua). Pedes flavescentes. Nares ovales. Iris fusca. Cauda xqualis.

Caput et pars colli, gula, jugulum et teetrices ala. rum majores nigra coeruleo et viridi nitentia. Occiput cristâ concolor dependente. Dorsum, tectrices minores, pectus et abdomen rosea vel pallide incarnata. Remiges fuscr latere interiore einerascentes, exteriore et apice saturatiores, viridi nitentes. Rectrices nigrx, nitentes; Teetrices inferiores caudx nigra marginibus albis. Femora nigra.

Fonina: fusca ubi mas niger. Crista minor. 


\section{GENUS XVIII. \\ A M P E L I ${ }^{*}$ )}

$\boldsymbol{R}_{\text {ostrum breve, rectum, convexum, basi latum, }}$ unde faux ampla, subdepressum**). Maxilla superior aliquantum longior, apice decurvatum, utrâque pone apicem emarginatâ.

Sares ovales plumis setosis, rigidis, teetx.

Lingua cartilaginea tenuis, lata, lanceolata, apire bifida.

Pedes ambulatorii, breviusculi,

Vičnus: Insecta, Baccre.

\section{AMPELIS GARRULUS,}

DiAGN: Rubescenti-griseus abdomine pallidiore. Crissum fusco-rufum. Vertex cristatus. Remiges secundarix appendicibus membranaceis coccineis.

\section{SYNONYMA.}

Ampelis Garmulus, Lin. Syst. Nat. I. p. 297. I. Retz. Faun. p. 23I. n. 208. Gmel. Syst. I. p. 833, Lath. Ind. Orn. I. p. 363. 12. I.

*) Rostri iormâ ad Muscicapas proxime accedit.

**) Ab apice conspefum fere trigonum. 
Lanius Garvulus, Lin. Faun. Sv. p. 28. n. 82.

Bombycilln Bokcnica, Briss. Orn. II. p. 333. n. 63.

Lc Yasenr de Bohene, Buff. Ois. III. p. 429. t. 26. Pl.

enl. 26I. Vers. Germ. Otto IX. p. 22 I cum fig.

IVaxen Chatterer, Pen. Brit. Zool. I. p. 3I4. n. II2.

tab. 49. Arct. Zool. 2. p. 346. 11. 207. Lath. Syn.

III. p. 9I. I. Vers. Germ. Bechst. III. p. 86. n. I. Seidenschwanz, Bechst. N. D. 2. Auf. 3 B. p. 410. tab. 34. fig. I.

Siccis: Sideriswans.

\section{DESCRIPTIO.}

Longit. avis 8 poll. Alr extens 14 poll. Rostr. a fronte ad apicen $\frac{3}{3}$ poll. A fexura alx ad apicem $4 \frac{x}{2}$ poll. Cauda $2 \frac{3}{5}$; Tibia $\frac{6}{8}$ poll. Digit. med. $\frac{6}{8}$. poll. postic. $\frac{0}{\mathrm{I} \delta}$ poll.

Rostrum et pedes nigri. Iris rufo-fusca. Crista verticis. Cauda fere æqualis.

Tota avis pennis mollibus, serieis vestits. Frons et erissum saturate rufa. Circa basin rostri supra linea et per oculos ad occiput fascia nigra. A maxilla inferiore stria albida inter fasciam jam dictam et gulam nigram. Ceterum corpus rufescenti-cinerenm, versus caput magis in rufum, versus caudam in canum vergens. Abdomen albidum. Remiges primores nigrx; $2-4$ latere exteriore apicis macula longitudinali alba ; sequentes maculâ pallide flava; secundarix cinereo - nigricantes, apicis exteriore latere macula albâ, membranula oblongo-ovata coceinea terminatae. Tectrices proximæ Remigum primorum nigræ apicibus albis. Rectrices versus basin cinerea, versus apicem nigra, apie jpso latius fiave. 
Femina: Nigredo gula minor; apices caudx flavi zingustiores et pallidiores; appendices Remigum ad summum quinque.

Var.c. Rostrum album versus apicem nigrum. Corpus totum fuligiroso-album, capite saturatiore, fronte et crisso fusco-rufis. Remiges albr apice lutex, secundarix apendicibus membranaceis coccineis. Rectrices albidæ apicibus luteis. Mus. Upsal. Amp. Garrulus Var. Wet. Ac. H. I808. p. 197. Tab IV.

$V \pi r$. . Alba, corpore subtus , dorso medio et tectricibus alarum fuscescenti-nebulosis. Gula et lora nigra. Remiges ut in primo descripto; sic etiam cauda, sed rectrices intermedix albr apicibus Inteis.

Mus. Payk.

\section{HISTORIA.}

In arctois regionibus xstivat. Mensibus Novembri et Deeembri catervarim Scaniam transmigrat. Baccis imprimis Sorbi delectatur, quibus allecta laqueis (Donor) simul cum Turdis capitur- Stupida, vorax et parum timida avis. Verno tempore revertens vescitur Insectis, quibus more Muscicape potitur. 


\section{GENUS XIX. \\ M U S C I C A P A.}

Rostrum breve, rectum, trigonum basi dilıtatum, maxilla superior longior apice aliquantum in. curvata, pone apicem emarginata.

Anguli oris vibrissis nigris, antice vergentibus, superne ciliâti.

Nares rotundarx plumis setosis obrectx. Lingua.

$P_{e d e s}$ ambulatorii *).

Vičus Insęta, præsertim Lepidoptera, Hymenop. tera et Diptera-

LXXXX. MUSCICAPA GRISOLA.

DIAGN: Supra fusco-grisea, subtus albida, collo inferiore et pectore striis longitudinalibus griseo - fuscis.

\section{SYNONYMA.}

Muscicnpa Grisoln, Lin. Syst. Nat. I. p. 328. n. 20. Retzii Fauna Sv. p. 25I. 1. 23 I. Gmel. Syst. I. p. 949. Lath. Ind. I. p. 467. n. I.

Motacilla Ficedula, Lin. Faun. Sv. p. 90. n. 251. (Nee tamen Syst. Nat. qux ad sequentem speciem pertinet).

*) Digiti non longius fissi quam in ceteris Passeribus. Extimus semper medio ad primum fere articulum eonnexus. 
Muscicapa, Briss. Orn. II. p. 35\%. I. t. 35. f. 3.

Le Gobemouche, Buff. Ois. IV. p. 517. t. 25. f. 2. Vers.

Germ. Otto XIV. p. 10. cuin figura. Pl. Enl. 565. f. I.

Spotted Flycaicher, Pen. Brit. Zool. I. p. 350. n. 134. Lath. Syn III. p. 323. n. I. Vers. Germ. Bechst. III. p. 3 I 4. 12. I.

Gefleckter Fliegenfünger, Bechst. N. D. 2 Auf. 3. B p. $42 I$.

\section{DESCRIPTIO.}

Longit. avis $6 \frac{2}{3}$ poll. Alr extensæ $10 \frac{\pi}{2}$ poll. Rostr. a fronte ad apicem $\frac{1}{2}$ poll. A flexura alx ad apicem $3 \frac{3}{8}$ poll. Cauda $2 \frac{2}{8}$ poll. Tibia $\frac{5}{8}$ poll. Digit. med. $\frac{9}{16}$ poll- Digit. post. $\frac{3}{8}$ poll.

Rostrum nigrum, basi subtus pallidum. Anguli oris et os intus pallide lutea. Nares ovato-rotundatæ, pervix. Supra nares et sinus oris vibrissis nigris antice vergentibus. Iris saturate fusca. Pedes cum ungvibus, nigri.

Caput supra griseum maculis saturatioribus ad ra. ches, pennis frontisalbido marginatis. Collum supra, dorsum, scapulx et uropygium fusco-grisea. Subtus avis alba striis longitudinalibus fusco-griseis ad latera gulx, in jugulo et pectore. Gula, abdomen et crissum immaculata alba*). Remiges et tectrices saturate fusca, secundarix cum tectricibus majoribus margine exteriori griseo-albx. Tectrices inferiores et hypochondria albide fuscescentia, sordide lutescenti tincta. Cauda aliquantulum bifurca. Rectices fuscx,

*) Obs. Linne et omnes, qui post illum sine autopsia descripserunt, dicunt Crissu rufeseens, quo nihil falsius. 
extima magis griseo-fusca; margine exteriore a basi pallide grisex.

Feminn: aliquantum minor pedibus nigro-fuscis. Preterea vix a Mare coloribus distinguenda.

Pullus nuper volatui aptus: Rostrum cinereo-fuscum. Sinus oris albidi. Pedes grisei. Supra fuscus maculis numerosis albide-testaceis. Subtus albus, gulâ, jugulo et pectore sordide - fusco nebulosis.

\section{OBSERV.}

Sensim maculie supra corpus evanescunt; pone oculos et in apicibus majorum alarum teetricum diutius manent. Remiges in. timx inargine exteriore latius pallide testacez.

\section{HIS T ORIA.}

Frequens certis locis. Migratoria, abit versus medium Mensis Septembris. Redux post initium M. Maji. Quamdiu transmigratio durat, in hortis Sca. nir occurrit. Summa semper petit cacumina, sono pipilante Ist, Ist! se prodens. Insecta forte prætervo. lantia ocissime arripit, iisque arreptis, in ramulum, unde devolarat, revertitur, prædam consumtura. Nidulatur in sylvis; vix tamen in Scania, ubi post medium Julii occurrit, sed ante hoc tempus haud visa, In aliis Svecix provinciis ex. gr. in Hallandia nidulantem inveni. Apud nos videtur bis per annum nidificare. Pullos enim volatui aptos jam medio Junio inveni. Feminam ova parientem eodem tempore in nidulo cepi. Pennz vero ita detritæ, ut abdomen fere calvum esser, indicare mihi videbantur aviculam jam antea, hac astate, ora et pullos forisse. Nidum e muscis, straminibus et radiculis graminum construetum, intus lanî et plumulis stratum in truncis et radicibus putrefactis cavis haud longe ab aqua, ponit. Ora 5 palli- 
de-grisea maculis pallide russ, presertim in extremitate crassiore. Mense Augusto et initio Septembris in sylvis Scaniz et Hallandiz \&c. copiosa.

\section{LXXXXI. MUSCICAPA ATRICAPILLA.}

Dragn: Jhas: supra niger fronte, maculâ alarum Redricibus duabus lateralibus extus et corpore subtus albis.

Femina: Supra fusca, fronte, macula alarum, Rectricibus tribus lateralibus extus et corpore subtus albidis.

\section{SYNONYMA.}

Muscicapa Atricapilla, Lin. Syst. Nat. I. p. 326. n. 9. Retz. Faun. Sv. p. 250. n. 230. Gmel. Syst. Nat. I. p. 935. Latl. Ind. Orn. I. p. 467.

Motacill , Lin. Faun. Sv. ed. Ima p. 86. n. 230. tab. I. fig. 229. Ed 2 Tab. I. f. 256. $\sigma^{\pi}$ mas, $L^{\circ}$ junior avis.

Motacilla Ficzdula, Lin. Syst. Nat. I. p. 330. n. IO (non Diagnosis, qua ad antecedentem pertinet, sed Descriptio)*).

* Mira circa hanc arem apud Linné, confusio, cajus causam si quæris hæc est. Cel. Leche avem hujus speciei ad Simonstorp in Scania fixit anno 1730 (Adversaria Lechei.) descriptionemque ejus et forte iconem Linneo obtulit. Linne qui nec hanc nec Motac. Atricapillam ipse viderat, iconem datam Motac. Atricapille n. 229. adjunxit (in prima fauna editione.) adferens tamen descriptionem Muscicapx Atricapill sub No. 230 . In secunda Editione Faunæ hunc errorem eo modo mutavit ut Num 230 prime Editionis (veram Musc. Atricapillawi) cum sua Moracilla Rubetra conjungeret, et iconem ejus Motacilla Atricapille adderet. Demum in XIIma Systematis Editione Num: 230. primae Editionis Faunae, ut wistinctam Speciem quidem retinuit: sed iconem editionis secnndae N, 256 . plane omisit. 
Muscicapa Nigra, Briss. Orn. II. p. 381. n. 13.

Le Gohe Mouche de Lorrnine, Buff. Ois. IV. p. 520. PI. enl. 565. f. 2. Vers. Germ. Otto XIV. p. I7. fig: infer. (Mas).

Pied Flycatcher, Pen. Brit. Zool. I. p. 351. 11. 135. Arct. Zool. II. p. 39I. B. Lath. Syn. III. p. 324. n. 2. Vers. Germ. Bechst. III. p. 3 I6. n. 2.

Schwartzriickiger Fliegenfünger, Beehst. N. D. 2 Auf. 3 B. p. 43 I. (Avis secundo ætatis anno).

Der Schzoartzgraue Fliegenfünger, (Musc. Muscipeta) Bechst. 1. c. p. 435.

Becfigue, Briss. Om. III. p. 369. Buff. Ois. V. p. I87.

Vers. Germ. Otto. XV. p. 196. cum figura. Pl. Enl, 668. f. I.

\section{DESCRIPTTO.}

Longitudo avis $5 \frac{3}{8}$ poll. Ala extense 9 poll. Rostrum a fronte ad apicem $\frac{5}{16}$ poll. A flexura ala ad apicem $3 \frac{x}{8}$; Cauda 2 poll. Tibia $\frac{6}{8}$ poll. Digit. med. $\frac{5}{8}$ poll.; post. $\frac{7}{16}$ poll.

Rostrum nitide atrum. Iris Fuser. Pedes nigri.

Tota avis supra nigra cervice et uropygio fusco mixtis, fronte alba. Avis subtus nivea. Remiges saturate fuscx; 5 -I5 basi albx; intimx tres (I6-18) albæ latere interiore versus apicem nigræ. Tectrices minores nigro-fusca, ex proximis intima apice albx, unde macula magna alba in ala composita oritur.

Rećtrices: nigræ; extimxduxlatere exteriore. A bssi fere ad apicem albr.

Variat: Maculis frontalibus binis albis.

Femina: Frontis macula minor, sordide alba.

Avis supra griseo-fusca. Subtus alba, fuscescenti. prrsertion in pectore, levirer tinctr. Remiges saturate fusca , 5 - I 5 latere exteriore juxta basin sordide albz: 
intimx tres a basi ad medium, et margine exteriore la. tius albr. Teetrices minores fuscr, majorum intima apice albæ. Rectrices nigræ, extimæ tres nigro-fuscr latere exteriore a basi versus apicem albo. Teatrices caudx superiores nigrae.

Funior Avis: Frons, caput supra, cervix, dorsum, scapulz ct alarum tectrices minores griseo-fusca (vix olivaceo tincta). Latera capitis fuscescentia luteo immixto. Collun antice, alarum tectrices inferiores, superior pectoris pars et hypochondria sordide lutescentialbida. Abdomen et crissum alba, Remiges nigricantes, 5-I5 latere exteriore juxta basin luteo-albida; tedrices majores apice et intima tres remiges margine exteriore lureo-albida, unde fascia angusta luteo-albida in alis complicatis oritur. Reftrices nigricantes in aliis dux, in aliis tres ultime latere exteriore albx.

\section{OBSERV.}

Vernali tempore soniores aves ita sunt colorata nt primo descriptx. Pullis vero exclusis deplumant, et post deplumationetn omnes, marcs, fomine et juniores fere similes sunt, ita ut autumnali tempore nullam tatn supra nigram et subtus pure albam invenias ut marem primo descriptum. Hic autem griseofuscus color in junioribus, etian proximo vere conspicitur, et ex his, nova species, a quibusdam Ornithologis, constituta est.

\section{HISTORIA.}

Migratoria. Nas ad nos revertitur circa finem $M$. Aprilis, tuncque in hortis Scanix occurrit, femina aliqvantum serius advenit. Circa medium Majum hortos relinquit et nidificatura in sylvas se confert. Mas arbori insidens bene cantat. Nidulatur in cavis arboribus, inter radices truncorum, in Scania, Hallandia, Ostrogothia \&̈c. Medio Junio nidos cum, ovis 6 coe- 
rulescenti-albis, inmaculatis, inveni. Jam post medium Julium pullos volatui aptos vidi. Post hoc tempus præsertim m. Augusto in sylvis copiosissime occurrit et mutatione pennaruin facta, omnes, mares, feminæe et pulli similes sunt, nec ulla tan superne atra subtusque nivea occurrit ut mares vernali tempore. Ex infimis arborum ramis, qvibus plerumque insidet, insęta præxtervolantia celerrime arripit, et captâ prædâ in ramulum revertitur. Timida et fixu difficilis. Alas continuo et celerrime movet. Juxta medium m. Septembris sensim abit. 


\section{GENUS . XX。 \\ S A X I C O L A, Meyeri.}

$\boldsymbol{R}_{\text {ostrum rectum, tereti-compressum, basi dila- }}$ tatum. Maxilla superior longior, pone apicem decurvatum, emarginata.

Anguli oris vibrissis antice vergentibus superne ciliati.

Nares patulæ, rotundato-ovales.

Cauda brevior, subæqualis.

$p_{e d e s}$ ambulatorii.

\section{SAXICOLA RUBETRA.}

Diagn. Supra nigricans marginibus pennarum griseo-ferrugineis. Macula alarum et stria supra oculos alba, jugulum et peetus luteo-rufa.

\section{SYNONYMA.}

Motacilla $R_{u b l}$ betra, Lin. Fam. Sv. p. 93. n. 255. Retz. Faun. p. 260. n. 243. Lin. Syst. Nat. I. p. 332. n. I6.

Rubetra Major sive Rulicola, Briss. Orn. III. p. 432. n. 26. t. 24. f. I.

Sylvia Rubetra, Lath. Ind. 1. p. 525. n. 58.

Saxicola Ruberra, Meyer Vögel Liv- und Esthl. p. I28. 
Grand Traquet ou Tarier, Buff. Ois. V. p. 224. P1. enl. 678. f. 2. Vers. Germ. Otto XV. p. 252.

Whin-chat, Pen. Brit. Zool. I. p. 385. n. I58. Lath. Syn. IV. p. 454. n. 54. Vers. Germ. Bechst. IV. p. 45 I. n. 54.

Braunkehliger Steinschmätzer, Bechst. N. D. 2te Ausg. 3. B. p. 684 .

Svecis: Busksqwïtta.

\section{OBSERV.}

Huc ut femina pertinet Alauda Campestris Lin. Faun. Sv. p. 77. n. 212. quod miror neminem Ornirhulngorum athue vidisse, quamvis Descriptio est inter optimas in Faund Linnei Svecica. Magis tamen mirandum cel. virns Gmelin, Latham \&c. hanc Diagnosin in Al. Campestrem imnutatam retinuisse.

\section{DESCRIPTIO.}

Longitudo avis 5 poll. Alæ extensæ $9 \frac{\pi}{2}$ poll. Ro. str. $\frac{3}{3}$ poll. A flexura ali ad apicem 3 poll. Cauda 2 poll. Tibia $\frac{7}{8}$ poll. Digit, med. $\frac{6}{8} ;$ postic. $\frac{T}{2}$ poll. Rostrum nigrum. Nares ovatx. Sinus oris nigri, vibrissis nigris supra ciliati, Iris griseo-fusca. Pedes nigri.

Caput supra Dorsum et Scapulæ nigra pennis griseo. ferrugineo marginatis. Uronygium griseo-ferrngineum maculis nigris. A naribus supra oculos ad nu. cham stria alba. $A b$ angulo oris sub oculis ad latera Colli fascia lata nigra, rufo et albo interdum immix. to. Gula alba dimittit lineam albam ad latera juguli, Jugulum et pars superior pectoris dilute flavo - ferruginea. Inferior pars pectoris et abdomen alba; hypo. chondria et crissum favescenti-rufo leviter tincta. Femora albida maculis parvis nigricantibus. Remiges saturate fusce extus griseo-ferrugineo, intus albido 
marginatx; primores basi alba tectricibus proximis albis basi et apice nigris; secundaria apice pallidæ tectricibus nigricantibus, interioribus semi-albis vel albis, unde macula oblonga major in ala composita. Rectrices nigro fuscr pallido marginat:; par intermedium basi album, ceterx a basi supra medium alba.

Fenina: supra saturate fusca marginibus pennarum rufescenti-griseis. Fascia supra oculos rufescenti-albida. Macula alarum alba parva aut fere nulla. Fasciâ sub oculis caret, sed tempora habent maculam fuscorufam.

Pullus ante primam deplumationem: Supra nigricans; stria ad rachin singulze penmæ dilute ferruginea, marginibus rufo-ferrugineis. Stria supra oculos, gula, jugulum et pectus sordide ferrugineo-flavescentia, fusco immixto. Remiges cum tectricibus nigræ ferrugineo marginatr. Rectrices a basi ad medium albæ, dein nigrx rufescenti-albido fimbriatr.

\section{HISTORIA.}

Migratoria ad nos renit versus finem Mensis Aprilis (circa d. 25 Apr. prima redit.) astate in campis, sylvis adjacentibus, inter frutices et in agris vulgaris. Plerumque in summo cacumine fruticis vel cardui cujusdam sedit. Cantus haud insvavis, vesperi et mane pracipue audiendus. Nidum ex culmis et foliis graminum construfum, pilis et setis intus stratum, in fruticibus haud multum a terra elevatum ponit. Ova 5 pallide coerulea, immaculata. Vescitur Insectis qvibus fere more Muscicapte potitur. Abit Mense Sep. tembri. 


\section{LXXXXIII SAXICOLA OENANTHE.}

Diagn. Dorsum canum, frons, linea supra oculos ct uropygium alba. Fascia per oculos nigra. Cauda alba apica nigra.

\section{SYNONYMA,}

Motucilla Oeranthe, Lin. Faun. Sv. p. 93. n. 254. Retz.

Faun. 259. 11. 242.

Motacilla Oenanthe, Lin. Syst. Nat. I. p. 332. n. I5.

Fabr. Faun. Groenl. p. 122. 11. 84.

Sylvia Oenanthe, Lath. Ind. Orn. I. p. 529. n. 79.

Vitiflora, Briss. Orn. III. p. 449. n. 33.

Cul-blanc, Vitrec ou Motteux Buff. Ois. V. p. 237. Pl.

Enl. 554. f. I. 2. Vers. Germ. Otto XVI. p. 5.

f. I. 2.

Whent-Ear, Pen. Brit. Zool. I. p. 383. 11. I57. Arct.

Zool. II. p. 420. P. Lath. Syn. IV. p. 465. n. 75.

Vers. Germ. Bechst. IV. p. 460. n. 75. Sup. p. 182. Saxicoln Oenanthe, Meyer Vögel Livl. u. Esthl. p. 127. Weissschwänziger Steinschmäzzer, Bechst. Nat. D. 2te Auf. 3ter B. p. 675.

Syecis: Stensquätta.

\section{DESCRIPTIO.}

Longit. avis $6 \frac{x}{2}$ poll. longa; alx extensix 12 poll. Rostr. $\frac{2}{13}$ poll. A flexura alx ad apicem 4 poll. Cauda $2 \frac{2}{3}$ poll. Tibia $1 \frac{\frac{7}{8}}{8}$; Digit. med. $\frac{6}{3}$; Digit. post. Iㅇ poll.

Rostrum, pedes et iris nigra. Anguli oris nigri, vibrissis nigris supra ciliati. Nares ovatx, nudx. 
Caput supra collum, Dorsum et Scapula paliide ci. nerea mixtura levissima rufescente. Frons albida dimitrit lineam albam supra oculos. A naribus per oculos fascia nigra, quæ in temporibus magis dilatatur. Subtus avis alba, in jugulo et pectore tinctura levissima ferrugineo-favescenti. Alx nigra. Remiges medix extus pallide griseo tenuiter marginatx; secundariæ apice pallidx. Tectrices alarum superiores nigræ, inferiores et femor pennis nigris albo fimbriatis. Rectrices a basi supra medium (ad apicen alx claus $x$ ) niver, dein nigrr; par medium nigro-fuscum a basi ad tertiam partem album. Tectrices caudæ superiores alba.

Feniria: aliquantum minor, caret albedine frontis et fascia per oculos ad tempora nigra, cujus loco in temporibus macula minor fusco-rufescens. Caput et Dorsum sordide cinerea, rufescenti tincta. Gula, jugulum et pectus sordide ferruginescenti-lutea. Alæ fuscæ ubi Maris nigra.

\section{HISTORIA:}

Migratoria. Redux ante medium Mens. Aprilis. In campis ad lapides et sepes æstate vulgaris. Agilis admodum, caudam erigit et expandit, corpusque totum sursum et deorsum clamitans movet. Nidulatur in sepibus et lapidum acervis. Nidus ex culmis et straminibus construetus, plumis, pennis, setis \&c. intus stratus. Ova 5- 6 alba, coerulescentia, immaculata. Vescitur Insectis, presertim Coleopteris et Dipteris. 
GENUS XXI.

\section{S Y L V I A.}

Rostrum rectum, subtenue, subulato-acuminatum, mandibulis fere æqualibus, superiore (plerumque) emarginatâ.

Nares obovatæ, nudæ.

Lingua apice lacera.

Pedes ambulatorii, mediocres, ungue postico mediocri, curvato.

Cauda modica.

\section{DISSECTIO I.}

Rostrum robustiusculum, altius quam latius, compressum. Pedes robustiusculi.

\section{SYLVIA LUSCINIA。}

Diagn. Supra rufo-grisea rectricibus fusco rufis, subtus griseo-albida.

\section{SYNONYMA.}

Motacilla Luscinia, Lin. Faun. Sv. p. 88. n. 244. Retz. Faun. p. 252. n. 232. Lin. Syst. Nat. I. p. 328. Gmel. Syst. I. p. 950. Briss. III. p. 397. I3.

Sylvia Luscinia, Lath. Ind. I. p. 506. I. 
Le Rossigrol, Buff. Ois. V." p. 8I. t. 6. f. I. Pl. enl.

615.2. Vers. Germ. Otto XV. p. 7. cum fig.

Nightingule, Pen. Brit. Zool. I. p. 365. 11. I54. Aret.

Zool. II. p. 416. A. Lath. Syn. IV. p. 408. I.

Vers. Germ. IV. p. 408. Sup. p. I80.

Die Nachtigall, Bechst. N. D. 2 Auf. 3. B. p. 476.

Svecis: Näcktergal.

\section{DESCRIPTIO.}

Longit, avis $6 \frac{3}{8}$ poll. Alx extensæ $10 \frac{2}{8}$ poll. Ro. str. $\frac{x}{2}$ poll. Cauda $2 \frac{3}{4}$ poll. extra alas extenditur $I \frac{r}{2}$ poll. Tibia $1 \frac{7}{8} ;$ Digit. med. $\frac{7}{8}$; Dig. post. $\frac{0}{15}$ poll.

Rostrum aliquantum robustum, basi subdilatatum, versus apicem compressum; maxilla superior fusca aliquantum longior pone apicen emarginata; maxilla inferior pallida apice fuscescenti. Nares ovato-rotundatx. Anguli oris, faux et lingua lutea. Iris obscure fusca. Pedes cinereo-fusci. Cauda subrotundata.

Caput, cervix, dorsum et alæ supra cinereo-fusca, rufo tincta. Uropygium et cauda fusco-rufa. Gula, abdomen et crissum alba. Collum antice, pęus et hypochondria cinerascentia.

\section{HISTORIA.}

Migratoria ad nos venit primis diebus Mens. Maji. In frondosis ubique fere Scanix invenitur, numerosius ad Beckaskog, Segesholm, Torup, Engelholm \&c. etiam in Blekingia. Habitat praterea in Oelandia, Gottlandia, Svartsjölandet, Fullerön, Uplandia \&c. Ad occidentalem patrix plagam vix supra Scaniam adscendit, jugum (Hallandsàs dictum) vix transmigrans. Numerus Lusciniarum ita tamen apud nos imminuitur, ut plurimis locis ubi olim frequemtes fuerunt, nunc 
rara aut nulla occurrat. Nidulatur in fruticibus pro. pe terram initio Mens. Junii. Ova ponit quinque sordide olivacea. Dum ovis incubat femina, Mas in frutice adjacente cantat. Cantus celebratissimus et eximius (quem litteris exprimere conatus est Cel. Bechstein 1. c.) nonnunquam etiam interdiu, sed plerumque im. becillior exauditur: nocke vero silentium et somnum plurimis animantibus imponente, cantus ejus fit plenus, gravis, acutus, creber, extentus, uhi visum est, vibrains, summus, medius, imus. (Plin.) Pullis exclusis, quod plerumque fit circa d. XX M. Junii, cantus silescit. Uterque parens Larvis et Inseetis pullos unâ nutriunt.

\section{VC. SYLVIA NISORIA.}

(Tab. V.)

Diagn. Supra saturate cinerea, subtus alba cinereo undulata. Irides aurantiæ.

\section{SYNONYMA.}

Die gesperberte Grasmïcke, Sylvin Nisorin, Bechst. N. D. 2 te Aufl. 3. B. p. 547.

Mus. Payk. Mus. Upsal.

OBSERV.

Bechstein 1. c. ut Synonymum adfert Mot. Ficedulam Lin. Faun. p. 90. ก. 25 1. quod tamen miniux ad hanc, sedirevera ad Muscicapam Grisolin pertinet.

\section{DESCRIPTIO.}

Lusciniam magnitudine excedit, ideoque inter indigenas sui generis facile maxima. Ultra 7 poll. longa. Rostrum fere $\frac{5}{8}$ poll. Cauda fere 3 poll. extra alas ad I $\frac{x}{2}$ poll. extenditur. Tibia I poll. 
Rostrum subulatum, leviter compressum, fuscum, mandibula inferiore basi pallida. Iris aurantia. Pedes validi, grisei, digito postico ungue forti et adunco armato.

Singularis similitudo coloris cum Falcone Niso, etiam in Iridibus obscrvanda. Avis supra saturate cineyea, oculorum orbitae præsertim ad rostrum nigricantes. Subtus tota alba undulis seu lineis transversis cinereis in gula et jugulo copiosissimis. Remiges fusca margine dilutiores, secundarix albedine terminatz. Tectrices majores apice albx. Rectrices xquales cinereo-fusce I-I. aliquantulum breviores, introrsum versus apicem dimidiato-albx. 2-2. macula magna in apice alba; reliqux albo leviter terminatx, intermedis imnaculatis. Variat Rectricibus I - I. tantum in apice albis.

\section{HIST ORIA.}

Rara. Migratoria. In Scania prope Ifvetosta*) ubi cum conjuge in alnetis prati hamidi habitat. Avis timida; inquieta, alte et celeriter volat abrupte cantando. Pullis exclusis per familias in alnetis inrenitur. Homine ad pullos appropinquante, parentes triste vociferantes ab uno in alterum fruticem, volitant; fere ut Ianius Collurio.

\section{SYLVIA ATRICA PILLA.}

Dragn. Supra saturatius, subtus pallidius cinerea. pileus maris niger, femine fusco-ferrugineus.

\section{SYNONYMA.}

ALtacilla Atricapilla, Lin. Faun. Sy. p. 93. n. 256. Retz. Faun. p. 260. 11. 244. Lin. Syst. I. p. 332. n. I8. Gmel. Syst. I. p. 970. 
Syluia Atricapilla, Lath. Ind. I. p. 508. 11. 6.

Curruca Atricapilla, Briss. III. p. 380.6.

$L_{a}$ Fauvette it tete noire, Buff. Ois. V. p. 125. t. 8. $f$.

1. Vers. Germ. Otto XV. p. 75. cum 2. fig.

Blackcap, Pen. Brit.Zool. I. p. 374. n. I48. Arct.Zool.

II. p. 4I8. F. Lath. Syn. IV. p. 4I5. n. 5. Vers.

Geim. Bechst. IV. p. 415.

Der Mönch, Bechst. N. D. 2te Aufl. 3. B. p. 5 I2.

Svecis: Srarthätta.

\section{DESCRIP TIO.}

Longit. avis $6 \frac{1}{2}$ poll. Alzextensx $9 \frac{3}{3}$; Rostr. $\frac{3}{3}$ poll. Cauda $2 \frac{1}{2}$; Tibia $\frac{7}{8}$; Digit. med. $\frac{8}{8}$; digit. post. $\frac{x}{2}$ poll.

Rostrum supra nigricans subtus griseo-coerulescens. Nares obovatz. Faux pallide incarnata. Iris brunnea. Pedes plumbeo-fusci.

Caput supra a fronte per oculos ad nucham nigrum. Cervix, latera colli et capitis pallide cinerea. Dorsum et Scapula cinerea, olivaceo tincta. Avis subtus pallide cinerea, gula, abdomine et crisso albidis. Remiges fusce, olivaceo extus tinctr, primx dure extus albo tenuiter marginats. Cauda xqualis rectricibus acuminatis fuscis olivaceo marginatis.

Femina: Caput supra ferrugineo-fuseum. Dorsum rufo-griseum olivaceo tinctum. Genze et gula pallide cinerex. Pe?tus et hypochondria pallide grisea. Ab. domen album, rufescenti tinctum.*)

\section{HISTORIA.}

Passim. Migratoria. In Scanix hortis et sylvis circa finem M. Maji redux rarius tamen occurrit. Nidula-

*) Inventa cse Scloperoque occisa a Cienerosiss. Comite Trolle Waitheister, cui Historiam avis Descriptionemque debeo, १uique specimine descripto Museun Payk. donavit. 
tur in fruticibus. Ova 4-6 lutescenti-alba ferrugineo et fusco maculata. Vescitur Insectis, Larvis et Baccis.

\section{VCII. SYLVIA HORTENSIS,}

\section{Bechst.}

Diagn: Supra cinereo-fusea subtus cinereo - albida, peEtore leviter fulvescente. Palpebri albz. Pedes plumbei.

\section{SYNONYMA.}

Motacilla Salicaria, Lin. Faun. Sv. p. 90. 11. 249. Motacilla Hippolais, Tengm. Wet. Ac. Handl. I783. p. 53. Retz. Faun. p. 255. n. 236. et Descriptio pricr sub Mot. Salicaria p. 256. qux ad M. Salicariam Lin. et ad M. Hippolaidem Tengm. peitinet. Sylvin Hortensis $\beta$, Lath. Ind. I. p.507. n. 3. nec tamen $M$. hortersis Gmelini, qux forte $M$. Sylvia Lin. Sylvin passerina, Lath. Ind. I. p. 508. $11_{\mathrm{r}} 5$. Curraca Minor, Briss. Orn. III. p. 374. 3. Passerinette, Buff. Ois. V. p. I23. Pl. enl. 579. f. 2.

Vers, Germ. Otto XV. p. 72. cum fig.

Pettychaps, Lath. Syn. IV. p. 4I3. n. 3. et Vers. Germ. Bechst. IV. p. 4I2. n. 3. non prior, sed posterior, quam in Lancashire non raram dicit Latham.

Die Graue Grasmiicke, Bechst. N. D. 2te Auf. 3. B. p. 524.

\section{OBSERV.}

Omnes fere Ornithologi in Salicaria Lin, citanda hallucinan. iur. At vero aut hac est aut nulla distineta Species. Cel. Tengmalm, qui 1. c. avem nostram luculenter descripsit, dicit esse in Fauna confusionem Mr. Hippolaidis et Salicaria; nec ta- 
men in quo confusio proprie consistat dicit, nee confusionem correxir. Nomina solum mutavit, es temere. Nam M. Hippolaidem Lin. non esse Hippolaitem Tengmalmi, quis non videt? Talem nominum mutationem etiam Mot. Syluia et Curruea fecis Tengmalm, quod infra ostendam. Avicula nostra vix accuratius describi porest quam a Brissonio descripta est loco cirato, quem ad suan $P_{\text {asserinette adfert Buffon. Quz vero de moribus }}$ ejus adfert Buff. observationibus certe debentur, autunno ina stitutis (I11. Wachtm。 in litt.)

\section{DESCRIPTIOO.}

Longit. avis fere 6 poll. Alz extensx 9 poll. Ro. str. $\frac{3}{8}$, a flexura alx ad apicem 3 poll. Cauda 2 poll. Tibia $\frac{6}{8}$ poll. Digit. med. $\frac{\pi}{x} \frac{\pi}{6}$; Digit. post. $\frac{x}{2}$ poll.

Rostrum supra corneo-fuscum, subtus pallidum, altius quam latius, maxilla superior aliquantum longior, pone apicem excisa. Nares oblongo-ovatr, sura sum subcurvatx. Fanx pallide grisea. Anguli oris pallidi vibrissis nigris brevioribus ciliati. Iris rufescenti-fusca. Pedes plumbei, unguibus griseo-fuscis. Cauda æqualis.

Avis supra cinereo - fusca levissime in olivaceum in. clinans. Palpebrx albx. Remiges fuscr, apice tenu. iter pallido cinct: margine exteriore cum tectricibus dorso concoloribus. Rectriees cinereo-fusce, vix dor. so obscur:ores, margine exteriore et apice tenuiter albido cinctx. Tętrices alarum inferiores dilute flavescentes. Gula, pectus, hypochondria cano-fuscescen. tia vix in flavescens inclinantia. Abdomen et crissum alba.

\section{HISTORIA.}

Migratoria. Redux jam medio Miajo cum forescunt Prunus cerisus et Tulipa sylvestris, in hortis cantat, 
et abit medio Septembris. Ubique in Srecix Australioris hortis et sylvis frondosis. Etian in Hallandia et cirea Holmiam hand rara. Tempore vernali et æstate per totam diem alta roce egregie cantat. Vescitur Inseetis et Larvis. Nidulatur in densis fruticibus ex. gr. Juniperi, Cratagi \&e. Nidum haud multum supra teram elevatum culmis graminum exsiccatis constructųm, setis equinis stratum, Mense Junio inveni. Ova $4-5$ cinereo-alba maculis in aliis minoribus in aliis majoribus fušco-olivaceis. Sonus, quem edit hoste adpropinquante: Tack-Tuck-Tack! fere audit. Abit circa medium Septembrem.

\section{SYLVIA CINEREA, Bechst.}

Diagn. Supra cinerco-fusca remigibus latere exreriore ferrugineis, subtus albida, Reetrice exrima extus longirudinaliter oblique alba, secunda apice alba.

\section{SYNONYMA.}

Motacilla Sylvia, Tengm. Wet. Acad. Handl. 1783. p. 53. Descriptio sub Mot. Hippol, in Faun. Sv. Lin. p. 90. 1. 248.

Sylvin cineren, Lath. Ind. I. p. 5 I4. n. 23.

Fauvette grise, Buff. Ois. V. p. II7. Vers. Germ. Otto XV. p. 9I. cum fig. Pl. enl. 579. t. 3.

White-Thront, Pen. Brit. Zool. I. p. 387. n. І 60. Aret. Zool. II. p. 422. S-Lath. Syn. IV. p. 429. n. I9. Vers. Germ, IV. p. 428. n. I9.

Die Fahle Grasubicke, Bechst. N. D. 2te Auf. 3. B. p. 534. 


\section{OBSERV.}

Hane aviculam nullibi in Fauna Suec. Lin. probe descrip. tam video, nisi Descriptione sub n. 248. (Diagnosis tamen hue non pertinet.) Sed quia Linné hanc avem descripturus, illam ita similem invenit antecedenti, ut feminam ejus putarer; non possum quin credam Mot. Carratani Lin. Faun. 247 . huc pertinere. Hanc meam opinionem quoque probanc Aves Pifke Rudbeckii, qua semper sunt refugio illi, qui Synonymiam Linseanam extricare voluerit. Igitur in fol. 61, cel. Rudb. optimam hujus avicule dedit iconem eâdem exacte Synonymiâ, quam sub Mot. Cur ruca adferc Linné. Hoc probato restat, ut Curtucam Aułorum ind Faun. Lin. indagemus. Hanc sub. no 250. (Mor. Sylvia) quzrendam puto. Color enim et Magnitudo (vix Trochilo major) in nullam melius quam in hanc qquadrant. Pl. enl. 579. f. 3. aviculam bene refert. Descriptio vero Buffonii corrupta et mores hujus avis cum sequentis confusi. Synonymi, quis ex Pennant et Bechstein citavi, optima sunt.

\section{DESCRIPTIO.}

Longit, avis 6 . poll. Ali extense $8 \frac{\pi}{2}$ poll. Rostr. $\frac{7}{16}$ poll. Tibia fere I poll. Digit. med. $\frac{6}{3} ;$ Digit. post. $\frac{x}{2}$ poll. Cauda $2 \frac{\mathrm{r}}{2}$ poll. Extra alas extenditur $1 \frac{5}{8}$ poll.

Rostrum supra fuscum, subtus pallidum, maxilla superiore parum longiore, vix excisa. Anguli oris et os intus flavescentia vibrissis quatuor nigris. Nares ob. longa sursum curvatx. Iris dilute avellana. Pedes futm sco-flavescentes unguibus cinerascentibus.

Caput supra ei Cerrix cinerascentia. Dorsum cinereo-fuscum rufescunti levirer tinctum. Remiges fusce, primores tres margine exteriore tenuiter albie, secind a $^{-}$ vice cum tectricibus majoribas ferugineo late marginatx, ita ut ala complicata terruginca apparent. Tems porum macula dorso concolor; preterea latera capitis et colli cinerea dorso pallidiora, Subtus avis atbita cum rufescentis mixtura in jugulo, lattribus peftoris 
et crisso. Gula et abdomen alba. Alæ ad basin subtus albæ maculis parvis fuscis. Cauda rqualis; Rectrices fuscx, extima aliquantum brevior cinerascens, maculà longâ, alba fere a basi exterioris marginis ad apicem ubi latior totam occupat pennam; secunda apice alba.

\section{HISTORIA.}

Migratoria redit circa Medium Mens. Maji. In Iucis, hortis, sylvis et truticetis haud rara. Agilissima et inquietissima avis. Egregie cantat. Cantans sæpe sicut Anthus Arboreus e cacumine arboris vel fruticis in aëren volando ascendit et cantu finito in sedem re. vertitur. Homine ad nidum ejus adpropinquante ira-

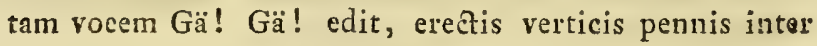
frutices saliens. Nidulatur in frondosis fruticibus. Ova virescenti-alba punctis olivaceis et fuscis. Vescitur insectis et Larvis.

\section{IC. SYLVIA CURRUCA.}

DIAGN. Supra rufescenti-grisea capite cinerascente, macula temporum obscuriore. Subtus alba. Rectrix extima latere exteriore et apicis margine interiore alba.

\section{SYNONYMA.}

Motacilla Syluia, Lin. Faun. Sv. p. 90. 11. 2.50? (Vide observ. in Synon. antecedentis).

Mot. Curruca, Tengm. Wet. Ac. Hand. 1783. p. 53. Sylvia Curruca, Lath. Ind. I. p. 509. n. 9.

Carruca garvula, Briss. III. p. 384. 11. 7.

Fauvette babillarde, Buff. Ois. V. p. I35. P1. enl. 580. fig. 3. Vers. Germ. Otto XV. p. 97 cum fig. (Descriptio ad antecedentem pertinet.) 
Balbling Wabler, Lath. Ind. Om. IV. p. 417. n. 6 . Ver's. Germ. Bechst. IV. p. 4 I 7.

Das Millerchen, Syluid garula, Bechst. N. D. 2te Auf. 3 B. p. 540.

\section{DESCRIPTIO.}

Longit. atis $5 \frac{2}{3}$; Alx extensiz 8 poll. Rostr. $\frac{3}{3}$ poll.

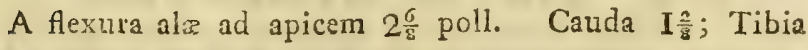
$\frac{5}{\frac{5}{5}}$ poll. Digit. med. $\frac{5}{8}$ poll. postic. $\frac{x}{2}$ poll.

Rostrum compressum, acutum, supra nigricans, subtus pallide coerulescens; maxilla superiore aliquantum longiore, pone apicem partm excisa. Anguli oris pallidi. Os intus et lingua bifida, pallida. Nares oblongx, sursum curvate, vibrissis supra sinus oris 3.4 nigris. Iris constat annulis binis, quorum exterior albidus, interior rufus. Pedes plumbeo-fusci.

Caput supra cinereum, fuscolevissime tinctum. Dor. sum et alarum tectrices pallide cinereo-fusca leviter rufescenti tirea. A naribus per oculos fascia cinereofusca, qux latior est et obscurior pone oculum. Palpebra inferior alba. Avis tota subtus alba; in gula jugulo et abjomine pure, in pectore, alarum tectricibus inferioribus et crisso levissime in rufescens inclinans. Remiges et majores teetrices fuscr irufescentigriseo tenuiter marginatx. ReĈrices fusca, extima aliquantulum breviore, pallidiore, latere exteriore et margine interiore ad apicem alba, secunda apice albo unaryinata; medix binx albıdo, reliqux rufescenti. -griseo tenuiter marginata. Obs. Variat Rectrice 1-I dimidiato oblique albida prater lineam fuscam secundum xachin.

\section{HISTORIA.}

Migratoria. Circa XX d. M. Maji in Scanix hortis ef fruticetis redux obvenit. Inter frondes arborum Nilsson-Ornith. 
Insecta et Larvas lectura salit avis agilis et inquieta. Suavi sed submissa voce cantat. Nidulatur in fruticibus. Ova 4.5 alba, extremitate obtusiore maculis coerulescentibus et brumneis. Frigore instante domos intrantem vidi. Abit circa medium Septembrem.

\section{SYLVIA RUBECULA.}

Dingn: Supra sordide olivacea, gula er pectore fulvo-rubris.

\section{SYNONYMA.}

Motacilla Rubecula, Lin. Faun. Sv. p. 95. 11. 260. Retz. Faun. p. 264. n. 249. Lin. Syst. I. p. 337. Gmel. Syst. I. p. 993.

Sylvia Rubecula, Lath. Ind. I. p. 520. n. 42. Rubecula, Briss. Orn. III. p. 418.

Rouge-gorge, Buff. Ois. V. p. I96. t. II. Vers. Germ. Otto XV.p. 209 cum fig. Pl. enl. 36I. f. I.

Red-Breast, Pen. Brit. Zool. I. p. 372. n. 147. Aret.

Zool. II. p. 4I7. D. Lath. Syn. IV. p. 442. 11.. 38.

Vers. Germ. Bechst. IV. p. 442. n. 38.

Rothehlichen, Bechst. N. D. 2. Auf. 3. B. p. 579. Svecis: Rotgel, Scanis. Rödbrösta.

\section{DESCRIPTIO.}

Longit. avis $5 \frac{8}{2}$ poll. Alz extensa 9. poll. A flexura alx ad apicem $2 \frac{6}{8}$ poll. Cauda $2 \frac{2}{3} ; \operatorname{Rostr} \frac{3}{\frac{3}{3}}$; Tibia I. poll. Digit. med. $\frac{5}{8}$, postic. $\frac{1}{2}$ poll.

Rostrum nigro-fuscum altius quan latius, máxilIa superiore prominula, pone apicem emarginata. Nares ablongx sursum curvatx. Anguli oris et os 
intus flavescentia. Iris fusca. Pedes griseo-fusci un. guibus obscurioribus. Cauda æqualis.

Caput supra, cervix, dorsum sordide olivacea. Fascia frontalis, gula cum capitis lateribus usque ad oculos, jugulum et superior pectoris pars fulvo-rubra, qui color in colli lateribus coerulescenti-cinereo cingitur. Pars inferior pectoris et abdomen alba. Crissum sordide album. Remiges et acuminatx Rectrices fuscre latere exteriore Dorso concolores. Tectrices majores Remigum interiorum (interdum) apice maculis parvis flavescentibus. Teetrices inferiores fuscescentes, luteo intermixto.

Avis junior: ante primam pennarum mutationem : Caput supra et ad latera, Dorsum et tectrices minores olivacea, singula penna macula flavescenti, fusco terminata. Remiges et Rectrices ut in adultis, sed magis olivaceo marginatx. Gula et pectus sordide flavescentia, pennis fusco terminatis. Abdomen et crissum sordide albida.

\section{HIS T ORIA.}

Migratoria, sed inter primas, quæ adveniunt et postremas, qux abeunt. Jam ad finem M. Martis in hortis Scaniz invenitur, et frigore tum oppressa haud raro domos quærit. Verno tempore frutices et arbores hortorum frequentans suavi sed demissa roce cantat, etian autumno, ante abitum, in hortis cantat. Estate sylvas densas et frondosas nidifieatura petit. Nidulatur in terra inter radices arborum, in truncis \&c. Nidus extus ex muscis, intus ex culmis graminum constructus. Ova 5 sordide albida punctulis rufo-fuscis extremitate obtusiore confuentibus. Pulli volatui apti jan initio Julii occurrunt. Victitat Insectis, Larvis, lumbricis et autumnali tempore Baccis 
Sorbi, quibus allecta in Laqueis inter Turdos capitur. Usque ad finem M. Decembris in hortis obvenit.

\section{DISSECTIO. II.}

Rostrum temuissimum, teres, acutum, basi lito tius quam altins. Pedes graciles.

\section{SYLVIA PHOENICURUS.}

Diagn. Mas: Frons alba. Caput et dorsum coeruleo-cinerea. Gula et jugulum nigra. Pectus et cauda rufa rectricibus intermedis fuscis.

Femina: Supra rufescenti-grisea; gula et abdomen albida; pectus sordide ferruginescens.

\section{SYNONYNA.}

Motacilla Phoenicurus, Lin. Faun. Sv. p. 94. n. 25\%. Retz. Faun. p. 26I. 11. 245. Lin. Syst. Nat. 335. 11. 34. Gmel. Syst. I. p. $98 \%$.

Sylvia Phoenicurus, Lath. Ind. I. p. 5 II. 11. I5. Rossignol de muraille, Buff. Ois. V. p. I70. t. 6. f. 2 . Vers, Germ. Otto XV. p. I65. cum fig. 2. Pl. enl. 35I. f.I. (Mas.) 2 (fem.)

Ruticilla, Briss. Om. III. p. 403.

Redstart, Pen. Brit. Zool. I. p. 37 I. n. I46. Aret.

Zool. II. p. 4I6. B. Lath. Syn. IV. p. 42I. II.

Vers. Germ. 42I. II.

Gartenrothschsoinzchen, Bechst. N. D. 2te Auf. 3. B.

p. 607 .

Femina: Succis Rödstjert.

MLotacilla Erithacus, Lin. Faun. Sv. p. 94. n. 258.

Retz. Faun。 p. 262. n. 24\%. Gmel. Syst. I. p. 988. Syluia Evithasis, Tuatli. Ind. T. p. 513 . 
Red-tail, Lath. Syn. IV.p. 425. n. I4. Vers. Germ. IV. $\mathrm{p}$ 42.5. 11. I4. sc. prius descripta seu femina; avis qux pro Mare huic adjungitur, alia est et forte ut Bechst. putat femina annua Sylv. Svecice. Svecis: Rödbuk.

Azis Zunior: Motacilln Tithys, Lin. Faun. Sv. I. Edit. p. 85. n. 227. (Obs. Bechst. N. D. adfert hoc sy. nonymum ad suam $S$. Tithyn, ad quam minime tamen pertinet, sed revera ad juniorem Phoenicurum.)

\section{DESCRIPTIO.}

Longit. avis fere $\sigma$ poll. Alx extensæ $9 \frac{\pi}{2} ;$ Rostr. 7 poll. A flexura ale ad apicem 3 poll. Cauda $2 \frac{3}{3}$; Tibia $\frac{7}{8}$ poll. Digit. med. $\frac{5}{8}$; postic. $\frac{x}{2}$ poll.

Rostrum nigrum, tereti-acutum, maxilla superiore prominula apice deflexa, vix emarginata. Os intus et anguli oris flava. Nares ovatx. Iris nigricans. Pedes nigri, cum digitis, graciles. Cauda subrqualis vix rotundata.

Frons alba, supra basin rostri nigra. Vertex, cervix, dorsum et scapulæ coerulescenti-cinerea (rufo interdum leviter immixto) Gula, jugulum, latera colli et capitis usque per oculos nigra. Pectus, hypochondria, tectrices alarum inferiores, Uropygium rufo-fer. ruginea. Abdomen album. Crissum dilute ferruginescens. Remiges fuscx dilute ferruginescenti tenuiter extus marginatæ. Rectrices rufo-ferruginex, intermediis duabus fuscis, basi et margine exteriore rufo. ferrugineis.

Femina: a mare coloribus valde differt. Supra grisea rufescenti tineta. Genæ, gula et jugulum sordide alo bida. Pectus, tectrices alarum inferiores et hypochondria sordide ferruginescentia. Abdomen et crisums 
Mbida, levissime ferruginescenti tincta. Remiges et Rectrices ut in Mare.

Avis junior: quaiis autumnali tempore in sylvis et hortis una cum parentibus, frequens occurrit. Stupra cinereo-fusca, rufo leviter tincta, Uropygio ferrugineorufo. Pectus et hypochondria fuscescentia, flavescenti tincta. Abdomen et gula albida. Crissum pallide fulvescens. Remiges nigricantes, prxsertim interiores pallide ferrugineo marginatx. Rectrices ferrugineo. rufx; par intermedium nigricans margine exteriore rufo - ferrugineo.

Mas junior post primam penuarum mutationem: Frons albida fusco tincta. Vertex, cervix, Dorsum coerulescenti-cinerea fusco saturatius tincta. Gula et jugu. lum pennis nigris pallide cinereo terminatis. Pectus ferrugineum albo pulchre undulatum. Abdomen medium album. Crissum flavescenti-albidum. Remiges et Rectrices ut in proxime antecedenti.

\section{HISTORIA.}

Migratoria. Redux ad finem M. Aprilis. Verno cempore in hortis, sepibus vivis et in fruticetis frequens. Cantus haud insuavis. perpetuo fere ocissime candam concutit avis agilissima. IEstivat in syivis den. sioribus et hortis, ibique in cavis arborum et murorum, inter lapides sepium \&re. nidulatur. Ova 5 -8 pallide coerulescenti-viridia. Homine ad nidum vel ad pullos appropinquante sibilantem sonum edit: huit! huit! et magis in affectu: huit Ké-Ké! huit Ké Ké Ǩe! Auiumnali tempore ante dimigrationem, parentes cun pullis in sylvis et hortis sune frequentes. Abit post me. dium Seprembrem. 


\section{SYLVIA. TITHYS, Lath.}

Diagn. Supra saturate cinerea. Gula, jugulum et superior pars pectoris nigra. Rectrices rufx, inrernediis binis fuscis.

\section{SYNONYMA.}

Sylvin Tithys, Lath. Ind. Orn. I. p. 5I2. n. I6.

Motacilla gibraltariensis, Gmel. Syst. I. p. 987. atrata - Gmel. 1. c. p. 988.

Grey-Redstart, Lath. Syn. IV. p. 423. n. I2. Vers. Germ. Bechst. IV. p. 424. n. I2.

Black-Redtail, Lath. Syn. 1. c. p. 426. n. 16. Vers. Germ. 1. c. p. 426.

Hausrothschwänzchen, Bechst. N. D. 2te Auf. 3. B. p. 597. tab. XVIII.

Mus. Payk. Mus. Holm.

O BSER V.

Errant qui Mot. Tithyn Faun. Sv. I Ed. n. 227 ad hane avem referant. Hoc quisque videre potest, qui vel obiter descriptionem in Fauna Sv, datam inspiciat. Est vero Linnei Tithys junior Phoenicurus, qualis autumno frequens in Sylvis occurrit. In secunda editione avem hanc ob caussam exclusit Linné, et in XII Systematis editione eam ad Phoenicurum, cui pertinet, quamvis non ut femina, retulit. Liuné nostram avem non novit.

\section{DESCRIPTIO.}

Magnitudo Sylvic phoenicuri. Caput supra, cervix, Dorsum, uropygium saturate coeruleo-cinerea. F2scia supra basin rostri, latera colli usque ad oculos, Gula, jugulum et superior pectoris pars nigra, pectore undulis cinereis. Hypochondria cinerea. Abdomen medium albidum. Crissum luteo-rufescens. Kemiges fusce cinereo marginate. Tectrices superiores 
caudæ et Rectrices Inteo-ferruginex, pari intermedio fusco.

Fenina: Corpus obscure cinereum fere nigricans; abdo. men griseo variegatum. Remiges fusce extus aibido marginatæ. Reçrices ut in Mare.

\section{LOCUS.}

Novitia hæc Ornithologiæ Svecia rarissime apud nos occurrit. Specimen prope Upsaliam ab Illustriss. Comite Trolle Wachtmeister verno tempore sclopeto occisum est.

\section{SYLVTA SVECICA.}

Diagn. Supra cinereo-fusen. Gula coerulea. Cauda basi ferruginea, apice nigricans.

\section{SYNONYMA.}

Motacilla Svecica, Lin. Faun. Sv. p. 94. 11. 259. Retz. Faun. 263. n 248. Lin. Syst. I. p. 336. 37. Gmel。

Syst. I. p. 989.

Cyanecula, Briss. III. p. 4I3. I9. (Mas.)

Cyanecula Gibraltariensis, Briss. III. p.4I6.11.20. (fem.) Sylvia Svecica, Lath. Ind. I. p. 52I. n. 43.

In Gorge bleue, Buff. Ois. V. p. 206. t. I2. Pl. enl.

610. f. I. 2. 3, Vers. Germ. Otto XV. p. 225. cùm 2. fig.

Blue throated Warbler, Lath. Syn. IV. p. 444. n. 39.

Vers. Germ. Bechst. IV. p. 444. 11. 39. Pen. Arct. Zool. II. p. 4I7. E.

Blarkehlchen, Bechst. N. D. 2 te Auf. 3ter B. p. 589.

\section{DESCRIPTIO.}

Longit. avis $5 \frac{\mathrm{x}}{2}$ poll, Rostr, $\frac{3}{3}$; A flexura alr ad apicen $2 \frac{5}{8}$ poll. Cauda fere 2 poll. Tibia $I \frac{1}{8} ;$ Digit. med. $\frac{\sigma}{8}$ poll; postic, $\frac{x}{2}$. poll. 
Rostrun nigrum, gracile, tereti-compressum. Anguli oris lutei. Iris fusca. Tibiz incanatze digitis nigricantibus.

Caprt supra, ceivix, Dorsum, scapula cinereo-fus sca. A naribus ad oculum stria albida. Tempora fusco-rufescentia. Collum antice a gula ad sternum pulchre nitide-coeruleum fascia nigricante terminata. Pectus anterius ferrugineum. Abclomen albidum. Crissum albo-ferruginescens. Remiges fuscæ cinereo-fusco marginatx. Refrices a basi ad medium ferruginex, ceterum nigricantes; par medium fuscum.

Fenina: Gula albida ad latera stria utrinque longitudinali nigra. Jugulum striis binis cocruleis. Pedes in. carnati.

\section{LOCUS.}

Habitat in Salicetis et alnetis Norrlandia et Lappo. rix, dimigrationes per Finlandiam instituens, in inferiope Svecia nunquam visa.

\section{SYLVIA MODULARIS.}

DIAGN: Supra rufo-brunnea nigricante maculata. Caput, collum et pectus sordide coerulescenticinerea, capite et collo supra fusco striatis.

\section{SYNONYMA.}

Motacilla Modulavis, Lin. Faun. Sv. p. 89. n. 245. Retz. Faun. 25.3. 17. 233. Lin. Syst. Nat. I. p. 329. n. 3. Gmel. Syst. I. p. 952.

Syluit Modularis, Lath. Ind. I. p. 5 I I. n. I3. Curruca Sepiaria, Briss. Orn. III. p. 374. I2. Traine-buisson, Buff. Ois. V. p. I5I. Pl. enl. 6I5. f. I. Vers. Germ. Otto IV. p. I29. cuin fig. 
Hedge Spirrow. Pen. Brit. Zool. I. p. 376. n.' I50. Aret. Zool. II. p. 4 II8. H.

Hedge-Warbler, Lath. Syn. IV. p. 4I9.' n. 9. Vers. Germ. Bechst. IV. p. 420. n. 9.

Brannelle, Bechst. N. D. 2te Auf. 3. B. p. 6I7. Svecis: Järnspart.

\section{DESCR IP TIO.}

tongit. avis 6 poll. Alr extensæ $8 \frac{7}{2}$. Rostr. $\frac{7}{1} \frac{\gamma}{\gamma}$ poll. Cauda $2 \frac{2}{8}$ poll. extra alas complicatas $1 \frac{\pi}{2}$ poll. extendi. tur. Tibia $\frac{7}{8}$ poll. Digit. med. $\frac{6}{5}$; postic. $\frac{2}{15}$ poll.

Rostrum nigrum, teres basi aliquantum dilatatum, mandibula superiore prominula apice deflexa. Nares oblongx membrana fornicata semitectx. Os intus pallide fiavum. Iris fusco-rufa. Pedes incarnato-flavicantes.

Caput et collum supra einerea fusco striato-macula. ta. Dorsum et scapulx rufo-brunnea maculis nigrofuscis (Dorso Fring. Domestica fere simile). Uropygium griseo-brumneum immaculatum. Gula sordide albida. Jugulum, later colli, superior pars pectoris ef tectrices alarum inferiores coerulescenti - cinerea. Macula temporum rufa albido lineata. Abdomen albidum. Crissum albidum maculis acutis fuscis. Remiges saturate fuscr ferrugineo marginatip. Tectrices proximæe apice (plerumque) maculis minutis albidis. Rectrices saturate fusce, pari intermedio dilutiore marginibus ferruginescentibus,

\section{HI S TORIA.}

Haud vulgaris. Migrant pleræque, hybernant nonnulla non solum in Scania sed etiam in Hallandia \&c. *)

3) Juxta Lund in Scania hicme haud raro capiuntur inter Fring gilles. 
Autumno qux abeune, initio M. Aprilis redeunt et in hortis sepibusque vivis Scaniae occurrunt. Dulci sed submissa voce cantant. Victitant Insectis et seminibus.

\section{DISSECTIO III.}

Frons strite-acuminata, depressit. Rostrum elongatum, in pleriscrue dilatatum. Pedes robustius. culi. Cauda rotandata.

\section{SYLVIA HIPPOLAIS.}

Diagn, Supra cinereo-olivacea, subtus pallide flava. Cauda aqualis. Nares rotundatre. Pedes plumbei.

\section{SYNONYMA.}

Motacilla Hippolais, Lin. Faun. Sv. F. 90. n. 248. (Diagnosis nee tamen Descriptio, qux, ut ipse suspicatur, ad suam Currucani n. 247. pertinet. Vide Obs. sub Sylv. cinerea.)

Motac. Salicaria, Retz. Faun. p. 255. n. 237. cum Descriptione Pennantii ; Descriptio Linnei ad $M$. S $S_{\text {- }}$ licarian Lin. i. e. ad Hortcusem meam pertinet.

- Curruca Artndinacea, Briss. Orm. III. p. 378. Favette des rosenux, Buff. Pl. enl. 58I. f. 2. CObs. Descript. in Hist. Ois. V. p. I42. Otto XV. p. II2. potius ad sequentem pertinere videtur.)

Pettychinps, Pen. Brit. Zool. I. p. 376. n. I49. Obs. Pettychaps Lath. Syn. ad meam S. Hortensem pertinet.

Bastard nachtigall, Bechst. N.D. 2te Auf. 3. B. p. 553.

OBSER V.

Plura Synonyma certe! exrrica:e non valeo. Sxpius enim hiec cuin $S$. Trochilo, sapius cum alis confusa. Adfert quidem 
Cel. Bechstein 1. c. The lesser Pettycheps, Lath. Syn. IV. p. 4 I . hac vero avis tutn descriptione tum margis historiâ ad S. Trochilum pertinere mihi viderur. Citavit etian Bechst. La Fanvette Buff: Ois. V. p. 117 . quæ huc minime pertinet.

\section{DESCRIPTIO.}

Longit. avis $5 \frac{7}{2}$ poll. Alæ extensa 9 poll. Rostr. $\frac{7}{16}$ poll. A flextra ala ad apicem 3 poll. Canda 2 poll. Tibia $\frac{7}{8}$ poll. Digit. med.? $\frac{3}{8} ;$ digit. post. $\frac{x}{2}$ poll.

Caput fronte acuminata depressa. Rostrum rectum, obtusiusculum, præsertim basi depresso. dilatatum*), stpra incarnato - fuscum subtus flavescent. - incarnatum marginibus et oris angulis luteis, vibrissis 3.4 nigris. Mandibulæ æquales superiore vix emarginata. Nares patulæ rotundatx. Os intus croceum. Iris rufo-fus. ca. Pedes plumbei unguibus griseo-fuscis.

Caput supra, cervix, dorsum et tectrices alarum minores cinereo-olivacea. Stria pallide fava a naribus ad oculum. Falpebrx pallide favx. Gula, jugulum, pectus et abdomen dilute-flava, crisso et tectricibus aiarum inferioribus pallidioribus. Remiges saturate fusex, primores tenuius, secundarix latius luteo-viridi extus marginatx. Rectrices zquales, saturate fu. scre, extima pallidiore, albo tenuiter fimbriata, ceteris intus albo-extus pallide virescenti marginatis.

\section{HISTORIA.}

Migratoria, revertitux circa medium Majum. Avis. carta et inquieta haud rara est in hortis, fruticetis et ad margines sylvarum in inferiore Svecia. In magnis et densis sylvis rarius occurrit. Cantus egregius et

*) Rostro Muscicapx quodammodo simile. 
varia modulationis plenus, quem qui attentius exaudit, ex sonis variarum avium compositum reperiet. Recog. noscet enim in eo sonos Hirundinis Sturni, Fringille Conzabine, imo vero Sternce hivundinis et Trinzge Calidris. Vox quam edit cum homo vel alius hostis ad nidulum adpropinquat, his fere syllabis exprimi potest: Tack-Tack-Tack! et magis irata: Tăck-ăky̆ŏ-Tăckăhȳohȳ! Nidum pulcherrimum obconiformem, in fruticibus frondosis, duas fere ulnas super terram, medio Junio inveni. Foliis culmisque graminum et amentis Salicum artificiose erat constructus, extus pulcherrime ornatus subtili et albo cortice extimo Betulx, intus setis equinis stratus. Ova 5 pulcherrima, rubescenti-albida punstis et venis saturate sangvineis, presertim circa extremitatem crassiorem.

\section{SYLVIA ARUNDINACEA。}

\section{(Tab VI.)}

Diagn. Supra ciacreo-fusca, subtus alba ferrugine. scenti tincta. Cauda subcuneiformis, Rostrum elongatum. Frons depressa.

\section{SYNONYMA.}

Motacilla Artndinacen, Lightfoot Philos. Transadt. Vol. LXXV. anno I785. P. I. p. 8. fig. I. (bona.) Gmel, Syst. I. p. $992 *$ ).

Sylvin Arundintacen, Lath. Ind. Orn. I. P. 510.11 .12$. Der Spitzkopf, Bechst. N. D. Ima. Edit. 4. B. p. $66 \%$. Der Spitzkopf mit der Schwanzbinde, Bechst. 1. 6. 669.

*) Gmel. et Lath. in magnitudine avis exponenda hallucinantur; Lightf, l. c. dicit maris longitudinem esse $5 \frac{T}{2}$ poll. femine 5 Loll, sed laticadinein $7 \frac{\pi_{2}^{2}}{2}$ poll. 
Bouscavle de Provence, Buff. Ois. V. p. 134. Pl. enl. 655. f. 2 ?

Reed Wrcen, Lath. Syn. IV. p. 6I5. n. I5I. Vers. Germ. IV. p. 560.

Der Teichlaubvogel, Bechst. N. D. 2te Auf. 3. B. F. 566 .

\section{OBSERV.}

Specimen quod descripsi in Mus. Reg. Acad. Holm. asservatur, in Svecia occisum. Notatu sane dignum est quod hoc exemplar in reftricibus mediis talen habeat fasciam pallidioren (quamis obsoleram) qualem semel vidit Cel. Bechst. et quæ illum induxit ut novam constitueret speciem. Unde hæc sit, nescio. sed in arundinetis quarenda est avis.

\section{DESCRIPTIO.}

Longit. Avis 5 poll. Rosti. $\frac{7}{2}$ poll. A flexura ale ad apicem $3 \frac{\pi}{3}$ poll. Cauda 2. poll. Tibia fere $I$. poll. Digit. med. $\frac{\pi}{\frac{1}{6}}$; Digit. post. $\frac{9}{16}$.

Caput fronte acuminata et depressa. Rostrum longum, subtus teres, luteo-incarnatum, supra nigro-fuscum carina angulatum, maxilla superiore aliquantum longiore vix emarginata, apice obtusa, defexa. Supra angulos oris vibrissa quatuor nigra, breviores, reflexx. Nares ovales. Pedes validiusculi unguibus, præsertim postico, robustis, compressis. Cauda cuneato-rotundata, rectricibus acutiusculis, prasertim intermediis duabus longioribus.

Tota avis supra griseo-fusca olivaceo tincta, capite saturatius, uropygio pallidius. A naribus supra oculos stria albicans, in hoe specimine vix conspicua. Gula alba. Avis subtus albida tinetura ferruginescenti-lutea in petore, hypochondriis et crisso. Remiges saturate fuscr marginibus pallidioribus. Tectrices in- 
feriores sordide alba. Rectrices saturate fuscx, latcribus pallidiore, apicibus fere albido marginatis.

$$
\text { OBSERV. }
$$

Magnitudine et habitu S. Hipjolaili sinillima, a qua tamen his fere differt. I tho Rostrun ad basin aliquantum estangustius, et carina superne longirtdinalis a basi inter nares ad apicem magis conspicua. Maxilla superior quoque longior et apice deflexa. 2do Ungues majores sunt, præsertim postico valido, obtuso et compresso. 3rio Cauda fere cuneiformis, ato Color dissimilis.

\section{DISSECTIO. IIII}

Rostrum brevius, tenue, subcompressum. Pedes graciles.

\section{SYLVIA SCHOENOB压US,} mihi.

MOTACILLA SCHOENOBIENUS,

Lin. Faun. Sv.

Diagn. Capur supra nigricans striis griseo-olivaceis. Dorsum griseo-olivaceum maculis fuscis; uropygio sordide ferrugineo. Stria albida supra oculos. Subsus ferruginescenti-alba. Cauda rotundata.

\section{SYNONYMA.}

Moracilln Sehoenobcenus, Lin. Faun. Sv. p. 89. n. 246. Retzii Faun. p. 253. n. 234.

Syluia Salicaria, Lath. Ind. 1. p. 516. (Deseriptio), Sedge, Pen, Brit. Zool. I. p. 3SI. M. I55. (Bene descripta.) 
Seage Wirbler, Lath. Syn. IV. p. 430. 11. 21. Vers。 Germ. Bechst. IV. p. 43 r. 11. 2r. Aret. Zool. II. p. 419. $\mathrm{M}-$

Der Schilfsünger, Sylvial Phragmitis, Bechst. Nat。D. 2te Altf. 3. B. p, 633.

Svecis: Säfstigare.

OBS ERV.

Hac est avis, quamsub nomine Motac. Schoenobani intel. ledam voluit Linne in Fauna Sv. Descriptio Linneana brevior qquidem est et minùs exacta. Linné vero avem numquam ipse vidic. Cel. autem Leche, a quo descriptionem sumsit, hanc multo magis accuratan et perfectain in Alversariis suis, gnux ad manus sunt, reliquic. Ex his igitur liquet avem hanc nostram csse eandem ipsam, quam nomine Schoenobceni descripserit Linne. Cel. Pennant, ( $q u i$ nostram avem bene descripsit), et omnes fere post illum Ornithologi entmerarunt inter Synonyma ejus Motae. Solicarian Lin., quo tamen wihil falsius.

\section{DESCRIPTIO.}

Longit. avis 5 poll. Alx extensa $7 \frac{T}{2}$ poll. Rosti. 告 poll. Extra alas extenditur $I \frac{2}{8}$ poll. Tibia $\frac{7}{8}$; Digit. med. $\frac{5}{8}$ poll. Digit. post. $\frac{x}{2}$ poll.

Avis gracilis fronte acuminata. Rostrum acutissimum, rostro Sylvice Svecice simile, supra carinatum nigro-fuscum, versus apicem compressum; subtus basi pallidum. Nares ovato rotundatz. Os intus aurantinm. Lingua apice lacera, basi supra maculis binis fuscis notata. Iris castanea. Pedes sordide flavescentes, plantis subtus luteis unguibus pallide fuscis, postice majori, curvato, intermedio rectiusculo.

Caput supra fusco-nigrum striis longitudinalibus griseo-olivaceis proxsertim in medio pileo, ita ut faseia supra-superciliaris utrinque nigra immaculata ex- 
sistat. Dorsum saturate fuscum marginibus pennarum latioribus griseo-olivaceis. Dorsum posticum et tectrices caude superiores sordide lutescenti - rufa, imma culata. A naribus, supra oculos versus nucham stria albida; per oculos stria fusca pone oculos latior rufescens. Subtus avis sordide alba, rufescenti-luteo tincta in jugulo, hypochondriis et crisso. Alæ extenszob. tusx. Remiges fuscx, ultima extus albido marginata, interiores cum tectricibus griseo-olivaceo marginatæ. Tectrices inferiores albidx luteo tinctx. Cauda rotur. dato-cuneiformis (explicata angulum format obtusum). Rectrices apice rotundato fuscx, pallidius apice marginatz.

Pullus vix volatui aptus: Supra testaceus maculis longitudinalibus nigris pricipue in capite. Linea supra oculos pallide fiavescens. Remiges et Rectrices nigricantes, testaceo latius marginatr. Subtus albidus tinctura levi ferrugineo - flavescenti.

\section{HISTORIA.}

Migratoria. Locis paludosis ad margines Lacuum et fluviorum inter Arundines et Equiseta \&c. in Scania et Uplandia avem vidi. Inter arundines saliens variis modulationibus interdiu et in multam noctem cantat. Cantans arundines sxpe relinqvit et in ärem, Alauda inftar, sed brevius, adscendit. Cantus laaud insuavis c Cantu Mot. flave., Hirund. rustica et quibusdam so. nis stridentibus compositus videtur. Nidum nondum. inveni.

\section{SYLVIA SIBILATRIX.}

(Tab. VII.)

Diack. Supra flavescenti-viridis. Supercilia lutea. Fascia per oculos fusca, Gula et jugulum $\mathrm{Al}$. Nilisen Oruith. 
vescentia, Abdomen et crissum alba. Nares ovata.

\section{O BSERV.}

Cel. quidem Bechstein aviculan hancee et quodd colores ct guoad mores optime descripsit; Synonymiam vero quam adfert, approbare non possum. Recitat igitur smo Motacillam Trochilum Gmel. Syst I. p. 995. n. 49. (marem). Gmelin autem 1. c. nullam adfert coloris differentiam inter Marem et feminam. Avis ejus eadem est atque illa, quam sub hoc nomine intellectam voluic Linné. 2do Posillot ou Chantre, Buff. Ois. V. p. 344. Pl. enl. ก. 65 I. f. I. Vers. Germ. Otto XVI. p. 20r. Buffon 1. c. aperte confundit historiam hujus avis cum sequence. Otto vero 1. c. p. 211. nomine Sänger aviculam nostian descripsisse videtur. 3 tio Yellozy Wren, Lath. Syn. IV. p. 412. n. 147. Descriptio quidem coloris tam brevis est, ut inter aves tam sibi similes pexdiffeile sit videre, ad utram pertineat. Mores vero et cantus potius ad Trochilum pertinent. Quic vero cun ita sint, equidem hæc vera habeo.

\section{SYNONYMA.}

Grimer Lnubvogel, Sylvin Sybilntrix, Bechst. N. D. 2te Auf. 3. B. p. 56I.

The Wood Wren, Lamb. Transact. of Lin. Soc. Vol. II. p. 245.

Sylvia Sylvicola, Montagu Transact. Lin. Soc. IV, p. 35.

\section{DESCRIPTIO.}

Longit. avis 5. poll. Ala extensa $8 \frac{x}{2}$ poll. Rostr. $\frac{3}{\mathbf{x}^{6}}$ poll. Cauda 2. poll. Tibia $\frac{6}{8}$ poll. Digit, med. $\frac{x}{2}$ poll ; postic. $\frac{7}{T^{0}}$ poll.

Caput fronte Atricts. Rostrum basi latiusculum versus apioem compressum, marginibus tamen haud in- 
flexis, supra fuscum, carinatum, subtus basi et mar. ginibus incarnatum. Maxilla superior longior pone apicem deflexum emarginata. Nares ouatae. Os in. tus pallide favum et lingua bifida. Anguli oris vibris. sis nigris ciliatæ. Iris avellana. Pedes griseo-fusci plantis sordicle luteis.

Supra pulcherrime viridis capite et uropygio præser"s tim favo tinctis, A naribus supra oculos versus nu. cham stria flava, pone oculum pallidior. Per oculos stria fusca, qux latior est et saturatior pone oculos. Gula et collum antice pallide flava. Pectus, abdomen et crissum pulchie alba. Remiges nigro-fuscx margine exteriore flovescenti virides; interiores apice tenuiter albidx. Tectrices majores nigro-fusc marginibus flavescenti-virides. Alæ subtus secundum marginem flavæ punctis nigris. Canda bifurca. Rectrices satu. rate fusca; extima margine exteriore pallida, ceter flavescenti-viridi extus marginatr.

Femina: Mari similis coloribus tamen aliquantum pallidioribus.

Pullus: Rostrum supra fuscum, subtus incarnato-luteum. Anguli oris et os intus flava. Nares oblongo-ovales. Iris fusca. Pedes pallide grisei, plantis luteis. Cauda bifurca. Colores pennarum ut in femina.

\section{HIS T ORIA.}

Migratoria. Rara. In sylvis frondosis, præcipue in fagetis Scania ex gr. ad Roslätt, Billinge \&c. Hollan. dice ex gr. ad Fröllinge hanc pulcherrimam aviculam inveni. Magnitudine et coloribus cum S. Trochilo fa. cile confunditur. Cantu vero singulari ab omnobus congeneribus eriam eminus dignoscitur. Inter fiondes cacuminum fagorum saliens agilissima avicula se prodit voce extenta et trifti: dju! dju! dju! quain sequitur 
cantus alacris et sonorus Ippfippfippferrrr! Cantus maris fere continuus nec nisi devoratione Insectorum et Larvarum, ques inter frondes legit, interruptus. Cantans jam ramulo insidet expansa cauda et demissis alis; jam ab uno in aliud arboris cacumenvolitat. Nidulatur inter radices truncorum, vel juxta aliam quandam eminentiam in ipsa terra. Nidum fine arte ex Muscis et radiculis constructum intus culmis graminum stratum, apertura laterali, (nidulo $S$. Trochili fere similem) initio M. Julii inveni. Ova 6 oblongo-ovata, parva, pallide grisea rufo et fusco maculata atque striata. Marem juxta nidum canentem jam pullis exclusis audivi. Parentes prolem teneram Insectis et Larvis nusriunt.

\section{SYLVIA TROCHILUS.}

Diagn. Supra cinereo - virescens, superciliis pallide flavescentibus, subtus albido flavescens. Nares oblongæ, subcurvatæ; pedes luteo-fusci. Ala. rum flexura subtus flava.

\section{SYNONYMA.}

Motacilla Trochilus, Lin. Faun. Sv. p. 96. n. 263. Retz. Faun. Sv. 266. n. 252. Gmel. Syst. I P. 995.

Mot. Acredula; Lin. Faun. Sv. p. 96. n. 263. Sylvia Trochilus, Lath. Ind. I. p. 550. Afilus, Briss. Orn. III. p. 479.

Ie Chantre, Pl. enl. 65I. f. I.

Yellow -Wren, Pen. Brit. Zool. I. p. 378. n. I5I. Lath. Syn. IV. p. 4I2, 1. I47. Vers. Germ. Bechst。 IV. p. 499. 
Der Fitis. Bechst. N. D. 2 te Auf. 3. B. p.' 643. Svecis: Sparfkung, Skogsknett.

\section{DESCRIPTIO.}

Longit. avis 5. poll. Rostr. $\frac{3}{8}$; Cauda bifurca 2 poll. Ala extensa $8 \frac{5}{8}$ poll. Tibia $\frac{13}{16}$ poll. Digit. med. $\frac{5}{8}$ poll.; postic. $\frac{x}{2}$ poll.

Rostrum antice compressum marginibus inflexis, maxilla superiore parum longiore fusca, apice deflexa, pone apicem emarginata, inferiore flavescenti-fusca. Nares pervix oblongæ, sursum subcurvatæ. Anguli oris, faux, lingua et margines maxillarum flava vibrissis 3. nigris. Pedes flavescenti-fusci, plantis fla. vis, ungvibus flaves enti - fuscis.

Supra cinereo - viridis. A naribus supra oculos versus nucham stria flavescenti-alba. Stria fusca inter rostrum et oculos. Tempora fuscescentia. Gula albida. Jugulum et pectus albida flavo intermixto. Ab. domen album. Crissum dilute flavum. Remiges fu. scæ, margine exteriore olivacex. Alæ ad basin subtus flavx (fusco interdum maculatx). Rectrices fuscx, margine interiore pallidæ, exteriore tenuiter olivacex.

\section{HIST ORIA.}

Migratoria. Avis parva, gracilis, inquieta ad nos redit circa d. XX. Mens. Aprilis. Per totam astatem in sylvis et hortis frondosis frequens, autumno copiosa. Sono sibilante huit! huit! se prodens. Cantum primo acutum, tum submissius tristem his litteris bene expressit Bechst. 1. c.: Didi, Duhu, dehi, zia, zin! Nidum intermuscos in ipsa terra, fornicatum apertura laterali rotundata, culmis constructum, pennis et plumis stratum Mens. Junio inveni. Ova 6. albida macu- 
lis striisque violaceis. Pulli supra magis virides quain parentes, subtus pallide flavescentes, Remigibus apice albidis.

\section{SYLVIA REGULUS.}

Diagn. Supra virescens vertice flavo. Remiges fü sca latere extcriore flavo-virescentes.

\section{SY NONYMA.}

Motacilla Regulus, Lin. Faun. Sv. 95. n. 262. Retz.

Faun. 265. n. 25 I. Gmel. Syst. I. p. 995. n. 48. Sylvia Regulus, Lath. Ind. 1. p. 548. 11. I52.

Calendula, Regulus cristatus vulgo dičta, Briss. Orn. III. p. 579.

Roitelet huppé, Buff. Ois.V. p. 363. t. I6. f. 2. Pl. enl. 65 I. fig. I. Vers. Germ. XVI. p. 234.

Gold-crested Wren, Pen. Brit. Zool. I. p. 379. 11. 153.

Lath. Syn. IV. p. 508. n. I45. Vers. Germ. Eechst.

IV. p. 496. Aret. Zool. II. 11. 32I.

Das Goldhähuchen, Bechst. N. D. 2te Mut. 3. B. po 655.

Svecis Kongsfogel. Scanis Fogelkong. Uplandis Busklus.

\section{DESCRIPTIO.}

Inter aves Europæas facile minima vix 4. poll. Ionga.

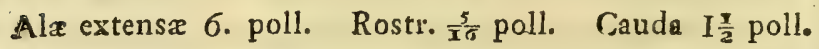
Tibia $\frac{\pi}{1} \frac{\pi}{6}$ poll. Digit. med. $\frac{\pi}{2}$ poll. Digit. post. $\frac{7}{16}$ poll.

Kostrum nigrum, rectum, tenue, acutum, maxillis xqualibus. Nares ovatx, penna rigida nigra tefix. Os intus flavum. Anguli oris vibrissis 3. vel 4. nigris. Pedes luteo-fusci. 
Macula magna verticis crocea (in femina lutea), fa. sciâ nigrâ utrinque marginata. A naribus supra ocalos stria alba. Latera capitis cinerascentia. Cervix et dorsum virescentia. Gula albida. Jugulum, pectus et abdomen sordide albida, tinetura fusco-flavescente. Tectrices alarum fuscæ, majores apicibus a'bis. Re. miges fuscr, margine exteriore (in quibusdam interrupte) flavo-virescente, sccundariz basi alb $x$; intim $x$ apice albx. Rectrices fusca, extus virescenti marginatx.

\section{HIS T OR I A.}

Permanens. Frequens. Estivat et hybernat in pinetis Srecix superioris, autumno hortos et sylvas Scanix frondosas visitat. Avis agilis, parum timida. Cristam parvulam pro lubitu erigit et demittit. $A b$ insectis corunque ovis sylvas purgat utilis avicula.

\section{SYLVIA TROGLODYTES.}

DIAGN. Supra fusco - rufescens obsolete nigro-undulata. Remiges exrimæ nigro-tessellatæ. Cauda cuneiformis lineis transversis fuscis.

\section{SYNONYMA.}

Motacills Troglodytes, Lin, Faun. Sv. p. 95. n. 26r. Retz. Faun. 264. 11. 250. Gmel. Syst. I. p. 993. n. 46.

Sylvin Troglodytes, Lath. Ind. I. p. 547. 1n, I48. Regulus, Briss. Orn. III. p. 425. n. 24. Troglodyte, Buff. Ois. V. p. 352. tab. I6. f. 1. Pl. enl. 65 I. f. 2. - Vers. Germ. Otto XVI. p. 215. 
Wren, Pen. Brit. Zool. I. p. 380. n. I54. Lath. Syn.

IV. 506. 13. 143. Vers. Germ. IV. p. 493. Arct.

Zool. II. No. 322.

Zaunkönig, Bechst. N. D. 2te Auf. 3. B. p. 666.

Svecis: Gärdsmyg, Tummeliten, Tummelilla.

\section{DECRIPTIO.}

Longit. avis $4 \frac{3}{8}$ poll. Alæ extens $6 \frac{3}{8}$ poll. Rosti.

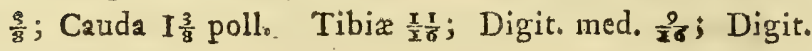
post. $\frac{1}{2}$ poll.

Caput fronte acutissima. Rostrum nigro fuscum, basi subtus flavescens, tenue, acutum, compressum subdeflexum, maxillis fere æqualibus. Nares oblongx. Anguli oris et os intus flava. Pedes et digiti, quorum posticus robustior, fusci.

Collum supra et Dorsum rufo-fusca striis transversis nigro-fuscis, capite saturatiore, unicolore. Supra oculos linea albida. Gula, jugulum et pectus rufescenti-cinerea. Abdomen et crissun rufescentia striis transversis nigro-fuscis. Tectrices canda inferiores maculis albidis. Remiges fusca, primores (6.) latere exteriore fusco albidoque tessellatæ, ceter $x$ latere exteriorè dorso concolores striis transversis fuscis. Tec. trices alarum minores punctis quibusdam (4-6) albis. Femina saturatius rufo-fusca striis transvarsis in dorso vix conspicuis.

\section{HIS TORIA.}

Manens. Passim. Estivat in pinetis montuosis. Autumno et hyeme in dumetis et aliis fruticibus densis et humilibus prope terram, etiam inter lapides salit sepitque avicula admodum agilis. Caudam brevem, 
erectam fert. Vox pro magnitudine avis magna. Cantus suavis et alacris. Nidulatnr inter radices arborum, inter acervos ramorum aridorum \&ç. Nidus artificiose constructus extus ex muscis, intus plumis, lana et se. tis stratus, globosus, apertura laterali parva rotunda. ta. Ova 7.8 rotundato - ovata, alba punctis rubris. Vescitu: Insecris eorunque ovis. 


\section{GENUS XXII. \\ M O T A C I L L A.}

Rostrum gracile, reres, apice subulato.

Nares ovato-rotundatx, nudx.

Lisgzua apice lacera.

Pedes ambulatorii, longi, graciles, ungvi postico in

aliis longiore, in aliis breviore, curvato.

Carda elongata.

Locus juxta aquas.

Ditơus Insecta.

\section{MOTACILlA ALBA.}

Diagn: Dorsum cinereum. Frons, latera colli ef capitis abdomenque alba. Verrex, occipur et collum antice nigra. Cauda nigra extimis duabus reetricibus dimidiato oblique albis.

\section{SYNONYMA.}

Motacilla alha, Lin. Faun. Sv. p. 92. 11.252. Retz. Faun. p. 256, n. 239. Lin。Syst。 I. p. 33 I. II. Gmel. Syst. I. p. 960. Briss. III. p. 46I. n. 38. Lath. Ind. I. p. 50I. n. I.

La Lavandiere, Buff. Ois. V. p. 25I. t. I4. f. I. Pl. En1. 652. f. I. (senior), fig. 2. (junior post de. plumationem.) Vers. Germ. Otto XVI. p. 28. cum duabus fig: 
Motac. cincrea, Briss. III. p. 465. Gmel. Syst. I. p. 96I. et.

In Bergeronette, Buff. Ois, V. p. 26I. Pl. enl. 674. I.

Vers, Germ. Otto VI. p. 46. cum fig. (junior ante deplumationem.)

White Wagtail, Pen. Brit, Zool. I. p. 36I. n. I42. tab. 55. Arte Zool. II. p. 396. B. Lath. Syn. IV. p. 395. I. Vers. Germ. Bechst. IV. p. 396. Suppl, p. 178.

Svecis: Ärla, Sädesärla。

\section{DESCRIPTIO.}

Longit. avis $7 \frac{T}{2}$ poll. Alic extensix II poll. Rost. go poll. A flexura ale ad apicen $3 \frac{\pi}{2}$ poll. extra alas extenditur ${ }_{2}^{r}$ poll. Tibia I poll. Digit. med. $\frac{\sigma}{3}$ poll. Digit. post. $\frac{0}{16}$ ut in Sylviis brevis et curvatus.

Rostrum nigrum mandibula superiore longiore, pone apicem aliquantum emarginata. Nares ovato-oblongæ. Iris fusca. Pedes nigri.

Frons, capitis et colli latera alba. Vertex, cervix, gula et jugulum usque ad sternum nitide nigra. Dorsum, Scapulx, tectrices alarum minores, latera pecto. ris et abdominis coerulescenti-cinerea. Pećtus, abdomen et crissum alba. Remiges saturate fuscx, margine exteriore cinerascentes, interiore ad basin albx. Remiges I6 (longitudine prima) I7 et 18 latere exteriore, latius albx. Teetrices longiores utriusque ordinis nigrescentes, apice et margine exteriore albx, unde fascix dux obliquo-transversx et strix quxdam albx in ala complicata. Reßrices nigrx extimx dux nivex, basi et plurima parte marginis interioris fusca. Par medium margine exteriore interdum albo. Teetrices caudæx superioris nigræ marginibus cinerascentibus. 
Femisa differt colorbus minus vividis. Scil. Albedo frontis et laterum capitis sordida, nigredo verticis et occipitis cinereo intermixto. In ceteris Mari similis. Avis junior ante primam deplumationem ab utroque pa. rente differt. Caput supra, cervix, dorsum sordide cinerea, capite obscuriore. A naribus ad nuchan supra oculos fascia nigricans. Latera colli ct capitis cinerea, fusco alboque immixto. Tota subtus sordide alba. In utroque juguli latere fascia nigricans descen. dit, seque conjungit in lunula transversa, nigricante supra sternum. Cetera fere ut in parentibus.

\section{OBSERV.}

Hic color post primam deplumationem itâ inutatur, ut Mense Septembri, omnes fere aves hujus Speciei sequentem habeant colorem: Supra coeruleo-cinerea, in olivaceum vix tendens. Frons albida demittit lineam aibam supra oculos. Gula et jugulum pure alba. Tempora cinereo-fusca. Ad latera juguli macula nigricans. In inferiore juguli parte macula magna lusaris nigra.

\section{HISTORIA.}

Migratoria in Scaniam venit primis diebus M. Aprilis. Ad ripas rivulorum et lacuum, in plateis et areis, in pascuis et pratis currens, cibum sibi quærit. Vernali tempore aratrum quoque sequitur. Victitat Insectis et Larvis. Nidulatur in catervis lapidum et trun. corum, in cavis arborum et murorum \&c. Ova 5-6 coerulescenti-alba maculis obscuris. Accipitres persequitur, aliasque aves clamore suo, sicut Hirundo, prxmonet. Caudam perpetuo movet. Abit M. Octobri. Initio Novembris adhuc unum vel aliud individuum in Scania obvenit. 


\section{MOTACILLA FLAVA.}

DingN. Supra olivacea, subtus flava. Rectrices duæ !aterales plus quam dimidiato oblique albx. Unguis posticus longus.

\section{SYNONYMA.}

Motacilla Flava, Lin. Faun. Sv. p. 92. 11. 253. Retz. Faun. p. 257. 11. 240. Lin. Syst. Nat. I. p. 33 I. n. I2. Gmel. Syst. I. p. 963. Lath. Ind. I. p. 504. n. 8.

Motacilla verma, Briss. Orn. III. p. 468.40.

Bergeronette de printemps, Buff. Ois. V. p. 265. tab. I4. fig. I. Vers. Germ. Otto XVI. p. 54. cum fig. Pl. enl. 674, 11. 2 .

Yellorv Wagtail, Pen. Brit. Zool. I. p. 362. n. 143. ArE. Zool. II. p. 396. F. Lath. Syn. IV. p. 400. 6. Vers. Germ. Bechst. IV. p. 402.

Mot. Boarula, Lin. Mant. Piant. p. 527.

Gelbe Bachstelze, Bechst. N. D. 2te Au1. 3. B. p. 466. Svecis, Gulärla.

\section{OBSERV.}

Mot. Boarula Iath. Ind. I. p. 502, et Bechst. 1. c. p. 459. qua cauda longiore et prasertim ungvi postico breviore curvato (ut in $M$. Alba) gulaque maris nigra a $M$. flava satis dignoscitur, nunquam in Svecia inventa est. Motacilla qux in Manc, Plant. Iinnei 1. c. Bosrmla vocatur, quaque Boves tota die sequitur, Conopsides calcitrantes et irritantes colligens, nulla alia est? quam $M$. flava pracipue junior.

\section{DESCRIPTIO.}

Longit, avis fere 7. poll. Alz extensz $10 \frac{\pi}{2}$ poil. Rostr. $\frac{x}{2}$ poll. A flexura ala ad apicem $3 \frac{2}{y}$ poll. Can! 
da $2 \frac{5}{8}$; extra alas compositus $I \frac{6}{3}$ poll. Tibia I poll. Digit. med. $\frac{x \hat{3}}{x \frac{5}{6}}$ poll. Digit. post. $\frac{\pi}{8}$.

Rostrum teres, acutum, nigricans, basi subtus paliidius, mandibulis xqualibus, superiore excisa. Nares ovate. Fara pallide incarnata. Iris fusca. Pedes nigricantes. Unguis posticus $\frac{7}{16}$ goll. longus et modice curvatus.

Caput supra coerulescenti-cinereum, interdum oliva. ceo immixto. A naribus supra oculo versus nucham linea alba. A sinu oris per oculos ad tempora stria nigricans, sub oculo, albo interrupta. Dorsum olivaceum uropygio magis fiavescente. Gula et crissum Havescenti-alba. Præterea tota avis subtus pulcherrithe flavamaculis obscuris, pluribus paucioribusve (raro nullis) in jugulo. Remiges fusca (quarum I6ta longitudine I. 2. 3.) margine interiore ad basin albx, intimx tres aut quatuor longx, acuta, molles, margine exteriore albide-favcscentes, id quod facit fascias binas obliquas et strias quasdam longitudinales albo - flavescentes in ala complicata. Tectrices alarum inferiores fuscescentes, flavo tinctz. Rectrices exteriores duæ albæ, basi et ad marginem interiorem oblique nigro-fuscx; tertia margine exteriore tenuiter alba, reliqux nigro-fusca, immaculatx.

Femina: Supra cinereo-olivacea; Gula et jugulum sordide albida, tincturâ flavescenti levissima. Abdomen et crissun fava, neque tam splendida ac Maris.

Funior, qualis Mense Julio frequens occurrit: Rostrùm supra fuscum, subtus pallidius. Pedes fusci. Caput et Dorsum supra fusca olivaceo tinctas Supra oculos stria flavescenti-alba pone oculos latior. Supra hanc fascia nigricans. Per oculos ad tempora fascia dilute fusca. Remiges et Rectrices ut in adultis. Avis subtus sordide iutescenti-albida. Ad latera juguli stria 
nigra utrinque descendit, in superiore pectoris parte se jungit et maculam majorem nigricantem format. $\mathrm{Hz}$ vero strix et hac macula sensim atate evanescunt*).

Var. a. Forma et magnitudine M. flava.

Capur nigrum, cinereo postice immixio. Dorsum, Remiges et cauda ut in M. Pava. Gula albida punctis nigris. Tota præterea fava, in inferiore juguli parte maculis fuscis fere confuentibus. Mus. Payk.

\section{OBSERV.}

Hæc Motacilla ad Enonteckis et Enara Lapponiz habitat, neque $M$. Flava, (vulgaris) illic occurrit. A $\bar{I}$. Borruld distinguitur cauda breviore, et præsertim ungue postico longiore, modice curvato. An distineta Species? An pro climate varictas?

$V a r$. 3 . Habitus Mot. flave junioris: Supra grisco-fusca olivaceo tincta. A naribus ad superiorem oculi marginem stria alba. Renigts obscuro-fuscr, intime tres margine cxteriore albidæ. Ala fascia duplici obliqua alba. Subtus tota alba, jugulo, anteriore pectore et crisso lutescenti tinEtis. Cetera ut in $M$. fliva. (Tabula nostra VIII.)

Mus. Holm.

Est M. Tschutschensis Ginel. Syst. 1. p. 962, cetera. rumque. Prope Stockholm occisa.

\section{HIST ORIA.}

Migratoria in Scaniam advenit serius quam $M$. Alba scil. circa vigesimum diem M. Aprilis. Vernali tem-

*) Cel. Bechstein has maculas numquam observavit, ei Fiutas aves illis predicas juninies esse Bourtilas. Apud nos vero, ubi frequens esi $M$. flava, M. vero Bcarula nunqquam visa, frequences occurrunt in pascuis ec in foeno strato, inter se. niores, a quibus quoqus aluntur. In nido illss q̨uog̨ur vidi. 
pore aratrum sæpe sequens Insecta et Larvas legit. Estate 'in pratis depressis et inter Segetes nidulatur. Ova ponit 5-6 sordide virescenti-grisea, maculis fuscescentibus. Pulli per totam xstatem parentes sequuntur. In pratis humidioribus, in foeno strato, in pascuis inter ores, equos et boves currens, gregaria sxpe Insecta et Larvas consumit. Ad medium usque Septembrem in tesquis et pratis Scaniz depressis obvenit. 


\section{GENUS XXIIx。 \\ A N T H U, Bechst.*)}

Capue versus rostrum elongato - coarctatum.

Rostrum gracile, subteres, subularum maxillis mar. gine aliquantum inflexis, superiore longiore, pone apicem emarginata.

Nares ovaræ, nudx, superne membrana fornisata semitedx.

Jiing sa bifida vel lacera.

$p_{e d e s}$ ambulatorii, digito postico plerumque longo,

magis minusve curyato.

Locus: prata depressa, litora, sylvæ.

Vicfus: Insedia et Larva.

\section{DISSECTIO I.}

Ungue postico lorgo modice curvato. Rostro tenuissime.

CXIV. ANTHUS PRATENSIS, Bechst.

Diagn. Supra olivacea nigricante maculata. TeEtrin ces alarum majores apice albida. Subrus alba

*) Genus Alauda Linzei, natura duce, induo Genera, Anthum scil. et Alaudam abit. Antlins continctur Speciebus aliis quus ad Motacillas, aliis qua ad Alardas propius accedunt. In seric igitur naturali inter hacc duo gencra optume collocatur.

Nilsse7s Qrnith.

Q 
jugulo et pectore maculis oblongis nigricantibus. Rectrices nigricantes, extima extus dimidiato oblique alba; secunda macula cuneiformi alba. Rostrum gracillimum. Ungvis posticus digito longior.

\section{SYNONYMA.}

Alauda Pratensis, Lin. Faun. Sv. p. 76. n. 210.

Alnuda sepiaria, Briss. Orn. III. p. 347.

L'Aloutte Pipi, Buff. Ois. V. p. 39. Vers. Germ. Otto XIV. p. 218. Pl. enl. 66I. f. 2.

Tit - Lark, Will. Orn. p. 206. (Bene descripta) Pent. Brit. Zool. I. p. 357. n. I38. (Descriptio optima; historia vero cum Antho arboreo confusa.) Lath. Syn. IV. p. 374. n. 5.

Wiesen-Pieper, Anthus pratensis Bechst. N. D. 2te. Aufl. 3. B. p. 732 .

Svecis: Anglärka.

\section{OBSERV.}

Improbus esset labot et inutilis onnes Scriptores retensendi. Longe vero facilius est avis colores et noreg describere quam Synonyma apud Austores rite eruere, quod quidem sepius fieri omnino non potest, quia Ornithologi aves, quas descripserunt, ipsi ignorarunt. Eadem avis pluries descripta, confusi mores, composita historia. Eriam accuratissimus natura observator Bechstein in prima editione Naturgesch. Deurschl. et in Commentariis ad versionem Germ. Lathami Synopsis confudis Species hujus generis distinguendas. Hos vero errores in 2da editione Nat. Deutschl. natura duce, correxit.

\section{DESCRIPTIO.}

Avis gracilis, inter congenteres Svecanas minima, 6. poll. longi. Alz extense circe IO. poll. Rostr. 
$\frac{1}{2}$ poll. A flexura aliz ad apicem 3. poll. Cauda $2 \frac{2}{s}$ poll. Tibia $\frac{7}{3}$ poll. Digit. post. $\frac{6}{3}$ poll. ungue $\frac{3}{3}$ poll. Digit. med, $\frac{6}{8}$ poll.

Rostrum tenuissinum, acututin, compressum margi. nibus aliquantum inflexis. Maxilla superior nigricans, parum longior, pone âpicem emargitiata, inferior rufoIutea apice fusco. Nates rotunduto-ovales, nudæ, superne membranâ fornicatâ. Anguli ờis eminentes lutei vibrissis 3. nigris brevioribus. Os intus pallide griseo-incarnatum. Lingua lacera. Iris brumea. Pedes pallide incarnati, Digitis fuscescentibus, unguibus fuscis, quorum posticus longus et parum curvatus.

Caput supra, cervix, Dorsun et scapulæ olivacea maculis nigricantibus, quarum minores in capite, majores in dorso (i. e. singula penna nigricans margine latiore griseo - olivacea.) Uropygium ${ }^{2}$ griseo - olivaceum immaculatum. Stria luteo-alba supra oculos. Tempora fuscescentia lineis albis. Avis subtus sordide alba jugulo et pectore tincturâ sulphur'ea. Ad latera g'ulx decurrunt macula fuscr in striam fere junctx; qux maculir in jugulo et superiore pectore magis sunt distinetx, nigricantes, ovales, et in hypochondriis ob. longx. Abdomen et crissum sordide alba. Remiges nigricantes, quarum primà margine exteriore tenuiter alba, ceter margine exteriore viridi-luteo, in intimis tribus latiore, $1-4 x q u a l e s, 5-15$ breviores, apice excisæ, I 6 ta cum qrattor primis æqualis, mollior (Etiam in hoc Mot. flave similis). Tectrices minores nigticantes tenuiter olivaceo marginatx. Teetrices ma* jores utriusque ordinis nigricantes apice sordide albo marginatie, unde strix binx obliqux albidx in ald oritutur. Teetrices inferiores fuscescentes tinctura 1utea. Cauda aliquantum bifurca nigricans; Rectrix extima ab apice supra medium extus oblique alba; se-

Q 2 
cunda maculâ apicis cuneiformi albâ juxta rachin ad latus interins. Ommes vero margine exteriore tenuiter luteo - virescentes.

Fenina: Supra magis nigro-fusca, (ubi mas nigricans) marginibus pennarum griseo-olivaceis. Subtus sordi. de alba tincturâ sulphureâ vix conspicuâ.

Avis junior: Rostrum fuscum basi subtus incarnatum. Pedes pallide lutescentes digitis magis fuscescentibus, subtus luteis.

Avis supra nigra pennis luteo-viridi latius margina. tis. Uropygium olivaceum maculis obsoletis nigricantibus. Collum antice et pectus pallide lutea maculis minoribus, crebris, oblongis, nigricantibus. Abdomen et crissum sordide alba. Alx nigricantes marginibus pennarum luteo-viridibus, et striis binis ob. liquis pallide luteis. Rectrix extima dimidiato-oblique alba, secunda apice macula cuneiformi alba, tertia ultimo apice alba; ceterum nigræ marginibus luteo-viridibus, in intermedio pari latioribus.

\section{HISTOR IA.}

- Migratoria. In Scania, Hallandia, Uplandia \&c. passim frequens. Mature adrenit nive nondum nisi locis humidis soluta. In pratis depressis, gregaria, quamvis dispersa, jam ante finem Mensis Martis oecurrit. Hominem adpropinquantem supervolitat sono sibilante: Ist, Ist - Ist, Ist, Ist! (Singulum Ist ! edens, alas componit, unde volatus alternis vicibus jam altion jam demissio:). Nive jam soluta in prata et tesqua juniperis et grumulis adspersa, nidificandi caussâ abit. Nidulatur in jpsa terra in grumulo $v+l$ sub frutice Juniperi. Ova $4-6$ coerulescenti - alba maculis rufis; pulli Larvis et Insectis a parentibus aluntur. Cum homo vel alius hostis ad nidulum adpropinquat, mater- 
cula ejulantem et tristem edit sonum, qui pullis educatis nunquam exauditur. Tempore propagationis Mas e frutice vel grumulo quodam in aürem adscendit, sono Tick! Tick! Tick, certaque altitudine attacta librans se expansis alis demittit, cantu ocius et alacrius continuato. Pullis exelusis et educatis per familias campos depressos et arva frequentat, sono Gick! Gick! in ä̈re se prodens. Hoc (autumnali) tempore arboribus quoque insidet, id quod verno tempore nunquam vidi. Vescitur Insectis et Larvis. Versus finem M. Septembris et initio Octobris sensim abit.

\section{ANTHUS RUPESTRIS, mi $i$.}

(Tab. IX. fig. I senior fig. 2 junior.)

Diagn. Supra fusco-cinerea maculis obsoletis saturatioribus. Subtus sordide albida maculis fuscescentibus, Reatrice extima extus oblique sordide alba. Rostrum nigrum. Pedes nigro-fu• sci. Unguis posticus longus, curvatus.

\section{SYNONYMA.}

Alanda petrosa, Rocklark, Montagu Transact. of Lin, Soc. Vol. IV. p. 4 I.

Dusky Lark, Lewin Brit. Birds, vol. 3. pl. 94. Alauda olscura, Lath. Ind. Orn. I. p. 494. 7. (junior). Alauda pratentsis, variet. Pen. Brit. Zool. fol. pl. P. I. (junior).

Anthus aquaticus, Wasserpieper, Bechst. N. D. 2te Aup. 3. B. p. 745 .

\section{OBS ERV.}

Primus qui hanc avem ut distinctam Speciem distinge descripsit, est cel. Lewin 1. c. Cel. autem Latham Specimen hujus speci- 
ei recepit ab ipso Lewin, eamque Alaudam obscrram appellavit. Hoc idem Specimen a Lathamo communicatum est celeberrimo M.ntagu, qui postea (anno r79r.) avem in rupibus Provincix South Wales invenit, eanque 1. c. Alaudam petrosam appellavir. Ex his itaque liquet, $A l$. obsckram Lath. eandem esse ac. Al. petrosam Mont. Bechstein, qui 1. c. eandem descripsit gvem, aliam adfert Synonymiam minime vero accuratam. Quamvis itaque avis jandudum descripta est, necessarium tamen mihi visum est nom vum illi eligere Nomen; cum antea nullum aptum sit electum. Anthws enim aquaticus quo a Bechstein appellarur, non magis huic quam Anth. pratensi competit. Bechst. svem non nisi præecrmigrantem cognovit. Nescio vero quo jure Avis petrosa dici possit. Nomen autem quod elegi, indicat locum, in quo avis nascitur et ubi semper nisi sub dimigrationibus versatur. Spipoletta Will, Orn. (Ang.) p. 209. E. X, et Alauda Spinoletta Scup. Ann, 1. p. 128. potius ad hunc quam ad Anthum campestrem pertinere videtur.

\section{DESCRIP TIO.}

Longit. avis $6 \frac{6}{8}$ poll. Alæ extens $\mathrm{I} I \frac{3}{8}$ poll. Rostr. a fronte ad apicem $\frac{0}{5 \sigma}$ poll. A sinu oris ad apicem $\frac{6}{8}$ poll. A flexura ale ad apicem $3 \frac{\mathrm{T}}{2}$ poll. Cauda $2 \frac{\mathrm{t}}{2}$ poll. Tibia I poll. Digit. med. $\frac{7}{8}$; Digit. postic. $\frac{7}{8}$; unguis postic. $\frac{7}{16}$ poll.

Rostrum nigrum, basi suhtus vix pallidum, tenue, acutim, compressum marginibus aliquantum inflexis. Maxilla superior parum longior pone apicem emarginata. Lingua apice lacere. Faux griseo-incarnata maxillis intus nigricantibus. Nares perviæ, ovales. Iris avellana. Pedes nigro-fusci, pro magnitudine avis magni et crassi, unguibus nigris, quorum posticus longitudine digiti modice curvatus.

Avis supra saturate cinerea, in dorso leviter fusco maculata. Stria albida supra et pone oculos. Tempora fuscescentia lineolis albidis. Subtus avis sordide albida luteo vix tincta maculis cinereo-fuscis ad latera 
gulx et juguli, in pectore et hypochondriis. Remiges fuscr, quarum extimx extus albo, ceteræ pallide griseo marginatx. Tectrices fuscx, proximx extus grisex, penultimx apice albidx. Teetrices inferiores fuscescen. tes tinçurâ lutescenti. Cauda aliquantum bifurca. Rectrices nigricantes, extima latere exteriore et oblique per apicem sordide alba; secunda ultimo apice pallida; par medium cinerascens.

Avis junior: Supra saturate olivacea maculis in Dorso obsoletis nigricantibus, subtus flavicans maculis in lateribus colli et in pectore fuscis. Remiges nigricantes, primores margine exteriore tenuiter luteo-albæ, secundarix extus olivaceo latius marginatæ. Tectrices minores nigricantes olivaceo marginatr, majores nigricantes margine exteriore olivacez apice albidx. Rectrices nigræ olivaceo extus tenuiter marginatæ, extima extus oblique sordide alba, secunda apice ultimo albida. Rostrum et pedes fusci plantis et basi rostri subtus luteis.

\section{HIS TOR I A.}

Migratoria. Habitat in rupibus et saxis, juxta lito. ra maris et in ipso mari sitis. Inter Ahus et Sölfivits. borg Scanir in saxis litoris numerosam invenit Illustriss. Comes Trolle Wachtmeister, mihique benignissime communicavit. Ad Torekow, in Hallands - Wäderö et in litore petroso Hallandix, frequens quoque occurrit. Eminus obscurior apparet quam congeneres. Vox et cantus fere similis Anthi pratensis. Sicut ille, Mas in aërrem cantans adscendit, et immotis alis in rupem quandam se demittit. Nidulatur in rupibus inter granula. Ora 5. sordide alba maculis fuscis, in extremitate craso siore saturatioribus et fere confluentibus, Vescitur Ina* sectis et Larvis. 


\section{DISSECTIO II.}

Ungue postico brevi minus vel magis curvato. Rostro robustiusculo.

\section{ANTHUS CAMPESTRIS.}

Diagn, Supra dilute cinereo - fusca in olivaceum inclinans. Tænia ferruginescenti - alba supra oculos. Subrus albicans striis in pectore fuscis. Rostrum validum, elongatum. Unguis posticus brevis rectiusculus.

\section{SYNONYMA.}

Alauda novalitin, Frischs Vögel Deutschl. Tab. I5. f. 2. b.

Anthus campestris, Bechst. N.D. 2te Auf. B. 3. p. 722. t. 2 .

Alauda campestris, Briss. Orn. III. p. 349. 5. In Spipolette, Buff. Ois. V.p. 43. Vers. Germ. Otto XIV. p. 224. cum. fig.

Mcadow - Lark, Lath. Syn. IV. p. 378. 11. IO. Vers. Germ. Bechst. IV. p. 379.

Svecis: Fülelärdka.

\section{OBSERV.}

Fac inter Synonyma mihinota sola sunt digna qua adferantur. Neque enim difficile est videre quamnam avem ante oculos habuerit Ill, Linné, cum Alardam campestrem n. 213 in Faun. Svec. describeret. Nimirum hoc nomine egregie descripsit Saxicolam (Motacillam Lin.) rubetram, fem. Mirandum itaque sane est, laudatos illos Ornithologos, Gmelin, Latham plures ut taccam, non solum Synonyma Linneana, sed etiam falsain illam Diagnosin retinuisse. Neque in ultima Faunæ Svecica editione inelius rei consultum est, cum Descriptioni Linneanæ (quæ sal- 
rem ex unica Specie facta est) varia sunt addita et immixta, quz a Pennanti Field-Lark (Brit. Zool. I. 11. 139) desumta sunt. Hæc voro Ficld-Lark neque ad cam speciem pertinet, quam ante oculos habuerir Linné, cum Al. suam campestren decriberet, neque ad hanc nostram. Est igitur hæc Species re vera Faunæ nostræ novitia, quæ benevolentix et favori in Scientiam Naturalem Illustriss. Comitis \&c. T. Wachtmeister debetur. Frisch 1. c. avem bene depinxit, quam et Bechstein 1. c. optime descripsit.

\section{OBSERV.}

Alauda Spinoletta, que a Lathamo huic jungitur, variorum Auctorum, ad Anthun rupestrem pertinere viletur. Pispoletta Cetti Uc. di Sard. p. 158. ad Anthnu pratensen zeferenda.

\section{DESCRIPTIO.}

Longit. avis $6 \frac{T}{2}-7$ poll. Alx extens $x$ I I. poll. Rostr. a $\sin u$ oris ad apicem $\frac{6}{8}$ poll. A fronte $\frac{x}{3}$ poll. A flexura alx ad apicem $3 \frac{5}{8}$ poll. Cauda $2 \frac{5}{8}$ poll. Tibia $I$. poll. Digitus med. $\frac{5}{8}$ poll Digit. post. fere $\frac{5}{8}$ poll. Ungue postico $\frac{2}{8}$ poll.

Rostrum elongatum, basi robustius quam in antece. dentibus, versus apicem aliquantum compressum marginibus inflexis, supra fuscum, subtus albidum, apice fusco. Maxillx æquales superiore pone apicem excisa. Os intus pallide inearnatum lingun apice bifida. Nares nudic: rotundate. Anguli oris pallide incarnati; vibrissis 3. 1. 4. brevioribus nigris. Iris fusca. Pedes pallide incarnati. Unguis posticus digito brevior, fere rectus.

Corpus supra dilute cinereo fuscum in olivaceum tendens maculis longitudinalibus fuscis juxta raches pennarum in dorso et capite. Uropygium immacula. tum ut etiam tecirices cnudx superiorcs, quarum binx nltra caudic medium clongatix et acuminatre. A naribus supra oculos versusnucham tænia fermginescenti - alba. 
Lora fusca. Tempora fuscescentia. A sinu oris sub oculis ad latera gulæ linea nigricans. Avis subtus sordide alba tincturâ, præsertim in pećtore, ferrugineo-lurcâ. Latera juguli et pectus maculis parvis rarioribus longitudinalibus fuscis. Remiges fuscæ, primores te. nuiter, secundariæ latius margine exteriore ferruginescenti albidx. Tectrices majores saturate fuscx margine et apice ferruginescenti-albæ. Tectrices inferiores albidx, luteo tinçæ. Cauda subæqualis. Rectrices fusca, prima fere a basi extus dimidiato oblique alba, secunda a medio extus oblique alba, intermedia bina cinereo - fusex, magis quam ceteræ acuminatæ (fere lan. ceolatr).

Fenina distinguitur tinctura pectoris ferruginescenti - lute vix conspicua, et pectore fere immaculato striis mis fusco-griseis.

Avis junior magis variegata vel undulata. Supra saturate fusca vel nigricans pennis testaceo fimbriatis. Stria flavescens supra oculos. Remiges nigricantes quarum prasertim interiores et tectrices proximæ testaceo latius marginatx, apicibus pallidioribus. Tectrices minores et penultima nigricantes testaceo-albo latius marginatx. Refrices nigrz testaceo marginatx, ultima et se. eunda extus oblique albidx. Subtus avis sordide alba. Lineola ad latera gulæ utrinque nigra pectore luteo tinc to, nigro maculato.

\section{HISTORIA.}

Migratoria. In Scanix campis arenosis hre Species rarius occurrit. Illustriss. Comes T. Wachtmeister illam in Svecia primus invenit prope Prædium sum Arup

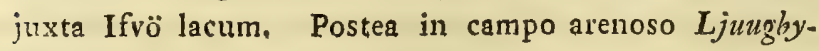
hed dicto, et juxta Torekow, haud longe a mari, a me visa. Altissime volitans voce sonora Zivoly! Zivoly! 
crebrius edita, se prodit qurrenti. Raro arboribus in, sidet. In ipsa tèrra Insecta et Larvas colligens tamquam Sturnuscurrit. Motacillae instar candam mover. Alau. da avensi eminus fere similis. Collecta messe in agris interdum per familias obvenit.

\section{AN'THUS ARBOREUS.}

Diagn. Supra griseo olivacea fusco-maculara. Tectrices alarum majores apice griseo-albæ. Subtus alba jugulo et pectore ferruginescenti-lureo rinctis, fusco longitudinaliter maculatis. Rectrices fuscr, extima extus dimidiato alba, secunda apice alba. Rostrum breve validiusculum. Unguis posticus digito brevior, curvatus.

\section{SYNONYMA.}

Die Pieplerche, Bechst. Nat. D. I. edit. B. 4. p. 135. Lath. Syn. Vers. Germ. IV. to 52. fig. I. (fig, mediocris):

Der Bammieper, Anthus Arboreus, Bechst. N. D. 2. Auf. 3. B. p. 706. tab. 36. f. I.

Alauda Trivialis, Lin. Syst. Nat. p. 288. 11. 5. (quoad mores huc pertinet), Lath. Ind. I. p. 493. n. 6. Gmel. Syst. I. p. 796.

Alauda minor, Gmel. I. p. 793.

The field Lark, Pen. Brit. Zool. I. p. 358. n. I39.

(Bene descripta).

Lesser Field Lark, Will. Orn. p. 207.

Farlouse ou Alouette de prés, Buff. Ois. V. p. 3I. t. 3. PI. enl. 660. f. I. Vers. Germ. Otto XIV, p. 203. cum fig.? Eins Pommersche Lerche: Otto 1. c. p. 
2I I. (Hoc loco nostra avis optime a Cel. Otto est descripta.)

Svecis: Piplärka.

\section{OBSERV.}

Hæc sunt Synonyma qux cum certitudine quadam colligere potui, Confusa enim sxpissime est aut Descriptio aut Historia hujus avis et plerumque eum Antho pratensi. An Sylvin Locustella Lath. Ind. 1. p. 5 I 5. n. 25. huc pertineat (ut Bechst. 1. c. putat) dubito. Neque enim hæc, nec alia hujus generis habet cardam cuneatam (Lath. 1. c. nec in media et densissima parte fruticum se celat unde difficillime fugatur (Pen. Brit. Zool. n. 156. Grasshopper Lark) nec minor est Regulo non cristato (i. e. Sylv. Trochilo) ut Willugh. 6. p. 207 dicit de sua Titlark Shat sings like a Grasshopper, unde ceterorum Locustella desumta est. Anne Sylvia modnlari vel Schoenobuno hac confusio origirem debet? Alduda Goperisis, Mus. Carls. forte huc pertinet, quod tamen certe affirmare non audeo. Specimen xre incisum inter reliquias Musei Carlsoniani invenire non potui. Al. obsczra Lath. minime ad hanc, ut Beehst. putat, sed ad Anth. rupestren pertinet. Vide supra p. 245.

\section{DESCRIPTIO.}

Longit. avis $\frac{6}{8}$ poll. Alæ extensæ I2. poll. Rostr. $\frac{7}{\bar{x} \sigma}$ poll. A flexura alæ ad apicem $3 \frac{1}{2}$ poll. Cauda $2 \frac{\pi}{2}$ poll. extra alas extenditur $I \frac{3}{8}$ poll. Tibia $\frac{7}{8}$ poll. Digit. med. $\frac{6}{8}$ poll. Digit. postic. $\frac{5}{8}$ poll. ex quo unguis 家 6 poll.

Rostrum breve, validiusculum; maxilla superior obscure fusca, emarginata, aliyuantum longior; inferi. or pallide incarnata apice fusco. Nares ovatr, nuda. Sinus oris eminentes pallidi. Os intus pallidum. Iris fusca. Pedes sordide albescentes. Unguis posticus brevis, curvatus. Cauda aliquantum bifurca Rearicibus acuminatis. 
Avis supra griseo - olivacea maculis longitudinalibus saturate-fuscis, in capite minoribus, in dorso majoribus. Uropygium fere inmaculatum, fusco-grisescens. A naribus supra oculos stria albicans. Tempora griseo - fusca. Corpus subtus a gula ad abdomen testaceum s. pallide ferruginescenti-luteum, maculis nigro. fuscis ir juguli lateribus striam utrinque descendentem formantibus, in peciore superiore oblongis fere triquetris, in hypochondriis lintaribus. Abdomen et crissum alba. Femora extus fusca flavo tincta. Remiges saturate fusca, primores I- 4 margine exteriore tenuiter alba, ceteræ inprimis intima olivaceo marginatx. Tectrices minores olivaceo-fusca; majores fuscæ mar. gine olivacex, apice albe, unde trnia alarum duplex alba oritur. Reetrices fusca, extima extus dimidiato oblique alba; secunda maculâ apicis parvâ, cuneiformi alba (qua interdum caret, apice ultimo tantum albo) ceterx olivaceo marginatix, intermedix latius.

Fenina: Mari fere, similis sed aliquantum pallidior. Avis junior: Juniori Antho pratensi (cujus Descriptionem vide supra) similis. Distinguitur tamen: Rostro breviore et robustiore; maculis pectoris majoribus; Rectricibus apice acuminatis, et inprimis ungue postico Digito breviore et curvato.

\section{HISTORIA.}

Migratoria. Frequens. Serius advenit et maturius abit quam Anth. pratensis. Redux circa medinm M. Maji in arboribus hortorum et pratorum primo occurrit. Fistivat in sylvis. Mas e cacumine arboris in ärrem adscendens, et paulo post in eundem plerumque locum expansis et immotis alis se demitrens, cantum suavem et sonorum modulatur. Hoste ad niduiun adpropinquante tristem et ejulantem vocem edunt paren- 
tes. Nidulatur in ipsa terra inter Gramina. Nidus extus graminibus exsiccatis constructus, intus setis equinis et pilis stratus. Ova 5. grisea maculis rufo-fuscis. Vescitur Insectis et Larvis. Autumnali tempore, ante abitum, agros gregatim frequentat, et sonum pipientem, qui alio tempore nunquam anditur, volans edit. 


\section{SUBDIVISIO}

Maxillis integris.

\section{GENUS. XXIV. \\ A L A U D A.}

\section{Caput subovatum.}

Rostrum cylindrico - subulatum, rectum manaibulis æqualibus, integris, basi deorsum dehiscentibus. Nares ovatæ, plumis setaceis recræ.

Lingua bifida.

Pedes ambulatorii; unguis posticus digito longior, rectus, apice tantum paulo curvatus.

Locus: Agri, arva, nemora.

Victus: Semina et Insecta.

\section{ALAUDA ARVENSIS.}

DiAGN. Supra griseo-rufescens maculis fuscis; subtus rufescenti-alba, pectore maculis minutis fuscis. Cauda longa bifurca. Rectrice extima exrus supra medium oblique alba, secunda latere exteriore et apice alba.

\section{SYNONYMA.}

Alauds aryensis, Lin. Faun. Sv. p. 76. n. 209. Systo Nat. I. p. 287. n. I. Lath. Ind. I. p. 49 I. n. I. Retz. Faun. p. 202, n. 193. Gmel. Syst. I. p. 79 I. 
Alauda, Briss. Om. III. p. 335. I.

L'Aloucte, Buff. Ois. V. I. t. I. Vers. Gem. Otto XIV.

p. 152. cum. fig. Pl. enl. 363. f. I.

Sky-Lark, Pen. Brit. Zool. I. p. 353. t. 55. Lath. Syn.

IV. p. 368. n. I. Vers. Germ. Bechst. IV. p. 369. I. Feldlerche, Bechst. N. D. 2te Auf. 3. B. p. 755 .

Svecis: Lärka, Sånglärka.

\section{DESCRIPTIO.}

Longit. ayis $7 \frac{t}{2}$ poll. Alæe extense I $4 \frac{T}{2}$. Rostr. $\frac{5}{2}$ poll. A fexura ala ad apicem $4 \frac{5}{2}$ poll. Canda fere 3. poll. Extra alas plus quan I. poll. extensa. Tibia I. poll. Digit med. $\frac{7}{3}$ poll. Digit. post. I; monis postic. $\frac{5}{8}$ poll.

Rostrum robustiuseulum, teres, supra converum, maxilla superiore fusca, integra, inferiore albido apice fusco. Anguli oris eminentes, lutei. Os intus pallide incarnatum. Lingua obtuse bifurca. Nares ovales plumulis setosis teet:. Iris griseo-fusca. Pedes fusco-incarnati, unguibis fuscis, postico apice albo.

Caput, Dorsum et scopula saturate fusca marginibus pennaram rufescenti-vel albide-griseis. Uropygium et tectrices alarum minores rufescenti-grisea fere immaculata. Genie et stria supra oculos sordide alba. Tempora fusca rufescentia. Aris subtus sordide alba, collo et pectore antice ferruginescenti-luteo tinctis, maculis parvis fuscis cuneiformibus, fere striatis. Re. miges saturate fuscæ; extimæ tres fere aquales, margine exteriore albr; 5-I5 apice albr, excise, margine exteriore forruginex; 15 longitudine quintx, cum sequentibus, margine pallide griser. Tectrices majores fusce, margine exteriore rufescenti-griseo, apice albido. Cauda longa, bifurca, Rectricibus nigricantihus acũtis, extima cxtus ab apice supra medinm obli- 
que alba, secunda latere exteriore per totam longitudi. nem et margine interiore ad apicem alba. Par interme. dium fuscum extus rufo-griseo, intus ferrugineo latius marginatum.

Femina: minor: 7. poll. longa; fere similis Mari, pectore tamen pallidiore.

Funior avis: magis variegatus, ab aliis Speciebus optime dignoscitur rostro et ungue postico.

\section{OBSERV.}

Vertex Maris pennis longioribus est instructus, quas prolubitu in cristam bievem erigere potest. Tempore propagationis hoc sxpissime facit. Talem vero Alatdan Aucupes Scanix appellant Topplärka ct dicunt hare melius cantare quam illam, qure crista caret. Et hoc jure. Aliam Al. cristatam nec in Svecia nee in Selandia inveni, nisi ab exteris allatum.

\section{HISTORIA.}

In agris, arvis et campis frequens. Mensibus Septembri et Octobri gregatim $a b$ uno in alterum agrum errat. Sensim evanescit. Manent tamen nonnullx in Scanix agris in multam hyemem. Mature redit. Jam Mense Februario, aurâ leniore in aëre supra agros se librans, cantu dulci ver latum adnunciat.

Ecce suum Tirili, Tirili, Tiritirtiti tractim

Candida per vernum cantat Alauda solum.

Etiam terra insidens cantat. Nidulatur in agris $\mathbf{P} \mathbf{L}$ campis. Fidum graminibus siccis constructum pilis stratum in ipsa terra ponit. Ova 5. albo-grisea punctis et maculis griseo-fuscis. Vescitur Seminibus, Insectis, Larvis et satis. 


\section{ALAUDA ARBOREA.}

DIAGN. Supra pallide testacea, nigro maculata Seria albida supra oculos caput cingens. Subrus albida pectore maculis nigris. Cauda brevis æqualis.

\section{S YNONYMA.}

Alauda arboren, Lin. Faun. Sv. p. 77. n. 211. Retz. Faun. p. 22I. 1. 195. Lath. Ind. I. p. 492. Gmel. Syst. I. p. 793. n. 3. Briss. Orn. III. p. 340. t. 20. f. I.

Alouette des bois ou Cujelier, Buff. Ois. V.p. 25. Vers.

Germ. Otto XIV. p. I90. Pl. enl. 660. f. 2.

Wood-Lark, Pen. Brit. Zool. I. p. 356. n. I37. Lath.

Syn. IV. p. 36I. n. 3. Vers. Germ. Bechst. IV. p. 373. 1. 3.

Alauda nemorosa, Gmel. Syst. I. p. $79 \%$. n. 21. Al. cristatella, Lath. Ind. Orn. I. p. 499. 13. 26.

Lulu, Buff. Ois. V. p. 74. Vers. Germ. Otto XIV. p. 277. Pl. enl. 503. f. 2.

Lesser Crested Lark, Pen. Brit. Zool. I. p. 360. n. I44. Lath. Syn. IV. p. 39I. n. 24.

Baunlerche, Bechst. N. D. 2te Auf. 3. B. p. $78 \mathrm{I}$. Svecis: Trädlärka.

\section{DESCRIPTIO.}

Longit. avis 6. poll. Alæ extensæ J2 poll. Rostr. $3 \frac{7}{3}$ poll. A flexura alx ad apicem $3 \frac{6}{8}$ poll. Cauda 2. poll.; extra alas $\frac{1}{2}$ poll. extenditur. Tibia $\frac{7}{8}$ poll. Digit. med. fere $\frac{\sigma}{8}$ poll. Digit. post. $\frac{\sigma}{8}$ poll. Ungv。 post. $\frac{7}{16}$ poll.

Rostrum tenuius quam antecedentis, tereti-compressum, supra fuscum, subtus pallidum. Pedes pallidi. 
Unguis posticus pallidus, compressus, rectus, apice subincurvatus.

Quoad habitum brevior et cressior quam $A l$ arvensis. Caput, Dorsum et Scapulx supra nigricantia marginibus pennarum griseo-ferrugineis. Uropygium et Tectrices caudx supra rufo-grisea immaculata. Vertex pennis longioribus instruens, quas in cristam erigere potest avis. A naribus supra oculos ad nucham fascia albida Pileum cingit. Macula Temporalis fusco-rufa postice fere nigra. Subtus aris sordide alba maculis nigricantibus cuneiformibus in jugulo minutis, in su* periore pectoris parte aliquantum majoribus. Gula, Abdomen et crissum immaculata. Latera colli et nucha albida maculis nigricantibus, unde "Collum fere an. nulo albo cinctun," Lin. Fan. So. Remiges nigro-fuscx apice albicantes: primores (5) margine exteriore tenuitcr albx, ceterx pallide ferruginer. Teetrices minores rufo-grisex, majores remigum primorum nigricantes npicibus rufo-glbescentes. Ad marginem alæ clausx macula albr. Cauda rqualis, brevis, nigra, Rectricibus late obtusis extimấ ab apice fere ad medium albá, fusco immixto, secunda et tertia macula apicis obliqua alba; pare intermedio rufo-griseo, ad rachem tantum fusco.

Temina: Mari fere similis, sed color ejus sordide albi. dus vel luteo-albidus, in hac purius albus.

\section{HISTORIA.}

Mighratoria. Mature redit, jam ad finem M. Martii. Rost medium M. Septembrem versus meridiem dimigrans, agros Scanix gregarin frequentat. Volnns continuo Daditgoi! clamat. Volatus arcuatim sltior et demissior. FHativat in memoribus, precipue pinetis Hal- 
landia, Smolandir \&c. Mas cantans e cacumine arboris in aërem altissime adscendit; etiam cacumini arboris insidens. Nidulatur in terra sub frutice juniperi $\&$ c. Ova 4-5. saturate grisea maculis fuscis prasertim circa extremitatem obtusiorum. Vescitur Insectis et se. minibus. 


\section{GENUS XXV. \\ C I N C L U S.*)}

Rostrum rectum, tenue, acurum, dorso fere carinaro, maxillis margine inflexis, superiore pone apicen decurvatum parum excisa. Nares lineares, membrana et pilis fere tectx. Caput parvum.

Lingua plana, apice bifida.

Pedes ambulatorii.

Oculi altius sub vertice positi.

\section{CINCLUS AQUATICUS.}

Diagn. Nigricans, collo antice et pectore albis.

\section{S YNON YMA.}

Sturnus Cinclus, Lin. Faun. Sv. 78. n. 214. Amoen, Ac. IV. p. 594. Retz. Faun. Sv. p. 225. 12. 20. Gmel. Syst. I. p. 803. n. 5.

Turdus Cinclus, Lath. Ind. Orn. I. p. 343. n. 57.

Merula aquatica, Briss. Orn. V. p. 252. I9.

*) Hoe Genus omnina a Sturuis separandum. Formâ rostri propius ad Turdos accedit, variis vero notis distinguitur. Quoad habitum ad Passerum ordinem pertinet, quoad vivendi rationem potius ad Grallas accedit. Etiam femora vis usque ad genua pennis tecta, transitum ad Aves aquaticas demonstrant. 
Merle d' enu, Buff. Ois. VIII. p. I34. t. 11. P1. enl. 940. Vers. Germ. Orto. XXVIII. p. 246. cum. fig. Witer-Ouzel, Pen. Brit.Zool. I. p. 3 I2. 11. II I. Arct. Zool. II. p. 332. B. - Lath.Syn. III. p. 48. 50. Id. Sup. p. 142. Ver's. Germ. Bechst. III. p. 45. 11. 50.

Der Wrissersehwätzer, Cinclus aquaticus, Bechst. N. D. 2te Auf. 3. B. p. 808 .

Suecis: Wattnstare, Strömstare.

\section{DESCRIPTIO.}

Longit. avis $7 \frac{r}{2}$ poll. Alæ extensæ 13 poll. Rostr. e fronte $\frac{5}{8}$ poll. A flexura alx ad apicem 4 poll. extra alas $I_{8}^{2}$ poll. Tibia $I \frac{\pi}{8}$ poll. Digit. med. I. poll. postic. $\frac{5}{8}$ poll.

Rostrum nigrum. Iris pallide fusca; palpebrx alba. Pedes antice fallide grisei, postice et subtus nigro-fu. sci. Ungues fusci.

Magnitudine fere Sturni, capite tamen minore, colpore crassiore, alis et cauda brevioribus. Caput, collum supra ef ad latera brunneo-fusca (fusco-castanea). Dorsum, Scapulx, alarom teclrices et uropygium pennis cinereis nigro latius fimbriatis; Gula, collum antice et pectus ad medium pure alba. Ceterum pectus et abdomen nigra castaneo tincta. Hypochondria et crissum nigro-cinerea. Remiges nigro-fuscr, secundaria extus cinereo marginatx; Cauda æqualis Rectricibus obrusis nigrieantibus, cinereo cinetis.

Fomina Mari similis sed dignoscitur capite et collo supra magis griseo-fuscis; collo antice et pectore sordide albis. 


\section{HIST ORIA.}

Juxta fuvios lapidosos et ad cataractas superioris Srecix passim. Non nisi hyeme in Scania invenitur. Solitaria nee nisi propagationis tempore per paria occurrit. In aquas ad eaput usque descendit cibum qux. situra. Urinatur quoque summa dexteritate licet fissipes. Hieme cantat. Nidulatur jam M. Martio, inter lapides ad fluviorum ripas. Ova 4-6 alba. Vescitur Insectis aquaticis eortmque Larvis. 


\section{GENUS XXVI. \\ $S T U R \quad N \quad U$ S.}

Rostrum reCum, subulatum, angulatum; depressum, obtusiusculum, mandibula superiore integerrima marginibus patentiusculis.

Nares oblongx, supra marginatæ.

Lingua emarginata, acuta.

$p_{\text {edes ambulatorii. }}$

\section{STURNUS VULGARIS.}

DiAgn. Niger purpureo, viridi et coeruleo fulgore nitens, maculis parvis albidis adspersus.

\section{SY NONYMA.}

Stumus Vulgaris, Lin. Faun. Sv. p. 77. n. 213. Retz. Faun. p. 224. 1. I99. Gmel. Syst. Nat. I. p. 80I. Lath. Ind. Om. I. p. 23 I. n. I. Briss. Orn. II. p. 439.

L'Etournenu, Buff. Ois. III. p. I76. t. I5. Pl. enl. 75. Stare or Starling, Pen. Brit. Zool. I. p. 299. n. 104. t. 46. Are. Zool. II. p. 33I. A. - Lath. Syn. III. p. 2. Suppl. p. I37.

Svecis: Stare.

\section{DESCRIPTIO.}

Longit. avis $8, \frac{2}{2}$ poll. Alx extense 15 poll. Rostr. I poll. A Alexura alx ad apicem 5 poll. Cauda $2 \frac{\pi}{2}$ 


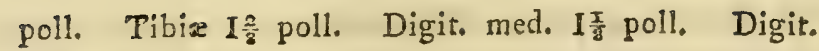
post. 7 ' poll.

Rostrum basi supra ad mares pennis teeturn. Pedes robustiusculi, et unguis posticus præsertim robustus a a. Rias annosus, tempore propagationis:

Rostrum pallide luteum, basi subtus album (in ave mortua nigrescens). Iris avellana. Pedes lutco-incarnati.

Corpus totum (pennis longis acutis tectum) nigrum, capite supra et collo subtus purpureo nitentibus. Dorsum, scapulx et superior pectoris pars viridi (æneo) colore nirentia. Ceterum pectus et alarum tectrices majores fulgore coeruleo. Collum supra, Dorsum et scapulæ apicibus pennarum testaceo-albis, unde maculis parvis trigonis adspersa. Crissum obscure nigrum pennis albido fimbriatis. Caput, collum subtus, peetus et abdomen immaculata. Remiges latere exteriore nigr: interiore fusca, versus apicem cinerex nigro fim. briatæ, margine exteriore pallide - testaceo tenuiter marginntx. Cauda fere xqualis Rectricibus nigricantibus cinereo supra tinctis, lateralibus extus pallide testaceo. marginatis. Tectrices caudiesuperiores rufo, inferiores albo, late marginatr.

3. Funior post priman deplumationem:

Corpus tegitur pennis haud ncuminatis. Rostrum supra nigrum, subtus fuscum. Pedes fusco incarnati. Nigęr minus nitens; Caput supra, Cervix, Dorsum et scapula maculis rotundatis pallide rufis. Corpus totum subtus nigrun maculis crebris albis in collo minoribus subrotundis, in pezore et abdomine majoribus transversim-ovalibus. Remiges et majores tęrices nigro-fusex margine éxteriore rufx. Rectrices nigricantes, cinereo tinct: rufo marginatx. 


\section{\%. Fnnior ante primam Deplumationem:}

Sapra fusco-fuliginosa immaculara Remigibus ferru. ginco marginatis. Subtus pallide grisea maculis crebris longitudinalibus griseo-fuscis. Gula albida.

J. Funior (Variet.?) ipso tempore deplunationis:

Caput collumque supra ét ad latera cinereo-fusca. Dorsum cinereo - fuscum, pennis nigris aneo colore nirentibus maculâ apicis albida, insertis. Uropygium nigrum pennis rufo marginatis. Gula alba. Jugulum albidum versus pectus vix fuscescens. Pectus et abdomen nigra maculis albis adspersa. Remiges et Rectrices ut in $\beta$.

Mus. Payk. Mense Julio occisa.

\section{HISTORIA.}

Frequens. Migratoria. Mature redux. Jam M. Martio in pratis depressis et ad rivulos Scanix caterva. tim occurrir socialis Avis. Mox in sylvas frendosas, se propagandi caussa, abit. Nidulatur in cavis arbo. zun ex. gr. Quercuum, Fagorum, Populorum \&c。 sxpe in una arbore plures. Etiam sub tectis xdium. Ova 4-7 pallide cinerascenti-viridia. Pulli jam M. Junio volatui apti sunt. Post hoc tempus gregatim in pratis et pascuis occurrunt. Vescuntur Insectis, LaY vis, Lumbricis \&c. Dorso avium insidentes Insecta deligunt. Inter arundines ad margines lacuum et fossarum vel in arboribus, innumeris fere gregibus perno Zant. Facile mansuescit avis. Docilis, aliarum avium cantus, et vocem humanam imitari discit. $M$. Oftobri abit.

\section{OBSERV.}

Zinne in Dissert. do Migrationibus Avium. p. $3 \mathrm{r}$, contendit Sturnos in Scania hybernare, quod nunquan, ne lenioribus qui- 
dem hyemibus, vidi. In Faun. Svec. 1. c. dicit: Discsdit post me.uian astatem in Scaniam campestrem. Ist quidem verum, Sturnum hoc tempore in Scaniam campestui frequenvem esse, sed in Uplandia quoque e. gr. ad Prædium Wallox-Säby, juxta finem M. Augusti maximas vidi carervas. 
GENUS XXVIY.

$P$ A. R U S

Rostrum rectum, breve, compressum, integerrimum, basi setis tectum.

Nares pennis setaceis tektr.

Lingan truncata, seris quatuor terminasa.

Pedes ambulatorii.

\section{PARUS MAJOR.}

DIAGN. Caput nigrum, temporibus albis, nucha viridi-lutea. Dorsum olivaceum, Pectus et abdomen flava, fascia in mediolongitudinali nigra.

\section{SYNONYMA.}

Parus Mnjor, Lin. Faun. Sv. p. 96. n. 265. Retz. Faun.

p. 268. n. 255. Gmel. Syst. I. p. 1006. Lath.

Ind. I. p. 562. I. Briss. III. p. 539.

Ia grosse Mesange ou Charhonniere, Buff. Ois, V. p. 392.

t. I\% Pl. enl. 3. f. I. Vers. Germ. Otto XVII. p. 29. cun. fig.

Grent Titmouse, or Ox-eye, Pen. Brit. Zool. I. p. 390.

t. 57. f. I. Lath. Syn. IV. p. 536. I. Vers. Germ. Bechst. IV. p. 53 I. n. I.

Die Kohlmeise, Bechst. N. D. 2te Anf. 3. B. p. 834. Suevis: Talgoxe. 


\section{DESCRIPTIO.}

Longit. avis 5. poll. Rostr. $\frac{3}{8}$ poll. A flexura alr ad apicem 3. poll. Cauda $2 \frac{x}{2}$ poll. Tibia $\frac{7}{8}$ poll. Digit. med. $\frac{5}{8}$ poll, Digit. post. $\frac{1}{2}$ poll.

Rostrum validiusculum, nigrum, reetum, tereti. compressum, acutum, mandibulis æqualibus. Nares rotundatz plumis setosis tectx. Iris fusca. Pedes cum unguibus, plumbei.

Caput splendidenigrum, Gula rigra. A jugulo nigro tenia utrinque concolor latera capitis alba cingit. Nucha pallide viridi-lutea. Dorsum et scapula pul. chre olivacca. Uropygium pallide cinereum. Pectus et abdomen virescenti-flava, stria longirudinali nigra, in abdomine latiore. Crissum medio nigrum lateribus albis. Remiges nigricantes, primores (oxceptis duabtis primis) a basi ad medium pallide coeruleo dehinc albido marginatx. Intimt latere exteriore lutescenti, apice albido marginatx. TeEtrices alarum pallide coernlex, majores apicibus albis. Rectrices nigricantes, extima longitudinalite: extrorsum oblique alba; secunda apice alba; medix binx coeruleo-tinetr; reliģux exisriore latere coerulescentes.

Fenina: Minor, coloribus minus vividis, striâ pectoris et abdominis nigra, breviore.

\section{HISTORIA.}

Manens. In sylvis et hortis ubique. Instante hyeme ad domos appropinquat, et interdum intrat. Agilis. sima avicula. Ferox, aves imbecilliores interdum $c r$ dit cerebrumque devorat. Vescitur præterea Insectis, Larvis, seminibus', etiam cadaveribus*). Nidulatu:

2) Intrat quoque Caveas, in quibus Caro et alis obsonia ven. tis exponuntur, linteis, quibus circumdantur, laceratis, Sic has aves totum fer Tetraonem Tetricem devolantes vidi. 
in cavis arborum, murorum \&e. Ova 8 et plura, lu. tescenti - alba, maculiis et striis rufescentibus.

\section{PARUS COERULEUS.}

Diagn. Frons aiba. Vertex coeruleus. Corpus supra olivaceum, subtus flavum.

\section{SYNONYMA.}

Parus coeruleus, Lin. Faun. Sr. p. 97. n. 267. Retz.

Faun. p. 269. 11. 256. Gmel. Syst. I. p. 1008.

Lach. Ind. Orn. I. p. 566. 11. I2, Briss. Orn. III. p. 544.2 .

La Mesange bleue, Buff. Ois. V. p. 4I3. pl. enl. 3. f. 2.

Vers. Germ. Otto XVII. p. 75. cum. fig.

Blise Titmouse, Pen. Brit.Zool. I. p. 39 I. n. I63. t. 57.

f. 2. Lath. Syn. IV. p. 543. n. IO. Vers. Germ.

Bechst. IV. p. 538. n. IO.

Dic Blanmeise, Bechst. N. D. 2te Auf. 3. B. p. 860 . Svecis: Blàmes.

\section{DESCRIPTIO.}

Longit. avis $4 \frac{\pi}{2}$ poll. Alr extens $7 \frac{\pi}{2}$ poll. Rostr. $\frac{2}{8}$ poll. A flexura alr ad apicem $2 \frac{\pi}{2}$ poll. Cauda $2 \frac{\pi}{2}$. Tibia $\frac{5}{8}$ polt. Digit. med. $\frac{9}{16}$ poll. post. $\frac{3}{2}$ poll.

Rostrum b:eve, compressum supra subtusque convexum, nigricans maxillis marginealbidis. Irss fusca. Pedes cum unguibus plumbei.

Frons et latera capitis alba. A rostro per oculos stria nigra, pone oculos ad cevicem saturate coerulea. Vertex coeruleus. Gula nigra demittit fasciam saturate coeruleam utrinque circa latera capitis ad cervicem. Occiput et inferior Cervicis pars, (versus Dorsum) al- 
bicantia. Dorsum viride. Pectus, latera 2udominis et crissum flava. Abdomen medium albescens striâ longitudinali coerulea. Alarum tectrices coerulea proximre apice albr, unde tænia darum alba. Remiges latere interiore nigricantes, exteriore coerulex, yrimores 4 margine exteriore, secundarix apice, albx. Cauda fere aqualis Rectricibus coeruleis, lateralibus extus albo merginatis.

Femina aliquantum minor, colore coeruleo minus pul. chro et stria abdominis vix conspicua.

\section{H IS T ORIA.}

Haud rara. Autumno et hieme hortos frequentat. Estivat in sylvis. Nidulatur in cavis arborum. Ova ponit 8-IO rubescenti-alba, rufo fuscoque maculata. Vescitur Insectis, Larvis, seminibus.

\section{PARUS CYANUS.}

Dragn. Dorsum pallide cceruleum; Caput et Conpus subcus alba. Fascia cervicis iransversa 52. turate coerulea. Cauda longa, rotundata.

\section{SYNONYMA.}

Parus cyants, Gmel. Syst. I. p. 1007. n. 16. Pallas Now. Comment. Acad. Petrop. T. XIV. p. 588. n. 8. t. 23. f. 3. Iepechin ibid. p. 498. n. I. t. 13. f. I. Lath. Ind. 1. p. 563. Retz. Faun. Sv, p. 26\%, n. 253.

Parus Salyensis, Mus. Carls. I. t, 25 *) Gmel. Syst. I. p. I008, 11. I\%.

7) Ex loo endem Specinine, quod adhuc in Afuseo Paylulliann invenitur, Descriprionem sumsi. 
Parus Knjasciok; Gmel Syst. Nat. I. p. IOI3.

In grosse Mesange bleue, Buff. Ois. V. p. 455. Vers.

Germ. Otto. XVIl. p. I53. 11. 5. cum. fig. - p. 174. 11. 8. et p. 84. Append. *)

Parus cocruleus major, Briss. Orn. III. p. 548. n. 3.

Azur Titnzouse, Pen. Arct. Zool. II. p. 426. C. Lath. Syn. IV. p. 538. n. 3. Vers. Germ. Bechst. IV. p. 533. 11. 3. et p. 553. 11. 30.

Die Lazir-Meise, Bechst. N. D. 2te Auf. 3. B. p. 865.

Mus. Payk.

\section{DESCRIPTIO.}

Inter P. cosmlenn et caudatum serie naturali collocandus. Paro cocruleo major, caudâ longiore, rostro breviore et crassiore, compresso, obtusiusculo, maxillis aqualibus pallidis presertin ad margines albidis. Pedes, cum unguibus acutis, nigro-coerulei.

Caput et tota avis subtus alba pennis paucis $2-3$ nio gro-coeruleis in medio pectore insertis. Stria nigra a rostro per oculos ad latera colli. Fascia lata cervicis transversa nigro-coerulea, pone quam initium Dorsi albescens. Dorsum et alarum basis albo-coertlescentia. Alæ coerulex apicibus Remigum secundariarum al. bis. Tectrices proxinn apicibus late albis, unde fascia alarum transversa et alia obliqua, lata, alba. Cauda longa, cuneato-rotundata, coerulea, Rectricibus lateralibus ( $I \cdot I ; 2-2 \& c_{0}$ ) extus dimidiato oblique albis.

*) Descriptio ex Mnseo Carlson. smmta. Figura vcro, qua ex eodem Musco desumta perhibetur, potius ad $P$. coersilemt pertinet. 


\section{LOCUS.}

Non nisi semel, quantum ego scio, in Svecia inventus, et quidem ad Prædium Säby in Sudermannia.

\section{PARUS CAUDATUS.}

Diagn. Cauda cuneiformis corpore longior. Capue album. Corpus supra nigrum albo et roseo varium.

\section{SYNONYMA:}

Lanius biarmicus, Lin. Faun. Sv. p. 29. 11. 84. cum. fig. Tab. I.

Lanius Caudatus, Lin. Faun. Sv. p. 28. 11. 83.

Parus caudatus, Retz. Faun. Sv. p. 27 I. 11. 259. Lath.

Ind. Orn. I. p. 569. Gmel. Syst. I. p. IOIO. n. II. Parnis longicaudus, Briss. Orn. III. p. 570. n. I3.

La Mesange ì longue queule, Buff. V. p. 437. t. I9. Pl. enl. 502. f. 3. Vers. Germ. Otto XVII. P. II 4. cum. fig.

Long-tailed Titmouse, Pen. Brit. Zool.I. p. 394 n. I66. Lath. Syn. IV. p. 550. n. I8. Sup. p. I90. Vers. Germ. Bechst. IV. p. 544. n. I8.

Der Schicvanzmeise, Bechst. N. D. 2te Auf. 3. B. p. 879.

Svecis: Altita, Swansmes.

\section{O BSERV.}

Parus biarmicus qui Bechst. 1. p. 889 . Scanix et in Faune Retz. p. 272. superioris Svecicz incola dicitur, nunquam in Svecia inventus est. Avis, quem Linne a Cel. Leche e Scania recepit et in Faun. Sv. n. 84. Lanium biarmicum appellavir, est revera Parus enudatus, quod tum ex Descriptione tum ex Icone saNilsson Oruith. 
tis liquet. Laninm cnudatum Lin. Faun. Sv. n. 83. descripsit Auctor ex figura nimis colorata, que inter Aves pie. Rudbeckii exstat (fol. I I 4 ).

\section{DESCRIPTIO.}

Longit. avis 6. poll. Alæ extensæ $7 \frac{\pi}{2}$ poll. Rostrum vix $\frac{2}{8}$ poll. A flexura alæ ad apicem $2 \frac{1}{2}$ poll. Cauda $3 \frac{\pi}{2}$ poll. Tibia $\frac{x}{2}$ poll. Digit. med. fere $\frac{1}{2}$; Digit. post. $\frac{5}{x^{6}}$ poll.

Rostrum breve, compressum, nigrum, basi pennis reversis tectum. Iris nigro-fusca palpebris margine luteis vel rubris. Pedes saturate fusci.

Caput supra et ad latera album. Cervix rufescens demittit striam concolorem utrinque in scapulas et ala. rum teetrices. Dorsum nigrum roseo et albido varium. Avis subtus alba, abdomine et crisso rufescentibus. Remiges primores nigricantes, secundarix fuscr albo late marginatx. Tectricesnigræ. Cauda longa, nigra, cuneiformis. Rectricibus tribus lateralibus fusc1s latetere exteriore et apice oblique albis.

Femina distinguitur striâ nigricante a rostro supra oculos ad nucham ductâ.

\section{HISTORIA.}

Passim. Manens. Parum timidus. Autumno eq hieme in sylvis frondosis, prasertim in alnetis, Estivat in sylvis. Nidum magnum, ovalem, extus ex Muscis et Lichenibus constructum, intus pennis stratum, aperturâ laterali rotunda, in ramo juxta truncum arbo. ris cujusdam ponit. Ova 9-12 alba, interdum rufo punctata. Fictus: Insecta, Larve \&c。 


\section{PARUS CRISTATUS.}

Diagn. Caput cristatum. Collare nigrum. Dorsum rufescenti-griseum. Abdomen albidum.

\section{SYNONYMA.}

Piarus cristatus, Lin. Faun. Sv. p. 97. n. 266. Retz. Faun. p. 268. 11. 254. Gmel. Syst. I. p. I005. Lath。 Ind. Onn. 1. p. 567 . n. I4. Briss. III. p. 558. n. 8 .

La Mesange huppée, Buff. Ois. V. p. 447. P1. enl. 502. f. 2. Vers. Germ. Otto XVII. p. I34. cum. fig. Crested Titmouse, Arct. Zool. II. p. 427. F. Lath. Syn. IV. p. 545. I2. Vers. Germ. Bechst. IV. p. 540. n. 12.

Die Houbenmeise, Bechst. N. D. 2 te AuH. 3. B. p. 869. Suecis: Tofstita, Tofsmyssa: Uplandis: Meshatt. Scanis: Toppmes.

\section{DESCRIPTIO.}

Longit, avis $4 \frac{\mathrm{T}}{2}$ poll. Alx extensæ $7 \frac{\mathrm{T}}{2}$ poll. Rostr. $\frac{5}{x^{6}}$ poll. A flexura alre ad apicem $2 \frac{2}{8}$ poll. Cauda 2 poll. Tibia $\frac{6}{8}$ poll. Digit. med. $\frac{1}{2}$ poll. Digit. post. $\frac{7}{x-6}$.

Rostrum nigrum, gracile, compressum. Nares ovales pennis setosis albis tectæ. Iris fusca. Pedes plumbei.

Vertex cristatus e pennis acutis fere pollicaribus, nigris, albo marginatis. Ceterum caput supra nigrum marginibus pennarum albis, unde frons $f$ re alba. Latera capitis cinereo-alba, macula pone aures semilunayi nigra. Gula et jugulum nigra. Ab inferiore juguli parte fascia nigra, utrinque ad nucham recurrens, col- 
Ium cingit. Dorsum et scapuli rufescenti-grisea. Pectus et abdomen alba. Latera abdominis, crissum, et caudæ tectrices inferiores rufescenti leviter tincta. Remiges griseo-fusc $x$ extus rufescenti tinctx, primores margine exteriore albidx. Cauda fere æqualis, Rectricibus griseo-fuscis, lateralibus versus basin extus rufescenti - albidis-

\section{HISTORIA.}

Habitat passim in pinetis Svecix, ex. gre in Hallanlandia, Smolandia, Uplandia \&c. Sono singulari Krrrit! Krrrit! eminus se prodit agilis avicula. Nidulatur in cavis arboribus. Ova 8.10 alba maculis in extremitate sanguineis. Veseitur Insectis eorumque ovis, Larvis, etiam seminibus Pini. Apud nos hyemat。

\section{CXXVII, PARUS ATER.}

Diagn. Caput et collum nigra, lateribus maculà magna alba. Stria occipitis alba. Dorsum cinereo-coeruleum. Pętus albidum.

\section{SYNONYMA.}

Parus ater, Lin. Faun, Sv. p. 97. n. 268.

Parus ater, Retz. Faun. Sv. p. 270. n.257. Gmel. Syst.

I. p. 1009. Lath. Ind. I. p. 564. 11. 8.

Parus atricapillus, Briss. Orn. III. p. 55 I. 5.

La Petite Charhonniere, Buff. Ois. V. p.400. Vers. Germ.

Otto XVIII. p. 44.

Colemouse. Pen. Br. Zool. I. p. 392. n. I64. t. 47, f. 3 Lath. Syn. IV. p. 540. 7. Vers. Germ. Bechst. IV. p. 536. ก. 7 .

Thmmenmeise, Bechst. N. D. 2 te Auf. 3. B. p. 853 . 


\section{DESCRIPTIO.}

Longit. avis $4 \frac{3}{8}$ poll. Alr extense 7. poll. Rostr. $\frac{5}{5}$ poll. A Aexura alie ad apicem $2 \frac{\pi}{2}$ poll. Cauda $I \frac{7}{8}$ poll. Tibia $\frac{5}{8}$ poll.; Digit. med. supra $\frac{\pi}{2}$; Digit. post. vix $\frac{T}{2}$ poll:

Fostrum subgracile, acutum, compressum, supra convexum, nigrum, marginibus maxillarum pallidis. Nares ovato-rotundata setis nigris tectx. Iris fusca. Pedes plumbei.

Caput supra et cervix nitide nigra, striâ nuchæ longitudinali alba. A sinu oris, sub oculo ad latera capitis et colli macula longitudinalis, magna, alba. Dorsum, scapulx et alarum minores tectrices coeruleocinerex. Uropygium in viride vergens. Gula et jugulum ad sternum usque nigra. Pectus et abdomen albida hypochondriis et crisso rufescenti tinctis. Re. miges saturate cinereo-fuscx extus tenuiter cinereo-albo intus albo marginatx, intimx apice albo maculat Rectrices cinereo-fuscæ extimæ extus versus basin albido marginat $x$.

\section{HIS T O R I A.}

Manens. In pinetis Sveciæ æstivat. Mense Octobri hortos Scanir per familias visitat. Nidulatur in ipsa terra aut in truncis cavis. Ova $6-8$ alba punctis rufis. Vescitur. Insectis eorumque ovis, qua exarboribus legit, unde sylvis utilis.

\section{PARUS PALUSTRIS.}

Diagn. Caputsuperne nigrum. Tempora alba. Dorsum rufescenti-griseum. 


\section{SYNONYMA.}

Parus palustris, Lin. Faun. Sv. p. 98. n. 169. Retz.

Faun. p. 270. n. 258. Gmel. Syst. Nat. I. p. 1009. n. 8. Lath. Ind. On. I. p. 56.5. n. 9. Briss. III. p. 555. 7 .

La Mesange de Marais, Buff. Ois. V. p. 403. Pl. enl. 3.

f. 3. Vers. Germ. Otto XVIl. p. 57. cum. fig.

Marsh Titmouse, or Black-Cap, Pen. Brit.Zool. I. p. 393. n. I65. t. 57. f. 4. Lath. Syn. IV. p. 54I. n.

8. Vers. Germ. Bechst. IV: p. 536. n. 8.

Die Sumpfmeise, Bechst. N. D. 2te Auf. 3. B. p. 873. Surcis: Entita. Tomlinge.

\section{DESCRIP TIO.}

Longit. avis $4 \frac{2}{8}$ poll. Alæ extensx 7 poll. Rostr. fere $\frac{3}{8}$ poll. A flexura alæ ad apicen $2 \frac{6}{5}$ poll. Canda 2 poll. Tibia $\frac{5}{8}$ poll. Digit. med. $\frac{x}{2}$ poll. post. $\frac{\pi}{2}$ poll.

Rostrum nigrum, tereti-compressum. Nares pennis setosis nigris tectr. Iris castanea. Pedes sordide plumbei.

Caput superne ad nucham nigrum. Genx et tempora alba. Dorsum rufescenti-cinereum. Gula nigra. Corpus præterea subtus sordide album, hypochondriis et crisso in rufescens vergentibus. Alz griseo-nigrx margine exteriore Remigum albido. Rectrices griseo. fuscæ lateralibus extus albido marginatis.

Feminn: gulâ nigrâ vix conspicuâ.

\section{H IST OR IA.}

Menens. Habitat in hortis et sylvis frondosis haud raro. Larvas, Insecta corumque ova ex arboribus de. 


\section{PASSERES. PARUS.}

ligit, unde hortis utilis avicula. Vescitur quoque baccis Sorbi, et sæpe in laqueis (Donor dictis) cum Turdis capitur. Nidulatur in eavis arboribus. Ova 8-I2 albo-grisea maculis rubris. 


\section{FAMILIA III. \\ I N C UR V I R O S T R E S.}

Rostram minimum, acutum, apice incurvum, basi latissimum et depressum; unde faux amplissima. Viezus: Insecta.

\section{GENUS XXVIII. \\ $H I R U N D O$.}

Rostruan minimum, apice incurvum, acutum, basi depressum.

Sinus oris vibrissis caret.

Rittus capite amplior.

Lingua brevis, lata, apice fissa.

Tibic breves.

Pedes ambulatorii vel prehensiles; unguibus acutissimis.

Caude plerisque forficata.

\section{DISSECTIO I.}

Digitis tribus anticis, uno postico.

CXXIX. HIRUNDO RUSTICA:

Diagn. Nigro - coerulescens, pectore, abdomine, et crisso albidis, fronte gulaque castaneis. Rec. 
trices nigræe, maculâ albâ, exceptis intermediis, notata.

\section{SYNONYMA.}

Hiruncio rustica, Lin. Faun. Sv. p. 98. 11. 270. Retz. Faun. p. 273. n. 261. Gmel. Syst. I. p. IOI5. Lath. Ind. Orn. I. p. 572. n. I.

Hirundo domestica, Briss. Orn. II. p. 486. I. Hirondelle de cheminee, Buff. Ois. VI. p. 59 I. t. 25. f. I. Pl. enl. 543. f. I. Vers. Germ. Otto XXII. p. I 19. cum fig.

Chimney or common Swallow, Pen. Brit. Zool. I. p.398. n. [68. t. 58. Arte. Zool. II. p. 429. n. 330. Lath. Syn.:IV. p. 56 I. Sup. p. I92. Vers. Germ. Bechst. IV. p. 554. n. I.

Die Ruchschivalle, Bechst. N. D. 2te Auf, 3. B. p. 902.

Svecis: Laduswala.

\section{DESCRIPTIO.}

Longit, avis $6 \frac{x}{2}-7$. poll. Alæ cxtensæ 13. poll. Rostr. a fronte $\frac{5}{\overline{1}}$ poli. $\Lambda$ flexura alx ad apicem fere 5 poll. Cauda $4 \frac{2}{3}$ poll. extra alas $I \frac{x}{2}$ poll. extensa. Tibia $\frac{1}{2}$ poll. Digit. med. $\frac{9}{x \bar{\sigma}}$; Digit. post. $\frac{3}{8}$ poll.

Rostrum nigrum, reêtum, basi depressum, latum, apice teresi-acutum maxilla superiore pone apicem decurvatum excisa. Nares ovales aliqvantum sursum curvatx. Os intus et lingua lutea. Iris fusco-castanea. Pedes nudi, fusci, unguibus acutissimis.

Frons et gula pulchre castaneo-rufa. Caput et collum superne, Dorsum et alarum teetrices nigra, coeruleo nitentia. Latera capitis et collum antice nigra. Peftus, abdomen et crissum alba, ferrugine- 
scenti levissime tineta. Remiges nigrx, latere interiore fuliginoso-fuscr. Cauda nigra forficata, Rectricibus, excepto pari intermedio, macula alba pone apicem noratis; extima utrinque longissima et angustissima.

Ferniza dignoscitur caudâ minus forficata, rectricibus extimis non tam elongatis.

Var. Hinindo rustica alba: Tota alba.

\section{HIS T ORIA.}

Habitat ubique ad ædes frequens. Migratoria redit primis diebus M. Maji et nos relinquit initio M. Septembris, australiores terras petitura*). Ante abitum innumeris gregibus inter arundines juxta aquas obvenit, Insecta aquatica, sola fere qux jam restant, lectura. Nidulatur in domibus sub tectis. Nidum se. miglobosum ex luto glutinoso et terra argillacea, straminibus intermixtis, pennis intus vestitum, apertura laterali præditum, muro vel trabi agglutinat. Ova ponit 4-6 alba, punctis fuscescentibus et violaceis. Vescitur Culicibus aliisque Insectis, qux volitans, in aëre et superficie aquæ prædatur. Volatus altior sere-

v) Non equidem ignoro plurimorum Ornithologorum opinionem de hibernis Hirundinum in lacubus, stagnis et paludibus. Hæc vero opinio, mea sententia, observationibus minus accurate habitis nititur. Nullum adhuc factum verum nec rclatum audivi, nec descriptum legi, quod dimigrationi Hirundinum absolute repugnet. Repugnat vero rationi. aviculam tam calido sanguine instructam, hibernum aquasum frigerem posse perferre. Eadem olim fabula de pluribus narrabatur avibus ex. gr. de Tringa vanello, de Charadrio campestri et Hiaticula, de Ardea Ciconia, de Colymbo aurito et aliis (vide Diss. Lin. de Migration. Avium). Cum vero angustia loci ine vetet fusius de hac re disputare, Lectorem ad ea delegare volo, qua egregia scripsit Cel. Bechstein in Naturg. Deutschl. 2 te Auf, 2. B. p. 122. et seqq. et 3 . B. p. 907 . et seqq. 
nam, demissior pluviosam tempestatem prrasagit vulgo. Sono acuto: Zifit! aliis aviculis hostem advenientem indicat, et, ut caveant, pramonet. Quem vero clamorem ut plurimum audiunt Columbr, Gallinx, alixque aves inermes, statim pastu relicto indicatum hostem circumspiciunt et refugium meditantur. Mirandus sane instinctus! Unde hane linguam intelligere didicit juvenis Columba, qux antea nec Falconem vidit nec Hirundinem audivit unquam? Non experientia sed natura illam docuit.

\section{HIRUNDO URBICA.}

Diagn. ReErices immaculatæ. Dorsum nigro-coerulescens. Uropyginn et corpus subtus alba.

\section{SYNONYMA.}

Hivundo urbica, Lin. Faun. Sv. p. 98, n. 27 I. Retz. Faun. p. 273. 18. 262. Gmel. Syst. I. p. IOI\%. Lath. Ind. I. p. 573. n. 3.

Hirundo rustica s. agrestis, Briss. Orn. II.'p. 490. 2. Hirondelle it cul blanc, Buff. Ois. VI. p. 6I4. t. 25. f.

2. Vers. Germ. Otto. XXII. p. I52. cum fig.

Le petit Martinet, Pl. enl. 542. f. 2.

Martin, Pen. Brit. Zool. I. p. 40I. n. I69. Arê. Zool.

II. n. 33I. Lath. Syn. IV. p. 564. 3. - Sup. p.

I92. Vers. Germ. Bechst. IV. p. 557. n. 2.

Die Hausschwalbe, Bechst. N. D.2te AuA. 3. B. p. 915. Svecis: Husswala.

\section{DESCRIPTIO.}

Longit. avis 5. poll. Alx extensx IO $\frac{1}{2}$ poll. Cau. da 2. poll. 
Rostrum nigrum, intus flavum. Iris fusca. Pedes Ianâ alba usque ad digitos vestiti, ungnibus griseis.

Caput supra nigrum. Dorsum nigrum coeruleo nitens. Uropygium, Tectrices caudx superiores, Gula, Pectus et abdomen nivea, collo subtus rufescenti tincto. Remiges nigro-fuser intimis tribus apice albis. Rectrices cocruleo-nigræ, immaculatr, exterio!es sensim longiores ita ut cauda sit minus quam in præec. dente forficata.

Fcmina: Gula sordide alba.

\section{HISTORIÁ.}

Migratoria serius aliquantum advenit maturiusque abit, yuam $H$. rustica, scilicet circa medium Majum. Ad $x$ des et templa frequens. Nidum ex terra argillacea firme constructum, pennis mollibus intus vestitum, sub subgrundiis muro adglutinat. Ova ponit 4.6 alba punchis fuscis. Vescitur Inseetis.

\section{HIRUNDO RIPARIA.}

Dịagn. Cinereo-fusca; Gula et abdomen alba. Pedes subnudi.

\section{SYNONYMA.}

Hirundo riparia, Lin. Faun. Sv. p. 99. n. 273. Retz. Faun. p. 274. n. 263. Gmel. Syst. I. p. IOI9. Briss. II. p. 506. 1. I2. Lath. Ind. I. p. 575. 11. 10.

L'Hirondelle de rivage, Buff. Ois. VI. p. 632. P1. Enl. 543. f. 2. Vers. Germ. XXII. p. 176. cum fig. Sand Martin or Shore-bird, Pen. Br. Zool. p. 402. n. I70. Arct. Zool. II. n. 332. - Lath. Syn. IV. p. - 568. 11. IO. Vers. Germ. Bechst. IV. p. 560. n. IO. 
Ujerschwalbe, Bechst. N. D. 2te AuA. 3. B. p. 922. Svecis: Strandswala, Backswala, Jordswala.

\section{DESCRIPTIO.}

Longit. avis 5. poll. Alæ extensz 9. poll. Rostr. $\frac{2}{8}$ poll. A flexura ala ad apicem $4 \frac{2}{5}$ poll. Canda $2 \frac{1}{3}$ poll. Tibia $\frac{\bar{x} \bar{\sigma}}{\text { poll. }}$ Digit. med. $\frac{5}{8}$ poll. Digit. post. $\frac{3}{8}$ poll.

Rostrum nigrum, depressum, basi latissimum, apice acutum, maxilla superiore pone apicem excisa. Iris fusca. Nares obliqure, oblongx. Pedes fusco-nigricantes, nudi, ad exortm digiti postici plumula nonnullæ.

Caput et corpus totum supra cinereo-fuscum (cinereo-murinum). Collum antice annulo cinereo-fusco cinctum. Gula alba rufescenti levissime tineta. Pectus, abdomen et crissum alba. Remiges et Rectrices saturate fuscr. Cauda minus forficata quam in congeneribus, alisque brevior.

\section{HISTORIA.}

Migratoria, revertitur circa ......, abit jam M. Augusto. In abruptis littoribus, arenosis precipitiis, sepibusque terreis fodit cuniculos horizontales profundos, in quibus nidulatur. Ova 6 alba, rufescenti-cineyeo-nebulosa. Victus: Inseeta.

\section{DISSECTIO II.}

\section{Digitis omnibus anticis,}

\section{HIRUNDO APUS.}

Diagn, Tota nigricans, Gula alba. 


\section{SYNONYMA.}

Hirundo Apus, Lin. Faun. Sr. p. 99. 11. 272. Retz.

Faun. p. 275. n. 264. Gmel. Syst. I. p. 1020.

Briss. II. P. 512. 11. I5. Lath. Ind. I. p. 582. n. 32 .

Le granl Martinet, Pl. enl. 542. f. I. Buff. Ois. VI. p. 643. Vers. Germ. Otto XXII. p. 193. cum. fig. $S_{\text {wift }}$, Pen. Brit. Zool. I. p. 403 . n. I7I. t. 58. Aret.

Zool. II. n. 334. Lath. Syn. IV. p. 584. n. 34.

Vers. Germ. Bechst. IV. p. 574. n. 34.

Thumarchwallo, Bechst. Nat. Deutsch. 2te Auf. 3. B. p. 929.

Svecis: Ringswala.

\section{DESCRIPTIO.}

Longit. aris $7 \frac{\frac{2}{\delta}}{8}$ poll. Alx extensx $6 \frac{\mathrm{T}}{2}$ poll. Rostr. a fronte $\frac{2}{8}$ poll. A flexura alæ ad apicem $6 \frac{2}{3}$ poll. Alx clausæx caudâ I. poll. longiores. Cauda fere 3. poll, Tibia fere $\frac{\mathrm{r}}{2}$ poll.

Rostrum nigrum minimum depressum basi latissimum, apice decurvatum. Nares oblongo-ovales. Iris castanea. Tibiz pennis plumosis nigricantibus antice tcetr. Digiti incarnato-fusci, omnes antice versi, intimo brevissimo. Ungues acutissimi nigri. Alx longissima. Cauda forficata.

Corpus totum nigricans. Gula alba.

Femina: Fusco-nigricans. Gula sordide albida.

\section{HISTORIA.}

Migratoria. Redit circa medium Majum. Sono acutissimo, sibilante $\mathcal{F} \cdot \mathcal{F} \cdot \mathcal{F}$ ! eminus quoque digno. scitur. Celerrime volat immotis fere alis. In excelsis 
locis templorum arcium et adium habitat. Nidulatur in cavis arborum et sub tectis, etiam in sylvis, in cavis arboribus. Ova $3-4$ latea, griseo obsolete variegata. Vescitur Inseetis. 


\section{GENUS XXIX. \\ C A P R I M L G US.}

$\boldsymbol{R}_{\text {osirum minimum, acurum, apice incurvum, basi }}$ depressum.

Sinus oris vibrissis rigidis.

RiËus amplissimus.

Lingua acuta, integerrima.

Cauda inregra, Rectricibus decem.

$P_{\text {edes }}$ breves, terradactyli, ungue medio latiusculo, intus serrato.

Apertara asrizsn ampla.

\section{CAPRIMULGUS EUROP EUS.}

Diagn. Corpus supra variegatum ex nigro, fusco, ferrugineo er albo; subrus albo - rufescens undulis fuscis. Cauda cinerea, fusco transversim $\mathrm{fa}$ sciata et variegata. Narium tubuli obsoleti.

\section{SYNONYMA.}

Caprimulgus Europeus, Lin. Faun. Sv. p. 100. 11. 274. Retz. Faun. p. 275. 1. 265. Gmel. Syst. I. p. IO27. Lath. Ind. Orn. I. p. 584. n. 5. Briss. Orn. II. p. 470 . I. t. 44.

L'Engoulevent, Buff. Ois. VI. p. 5I2. Vers. Germ. Otto XX. p. 5. cun fig.

Le Crapaud - Volant, Pl. enl. 193. 
Noćturnal Goat-sucker, Pen. Brit. Zool. I. p. 4I6. n. I?3. t. 59.

European Goatseucker, Ar7. Zool. II. p. 437. A. Lath. Syn. IV. p. 593. 5. Sup. p. 194. Vers. Germ. Bechst. IV. p. 580, n. 5.

Der Europäische Tagschläfer, Bechst. $N$. D. 2te Auf。 3. B. p. 939. tab. 39. t, 2.

Svecis: Nattskräfwa, Nattskärra.

\section{DESCRIPTIO.}

Longit. avis $10 \frac{6}{3}$ poll. Alæ extensx I. ped. $9 \frac{t}{2}$ poll. Rostr. a fronte $\frac{3}{3}$ poll. A sinu oris $I \frac{I}{8}$ poll. A flexura alx ad apicem $8 \frac{2}{2}$; Cauda $5 \frac{5}{5}$ poll.; extra alas extenditur fere 2. poll. Tibia $\frac{3}{8}$ poll. Digit. med. $\frac{7}{8}$ poll. Digit. post. $\frac{5}{x^{7}}$ poll.

Caput ratione corporis magnum, depressum. Rostrum parvim, nigrum, basi depressum, latum, apice tenue maxillis incurvatis. Faux amplissima, usque sub oculos se tendens, albido-lutea. Sinus oris Vibrissis rigidissinis nigris superne ciliati. Nares pro. minentes cylindio elevato. Lingua parva acutissima, palato adfixa. Oculi magni coeruleo-nigri. Aures ampla. Pedes breves, incarnato-fusci, tibiis antice quoad partem pennis tectis. Unguis intermedius larere interiore denticulis sex pectinatus. Caput et dor. sum cinerea undulis et punctis tentissimis fuscis, maculisque longitudinalibus nigris, presertim supra ca. put. Occiput et scapula maculis præterea pallide ferrugineis. $\Lambda$ maxilla inferiore ad latera colli decurrit fascia ferruginescenti-alba. Collum inferne et pecius anterius undulis tenuissimis cinereis et nigricantibus, masulis in medio collo ferrugineo-luteis. Abdomen cinereo-ferruginescens undulis crebris latioribus nigro-fuscis. Crissun pallide ferruginescens undulis Nissson Oraith. 
nigricantibus. Remiges nigro-fuscr, characteribus ferrugineo-luteis hinc inde notatis, apicibus cinereovariegatæ; extimæ tres maculâ magna alba, interiore latere; intimx Dorso concolores. Teatrices majores colore dorsi, maculis ovalibus pallide ferrugineis. Tecrices inferiores ferruginex undulis fuscis. Cauda fere aqualis rectricibus exterioribus parum brevioribus. Rectrices cinereo-fusco variegata, fasciis transversis (8 - 9) nigro-fuscis notatx; extimx binx (I. I - 2. 2.) apice alba.

Femina caret maculâ albâ in remigibus tribus primis et in rectricibus duabus extimis.

\section{H ISTORIA.}

In sylvis passim. Migratoria avis mature discedit, sero revertitur. Susurro nocturno de arbore se prodit. Interdiu quiescit; noctu Insecta quasitura evolat. Volatus valde pacatus. Nidificat ad prarupta umbrosaque montiun latera. Ova 2. sordide alba, fuscescenti maculata. 


\section{ORDO IV. \\ C O L U M B E, Lath。}

GENUS XXX.

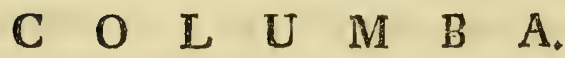

Rostrum mediocre, molle, tenue, rekum, sub. compressum; maxilla superiore versus apicem elevato-convexa, apice descendente obruso; inferiore ręta pone apicem subtus subgibba. Nares oblongæ, postice acuræ, membranâ molli tumida semireeta.

Lingua integra subtrigona.

Pedes breves.

\section{COLUMBA PALUMBUS.}

Diagn. Corpus coerulescens. Alæ maculâ longirudinali niveâ. Cauda pone apicem nigrum fasciâ subtus latâ albidâ.

\section{SYNONYMA.}

Columba Palumbus, Lin. Faun. Sv. p.75. n. 208. Retz. Faun. P. 219. 1. 19I. Gmel. Syst. I. p. 776. L2th. Ind. Orn. I. p. 60I. n. 32. Briss, Orn. I. p. 89. ม1. 6 . 
Le Pigcon ranuicr, Buff. Ois. II. p. 53 I. t. 24. Pl. enl. 316. Vers. Germ. Martini VI. p. 238. cum fig. Ring-Pigron Pen. Brit. Zool. I. p. 296. n. I02. Arct.

Zoo1. II. p. 329. B. Lath. Syn. IV. p. 635. n. 29.

Sup. p. 198. Vers. Germ. Bechst. IV. p. 620.11. 40. Ringeltauhe, Bechst. N. D. 2te AuA. 3. B. p. 949. Suecis: Ringdufwa. Oelandis: Sjutut.

\section{DESCRIDTEO.}

Iongito avis circa $I 7$ poll. Alie extensx $2 \frac{\pi}{2}$ ped. Rostr. $\frac{7}{8}$ poll. A flexura alx ad apicem 10. poll. Cau. da $\sigma \frac{6}{8}$ poll. extra alas extensa 2. poll. Tibia $I_{8}^{2}$ poll. Digit. med. I $\frac{5}{3}$ poll. Digit. post. fere I poll.

Rostrum rectum, compressum, Iuteum, maxilla superiore longiore, apice obtusa, dependente, fornicata; inferiore recta pone apicem subtus gibbo. Nares lineares, antice rima hiante, membranâ tumida, mollis incamata tectx. Orbita nuda cinereo-coerulescens. Iris pallide lutea. Cauda longa subrgualis. Pedes incarnati. Tibix antice sub genubus plumosx. Digiti usque ad basin fissi unguibus fuscis.

Corpus supra fusco-coerulescens, capite et uropy. pygio pallide coerulco-cinereis. Cervix viridi-coeruleo nitens, ad latera colli maculâ utrinque alba nitens, ovalis, magnitudine ovi columbini. Collum antice et pectus purpureo-einerea (rubicundo-cinerea). Abdomen et crissum pallidiora. Hypochondria et alarum tectrices inferiores pallide cinerea. Remiges nigricantes, primores margine exteriore albæ. Teetrices pri* xhores, majores ot minores nivex, unde macula longitudinalis magna, alba, juxta marginem alı clausz oritur. Rectrices versus basin cinereo - coernlescentes, versus apicem atr $x$, fascia lata albida in medio subtus. 
Femina: Minor, maculâ alba ad latera colli minore, pectore pallidiore.

Funior: Rostro et pedibus incarnatis. Iris griseo-albida. Cervix caret colore nitente, et maculâ ad latera albâ.

\section{HISTORIA.}

Migratoria. Ad finem M. Martis et ad initium Aprilis in Scaniam redit, ibique in hortis et lucis rarius occurrit. Mox vero in pineta superioris Svecix, se pro. fagandi caussa abit. Inter arborum ramulis nidulatur. Ova 2. rarius 3. alba ponit. Subole educata in Scaniam revertitur, ubi a medio Septembri innumeris gregibus querceta, fageta, et agros Secali cerenli consitos frequentat. Vescitur seminibus Pini, glandibus, seeali, tritico, piso sativo \&rc. Timida fixuque difficilis. Caro juniorum sapida.

\section{COLUMBA OENAS.}

Diagr. Corpus coerulescens uropygio pallide cinereo. Alæ fascia nigra duplici. Cauda pone apicem nigrum fascia subtus cinerea.

\section{SYNONYMA.}

Columba Ocnas, Lin. Faun. Sv. p. 75. 11. 207. (nee. ta. men Diagn. in Syst. Nat. I. p. 279. huc pertinet). Gmel. Syst. I. p. 769. 11. I. Lath. Ind. II. p. 589. n. I. Briss. On. I. p. 86. 11. 5.

L'Oenas ou le Pigeon Desertcur, Buff. Ois. II. p. 498. Vers. Germ. Martini VI. p. I55.

Stock-Dove or IVood-Pigcon, Will. Orn. (Angl.) p. 185. 
Srock-Dove or Wood-Pigeon, Pen. Brit. Zool. V. 2. ap. pend. A.

Stock-Pigeon, Lath. Syn. IV. p. 605. Vers. Germ. Bechst. IV. p. 589. n. 1.

Die Holzanube, Bechst. N. D. 2 te Auf. 3. B. p. 957. Svecis: Wilddufwa, Skogsdufwa.

\section{DESCRIPTIO.}

Longit. avis 13. poll. Alx extensæ 2 ped. 2 poll. Rostr. $\frac{6}{8}$ poll. A flexura alæ ad apicem 8. poll. Cauda $4 \frac{2}{y}$ poll. extra alas 1 . poll. Tibia $1 \frac{\mathrm{r}}{8}$ poll. Digit. med. I $\frac{2}{8} ;$ Digit. post. $\frac{5}{8}$ poll.

Rostrum lutescens vel albidum (interdum incarnatum) mandibula superiore longiore, apice descendente obtuso, inferiore recta, subtus subgibba. Nares lineares antice rima hiante, membrana tumida, molli, incarnata teftr. Iris fusca. Orbita nuda, pallide incarnata. Pedes saturate incarnati tibiis antice sub ge. 11 pennis tectis.

Corpus cinereo-coerulescens, collo supra viridi-coeiuleo et purpureo nitente, subtus et pectore purpurascente; capite supra, Dorso antico et scapulis fuscescenti - tinctis. Dorsum medium, Uropygium, tectrices alarum majores, abdomen et erissum pallide cinerea. Remiges primores nigro-fuscz, margine exteriore tenuissime pallidx, secundarix cinerex apice nigræ, intimx cinereo-fuscæ. Tectrices majores maculis majoribus nigris juxta medium latere exteriore, ex quibus atque ex apicibus Remigum intermediarum, fascia duplexnigra, in ala composita oritur. Cauda subrocundara Rectricibus cineris versus apicem magis ma. gisque niguis; fascia pone apicem semipollicari cinerea, presertin subtus conspicua.

Femina collo et pectore ininus niticis. 


\section{H IS T OR I A.}

Frequens. Migratoria redit ad finem M. Martis et in agris satis sylras adjacentibus per familias occurrit. Tempore propagationis in sylvas frondosas et acerosas per paria discedit. Nidulatur in cavis arboribus *). Ova 2. rarius 3. alba. Autumno gregatim aut solx aut crm Palumbis sociatr, in agris demersis aut Secali consiris occurrunt. Vescitur Secali Tritico, hordeo, avena, piso \&c.; etiam seminibus Pini, Cannabis. Men. se Octobri abit. Caro sapidissima.

\section{OBS ERV.}

Columba Domestica mansueta non huic Speciei, ut quidana pueant, crigirem delet, sed alii, qua inter rupibus habitat, viropygioque albo dignoscitur. Hæ vero duæ Species sæpius sunt confusx. Col. Oenas apud nos frequens est, Col. vero domestica fera nunquam, quartum ego scio, in Svecia inventa est. Cum vern wher rujes ivorvegia habitaie refertur; ${ }^{* *}$ ) fieri quidem pom test $n$ inter rupes quoque Svecix habitet, quamvis nondum ab Ornithologis observata fuerit. Diagnosis ejus hæc est.

\section{COLUMBA DOMESTICA.}

\section{Diagn. Coerulescens, uropygio albo, fasciis alarum} duabus caudxque apice nigris, Membrana nz-

*) Observ. Cel. Gmelin in Syst. Nat. 1. c. Latham in Syn. 1. c. et Pennant in ArEt. Zool. Germ. II. p. $306 \mathrm{~A}$. dicunt hane coltumban in Europa et Sibirix turribus ripisque rupestribus labitare et nidulari. Sed hoc, ni fallor, de sequente Specie (Columba Domestica), minime vero de hoc intelligendum esr.

**) Nulla enim alia esse potest, de qua ita habet Cel. Pontopp. in Norv. Nat. Hist. vers. Germ. Tom. II. p. 132: Auf den Inscln ander Secl üste in Rylylle finciet man cine Art wilde Tauben, die den zahmen ähnich sind, ausser dass sie alie einerley Farbe und blate glinzende Fe dern am Haise haben: mad dass sie ihre Nester in den Felsenritzen bnten. Sie sind niche so scheu, als die Holztäuben. 
rium tumidaalbescens. Longit. I, ped. Alæ exrensæ fere 2. ped.

A. COLUMBA DOMESTICA FERA。

\section{SYNONYMA.}

Columbr domestica, Gmel. Syst. I. p. 769. n. 2. Lath. Ind. Orn. II. p. 589. n. 2.

Columba livia, Briss. Orn. I. p. 82. n. 3. Columba saxatilis, Briss. 1. c. p. 84. n. 4. Columba rupicola, Raji Av. p. 63. n. II. Biset et Pigeon de rocke, Buff. Ois. II. p. 498. Pl. enl。 11. 5 IO. Vers. Germ. Martini VI. p. 155. 158. cum. fig.

Common or Rock-Pigeon, Pen. Brit. Zool. I. p. 290. n. IOI. $t .45$.

White-rumped Pigeon, Lath. Syn. IV. p. 605. n. 2.

Vers. Germ. Bechst. IV. p. 590. n. 2.

Biset-Pigeon et Rock Pigeon, Lath. Syn. 1. co n. 3. val. A. B.

\section{LOCUS.}

Habitat in rupium fissuris et præcipitiis.

b. COLUMBA DOMESTICA MANSUETA. Pltrimz varietates in Svecia, cultz hospitantur. 


\section{ORDO V. \\ G A I I N $\mathbb{E}$, Lath。 \\ GENUS XXXI.}

T $\quad E \quad T \quad R \quad A \quad O$.

Rosirum breve, conico-incurvum, fornicarum. Mhaceśa supra oculos nuda, papillosa.

Pedes plumosi.

DISSECTIO. $x$.

Pedibus ad digitos tantum piunosis.

\section{TETRAO UROGALLUS.}

Diagn. Cauda cuneato-rotundata. Axillæ albz.

Mas nigricans, supra tenuissime albo variegatus.

Gula barbata.

Femina supra nigro et ferrugineo transversim ur!dulata; subrus ferruginea postice nigro undulata.

\section{SYNONYMA.}

Tetrao Urogallus, Lin. Faun. Sv.p. 72. 11. 200. Faum. Retz. p. 207. n. 183. Ginel. Syst. I. p. 746. Lath. Ind. Orn. II. p. 634. n. I.

Urogallus major, Briss. Orn. I. p. 182. 
Coq de Erryere, ou Teras, Buff. Ois. II. p. I9r. t. 5.

Pl. enl. 73. 74. Vers. Germ. Martini,V. p. 7. cun 2. fig。

Wood-Grous, Pen. Brit. Zool. I. p. 262. 11. 92. tab. 40. 4I. Are. Zool. II. p. 3 I2. A. Lath. Syn. IV. p. 729. I. Vers. Germ. Bechst. IV. p. 693.

Grosses Waldhún, Bechst. N. D. zte AuA. 3. B. p. 1298.

Svecis: Tjäder.

\section{DESCRIPTIO.}

Longit. avis 2. ped. IO. poll. - 3 ped. Alæ extensi $3 \frac{1}{2}$ ped.

Rostrum lutescenti-album, robustum, incurvum. Iris aveilana. Nares penuulis nigris tectx. Peds us. que ad digitos pennis tect. Digiti marginibus pectiratim ciliati, cum unguibus, griseo-fusci.

Caput et collum nigra, albo tenuissime variegata. Pennæ occipitis longx. Sub gula fasciculus, barbx instar, e pennis longris, mollioribus, nigris. Supra oculos macula longitudinalis, nuda, papillosa, carminea. Palpebre rubro marginatx. Dorsum et uropygium nigra albo tenuissime variegata et undulata. Pectus nigrum viridi nitens. Abdomen nigrum maculis albis adspersum. Crissum pennis longis nigris apice albis. Latera Dorso similia. Femora nigra tenui albo undulata apicibus pennarum late albis. Tibire pennis pilosis griseo-fuscis albo variegatis dense tectx. Alæ supra saturate fuscr et rufo-fuscæ, nigro tenuiter undulatæ, tectricibus majoribus variegatis. Tectrices inferiores et axillx albx. In flexura alz macula alba. Remiges saturate fuscre vel nigricantes primores extus albo marginata, secundaric margine exteriore albo gri. 
senque maculatrs, apice albs. Rectrices XVIII. Latx, truncatæ, nigre punctis rarioribus albis.

Fenina multo minor: I ped. 9-10 poll. longa.

Rostrum nigricans. Caput nigrtum maculis ferrugineo-luteis. Collum ferugineo-luteum maculis rotundatis nigris. Dorsum, scapulix, uropycritum, tectrices alarum et caudx nigro-fusca ferrugineo transversion undulata. Gula ferrugineo-lutea. Pectus ferrugineun maculis interdum hine inde fuscis. Abumen ferrugineo-luteum undulis nigris. Crissum ferruginescentiluteum, nigro undulatin, apicibus pennarum albidis. Remiges prinores saturate fuscr latere exteriore maculis ferrugineis ; isecundarix tectricibus concolores. Cauda saturate ferruginea nigro fasciata, alba terminata. Femora et tibix ferrugineo-grisea macnlis fuscis.

Variet. a. Mas. Dorsum anticun album maculis ferrugineis, fusco tenuissime undulatis; posticum nigrum pennis albis insertss. Abdomen et cauda maculis albis. Tibir sordide albr. Act. Holm. 1807. p. 4.

E Dalekurlia.

Mus. Holm.

Var. $\beta$. Mas: Vulgari Minor. Cinereo-canus, capite et collo saturatioribus. Mus. Thunb. T. Eremita Wet. Ac. Hand1. 1789. p. 179. Ex Lapponia.

Var. $\gamma$. Femina: Supra sordide testacea undulis rarioribus albis, subtus testacco et albido undulata.

Mus. Thunb.

Vor. $\delta$. Fem: Tota sordide albida, fuscescenti nebulosa, Mus. Payk. Ex Enontekis Lapponix. Hybridas Varietates vide sub T. Tetrice.

Obs. Dignoscuntur omnes hujus varistates a varietatibus sequentis Specici, prěsertim caudâ rorundata.

\section{HISTORIA.}

In sylvis montunsis, precipue ubi mixtæ suntarbores accrosæ et frondose, frequens. Mense Aprili coit, 
"Gallis ita deletis id quod sape fit, ut Gallina vagan bunda et xstuans maritales amplexus frustra et anxie desideret, hec ipsa ecstasi interdum corripitur ut nudis manibus se prehendi capiatur." Ödm. Hac anxietate cum Tetrice interdum coit, unde nascitur T. Hybridus. Interdiu teræ plerunque insidet, noen in arboribus quiescit. Ova $8-12$ sordiảe alba maculis sordide luteis in terra ponunt. Vescitur xstate baccis et insectis, hyeme gemmis, baccis juniperi et foliis pino. rum. (Vide Historiam avis fusius expositam in Wet. Ac, Handl. I787. p. 201.)

\section{TETRAO TETRIX.}

DiAgn. Cauda bifurca Reetricibus lateralibus extrorsum curvaris. Macula alarum alba.

Mas: Niger; capite, collo, dorso et uropygio nitore coeruleo splendentibus.

Femina: Capur er collum ferruginea fasciis trans. versis nigris; Dorsum, uropygium et cauda nigra ferrugineo fasciata.

\section{SYNONYMA.}

Tetrao Tetrix, Lin. Faun. Sv. p. 73. n, 202. Retz. Faun. p. 208. 11. 184. Gmel. Syst. I. p. 748. Lath. Ind. II. p. 635. 11. 3.

Urogallus minor, Briss. Orn. I. p. I86. 2.

Petit Tetrix ou Coq de bruyeres à queue fourchue, Briss.

Ois. II. p. 2 IO. t. 6. P1. enl. I72. I73. Vers. Germ.

Martini V. p. 23. cum 2, fig.

Black Grous, Blackcock, Pen. Brit. Zool. I. p.'266. n. 93. t. 42. Ara. Zool. II. p. 3 I 4. C. - Lath. Syn. 
IV. p. 733. 3. - Sup. p. 2I3. Vers. Germ. Bechst. IV. p. 697. 11. 3.

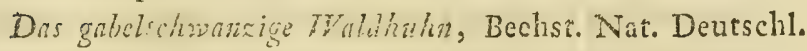
2te Auf. 3. B. p. I319.

Svecis: Orre. Scanis: Mas. Orrhane, Femina, Orhöna*

\section{DESCRIPTIO.}

Longit. I. ped. IO poll. Alx extensa 2 ped. 8 poll. Rostrum nigrum, breve, crassum, incurvum. Nares pennis minutis dense teetr. Iris coerulescens. Supra oculos macula nuda papillosa coccinea. Apertura auriun magna. Pedes ad digitos usque pennis tectæ. Digiti fusci marginibus peßtinatim ciliati.

Niger, capite, collo, Dorso et uropygio nitore coertleo splendentibus. Pennx scapulares teerricesque ala. rum minores ferrugineo tenuissime undulata, ceterx tectrices, alarum et caudie, nigrr. Uropygium albo tenuissime variegatum. Latera crissi albo undulata. Remiges breves, subincurve, obscure fusce, margine exteriore ferrugineo variegatæ, quinta et sequentes basi albx, qux albedo in sequentibus ita angetni ut (cum tectricibus proximis majoribus a basi ad medium albis) maculam magnam alban formet. Remiges interiores, cum suis tectricjbus, apice albo marginatx. Tectrices alarum inferiores albx. Cauda bifurca rectricibus XVIII nigris, exterioribus tribus utrinque extrorsum curvatis, ceteris brevioribus, albo tenuiter marginatis. Tectrices caudis inferiores longrx, albx. Femora et tibix albo-grisea, fusco maculata.

Femina minor, I ped. 7 poll. longa. Cauda minus quam maris bifurca, minusque lateriblis extrorsum curvata. Caput et collum ferruginea fasciis transversis nigris. Dorsum, Uropygium ế cauda nigra fasciis transversis ferrugineis. Pectus st crissum \&lba fasciis ferrugineis 
et nigris. Abdomen nigro-fuscum fasciis angustis ru. fesconti-aibis. Remiges primores obscure grisex, latere exteriore ferrugineo maculati, interiores a basi ad medium alba. Tecrices alarum superiores colore dorsi apicious hine inde albis. Tectrices candre inferiores longx, albx, fasciis rarioribus nigris ferruginco marginatis. Femora et tibix albo-grisex fasciis angustis fuscis.

\section{VARIETATES TETRICIS.}

Varict. a. Mas. Cincreo-fuscescens, crisso et fascia ala. rum obliqua; albis. Mus. Payk. Ex Uplandia.

Wartet. $\beta$. Fomina. Alba, undulis supra rarioribus obsolete-fuscescentibus. Cauda leviter bifurca. Tibix pennis sordide albis, obsolete fusco undulatis tecra. Wet. Acad. Handl. 1785. p. 230. - Mus. Carlss. III. t. 66. Obs. In hac igura, Cauda notatur rotundata, quod faisum est. - Prope Hedemora Dalekarlix interfecta est avis, quæ jam in Mus. Holm. asservatur.

Variet. $\gamma$. Mas: Hyliridus a Tetrice patre et Urogallina matre:

Magnitudo circiter Urogalli Femiac. Circa 2 ped. longus.

Rostrum caerulco-nigrum. Supra oculos macula nuda, coccinea, papillosa. Gula barbata (nec tamen tam prolixâ barba, quam in Urogallo). Cauda bifurca Rectricibus aliquantum extrorsum curvatis. Tibire teguntur pennis pilosis, grisso-fuscis, albo irroratis.

Caput et collum nigra coeruleo-nitentia. Dorsum, Scapulx et alarnm teftrices superiores nigricantia, rufescenti variegata. Abdomen nigrum, maculis hine inde albis. Axille et tectrices alarum inferiores albx. Reniges fuscr rachibus albidis, primores extus albo et ferrugineo variegata, interiores a basi ad medium albr 
maculis hinc inde fuscis, medietate exteriore ferrugineo-variegata apicibus albo marginatis. Refrices XVIII. nigrx, apice albo marginatz, exceptis duabus lateralibus. Tectrices caudx superiores ferrugineovariegatx apicibus albo marginatis. Tectrices inferiores nigræ, apicibus albis。

\section{OBSERV.}

Inter plurime qua vidi Specimina ${ }_{3}$ víx duo perfecte sibi similia inveni. Omnia vero dignoscuntur a Tetrice presertim gula barbatâ; ab Urogallo cauda bifurcâ; ab utroque colore et magnirudine.

\section{SYNONYMA.}

Tetrno Hybridus Lin. Faun. Sv, p. 72. Mus. Carls. I. I5. Wett. Ac. Handl. I744. p. I8I.

Tetrino intermedius, Langsdorff, Mem. de l'Aead. Petersb. III. I8II. p. 286.

Tetrno Medius, Meyer, Berlin Magaz. V. Jahrg. p. 337. Suecis: Rackelhane. Roslagis! Roslare.

Variet. J. Mas. Hybridus a Tetrice patre et Tetr. subalpino feminn: Magnitudo Tetricis Maris. Rostrum nigrum. Tibir pennis sordide albis usque ad ungues teetr. Cauda,bifurca (nec tamen falcata). Caput supra, Cer. vix et collare circa jugulum nigra maculis et punctis albis et cinereis. Dorsum fuscum albo tenuissime un. dulatum. Teetrices alarum albæ maculis fuscis albo tenuissime yariegatis. Tota avis subtus alba maculis nigris, in jugulo et abdomine fascias transversas fere formantibus. Thunb. Wet. Ac Handl, I808. p. I95. Tab. III. E. Wermelandia. Mus. Thunb. Varist. $\varepsilon_{0}$ Mas. Hybridus a Tetrice patre et Tctr. subal. pino femina:

Nilsson Orsith. 
Magnitudo et habitus $T$. Tetricis maris. Rostrum nigrum, formâ Tetricis. Macula supra oculos coccinea. Cauda bifurca, minus tamen, quam in Tetrice. Pedes ad ungues usque lanâ sordide albâ vestiti. Ungues rectiusculi, lati, subtus concavi. (Pedes itaque Tetr. subalpini, sed aliquantum majores).

Color Tetricis, sed collum maculis albis confuentibus. Alr, pectus et abdomen alba, maculis nigris. Cauda nigra Rectricibus mediis apice albis. Tectrices caudx inferiorcs albx, immaculatx, superiores nigre, apice alba. Mus. Caris, III. t. 6I. E. Norrlandia. Mus. Holm.

\section{OBSERV.}

Fx his videre licet hanc Speciem, nostro saltem sub coelo, pluribus esse obnoxiam varietatibus, e quibus ultimas tres ( $\gamma$. J. et .) ornithologis maxime notatu dignas censemus. 111. Lin.

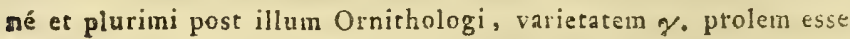
hybridam a Tetrice et Urogallina ortam existimans. Celeberrimi vero viri Meyer et Langsdorff 1. 1. c. 6, hanc avem pro distincta Specic habent; in quo tamen cum his cel. Ornithologis eonsentire non possum. Rationes omnes, quibus nicitur horum virorum opinio, recensere, ngustia loci me verat. Illis ramen paucis respondebo: Hybrida hæc proles existere non potest $r i s i$ in regionibus ubi unâ inveniuntur et Tetrices et Urogallina, et ubà Urogalli a venatoribus quodammodo deleri sunt* ${ }^{*}$ ). Si exstant femine, nihil contra opinionem meam probant: ex alis enim avium hybriditatibus uterque nascitur sexus, Si quis de his muptiis hybridis dubitat, eam ob caussam, quia nulla avis diyersa Speciei urget necessitas coěundi, illum ad ea delegare volo qua in Actis R. Ac. Sc. Holm. VIII, p. 201 (Om Tjäderfogler z dess wilda och tama tillstand) scripta sunt. Qui vero videc varietates 8 et (quarum illa in Mnseo Thunbergiano Upsalise

- Z Jae caussa est cur in dissitis tervis obvenime. 
hae in Museo Reg. Ac. Scient. Molm. athwc asservantur, qua. rumque hybrida a Tetrice el Tetr. subalpino origo, primo adspectu evidens \&c.); non diutius dubitare potest de libidine $T e$ tricis ad furtivos amores congeneribus instituendos semper paratissima. Vide praterea AAa Soc, Upsal, vol, V. p. 75.

\section{H IS T O R I A.}

In Sylvis prrcipue inter betulas et juniperos fre。 quens. Coit Mens. Martio et Aprili, ubi Mas sono gravi et rauco thalami sociam ad nuptias celebrandas appellat. Inter mares libidinosos de feminis sape pugna oritur. Nidulatur in terra. Ova $8-12$ sordide luteo-alba punctis ferrugineis. Vescitur xstate baccis, insectis at formicarum ovis, hyeme gemmis, amentis et precipue baccis juniperi.

\section{TETRAO BONASIA.}

DiAgn. Cauda cinerea nigro-variegata, fascia pone apicem album lata nigra, exeepris Rectricibus duabus intermediis. Gula maris nigra, feminx pallide ferruginea, fusco-maculata.

\section{SYNONYMA.}

Tetrao Bonnsia, Lin. Fauin. Sv. p. 73. n. 204. Retz。

Faun. 213. 11. 187. Gmel. Syst. I. p. 753. Lath.

II. p. 640. n. I4. Briss: Orn. I. p. I9I. 3.

Gclinotte, Buff. Ois. II. p. 233. t. 7. Pl. enl. 474. 475.

Vers. Germ. Martini p. 62. cum. 2. fig.

Hazel Grous, Aret. Zool. II. p. 317. F. Lath. Syn. IV.

p. 744. Vers. Germ. Bechst. IV. p. 707.

Schwarzkehlige Waldhuhn, Bechst. N, D. 2 te Aufl. 3.

B. p. 1338 .

Svecis: Hjärpe. 


\section{DESCRIPTIO.}

Longit. avis circa I. ped. Rostrum breve, con* vexum, nigrum. Nares plumis dense tectr. Iris avellana. Tibiz fere ad digitos tectr lana rufescenti. Digiti nudi marginibus pectinatis.

Caput et collum supra cinerea, rufescenti tinka: undulis numerosis fuscis. Supereilia nuda sanguinea. Gula nigra, fascia alba cincta. Pone oculos macula alba. Dorsum et alarum teetrices ferruginex maculis albis et nigris. Dorsum posticum et tectrices caudx cinerea ferrugineo et nigro immixtis. Corpus subtus albidum, pectore undulis confertis, abdomine, maculis majoribus cordatis fuscis. Remiges fusca latere exteriore pallide rufo variegatz. Cauda subrotundata Rectrieibus XVI. cinereis nigro-fusco punetstis et variegatis pone apicem album fascia lata nigra, qua caret par intermedium.

Femina: minor; gula non nigra ut in mare, sed pallide ferrugineo-lutea, fusco maculata. Praterea mari subsimilis.

Frict a. Diluta. In omnibus T. Bonasix feminx similis, colore tamen dilutissimo. Sic ubi illa nigra vel fusca, hac fuscescens, ita ut rudimentum vel fascire caudx conspicuum sit.

Tetr. Canus Mus. Carls. I. to I6. Lath. Ind. Onn. II. p. 640. Specimen in Mus. Payk. adhuc asservatum vidi。

\section{HIST ORIA.}

Regionum septentrionaliorum sylvas montuosas incolit. Nidulatur sub frutice. Uva IO- 12 pallide ferruginea, fusco maculata. Vescitur Baccis, insętis, arborum gemmis amentisque. Caro omnium sapidissima $*$ 。

-) Unde nomer Bonasia, quasi boua assa. 


\section{DISSECTIO II.}

Pedibus usque ad ungues, 'etiam subtus hyeme lanatis; digitis media astate nudirusculis. (Lagopodes. Svecis Ripor).

\section{TE'TRAO SUBALPINUS*),} mihi.

Diagn. Rectrices XIV. nigræ, apice albæ: Remiges omnes albæ. Hyeme albus. FEstate: Capue et collum fulva s, rufa nigro maculata. Dorsum nigrum, undulis ferrugineis.

\section{SY NONYMA.}

Tetrno Lagopus, Lin. Faun. Sv. p. 73. n. 203. Syst。 Nat. XII. I. p. 274.

Tetrno, (Rehusak) Lngopus, Martin Act. Soc. Phy. siogr. Lund. p. 150 (Specimen mutilatum) unde. Tetrao Lapponicus, Gmel. Syst. I. p. 75 I. n. 25. Lath. Ind. II. p. 640. et.

Tetrao Cachinnnus, Retzii Faun. Sv. p. 210 n. 185, White Grous, Lath. Syn. IV. p. 743. n. II. Pen. Aret. Zool. II. 11. I83. Edw. t. 72.

Rød grous, Lath. Syn. IV. Vers. Germ. IV. p. 709. n. 13. (Descriptio; mores vero cum sequentis confusi sunt).

Perdrix de Saules, Hearne Voy. Baye Huds. II. p. 260. Morast-Waldhuhu, Meyer, Vögel Liv, und Esthl. p. I52. Svecis: Ripa, Snöripa, Skogsripa. Norvegis: Skog Rypa, Lie Rypa; Dal Rypa. Lapponibus: Rehusak.

* Nomina inepta huirs et sequentis Specici mntare necessa. riun duxi. Alia mihi quarenti, ea maxime charaferustica visa sunt, qua loca avium natalia inslicarent; Sc. Hac in Subalpinis, -sequens in alpinis locis degit. 


\section{DESCRIPTIO.}

Mas: Longit. I6. poll. Alr extensæ 2. ped. 1. poll. Rostr. a fronte $\frac{7}{8}$ poll. A Hexura alx $\&$ poll. Cauda fere 5 poll. Tibia $I \frac{5}{8}$ poll. Digit. med $1 \frac{5}{8}$ poll. postic. $\frac{x}{2}$ poll.

Rostrum saturate corneum, robustum, apice incurvo, obtuso. Nares plumis tectr. Iris saturate fusca. Macula superciliaris nuda coccinea, superne marginata membranâ pectinatim dentara, præsertim tempore pruritus conspicua. Ungues longi, lati, pasum curvati, supra convexi. subtus concavi; tusci margine et apice albidi. Cauda zqualis.

A. Mas oc cisus d. I. M. Fuuii. Caput et collum saturate rufa, maculis parvis nigris, superne confertioribus. Pars colli inferior versus dorsum et pectus undulis nigris et fulvis: Mentum, palpebrx et narium tectrices alba. Dorso albo immixtæ sunt pennæ nigræ ferrugineo undulatx. Pectus, Abdomcn, crissum, pedes lanati, alæque subtus alba. Remiges albæ rachibus extimarum sex fuscis. Tectrices alarum albr, mediis quibusdam e nigro et rufo variegatis. Rectrices XIV. nigræ, apice et basi albæ; præterea duæ medir s. spuriz, alias obtegentes albæ. Tectrices long: rufx nigro variegati. Pedes usque ad ungues íplumosx, albr, sed digiti subtus nudi.

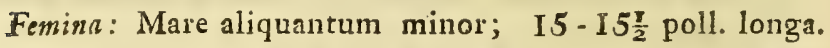
Alæextensæ 23 poll. v. 2 ped. Macula superciliaris coccinea nuda et membrana ejusdem marginalis dentata, minor quam in Mare. Pedes ut in Mare.

B. Fomina occisa d. I Mens. Funii: Caput et collum fulvo-ferruginea maculis nigris, prasertim superne confertis, ita ut pileus et cervix fere nigra sint maculis minoribus fulvis adspersa. Dorsum et uropygium nigra striis transversis andulisque fulvo ferrugineis et 
luteis. Pectus, Hypochondria er crissum fulva maculis transversis s. fasciis nigris. Abdomen album. Alx ut in Mare, Teetricibus tamen pluribus nigris fulvo undulatis. Reatrices XIV. nigra apice et basi albx; spurix dorso concolores. Pedes ut in Mare.

C. Mas medio Fulio: Caput, collum et pectus fulvo-ferruginea, gula et genis pallidioribus; vertice et cervice nigro maculatis; pectore striis, punctis et undulis nigris rarius adsperso. Dorsum et Scapulares, Uropygium et tectrices alarum medix nigra striis transversis undulisque confertis ferrugineis et (hinc inde) albidis. Tectrices alarum minimx (juxta marginem sitx) et remiges alba. Abclomen album. Crissum et lfemora rufo tincta. Reetrices XIV. nigrx, rufo tinctx, apice et basi albx. Rectrices spurix binx ceteris longiores e nigro et ferrugineo variegatz. Tectrices caudx inferiores ferruginea maculis nigris, apicibus albis. Tibix antice et ortus digitorum superne plumis pilosis sordide albis vestiti; Tibir postice ct digiti, nudi, supeme squamati.

D. Feninu medio Fulio: Similis feminx sub Litt. B. descript:e, undulis tamen dilutioribus fere albidis inter fulvas immixtis. Digiti ut in Mare, nuper descripto.

E. Pullus (d. I6 Julii) muper ad volandnm aftus: 7. poll. longus. Supra ex ferrugineo et nigro variegatus, maculis parvis albidis in alarum tectricibus; Remiges cinereo-fuscx primores extus pallide ferrugineo marginate et variegatæ. secundarix latius et saturatius extus ferrugineo marginatæ. Cauda rotundata Rectricibus XIV. ferrugineis maculis irregularibus, confertis nigris. Pedes usque ad ungues vestiti plumulis pilosis pallide ferruginescentibus.

F. Pullus (d. 7. Aug.) I3 poll. longus. Feminx Litt. B. fere similis, sed remiges 3.4. 5. fuscie sunt, extus 
ferrugineo variegatx. Reetrices nigrx brevissimæ (e vaginis erumpentes.) sed Rectrices spurix multo longiores, acuminatz, colore dorsi. Digiti subnudi.

Post medium M. Augustum pedes hujus avis sensim vestiuntur plumis densissimis albis. Ungues albescunt basi tantum fusco. Habitus astivalis variega. tus successive deponitur et hyemalis, albus iuduitur, his fere stadiis: Imo Pectus et Uropygium, teetrices alarum et Reĉrices spuriz in alba mutantur. 2do C8put et collum magis magisque pennis albis ornantur, et ferrugineis privantur. 3tio Dorsum albescit. Tota hæc pennarum mutatio ante finem Mens. Septembris peracta est. Ab hoc tempore et per totam hiemem avis est nivea rostro nigro Reetricibus XIV. nigris apice et basi albis, rachibusque Remigum sex extimarum fuscis exceptis.

\section{HIS TORIA.}

Inhabitat frequens Scandinaviam Septentrionaliorem a Finnmarkia usque ad 6I. latitudinis gradum, citra quem non longe descendit. Tesqua depressa et uliginosa, ubi sunt frutices Betula alba et nana, prasertim amat; in sylvis quoque obvenit. Latera alpium adscendit usque ad terminum sylvaticum (i. e. usque ubi desinit betula alba); nuda alpium juga nunquam visitans. Monogamiam celebrat, Coit mense Majo. Mas ad nuptias celebrandas feminam appellat voce hinniente, qua cachinnos quodammodo amulatur. Huic respon. det femina voce debiliore: Fack! Fack! quem feminx sonum imitantes venatores marem alliciunt. Evolans quoque vel terrefactus hos cachinnos edit Mas, saltem per xstatem. Femina plerumque evolat tacita. Nidulatur in terra inter fruticulos. Ova IO- 12 (usque ad 18. nonnunquam inventa, narrabant zustici) lurescenti-alba mactilis et punfis nigris irrorata. Pullis educatis plurimx familiz sosiatx ab uno in alterum 
iocum errant. Capitur laqueis, vel necatur globulis. Vescitur baccis, foliis, gemmis, seminibus. Caro sapida.

\section{TETRAO ALPINUS.}

DiAgr. Reetrices XTV, nigra, apice albr. Remiges alba. Hyems albus loris nigris. Fistate. Mas: Caput et collum nigricantia. Lora nigra. Dorsum nigricans fulvo ex cincreo tenuissime variegatum. Femina: nigra fasciis fulvis et albis.

\section{SYNONYMA.}

Tetrno Lagopus, varietas alpina, minor, Lin. Faun. Sr. p. 73. n, 203. Syst. Nat. XII. I. p. 274.

Tetrno Lagopus, Fabric. Faun. Groenl. p. I14. 11. 80. (exacte descripta) Lath. Ind. II. p. 639. n. 9.

Tetrao rupestris, Lath. Ind. II. p. 640, 11. II. Gmel. Syst. I. p. 75 I.

Tetrao (Keron) mutus, Montin, Acta Soc. Physiogr. Lund. 1. p. I53. (femina est, unde muta dicitmr). Ptarmigan Grous, Lath. Syn. vers. Germ. IV. p. 705. ก. 10.

Rock Grous, Pen. Ark. Zool. II. n. 184.

Perdrix de roches, Hearne Voy. baye Huds. II. p. 260. Schrevonldhuhn, Meyer Vögel Liv. u. Esthl. p. I63. Svecis: Fjällripe: Norvegis: Fjellrypa, Skare rypa; Fjellskarv; Lapponibus: Keron.

\section{DESCRIPTIO.}

Mas: Long. 14-14 $\frac{1}{2}$ poll. Alx extenst fere 2, ped. Rostr. a fronte $\frac{5}{8}$ poll. A ficurua alie ad apicem 8 poil.

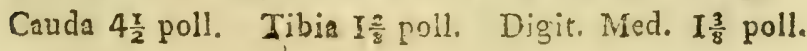


Rostrum nigrum, minus robustum quam antecedentis, maxilla superiore incurva, versus apicem acuminata, apice ipso truncato. Nares plumis tectx. Iris fusca. Macula superciliaris nuda coccinea, superne marginata membrana pectinatim dentata, prasertim tempore coitus conspicua. Ungues longi, lati, magis curvati, basi fusci, apice et marginibus pallidis.

Mns die I. M. Funii occisus: Caput et collum nigra; gula palpebraque inferiore albis. Supra basin rostri macula alba s. rufescenti-alba. Pone et infra aures ma. culis albis. Tempora punctis ferrugineis. Macula de restro per oculos pure nigra. Verter et occiput lineis transversis ferrugineis. Dorsum nigricans, ferrugineo et cinereo tenuissime undulstum et irroratum, pennisque albis insertis. Alæ nivex tectricibus quibusdam dorso contiguis colore dorsi. Raches Remigum sex primorum fusca. Pectus, abdomen, crissum, teetrices caudæ inferiores albæ, pennis nigris fulvo s. cinezeo variegatis in pectore et hypochondriis insertis. Cauda xqualis. Reetrices XIV. nigræ apice et basi alba; intermedix s. spurix bina albx, ceterx darso concolores. Pedes usque ad ungues plumis densis albis vestiti, digitis subtus nudis.

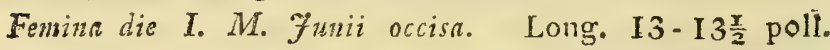
Caput supra nigrum maculis fulvis. Nares plumis albis refx. Gula luteo-albida, nigro maculata. Cọllum, Dorsum, Uropygium, caudx teetrices, Scapulares et tectrices alarum dorso contigux nigra fasciis iransversis fulvis er albis; (apice singulx pennæ albo). Collum inferius, pectus superius, et hypochondria fasciata nigro, fulvo et albo. Abdomen, crissum et inferiores caudx teatrices albx. Rectrices ut in Mare. 


\section{O B S E R V.}

Post hoc tempus magis magisque deponit avis pennas albas et induit nigras variegatasque. Mense Julio mutatione pennarum perata, avis ita piota est ue in Fann. Groenl. 1. c. describitur. Digriti hac tempestate nudi slint. lfense Aug. et Sept. habitu hyem li albo rensim induicur aris, ita nt inveniatur:

Hyeme: Nivea, macula nigra a roatro per oculos, presertim in Mare conspicua; $R$ ulricibs XIV. nigris apice ct basi albis; rachibus Reınigum sex primarum fuscis.

\section{HISTORIA.}

Inhabitat juga alpium Lapponicarum, nunquam, nisi frigore maxime intenso et alpibus nive profunda obrutis, in sylvas, vel in plonities descendens. Adscendit vero alpes xstate, usque inter nives. Amat loca petroșa. Quiescit in rupibus s. in nive. Monogamiam celebrat. Coit ante medium M. Junii. Mas thori sociam appellans s, terrefactus evolans, rhondjos sonat, sotertentis hominis s. ranx temporarix vocem æmulantes. Vocem feminx debilis I - ack! I ack! imitando mas stupidus allicitur, tempore pruritus. Femina plerumque tacite evolat. Nidulatur in terra inter Salices, Empetra \&c., Ova 8-10 fulvescenti-alba, maculis confertis nigris. Hyeme avis gregaria est. Caro parvi astimatur.

$$
\text { OBSERV. }
$$

In itinere Norvegico nestate nuper pruterlapsa, $(1816) \mathrm{in}$ stituto, hanc et antecedentem speciem, quarum Historia huc usque minus rite cognita fuit, in Alpibus Scandinavicis observandi occasionem habui. Obscrvationes Cel. Montin, quaruın illa ex Speciminc mutilato, hæc ex femina facte sune, causa fuerunt plurium errorum in scriptis Ornithologicis. 


\section{GENUS, XXXIT. \\ P. E R D I X.}

Rostrum breve, robustum, maxilla superiore incurvo-fornicata.

Nares margine prominulx.

Orbita papillosa.

Bedes nudi, plerisque calcarati.

\section{PERDIX CINEREA.}

Dragn. Sub oculis macula nuda papillosa. Corpus cinereo, rufo et nigro variegatum. Linea longitudinales albidx in verrice er scapulis. Cauda zufa reEtricibus mediis exceptis.

\section{SYNONYMA.}

Tetrno Perdix, Lin. Faun. Sv. p. 74, n. 205. Retz, Faun.

p. 214. 11. I88. Gmel. Syst. I. p. 757.

Perdi: cinered, Lath. Ind. Orn. II. p. 645. 11. 9. Briss.

I. p. 219.

Ln Perdrix grise, Buff. Ois. II. p. 40 I. Pl. enl. 27.(fem.)

Vers. Germ. Martini VI. p. I2, cum. fig.

Common Partridge, Pen. Brit. Zool. I. p. 274. n. 96.

Art. Zool. II. p. 3I9. A. - Lath. Syn. IV. p. 762.

8. Vers. Germ. Bechst. IL. p. 72 I. n. 8.

Gemeines Feldhuhu, Bechst. N. D. 2te Aufl. 3. B. P. I361.

Svecis: Rapphöns. Scanis: Åkerhơns.

\section{DESCRIPTIO.}

Longit. avis 12. poll. Alr extersz.... Rostrum $\frac{5}{8}$ poil. A flexura alz ad apicem $6 \frac{\pi}{2}$ poll. Cauda $3 \frac{3}{3}$; Tibia $1 \frac{\kappa}{8}$ poll. Digit. med. I $\frac{5}{8} ;$ Digit. post. $\frac{3}{8}$ poll. 
Rostrum coerulescens in olivaceum tendens. Iris rufo. fusca. Circa oculos poftice papillz rubrx. Nares squa. ma tecrx. Pedes grisco - incarnati; Mas calcare brevi inftruktus. Ungues cornei.

Frons, stria supra oculos ad nuchan et gula, rufa. Vertew olivaceo-fuscus lineis longitudinalibus luteo-albis nigro marginatss. Tempora et collum ad mediun pectus cinerea undulis tenuissimis njgris. Dorsun et uropy. gium cinerea rufo inmixto undulis tentissmis nigris, striisque latioribus transversis fuscis. Pennæ scapula. res tectricesque alarum rufo-griser undulis tenuissimis nigris, strià ad rachin longitudinali luteo-alba, nigro marginata et maculâ majori rufa ju larere ínteriore sin. gulx pennx. Peßtus maculâ castanea formam ferri equini referente. Latera pallide cinerea undulis tenu. issimis fuscis, fasciisque transversis latis rufis. Medihum abdomen album. Crissum rufescenti album. Re. miges breves, introrsum curvatr, fuscr, fasciis ferrugineo-luteis. Tectrices inferiores albr. Rcetrices XVIII; quarum I - 7. utrinque apice pallido, fusco variegato. Medir quatuor et teArices caudie superiores, longitu. dine caudx, lutescenti fuscoque tenuissime variegatx fasciis transversis castaneis.

Fenina: dignoscitur præcipne tectricibus alarum et seapulis fusco (non rufo) maculatis; macula pectoris castanea; qux in mare ferrum equinum refert, in fermina maculis sparsis concoloribus continetur.

Avis junior: plane caret nacula peetoris castanea. Pedes flavescentes.

\section{HISTORIA.}

Manens. Habitat gregaria, tempore vero pruritus per paria in campis, agris et fruticetis, agros adjacen- 
tibus Scanix, Ostrogothix, Uplandire \&c. *). Vescitur Insectis, seminibus, herbis, hyeme vero satis et præsertim baccis juniperi. In terra nidulatur: Ova I22 I sordicle virescenti-grisea; in fossulâ, straminibus pennirque parcius vestitâ, ponunt.

\section{PERDIX COTURNIX.}

Diagn. Corpus griseo-ferrugineum maculis nigris, striis longitudinalibus ferrugineo-albidis. Super oculos stria albida, aliaque in medio vertice. Cauda fusca striis transversis, rachibusque ferrugineo - albidis.

SYNONYMA.

Tetrno Coturnix, Lin. Frun. Sv. p. 74. 11. 206. Retz。

Faun. p. 215. n. 189. Gmel. Syst. 1. p. 765. Briss.

I. p. $247 . \mathrm{n}, 14$.

Perdix coturnix, Lath. Ind. Onin. II. p. 65 I. n. 28.

In Caille, Buff. Ois. II. p. 449. t. I6. PI. enl. I70

Vers. Germ. Martini VI. p. 79. cum fig.

The Ouail, Pen. Brit. Zool. 1. 1. 276. n. 97. Arct. Zool.

II. p. 320. B. Lath. Syn. IV. p. 779: n. 24. Suip.

p. 222. Vers. Germ. Bechst. IV. p. 735. n. 24.

Das kleine Foldhuhn oder die Hachtel, Bechst. N. D. 2te Aufl. 3. B. p. 1402.

Suecis: Wacktel.

DESCRTP TIO.

Longit. avis circa 7. poll. Nix extensæ 14 poll. Rostr. $\frac{3}{8}$ poll. Cauda $1 \frac{\pi}{2}$ poll. Tibia $1 \frac{7}{8}$; Digit, med. I. poll. Digit. post. $\frac{2}{8}$ poll.

*) Ante aliquot annos in Scania campestri frequentissima cıt. Anni vero 1814 gelida et nivosa hyems plurinam pareams exstirpabat, ita ut vix solitaria hinc inde jam obveniar. Hanc vero calamitatem in Scania campestri habitantes solx acceperunt. In Uplandia enim plurimas vidi greges astate nuper precerlapsa (1815). Inter frutices scilicer, quibus in Scania campestri carebant, victum et contra vim vento. rum præsidium obinebant. 
Rostum migricans. Nares rime longitudinales, membrana fornicata inflata teet $x$. Iris olivaceo-tusca. Pedes pallide incarnati calcare carent.

Caput supra tegitur pennis nigricantibus ferugineo late marginatis. A naribus supra oculos ad nucham stria lutes albida, postice latior, alia a fronte per medium verticem. Latera copitis albida rufo maculata. Cervix et dorsum, latera uropygii et tectrices caudz, maculis nigro-fuscis et ferrugineis, striisque longitudinalibus albidis vel albo-ferrugineis. Uropygium medium nigrum lineis transversis ferrugineis. Gula media nigro-fusca striis castancis binis cincta, quarum altera ex basi rostri, altera ex auribus descendit. Collum antice et pectus pallide ferruginea, lineola longitudinali albida in singula penna. Abdomen sordide albidum. Crissum et rectrices caudz inferiores ferrugineo-alba. Latera castanea stria lata longitudinali albida nigro tenuiter marginata in singula penna. Tectrices alarum rufescenti.g; isex; minores lineolis longitudinalibus albidis; majores striis transversis feruginescentibus. Remiges griseo-fuscre, striis transversis ferrnginescentibus. Tectrices inferiores ferruginescenti-albx. Rectrices XIV. deorsum curvatz fuscr striis transversis 4.s. 5. rachibusque ferruginescenti-albis. Femina: Gula albida; Pectore maculis nigricantibus; striis dorsi longitudinalibus pallide ferrugineis.

\section{HISTORIA.}

Migratoria avis habitat in agr.is Scaniz \&c. Inter se. getes xstate proditur sono singulari: Pickvervick-Pick: wrvick! Vescitur seminibus, satis, insectis. 


\section{Corrigenda:}

Pag. 9. Lin. 0. lege: Brit. Zool, II. App. g. 623. Art. Zool. II. p. $300 \cdot$ ก. 92 .

- 9. -19.1 . p. 200.13 .93$.

- 9. - 28. ndie: Digiti lutei.

- 10. - 18. lege: Norrlandia.

- 27. - 39. et lege: ex.

- 28. - 28. lege: Musvag.

- 28. - 29. I. p. 88. ad aniecedentem lineam pertizet.

- 3i. - j. leg. varietatem C.

- 33. - 3. sopra deleatur.

- 62. Synonymis adde: Strix Strisula, Tengm, ver. Ac. H. 1783 . P. $47 \%$

- 63. $-\times 5$. lege: cui,

- 70. - 20. 1. Nosten.

- 85. - 3.1. dimigrant.

- 90. - ro. 1. Caryocatades guttatss.

- 94. - post descriptionent Mavis, addes Femina vire scens ubi Mas fulvus, et fusca ubi ille niger.

- 118. - 12.1. Anatomica.

- 155. - $\tau \cdot$ leg. Cel. Meyer descripsit avem, qua ni fallor \&C.

$-177 .-26.1$. At vero.

-203. - 27. *) deleatur; Nota enim infra posita, ad ante= cedentem paginam pertinet.

-219. - 8. "Cauda rotundata", deleatur.

- 247. - 30 lege: gramina.

-256. - 8. 1. Sänglärka.

$-282 .-30$. lege: frigus.

- 300. - 25. 1, Buff.

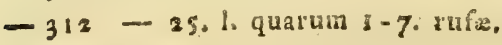

Cetera errata leviora ipse corrigat $\bar{B}$. Lector.

TYPIS H. F. POP P. 


\section{ORNITHOLOGIA}

S V E C I C A.

AUCTORE

\section{SV. NILSSON,}

PHILOSOPHITE DOCTORE,

IN ACAD. IUNDENSI ADJUNCTO E'T MUSEI RER, NATURAL. PRAFECTO; SOCIET. PHYSIOGR, LUND MEMBRO;

SOCIETATUM NAT. SCRUTATORUM WETTERAV.

ET MARPURG, GORRESPOND.

\section{PARS POSTERIOR.}

CuM II TABULIS ENEIS PICTIS.

\section{HAVNI E, M C CCXXI.}

APUD F. H. SCHUBOTHIUM, AULIE REGIJ BIBLIOPOLAM, 


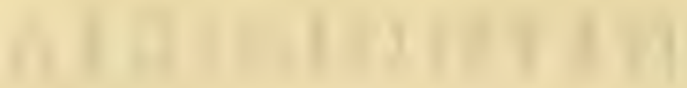

.

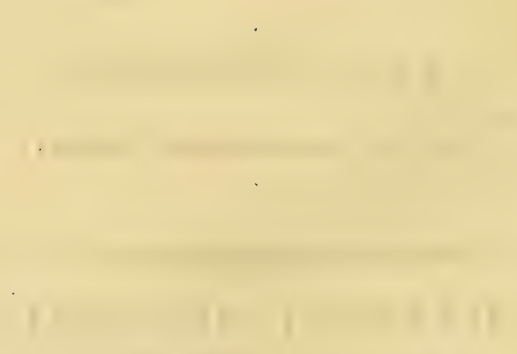


VIRO EXCELLENTISSIMO, GENEROSISSIMO,

\section{JOH. GABR. COMITI DE}

\section{TROLLE WACHTMEISTER,}

I REGNI PROCERIBUS UNI, A. H. REGIS REGNIQUE SVECIA JUSTITIE CANCELLARIO,

REGII ORDINIS DE STELLA POLARI COMMENDATOR. 

Cum in secunda quoque hujus operis parte elaboranda neque Tuis cohortationibus nec consiliis nobis defuisti, Vir Excellencissime! blanda illa spe ducimur, fore, ut æquo animo mentem interpreteris, quod hanc etiam partem, omni, qua par est, devotione, in tesseram grati animi Tibi dedicemus.

Permansurus, dum vixero, Excellentissimi Tui nominis

cultor devotissimus Sv. Nilsson. 
: 1 n 1 +

,

.

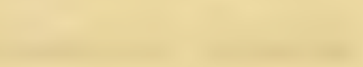

$\therefore: i$ 


\section{PREA T I O.}

Tandem, benevole Lector! ultimum Tibi Ornithologice Svecica Fasciculum; quique totius operis complexum claudit, tradimus, non quale voluimus, sed quantum pro virili in hac nostra rerum ratione prestare potuimus. Neque id fausti nobis atque multis Naturæ scrutatoribus contigit, ut quidquid ex ipsa Natura hausimus, id novissimis optimisque libris et elegantissimis iconibus comparare possemus.

Qui enim degit literasque is in terris sequitur, ubi omne literarum genus resque libraria floret, haud facile animo concipiet, quibus quantisque impedimentis ille literarum cultor 


\section{VIII}

in iis locis prematur; ubi penuria bibliothecarum et commercii literarii requo passu illi obstent.

Diutius mihi omnino ignoti fuerunt et novissimi et probatissimæ nota Scriptores Ornithologici; neque enim longiori tempore alii mihi suppeditarunt Auctores, cum quibus ipsam. Naluram conferrem, præter Linnæum, Pennantium et denique Bechsteinium, et quædam pauca aliorum scripta. Hinc plura pro novis habui, quæ meliora edoctus, jam aliis cognita accepi; hinc Naturæ arcana ea diligentia detegere non potui, qua, ducibus optimes notæ Autoribus, fieri potuisset.

Qui igitur melius de hoc nostro instituto sentire voluerit, ille haud fastigium disciplinæ suspiciet, quo proximis abhinc annis in Germania, Hollandia, Gallia \&c. Ornithologia pervenit; (quod, quamvis magnificum, in usum vertere non potui, quippe incognitum) sed omnia ad scientia statum, qualis mihi scribenti in Svecia obvenit, referre debet. 
Hac ctiam comparatio facta fuit; neque est quod diffitear, me plurima honesto illi Critico (et nomine mihi incognito) debere, qui non minus humaniter quam modeste priorem Operis partem in Annalibus Fleidelbergensium (anno 1818. Mcns. Junio. pag. 620 et sequ.) recensuit. - Persuasum milhi est, et istum, et alios integros judices facile esse reperturos, partem quoque alteram (si difficultatem rei memineris), non minori cura, sed majori sane experientia, esse elaboratam. Nam per quinquennium illud, quod, absoluta jam priori operis parte, effluxit, nihil neque operæ neque sumtus omisi, ut eam cognitionem perciperem, qua ad opus augendum quodammodo faceret. Copiam plurium Auctorum nobis paravimus, qui in priori parte conscribenda nos effugerunt; Musea plurima Patriæ ornithologica, et majora et minora, revisimus; insuper plurimis australis Sreciae provinciis peragratis, anno $x 816$, regiones peninsulæ Scandinavica areicas, prescrtim maritimas, visi- 
tavimus, ut aves boreales; suo quamque in domicilio cognosceremus.

Quod de avibus terrestribus in hoc iti-i nere notavimus, priori parti inserere ideo non licuit, quia, nobis redeuntibus, sub prelo jam desudaverat. Nam non nisi plagulam, quæ Lagopos pertractat iterum imprimendam curavi, quia peropportune mihi accidit, ut per omnem astatem has avium species, hucusque parum aut confuse cognitas, in jugis Septentrionalium sylvisque pernoscerem. Nonnullas alias, quas in aves terrestres redintegravimus observationes, in supplementum partis posterioris retulirnus; in qua etiam parte, in usum nostrum ea convertimus, que in hoc itinere de avibus aquaticis animadvertimus.

Quam operis rationem a principio nobis proposuimus, eam assidue persecuti fuimus. Ipsum enim instituti consilium hoc fuit, ut descriptionibus absolutis et Naturæ convenienter factis, eas avium species, quae in Srecia degunt, definiremus, atque ut certis synony- 
mis eas determinaremus aves, quas Linné ceterique Patria Ornithologi descripserunt. Quod nos etiam adsecutos fuisse speramus.

Quod ad species Linneanas determinandas adtinet, eosdem quoque fontes perscrutati fuimus, ex quibus Linné cognitionem hausit plurimarum avium, quarum in Fauna Svecica meminit. Nam si breves istas Linnæi descriptiones tantummodo consulueris, haud facile unaquæque avium species dignoscetur, quod longe aliter est in fontibus, quos laudavimus; hi vero sunt Rudbeckii aves pictoe et Lechei opus manuscriptum. Illæe constant figuris avium, vivo colore depictis et plerumque naturali magnitudine et præstanti arte, ut facile dignosci possint. His igitur ducibus figuris, quæ a Linnæo sæpius citantur, haud raro frcilius est videre, quammam avem ille intellctam voluerit, quam ex ipsis illius descriptionibus. In hoc ipsum Rrudbeckii opus Indicem cum commentariis criticis contexuimus, 
Aatis Acad. Scient. Holmiens. 1816. p. $21 \&$ 1817. p. 128. insertis.

Mauuscripta vero Lechiana multum lucis Faunæ Linneanæ sæpius adcommodant. Leche enim, qui eodem cum Linnæo tempore vixit; Ornithologia studium, auspice natura, sectabatur. Plura quoque Linnæo subsidia in Fau-: nam ejus adtulit, qux tamen hic iste, amicus brevitatis studiosissimus, nonnunquam ita amputabat, ut æegrius dignoscantur.

Exemplaria etian descriptionum et iconum ex plurimis avibus, quas ceteri nostrates reliquerunt, videre mihi licuit in Museis nostris adhuc servata.

Lucubrationes antecessorum ita adhibuimus, ut suum cuique tribueremus; quos vero errores offendimus, eos ingenue et uti literatum decet, sine ira et studio adnotavimus.

Sed risi plures mihi Fautores adfuissent; hic liber ne illa quidem rerum copia et perfelione, qua nunc gaudet, prodiret; in quibus primum sibi locum vindicat Vir ille Excel- 
lentissimus; idemque Naturæ scrutator sagacissimus, cui opus nostrum devotissime dedicavinus.

Quod vero maximis in Patria Museis uti mihi contigit, præter alios acceptum debeo Illustrissimo G. à PAYKULL, Libero Baroni, Aulæ Mareschallo et Equiti aurato; C. P. Thuneerg, Professori Upsaliensi et Commendatori fulgentissimo, et beatæ memoriæ O. Stwarz, Professori Holmiensi et Equiii aurato, qui singuli, pro liberalitate illa, qua hujus seculi vere literatos literarumque patronos dignosces, aditum ad suas in hac disciplina collectiones mihi libenter fecerunt, quo uno loco congesta ea exemplaria comparare potuimus, quæ in Natura non nisi separata deprehenderis.

Initio equidem historiam succinctam de fatis studii ornithologici in Svecia scribere constitui; re vero accuratius pensitata, mihi persuasum est, hoc modo magnitudinem libri; in nullum ipsius disciplinæ commodum, auc- 
tum iri. Pauci omnino in Svecia fuerunt, qui studio ornithologico ex Natura hauriendo unice incumberent; sed plurimi non nisi horas Botanicæ vel Entomologiæe surreptas huic nostræ scientiæ consecrarunt. Hinc plus justo aucta nominum congeries et synonymorum confusio necesse oriretur. Quare speravimus fore, ut nostræ cujuslibet avium speciei descriptiones, quantum fieri potuit exacta et absoluta, non modo non superfluæ, sed potius in id conferre videantur, it nova avium Syecicarum nomina fingendi libido quodammodo retineatur; quod si factum fuerit, neque nos frustra operam consunsisse putabimus.

Scribebam in Museo Academico Lundensi, die 6to Decembris 1820. 
OR N I T H O L O G I A S V E C I C A.

BARTIS POSTERIORIS

FASCICULUS PRIOR. 
TYYIS HARTV. FRIDR. POPP. 


\section{A VES SVECIA}

DIV. II. A QUATICAF.

\section{ORDO. GRALLIPEDES}

\section{SUBORDO I. CURSORES. (TRIDACTYLI)。} SECTIO I.

DIGITIS MEMBRANA AD BASIN JUNCTIS.

\section{G E N US. O T I S.}

$\boldsymbol{R}_{\text {ostrum breve, reltum; subconicum, compres- }}$ sum, maxilla superiore fornicata, apice subinHexo.

Nares ovatæ, prope se invicem sitæ.

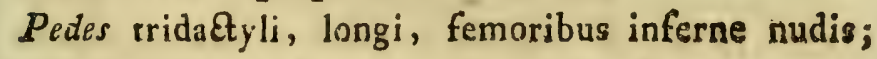
digitis juxta basin membrana junßtis.

\section{OTIS TARDA, Lim.}

Diagn: Corpus supra ferrugineum fasciis. er undulis nigris. Collum antice cinereum. Nias: mandibula inferioı $i$ urrinque mystacibus penna. tis ornata. 


\section{SYNONYMA.}

Otis tarda, Retz. Fin. Sv. p. 203. n. I78. Lin. Syst. 1.

p. 264. n. I. Gmel. Syst. I. p. 722. Lath. Ind.

II. p. 658. n. I. Briss. orn. V. p. I8. n. I.

Outarde, Buff. Ois. II. p. I t. I. vers. Germ. Martini

IV. p. 5. cum fig. Pl. enl. 245 (mas).

Great Bustard, Pen. Brit. Zool. I. n. 98. t. 44. Arct.

Zool. II. p. 32 I. id. Sup. p. 63. Lath. Syn. IV.

p. 796. vers. Germ. IV. p. 75I. n. I.

Der grosse Trappe, Bechst. Nat. D. 2te Auf. 3. B. p. I432. Meyer Vögel Liv. u. Esthl. p. I68.

Svecis: Trapp, Stortrapp.

\section{DESCR I P T IO.}

Inter maximas Sveciæ aves; major Gallopavone. Mas circa $3 \frac{r}{2}$ pedes long. et alæ ejus extensa 6 . ped.

Rostrum griseo-fuscum. Iris aurantia. Sub lingua foramen in saccum gularem pro aqua ducens *). Pedes sordide grisei. $M_{i s}$ ad latera maxillz inferioris utrinque mystaces pennaceas, longas (circa 5 pollicares), cinereo-albas gerit, quibus Femina caret.

Caput, collum et pectus pallide cinerea. Dorsum ferrugineum fasciis confertis nigris. Scapulares et tectrices alarum minores ferrigineo-lutex, undulis nigris. Abdomen, crissum et tectrices alarum majores albx. Remiges primores nigra, medix albx, intima colore dorsi. Rectrices XX. ferruginer, fasciis maculisque nigris, et apieibus luteo-albis. Plume omnes et apices Scaporum pulcherrime rosei.

*) Hunc Saccum descriptum et delineatum invenies in Schrift, der Berl, Gesellsch. Naturforsch. Fr. 1II. p. 376. t. 8. f. 7. a 
Femina minor, mystacibus destituta. Gula et latera capitis brunnea, collum antice cinereum. Vertex et Cervix dorso concolores; coloribus minus quam in Mare vividis.

\section{HISTORIA.}

Avis rarior, migratoria, ad nos redit mense Aprili. In campis Scanix arenosis et tesquis depressis xstatem degit, et per paria obvenit. Ova 2-3. pallide olivacea maculis fuscis ornata, in cavitate humi fovet. Finita xstate per farnilias abit. Valde timida est. Canibus Grajis interdum deprehenditur, cum propter molem corporis et brevitatem alarum non nisi prævio cursu in aërem se levare possit. Vescitur granis, oleribus, Insectis et Lumbricis. Caro, prasertim junio. rum, sapida.

\section{OTIS TETRAX, Linn.}

DIAGn: Corpus supra e ferrugineo et nigro varie. gatum; Capite colloque lavi. Mas: collum nigrum torque duplici, albo; Femina: collum colore dorsi, torque destitutum.

\section{SYNONYMA.}

Otis Tetrax, Lin. Fn. Sv. p. 70. 11. 196. Retz. Fn. P. 204. n. 179. Lin. Syst. Nat. I. p. 264. 13. 3. Gmel. Syst. nat. I. p. 723. Lath. Ind. II. p. 659. i1. 3 .

Otis minor, Briss. Orn. V. p. 24. 2. t. 2. f. I. 2. Fetite Outarde, Buff. Ois. II. p. 40. Pl. enl. t. 25. (mas.) IO. (fem.) Ver's. Germ, Martini IV. p. $4 \mathrm{~S}$. f. $80.8 \mathrm{I}$. 
Little Bustard, Pen. Br. Zool. I. 11. 99. Arê. Zool. II. p. 32 I. A. Lath. Syn. IV. p. 759. id. Sup. I. p. . 226. vers. Germ. IV. p. 703.

Der Kleine Trappe, Bechst. N. D. 2te Auf. 3. B. p. I446. Meyer Vögel Liv. u. Esthl. p. I69.

Svecis: Småtrapp.

\section{DESCRIPTIO.}

Magnitudo fere Tetroonis Tetricis; mas I8. poll. long. Rostr. I. poll. A flexura alæ ad apieem I2 poll. Tibia $2 \frac{6}{8}$ poll. Digit. med. I $\frac{x}{2}$ poll. Cauda 4. poll.

Rostrum griseo-fuscum, gallinacenm, maxilla superiore subfornicats, marginibus inflexis. Nares ovatz in dorso rostri sita. Pedes lutescenti-grisei. Caput et colluin lavia.

Caput supra nigrum striis ferrugineis. Tempora, genx et gula zufescenti-alba maculis minutis nigris. Collum nigrum torque albo, duplici, antice decurrente. Dorsum, Scapulares et teetrices alarum ex nigro et ferrugineo transversim variegata et undulata. Corpus subtus marginesque alaxum alba. Remiges primores apice nigra, basi albx; secundarix albx; intimx dorso concolores. Rectrices XVIII, quarum medix quattuor ferruginex, ceter albx, omnes irregulariter nigro fasciatz.

Mas junior: Seniori similis, præter collum, quod in hoc ferruginescens striis nigricantibus.

Femina: minor: I5 poll. longa; Collum colore dorsi, torque albo destitutum. Peetus superius et latera ejusdem ferrugineo-albida maculis transversis nigris Gula et jugulum albida immaculata. Dorsum tenuius quam inMare variegatum. Præterea mari fere concolor. 


\section{HIS TORIA.}

Inter rarissimas patrix aves. Habitat in arvis campisque sterilibus et lapidosis. Prope Ystad Seanize quotannis obvenit, seque ibidem propagat. Inter aves Fennicas quoque numeratur (Faun. Fenn. p. 27.) Polygsmiam amat. In cavitate agri ponit Ova 3-5 nitidiviridia. Vescitur granis, seminibus, insectis, vermibur. 


\section{GENUS. \\ CHAR A D I US.}

Rostrum capite brevius, subulatum, tenue, reerum, subteres, pone apicem compresso-incrassatum.

Nares lineares.

Pedes tridalyli, digito exteriori intermedio mem. brana juxta basin connexo.

Caput crassum fronte elevata.

CXLV.CHARADRIUS APRICARIUS, Lin.

Diags: Supra nigricans maculis fulvis et albidis。 Cauda fusca lineis transversis lutescentibus.

\section{SYNONYMA。}

Charadrius campestris, Nilss. Anal. Orn. Lund 1814. p. 3I. n. I8.

- euratus, Bechst. N. D. 2te Attf. 4. B. I Abth. p. 395. Meyer Vögel Liv. u. Esthlo p. I73.

Avis habitu astivali:

Charadrius apricarius, Lin. Fn. Sv. p. 67. n. I89. Lin. Syst. Nat, 1. p. 254. n. 6. Retz. Fn. p. 194. n. I67. Lath. Ind. Orn. II. p. 742. n. 5. Gmel. Syst. I. p. 687. Fabr. Fn. Groenl, n. 79. 
Pluvialis auren freti Hudsonis, Briss. Orn. V. p. 5I. 4. Le Pluvier dore à gorge noire, Buff. Ois. VIII. p. 85. vers. Germ. Otto XXVIII. p. 90-94.

Spatted Plower, Edw. III. t. I40.

Alwargrim Plover, Pen، Ar\&. Zool. II. 11. 398. id. Sup. p. 69. Lath. Syn. V. p. 198. 5. vers. Germ. V.p. I72.

Avis habitu autumnali:

Charadrizs pluvialis, Lin. Fn. Sv. p. 68. n. I90. Syst. nat. I. p. 254. n. 7. Retzii Fn. Sv. p. 195. n. I68. Gmel. Syst. I. p. 688. Briss. Orn. V. p. 43. I. t. 4. f. I. Lath. Ind. II. p. 740.

Pluvialis aurea minor, Eriss. Onn. V. p. 47. n. 2.

Pluvier doré, Buff. Ois. VIII. p. 81. Pl. enl. 904. vers. Germ. Otto XXVIII. p. 75.

Golden or green Plover, Pen. Br. Zool. II. p. 474. n. 208. t. 72. - Arct. Zool. II. n. 399. Lath. Syn. V. p. I93. I. vers. Germ. V. p. 167.

Scanis: Ljungwipa, Brockfogel; Hallandis: Lyngspole; Smolandis: Ljungspole, Myrpytta; Oelandis: Alwargrim. Dalekarlis: Åkerhöns. Lappis: Hutti.

\section{DESCRIPTIO.}

Longit. II - I I $\frac{\pi}{2}$ poll. Alx extensx 2 ped. A flexura alæ ad apicem $7 \frac{x}{2}$ poll. Tibir I $\frac{s}{3}$ poll. Digit. med. I $\frac{2}{8}$ poll.

Rostrum postice subteres, nigro-fuscum, antice nigrum, compressum marginibus inflexis; pone apicem superne et subtus aliquantum crassius. Nares lineares. Oculi magni, prominentes, nigri Iridibus 
fuscis. Pedes fusco-cinerei, digito postico semper destituti *):

Avịs habitu estivali:

Vertex, cervix, dorsum et Scapulares nigro-fusca vel nigra, maculis fulvis vel aurantiis et albidis pulcherrime adspersa. Tectrices alarum fuscx maculis dilutioribus (luteo-albidis). Frons alba dimittit fasciam albam utrinque supra oculos ad latera cervicis, juguli et pectoris. In pectore superiore fere concurrit, iterumque in hypochondris discurrit. Quas includit partes, basis rostri, genæ, jugulum, petus, abdo. men et crissum, nigra sunt, pennis pluribus vel paucioribus albis (in abdomine et crisso) insertis. Gula interdum albo, et genx luteo inmixtx. Remiges nigro-fuscz basi albe; primores acuminatz latere interiore ad medium albe, rachibus a medio versus apicem albis; secundarix obtusiores, apice albidx; intimx 3 - 4. longx, colore dorsi. Tectrices proxima Remigum primorum nigricantes apice albx. Tectrices inferiores albx, ad basin alx fusco nebulosæ. Rectrices nigro-fusca, lineis transversis ofto ad raches interruptis, fulvis, in lateralibus, albidis.

Femina: Mari similis, coloribus tamen minus vividis. Scil. Fascia alba, qux a fronte ad latera colli et pecto. ris decurrit sordidior, nec tam splendide alba. Col. lum antice, et corpus subtrs plures habet pennas albas immixtas.

Avis habitu autumnali :

Supra nigro-fusca maculis luteis vel aurantiis pulcherrime adspersa. Facies sordide alba, maculis mi-

7) Qui Omithologi doeuerunt hane speciem ungue postico brevi interdum esse instructam; avem, ni fallor. cum $V$ tho nelio melauogastro, cui simillina est, confuderant. 
nutissimis fuscis. Latera capitis et colli lutea, fusco nebulosa. Gula alba. Jugulum ez superior pars pectoris dilute griseo-fusca, luteo immixto. Reliquum pectus, abdomen et crissum alba. In ceteris prius descriptx similis.

\section{OBSERVATIO.}

Charadrium Apricarium et pluvialem Lin. unam eandemque constituere speciem, inter omnes jar constat. Ut argumentum vlterius, si necèssarium judicaretur, adferie possuın, me examinasse iconem Rudbeckianam, quam in Fauna Sv. citavit Linné; hanc vero iconem, xque ac figuram Edwardii allatam, avem nostram pennis astivalibus indutam perfe?te referre. Oelandorum Alvargrim eadem esr avis. Muratio autem colorum his fere stadiis peragitur: Mensibus Junii et Julii cun per paria juxta nidos vel pullos obveniunt aves, nigredo inferiorum corporis partinm maxime explieata est. Ab hoc tempore deplumantur aves et sensim hiemalem indunt colorem, ita ut singulx senio. res junioresque, quæ ad mens. Saptemb, et initium m. OAtobris remanentes occurrunt, sibi similes sint et Char, pluvialem Aucior. referant. Hyeme oras nostras relinquunt. Primo vere redeuntes, nigredine juguli, pøeforis et abdominis minus explicata ornatx sunt; imo vero occurrunt (presertim feminæ) qua hoc anni tempore, omni nigrédine in partibus allatis carere videantur. At qui pennas petoris et abdominis sejungit, videbit novas pennas vel niveas vel nigras e vaginis erumpentes, re vera adesse, quamvis in adspectum nondun pervenissent. His - wutem pennx, hiemali veste sensin posita, magis magisque ex. plicantur, avemque incubationis tempore, ut supra dixi, presertim ornant.

\section{HISTORIA:}

Migratoria avis jam in medio Mens. Aprilis redux regiones nostras visitat. Passim frequens. Esstate to. tam incolit Scandinaviam, non minus ericeta et tesqus Scanix, quam alpes Lapponix, in quibus usque supre terminum vegetationis arborex frequens per paris obve- 
nit et voce sibilante et sonora Hyyt! vel e longinquo se prodit. vescitur insectis et vermiculis. Ividulatur supra terram. Ova 3-5. olivacea, nigro maculata. Pullos volatui aptos jam medio Julio inveni. Ab hoc usque tempore dimigrationes versus terras australiores instituuntur. Post medium m. Augustum, et toto Septembri innumeris fere gregibus sociatx in campis et agris Scanix occurrunt. Sensim abeunt. Manent ronnulla ad frigus usque rigidum. Terra vero nive obruta, hx quoque oris nostris valedicunt.

\section{CHARADRIUS MORINEL- LUS, Lin.}

Diagn: Supra griseo-fuscus marginibus pennarum ferrugineis; pileus nigricans fascia alba cinctus; pectus ferrugineum fascia transversa alba.

\section{SYNONYMA.}

Charadrius Morinellus, Lin. Fn. Sv. p. 67. n. 188. id. edit. prim. I60. (junior). Retz. Fn. 194. n. I66. Gmel. Syst. I. p. 686. n. 5. Lath. Ind. II. P. 746. n. 17. Briss. Om. V. p. 54. 5. t. 4. f. 2.

- tataricus, Pall. Reis. II. p. 715. 11. 32.

- Sibiricus, Lepechin Reis. II. p. 185. t. 6.

Petit Pluvier ou Guignavd, Buff. Ois. VIll. p. .87. Pl. enl. 832. vers. Germ. Otto XXVIII. p. IOI. \&c. eum fig.

Dottrel, Pen. Br. Zool. II. p. 47\%. 1. 2 IO. Lath. Syn.

V. p. 208. vers. Germ. V. p. 182. 11. I4.

Der dumme Regenpfeifer, Bechst. N. D. 2te Auß. 4. B.

I. Abth, p. 406.

Svecis: Pomeransfogel. 


\section{DESCRIPTIO.}

Femint senior habitu veraali: Long. IO poll. Alx extensx I9 $\frac{x}{2}$ poll. Rostr. $\frac{5}{8}$ poll. A flexura alx ad apicem 6 poll. Cauda $2 \frac{T}{2}$ poll. Tibia $I \frac{T}{2}$; Digit. med. I poll.

Rostrum teretiusculum, nigrum, apice acuto. Nares lineares. Oculi magni iridibus fuscis. Pedes olivacei. Digitus medius exteriori ad primam articula. tionem membrana junetus.

Caput supra nigricans maculis minutis et striis griseis in anteriore ejus parte adspersis. Fascia pure alba supra oculos utrinque ad nuchan ducta. Lora nigricantia. Latera capitis infra oculos et gula alba. Corpus et alæ supra griseo-fusca, pennis scapularibus et alarum tectricibus ferrugineo marginatis. Collum antice saturate cinereum terminatur fascia pectorali transversa alba, nigricante superne marginata. PPectus et Hypochondria saturate ferruginea. Abdomen nigrum, postice et crissum femoraque alba. Remiges griseo-fusc:e, margine interiore a basi pallide cinerex; prima rachis alba, qui color extra rachin longitudinaliter parum extenditur. Intima tres longitudine fere primr, colore dorsi. Rectrices fusco-cinerex, versus apicem nigricantes, albo terminat:; exteriores quoque margine exteriore albæ. Tectrices alarum inferiores albx.

Mas habitu vernali: Long. $9 \frac{x}{2}$ poll. Caput suprenigricans marginibus pennarum ferrugineis. Fascia alba supra oculos ad nucham ducta, postice latior. Latera capitis infra oculos et gula albida striis fuscis minutis adspersa, Corpus et alx supra griseo-fusea rnarginibus pennarum ferrugineis. Collum antice sordide fusco-cinereum, undulis obsoletis rufis. Fascie su. 
pra pectus obsoleta albida, nigro superne marginats. Peetus ét hypochondria ferruginea albo immixto. Abdomen medium nigrum, maculis albis. Cet. ut in snteced.

Funior avis: Caput et dorsum supra nigricantia marginibus pallide rufis. Frons sordide albida maculis minutis fuscis: Fascia supra oculos et latera colli sordide rufescentia. Gula albida. Juguium sordide cinereum rufescente tinctum. Fascia transversa dilutior in pectore vix conspicua. Pectus dilute ferrugineum. Ab. domen album. Crissum albo-rufescens.

Senior avis habicu autumnali: Caput supra nigricans marginibus pennarum rufescentibus. Dorsum tegitur pennis partim fuscis marginibus subferrugineis, partim griseis marginibus pallidioribus. Facies albicans maculis minutis fuscis. Supra oculos versus nucham fascia sordide alba; infra hanc et pone oculum macula fusca. Gula alba. Jugulum cinereum fusco et rufescenti immixto. Ad basin sterni fascia transversa albida, nigro abrupte marginata. Pone hanc fasciam pectus est magis ferrugineum. Abdomen medium nigrum, pennis albis insertis. Crissum et tectrices cau. $d x$ inferiores albo-rufescentia.

\section{O BSER VATIO.}

Hrec Species eum aliis quibusdam ex ordine Grallipedum id cormunue habet, quod sexus feinininus major sit et uagis coloracus quam masculinus. Hoc non observarunt ornithologi, guare Feminaw pro Mare, et vice versa, descripserunt.

\section{HIS TORIA.}

Septemtrionales alpes æstate incolens, Gothiam verno et antumnali tempore transmigrat. Mensibus Aprili et Majo in arvis et campis Scanix per familias, 
rarius tamen, occurrit; inter initia mens. Junii in tesquis alpinis Dovrefjeld, supra terminum vegetationis arboreæ avem per paria observavi. Propagandi mo. dus et oeconomia zstiralis nondam satis nota sunt. Redux m. Angusto et Septembri, Seaniam iterum visi. tat aut sola aut cum Char. apricario associata.

\section{CHARADRIUS HIATICULA,}

\section{Lin.}

Diagn: Supra fusco-griseus, linea alarum obliqua, Frons, collare et corpus subtus alba. Fascia nigra, antice latior collum inferius cingens. Remiges plurima in medio longirudinaliter albx. Rostrum aurantium, apice nigro. Pedes lutei.

\section{SYNONYMA.}

Charadrius s. Hiaticula favipes, Rudb, av. pietz fol. 188.

Charadrius Hiaticsla, Lin. Fn. Sv. p. 66. n. 187. Retz. Fn. Sv. p. 193. n, I65. Lin. Syst. I. p. 743. n, 8. Gmel. Syst. I. p. 683. Lath. Ind. II. 743. n. 8. Le Plavier à collier, Buff. Ois. VIII. p. 60. t. 6. Pl. enl. 920, vers. Germ. Otto. XVIII. p. II7. et 129. cum fig.

The ringed Plower, Pen. Br. Zool. II. p. 479. n. 2 I $\mathrm{Y}$. Arct. Zool. II. p. 485. n. 401. Lath. Syn. V. p. 20I. n. 8. vers. Germ. V. p. I76.

Der buntschä̈blige Regenpfeifer, Bechst. Nat. D. 2te Auf. 4 B. I. Abth. p. 4 I4.

Halshand - Regenpfeifer, Meyer Vögel Liv. u. Esthl. p. 175.

Suecis: Strandpipare; Scanis: Sandrulling; ad Skanör Gryll. 


\section{DESCRIPTIO.}

Long. $7 \frac{1}{2}$ poll. Alz extens $x$ I $6 \frac{2}{8}$; A flexum alx ad apicem $5 \frac{2}{8} ;$ Rostr. $\frac{\frac{7}{8}}{8}$; Caud $2 \frac{1}{2}$ poll. Tibia $1 \frac{\frac{\pi}{8}}{3} ;$ Digit. med. $\frac{7}{8}$ poll.

Rostrum basi latius, ultra medium aurantium apice nigrum. Margo palpebrarum aurantius. Iris fusca. Cauda subrotundata cum alis xqualiter extenditur. Pedes lutei. Digitus med. basi adnexus extimo mem. branâ ad primam articulationem.

Supra basin rostri fascia inter oculos nivea; supra hanc faseia latior nigra. A basi rostri infra oculos ad tempora fascia nigra postice latior. Pone oculos macula alba. Gula et superior juguli pars nivea fasciam concolorem, postice angustiorem circa collum dimittit. Infra hanc fascia nigra, antice pollicaris, postice multo angustior, collum cingit. Prrterea tota avis subtus sivea. Vertex, occiput, dorsum et supcriores tearices fusco-grisea. Remiges nigro fusca, latere interiore a basi albida; primores I-4. rachi media alba; sequentes in medio juxta rachis latus exterius maculâ longitudinali alba, qux albedo ita in sequentibus augetur, ut 19. 20. 2I. plane sint albx. Intimx tres longitudine tertix, colore dorsi. Tectrices proxima remigum primorum nigricantes; remigum secund. fuscr, apicibus albis, unde linea obliqua alba in ala complicata oritur. Rectrices extimx, albx, interiore latere (interdum) macula fusca notatx; 2. 2-3 3. grisex, versus apicem nigricantes, apice et margine exteriore albæ; 4. 4-5.5. basi grisex, versus apicem nigricantes apice albx; 6.6 . immaculatz extus nigricontes. In Femina color niger non tam ater est, sed griseo intermixtus, nec fascia colli nigra tam lata. 


\section{HISTORIA.}

Migratoria avis post medium mens. Martis litora marina Scanix visitat. Per æstatem in litoribus tun Scanix tum regionum areticarum obvenit; frequentius vero in illis. Etiam Lacus frequentat. Celeriter currens sono sibilante thyk, thyk! se prodit et ab insequenti specie, vel eminus dignoscitur. Mense Aprili se propagat. Ova 4-5. altera extremitate acuminata, pallide lutescentia, punctis et maculis minutis nigris in arena ponit. Mense Octobri litora nostra relinquit, regiones australiores petitura. Caro sapida.

\section{CHARADRIUS MINOR,}

\section{Meyeri.}

DIAGN: Supra fusco-griseus; frons, collare et corpus subtus alba. Fascia nigra antice latior collum inferius cingens. Remiges immaculatæ. Rostrum gracile nigrum; pedes flavescenti-incarnati.

\section{SYNONYMA.}

Charadrius fulvipes, Rudbeckii aves pictx fol. I89*). Charadrius minor, Meyer Vögel Liv. u. Esthl. p. I76.

- curonicus, Beseke Nat. G. der Vögel Curlands p. 66. n. I34. Gmel. Syst. I. p. 692. n. 29. Lath. Ind. IL p. 750.

Charadritis fouviatilis, Bechst. N. D. 2te Auf. 4. B. I. Abth. p. 422.

*) Erat igitur hace avis jan Rudbechio nostro ut distindta species cognuta. 
Le petit pluvier à collier, Buff. Ois. VIII. p. 60. Pl. enl. 921 .

Der Alexandrinifche Sirandpfeifer, Otto in Buff. Vögel XXVIII. P. I34; cum fig.

\section{DESCRIPTIO.}

Præcedente, cui valde similis, miror; $6 \frac{\pi}{2}$ poll. long. Ala extensx I4; a flexura alx ad apicem $4 \frac{\pi}{4}$; Rostr. $\frac{\pi}{2}$ poll. Cauda $2 \frac{2}{8}$ poll. Tibiæ I. poll. Digit, med. $\frac{6}{B}$ poll.

Rostrum gracile totum nigrum; Iris fusca; margo palpebrarum aurantius. Cauda subrotundata, extra alas parum extenditur. Pedes fuscescenti- vel fiavescenti-incarnati. Digit. med. basi adnexus extimo membrana quoad primam articulationem.

Supra basin rostri fascia inter oculos alba; supra hanc fascia nigra. A sinu oris infra oculos ad tem. pora fascia nigra ducitur. Gula et superior juguli pars nivea fasciam albam postice angustiorem circa collum dimittit. Infra hanc fascia nigra, antice $\frac{6}{8}$ poll. lata, postice angustissima, collum cingit. Præterea rota avis subtus nivea. Vertex, occiput, dorsum et auperiores teetrices cinereo-fusca, vel porius rufescenti-grisea. Remiges primores fuscr, secundaria cinereo - fuscx, margine interiore a basi grisex. Prima tantum rachis alba, ceterx raches pennis concolores. Remiges 18-21. apice solum albidx. Intimx tres, longitudine fere primx, dorso concolores. Tectrices proxima Rem. prim. nigro - fuscx; secundar. fusco - cinerex, vix apice pallidx. Rectrices extimx albx latere interiore macula fusca notatx; 2.2-3.3. grisex, versus apicem nigricantes, apice et margine exteriore alba; 4. 4-5. 5. grisex versus apicem nigricantes, apice albz 6. 6. macula apıeis alba destitutio. 
Color Femina niger non tam ater est et nitens, sed griseus intermixtus, et fascia colli nigra angustior. In ceteris mari similis.

\section{HISOTRIA.}

Migratoria. Habitat astate passim in ripis fluviorum et lacuum arenosis, non mintas in inferiore quam superiore Svecia. Sono triste subsibilante: Kirw! - Kirzv! eminus quoque a præcedente dignoscitur. Ov\& 3-5 luteo-alba maculis nigris et fuscis ornata.

\section{CXLIX, CHA.RADRIUS ALBIFRONS,}

\section{Meyeri:}

DIAGN: Supra fusco-griseus. Frons, collare, collum antice et corpus subtus nivea. Rectrices binæ utrinque laterales albæ. Rostrum er pedes nigri.

\section{SYNONYMA.}

Charadrius littoralis, Bechst. Nat. D. 2te Auf. 4. B. I. Abth. p. 430.

- alexandrinus, Brün. orn. bor. app. p. 77.

\section{DESCRIPTIO.}

Longit. circiter 7 poll. A flexura alæ ad apicem $4 \frac{2}{8}$ poll. Cauda fere 2 poll. Rostr. $\frac{5}{8}$ poll. Tibia $I \frac{\pi}{3}$ poll. Digit. med. $\frac{\sigma}{8}$ poll.

Rostrum nigrum. Iris fusca. Palpebrarum margo luteus. Pedes coerulescenti-nigri.

Caput, Collum, dorsum et alarum tectrices fusco. grisea marginibus pennarum obsolete rufescentibus. Nilsson Ornith. Pars $I I$. 
Frons alba demittit fasciam albam utrinque supra oculos. Lora cinerea. Gula, jugulum et tota avis subtus nivea; collum superne cingitur torque albo, sub quo latera colli saturate cinerea sunt. Remiges primores latere exteriore et apice nigricantes, rachi prima tota nivea, ceterarum raches medio nivex. Remiges secundarix cinereo - fuscx marginibus et rachibus albis. Teetrices alarum proxina dorso concolores apice albis, unde fascia angustior alba in ala composita. Cauda equalis Rectricibus tamen binis intermediis aliquantum longioribus. I. I-2. 2. tota albx. 3. 3. alba, macula fusca juxta interiorem apicis marginem; 4. 4. pallide grisea, versus apicem fusco, apice albo marginata; 5. 5. eodem colore sed saturatius. 6 . 6 . saturate fusca immaculata.

\section{O BSERVATIO. I.}

Forte Fenizan est quam descripsi; avem enim vivam nonสำ vidi.

OBSERVATIO. 2.

Suspicor hanc avem inter Scanenses posse numerari, quamvis nondum, certa fide, in Scandinavia sit occisa. Quæ enim in Acitis Nidros. V. p. 153. tab. V. Charadrius Alexandrinus appellatur, potius, me judice, ad Calidvin grisean pertinet. 


\section{5 \\ GENUS. \\ H $\mathbb{E}$ M A T O P U S.}

$R_{\text {nstrum capire longius, robustiusculum, rectum, }}$ compressum, apice cunearo.

Nares lineares in sulco prope basin sitæ. $P_{e d e s}$ tridactyli, robusti, digitis fissis, exteriore intermedio membranâ basi connexo.

\section{H无MATOPUS OSTRALEGUS.}

Diagn: Niger,' corpore subtus, fascia alarum, dorso postico et caudæ medietate interiore albis.

\section{SYNONYMA.}

Hematopus Ostralegus, Lin. Faun. Sv. p. 69. 11. 192. Syst. Nat. I. p. 257. Retz. Fr. Sv. p. 197. n. I70. Lath. Ind. II. p. 752. Gmel. Syst. I. p. 694.

Ostralega s. Pica marina, Briss. Orn. V. p. 38. t. 3. f. 2. Hutitrier, Buff. Ois. VIII. p. I19. t. 9. P1. enl. 929.

Vers. Germ. Otto XXVIII. p 2 I4. cum fig.

Piel Oister-catcher, Pen. Br. Zool. II. p. 482. n. 2 I3. t. 74. Aret. Zool. II. p. 406. Lath. Syn. V. p. 2 I9. vers. Germ. Bechst. V. p. I93. t. 87 .

Der geschächte Austernfischer, Bechst. Nat. D. 2te Auf. 4. B. I Abth. p. 439. t. I2. Meyer Vögel Liv. u. Esthl. p. I72. 
Scanis: Strandskate. Gottlandis: Marspitt, Oelandis: Strandskjura.

\section{DESCRIPTIO.}

Long. I6 poll. Rostr. $2 \frac{\sigma}{8}$ poll. A flexura alæ ad apicem $10 \frac{1}{2}$ poll. Cauda $4 \frac{3}{8}$ poll.; Tibia 2 poll.; Digit. med. I $I_{2}^{x}$ poll.

Rostrum comperssum, robustiusculum, rectum, medio tamen superne aliquantulum depiessum, apice in cuneum verticalem compresso, totum aurantium. Palpebræ aurantix. Iris coccinea, Pedes robusti et crassi, pallide rubri; digitis callo marginatis, exteriore cum medio membrana juncto.

Caput, Collum usque ad sternum, Dorsum ad medium et alarum minores tectrices nigra. Macula interdum parva sub oculis et alia gularis semilunari-transversa, dorsum posticum, pectus, abdomen, femora et crissum alba. Remiges primores nigris, latere interiore a basi albx; secundarix albx; Tećtrices stiperieres medix nigra apice alba, majores alba, unde fascia obliqua alba in ala clausa oritur Tectrices inferiores albx. Rectrices medietate interiore albx, exteriore nigræ.

Funior: Rostrum aurantium apice nigrum. I is brunnea. Pedes pallide grisei. Macula gula alba vix conspicua. Color alarum niger sordidus in fuscum tendens.

\section{HISTORIA.}

Habirat in littoribus marinis, præsertim lapidosis usque intra circulum polarem. Lacus quoque sed rarius frequentat. Autumno abit, mense Martio ad oras nostras revertitur. Voce acutissime sonora et clamosa hostem indicat vigil avis; unde odiosa venatoribus, qui 
insidias aliis parant avibus, quibus de periculo moner. Perbene natat, unde, alâ ietu venatoris læsa, fugam natando quærit. Vietitat testaceis aliisque vermibus marinis, quibus recesso mari in littore potitur. Ova 2-3. lutescenti-grisea maculis fuscis irregulariter adspersis in arena littorali fovet. Caro, præsertim retracta pelle, edulis et sapida. 


\section{SECTIO II.}

Digitis plane fissis.

QENUS.

\section{A L I D R I S.}

$\boldsymbol{R}$ ostrum longitudine capitis, tenue, rętum, sub. teres, basi compressum, apice depresso-subincrassarum.

Nares parva oblongo - ovales.

Pedes tridactyli, plane fissi.

\section{CALIDRIS GRISEA, Meyeri.}

1) IAGN : Rostrum et pedes nigri. Corpus supra griv seum, rachibus nigris, subrus, cum facie, album.

\section{SYNONYMA.}

Charadrius Calidris, Lin. Syst. Nat. I. p. 255. n. 9.

Gmel. Syst. I. p. 689. Lath. Ind. II. p. 74I. n. 4.

Tringa arenaria, Lin. Syst. I. p. 25 I. n. I6. Gmel. Syst, I. p. 680 .

Calidris grisen minor, Briss. Orn. V. p. 236. 11. 17. to. 20. f. 2.

Sanderling, Buff. Ois. VII. p. 532. vers. Germ. Otto XXVII. p. 60. (fig. falsa.) 
The Sunderling, Pell. Br. Zool. II. p. 480. 11. 212. t. 73. Aret. Zool. II. n. 403. Lath. Syn. V. p. 19\%. id. Sup. p. 253. vers. Germ. V. p. I7I. 11. 4.

Der graue Sandlaitfer, Bechst. Nat. D. 2te Aufl. 4. B. I Abth. p. 368. Meyer Vögel Liv. u. Esthl. p. I77. Mus. Payk. Mus. Ups.

\section{DESCRIPTIO.}

Magnitudo Tringe alpine, $7-7 \frac{r}{2}$ poll. long. Rostr. $\frac{5}{3}$ poll. Rostrum compressum et pedes nigricantia. Iris brunnea.

Frons, genx et gula alba, stria a rostro ad oculos grisea. Vertex albide cincrascens striis minutis et punctis fuscis. Dorsum pallide griseum rachibus nigris. Uropygium et teetrices caudx superiores lateribus albis, medio griseo. Corpus subtus album. Remiges primores fuscx rachibus albis, secundarix a basi ad medium albx, exinde fusce, apiee albo marginatx. Teetrices alarum superiores fuscr; medix et majores apicibus albæ, unde stria slarum obliqua alba. Reetrices 12. quarum medix binx longiores fusex, ceterx griser, laterales pallidiores marginibus singularun albidis.

\section{OBSERVATIO.}

Similis Tringe Cinclo quoad colores; Differe tamen rostro breviore, magisque compresso, pectore immactlato et prascrtin pedibus digito postico plane destitutis.

\section{HISTORIA.}

Autumno et vere in littoribus marinis arenosis gregaria obvenit. Festatem sine dubio degit et se propa- 
gat in terris areticis *). Ovatio nondum cognita est. Vescitur vermibus et Insectis aquaticis.

*) Ni fallor, hac est avis que chavadrius alexandrinus in Actis Nidros. $V$. p. 153. t. $V$. f. $I$. appellatur, et qux maximis gregibus littora marina Norvegix ad "qudderen et Lister autumno frequentare refertur. Quodsi ita, non dubitandum est, quin domicilium avis astivum in plagis Scandinavin maxime borealibus sit quxrendum. 


\section{SUBORDO II.}

\section{VADATORES (Tetradactyli).}

\section{SECTIO I.}

Tibiis et femorum parte nuda longioribus;

Digitis anticis vel omnibus vel extimis duobus, salrem ad primam articulationem, membranâ junctis.

GENUS.

\section{R E C U R V I R O S T A.}

ostrum longissimum, gracile, depressum apice flexili, recurvato.

Nares lineares, pervix.

jedes longi, graciles, tetradaEtyli; digito postico brevi, anterioribus semipalmatis.

\section{RECURVIROSTRA AVO-}

\section{CETTA.}

Dragn: Alba, pileo, cervice et alis supra nigris maw culam albam includentibus. Pedes cærulei. Ro. strum nigrum. 


\section{SYNONYMA.}

Recurvirostra Avocetta, Lin. Fn. Sv. I9I. Retz. Fn. Sv.

I96. n. I69. Syst. Nat. I. p. 256. Lath. Ind. fII.

p. 786. Gmel. Syst. I. 2. p. 693.

L'Avocette, Buff. Ois. VIII. p. 466. t. 38. Pl. Enl. 353.

Vers. Gern. Otto XXXII. p. 81. cum fig.

Scooping Avoset, Pennr. Br. Zool. 2. p. 504. t. 8. Aret.

Zool. 2. p. 503. Lath. Syn. V. p. 293. Suppl. 263.

Vers. Germ. V. p. 263.

Der Blaufiussige Wassersäbler, Bechst. Nat. Deutschl. 4. B. I Abth. p. 450. t. 25. f. 2.

Oelnandis: Skärffäcka. Gottlandis: Alfit.

\section{DESCRIPTIO.}

Longit. avis I7 - I8 poll. Rostrum 3 poll. A flexura alæ ad apicem $7 \frac{x}{2}$ poll. Nuda pars femoris $I \frac{r}{2}$ poll. Tibix 3 poll.

Rostrum nigrum; Iris rufo-fusca; Pedes cinereocoerulei.

Corpus album capite supra et cervice nigris; alx et scapulares nigre maculam magnam longitudinalem albam includentes. Cauda alba.

\section{HIST ORIA.}

In littoribus Suecix australioris ex. gr. Gottlandix, Oelandix et Scanix rarius occurrit. Ova $2-3$ cinereoriridia maculis nigris in arena fovet. 


\section{GENUS VI. \\ P L A T A L E A.}

$\boldsymbol{R}_{\text {ostrum longissimum, robustum, depresso - pla- }}$ num, apice orbicutate dilatato.

Nares parva, oblongæ, juxta basin et se inviccm sita.

Facies ad partem nuda.

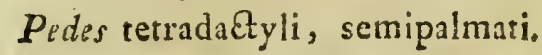

CLIII. PLATALEA LEUCORODIA, Lin.

DraGN: Corpus album, Gula nuda fulva; oeciput cristatum.

\section{SYNONYMA.}

Platnlea Lencorodin, Lin. Faun. Sv. p. 56. n. 160. Retz. Faun. p. I66. n. I28. Lin. Syst. Nat. XII. I. p. 23I. Gmel. Syst. I. p. 6I3. Lath. Ind. Orn. II. p. 667.

Platea, Briss. V. p. 352.1.

La Spatule, Buff. Ois. VII. p. 448. t. 24. Vers. Germ.

. Otto XXVI. p. 104. cum fig. Pl. enl. 405.

White Spoonbill, Br. Zool. II. p. 634. App. t. 9. Aret. Zool. II. p. 44I. A. Id. Supp. p. 66. Lath. Syn. V. p. I3. I. Vers. Germ. Bechst. V. p. I. 
Weisser Löfler, Bechst. N. D. 2te Auf. 4 B. I Abth. p. 4. Meyer Vögel Liv. und Esthl. p. I79.

Svecis: Pelikan.

Mus. Payk. Mus. Upsal.

\section{DESCRIPTIO.}

Ardeâ Cinereâ minor; 2 ped. 6 poll. lonģ. Ro. strum 8 poll. long. pone apicem fere 2 poll. latum. Tib. $5 \frac{x}{2}$ poll.

Rostrum nigrum, supra apicem macula fulva, depressum et planum, basi supra rugosum, apice orbiculate-dilatatum maxilla superiore sulco marginali circumdata. Iris rubra. Pedes nigri, semipnimati, membrana inter digitum extimum et medium majore. Lora, orbita et gula dilatabilis nuda, extensilis, fulva Occiput pennis elongatis ( 5 fere pollicaribus) acuminatis, dependentibus cristatum. Alæ clausæ apicem caudæ attingunt.

Mas: Totum corpus album, excepto pectore, quod macula ornatur luteo-rufescenti.

Femina: Alba macula pectoris fere destituta. Capite fere lxvi.

Funior Avis: Remiges primores apicibus nigris.

\section{HISTORIA.}

Migratoria avis æstate, rarius tamen, obvenit in plagis patriz borealibus, Lapponia et Westrobothnis, unde receptam in Museis citatis examinavi. Migrationes pèr Finnlandiam et Rossiam instituere videtur, quare nunquam in meridinnaliori Patria observata est. Nidifisat in arboribus excelsis juxta fuvios. Ova 3.4 alba subescenti-maculata ponere refertur. Vescitur piscieulis, ranis, alisque amphibiis et Insectis aquaticis. 


\section{OBSERV ATIO.}

Platalea pygmer Lin. cujus existentia a quibusdam Ornithologis recentioribus negatur, re vera existit. Specimen ipse vidi, et forte idem illud, quod in Museo Adolphi et in Systemate Naturæe descripsit Linné, adhuc in Mnseo Illustr. Thunbergii Upsalix asservatum. Thunberg avem iterum descripsit et delineandan curavit in Adis Acad. Scient. Holm. anno 1816. p. 194. Tab. VI. Hec vero Linneana avicula ad Genus Platalea, accuratius determinatum, non pertinet. Neque enim gula extensili, neque loris nec orbitis nudis gaudet; nec pedes ejus longi et semipalmati sunt th in genere Platalea. In omnibus, rostri forma exceptâ, cum Tringis probe convenir; quate, me judice, proprium genus in sequenti setione juxta Tringas collogandum, constituere deber, quod quideın genus mihi dicitur

\section{EURYNORHYNCHUS.}

Rostrum mediocre, subreres apice dilatatissimo, subangulato.

Nires parva lineares, juxta basin rostri, distantes. $p_{\text {eides }}$ breves digitis usque ad basin fissis.

\section{EURYNORHYNCHUS GRISEUS,}

\section{mihi.}

Diagn: Supra fuscoegriseus, subtus albus.

\section{SYNONYMA.}

Platnlea Pygmea, Lin. Syst. Nat. I. p. 23I. 11. 3. Lath. Ind. Orn. II. p. 669. 3. Thunberg Stockh. Wettensk. Acad. Handl. 1816. p. 194. t. VI.

\section{DESCRIPTIO.}

Magnitudo Tringe Temninckii. Rostrum pollicare, capite parum longius. Pedes tetradadyli, digito po- 
stico brevi, plane fissi. Caput supra fuscescens mar. ginibus pennarum albidis. Dorsum et alarum tectrices fusco-grisen striis ad raches saturatioribus. Avis subtus alba. Rcmiges fuscæ, secundariis pallidioribus albo late marginatis. Cauda alis compositis brevior, subfusca rectricibus lateralibus albidis.

\section{HISTORIA.}

Plane incognita, nec locus quidem natalis certa fide definiri potest. 


\section{GENUS. \\ C I C O N I A.}

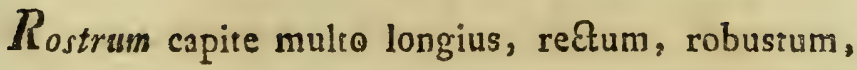
subcylindricum, compressum, acuminatum, maxilla superiore urrinque linea excavata er pone apicem emarginata, inferiore aliquantulum sursum curvata.

Nares lineares longæ, prope basin rostri in dorso sitx.

Orbita nuda.

Gula nuda, extensilis.

$P$ edes longi, tetradactyli, digitis brevioribus, a basi ad primam articulationem membrana connexis.

\section{CICONIA ALBA, Briss.}

DiaGN: Alba orbitis nudis, remigibus et scapularibus nigris; rostro et pedibus rubris.

\section{SYNONYMA.}

Ciconia Alba, Briss. Orn. V. p. 305. to 32. Bechst. Nat. Deutschl. 4 B. I Abth. p. 82.

Arden Ciconia, Lin. Fn. Sv. 162. Retz. Faun. p. 167. Lath. Ind. II. p. 676. Gmel. Syst. I. 2. p. 622. 
Ia Cicogne blanche, Buff. Ois. VII. p. 253. t. I2. Vers. Germ. Otto XXV. p. 3 I. cum fig. Pl. enl. 866. White Stork, Pen. Aret. Zool. 2. p. 455. Lath. Syn. V. p. 47. Vers. Germ. V. p. 25.

Weisser Storch, Meyer Vögel Liv. p. 185.

\section{DESCRIPTIO.}

Longit. avis 3 ped. 6 poll.

Rostrum et pedes rubra; orbita nuda nigra; Iris brunnea.

Caput, collum totumque corpus album, remigibus st pennis scapularibus nigris.

\section{HISTORIA.}

Habitat in meridionaliori Svecix plaga, in Scania vulgaris, in ceteris provinciis ex. gr. Hallandia rarior. Uplandiam tamen et superiorem Hallandiam interdum quoque visitat. Migratoria advenit ante medium Aprilem. Nidulatur super arbores demortuas et decollatas sylvarum, super teeta et spiramenta caminorum in vieis et prxdiis. Ova 3-5 ochraceo-alba. Magis adhuc pie quam vel Corvus Frugilegus vel Hirundo Rustica facetur; neque tamen plane innocens hospes; nam prxter Ranas, Lacertas et innocuos Natrices et angues, etiam pullos Perdicum et Anatum ova devorat.

\section{CICONIA NIGRA, Bechst.}

DIAGN: Nigricans pectore er abdomine albis. Ro. strum, gula et orbita nuda coccinea. Pedes rubri.

\section{SYNONYMA.}

Ciconia Nigra, Bechst. N. D. 2te Auf. B. 4. Abth. I. p. 96. n. 2. Meyer Vögel Liv. p. 186. 
Ciconin fissa, Briss. Om. V. p. 362.t. $3 \mathrm{I}$. Arden nigra, Lin. Fn. Sv. 163. Retz. Fn. I68 n. I3T. Lin. Syst. Nat. p. 235. Gmel. Syst. I. 2. p. 623. Lath. Ind. II. p. 677.

Cicogne noire, Buff. Ois. VIr. p. 27 I. Vers. Germ. Otto XXV. p. 60. Pl. enl. 399.

Black Stork, Penn. Arct. Zool. II. p. 456. Lath. Syn. V. p. 50.

Svecis: Swart Stork, Odens Swala.

\section{DESCRIPTIO.}

Longit. 3 pedes. Rostr. 7 poll. A flexura alx ad apicem ?? poll. Pars nuda femoris $2 \frac{\pi}{2}$ poll. Tibia 3 poll. Digit. med. 4 p. postic. 1 $\frac{2}{8}$ poll. Rostrum, Orbita et Gula nuda, coccinea. Iris brunuea. Pedes saturate rubri.

Caput, collum, scapulares, dorsum, ala et cauda i. e. totum corpus superne nigricans purpureo et viridi nitente. Pectus, abdomen et caudx inferiores tectrices, alba.

Funior: Rostrum, orbita et pedes olivacea. Caput et collum rufo-brunnea marginibus pennarum albidis. Corpus, alix et cauda supra fusco-nigricantia.

\section{HIS TORIA.}

Rarior apud nos et migratoria avis. In paludibus sylvaticis Scanix, Hallandix et Ostrogothiz a nobis visa. Vescitur pisciculis, ranis et Insectis. Nidtilatur in excelsis arboribus. Ova $2-3$ sordide alba, virescenti tincta. 
Rosirum capite parum longius, robustum, compressum, rectum, versus apicem subteres, apice subfornicatum; maxilla superiore basi urrinque linea excavata.

Nares fere in medio rostri sitæ, oblongo-ovales. Orbita et Lora pennis téta. Pedes longi, digito extimo membranâ medio basi adnexa; digito postico brevi, elevato.

\section{GRUS CINEREA, Bechst.}

DIAGn: Vertex nudus, papillosus, ruber. Corpus cinereum fronte, nucha, collo antice et remigigibus nigris; Pennis laxis laceris supra alas dependentibus.

\section{SYNONYMA.}

Grus Cinerea Bechst. N. D. 4 B. Abth. I. p. I03. Meyer Vögel Liv. P. I87.

Arden Grus, Lin. Syst. Nat. I. p. 234. Fn. Svec. I6I. Retz. Fir. p. I67. n. 129. Lath. Ind. II. p. 674. Gmel. Syst. I. 2. p. 620.

La Giue, Buff. Ois. VI. p. 29\%. t. I4. Vers, Germ. Otto XXV.p.89. cum. fig. 
Common Crane, Pent. Br. Zool. II. p. 629. tab. app. 6. Arß. Zool. II. p. 453. Lath. Syn, V. p. 50. Vers. Germ. V. p. I8.

Suecis: Trana.

\section{DESCRIPTIO.}

I.ongit. 3 ped. $8 \cdot 10$ poll. Rostr. a fronte $4 \frac{t}{2}$ poll. A flexura ale ad apicem 23 poll. Cauda 8 poll. Nuda pars femoris $4 \frac{r}{3}$ poll. Tibia $9 \frac{r}{2}$ poll. Digit. med. 4 poll, postic. I poll.

Rostrum nigro - virescens apice pallidius corneum. Tris brunnea. Pedes nigri digitis membrana submar. ginatis, extinoque juxta basin intermedio adnexo.

Frons pilis nigris vestita. Vertex subcalvus, papilInsus, ruber pilis raris adspersus. Lora nigra pilis dense vestita. Nucha, genæ, gula et collum antice infra medium nigricantia. Pone oculos stria lata alba utrinque per tempora in cervicm decurrit. Praterea corpus totum cinereum rachibus pennarum dorsi et textricum nigricantibus. Remiges nigrx. Teetrices ultimx (Lin.) cinereo-nigræ, longiores, laxæ, supra apicem alarum utrinque dependentes, radiis fibrosis. Cauda rotundata cinerea apice nigricans.

\section{H I S T ORIA.}

Migratoria, vere et autumno Scaniam transmigrat. In superioris Svecix paludosis se propagat; Ova 2 virescenti-grisea maculis fuscis. Vescitur herbis palustribus et segetibus consitis. 


\section{GENUS.}

\section{$A \cap R \quad D \quad E$ A.}

Rostrsm longum, refum, robustum, compressum, maxilla superiore utrinque linea excavata et pone apicem emarginata.

Nares prope basin rostri, latcrales, oblongx, membrana superne semitedt.

Lora calva.

Pedes terradactyli digito cximo mambrana interne. dio basi connexo.

\section{FAMILIA I.}

(Tenuicolles).

Rostrum capite nunlto longius, juwti basin haud compressum.

\section{ARDEA CINEREA, Lath.}

DiagN: Crista occipitis pendula nigra. Dorsum cinereo-coerulescens. Corpus subtus album ma= culis in collo antico et pefture nigris.

\section{SYNONYMA.}

Arder Cinerea, Lath. Ind. II. p. 69 I. Retw. Fn. Sr. p. 169. 11. I33. Bechst. N. D. P. 4. Abth, I. p. IO. Meyer Vorgel Liv. 180. 
Ardea Cineren, Lin. Fn. Sv. p. 59. 11. I65 (Junior).

Syst. Nat. I. p. 236. (Junior).

Aiden Mnjor, Lin. Syst. I. p. 236. Gimel. Syst. I. 2.

p. 627. (Senior).

Heron huppé, Buff. Ois. VII. p. 342. Vers. Germ. Otte

XXV. p. 172. cum figg. 2. Pl. enl. 755.

Hcron, Buff. Ois. VIr. p. 342. t. 19. Pl. enl. 787 (Jun.)

Common Heron, (male) Penn. Brit. Zool. II. p. 42 I.

Lath. Syn. V. p. 83. Vers. Germ. V. p. 54. (san.)

Common Heron, (female) Penn. Br. Zool. II. p. 42I. to

6I. (jun.).

Suecis: Hïger.

\section{DESCRIPTIO:}

Longit. 3. ped, 2 poll. Rostr. a fronte $4 \frac{6}{8}$ poll. A. flexura alr ad ejusdem apicem 18 poll. Nuda pars femoris $2 \frac{\pi}{2}$ poll. Tibia 6 poll. Digit. med $3 \frac{2}{8}$; po. stic. 2 poll.

Rostrum, lera et iris fulva; Pedes fusco-rufescentes. Pars femorum nuda testacea.

Frons et anterior pars verticis alba. Supra oculos utrinque stria nigra, quæ in cristam occipitis dependentem nigram sc conjungit. Collum album postice cinerascens, antice a juymlo ad medium pectus pennis longioribus acuminatis maculisque longitudinalibus nigtris notatis. Dorsum et alarum teetrices cyaneocincrea. De medio dorso et de scapularibus penne longx acuminatx argentei coloris utringue supra alas falcatim defexr sunt. Cauda saturate cinerea. Remiges nigræ, intimæ saturate cinerex. Margp alarum ct Alexura alba, justa quam in laterc colli macula magna nigra.. Corpus subtus album.

Funior: Crista brevis s. nulla. Pennis elongatis in collo ct alis destituta. Frons ct vertex cinerea. Maxilla supe- 
rior nigricans, inferior lutea. Pedes cinereonigrican. tes parte femorum nuda, lútea.

\section{HIS TORIA.}

Habitat in sylvis, quæ paludibus, Auviis et lacubus vicina sunt. In australiori Svecix parte vulgaris; in septentrionaliores quoque longrius adscendit (sic supra Nidrosiam Norvegix vidi) sed rarius. Migratoria, in Scaniam advenit jam fine M. Martis. Nidulatur in ex. celsis arboribus. Ora 3-4. colore thalassino. Vescitur piscibus, ranis \&c.

\section{OBSERV.}

Arrea Alba, qua in Fn. Sv. in Scania olim visa dicirur, nunquam postea ibidem inventa est. Neqque scio, an Linné ipse avem in Scania viderit, an ex relatis alior um inter aves Succicas retulerit. Neque enim inter aves pictas Rudbeckii occurrit, neque ejus mentio fir in Adversariis Lccke. Mllam igitur, ad plagas Europe australiores, ad Asiam et Afrucam percinentem, e Fauna Suecica exciudendam puto.

\section{ARDEA MINUTA.}

Diagn: Caput læve, et corpus supra, Remigesque primeres et Cauda nigra. Collum antice et tectrices alarum testacea.

\section{SYNONYMA.}

Ardea minutn, Lin. Syst. Nat. I. p. 240. n. 26. Gmel. Syst. I. 2. p. 646. Lath. Ind. II. p. 683.

Blongio de Suisse, Buff. Ois. VII. p. 395. Vers. Germ. Otto XXV. p. $30 \mathrm{I}$. cum fig.

Butor brun rayé - roux, Buff. 1. c. p. 424 et 425 . Litzle Bittern, Lath. Syn. V. p. 65. 


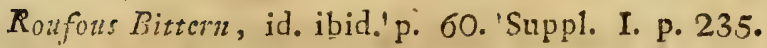

Dor Kieine Reiher, Bechst. N. D. 2te Auf. B. 4. Abth.

I. p. 71. Meyer Vögel Liv. p. I83.

\section{DESCRIPTIO.}

Longit. avis $13 \frac{x}{2}$ poll.

Lora nuda et rostrum luteum apice fusco. Iris iutea. Pedes lutescenti - virides.

Caput læve et Corpus supra, Remiges secundarix et cauda nigra viridi-nitentia. Latera capitis, collum et tectrices alarum rufo-testacea. Corpus subtus album, restaceo tinctum.

Funior: Rostrum apice et dorso fuscis. Fedes virides. Pileus niger. Dorsum nigricans marginibus pennarum rufescentibus. Latera capitis, colli, pectoris et teetrices alarum rufescentia maculis longitudinalibus fuscis. Gula'et crissum albida.

\section{HISTORIA.}

Nova hæc faunx nostræ incola in Westmannia occisa est. In palustribus locis habitat et nidulatur. Ova 5.6 alba. Vescitur pisciculis, ranis, insectis.

\section{FAMILIA II. \\ (Crassicolles).}

Rostrum capice partum longius, valde compressum, subdecurvatum.

\section{ARDEA STELLARIS, Lin.}

Diagn: Capur lieve. Corpus supra ferrugineum undulis et maculis transversis nigris; subrus pal- 
lidius maculis longitudinalıbus nigris. Penne colli tumida.

\section{SYNONYMA.}

Ardar Stcllavis, Lin. Fn. Sv. p. 58. n. I64. Syst. Nat. I. F. 239. Lath. Ind. Orn. II. p. 680. Retz. Fn. p. 168. n. I32. Gmel. Syst. I. 2. p. 635. A\&. Soc. Physiogr. Lund. I. p. 305.

Bitor, Buff. Ois. VII. p. 4 II. t. 2I. Vers. Germ. Otto XXV. p. 344. cum fig. Pl. enl. 789.

Bitterit, Penn. Br. Zool. II. p. 424. Aret. Zool. II. p. 45 I. Lath. Syn. V. p. 56. n. I7. Suppl. I. p. 234.

Vers. Germ. V. p. 33.

Dickhillsiger Reiher, Bechst. N. D. 2te AuA. B. A. Abth. I. p. 63. Meyer Vögel Liv. p. I82.

Svecis: Rördroin.

\section{DESCRIPTIO.}

Iongit, 2 ped. $3-4$ poll.

Rostri maxilla superior fusca, margine, maxilla inferiore et loris nudis, luteo-viridibus. Iris lutea. Pedes luteo-virides.

Caput supra et penna ejus laterales longx, nigra. Collum et corpus rufo-Iutea, collo undulis fuscis, in dorso et scapularibus maculis majoribus nigris margine rufo variegaris ornata; Pectus et abdomen maculis magnis longitudinalibus nigris. Tectrices alarum maculis undulatis nigris. Remiges nigricantes fasciis transversis testaceis. Caude testacco et fusco puleherrime variegata. 


\section{HIST ORIA.}

Rarior apud nos avis habitat in paludosis, arundinstis et juncetis. Viatitat piscibus, ranis, vermibus $\mathrm{et}$ insectis aquaticis. Nidulatux inter armalines.Ova 3 . 5 sordide viridiọ. 
GENUS.

\section{TANTA L U S.}

Rustrin longissimum, arcuatum, basi subtrigonum, dehine compressum, apice depresso - obrusum. Ficies a fronte per oculos nuda. Sacculas gularis nudus 1. Grta nuda extensilis. Nares oblongo-ovales, membrana ad partem teetx. Pedes tetradaceyli digiris a basi ad primam articula. tionem membrana junctis.

\section{TANTALUS FALCINELLUS.}

Dingn: Facies atro-viridis. Corpus castaneum, alis dorso et cauda chalybeo et violaceo nitentibus.

\section{SYNONYMA.}

Tantalus Falcirellus, Retz. Faun. p. I7I. n. J35. Lin. Syst. Nat. XII. I. p. 24 I. n. 2. Gmel. Syst. I. p. 648. Lath. Ind. II. p. 707. in. 14.

Numenius viridis, Briss. Orn. V. p. 326.4.

Courlis Verd, Buff:" Ois. Vill, p. 29. Vers. Germ. Otto

XXVII. p. I70.cun fig.

Courlis d'Italie, Pl. enl. 819.

Bay İbis, Lath. Syn. V. p. II3.'n, I2. Suppl. 67.

Vers. Germ. Bechst. V. p. 67. t. 8I, (junior). Pen.

Art. Zool. II. p. 460. A. 
Ninimersatt, Bechst. Nat. D. 2te Auf. 4 B. I Abth. p. 116.

\section{DESCRIRTIO.}

Longit. avis circa 2 ped. Rostr. 4 poll. $\Lambda$ flexura alx ad apicem I I $\frac{T}{2}$ poll. Cauda $4 \frac{\frac{\pi}{8}}{8}$ poll. pars nuda fe. morum fere 3 poll. Tibia $4 \frac{\mathrm{r}}{2}$ poll. Digit. med. $3 \frac{3}{8}$ poll., postic. I $\frac{5}{8}$ poll.

Facies nuda nigra viridi nitens. Rostrum olivaceofuscum. Iris brunnea. Pedes virescenti-fusci, ungvibus nigris. Digiti basi membranis, quarum exterior major, connexi. Cauda alis complicatis xqualiter ex. renditur.

Caput, collum corpusque subtus castanea, pectore et abdomine dilutioribus fulgore viridi et chalybeo nitentes. Alæ supra nigricantes, viridi et violace splendentes. Remiges et Reßtrices fulgore atro-viridi.

\section{HISTORIA.}

Rarissima apud nos avis. In Gottlandia interdum obvenit juxta paludes interiorum insulx partium. In Scania quoque, ubi descriptum specimen occisum est. Vescitur insetis et vermibus. 
GENUS.

\section{$N U M E N I U S$.}

Rostram longissinum, arcuatum, basi subrrigonum, dehine tereti-compressum, apice obtuso.

Faties pennis recta;

Nares lineares, laterales pervia, juxta basin rostri sita.

Pedes cerradactyli, digitis anticis basi ad primam articulationem membrana connexis *).

CLXI. NUMENIUS ARQUATA, Lath.

Diagn: Rostrum medietate exteriore incurvum. Corpus supra saturate fuscum marginibus penna. rum rufescenti-albidis. Collum et PeZtus rufescenti-albida maeulis fuscis acutis. Cauda fas. ciis fuscis et albidis.

\section{SY NONYMA.}

Scolopax arqvata, Lin. Faun. 5v. p. 59. 11. I68. Retz. Faun. I7I. n. I36. Lin. Syst. Nat. XII. I. p. 248. 11. 3. Gmel. Syst. I. p. 655.

Nunnenizs arquata, Lath. Ind. II. p. 1 IO. I.

*) Hac pracipne nota dignoscitur Nnmenius a Tringis quibasdam, qुua rostrum quequé habent curvatum. 
Nuntenius, Briss, Orn. V. p. 3II. I.

Courlis, Buff. Ois. VIII. p. 19. P1. en1. 818. Vers.

Germ. Otto XXVII. p. I45. cum. fig.

Commion Curlery, Pen. B1. Zool. II. p. 429. n. I76. to 63. Arê. 'Zool. II. p. 462. A. Lath. Syn. V.

p. II). Vers. Germ. V. p. 94. Id. Sup, p. 242. Grosser Brachvogel, Bechst. N. D. 2te Auf. 4 B.I Abth. p. 12I. Meyer Vögel Liv. u. Esthl. p.188. Svecis: Spof. Storspof. Scanis: Tullare, Längnäbba, Kourpa. Sudermanis: Windspole.

\section{DESCRIPTIO.}

Longit. avis circa 2 ped. Rosti. $4 \frac{7}{2}-5$ poll. A flex. ura al:x ad apicem I ped. Cauda $4 \frac{r}{2}$ poll. Nuda pars femorum $1 \frac{8}{2}$ poll. Tibir $3 \frac{3}{8}$ poll. Digit. med. $1 \frac{6}{8}$ poll., postic. $\frac{5}{8}$ poll.

Rostrum tereti-compressum, basi obtuse trigonum, subrectum, a medio ad apicen modice incurvun, fuscum, apice nigrum, basi subtus incarnatum. Iris brumea. Pedes saturate cinerei, digitis ad primam articulationem membranis (querum exterior major) connexis.

Caput supra dorsum et alarum tectrices nigro - fusca, marginibus pennarum dilute rufo-griseis. Avis subtus alba pallide griseo rufescenti, tincta maculis ad raches pennarum fuscis, in collo frequentissimis, in abdomine rarioribus. Dorsum posticum album, maculis sagittatis nigris. Gula et crissun alba immaculata. Remiges primores nigro. fusce, latere interiore albo trans. versim fasciati I-5, latere exteriore immaculata; $6-7$ \&c. latere exteriore maculis albis. Tectrices siarum inferiores albre fusco maculatx. Cauda et Teetrices ejus superiores fusco et albido transversim fascinta. Tectrices inferiores albz maculis fuscis. Femina Mare 
majoi rostro gaudet longiore. Margines pennarum non tain rufescentes sunt.

\section{HISTORIA.}

Habitat in pratis deprešsis et paludosis zstate vulga. ris. Migratoria in Scaniam redir juxta finem mens. Martis. Autumno dimigrans et verno tempore adve. niens littora marina gregation visitat. Late versus Septentrionem discedit. In insulis cnim Norvegix hanc avem usque ad Circulnm arcticum inreni. Neque tamen in Alpium latera tam alte adscendit, quam Species proxime insequens. Victus ejus sunt Insecta, Larve, Lumbrici, testacea minora. Nidulatur in pratis humidis, in tumulo et alia eminentia. Ova 4 olivacea maculis fuscis et nigris, prrsertim circa partem crassio. rem adspersa. Avis timida. Caro sapida.

\section{NUMENIUS PHOEOPUS.}

DIAGN: Rostrum in medio valde incurvum. Corpus supra saturate fuscum, marginibus pennarum dilutioribus, siria verticis longirudinali, albidâ. Ale supra albo maculatx. Cauda fasciis fusco: griseis et nigricantibus.

\section{SYNONYMA.}

Scolopax phoeopus, Lin. Faun. Sv. p. 60. n. I69. Syst. Nat. XII. I. p. 243. n. 4. Gmel. Syst. I. p. 657. Retz. Faun. p. 172. n. 137 *).

*) Mire illius nota diagnostica, que in Fauna Suec, huic avi. additur, et exinde in plurima scripta migravit, Calussam si 
Numenius Phneopus, Lath. Ind. II. p. 7IT. n. 6. Numenius minor, Briss. Om. V. p. 317.t. 27. f. I. Corlien on petit Courlis, Buff. Ois. VIII. p. 27. Pl. enl. 842. Vers. Germ. Otto XXVII. p J 6 I. cum fig. Whimbrel, Pen. Br. Zool. II. p. 430 . 11. 177. t. 64. Aret. Zool. II. p. 462. B. Lath. Syn. V. p. I23. Vers. Germ. Bechst. V. p. 98: 1. 6. Mittlerer Brachrogel, Bechst. N.D. 4. B. I Abth. p. 129. Regen-Brackvogel, Meyer Vügel Liv, u. Esthl. p. 189. Svecis: Sinïspof, Qvidbonde.

\section{DESCRIPTIO.}

Long. I6. poll. Alx extensx 2 ped. 7 poll. Rostr. eirca 3 poll. A flexura alæ $9 \frac{r}{3}$ poll. Cauda 4 poll. Nuda pars femoris I; Tibia $2 \frac{T}{\frac{1}{6}}$ poll. Digit. med. I $\frac{1}{2}$; post. $\frac{1}{2}$, poll.

Rostrum teres, coeruleo-nigricans, in medio prssertim arcuntum, apicc obtuso. Iris brunnea. Pedes plumbei unguibus nigris.

Pileus saturate fuscus, versus frontem rufescens, stria longituginali lutescenti - albida interrupta. Latera capitis, collum et pectus griseo-albida maculis fuscis longritudinalibus fere sagittiformibus, quz in hy. pochondria fascias transversales forment. Lora fusce. Gula alba. Dorsum anticum, scapulares et tectrices alarum saturate fusca, griseo-albo maculatim marginata. Dorsum posticum et crissum alba. Remiges

quis quarit, hxc est: In fnl. 202. inter Aves piças Rudm beckii, figura hujus Speciei obvenit manu sane rudiori con. feita. In hac maculæ dorsales exacte rhomboidales sunt, ita ut dorsum avis sabulau alentoriam tessellis obliqquis exaate representet. 
fusco-nigra lntere interiore maculis albis transversim ovalibus, primx 6 extus immacularæ, ceteræ maculis albis margine quoque exteriore. Tectrices proximx remigum primorum nigræ apicibus albis. Tectrices alarum inferiores alba griseo-fusco fasciatx. Cauda fusco-grisea fasciis transversis saturate fuscis anice albo. Tectrices caudae superiores albida fusciis fuscis; inferiores lutescenti-alba maculis griseo-fuscis.

\section{HIST ORIA.}

In australioribus patrix plagis, quas tantum trans. migrat, rara avis; in borealibus autem vulgaris. $\mathrm{Ha}$ bitat in pratis depressis et humidis. Incolit quoque latera alpium uliginosa usque ad terminum quo desinit Pinus sylvestris. Longius itaque versus boream procedit quam antecedens. Arboribus haud raro insidet. Ova in tumulo, tamquam antecedens ponit. 


\section{GENUS. \\ L I M O S A.}

Rostrum longissimum, flexibile, aliquantum re eurvatum et depressum, apice obtusum subdilatatum ; maxilla utraque fere ad apicem marginata. Notres juxta basin laterales, cuneiformes. Pedis longi femoribus altius nudis; Tibiis digito medio duplo fere longioribus.

Digitus medius extimo membrana junctus usque ad primam articulationem.

CLXIII. LIMOSA MELANURA, Leist。

Dragn: Supra griseo - fusca, nigro et ferrugineo immixto. Collum luteo-ferrugineum. Pedus undulis fuscis. Alæ nigricantes macula alba. Cauda nigra, basi alba.

\section{SYNONYMA。'}

Scolopax linirosa, Lin. Faun. Sv. p. 61. n. 172. Retz. Faun. p. 178. n. I45. Lin. Syst, I. p. 244. n. I3. Gmel. Syst. I. p. 666. Lath. Ind. II. p. 719. 11. I8. Limosa, Briss. Orn. T. V. p. 262 . I. I.

Limosá melaniıra, Meyer Vü̈gel Lív. tr. Esth1 p. I26. La Barge, Buff. Ois. VIr. p. 500. t. 27. Pl. enl. n. 874. Vers. Germ. Otto XXVI. p. 214 et $21 \%$. cum fig.

Nilsson Orxith. Pars It. 
Lesser Godwit, Pen. Br، Zool. II. p. 444، 17. 182.

Fodrella Snipe, Lath. Syn. V. p. 146. I7. Vers. Germ.

V. p. II8. - Arct. Zool. II. p. 467. 11. 375.

Olafs. IsI. II. p. 20 I. t. 48.

Dunkelfüssiger Wasserläufer, Bechst. Nat. D. 2te Auf. 4 B. I. Abthop. 244.

Mus. Payk. Lund. Upsal.

\section{DESCRIPTIO.}

- Longit. I5 poll. Rostr. 4 poll. A flexura alx ad apicem 8 poll. Cauda $3 \frac{2}{8}$ poll. Pars nuda femoris $I \frac{6}{8}$ ? Tibia fere 3 poll. Digit. med. $x \frac{\sigma}{8} ;$ postic. $\frac{x}{2}$ poll.

Rostrum rectum, aurantium, veisus apicem adscendens, nigrum, maxillis fere aqualibus apice incrassatis. Iris brunnea. Pedes fusci 1. nigricantes. Digitus extimus intermedio membranâ connexus ad primam articulationem.

Senior. Caput supra cinereum maculis minutis fuscis et ferrugineis. Orbita albida; Lora fusca. Corpus supra cinereo-fuscum marginibus pallidioribus ráchibus fuscis. Collum cinereo - ferrugineum punctis fuscis. Pectus undulis fuscis, ferrugineis et albidis. Abdomen, crissum et femora alba immaculata. Remiges nigricantes, primores quatuor latere interiore a basi albx; sequentes latere exteriore quoque a basi ad medium!albx; secundarix albx, apice tantum fuscx; intimx longitudine tertix, griseo-fuscx. Tectrices majores apice albr. Unde macula magna alarum alba. Reetrices nigr:x, basi et apice albx, qux albedo in pennis lateralibus ita procedit; ut sint fere totx albre. Teentces cauda superiores a basi ad medium albx, dehinc nigia. 
Funior. Caput supra griseo - fuscum striis ferrugineis. Facies griseo-rufescens. Dorsum et Scapulares griseofusca maculis majoribus nigris et minoribus ferrugineis. Dorsum posticum saturate fuscum. Collum dilute ferrugineum. Pectus dilute ferrugineum striis transversis fuscis. Abdomen et crissum alba. Remiges et Roctrices ut in antecedenti.

\section{HISTORIA.}

Habitat' in pratis paludosis, rarius in Scania, ubi vix nidulatur. Inter gramina refertur. ova 4 ponere olivacea maculis fuscis. Victitat Insectis et vermibus.

\section{LIMOSA RUFA, Briss.}

DIAGN: Supra saturate fusca maculis ferrugineis; subtus ferruginescens striis colli antici fuscis. Cauda fasciis transversis albis et fuscis.

\section{SYNONYMA.}

Scolopax Lapponica, Lin. Faun. Sv. p. 62. 11. I74. Retz. Faun. p. I79. Syst. Nat. I. p. 246. n. I5. Gmel.

I. p. 667. Lath. Ind. II. p. 718. n. I5.

Limosa rufa, Briss. V. p. 28 I. n. 5. t. 25. f. I. Meyer

Vögel Liv. u. Esthl. p. 196.

La Barge rousse, Buff. Ois. VII. p. 504. Pl. enl. 900.

Vers. Germ. XXVI. p. 237, 240.

Totanus rufus', Bechst. 1. c. p. 253.

Senior astate:

Scolopax agocephala, Lin. Syst. I. p. 246. n. I6. Lath. Ind. II. p. 719. n. 16. Gmel. Syst. I. p. 667.

D 2 
Limosa rufa major, Briss. Orn. V. p. 284. n. 6.

La grande Barge rousse, Buff. VII. p. 505. Pl. enl. 916.

Common Godwit, Lath. Syn. V. p. I45. n. I4. A. Vers. Germ. V. p. II7. b (var. A).'

Geiskopf Wasserläufer, Bechst. N. D. 2te Auf. 4 B. I. Abth. p. 234.

Avis Antumanli habitu:

Limosa grisea major, Briss. V. p. 272. tab. XXIV. f. 2.

Totanus leucophcus, Bechst. 1. c. p. 237.

Funior:

Totanus gregarius, Bechst. 1. c. p. 258. (junior, au* tumno).

Tringa gregaria, Otto in Buff. XXVI. p. 242.

$V$ cnatoribus Scanensibus: Augusti Snäppa.

\section{DESCRIPTIO.}

Long. I4. I5 poll. Rosti. $3 \frac{1}{2} \cdot 4$ poll. A flexurn ala ad apicem 9 poll. Cauda 3 poll. Nuda pars femoris I poll. Tibia $2 \frac{3}{8}$ poll. Digit. med. $I_{\frac{1}{2}}$ poll. post. $\frac{r}{3}$ poll.

Rostrum modice recurvatum, a basi ad medium fa. vescens 1. pallide incarnatum, apice nigrum, maxille superiore longiore apice depresso, subdilatato. Iris fusca. Pedes saturate cinerei. Digitus extimus intermedio membrana adnexus ad primam articulationem. Ungues nigri, breves, obliqvi, subtus concavi. Call= da alis brevior.

Caput supra, Dorsum et Scapulares saturate fusca maculis pallide griseis et ferrugineis. Alarum tectrices fusco-grisex marginibus albidis. Dorsum posticum album maculis rarioribus cordatis fuscis. Fascig 
albo-ferruginescens a rostro supra oculos; sub qua alia fusca. Collum antice et peçus luteo-ferruginea striis longitudinalibus fuscis. Abdomen album. Crissum album ferrugineo tinctum. Remiges primores nigro.fuscx, latere interiore griseo et albo tenuiter variegatx; secundaria cinereo - fuscx albo marginatr; intimx longx, fusce marginibus pallidis. Teetrices inferiores albx, maculis griseis. Canda cinereo-fusca fasciis transversis et apice albis.

\section{OBSERVAT IO.}

Coloribus adınodum variat pro xtate et anni tempestate. Variat quoque restri longitudine, in junioribus breviore.

$V a r$. . Supra saturate fusca maculis ferrugineis. Tota subtus saturate ferruginea, maculis in abdomine ct crisso albis (Avis annosa, estate capta). $V a r$. $\beta$. Supra fusca marginibus pennarum pallide griseis 1. albidis. Subtus alba collo antice lutescenti et pectore cinereseenti tinctis, striis minutissimis fuscis (avis senior atumunalis).

$V a r . \gamma$. Supra nigro-fusca marginibus pennarum pallide ferrugineis. In tectricibus alarum hi margines latiores, ita ut pallide sint ferruginex striis longitudinalibus fuscis. Collum antice et pectus pallide cinera. scentia ferruginescenti tincta striis minutis fuscis. Gula, abdomen, crissum et uropygium alba. Hypochondria alba maculis fuscis. Re@trices albæ tæniis transversis fuscis, apice lutescentes (autumnalis junior).

\section{OBSERVATIO.}

Tringa squatarola $\mathrm{Lin}$. Fn. Sv., cel. Lecheo et venatoribus Scanensibus Augusti Snäppa, thuc pertinet, Vide infra Artic. Vanellis melanogaster. 


\section{HIS T ORIA.}

In plagis borealibus æstatem degit; australiores patrix regiones vere et autumno transmigrans juxta palu. des rarius occurrit. Autumno quæ obveniunt, a venatoribus Scanensibus Augusti Snäppa appellantur. Viditat Testaceis, vermiculis et Insectis. 
GENUS.

\section{G L O T T I S.}

$\boldsymbol{R}_{\text {ostrum longum, durum, robustiusculum, basi }}$ compressum, sursum in medio curvatum maxillis*) dehinc teretibus, apice acutis et porrectis.

Nares juxta basin sitx, lineares.

Pedes longi. Digiti membrana basi connexi.

CLXV. GLOTTIS SEMIPALMATA, mihi.

DIAGN : Supra pallide grisea maculis nigro-fuscis; subtus lutescenti alba. Remiges primores nigræ basi albæ; secundariæ albæ. Cauda fasciis fuscis et cinereis. Pedes semipalmati.

\section{SYNONYMA.}

Scolopax semipalmata, Lath. Ind. II. p. 722. Gmel. Syst. I. p. 659.

Semipalmated Snipe, Aret. Zool. II. p. 469. n. 380. tab. XX. - Lath. Syn, V. p. I52. 12. 22. Vers. Germ. V. p. I23. 21. 22.

*) Frasertim maxilla inferior. 
Anne huc pertinet The cinereous Godwoit, Pen. Br. Zool. II. p. 442 . 17. I80, t. 66 ?

\section{DESCR IP TIO.}

Longit. circa I4 poll. Rostr. $2 \frac{2}{8}$ poll. A flexura alx ad apicem $7 \frac{\pi}{2}$ poll. Cauda $2 \frac{5}{8}$ poll. Nuda pars

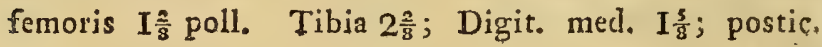
ix poll.

Rostrum fuscum, robustum, compressum, in medio aliquantum recurvatum, maxillis dehine teretibus , acutis, fere subulatis. (Rostro sequenti simili, sed robustius). Pedes fusci. Digiti semipalmati, membrana exteriore tamen majore) et secundum totam longitudinem marginati.

Var. A. Caput, cervix et jugulum fusco-cinerea, striis parvis albidis. Dorsum et alarum tectrices fusca marginibus pennarùn pallide griseo 1. albido maculatis. Peetus, abdomen et crissum alba, lutescenti leviter tincta. Remiges primores nigricantes, a basi ad medium albae. 5-IO albo quoque marginatx; secundarix albx; intimx longx, colore dorsi. Cauda xqualis. Rectrices laterales albæ, versus apiccm cinereofusco variegatx; intermedix basi albx, versus apicem cinereo-fuscr fasciis transversis luteo-albis. Tectrices caudx superiores albx, lituris cinereis cum margine parallelis-

Vai. B. Caput et collem striis albis fuscisque. Dorsum et alarum tectrices cinerea maculis majoribus nigris, transversis 1. sagittatis; subtus tota alba lutescenti tineta maculis in jugulo et pectore parvis rotundatis fuscis, in lateribus pectoris fasciis transversis fuscis. Gula alba immaculata. Cauda cinerea nigro transfersin fasciata - Cetera ut in priore descripta. 
OBSERVATIO.

Specimina descripta, quorum alterum ex America remissum est, alterum in Uplandia Svecia provincia occisum, in Museo ditissimo Iil. Barunis Paykuld, servantur, Quoad formas et proportiones partiun constantiorum excellenter congrumb ; quoad colores aliquantum differunt, et ni fallor var. B. junior est avis, vel saltem habitu autumnali vęstita,

\section{GLOTTIS CHLOROPUS, miki.}

DiAgn: Snpra sarurate fusca marginibus pennaruna luteo-albidis. Subtus alba. Remiges primores nigro-fuscæ; secundariæ fusco - cinereæ, latere interiore albo variegate. Cauda alba, fusco transversim striata.

\section{SYNONYMA.}

Scolopnx Glottis, Lin. Faun. Sv. p. 6I. n. I7I. Syst. Nat. I. p. 245. n. 10. Gmel. Syst. I. p. 664. Lath. Ind. Ir. p. 720. Retz. Faun. p. I77.

Limosa grisea, Briss. V. p. 267. n. 2. t. 23. f. I. La Barge grise, Buff. Pl. enl. 876 .

Totanus Glottis, Bechst. N. D. 2te Auf. 4 B. I Abth. p. 249.

Totanus Chloropus, Mey. Vögel Liv. u. Esthl. p. 198. Totanus fistulans, Bechst. 1. c. p. $24 \mathrm{I}$.

Green Shank, Pen. Br. Zool. II. p. 445. n. I83.

\section{DESCRIPTIO.}

Longit. I3 $13 \frac{\pi}{2}$ poll. Rostr. $2 \frac{\pi}{2}$ poll. A fiexura alze ad apicem 7 poll. Cauda $2 \frac{\pi}{2}$ poll. Tibia $2 \frac{\pi}{2}$; 
nuda pars femoris $I \frac{x}{2}$ poll. Digit. med. $I \frac{s}{s}$; postic. $\frac{2}{8}$-poll.

Rostrum cinereum robustiusculum, compressum, in medio sursum curvatum, maxillis medietate exteriore teretibus (marginibus inflexis) apice acutis nigro - fuscis. Pedes cinereo-olivacei. Digitus exterior inter. medio membrana basi connexus.

Caput collumque supra et Dorsum fusca marginibus pennarum albis 1. Iuteo-albidis. Frons, latera capitis et colli alba striis longitudin. fuscis. A rostro supra oculos fascia alba et alia fusca ad oculos. Dorsum posticum, Gula, Peêus, Abdomen et crissum alba. Remiges primores nigro-fusca, prime rachis nivea, I-5 immaculata; 6,7 \&c. apice albo marginatx; secundarix fusco-cinerex margine interiore albo varix; intimx longæ, colore dorsi. Tectrices alarum inferiores, Cauda alba lineis transversis fusois; in lateralibus rarioribus.

\section{HIS TORIA.}

Habitat æstate in plagis patriæ borealibus ad lacus; paludes et flurios. Autumno et vere australiores transmigrans, in Scania interdum obvenit. Timida admodum, evolans vocem sibilantem Tudut! fere sonantem edit. Vescitur pisciculis et Testaceis. 


\section{GENƯ \\ T O T A N U S, mihi.}

Rostram longum (capite aliquantum longius), gracile, debile, rectum, maxillis apice duris, (plerisque) acuminatis et inflexis.

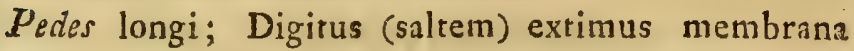
intermedio connexus ad primam articulationem.

CLXVII. TOTANUS FUSCUS, Bechst.

DIAGN: Supra saturate fusca undulis vel maculis albis, subtus cinereo-fusca albo-undulata. Cauda fusca lineis transversis albis.

\section{SYNONYMA.}

Avis habitu astivali:

Scolopax fusca, Lin. Syst. I. p. 243. 11. 5. Gmel. Syst. I. p. 657. Lath. Ind, II. p. 724. n. 35.

Limosa fusca, Briss. V. p. 276. t. 23. f. 2. (optime descripta).

La Barge brune, Buff. Ois. VII. p. 508. Pl. enl. 875. (figura optima). - Vers, Germ. Otto XXVI. y. 253-255.

Dusky Snipe, Pen. Arct. Zool. II. p. 47I. C. - id. Sup. p. 68. - Lath. Syn. V. p. 155. n. 30. Vers. Germ. V. p. I26. 
Toramus fuscus, Bechst. N. D. 2te Auf. 4 B. I Abth. p. 2 I2.

Tringa atra, Gmel. Syst, I. p. 673. n. 26. Lath. Ind. II. p. 738 .

Avis habitu autumnali:

Scolopax fusca, Tengmalm Wet. Acad. Handl. 1783. p. 48. Retz. Faun. p. 173.

Totanus maculatus, Bechst. N. D. 2te Auf. 4 B. I Abth. p. 203.

Scolopax Totanus, Gmel. Syst. I. p. 665. 11. 12.

Totanzs natans, Bechst. 1. c. p. $22 \%$.

\section{DES CRIPTIO.}

Longit. avis I2 - I3 poll. Rostr. $2 \frac{3}{8}$ poll. A flexura alæ ad apicem $6 \frac{\mathrm{r}}{2}$ poll. Cauda $2 \frac{5}{8}$ poll. Pars nuda femoris $I \frac{r}{2}$ poll. Tibia $2 \frac{3}{8}$ poll. Digit. med. $I_{\frac{\gamma}{2}} ;$ postic. $\frac{3}{8}$ poll.

A. AVIS SENIOR HABITU ESTIVALI:

Rostrum gracillimum, reetum, acutum, nigrum, maxilla inferiore basi incarnata, superiore parum longiore apice inflexo. Iris nigro - fusca. Pedes rubri. Digitus extimus intermedio membrana junctus ad primam articulationem.

Caput et collum tota saturate fusca. Dorsum et alæ supra saturate fusca vel nigricantia marginibus pénnarum albis. Pectus, Abdomen et crissum fusca cinereo rincta undulis angustis albis rarioribus. Dorsum posticum album. Remiges primores fuscx latere interiore albo-variegatx, rachi prima nivea; seeundarix fuscocinerex fasciis albis. Tectrices alarum inferiorcs albr. Cauda ejusdenque tectrices lineis transversis albis et fuscis fasciztz. 


\section{B. A.VIS HABITU AUTUMNALI:}

Magnitudo, forma et proportio partium exacte precedentis. Rostrum fuscum subtus basi incarnatum. Iris nigro - fusca. Pedes virescenti-lutei.

Caput supra et cervix cinereo-fusca striis minutis albis. Dorsum, Scapulares et alarum teetrices saturate fusca maculis albis, in dorso minoribus, in alis aliquantum |majoribus et frequentioribus. Dorsum posticum album. Fascia alba a naribus supra oculos. Lora fusca. Orbita alba. Genx et jugulum alba striis minutis fuscis. Gula alba immaculata. Pectus et abdomen albiea undulis numerosissimis einereo-fuscis. Remiges primores nigro-fusce latere interiore albido variegatæ, prima rachi nivea; secundarix cinereo-fuscx, latere exteriore maculis, interiore fasciis albis numerosis. Tectrices inferiores albæ maculis justa marginem fuscis. Canda ejusque tectrices superiores cinereo. fusca lineis transversis numerosis albis fasciata. Tactrices inferiores albz fasciis transversis fuscis.

\section{HISTORIA.}

Habitat astate in terris borealibus. Norvegia et Lap. ponix, ibique ad ripas fluviorum et lacuum inque pa. ludibus degit. Demigrans autumno in Scania quoque obvenit. Vescitur vermiculis et testaceis minoribus. Jam initio mensis Augusti pullos simul cum parentibus per familias hine inde errantes intra circulum pos larem vidi - ibique nidulari quotannis referebatur avis. Neque ovorum numerum nee colorem quisquam mihi certa fide determinare poterat. 


\section{CLXVII. 'TOTANUS CALIDRIS, \\ Bechst.}

Diacn: Supra fusco-cinerea maculis striisque nigris, subtus alba maculis longitudinalibus nigris. Remiges secundariæalbr. Rectrices albo nigroque transversim fasciatx. Pedes rubri.

\section{SY NONYMA.}

Totanus Calidris, Bechst. N. D. 2te Auf. 4 B. p. 2 I6. Scolopax Totanus, Lin. Fn. p. 59. 11. I6\%. Scolopax Calidris, Lin. Syst. I. p. 245. n. II. Retz. Faun. p. 177. n. I44. Gmel. Syst. I. p. 664. Lath. Ind. II. p. 722. 11. 25.

Totanus ruber, Briss. Orn. tom. V. p. I92. n, 4.

Le Chevalier à pieds rouges, Buff. Ois. VII. p. 5I3. t. 28. Vers. Germ, Otto XXVII. p. I2. cum fig. Pl. enl. 845.

Red Shank, Pen. Br. Zool. II. p. 446. n. I84. t. 65.

Lath. Syn. V. p. I50. Vers. Germ. I2I. n. 20.

Totanusstriatus, Briss. Orn. V. p. 196. t. I8. f. I.

Le Chevalier rayé, Buff. Ois. VII. p. 5I6. Vers. Germ,

Otto XXVII. p. 2I. cum fig. Pl, enl. 82\%.

Funior: Tringa striata, Lin. Syst. I. p. 248. n. 5. Lath. ind. II. p. 733. n. 24.

Tringa Ganbetta, Lin. Fn. Sv. p. 63. n. I77. Lin.

Syst. Nat. p. 248. n. 3. Gmel. Syst. J. p. 67 I.

Observatio. Sed The Gambet, Pen. Br. Zool. II. p. 465.

I. 70. non ad hane Speciem pertinet; est enim junior Totanus Pugrax.

Vesatoribus: Rëdbena. 


\section{DESCRIPTIO.}

Avis habitui cestivali:

Longit. II poll. Alre extensx 20 poll. Rostr. I $\frac{6}{6}$ poll. A flextra alr ad apicen 6 poll. Tibir 2 poll. Digit. med. I $\frac{3}{8} ;$ postic. $\frac{5}{x^{6}}$ poll. Nuda pars femoris fere I poll.

Rostrum gracile, rectum, acutum, nigrum, basi subtus rubrum; mandibula superiore parum longiore, apice inflexa. Nares lineares, pervix. Pedes pallide rubri unguibus nigris. Digitus extimus majori, intimus minori membrana intermedio adnexus.

Caput supra nigricans striis fusco - cinereis. Collum striis longitudinalibus nigris, marginibus pennarum postice griseis, antice albis. Dorsum anticum, Scapulares et techrices dorso contigux fusco-cinerea ma. culis et lineis ad raches, striisque transversis nigris. Dorsum medium album. Gula, jugulum, pectus et abdomen alba maculis parvis longitudinalibus nigris, in jugulo frequentioribus, in abdomine raris. Remiges $5-\sigma$ primores nigr $x$, latere interiore a basi albid $\approx$, rachi primæ alba; sequentes fusce latere interiore alba apicem versus albæ fusco undulatx; secundaria alba. basi fuscr. Rectrices et Tectrices earum superiores er inferiores alba (medix Rectrices pallide grisex) nigro transversim fasciatz.

'Avis junior: Pedes et basis rostri sordide lutea. Caput supra et Dorsum fusca marginibus pennarum pallide Inteo-griseis; subtus alba jugulo et superiore parte pectoris cinerascentibus, maculis parvis longitudinalibus fuscis.

\section{HIST ORIA.}

Migratoria avis redit jam fine mensis Aprilis. Habitat æstate in paludibus, in littoribus marinis, juxta 
Aumina, lacus, fossas turfosas et stagna vulgaris, 11011 solum in Svecia mexidionaliori, sed etiam in Norvegia et Lapponia. Latera alpium incolit usque in regione betulina. Nidulatur inter gramina locis nliginosis. Ova 4 olivacea maculis fuscis extremitate cras* siore confluentibus. Hostem ad nidum vel ad pullos adpropinquantem, clamore acuto et sonoro supervolitat. Vescitur Insectis, vermiculis, testaceis. Caro sapica.

\section{TOTANUS OCHROPUS, mihi.}

DiAGN: Supra saturate fusca viridi nitens, punctis pallidis adspersa; subtus et supercilia alba. Rectrices laterales albæ immaculatæ; ceteræ albre fasciis versus apicem nigris。 Rachis prime Remigis penna concolor. Pedes cinerei.

\section{SYNONYMA.}

Tringa Ochropts, Lin. Fn. Svi p. 64. n. 180. (cauda color erronee descriptus). Syst. Nat. I. p. 250. n. I3. Retż. Fn。 p. I86. 1n. I54. (Descriptio eironea). Lath. Ind. II. p. 729. n. I2. Briss. Orn. V. p. I77. t. I6. f. I. Gmel. Syst. I. p. 676.

Tringa'Glireoln, Otto 1. c. p. 73.

Le Becasseau ou Culblanc, Buff. Ois. VII. p. 534. Vers.

Germ. Otto XXVII. p. 62-73. cum fig. Pl. enl. 843.

Tringa littorea, varietas, Brunn. Bor. p. 53. 1. I79.

Green Sindpiper, Pen. Br. Zool. II. p. 168 n. 201 . Aret. Zool. II. n. 389, Lath. Syin. V. p. 170. 1. 12. Vers. Germ. V. p. I4I. 
Puiktirter Strandläufer, Bechst. N. D. 2te Auf. 4 B. I Abth. p. 283. Meyer Liv. u. Esthl, p. 202.

Venatoribus perperam: Enkel Beckasin。

\section{DESCRIPTIO.}

Longit. $8 \frac{\gamma}{2} \cdot 9$ poll. Ala extensæ I8 poll. Rostr. I $\frac{3}{8}$; A fiexura alæ ad apicem $5 \frac{\mathrm{T}}{2}$; Canda 2 poll. Nuda pars femoris $\frac{5}{8} ;$ Tibix $I \frac{3}{8} ;$ Digit. med, II $\frac{\pi}{8} ;$ postic. $\frac{3}{3}$ poll.

Rostrum gracile, rectum, acutum, viridi-fuscum, apice nigro: maxilla superiore aliquantum longiore apice parum inflexa. Iris fusca. Pedes pallide plunbei. Digitus extimus intermedio membrana junctus ad primam articulationeun.

Caput supra, Dorsum, Scapulares et alarum tectrices saturate fusca, dorso, scapulis et alarum teetricibus viridi nitentibus, punctis pallide testaceis adspersis. Uropygium medium nigro-cinereum. Teètrices caudæ superiores albæ, basi nigricantes. Fascia alba supra oculos. Lora fusca. Genæ, latera colli collum. que antice alba maculis minutis fuscis, superne acuminatis. Gula, peetus et abdomen alba immaculata. Remiges primores eum teetricibus proximis nigra uni. colores rachibus splendide nigricantibus; secundaria nigro-fuscr unicolores; intimx longx, molles, colore dorsi maculis parvis triquetris pallide testaceis marginatx. Alarum tectrices inferiores nigricantes undu* lis albis pulchre ornatx. Cauda cum alis complicatis xqualiter extenditur. Reetrices laterales I-I plane albæ, 2. 2 albæ macula nigra in latere exteriore versus apicem; lix macula nigrx in mediis quoad numerum augentur ita ut 6 . 6 . sint albre fasciis $5-6$ nigris. Tectriees inferiores albr, immeculatx, Avis hibith astivali.

Nilsson Orwith. Pिars $i \dot{I}_{b}$ 


\section{OB SERVATIO.}

Moschum olet, etiam diu post mortent farcta et exsiccata.

\section{HISTORIA.}

Migratoria avis ad nos redit post medium Aprilem. Ad margines fossarum, stagnorum, lacuum in arena vel luto siccato passim. Timida evolat sono acute sibilante. Ova 3.5 albescenti viridia maculis fuscis.

\section{TOTANUS GLAREOLA, miki.}

DragN: Supra fusca maculis parvis albis; Pectus, abdomen et supercilia alba. Rectrices laterales extus maculis fuscis; media albo et fusco fasciatæ. Rachis primæ Remigis nivea; Pedes fla vescenti-virides.

\section{SYNONYMA:}

Tringn Glureolit, Lin. Fn. Sv. p. 65. n, 184, Gmelo Syst. I. p. 677. Lath。 Ind. II. p. 730. 12. 13. Wood Sandpiper, Lath. Syn. V. p. 172, n. 13. Vers。 Germ. V. p. 143 .

Wald-Strandläufer, Bechst. N. D. 2tè Auf. 4 B. I Abth. p. 291. Meyei: Liv، u。 Esthl, p. 203.

Funior:

Tring Littored, Lin. Faun. Sv. p. 66. n. I85. Gmel。 Syst, I. p. 677 B. Lath. Ind. II. p. 73I. 11. I5。

\section{OBSERVATIO, I.}

Synonyma hujus, et antecedentis speciei sxpissine confusa sunt, etian ab Ornithologis recentissimis. Bechst. 1. c. p. 286 ef Meyer 1. c. p. 203 Tringan littorean Fn. Sr. cum Tringa 
Ochropo conjungunt, Sed Tringam littoream Finne Svecice ת. 185 non ad Ochropurin sed ad Glareolam pertinere, cuique descriptionem in Fauna perlegenti, ut videtur, peršpicuun erit "Teitrices inferiores ala exalbida lineis transwersis cinereis; Remiges setundarie apite albescunt; Remex primus rachi nivea gaudet ${ }_{t r}$ \&c. excellenter in hanc, ininime vero in Ochropum quadrant. Tringd bittored Otto $I_{0} c_{0}$ p. 24. cum fig. est junior Totanus Pिigniax。

\section{OBSERVATIO. 2.}

Tringam Glareolatn Retz. Fn, p. 186. n. 155. quxe a pletiso que ad hanc jam descriptam speciem ducitur, citare equidem non potui; Plurima enim hujus descriptionis ex Lechei Halfent kel Beckasin (in Adversariis) depronta sunt, er ut perspicutus cst, ad Scolopacen Gallittulant pertinent.

\section{DESCRIPTIO.}

Long. $7 \frac{\hat{\sigma}}{8}-7$ poll. Alæ extensæ $15 \frac{\hat{\sigma}}{3} ;$ Rosti. I $\frac{x}{8} ; A$ flexura alx ad apicem 5; cauda 2 poll. Nuda pars femoris $\frac{\sigma}{8}$; Tibix $I \frac{1}{2}$; Digit. med. $I \frac{2}{8}$; postic. $3 \frac{\pi}{8}$ poll.

Rostrum gracile, rectum, subteres, nigrum, basi subtus virescente. Maxilla superiore aliquantum lon. giore, apice parum inflexo. Iris fusca. Pedes fla. vescenti-virides. Digitus extimus intermedio membrana basi-junctus.

Caput supra, Dorsum, Scapulares et alarum tectrices fusca maculis albis plerumque triquetris ad margines pennarum. Collum supra fuscum striis longitudinalibus albis. Fascia alba supra oculos. Lora fusca. Genx, latera colli, collum antice et superior pars pec. toris alba maculis fuscis numerosissimis. Latera pectoris alba striis transver'sis fuscis. Ceterum peetus, abdomen, crissum et uropygium alba, immaculata. Tectrices caudx superiores albr, longiones maculis apice fuscis. Remiges saturate fuscr, prime rachis 
nivea; secundarix albo marginatæ latere interiore ma. culis albis !obsolete fasciato; intimx longx, molles, colore dorsi maculis margine triquetris, albis. Tectrices alarum inferiores albr maculis transversis fuscis. Cauda cum alis complicatis aqualiter extenditur. Rectrices medix binæ longiores, acutx; laterales (I - I. 2 2.) albre maculis quinque fuscis in latere exterioie; ceteræ fasciis albis et fuscis alternis. Teetrices inferiores longitudine Reetricum, albæ lineis et maculis fuscis.

Avis junior: Maculx superiorum partium rufescentes; collum antice et peetus superius cinerascenti-fusco nebulosa.

OBSER VATIO.

Dignoscitur hæc Species ab antecedenti, quacum sæpius confusa fuit: Colore Tectricum alarum inferiorum; rachi prime Remigis nivea; Remigibus secundariis albo cinctis; Rectricibus dusbus mediis longioribus acutis; Tectricibus cande inferioribus macuintis of .

\section{HIS TOR IA.}

Habitat in paludibus sylvaticis, pratisque depressis passim. Nidulatur non minus in Scania quam in superiore Svecia et in Norvegia. Ova vero nondum inveni.

CLXXI. TOTANUS HYPOLEUCOS, mithi.

DIAGN : Supra fusca olivaceo nitens rachibus striisque transversis nigris; subtus alba collo cinerascente lineis fuscis. Remiges plurime macula magna alba in medio lateris interioris. 


\section{SYNONYMA.}

Tringa Hypoleucos, Lin. Fn. Sv. p. 65. n. I82. Retz. Fn. Sv. p. 188. n. 157. Lin. Syst. 1. p. 250. Lath. II. p. 734. n. 28. Gmel. Syst. I. 2. p. 678.

Tringa Canutus, Lin. Fn. Sv. p. 65. 1. I83. Retz. Fu.

p. 189. n. I58. (Descriptio bona).

In Guignette, Buff. Ois. VII. p. 540. Vers. Germ. Otto XXVII. p. 77. cum fig.

Petite Alouette de mer, Pl. enl. 850.

Conmon Sindpiper, Pen. Br. Zool. II. p. 470. 11. 204.

t. 7I. Lath. Syn. V. p. I78. Vers. Germo; V. p. 148.

Trillernder Strandläufer, Bechst. N. D. 2te Auf, 4 B. I Abth. p. 295. Meyer \. c. p. 204.

Svecis: Strandsittare.

\section{OBSERVATIO. I.}

Tringan Canutum Lin. Fn. Sv. et Retz. Fn, ad hanc Speciem pertinere ex Descriptione liquet. Tringa $C_{\text {anutus }}$ vero Lin. Syst. Nat. et ceterorum antorum longe alia est avis, Vide Art, Tringa ferruginea jun.

\section{OBSERVATIO. 2.}

Bechstein 1. c. p. 302 , et Meyer 1. c. p. 204. nomine Tringa Cincli hanc Speciem describunt et Linneum citant, sed temere. Cinclus enim Linnai, ut infra monstrabo, longe alia est, numquan cum hax Specie confundenda, Avis. Vide Art. Trings Cinsclus.

\section{DESCRIPTIO.}

Longit. $7 \frac{\mathrm{r}}{2}$ poll. Rostrum $\mathrm{I} \frac{1}{8} ;$ alx extens $\mathrm{I}$ ped.

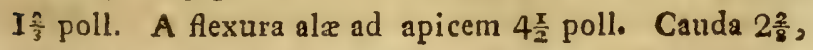
Tibir I poll. Digit. med. I poll. postic. $\frac{2}{3}$ poll. 
Rostrum tenue, rectum, tereti-compressum, grie seum apice nigro, maxillis fere æqualibus apice aliquantulum infléxis. Pedes virescenti-cinerei. Digitus extimus interrmedio membranâ junctus ad primam articulationem,

Caput, Dorsum, Scapulares et tectrices alarum calt: dæque superiores grise - fusca viridi nitentia; in capite et dorso antico striis longitudinalibus fuscis ad raches; in dorso postico, alarum caudxque tectricibus et in uropygio striis quoque transversis nigro-fuscis. Stria parva alba a rostro super oculos, aliaque fusca inter rostrum et oculum. Gula, pectus et abdomen alba immnaculata. Collum inferius antice album, lineis longitudinalibus, brevibus, fuscis, Remiges nigro-fusc primr immaculata rachi pone apieem albicante; 2 - I0 maculâ alba in medio vexilli interioris; I I - I 8 in me. dio et apice albx; intimx longx, molles, colore dorsi. Cauda cuneato-rotundata, Rectrices medir qvatuol colore dorsi striis transversis fuscis; ceter $æ$ albæ nigra fasciatx apice albo. Tectrices alarum inferiores albx, ad flexuram alæ fusco maculatz.

Jusnior: Fascix nigræ pone apices pennarum dorsi angua stiores sunt et fimbriis Intescentibus marginatx. Col. lum antice cinerascens lineis fuscis vix conspicuis. Rectrices mediz fasciis nigriçantibus destituţ.

\section{HISTORIA.}

Habitat ad lacus, fluvios, stagna aliasque aquas dulg eas ubique frequens. In littoribus marinis raro obvenit. Ad nos venit fine mensis Aprilis, Caudam Mo= tacillx instar motitat. Ova 4.5 pallida, luteo-virescentia, punctis et maculis fuscis adspersa, presertim in extremitate crassiose. 


\section{CLXXII, TOTANUS PUGNAX*).}

Dingn: Rostrum pone apicem depresso-incrassatum. Ungues longi, acuti. Remiges fuscæ vel nigræ rachibus albis, teetricibus minoribus fusco-cinereis. Rectrices cinereæ, laterales tres immaculata. Abdomen et crissum alba.

Mas vere: Papillis faciei granulatis, carneis, et collari e pennis longis ornatur.

Femina vere: Grisea maculis nigris.

Avis Autumno: Supra nigra marginibus pennarum ferrugineis. Collum et pectus cinerascentia, rufescenti tincta, Fascia alarum obliqua alba,

\section{SYNONYMA.}

Tringa pugnax, Lin. Faun. Sv. p. 62. 11. 175. Retz. Faun. p. 179. 11. I47. Syst. Nat. I. p. 247. n. I. Gmel. Syst. I. p. 669. Briss. V. p. 240. 18. t. 22. f. I. 2. Lath. Ind. II. p. 725.

Combattant ou Paon de neer, Buff. VII. p. 52I. 't. 29. PI. enl. 305. 306. Vers, Germ. Otto XXVII, p. 34 cum fig.

Ruff and Recue, Pell. Br, Zool. II. p. 457. n. 192. t. 69. Arê. Zool. II. p. 479. A. Lath. Syn. V. p. I59. I. Vers. Germ, V. p. I3 I.

*) Hac avis quasi in medio est inter Totanos et Tringas. Fia birus enim Totani est; forma rostri Tringec. Magis viva: est Tringis, minus fortasse Totanis, si macem verno tempore exceperis. Cun Tringis non bene jungitur; probius cuin Totanis. Forte vero rectius propriun constituat genus. 
Kämpfender Snndläufer, Beshst. N. D. 2te Auf. 4 B. I Abth. p. 266. Meycr Vögel Liv. u. Esthl. p. 201.

Avis habitu autumnali et junior:

Tringa grenovicensis, Lath. Ind. II. p. 731. n, I6.

Tringa rufescens, Bechst. 1. c. p. 332.

Totanus cinereus, Briss. Om, V. p. 203. t. I7. f, 2.

Chevalier varié, Buff. Ois. VII. p. 517. Pl. enl. 300. (junior).

Tringa variegata, Brünn. Bor. p. 54. n. I81. Femina senior:

Chevalier Commun, Buff. Ois. VII. p. 5 IT. Pl. enl. 844. Vers. Germ. Otto XXVII. p. 7. fig. Suçis: Brushane.

\section{DESCRIPTIO.}

Mas major feminâ, Longit. I2.I3 poll. Alæ ex tensæ 2 ped. Rostr. I $\frac{T}{2}$ poll. A flexura alx ad apicem $7 \frac{r}{2}$ poll. Cauda $2 \frac{r}{2}$ pall. Nuda pars femoris $I \frac{r}{2}$ poll. Tibia $2 \frac{\pi}{8}$ pall. Digit. med. I\% poll. postic. 몽 poll.

Rostrum rectum basi compressum, pone apicem depresso - incrassatum. Colore variat jam fusce, jam carneo, jam luteo, apice nigro, basi subtus colore pedum. Iris fusca. Pedes longi, jam aurantii, jam crocei, jan viridi-lutei et juniorum autumno viridicinerei. Digiti margine serrati; extimus intermedio membrana connexus ad primam articulationem. Ungues nigri, compressi, longiores et acutiores quam in congeneribus.

Mas vernali et estivali habitu:

Facies papillis granulosis carneis, et collum ornatur sollsi e pennis longis utrinque divergentibus. 
Variat coloribus ita ut inter magnum numerum 'vix duo sibi perfecte siniles inveniantur. His tamen notis omnes fere conveniunt: Remiges sunt fusce vel nigricantes rachibus albis. Teetrices alarum fusco-cincrex. Rectrices cinerex intermediis pone apicem maculis vel fasciis nigris, lateralibus immaculatis. Abdomen et crissum alba. Præterea innumeræ obveniunt varietates; sunt tamen præcipux:

a) Corpore nigro, nitore violaceo vel purpureo. Collo antice undulis ferrugineis. Dorsi pennis griseomarginatis.

b) Collari ferrngineo undulis nigricantibus, corpore supra nigro maculis ferrugineis et albis.

c) Collari albo undulis nig:icantibus; Dorso fusco ferrugineo et albo immixtis.

d) Collari albo, Dorso et pectore nigris æneo nitentibus.

FEMina minor, 10 poll. longa. Alæ extensx $19 \frac{\mathrm{T}}{2}$ poll.

Habitu estivali. Rostrum nigricans. Pedes oliva. ceo-lutei.

Corpus supra pallide griseum maculis nigris, (singula penna nigra margine pallide vel rufescenti-griseo). Collum antice et latera pectoris rufo-cinerascentia pennis nigris immixtis. Abdomen et crissum alba. Remiges saturate fuscr rachibus albis, latere inreriore versus basin albo-cinerex, secundarix cinereo-fusca apicis margine exteriore albo. Rectrices fusco-cinerex, laterales pallidiores apice albido marginatz.

Avis habitu nutumnali:

Rostrum nigricans basi subtus et pedibus jam vire* scenti-luteis jam viridi-cinereis.

Caput supra rufum striis nigris. Collum postice, Dorsum et Scapulares nigra marginibus pennarum ferrugineis. Facies grisco-alba punctis fuscis. Gula 
sordide alba immaculata, Jugulum et pectus superius pallide cinerea rufescenti tincta. Pectus, abdomen, crissuin, tectrices inferiores caudæ et laterales superiores alba. Remiges nigricantes rachibus albis, se. cundaria albo marginatx, intima colore dorsi; tectrices alarum saturate cinerex, medix pallide-ferrugineo marginatæ, majores apice albæ. Rectrices fusco-cinerex, laterales tres apice albo marginatæ, cetra apice testaceo maculis vel fasciis nigris,

\section{HISTORIA.}

Habitat in pratis depressis paludosis et uliginosis per totam Scandinaviam tam in Scania quam in Lapponia passim frequens. E migratione jam initio mensis Aprilis redeuntes, de feminis inter se vehementer pug. nant mares. Femina 3-4 fovet ova griseo-alba rufo maculata. Pullis educatis plures familix associatæ itinera versus australes terras instituunt. Hoc tempore maximis gregibus haud raro obveniunt in littoribus nostrị marinis*).

* Intra circulum arCicum adhue medio mensis Augusti (1816) degens, prima hrc erat avis, quam e Septentrione vergus meridiem gregatim tendentem observabam. 


\section{GENUS. \\ VA N E L L U S *)}

Rostrum capite brevius, tenue, compresso-teres, pone apicem acutum, compresse-incrassatum. Nares lineares, laterales.

Pedes Tibia digito medio longior. Digitus extimus medio membrana a basi ad primam articulario. nem connexus. Posticus brevis elevatus.

Capui crassum fronte elevata.

\section{VANELLUS CRISTATUS,} Bechst.

Diagn: Caput cristarum et collum antice nigrum. Dorsum obscuro-viride. Cauda nigra, medierate interiore, abdomine et pectore albis.

\section{SYNONYMA。}

Tringa vanellus, Lin, Faun. Sv. n. I76. Syst. Nat. I. p. 248. 1. 2. Retz. Faun, p. I80. 11. I48. Gmel. Syst. I. p. 670, 2. Lath. Ind. II, p. 726. n. 2. Briss. V. p. 94. I. t: $8, \mathrm{f}, \mathrm{I}$.

Le Vrnnenu, Buff. Ois, VIII. p. 48. t. 4. Pl. enl. 242 。

Vers, Germ, Otto XXVIII. 5. cum fig.

*) In serie naturali proxime ad Chavadrios accedit hoc Genur. 
Laprping, Br. Zool. II. n. I90. Arct. Zool II. p. 480. D. Lath. Syn. V. p. I6I. n. 2. Vers. Germ. Bechst. V. I 33.

Vanellus cristatus, Bechst. N. D. 2te Auf. 4 B. I Abth. p. 346. Meyer Vögel Liv. u. Esthl. p. 2 II. Svecis: Wipa, Toppwipa.

\section{DESCRIPTIO.}

Long. I2 $\frac{1}{2}$-I3 poll. Alx extens 2 2. ped. 5 poll. A flexura alæ ad apicem 9 poll. Rostr. I poll. Cauda fere 4 poll. Nuda pars femoris $\frac{6}{2}$ poll. Tibia 2 poll. Digit, med. cum ungue $I_{\frac{3}{8}}$ poll. postic. $\frac{2}{3}$ poll.

Rostrum nigrum. Osuli magni prominentes iridibus avellanis. Pedes incarnati unguribus nigris. Digiti marginati, extimus medio ad primam articulationem membranâ connexus.

Crista occipitis $3-4$ pollicaris e pennis elongatis tenuibus, dependens, apice adscendens. Vertex, crista, frons, genæ et collum antice a gula ad sternum nigra. Supra oculos stria alba nigro immixto, et sub oculis stria nigra. Latera capitis de cetero, et colli alba. Dorsum et Scapulares obscure viridia, nitentia pennis purpureis ad latera insertis. Uropygium satu. ratius viridi-fuscum, in purpureum inclinans. Tec. rices alarum atro - virides chabybeo colore nitentes. Remiges nigrx, extimæ tres vel quatuor pone apicem albidx; secundarix basi albx; intimx colore dorsi. Tectrices inferiores Remigum secundariarum albæ; primarum nigra. Pectus et abdomen alba. Crissum et tectrices caudx inferiores pallide rufescentia. Cauda squalis exteriore medietate nigra, interiore alba apice pallido, rectricibus extimis albis jam immaculatis, jam macula nigra in latere interiori pone apicem. Tectriecs cauda superiores proxima, sufa. 
Femrna dignoscitur crista breviore, gulâ et jugulo albo maculatis.

Junzor: Rostrum nigrum. Pedes fusco-virides. Pileus nigro-viridis crista brevi vix pollicari. Gula et jugulum alba. Latera capitis sordide albida. Stria sub oculis nigra. Initium pectoris nigrum. Dorsum et Scapulares obscure viridia nitentia, marginibus pennarum sordide luteis; alarum tectrices insuper obscuriores pennis subpurpuyeis immixtis. Cetera antecedentis.

\section{HIST ORIA.}

Habitat in pratis depressis et palustribus; in cam. pestribus quam sylvaticis locis longe frequentius obve. nit. In Scania campestri copiose. In Hallandia rarius. In provinciis superioris Sveciz vix umquam obvenit. Mature ex migrationibus redit jam circa vigesimum diem Mensis Februarii. Vietitat Insectis, vermiculis et restaceis. Nidulatur in pratorum grumulis. Ova 3.4 olivacea maculis majoribus minoribusque nigris, in extremitate crassiore confluentibus.

\section{VANELLUS MELANOGA. STER, Bechst.}

Dragn: Supra nigricans maculis transversis albis。 Subrus nigra (senior); vel alba peetore griseo nebuloso (junior). Cauda alba fasciis nigris, Pennæ axillares longæ, nigræ.

\section{SYNONYMA。}

Yunellus Melnnogaster, Bechst. N. D. 2te Auf. 4 B. I Abth. p. 356. Meyer Vögel Liv. u. Esthl, p. 2 12 
Senior:

Tringa Helvetica, Lin. Syst. 1. p. 250. n. I2. Gmel.

Syst. I. p. 676. Lath. Ind. I1. p. 728. n. I0.

Vamellus Helvericus, Briss. V. p. 106. 4. t. 10. f. I.

$V$ annedu de Suisse, Buff. VIII. p. 60. Pl. enl. 853.

Vers. Germ. Otto XXVIII. p. 25 et 30 . cum fig. Swiss Sandpiper, Arct. Zool. II. p. 396. Lath. Syn. V. Funior:

p. 167. 10. Sup. p. 248. Vers。Germ.V. 138.

Tringa Varia, Lin. Syst. I. p. 252. Gmel. Syst. I. p. 682.

Tringa Squatarola, Gmel. Sysst。 I. p. 682, Lath. Ind. II. p. 729. Bechst. N. D. 2te Auf. 4 B. p. 360.

Varellus griseus, Briss.V. p. IOO. 2. t. 9. f. I.

Vanrien Pluvier, Buff. VIII. p. 68.

Vanneau Gris, P1. enl. 854. Otto XXVIII. p. 50.58. Vanneau Varié, Pl. enl. 923.

Grey Sandpiper, BroZool. II. p. 456. is. I9I. Lath。 Syn. V. p. 168, n. II. Sup, p, 248. Vers. Germ。 V. p. I39. n. II. cum var. A.

\section{OBSERVATIO.}

Tringa Squatarola, Lin. Faunx n. 186. hue non pertinet. Descriptio enim a Domino Leche recepta est sed nimis mutilata. Dornini Lechei Angusti Snäppa a Linneo citata, est Limosa Rufa habitu autumnali, qua in Lechei Adversariis hoc inso nomine complete deseripta invenitur. Hæc quoque nota in Fauna "Pedes incisuris transyersis, ostendic Linnei avem non ad Variellos, pere tinere.

\section{DESCRIPTIO.}

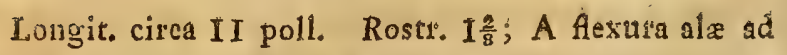
apicem 8 poll. Cauda 3 poll. Nuda pars fenoris 
fere I poll. Tibia 2 poll. Digit。 med. $I \frac{3}{8}$, posticus brevissimus.

Rostrum nigrum. Iris fusca. Pedes obscure grisei reticulati. Digiti marginati, exteriore intermedio membranẩ connexo ad primam articulationem. Digitus posticus brevissimus, vix nisi rudimentum digiti ungue minutissimo aitmatuin.

\section{Mas senior habitu vernali:}

Caput supra et Cervix griseo-albida fusco-maculata, Gula, laterà capitís inferiora, jugulum, pectus et abdomen nigra. Frons alba dimittit fascian concolorem supra oculos ad latera colli et pectoris. Crissum et teetrices caudx inferiores alba. Dorsum, Scapulares et tectrices alartum nigricantia apicibus pennarum albis, unde maculæ transvers $x$ albæ. Remiges primores nigre rachibus albis, latere interiore a basi albo; in qvinta oritur macula in medio juxta itachin alba, qua in octavà \&c. ad marginem exteriorem extenditur; secundaria cinereo - fuscex mårgine albx. Tectrices infe. riores albx axillaribus elongatis nigris. Cauda æqualis; Rectricibus albis nigro fusco transversim fasciatis: exterioribus albis latere exteriore tantum maculatis.

Fentina senioy:

Caput supra, cervix et dorsum griseo - fuscà et nigricantia apicibus pennarum albis. Teetrices alarum grisex albo maculatx et marginatio. Frons, supercilia, crissum ct latera colli pectorisque alba. Gula, jugulum et pectus nigricantia pennis albis inspersis. Tec. trices caudx superiores albx apicem versus fusco faso ciatæ.

Avrs habitu autumnali:

Caput supra et Dorsum fusca maenlis albis vel luteo. albidis. Frons, genx et gula alba immaculata. Sulb. 
tus alba collo antice griseo - fusco striato et nebuloso. Pennx axillares elongatx nigra. In Ceteris similis antecedenti.

\section{HISTORTA.}

Fstivat in Regionibus borealibus; autumno Scaniam transmigrans in littoribus marinis rarius obvenit. In omnibus fere eum Charadrio Aurato convenit. Difo fert tamen digito postico, quamvis brevissimo. 


\section{SECTIO II.}

Tibiis et parte nuda femorum brevioribus; digisis ad basin plane fissis.

GENUS,

\section{O R I N E L L A.}

Rostrum capire brevius, durum, rectum, basi robustiusculum, compressum, apice teres acuminatum.

Nares oblongæ, patulæ, superne membrana semitectre.

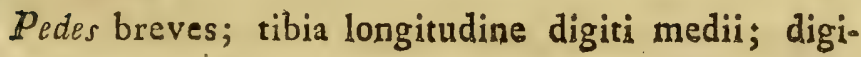
tis plane fissis.

CLXXV. MORINELLA' COLLARIS,

$$
\text { Mey. }
$$

DraGn: Facies alba. Collare nigrum. Cauda nigra, medietate interiore et apice alba. Pedes aurantii; Rostrum nigrum.

\section{SYNONYMA.}

Morineilla Collaris, Meyer Vögel Liv. Esth1. p. 210. Tringa Interpres, Lin. Fn. Sv. n. I78. Syst. Nat. I. p. 248. Itin. Gottl. p. 2I7. Retz. Fn. Sv. I82, n. Nilson arnith. Pars II. 
150. Lath. Ind. II. p. 738. n. 45. Ginel, Syst. I. p. $67 \mathrm{I}$.

Tounepierre, Buff. Ois. VIII. p. 130. t. IO. vers. Gern. Otto XXVIII. p. 236. cum 4 figg.

Coulon-Chaud, Pl. enl. 856.

Hebridal-Sandpiper, Pen. Br. Zool. II. p. 467. n. 200. Turaestone, Br. Zool. 1. c. n. 199. Edw. t. I4I. Lath.

Syn. V. p. 188. 37. Sup. p. 249. et p. 190. n. 37. var. A.

Steindrehender Sandlaufer, Bechst. N. D. 2 te AuA. 4 B. I. Abth. p. 335 .

Gottlandis: Tolk.

\section{DESCRIPTIO.}

Long. 9 poll. Alr extensi I9 poll. Rostr. $\frac{7}{8} ; A$ Aexura alæ ad apicem 6 poll. Cauda fere $2 \frac{x}{2}$ poll. Tibia I. Digit. med. I poll. postic, fere $\frac{3}{8}$ poll.

Rostrum nigrum basi fuscescens. Iris brunnea. Pedes aurantii anguibus nigris.

Frons, genæ et tempora alba. Vertex nigricans marginibus pemnarum griseis vel albis. A basi rostri superne ad oculos stria nigra; sub oculis macula nigra demittit fasciam, quæ jungitur in jugulo. Gula alba. A jugulo nigro fascia nigra, instar collaris, collum cingit; Cervix, sub hoc, alba. Collum antice usque sd sternum, nigrum pennis albis immixtis. Dorsum anticum et scapulares superiores nigra maculis fuscis et ferrugineis; Scapulares inferiores niver. Dorsum posticum et tectrices caudx proximx albx; tectrices caudr breviores nigra. Pectus, abdomen, crissum, tectrices alarum et caudx inferiores alba. Remiges nigricantes latere interiore a basi albr; a sexta omnes latere exteriore usque ad medium albx; qux albedo it 
angetur ut 18-19. fere totx sint albx. Remiges tertii ordinis elongatæ, colore dorsi; tectrices minores fuscr marginibus pallidioribus; têtrices proximæ nigricantes apice et latere interiore albæ. Cauda xqualis; rec. trices nigrex, medietate interiore et apice albx; extima alba maculâ nigrâ in latere interiore pone apicem.

Femina dignoscitur coloribus minus vividis; Caret quo. que maculis ferrugineis dorsi et scapularium.

\section{HIS T ORIA.}

Habitat in insulis et litoribus maris Balthici, non minus quam in insulis maris Norvegici usque ad circulum areticum, passim. Nidulatur in arena. Ova 3-4. cinereo-virescentia maculis fuscis. 


\section{GENUS. \\ T R I $N$ G A, mihi.}

Rostrunn mediocre (capite aliquantum longius vel brevius), subreres, tenue, debile, apice depresseincrassato, recto vel parum incurvato. Pedes breves, digiris usque ad basin fissis.

$$
\text { EAMILIA Ima。 }
$$

Rostrum refium capite fere longius, apice incrassaso. Cawda aqualis.

CLXXVI. TRINGA FERRUGINEA;

$$
\text { Mey. }
$$

Senior habitu cestivali:

DIAGN: Supra nigricans maculis ferrugineis, subtus ferruginea. Cauda æqualis cinerea. Rostrum rectum.

\section{SYNONYMA.}

Tringa ferruginea, Meyer Vögel Liv. u. Esthl. p. 20\% Tringa Islandica, Lin. Syst. Nat. XII. I. addend. Lath. Ind. II. p. 737. n. 39. (Synonyma cun Tr. subasquata confusa) Gmel. Syst. I. p. 682. n. 24.

Red Sundpiper, Pen. Brit. Zool. II. Po 469. 13. 202. to 72. Axk̂. Zool. II. ' n. 393. 
I. Vomneau gris de fer, Ascanius Icon. rer. Nat. I. p. 7. t. 9. (fig. bona).

\section{DESCRIPTIO.}

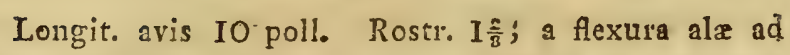
apicem $6 \frac{1}{2}$ poll. Cauda $2 \frac{2}{8}$ poll. Nuda pars femoris $\frac{\pi}{2}$ poll. Tibia $I_{\frac{2}{3}}$ poll. Digit. med. I poll. postic. $\frac{5}{3}$ poll.

Rostrum nigrun, rectum, basi compressum, apice obtusum aliquantum incrassatum. Iris fusca. Pedes breves, nigri, digitis ad basin fissis.

Caput supra et cervix fusca marginibus pennarum ferrugineis. Dorsum et Scapulares nigro-fusca marginibus pennarum griseis et ferrugineis, maculisque rotundatis ferrugineis; præsertim in pennarum scapularium marginibus pone apicem albidum per paria positis.

Uropygium et tectrices caudx superiores alba 1 . ferruginea trniis transversis fuscis. Tectrices alarum cinereo - fuscx, proxims juxta apicem albo marginatx. Remiges primores nigro-fusca latere interiore cinerascentes rachibus albis; 4-6 margine exteriore a basi albx: secundaria cinereo fusc $x$ albo latius marginatx. Teetrices inferiores albæ. Cauda xqualis, cinerea, Rectricibus extimis tenuiter albo marginatis. Tota avis subtus ferruginea, striis paucis (interdum nullis) transversis fuscis in pectore. Crissum albidum, ferrugineo tinctum, striis transversis fuscis.

Var. A. forte junior: Latera capitis, gula, collum antice, pectus et abdomen pallidius ferruginea maculis albis in peetore et abdomine inspersis. Latera fusco mect:lata. Crissum album striis fuscis. 


\section{Avis habitu hiemali:}

DIAGN: Supra cinerea lineis semilunaribus nigris et albidis; subtus alba pectore cinerascente, plerumque macularo. Cauda cinerea tedricibus su. perioribus albis, nigro fasciatis.

\section{SY NONYMA.}

Tringa cinerea, Brün. Orn. bor. p. 53. 11. 179. Retz.

Faun. p. 191. 11. 162. Lath. Ind. II. p. 733.n. 25.

Ginel. Syst. I. p. 673.

Ashcolored Sandpiper, Pen. Brit. Zool. II. p. 462. n. 194. Arct. Zool. II. 11. 386. Lath. Syn. V. p. I77. n. 22. vers. Germ. V. p. 148.

Tringa grisea, Gmel. Syst. I. p. 68I. Lath. Ind. II. p. 733. 11. 23. Retz Faun. p. 19 I. n. I6I.

Cnlidris grisen, Briss. V. p. 233. I6. t. 2I. f. 2. Maubeche grise, Buff. VII. p. 53 I. Pi. enl. 366. Grisled Sandpiper, Lath. Syn. V. p. I75. 20.

\section{OBSERVATIO.}

Tringa Canutus, Gmel. Syst. I. 2. p. 679. (Descr.) huc pertinere videtur, sed $T r$. Canutus Lin. Fn. Sv. ad Totanum $\mathrm{Hy}$ polencum, ut supra ostendi, est referenda. The Knot Penri. Br. Zool. II. p. 46I. sine dubio ad hanc speciem pertinet, sed Synonymia est cum Tringa maritima confusa. In Willughby orn. (ang1.) p. 302. Artic, Knot, nostra avis melius descripta est.

\section{DESCRIPTIO.}

Pileus saturate fuscus marginibus pennarum cinereis s. albidis. Cervix cinerea striis fuscescentibus. Dorsum, scapulares et tectrices alarum minores cinerca ; lineâ nigrâ singulam pennam cingente intra marginem albidum. Subtus avis alba pectore cinerascente, 
immaculato vel maculis parvis fuscescentibus adsperso. Remiges primores nigricantes, latere interiore versus basin cinereo-albx rachibus albis; secundariæ cinereofusce; omnes a quarta margine exteriore albo. Tectrices proximx remigum primorum nigræ apice albo marginatx; Tectrices proximæ remigum secund. cine. rex apice alba. Cauda rqualis, cinerea, Rectricibus intra marginem tenuem albidum linea fusca fimbriatis. Tectrices caudx superiores albæ maculis transversis vel fasciis nigris.

A. Junror: Supra sordide cinerea marginibus pennarum luteis; subtus alba pectore fusco naculato, sordide luteo leviter tincto.

\section{HISTORIA.}

Habitat astate in regionibus arcticis Sveciæ et Noyvegia. In paludibus et juxta lacus interiorum regionum. nuptias celebrat. Autumno abiens et vere rediens littora marina frequentat. Haud timida est. Vescitur vermibus et insectis. Caro sapida.

\section{FAMILIA. IIda.}

Rostrusn capite longins, apice incurvatum, sub. depressum. Rectrices intermedice bince longiores, áctminatce.

\section{TRINGA MARITIMA,}

\section{Briinnichii.}

DIAGN : Supra nigricans marginibus pennarum aibis vel cinereis. Caput et collum usque ad stcrnum saturate cinerea. Reatrices laterales tres cinc- 
rex margine albo; intermedix cum uropygio nigræ, immaculatæ. Rostri basis et pedes viridi-lutei.

\section{SYNONYMA.}

Tringa maritima, Brünn. Orn. bor. p. 54. n. I82. Lath. Ind. II. p. 73I. n. I8. Gmel. Syst., I. 2. p. 678. Transact. Lin. Soc. IV. p. 22.

Tringa striata, Retz. Fn. Sv. p. I82. n. I5I. Fabric. Fin. Groenl. p. 107. Zool. Dan. t. I22. vol. IV. p. 2. (In synonymia confusa est cum Tot. Calidri).

Scluinger Sandpiper, Lath. Syn. V. p. 173. n. I5. Arct. Zool. II. p. 480.

Fjöre Pist, Ström. Act. Nidros. III. p. 440. ta VII.

Leem. Lapp. P. 254. not. I03.

Fjarekurv, Pontopp. Nor. Nat. Hist. vers. Germ. II. p. 138.

\section{OBSERVATIO.}

Cel. Retzius 1. c. nostram avem descripsit; Diagnosis vero, quân dedit ad Tringalin striatam Lin. Syst. Nat, I. p. 248 , pertinet. Hxc vero Linneana avis ad Totanum striatum Brissonii, eum Totano Calidri nostro identicun, est referenda.

\section{DESCR IP TIO.}

Longit. avis 8 poll. Alæ extensæ I5 poll. Rostr.

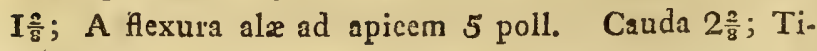
bia $\frac{7}{8}$ poll. Digit. med. I poll. Digit. postic. $\frac{2}{8}$ poll.

Rostrum tenue subteres, basi compressum, apice obtusum aliquantulum incrassatum, fusco-nigrum, besi supra et subtus viridi-luteum. Nares juxta basin 
rostri, parvae, lineares. Os intus pallidum. Pedes viridi- lutei unguibus fuscis.

Caput et collum supra saturate cinerea maculis in vertice nigris. Latera capitis et collum antice saturate cinerea marginibus pennarum versus sternum albis. Palpebrx albx, a rostroad oculum stria saturatior fusca supra quam (interdtmn) stria alba. Gula albida. Dorsum, scapulares et alarum tectrices nigra vel nigrofusca marginibus pennarum cinereis vel albis, unde, prrsertim alæ, supra undulatr. Uropygium et tectrices caudx supcriores nigrix, vix apice albidx. Pecrus et abdomen alba maculis cinereo-fuscis majoribus præsertim ad latera pectoris. Crissum et tectrices caudx inferiores alba striis longitudinalibus fuscis. Remiges nigræ latcre interiore in cinereum vergente rachibus albis. Primores usque a quarta margine tenui albo cinguntur; sequentes ad basin albescunt, qux albedo ita in secundariis augetur ut $\mathbf{I} \sigma-18$ fere sint totæ albæ; intime tres longe colore dorsi. Tectrices proxima apice latius albr; unde linea obliqua alba in ala composita oritur. Tectrices inferiores albr maculis juxta marginem ala cinereo-fuscis. Rectrices I. 2. 3. xquales cinerex margine albo; 4. 5. aliquantum longiores fuscr margine albido; 6. 6. nigro-fuscæ immaculatx.

OBSERVATIO.

Variat pro anni tempore. Estate minus striata, narn coispus supra pallidius est, marginibus pennarum obsolctioribus; pectus et abdomen albiora minusve maenlata. Junior avis maculns habet minutas testaceas in vertice et interscapulio hine inde sparsas.

\section{HISTORIA.}

Incolit boreales Scandinavix plagas. Per æstatera degit in littoribus lasum et interiorum maris sinumm 
ex. gr. Finmarkix et Lapponix ubi nidulatur sub fine M.Maji et initio Junii. "Ova 4-6 pallida maculis obscuris altera extremitate attenuata ${ }_{\text {" }}$ (Fabr.). Autumno et hieme littora maris exteriora et insulas plerumque gregaria frequentat. Littora scopulosa et ardua tunc temporis amat, ubi in saxis innumerx. Lepades, aliaque animalcula marina inter fucos degunt. Regionem illam præcipue incolit qux adfluente mari inundatur, refuente denudatur (fjere Norvegis) unde in Norvegia fjare mus appellatur. Parum timida. Evolans so: num plyt! plyt! repetit, unde nomen fjare plyt. Caro sapida.

\section{TRINGA ALPINA, Lin.}

Diagn: Supra nigra marginibus pennarum ferrugineis. Subtus albida maculis nigris in pectore (plerumque) confluentibus. Cauda cinerea. Rectricibus binis mediis longioribus, acuminatis, fuscis. Rostrum et pedes nigri.

\section{SYNONYMA.}

Tringa Alpina, Lin. Faun. Sv. p. 64. 11, I81. Retz. Faun. p. I85. n. I53. Olafs. Itin. Isl. p. 58 I. t. 4I. Lin. Syst. Nat. I. p. 249. n. II. Gmel. Syst. I. p. 676. Lath. Ind. II. p. 736. n. 37 . Bechst. Nat. D. 2 Aufl. 4 B. I. Abth, p. 323. t. 28. f. I. Cinclus Torquatus, Briss. orn. V. p. 2I6. n. II. t. I9. f. 2 .

Le Cincle, Buff. Ois. VII. p. 553. PI. enl. 852.

L'Aloutette de mer, Buff. Ois. VII. p. 548. Pl. enl, 85 I. vers. Germ. Otto XXVII. p. 105. cuin fig. La Brunette, Buff. Ois. VII. p. 439. 
Dunlin, Pen. Buit. Zool. II. p. 47T. n. 205. Aré. Zool. II. 11. 391. Lath. Syn. V. p. 185. 33. Id. Sup. p. 249.

Veränderlicher Brachvogel, Bcehsr. 1. c. p. 140. (in Descriptione cum sequente confusa).

Tringa variabilis, Mey, V. Liv. u. Estp1. p. 208.

\section{OBSERVATIO.}

Hre 'species Tringa Variabilis a ce\}. Bechstein appellatur, quia inutationum hujus et sequentis speciei hanc solam accusat. Non vero magis est variabilis quam plurime congeneres. Nomen itaque Lingnnum cum alio non magis apto mutare, non squum duxi.

\section{DESCR I P T IO.}

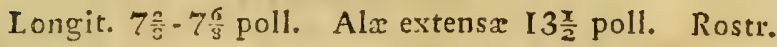
$I \frac{2}{3} ;$ A flexura alæ ad apicem $4 \frac{3}{8}$; Cauda $I \frac{6}{8}$; Tibix I. Digit. med. $\frac{7}{8}$; postic, vix $\frac{2}{3}$ poll.

Rostrum nigrum, tenue, subteres, basi compressum, versus apicem aliquantum incurrum, depressum, obtusum. Nares lineares, juxta basin rostri. Iris fusca. Pedes nigro-fusci.

Habitu vernali:

Caput supra, Dorsum et seapulares nigra, marginibus pennarum ferrugineis pracipue in Senpularibus latis et irregularibus. Facies albida striis minutis et punctis fuscis adspersa. Collum antice album (ferrugineo interdum tincto) maeulis parvis numerosis nigris. Pectus album maculis magnis nigris, sxpe confluentibus. Abdomen, crissum, Hypochondria, Tectrices caudx inferiores et laterales superiorum alba immaculata. Tectrices alarum cinereo-fuscx marginibus pallidioribus, rachibus saturatioribus; proximx apice albs. Remiges nigro-fusex latere interiore a basi cine:?o- 
albz; 5.8 margine exteriore albo; secundaria latere interiore a basi et margine exteriore apicis albx; intimæ tres elongatæ, acuminatx, cinereo-fusca. Uropygium et tectrices Caudx superiores medix, nigrofusca, immaculata. Rectrices I 5 zquales, pallide cinerex, vel potius albido-fuscescentes; 6.6 . longiores, acuminatz, saturate fuscæ.

Habitu autumnali:

Vertex niger marginibus pennarum rufis. Cervix cinerea striis fuscis. Dorsum et Scapulares cinerea, pennis nigris ferrugineo marginatis, hinc inde insertis. Corpus subtus album pectore sordido, maculis parvis fuscis.

\section{HISTORIA.}

Habitat in paludibus, tum camporum Scania, tum Alpium Lapponir passim frequens. In Scaniam advenit juxta finem mens. Martii. Nidulatur inter gramina. Ova 4. pallide virescentia maculis brunneis et nigris extremitate crassiore majoribus adspersa. Avis haud timida. Nutumno dimigrans in litoribus marinis gregaria obvenit.

\section{TRINGA SUBARQUATA*)。}

Dracn: Supra nigra maculis ferrugineis; subeus rufa, Tectrices cauda albæ nigro maculatæ.

*) Sunt qui hane avem ad Numenios numerent. Hi vero Ornithologi, si sibi consentanei esse velint, non solum hane Specietu sed rotam familiam inter Numeutos collocent. Conveniunt enim inter se he quatuor Specics, non solum rostri pedumque formâ, sed in toto quoque habita et in moribus, quibus vero omnibus a Nmmeniis valde differunt. 
Cauda cinereo - fusca basi et rachibus albis. Rostrum longum subarcuatum.

\section{SYNONYMA.}

Scolopax Subarquata, Güldenst. nov. com. Petrop. XIX.

p. 47I. t. 19. Gmel. Syst. I. 2. p. 658.

Tringa Islandica, Retz. Fn. p. 192. n. 163.

Tringa ferruginen, Brün. bor. p. 53. n. 180.

Nunnenius ferrugineus, Mey. Vögel Liv. p. 190. - Subarquata, Bechst. N. D. 2te Aufl. 4 B. I Abth. p. 135.

\section{DESCRIPTIO.}

Long. 8 poll. Rostr. I $\frac{x}{2}$ poll. A Rezura aire ad apicem 5 poll. Cauda $1 \frac{7}{8}$ poll. Tibia $I \frac{7}{8} ;$ Digit. med. fere I poll. postic. $\frac{2}{3}$ poll.

Rostrum nigrum, tenue, subteres, basi compressum, apice incurvum, depressum, obtusum. Iris fusca. Pedes nigricantes.

Vertex, dorsum et scapulares nigra maculis ferrugineis et albielis. Collum, pectus et abdomen rufa pennis albis insertis; cervice maculis fuscis. Remiges fuscæ; primores nigricantes; 6-9. margine exteriore albx; secundarix basi et margine albx; intimx longx, acuminatx, fusex marginibus pallidis. Teetrices proximx apice albæ, minores cincreo-fusca marginibus pallidis rachibus fuscis. Teetrices caudz superiores et inferiores albz maculis transversis nigris. Rec. trices I-5 cinerex, margine interiore et basi albs; intermedix binx longiores, acuminat: cinereo-fuscx, margine tenui et rachi albis.

Var. habitum mutans: Vertex, cervix, Dorsum nigricantia ferruginescenti maculata, pennis fusco, cincreis 
immixtis. Tectrices alarum fusco-cinerex. Avis subtus alba maculis in pectore fuscis pennisque ferrugineis hine inde insertis.

Mus. Lund.

\section{HISTORIA.}

Incolit astate boreales patrix plagas; australiores verno et autumnali temporibus, migrans et rediens, visitat. Mens. Septembri et Oetobri in littorizus marinis Scaniz obvenit. Ova nondum vidi.

\section{TRINGA CINCLUS, Retzio.}

DIAGN : Supra cinereo-fusca rachibus saturatioribus; capite pallidiore; subtus alba collo antice cinerascente striis minutis.

(Tab. X.)

\section{SYNONYMA.}

Tringa Cinclus, Retz. Fn. I90. n. I59. Lin، Syst. I. p. 25I. n. I8.

Cinclus, Briss. Orn. V. p. 2 II. t. I9. f. I. (Synony: mum optimum; Descriptio et figura egregix).

Purre, Lath. Syn. V. p. I82. n. 30. Pen. Br. Zool. II. p. 472. n. 206. (fig. t. 7 I. nec tam bona quam icon citata Brissonii).

Mus. Payk.

\section{DESCRIPTIO.}

Long. circa 7 poll. Rostr. I $\frac{2}{8} ; A$ flexura ale ad apicem $4 \frac{1}{2}$; Cauda $2 \frac{1}{8}$; nuda pars femoris $\frac{x}{3}$; Tibia I 
poll. Digit. med. I poll. Digit. postic. brevis, ungue instructus*).

Caput supra, cervix, dorsum et scapulares cinereofusca. Cervice pallidiore striis ad raches saturatioribus fuscis. Frons, latera eapitis et colli albida striis minutis in collo fusescentibus. Inter rostrum et oculos macula fuscescens, supra quam stria albida. Tota avis subtus alba collo antice cinerascenti colore tincto, striisque minutis fuscescentibus leviter maculato. Remiges nigro-fuscre, rachibus albis; extimx qvatuor vel quinque latere interiore medio cinerex, apice saturatiores; sequentes margine exteriore in medio albx, qui color albus ita sensim augetur ut intimx inter secundarias plane sint alba, maculâ interdum pone apicem fusca. Tectrices alarum cinereo - fuscæ marginibus dilutioribus et ad raches striis saturatioribus. Tectrices proxim apice albæ, unde stria alba in ala oritur. Cauda æqualis præter Rectrices binas intermedias, qux longiores sunt et aeuminatx, latereque interiore saturate fuscx, exteriore cinerex; ceteræ albo. fuscescentes rachibus albis. Tectrices caudx superiores saturate fuscx; inferiores alba, longitudine caudæ.

\section{OBSERVATIO.}

Hree avis proxime accedir ad Tringan alpinam, cujus pro xtate varietaten forte habebit is qui avem ipsam non viderit. Sed preter alias differentias cum coloris, tmm præcipue formæ, ex Descriptionibus urrinsque eruendas, differt quoque capite insigniter majori. Alii Ornithologi (Eechst. Meyer.) nomine Trinsga Cincli describunt Totanzm Hypolencim, ad quem Tringa

*) Hac pracipue nota differt a Calidri grisen, Vide cbsery, in pag. 23. 
Cinclus Linnei minime pertinet. Tr. enim Cinclus Lin, a Btissonii Cinclo desumta est, qui nostram avem optime refert.

\section{HISTORIA.}

Estatem, sine dubio, in terris borealibus degit, autumno et hyeme littora nostra invisit. In littoribus Roslagix et in Insulis maris Balthici inventa est. Propagatio et consuetudines nondum bene notx sunt.

\section{FAMILIA. IIItia.}

Rosirum retusm, capite brevius; Recirices sintersnedice bine longiore: acuminata.

\section{TRINGA.TEMMINCKII, Leisl.}

Dragn: Supra nigro - fusca maculis rufis; subtus alba collo antice grisescente, fusco maculato. Rachis prima tantum remigis nivea. Rętrix extima pure alba; intermediæ longiores fuscæ.

\section{SYNONYMA.}

Tringa Temminckii, Meyer Liv. p. 205.

Tringa' pusilla, (senior). Mey. On. Taschenb. II. p. 39 I. n. 7.

Tringa pusilla, Bechst. N. D. 2 te AwA, 4 B. I Abth. p. 308.

\section{DESCRIPTIO.}

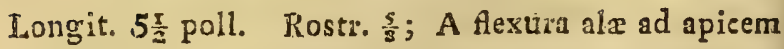
fere 4 poll. Cauda fere 2 poll. Tibia $\frac{5}{8}$; nuda pars femoris $\frac{2}{8}$; Digit. med. $\frac{5}{8}$; postie. $\frac{3}{26}$. 
Rostrum rectum apice vix incurvo, subteres, in. crassate-acuminatum, fuscum apice nigro. Pedes virescenti- cinerei.

Caput supra, Dorsum et stapulares nigro - fusca marginibus pennarum rufis. Cervix fusco-cinerea ma. culis fuscis. Uropygium cinereo-fuscum immaculatum. Frons, genæ et gula albida. Latera colli et capitis, collumque antice usque ad pectus cinerascentia maculis minutis fuscis. Peetus, Atdomen, cris. sum et tectrices caudx inferiores alba, immaculata. Teetrices alarum cinereo-fuscx apicibus pallidis. Remiges nigro - fuscx rachi primr tantum nivea, ceterarum magis fuscescentibus; primores a tertia, margine exteriore alba; secundarix apice albo marginatx; intimx tres longx, fuscæ, marginibus rufs. Teetrices proximæ apicibus albis. Reetrices extimæ (I. I.) albǽ 2-2. albæ margine exteriore cinerascente, qui color cinerascens magis magisque augetur in sequentibus; par medium fuscum ceteris longius.

Variet. Supra fusco-cinerea maculis nigris, marginibus ferrugineis. Facies, collum antice et pectus sordide alba maculis minutis fuscis. Gula, peetus inferius et crissum pure alba. cet. ut in anteced.

\section{HISTORIA.}

In borealibus Europæ plagis astatem degit et se propagat. Mens. Junii hanc avem per familias versus septemtrionem migrantem, juxta paludes et lacus Norvegix interiores, observavi. In medio M. Julii avem juxta Nidrosiam Norvegix occisam vidi, unde in his regionibus se propagare mihi videtur. Autumno au. straliores terras petens littora Svecir australioris gregnria visitat. Vescitur insectis et vermiculis.
Nilsson Ornitho Pars IJ.
$G$ 


\section{TRINGA MINUTA, Leisl.}

Diagn: Supra nigro-fusca marginibus pennarum ferrugineis et albis; subtus alba. Raches remigum omnium albr. Rectrices tres larerales pallide grisex; intermediæ fuscæ, longiores.

\section{SYNON YMA.}

Tringa minuta, Mey. Liv. p. 206. in obs. Tringa pusilla (junior.), Mey. Orn. Taschenb. II. p. 391.

Tringa pusilla, Retz. Fn. (Descr.) p. 193.

\section{OB SERVATIO.}

Tringa pusilla Lin. quæ corpore subtus rufescente dignoscitur, a Cinclo Dominicensi Ulesumta est, nec inter aves Europass numeranda.

\section{DESCRIPTIO.}

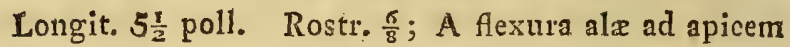
$3 \frac{6}{8}$; Cauda $1 \frac{\pi}{2}$; nuda pars femoris $\frac{5}{8}$; Tibia $\frac{7}{8}$; Digit. med. $\frac{5}{8}$; postic. $\frac{3}{16}$ poll.

Rostrum rectum subteres, basi compressum, apice incrassatum, nigrum; Pedes nigri.

Caput supra, Dorsum et tectrices alarum nigro-fusea marginibus pennarum ferrugineis et hine inde albis. Cervix cinerascens. Frons, genæ et tota avis subtus alba, tinctura in peetore sordide lutescenti, levissima. Stria inter nares et oculum fuscescens. Remiges nigrofuscx rachibus omnium albis; secundarix latere interiore basi albx et apice albo marginatx; intimæ longx, ferrugineo marginatæ. Teetrices proximæ apici albæ. 
Uropygium et tectrices caudx superiores nigra. Rectrices tres vel quinque laterales pallide grisex, albo marginate; intermedix longiores fusci, marginibus apicis ferrugineis.

Var. Supra cinereo-fusca rachibus fuscis apicibus pennorum albidis. Frons, latera eapitis et tota avis subtus alba striâ fusca inter rostrum et oculos. ceto sim. anteced.

\section{HISTORIA.}

FEstivat in plagis borealibus. Autumno et vere in littoribus marinis et juxta aquas tum Scanix tum Norvegia gregaria obvenit. De oeconomia nihil constat. 


\section{GENUS. \\ $S$ C O L O P A X.}

Rostrem longum, rętum, basi compressum, extus teres, sulcatum, molle, pone apicem punctis excavatis rugosum (in ave mortua); apice duro, lavi, superne et inferne linea longitudinali excavata; maxilla superiore longiore, apice subtus gibbo.

Nares juxta basin rostri, laterales, lineares.

Oculi magni, sub vertice siti.

Pedes: nuda pars femoris brevis; Tibia brevior (plerisque) quam digitus medius. Digiti ad basin fissi.

CLXXXIII. SCOLOPAX RUSTICOLA, Lin.

Drack: Occipus nigrum fasciis transversis ferrugineis. Corpus transversim variegatum e nigro, rufo, ferrugineo et griseo. Cauda nigra apice supra cinereo, subtus albo.

\section{SYNONYMA.}

Scolopax Rusticola, Lin. Fn. Sv. p. 60. n. I70. Retz。 Fn. 174. n. 139. Lin. Syst. I. p. 243. 6. Gmel. 
Syst. I. p. 660. Lath. Ind. II. p. 7I3. n. I. Briss. orn. V. p. 292. I.

La Becasse, Buff. Ois. VIr. p. 462. t. 25. Pl. enl. 885.

Otto XXVI. p. T29. cum, fig.

Woodcock, Pen. Br. Zool. II. p. 433. n. I78. t. 65. Arct. Zool. Ir. p. 470. Sup. p. 68. Lath. Syn. V.

p. I29. I. vers. Germ. V. p. I03.

Waldschnepfe, Bechst. N. D. 2te Auf. 4 B. I Abth. p.

I58: Meyer Vögel Liv. u. Esthl. p. 192.

Svecis: Morkulla.

\section{DESCRIP TIO.}

Long. I $3 \frac{x}{2}$ poll. Rostr. 3 poll. A flexura ale ad apicem fere 8 poll. Cauda 3 poll. Tibia $I \frac{2}{8} ;$ Digit. med. I $\frac{T}{2} ;$ postic. $\frac{3}{8}$ poll.

Rostrum pallide incarnatum apice nigrum. Nares oblongx parvx, membrana tectr. Oculi magni, prominentes, retrorsum sub vertice siti, nigri iridibus fuscis. Pedes griseo-incarnati femoribus fere ad genua pennis tectis; unguibus fuscis, postico brevissimo.

Caput supra antice cinereum fusco et rufo immixtis, postice nigrum fasciis tribus transversis, angustis, pallide ferrugineis. $\mathrm{Ab}$ angulo oris ad oculos fascia nigra, aliaque sub auribus. Dorsum et ala supra rufa maculis majoribus undulisque transversis nigris et maculis cinerascentibus albisque. Uropygium rufum undulis nigris. Gula alba. Collum pallide rufum undulis numerosissimis nigris. Pectus, abdomen et crissum sordide albida, rufo tincta, undulis nigris. Remiges fuscr margine utroque (primores) rufo-maculatx, secundarix fasciatr. Cauda rotundata. Reetrices XII. nigra, margine exteriore rufo maculate, apicibus supra cinereis subtus albis pulchre nitentibus; tectrices inferiores luteo - rufe apicibus albis. 
Var: Albida, rufescenti supra maculata, subtus undulata.

Mủs. Payk.

\section{HISTORIA.}

Migratoria avis redit in Scaniam M. Martis, si terra jam denudatur, vel Aprili, tumque in fruticetis uliginosis passim frequens obvenit. In Scania rarius æstatem degit; in superioris vero Svecire et in Norvegiz sylvis montosis ad ipsam terram, sub frutice, ova fovet. Ova 3-4 sordide lutescentia rufo maculata. Caro sapidissima.

\section{CLXXXIV, SCOLOPAX MAJOR,}

\section{Lath.}

DIAGN : Vertex niger tænia longitudinali pallide ferruginea. Peetus et abdomen sordide alba, undulis fuscis. Teetrices alarum maculis albis. Rectrices XVI, quarum mediæ VIII. nigra, apice rufx: laterales utrinque IV. albæ, basi nigro maculatæ.

\section{SYNONYMA.}

Scolopax major, Lath. Ind. II. p. 7 I4. n. 4. Ginel. Syst. I. p. 661 .

Scolopax paludosa, Gmel. Syst. p. 66I. n. 35. Lath. 1. c. n. 3. Retz. Fn. p. 175.

Great Snipe, Pen. Br. Zool. II. p. 450. n. I88.

Scolopax media, Frisch Vögel to 228.

Berassine des Savannes, Buff. Ois. VII. p. 48I. Pl. enl. 895. vers Germ. Otto XXVI. p. I62. cum fig. 
Mittel-Schnepfe, Bechst. N. D. 2te Auf. 4 B. I Abth.

p. 180. Meyer Vögel Liv. u. Esthl. p. 193.

Sveiis: Dubbel Beckasin.

\section{DESCRIPTIO.}

Lon'g. II - I2 poll. Alx extensæ I9 $\frac{1}{2}$ poll. Rostr. $2 \frac{r}{2}$; A flexura alx ad apicem $5 \frac{\sigma}{8}$; Cauda 2 poll. Nuda pars femoris $\frac{T}{2}$ poll. Tibia $I \frac{1}{2}$ poll. Digit. med. $I \frac{9}{8}$; postic. $\frac{1}{2}$ poll.

Rostrum subincarnatum apice nigrum basi subtus pallidum. Iris fusca. Pedes cinereo-virides.

Caput supra nigrum maculis minutis rufis; tænia longitudinali pallide ferruginescente a fronte per verticem ad occiput, fasciaque ejusdem coloris supra oculos ducta. Lora nigro-fusca. Collum ferruginescenti - griseum maculis numerosis fuscis. Gula albida, immaculata. Dorsum et seapulares nigra, cupreo colore nitentia marginibus latis pallide rufescentibus (qui fere strias quatuor longitudinales pallide rufas formant), et undulis transversis maculisque rufis. Uropygium nigrum maculis frequentioribus pallide rtrfis. Teetriccs superiores alarum nigrre, apicibus, et fasciis minorum pallide rufis; apicibus majorum late albis. Tectrices inferiores albæ fasciis transwersis nigris. Pectus, Abdomen et femora sordide alba fasciis maculisque undulatis nigris. Remiges fusca, prima rachi alba; secundarix apice albido marginatz. Cauda parum rotundata. Rectrices XVI. quorum medix VIIL. nigræx, versus apicem luteo-rufx lineâ pone apicem album nigrâ; laterales albx basi nigro maculatæ.

Mas aliquantulum minor Foninîn, genisque et jugulo magis maculatis. 


\section{HISTORIA.}

Habitat in paludibus et pratis depressis Scanix fere ubique. In superiore Svecia quoque inventa est. Muta evolat; volatus depressior et minus celer quam sequentis. Niduatur inter gramina- Ova $3-4$. oblongo-ovata, lutescenti - grisea maculis nigris et fuscis prxcipue circa extremitatern crassiorém confluelitibus. Caro sapidissima.

\section{SCOLOPAX GALLINAGO,}

\section{Lizz:}

DraGn : Vertex niger tænia longitudinali pallide ferruginea. Pectus et abdomen alba immaculata. Remex prima latere exteriore alba. Reetrices XIV. basi nigræ versus apicem ferruginex; undulâ pone apicem nigra.

\section{SYNONYMA.}

Scolopax Gallinago, Lin. Fn. Sv. p. 6I. n. I73. Syst。 nat. I. p. 244. 7. Retz. Fn. p. I75. 11. I4I. Lath。 Ind. II. p. 7I5. n. 6. Gmel. Syst. I. p. 662. 7. Briss. V. p. 298. 8.

Becassine, Buff. Ois. VII. p. 483. t. 26. Pl. enl. 883. Orto XXVI. p. I74. cum fig.

Common Snipe, Pen. Br. Zool. II. p. 448. no I8\%. to 68. Arct. Zool. II. 11. 366. Lath. Syn. V. p. I34。 n. 6. vers. Bechst. V. p. 108.

Feerschnepfe, Bechst. N. D. 2 te Auf. 4 B. I Abth. p. I85. t. 7. Meyer Vögel Liv, u. Esthl. p. I94. Svecis: Enkel Beckasin. Scanis: Horsgök。 


\section{DES CRIPTIO.}

Longit. $10 \frac{r}{2}$ poll. Rostr. $2 \frac{1}{2}-2 \frac{6}{8}$ poll. A flexura alæ ad apicem $5 \frac{\pi}{2}$, nuda pars femoris fere $\frac{r}{2}$ poll.

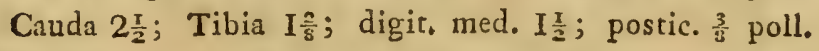

Rostrum pone apicem depressum, subdilatatum, punctis excavatis rugosum; a basi ultra medium superne fusco-incarnatum subtus pallidius, læve; apice ipso quoque lævi. Iris fusca. Pedes virescentiplumbei.

Caput supra nigrum maculis minutis rufis, tænia longitudinali pallide rufescenti a fronte per verticem ad nucham ducta, fasciaque ejusdem coloris utrinque supra oculos. Lora nigro-fusca. Macula aurium fuscescens. Collum pallide rufescenti-griseum maculis numerosis fuscis. Gula albida immaculata. Genæ albidx fusco punctatx. Dorsum et Scapulares nigra, striis quatuor longitudinalibus, latioribus, albo rufescentibus, et lineis transversis undulatis, rufis, variegata. Dorsum posticutn nigricans macnlis transversis albidis. Tectrices caudx griseo-ferruginex, lineis undulatis et maculis nigris. Pectus et abdomen alba immaculata. Hypochondria alba fasciis transversis fuscis. Remiges nigræ; prima latere exteriore albo, secundarix apice latius albæ, intimæe striis transversis rufis. Teetrices nigra; majores apice albo marginata; minores (interdum) rufo variegatix; apice rufescentialbx; minimx immaculatx. Tectrices inferiores et axillares albr fasciis griseo-nigris. Cauda rotundata. Rectrices XIV. medietate interiore nigrx, exteriore luteo-rubræ striis transversis nigris. Laterales I. I. albre ubi cetere luteo-rubrx.

$F$ ir. Albida, maculis rufescenti - fuscis nebulosa.

Mus. Payk. 


\section{HISTORIA.}

In palustribus locis frequens. Usque intra circuIum areticum eam quoque audivi et vidi; E Groenlandia apportatam habui. Migratoria in Scaniam redit jam mens. Martio. Nidulatur inter gramina. Ova fovet 4. olivacea maculis brunneis et nigris adspersa.

\section{SCOLOPAX PAYKULLI,} miki.

DIAGN : Supra fusca marginibus pennarum ferrugineis; subtus alba; peltore sordide lutèscenti, fusco maculato. Cauda cum teétricibus alba, fusco fasciata.

(Tab. XI.)

\section{OBSERVATIO.}

Singularis hec Species quoad magnitudinem et habitum multa habet cum Scol. Gallinagine communia; distinguitur autem non solum colore, sed etiam formis partium constantissimis, rum $a b$ hac, tum ab omnibus congeneribus. Præsertim singularis est membrana illa qux externum digitum cum medio jungit usque ad primam articularionem. Etiam hac distribucio colorum caude et dorsi postici, in hoc Genere plane insolita est. Multa itaque cum Totano, plurima vero cum Scolopace commurnia habet. Neque vero ex bis compositam quis putet. Ipse enim mortuam avem diligenter examinavi, et Illustris Possessor adseveravi pellem, e Lapponia missam, sub Ipsius oculis fuisge farctam. Novam igitur et distincam hanc Speciem Fautoris mei, Zoologi inclytissimi; Liberi Baronis \&c. G. de Prykull nomine, ornatam volui, 1

\section{DESCRIPTIO,}

Rostrum recum longum, basi aliquantulum compressum, pone apicem punctis excavatis rugosum, 
depressum, apice supra et infra linea longitudinali excavata. Pedes lutescentes; digitas extimus melio membrana connexus a basi ad primana articulationem.

Caput supra nigro - fuscum marginibus pennarum ferrugineis. Fascia albida a rostro supra oculos, aliaque fuscescens inter rostrum et oculum. Latera capitis sordide albida punctis fuscis. Gula, pectus et abdomen alba immaculata. Jugulum et peêus superius sordide albida luteo tincta maculis et striis minutis fuscis adspersa. Dorsum, scapulares et Remiges intimæ fusca marginibus pennarum luteo-ferrugineis. Alarum teatrices minores cinereo-fuscr, marginibus pallidis; majores apice alba, unde stria alba per alas. Remiges nigro-fuscx, prima rachi alba; 5-9. albo marginate; secundarix fusce, ad raches albre marginibusque latis albis; intimx longx fuscx, rufo ad margines variegatæ. Dorstim posticum (si sejunguntile penna) album. Uropygium album maculis parvis nigricantibus. Tectrices caudæ superiores et inferiores albe undulis transversis, numerosis, nigricantibus. Rectrices XII. fasciis transversis numerosis albis et ftscis, intermedir apice lutescentes.

Mus, Payk.

\section{LOCUS.}

Habitat in Lapponia paludibus unde Specimen descriptum recepit Gener. Possessor. De moribus nihil constat.

\section{SCOLOPAX GALLINULA,} Lin.

DIAGN: Caput supra lurco - rufum fascia longitudinali, lata, nigra. Abdomen album. 
Cauda nigro-fusca Re@ricibus XII margine rufo variegatis.

\section{SYNONYMA.}

Scolopax Gallinaln, Retz. Fn. p. I76. 11. I42. Lin. Syst. I. p. 244. n. 8. Lath. Ind. II. p. 715. n., 8. Gmel. Syst. I. p. 662.

Gallinngo minor, Briss. Orn. V. p. 303. n. 3.t. 26. f. 2 . La petite Becassine, Buff. Ois. VII. p. 490. Pl. enl. 884. Otto XXVI. p. I88. et 193 cum fig.

Fack Snipe, Pen. Br. Zool. II. p. 45 I. n. 189. t. 68. Ark. Zool. II. n. 367. Lath. Syn. V. p. I36. 8. vers. Bechst. V. p. IIO.

Moorschnepfe, Bechst. N. D. 2te Auf. 4 B. I Abth. p. 196. Meyer Vögel Liv. p. 194.

Venatorib. Scaneusibus: Hàrsnäppa vel Halfenkel Beckassin.

\section{DESCRIPTIO.}

Longit. $7 \frac{1}{2}-8$ poll. Rostr. I $\frac{5}{8} ;$ A flexura alx ad apicem $4 \frac{\pi}{2}$; Tibia I poll. Digit. med. I $\frac{T}{8} ;$ post. $\frac{2}{8}$ poll.

Rostrum basi sordide lutescens, compressius et altius quam in congeneribus, apice nigrum sub-depresse dilatatum, punctis excavatis rugosum, maxilla superiore longiore, apice subtus vix gibba. Iris fusca. Pedes virescenti - incarnati.

Caput supra fascia longitudinali lata nigra a fronte per verticem ad occiput ducta; ad utrumque hujus la. rus supra oculos stria luteo-ferruginea, nigro unaculata. Lora nigro-fusca. Cervix fusco-rufa punctis albis. Gula alba inmaculata. Latera colli, jugulum et superior pars pectoris alba ferrugines tinęa maculis 
fuscis fere confluentibus. Dorsum nigrum viridi et purpureo nitens, rufo transversim variegatum, fasciis quatuor luteo-ferrugineis, intermediis duabus latioribus. Pectus inferius, abdomen et femora alba immaculata. Remiges nigro-fuscr, primeres inmaculatx, secundarix apice albr; intimx dorso concolores. Tectrices alarum nigricantes marginibus albis. Caude euneiformis. Rectrices XII. acuminatæ, nigro-fusca apicibus rufis, marginibus rufo variegatis.

\section{HISTORIA.}

Habitat in pratis depressis et paludibus passim. Autumno migrat, vere redit. Propter volatum celerrinum, huc et illuc ductum, non facile figitur, quamvis cominus sir. Nidulatur in graminosis. Ova 4-5. virescenti-albida fusco maculata. Vescitur vermiculis et Insectis: 


\section{GENUS. \\ R A L L U S.}

Rostrum capite longius, subularum, aliquantum decurnatum, basi compressum, apice teres.

Nurés lineares, laterales, pervix, membrana'semitecta.

pedes: nuda pars femoris brevis; digiti mediocres, plane fissi.

CLXXXVIII. RALLUS AQUATICUS, Lin.

DiaGN: Supra niger marginibus pennarum rufis; subrus cinereus; hypocondria nigra fasciis transversis albis.

\section{SYNONYMA.}

Rallus aquaticus, Lin. Fn. Sv. p. 70. n. 195. Syst. nat. I. p. 262. n. 2. Retz. Fn. Sv. p. 202. n. I76. Lath. Ind. II. p. 755. n. I. Zool. Dan. tab. I50. AEta Nidros. II. p. 340. t. I2. Gmel. Syst. I. p: 712:

Le Rale d'enu, Buff. Ois. VIII. p. 154. t. I3. PI. enl. 749. Vers. Germ. Otto XXIX. p. 20. cum fig. Water Rail, Pen. Br. Zool. II. p. 484. to 75. Lath. Syn. V. p. 227. vers. Germ. V. p. I98. 
Der Wasser-Ralle, Bechst. N. D. 2te Auf, 4 B. I Abth. p. 465.

\section{DESCRIPTIO.}

Longit, 9-IO poll. Rostr. I I poll. A flexura alx ad apicem 5 poll.

Rostrum fuscum, maxilla inferiore rubra. Iris aurantia. Pedes sordide incarnati.

Caput supra, Cervix et dorsum nigra marginibus pennarum rufis, Latera capitis, collum antica, pectus et abdomen cinerea, undulis obsoletis rufis. Gula albida. Hypochondria et axillares nigra, striis transversis albis. Remiges nigro-fuscx; intimx dorso concolores. Cauda-brevis cuneiformis. Rectrices nigrz olivaceo-marginatx. Tectrices caudx inferiores nigrax, apicibus late albis.

\section{HIS TORIA.}

Migratoria et rara avis habitat inter arundines, gramina et equiseta ad margines aquarum. Vescitur Insectis, vermiculis et herbis aquaticis. Nidulatur inter arundines. Ova fovet $7-10$ olivacea maeulis fuscis. 
GENUS。

\section{G A L L I N U L A, Lath.}

$\mathbb{R}$ ostrum breve, conico •subulatum, compressum, supra subrusque apice æqualiter in acumen decrescens; basi superne fronte magis vel minus calva.

Nares oblongæ, pervix, laterales, pone medium rostri sitx.

Pedes: Digitis fissis, medio longitudine tibia vel longiore.

CLXXXIX. GALLINULA CREX, Lath.

DIAGN : Supra nigricans marginibus pennarum cinereis et rufis. Abdomen album. Alæ rufæ.

\section{SYNONYMA.}

Gallinula Crex: Lath. Ind. II. p. 766. 11. I. Rallus Crex, Lin. Faun. p. 7 O. n. I94. Syst. Nat. I. p. 26I. n. I. Retz。 Fn. p. 20I. n。 I75. Gmel. Syst. I. p. 7 II. I.

Ortygonetra, Briss. Orn. V. p. I59. 3. t. I3. f. 2. $R_{0 i}$ des Cailles, Buff. Ois. VIII. p. I46. to I2. Pl. enlo 750. Otto XXIX p. 8. cum fig. 
Crnke Grallinule, Pen. Br.Żool. II. p. 487, n. 216. t. 75. Aret. Zool. II. p. 492. n. 4 II. A. Lath. Syn. V. p. 250. I. vers. Bechst. V. p. 220.

Wiesen Knarrer, Bechst. N. D. 2te Aufl. 4 B. I Abth. p. 470. Meyer Vögel Liv.p. 2 I3.

Svecis: Kornknarr, Wachtelkung; Scanis: Angsnärpa; Gottlandis: Seidreifver. Westrogothis: Bjuggbit.

\section{DESCRIPTIO.}

Longit. IO poll. Alz extensi I $8 \frac{\mathrm{r}}{2}$ poll. Rostr. a fronte $\frac{6}{2} ; A$ flexura alæ ad apicem $5 \frac{r}{2}$ poll. Cauda

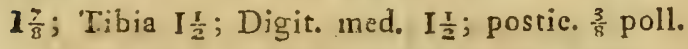

Rostrum supra fusco-incarnatum, subtus palliditts. Iris brunnea. Pedes pallide plumbei, femoribus parum supra genna nudis. Corpus valde compressum.

Caput supra, Dorsum, scapulares, remiges intimx et tectrices caudx superiores nigra, lateribus pennarum cinereo, apicibus olivaceo marginatis. Supra oeulos stria cinerea postice latior. Per oculos stria obsoletior brunnea. Gula albida. Prxterea collum antice et pectus pulchre cinerea, juxta latera rufo tincta et obsolete undulata. Abdomen medium album fasciis ob. soletis rufis. Hypochondria,- femora et crissum rufa, fasciis transversis albis pulchre ornata. Teetrices alarum superiores rufx. Remiges saturatius rufæ rachibus fuscis, extinâ extus albida. Tectrices interiores secundariarum, maculis minutis transversis albis. Cauda rotundata. Reetrices nigro fuscx, marginibus latis rufo-cinereis. Teetrices inferiores alba, basi rufæ.

Zunior: Supra griseo-rufa maculis nigris. Gula et abdomen medium alba. Collo antice, pectore, crisso et femoribus pallide rufis, Alæ et cauda ut in anteced. Nilsson Ornith, Pars II. 


\section{HIS TORIA.}

Mrgratoria avis redit in Scaniam post medium $\mathrm{Ma}$ jum. Incolit rstate Scandinaviam a Scania maxime meridionali usque intra circulum Arcticum ex gr. Insulas juxta litora maris Norvegici sitas. Inter segetes agrorum et gramina pratcrum presertim locis depressis, et arboribus destitutis degit, ibique sono insigni gemino: crax-crax! frequenter repetito, se prodit. Vescitur Inseetis, Lumbricis, seminibus. Nidulatur ad terram. Ova 7-10 rufescenti-lutea, maculis et punctis rufis.

\section{GALLINULA PORZANA, \\ Lath.}

Dragn: Fusco-olivacea punetis striisque albis adspersa. Frons et gula cinerea. Abdomen et tectrices caudæ inferiores albiảæ.

\section{SYNONTMA.}

Gallimula Porzma, Lath. Ind. II. p. 772 n. 19.

Rallus Porzana, Lin. Syst. I. p. 262. 3. Gmel. Syst. 1.

2. p. 7 12. Retz. Fn. p. 202. n. I77.

Le petit Rale d'eau ou le Maroutette, Buff. Ois. VIII. p.

I57. P1. enl. 75I. vers. Germ. Otto XXIX. p.

3I. cuin. fig.

Rallus aquäicus minzor, Briss. Orn. V. p. I55. 2. t. 13. f. I.

Spotted Gallinule, Pen. Br. Zool. II. n. 2I5. Arct.Zool.

Sup. p. 69. Lath. Syn. V. p. 264. n. I8. vers.

Germ. V. p. 233.

Punktirtes Rohrhnhn, Bechst. N. D. 2te Anf. 4. B. I A $\operatorname{arh.~p.~478.~Meyer~Vögel~Liv,~u.~Esthl.~p.~} 216$. 


\section{DESCRIPTIO.}

Long. 9. poll. Alæ extensa I5 poll. Rostr. $\frac{6}{8}$ poll. A flexura alx ad apicem $4 \frac{6}{8} ;$ Canda fere 2 . Ti-

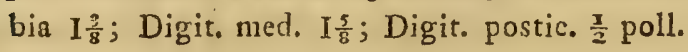

Rostrum supra luteo-viride, basi rubro; subtus luteum apice fusco. Iris fusca. Pedes luteo-virides.

Frons, supercilia et gula cinerea, vertice et nucha nigris marginibus pennarum olivacco-rufis. Cervix nigricans olivaceo immixto maculisque minutis albis adspersa. Dorsum nigrum marginibus pennarum latis olivaceis, lineis longitudinalibus, albis, ornatis. Tectrices alarum olivacex, maculis parvis vel striis transversis albio, nigro marginatis notatz. Remiges olivaceo-fuscx, immaculatx; margo alx et Remex prima extus albida. Cauda cuneiformis, Reetrices nigrx, olivaceo fimbriatz, intermedix binæ marginibus tenuibus, albis. Collum antice et pectus cinereo-olivacea, maculis parvis albis adspersa. Hypochondria et femora olivacea striis transversis albis, nigro marginatis. Abdomen medium album. Tectrices canda in. feriores lutescenti - albz.

Funior: Macula inter oculum et rostrum saturate fusca. Gula albida, maculis parvis, obsoletis.

\section{HISTORIA.}

Migratoria et rara avis habitat inter arundines et gramina ad margines lacuum et paludum per Sveciam passim. Vescitur inse?tis et herbis aquaticis. NiduJatur in grumulo inter gramina et arundines. Ova 912. pallide lutescentia maculis parvis et punctis rufin, aliisque obsoletis cinereis ornata. 
CXCI. GALLINULA CHLOROPUS,

\section{Lath.}

Diagn: Cinereo - nigra dorso et alis olivaceo - fuscis. Margo alarum et tectrices caudx inferiores alba. Frons calva rubra. Pedes olivacei.

\section{SYNONYMA.}

Gallinula chloropus, Lath. Ind. II. p. 770. n. I3.

Gallinala major, Briss. Orn. VI. p. 3. I. t. I.

Fulica chloropus, Lin. Syst. I. p. 258. no 4. Retz. Fn。

p. 200. Gmel. Syst. I. p. 698.

Common Gallinule, Pen. Br. Zool. II. p. 489. n. 217.

t. 77. Arct. Zool. II. p. 492. 11. 4 II. Id. Sup.

p. 69. Lath. Syn. V. p. 258. I'2. vers, Bechst.

V. 227.

Poule d'eau, Buffo Ois. VIII. p. 17I. t. 15. Pl. enl. 877. Otto XXIX. p. II 7. cum fig.

Grinfiussiges Meerhuth, Bechst. IN. D. 2te Auft. 4 B. I Abth. p. 489.

Rohrhuthit, Meyer Liv. p. 2 I5.

\section{DESCRIPTIO.}

Longit. $12 \frac{\pi}{2}-13$ poll. Rostr. a sinu oris $I \frac{r}{8} ; A$ Acxura alæ ad apicem? poll. Cauda 3 poll. Tibia I $\frac{7}{8}$ poll. Digit. med. $2 \frac{7}{8}$; postic. I poll.

Rostun cum fronte calva, rubrums, apice luteo. Iris rufa. Pedes luteo-virides, armillis rubris.

Color capitis et colli niger in peclore et abdomine in obscuro-cinereum transit. Abdomen medium albo nebulosum et strix hypochondriorum hinc inde albre. Dorsum, scapulires, teztrices alarum et caudx superiores olivacco-fusca, nitida. Remiges saturate fuscre 
Extima extus tenuiter alba. Intimx dorso concolores. Cauda nigra. Teetrices inferiores laterales albs, media nigra.

\section{HISTORIA.}

In superiore et inferiore Svecia passim. Habitat inter arundines et Equiseta juxta flumina, lacus et stagna, præcipue insylvaticis. Natat quamvis fissipes. In arundinetis nidulatur. Ova 6.8 olivacea maculis vufis. Vescitur insectis, vermibus et herbis aquaticis. 
ORDO II.

\section{P I N A T I P D E S.}

\section{SECTIO I.}

Mlembrana marginali đigitorum lobatt.

GENUS.

\section{PHALAROPU S*)}

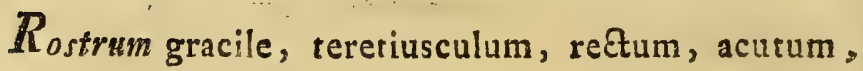
apice inflexo.

Nares lineares, parvæ, juxta basin, laterales. Pedes tetradactyli. Digiti antici ad medium palmari, dehine membrana lobata marginati.

CXCII. PHALAROPUS RUFUS, Bechst.

DiAGN: Rostrum pone apicem depresso-dilatarum. Corpus supra fulvo nigroque macularum, subtus rufum.

"In serie naturali hoc gentrs ad Totanos proxime accedit. 


\section{SYNONYMA.}

Phalaropus rufus, Bechst. N. D. 2te Auf. 4 B. I Abth. p. $38 \mathrm{I}$.

Tringa fulicaria, Lin. Syst. I. p. 249. n. 10. Brünn. Orn. bor. p. 5 I. n. I72.

Tringa hyperborea, Gmel. Syst. I. p. 676. n. 9. var. b. Le Phalarope rouge, Buff. Ois VIII. p. 225.

Ie Phalarope roussatre, Briss. Orn. VI. p. 20. Edw. Av. t. I 42.

Red Phalarope, Lath. Syn. V. p. 27 I.

\section{DESCRIPTIO.}

Longit. avis circiter $8 \frac{\pi}{2}$ poll. Rost, $\frac{7}{8}$ poll. A flexura alx ad apicem 5 poll." Tibia $\frac{6}{8}$ poll. Digit. med. $\frac{7}{2}$.

Rost:um fulvum apice nigro basi subteres pone apicem depresso-dilatatum apice acuto. Iris rufo-lutea. Pedes nigro - virides. Femora seminuda. Digiti a basi ad medium membrana juncti, dehine membrana lobata marginati.

Facies et pileus cinerea, hic obscurior. Genx et tempora alba. Dorsum maculis nigris et sordide luteis, i. e. pennis nigris marginibus latis rufo-luteis. Urapygium album maculis transversis nigris. Collum, peĉus, abdomen, crissum, tectrices caudæ inferiores et superiores saturate rufa. Teetrices alarum saturate cinerex, albo marginatx; majores apicibus latius alux, unde fascia alba in ala complicata. Remiges prinores nigro-fuscx rachibus albis, secundarix intus et apice albx: Cauda cuneato-rotunàa. Reôricibus fuscis; marginibus rufo-griseis. 


\section{HISTORIA.}

Habitat et se propagat in regionibus maxime bores. libus; vere et autumno demigrans et rediens, raris. sime obvenit in litoribus nostris marinis. Oeconomi avis adhuc plane incognita est.

CXCIII. PHALAROPUS CINEREUS, Briss.

DIAGN : Rostrum gracillimum. Corpus supra nigricans marginibus ferrugineis. Gula, pectus et abdomen alba, Latera colli (in mare) rufa.

\section{SYNONYMA.}

Phalaropus cinereus, Briss. Orn. VI. p. I5. 2.

Tringa hyperborea, Retz. Fn. Sv. p. 183. 1. 15. 2. Lin.

Syst. I. p. 249. n. 9. Gmel. Syst. I. 2. p. 675.

Phalnropus hyperboreus, Lath. Ind. Orn. II. p. 775. n.

I. (mas).

Tringa lobata, Fabr. Fu. Groenl. p. 109.

Le Phalarope cendré, Buff. Ois. VIII. p. 224. Pl. enl.

766. vers. germ. Otto XXX. p. III. cum fig.

Cock loot-footed Tringa, Edw. t. 143.

Red Phalarope, Lath. Syn. V. p. 27 I.

Le Coq d'Odin, Ascan. Icon. rer. nat. II. p. 8. t. 20.

Rothhälsiger Wassertreter, Bechst. N. D. 4 B. I Abth. p. 372.

Juмror: Phalaropus fuscus, Briss. Orn. VI. p.. I8. n. 3.

Lath. Ind. II. p. 776. n. 4. Edw. Av. t. 46.

Tringa lobata, Lin. Fn. Sv. p. 64. n. 179.

Phalaropus lobatus, Lath. 1. c. p. 776. n. 2. 


\section{DESCRIPTIO.}

Logit. $7 \frac{1}{2}$ poll. Rostr. $\frac{7}{8}$ poll. A fexura ala ad apicem $4 \frac{2}{6}$ poll. Cauda $2 \frac{1}{8}$ poll. Tibia $\frac{6}{8} ;$ Digit. med. $\frac{6}{3}$; postic. $\frac{3}{T^{3}}$ poll.

Rostrum nigricans, gracillimum, maxillis acutissimis, supêriore paulo longiore, apice infexa. Femora seminuda et pedes plumbei.

Caput, cervix, dorsum et teetrices alarum cinereo. nigra, dorso anteriore rufo maculato. Albedo Gulx ad latera extenditur. Jugulum et latera colli rufa. Palpebre albx. Pectoris latera cinerea. Pectus, ab. domen et crissum alba. Remiges nigræx primores rachi alba, secundarix basi et margine apicis albx. Teetrices proximx apice albæ, unde stria alba in ala. Cauda nigricans apice rufo marginata.

A. Caput et dorsum nigro-fusea fulvo hine immixto. Lateribus capitis colo: luteus inmixtus. Gula alba. Juguluin et latera colli ferruginea - cet. sim. antec. B. Pileus et latera capitis per oculos cinereo-fusca, rufo immixto. Frons, genx, gula, jugulum et latera colli alba. Dorsum et scapulares nigro-fusca marginibus latis ferrugineis; Teetrices alarum cinereo-fuscr, majores albo et ferrugineo marginate, proximæ apice albx. Collum antice cinerascens; prxterea tota sub. tus alba.

\section{HISTORIA.}

Habitat in regionibus Svecix et Norvegix septemtrionalibus. Fistaten degit juxta lacus et alias aquas dulces. Nidulatur quoque in insulis maris Norvegici. Vere ex autumno Svecian australiorem transmigrans, rarius obvenit. 


\section{GENUS. \\ F U L I C A.}

Rostram breve, crassum, rętum, compressum, apice supra subiusque æqualiter acuminatum.

Nares lineari-ovales, in medio rostro sitx.

Frons usque ad vericem calva colorata.

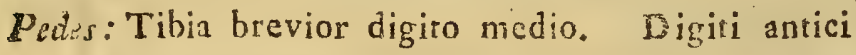
membrana lobata marginari unguibus longis acutissimis.

\section{FULICA A TRA.}

Dragn: Supra nigricans subtus cinereo-coerulea, capire et collo nigris, fronte calva alba.

\section{SYNONYMA.}

Fulica atra, Lin. Fn. Sv. 193. Retz. Fn. Sv. p. 199.

n. I7 I. Lin. Syst. I. p. 193. Lath. Ind. II. p. 777. Gmel. Syst. I. p. 702.

Fullica aterrima, Retz. Fn. p. I99. n. I72. Gmel. Syst.

I. n. 703 (inas senior).

La foulque ou morclle, Buff. Ois. VIII. p. 21 I. t. I8.

Pl. enl. 197. vers. germ. Otto XXIX. p. 2\%6. cuin fig.

La grande foulque, Buff. Ois. VIIL. p. 220. 
Common Coot, Pen. Br. Zool. Il. p. 494. to 7\%. Aret. Zool. II. p. 496. Lath. Syn. V. p. 275. vers. Germ. p. 243.

Great Coct, Lath. Syn. V. 277.

Schrvarzes Wasserhuha, Bechst. N. D. 2te AuA. 4 B. p. $51 \mathrm{I}$.

Fulica Ethiops, Mus. Carls. fasc. I. tab. I3.

Svecis: Wattenhöns, Sjöorre.

OBSERVATIO.

Fulicam leucorycem Mus. Cars. I. t. 12. avem esse composisam ex corpore Fulice otra et alis Ttivionis sabalpini; jam omnibus notum existimo.

\section{DESCRIPTIO.}

Longit. I7.poll. Rostr. I $\frac{3}{8}$ poll. A flexura alx ad apicem 9 poll. Cauda $2 \frac{2}{3}$; Tibia $2 \frac{\pi}{2}$ poll. Digit. med. $3 \frac{5}{8}$; post. $I_{\frac{3}{3}}$ poll.

Rostrum et frontis callus ovatus, alba. Iris rubra. Pedes fusco-olivacei, armillis fulvis.

Caput et collum atra. Dorsum, scapulx et tectrices alarum nigra cinereo tincta. Peetus et abdomen saturate coeruleo - cinerea griseo s. rubescenti immixto. Crissum er tectrices caudx superiores et inferiores nigræ. Cauda rectricibus XII. nigris griseo obsolete marginatis. Remiges primores nigro-fusca latere exteriore cinereo tineta; extima extus tenuiter alba; secundaria saturate cinerex apieibus albis. Tectrices alarum inferiores nigro-cinerex.

\section{HISTORIA.}

Migratoria aris habitet in stagnis, lacubus et fossis inter arundines et Equiseto liaud rara. Ex. or. in 


\section{PINNATIPEDES. FULICA.}

Scania juxta Landscrena in fossis castrensibus; in fos. sis turfosis per totam fere campestrem plagam. agre volat et ambulat, libentins natat. Terrefåta, motis celeriter alis, aquam pedibus pulsans hostem effugit. Nidulatur in arundinetis. Ova $6-10$. Vescitur Insectis et herbis aquaticis. Caro haud sapida, nec nisi protracta pelle, assa comeditur. 
SECTIO II.

Membrana marginali digitorum intogra.

GENUS.

\section{P O D I C E P S.}

Rostrum mediocre (capite jam aliquantum longius jam brevius), rętum, subulatum, compressum, acurum.

Nares oblongx, laterales, pervix, postice membrana tectx.

Pedes compressi, tetradactyli digitis anterioribus basi membrana connexis dehinc membrana integra, lobiformi, marginatis. Tibia postice bifariam serratz.

Cauda nulla.

\section{PODICEPS CRISTATUS, Lath.}

Diagn: Pileus, crista occipitalis et collare nigra. Facies alba lateribus capitis rufis. Collum antice, corpus subtus et remiges secundariæ alba.

\section{SYNONYMA.}

Podiceps cristatus, Lath. Ind. II. p. 780. I. Retz. Fn. Sv. p. I5I. n. 110. 
Colymbus cristatus, Lin. Syst. Nat. I. p. 2?2. Fn. Sv. 15 I. Gmel. Syst. I. 2. p. 589. - cornutus, Briss. Orn. VI. p. 45. n. 4. t. 5. f. I.

Le Grêhe cornu, Buff. Ois. VIII. p. 235. t. 19. Pl. enl, 400. vers. Germ. Otto XXX. p. 23 cum fig. Crested Grehe, Pen. Br. Zool. II. p. 497. Are. Zool. II. p. 498. Lath. Syn. V. p. 28 I. vers. Bechst. V. p. 249.

Der gehä̈ubte Steissfuss, Bechst. N. D. 2te Auf. 4 B. 2 Abth. p. 533. Meyer Liv. p. 220.

Funior:

Colynbus urinator, Gmel. Syst. I. p. 593.

Colymbus cristatus, Briss. Orn. VI. p. 34. n. I.

Le grểe huppé, Buff. Ois. VIII. p. 233. Pl, enl. 944. et 94 I.

Tippet Grebe, Lath. Syn. V. p. 283.

\section{DESCRIPTIO.}

Long. $19 \frac{T}{2}-20$ poll. Rostr. a fronte $I \frac{7}{8} ; \mathrm{ab}$ angulo oris $2 \frac{x}{2}$ poll. A fexura alx ad apicem 7 poll. Tibia 2; Digit. med. 3 poll.

Rostrum reetum, tereti-compressum, acutum, maxilla superiore fusca margine et maxilla inferiore incarnatis, apice albido. Iris coccinea. Pedes extus nigricantes, intus albescentes maculis in articulationibus óbscuris. Lora nuda rubra.

Caput supra et crista occipitalis bipartita, nigra. Gula, genæ et supercilia alba. Latera capitis inferiors et jugulum rufa, collari dependente nigro ornata. Collum postice fusco-cinereum. Dorsum, scapulares et alæe supra nigricantia marginibus pennarum rufescentibus vel cinerascentibus. Collum antice, pectus, abGomen alba, argenteo nitentia. Latera pectoris et ab. 
dominis rufa, fusco immixto. Reniges primores fuscx, secundarix albx rachibus a basi fuscis; intima fuscx. Margo alx superior albus; ceterum tectrices alarum cinereo - fuscr.

\section{HISTORIA.}

Habitat æstate in lacubus, amnibus et stagnis; in Scania haud rara. Nidum ex culmis junci, scirpi et aliorum graminum, in aqua natantem struit. Ova 4. oblonga, allida, sordide rufescenti tincta. Vere et autumno in mari obvenit. Vescitur Piscibus, insectis et vermibus aquaticis.

\section{PODICEPS RUBRICOLLIS,}

DiAgn: Pileus et crista occipitalis brevis, truncata, nigra. Gula et latera capitis inferiora cinerea. Collum lxve, rufum.

\section{SY NON YMA.}

Podiceps rubricollis, Lath. Ind. II. p. 783. Retz. Fn, Sv. p. I53. n。 II3.

Podiceps subcristatuls, Bechst. N. D. 2te Auf, 4 B. 2 Abth. p. 546. Meyer Liv. p. 22 I.

Colymbus rubricollis, Gmel. Syst. I. 2. p. 592.

Colymbus subcristntus, Gmel. 1. c. p. 590.

Le grểb à joues grises, Buff. Ois. VIII. p. 24I. Pl. enl. 93I. vers. Germ. Otto XXX. p. 45. cum fig.

Red-necked grebe, Lath. Syn. V. p. 288. Sup. p. 260. t. II8.

Funior:

Colymbus parotis, Sparm. Mus. Carls. I. t. 9. Gmel. Syst. I. p. 592. Lin。 Fn. Sv. n. I52, (A2.IA).

Nisisson Onnitlo Pars $I I$. 


\section{DESCRIPTIO.}

Longit. I $\sigma \cdot I \sigma \frac{\mathrm{T}}{2}$ poll. Rostr. a fronte $I \frac{\hat{\sigma}}{3} ; \mathrm{Ab}$ an. gulo oris fere 2 poll. A flexura alr ad apicem 7 poll. Tibia 2 poll. Digit. med. $2 \frac{\sigma}{3}$ poll.

Rostrum nigrum basi luteum, brevius et aliquantum robustius quan anteeedentis; basi minus, apice vero magis compressum et altum. Pedes extus nigri, intus virescenti - lutei.

Captut supra, crista brevis occipitalis truncata et cervix nigra, nitentia. Gula, genæe et superius juguIum cinerea, albido cincta. Collum antice et ad latera, superiorique pectoris pars, rufa, nitentia. Dorsum, scapulares et alr supra nigricantia marginibus pennarum hinc inde rufo-cinerascentibus. Pectus inferius et abdomen alba, argenteo nitentia. Latera pectoris et hypochondria fusco - nigricantia. Remiges nigrofuscx; secundaria albærachibus fuscis, intime nigrofuscx.

Avis junier: Caput læve, dorsum, cervix, supra nigrofusca. Latera capitis inferiora alba, fusco longitudinaliter striata. Gula alba. Jugulum rufum. Péctus Et abdomen sordide argentea. cet. ut antec.

\section{HISTORIA.}

Habitat æxtate in laeubus et stagnis Scanix, ibique nidulatur. Vescitur pisciculis, amphibiis et insectis aquaticis. Ova $3-4$.

\section{PODICEPS AURITUS, Lath.}

DIAGN : Pileus, crista occipiralis brevis, collare et gula nigra; crista aurium ferruginea. Jugulum rufum. Abdomen et Remiges secundaria albæ. 


\section{SYNONYMA.}

Podiceps nuritus, Lath. Ind. II. p. 78I. 11. 3. Retz. Fn. p. I52. N. III.

Colymbus auritus, Lin, Syst. I. p. 222. Fn. Sv. p. 53. 11. 152. Gmel. Syst. I. 2. p. 590, Briss. Orn. VI. p. 50. n. 6 .

Sef Önd, Mohr Isl. Nat. hist. p. 40. t. 2. Olaf. Itin. p. 550.

Eared Grebe, Latl, Syn. V. p. 285. Pen. Br. Zool. II. p. 500. t. 79. Aret. Zool, II. p. 499. Edw. t. 96. f. 2 .

Geöhrter Steissfuss, Bechst. N. D. 2te Auf, 4 B. 2 Abth. p. 552. Meyer Liv, p. 222.

\section{OBSERVATIO,}

Inter aves Rudbeckii pictas optima hujus avis icon exstat, quam miror Linné in Fn. Svo no 152 .| lnon citasse, cum tamen eandem fere Synonymiam ac Rudbeckius adferat.

\section{DESCRIPTIO.}

Long. I2 poll. Rostr. $\frac{7}{8} ;$ A flexura ala ad apicem $5 \frac{\pi}{2}$ poll. Tibia $I \frac{s}{8} ;$ Digit, med. $I \frac{7}{8} ;$ postic, $\frac{3}{8}$ poll.

Rostrum compressum, acutum, nigrum; maxilla inferior subtus subrecurvata. Iris et palpebra cinnabarinx. Pedes extrorsum nigricanti-cinerei, introrsum pallide grisei. Latera capitis et gula pennis longioribus tumidis, prasertion pone oculos. Caput nigrum stria pone oculos ferruginea. Cervix, dorsum nigricantia, Jugulum ferrugineun. Pectus et abdomen alba. Remiges primores sex fuscr, reliqux et secundarix alba; intimæ nigro fusce. 


\section{HISTORIA.}

Habitat in lacubus, stagnis et fltwiis, in Scania re. rius. Nidificat inter arundincs in aqua crescentes. Ova 3-4. albida, lutescenti tincta. Victus sut praced.

\section{PODICEPS OBSCURUS,}

\section{Lath.}

Diagn: Supra nigro. Fuscus capite levi. Gula, genx, abdomen et Remiges secundarix albr.

\section{SYNONYMA.}

Podiceps obscurus, Lath. Ind. II. p. 782. n. 4. Rctz, Fin. Sv. p. I53. n. II2.

Colymbus mizror, Briss. Orn. II. p. 56. n. 7.

Colymbus obscurus, Gmel. Syst. I. 2. p. 592.

Le petit grêbe, Buff. Ois. VIII. p. 232. Pl. enl. 942.

vers. Germ. Otto XXX. p, I6. cum fig.

Dusky Grebe, Pen. Br. Zool. II. p. 50I. t. 78. f. I. Lath. Syn. V. p. 286 . n. 5. vers. Bechst. V. p. 254- Edw. 96. f. I.

Dunkelbrauner Steisfuss, Bechst. N. D. 2te Auf. 4 B. 2 Abth. p. 559.

\section{DESCR I P TIO.}

Long. II poll. Rostr. $\frac{7}{8}$; A flexura alæ ad apicene 6 poll. Tibia $I \frac{3}{8} ;$ Digit. med. 2. postic. $\frac{3}{8}$ poll.

Rostrum rectum, compressum acutum, maxilla in. feriore aliquantum recurvata. Nares ovato-lineares. Pedes extus fusci, intus cinerascentes.

Caput supra. cervix, Dorsun et alæ nigricantia. Gula et latera capitis inferiora alba. Pectus et abdomen nitide alba (argentea) jugulo cinerascente. Remiges prinores et intima fusca, medix alba, unde 
macula alarum vel stria alba oritur. Tectrices alarum inferiorcs albx. Hypochondria alba, fusco maculata.

\section{IISTORIA.}

Rara apud nos avis habitat in aqua dulci et juxta litora marina. Nidificat in arundinetis. Ova 3.4. victus ut congenerum,

\section{PODICEPS MINOR, Lath.}

DIAGN: Supra einereo-fuscus, rufescenti tinctus, capitelrvi; subtus rufescenti-albidus. Remiges fuscæ, secundariæ latere interiore et apice albw.

\section{SYNONYMA.}

Podiceps minor, Lath. Ind. II. p. 784.n. 9. Retz. Fn. Sv. p. I54. n. II 4.

Colymbus minor, Gmel. Syst. I. 2. p. 59I. - fuviatilis, Briss. Orn. VI. p. 59.

Le Grêbe de rivière ou Castagnвux, Buff. Ois. VIII. p. 244. t. 20. Pl. enl. 905. vers. Germ. Otto XXX. 5 I. cum fig.

Litzle Grebe, Pen. Br. Zool. II. p. 50I. Syn. V. p. 289. Kleiner Steissfuss, Bechst. N. D. 2te Auf. 4 B. p. 565 .

\section{DESCR IP TIO.}

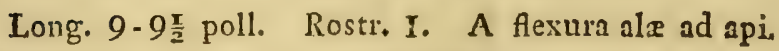

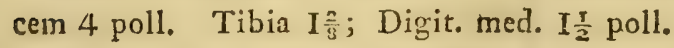

Rostrum robustum, subulatum, parum compres. sum, nigricans, basi subtus et lora nuda albida. Pe. des extus fusci intus incarnati. 
Caput supra, dorsum et alæe cinereo - fusca rufescenti tincta. Gula et abdomen alba. Latera capitis, collum antice, pectus et latera albida, rufo tincta. Remiges fuscr, primores immaculatr; secundaria apice, et laterę interiore albida,

\section{HIST ORIA.}

Rarior avis habitat in lacubus, fuviis et stagnis, Inter arundines nidum natantem struit. Ova 4. albida, rufescenti tincta. 
OR N I T H O L O G I A

S V E C I C A.

PARTIS POSTERIORIS

FASCICULUS POSTERIOR. 



\title{
ORDO. PALMIPEDES.
}

TRIBUS PRIOR: Pedibus tridactylis.

\author{
GENUS: \\ A L C A.
}

$\boldsymbol{R}_{\text {ostrum breve, compressissimum, transversim }}$ sulcatum, margine supra et subtus acuto. Nares lineares, marginales, laterales, nudx, vel plumis tectæ et juxta basin rostri sitæ. Pedes breves, retrąi, (postice siti) unde incessus fere erectus.

Alce breves; Remex prima longitudine secunda.

\section{ALCA TORDA, Limn.}

DiaGN: Rostrum sulcis quatuor nigrum, fascia alba. Pedes nigri. Cauda cuneiformis. Linea alba a rostro ad oculos aliaque alarum transversa.

\section{SYNONYMA.}

Alca Torda, Lin. Faun. Svec. p. 49. n. I39. Syst. nat. I. p. 210. Retz. Faun. p. I39. n. 96. Gmel. Syst. I. p. 55I. Lath. Ind. II. p. 793. n. 5. Briin. Orn. bor. p. 25. n. IO0. Ödmann N. Acta Acad. Scient. Holm. I788. p. 214. Fabric. Fn. Groenl. p. 78. Nilsson Ornith. Pars 11 . $\mathrm{K}$ 
Alca Balthica, Brünn. bor. IOI. (fem.)

Razorbill, Pc1. Brit. Zool. II. p. 509 t. 82. Arct. Zool.

II. p. 509. n. 425. Lath. Syn. V. p. 3 I9. Edw.

Av. VII. t. 358. f. 2.

Pingouin snacroptère, Temminck Manuel d'Ornitholo.

gie p. 6I6. - Buff. Pl. enl. 1003 (mas). I004. (fem.)

Tord Alke, Bechst. Nat. Deutschl. IV. p. 7 II.

Tordmulen, Svensk Zoologi II. B. 2. H. t. 44. Avis junior:

Alca Picn, Lin. Syst. I. p. 210. 11. 2. Retz. Faun. p. 140. 11. 97. Gmel. Syst. I. p. 55 I. Fabric. Groenl. p. 79. n. 5I. - Alca minor Briss. Orn. VI. p. 92.

t. 8. f. 2. (mas junior) - Aica unisulcata Briinn. bor. p. 25. n. 102. - Blackbilled Auk, Pen. Aret. Zool. II. p. 5IO. n. 426. Lath. Syn. VI. p. 320. Svecis: Tordmule; Blekingis: Turemule; Goxtlandis: Tord; Angermannis: Mule.

\section{DESCRIPTIO.}

Longit. I6 - I $6 \frac{\mathrm{I}}{2}$ poll. Rostrum a fronte $I \frac{3}{8} ;$ a sinu oris 2 poll. Cauda $3 \frac{\pi}{2}-4$ poll. Tibia $I \frac{2}{3}$ poll. Di. git. med. 2 poll.

Obs. Differt magnitudinc; Specimen cepi, cujus erat Longit. J 8 poll. Alæ extensæ 28 poll. Rostr. a fronte $I_{\frac{1}{2}}$ poll. a sinu oris $2 \frac{2}{8}$ poll. Tibix $I \frac{\pi}{2}$; Digit. med. $2 \frac{3}{8}$ poll.

Rostrum nigrum, valde compressum, dorso maxillx superioris semicirculari apice adunco (maxilla inferiori in medio gibba), lateribus sulcatum, sulcis 3.4 fasciaque transversa alba. Iris brunnea. Pedes nigri. Ala plicata basin caudx attinens. Cauda cuneiformis, Rectricibus medis acuminatis. 
Imo Mas senior: Caput et collum totum, dorsum, alx et cauda nigra; Linea a dorso rostri ad oculos; Peetus, abdomen, crissum, teetrices alarum et caudx, remigesque secundarix apice alba. (unde linea transversalis alba in ala complicata oritur).

2do Femina scnior: Caput et collum supra nigra, lineâ albâ a rostro ad oculos destituta. Gula, tempora et jugulum alba cet. color ut in Mare.

3tio Avis junior: Similis fere, quoad colores, seniori feminx, a qua tamen differt: rostro minori minusque alto, unco et sulcis fere destituto. Præsentia liner albx inter rostrum et oculos sexum masculinum et absentia femininum mature indicat.

\section{H IS TORIA.}

Habitat passim in utroque Scandinaviæ mari. Nidulatur in insulis passim gregaria, ex. gr. in Carlsön Gottlandix, in Bonden Angermannix (Lin.), in Nïmdön Sudermannix (Ödm.); in insulis quoque maris norvegici, frequentius tamen in regionibus arcticis. Hieme meridiem versus migrans oras quoque Scanire et Blekingix frequentat: neque raro hoc anni tempore a piscatoribus Oeresundicis in retibus, qux piscibus tensa sunt, capitur et ad urbes venalis deducitur. Victitat piscibus minoribus, præsertim Clupeis, et Crustaceis. Mense Majo proli augendæ inserviens, in patrias rupes revertitur. Ovum unum, pro magnitudine avis magnum, albidum, extremitate crassiori nigra, ceterum nigro maculatum, in rupis cujusdam fissura, vel foramine, vel in ipsa rupe, sine ullo strato, ponit.

\section{ALCA IMPENNIS, Lisi.}

Diagn: Rostrum et pedes nigri; macula ovata alba utrinque ante oculos: Alæ minutr. 


\section{SYNONYMA.}

Alca impennis, Lin. Syst. I. p. 210. 3. Faun. Sv. p. 49. 11. I40. Retz. Fn. p. I40. n. 98. Fabr. Fn。 Groenl. p. 82. n. 52. Gmel. Syst. Nat. I. 2. p. 550. Brünn. Bor. 105.

Great Auk, Pen. Brit. Zool. II. p. 507. tab. 8I. Arct. Zool. II. p. 509. n. 424. Edw. Av. III. t. I47.

Grand Pingoin, Buff. Ois. IX. p. 3 I3. t. 29.

\section{DESCR IP TIO.}

Magnitudo Anseris domestici, collo breviore.

Rostrum nigrum ancipiti-compressum, sulcis quatuor vel pluribus obliquis. Pedes nigri. Alæ minutæ, volatui ineptr.

Caput, gula, jugulum, dorsuin et alæ nigra, macula majori, ovata, utrinque inter rostrum et oculum.

Apices remigum secundariarum totaque pars inferior corporis alba.

\section{OBSERV.}

Pro atate colores fere eodem modo ac antecedens mutat, ita tamen ut linea illins alba maculan hnic formet, et moles corporis semper sit duplo major.

\section{H IS T ORIA.}

Habitat in pelagoboreali. Raro ad litora insularum Eahusia ) appropinquat, neque, quantum scio, ibi nidulatur. Ovum unicum eodem fere colore ac alca Tor.

*) Ante aliquot annos Specimen hajus Speciei juxta Marstrand occisum fuit. 
de, sed magnitudine multo majori, in rupibus a lit. tore remotis ponere refertur. Cetcrum historia hujus Speciei nondum bere explicata est.

\section{ALCA ARCTICA, Lin.}

Diagn: Rostrum rubrum basi plumbeum. Pedes aurantii. Gula et latera capitis cinereo-alba. Remiges secundariæ immaculatæ. Cauda xqualis brevis.

\section{SYNONYMA.}

Alca Arctica, Lin. Syst. I. p. 2 I I. n. 4. Fin. Suec, p. 49. n. I4I. Retz. Fn. I4I. n. 99. Fabr. Groenl. p. 83. 11. 53. Gmel. Syst. I. p. 549. Lath. Ind. II. p. 792. 11. 3. Brünn. Bor. p. 23. 11. 103.

Fratercula, Briss. Orn. II. p. 380.

Puffin Auk, Penn. Brit. Zool. II. p. 5I2. Aret. Zool.

II. p. 5 I I. n. 427 et forte 11. 428. Edw. Av. VII. t. 358. f. I.

Mncareux moine, (Mormon fratercula) Temm. Man. d'Ornith. p. 6I4. - Le Macaveux Buff. Ois. IX. p. 358. t. 26. Pl. enl. 275.

Der Arktische Alk, Bechst. N. D. IV. p. 723.

Siecis: Grönländsk Papegoja; Norw. Lunda, Lumne, Lundfogel.

\section{DESCR IPTIO.}

Longit. I3 $\frac{x}{2} \cdot I 4$ poll. Alx extense 23 poll. Rostrum a fronte $I \frac{6}{8}$. A flexura alre ad apicem $6 \frac{T}{2}$; Tibia I poll. Digitus med. $I \frac{6}{8}$ poll,

Rostrum compressissimum robustum, eadem altitudine ac longitudine, supra subtusque in acumen ferc 
zqualiter decrescens; medietate exteriore rubra (sanguinea) interiore plumbea; subtus, basi et linea transversali media albis. Maxilla superior sulcis tribus, inferior duobus. Sinus oris macula nuda lutea. Macula triangularis nuda plumbea oculos cingit. Iris pallide grisea. Pedes luteo-rubri unguibus acutis et arcuatis nigris. Cauda longitudine alarum, xqualis.

Pileus, collare latum, dorsum, alæ et cauda nigra nitentia, Remigibus in fuscum vergentibus. Gula et latera eapitis usque supra oculos et pone aures pallide cinerea. Pectus, abdomen et crissum tectricesque caudæ et alarum pure alba.

Auis Funior: Rostrum multo minus, lateribus fere lævibus, fusco-luteum. Pedes sordide rubri. Cnput su. pra et torques colli nigro-fusca. Gula et latera capitis sordide cinerea. Corpus subtus cinerascenti-album.

\section{HISTORIA.}

Hieme interdum oras Bahusiæ visitat; æstate oceanum arcticum insulasque ibi sitas incolit. In his insulis ex. gr. in Lovumnen et Fugelöen (juxta oras Nord. landix Norvegicx sitis) in insulis Lofodensibus et in quibusdam aliis ad Finnmarkiam pertinentibus, hæc avis innumerandis gregibus collecta xstatem degit et se propagat. Oeconomiam ejus, qualem ipse in insula Lovunnen obscrvavi et ab incolis audivi, talem describam: Advenit avis juxta insulam allatam post initia Mensis Maji, neque statim terram visitat, sed in mari degit, usque dum omnis nix in terra evanuerit. Jam vero domicilia priora requirit. Est vero in his plagis semper fere regio ad radices altissimorum inontium et in infimis eorum lateribus sira, quæ saxis et lopidibus de summo culmine rupium fractis et dejectis, horrid 
est et fere impervia. Hæc regio ab incolis Ore appellatur (Islandis Urd audit). Heic in forminibus et fissuris inter lapides Alca e gramine siccato et ex plumis nidum sibi præparat, in guo ovum unicum album magnitudine ovi Anatis domestica ponit. Quamvis gregaria nidulatur, et quamvis plures sæpe in eodem foramine ova fovent; tamen monogamiam celebrat et uterque sexus mutuo non solum incubat, sed prolem quoque mutuo cibat*). Alimenta, qux ad pullos apportant, sunt pisciculi in alto mari urinando collecti, prrsertim Amodytes Tobianus et Clupen Encrasicolus. Hoc labore prasertim occupatæ sunt Mense Julio, ubi continuo alix evolant, alix domum revertuntur et multitudine sua examen apium haud male referunt. In aëre alis agitato continuum susurrum quasi vento vehementi excitant. Juxta medium Mensis Augusti pulli volare possunt, et circa Festum Sti Bartholomæi (die 24 Aug.) his oris omnes cum parentibus valedicunt, et versus plagam occidenti-meridionalem volatum tendunt. $\mathrm{Ab}$ insulanis capitur canibus, qui intrantem inter lapides avem observant et egredientem apprehendunt. Hanc venationem per totem æstatem agunt innumerabilemque avium cohortem quotannis hoc modo capiunt. Caro præsertim juniorum sapida est. Plumis sicut Anatis Mollissima utuntur. Pennæ quoque in usu sunt.

*) It a referunt Insulani. Inter quinque vero aves, quas dissecui, nullum Marem inveni. Intel viginti, quas examinavi, in nulla inveni illum mucronem membranaceum, quo secundum Fabriciuin instructa est palpebra superior. 
GENUS.

U $R$ I A.

$\boldsymbol{R}_{\text {ostrum breve, subulatum, compressum, læve, }}$ supra convexum, maxilla superiore apice parum curvata, inferiore in medio subtus subangulata. Nares laterales, pervir juxta basin rostri in fossula sitx, membrana plumosa semitectx.

$P_{e d e s}$ breves, retracti (postice positi) ungvibus curvatis, acutis.

Alce breves, Remige prima ceteris aliquantulum longiore,

\section{URIA TROILE," Lath.}

DIAGN: Supra cinereo-fusca, subtus remigibusque secundariis apice albis. Rostrum longitudine fere capitis.

\section{SYNONYMA.}

Uria Troile, Lath. Ind. II. p. 796. Retz. Faun. p. I49. n. 109.

Uria Lomvia et Troille, Brünn. Bor. p. 27. n. I08, I09.

Colymbus Troile, Linn. Syst. I. p. 220. Fn. Sv. p. 52. n. I49. Gmel. Syst. I. p. 585.

Le Guillemot, Buff. Ois. IX. p. 350. t. 25. Pl. Enl. 903 . 
Langvige et Stuttnefin, Olavs. et Pov. Reis. in Isl. I.

p 355. tab. 2I, 22.

Guillemot à Capuchon, Temm. Man. d'Orn. p. 606. Foolisch Guillemot, Lath. Syn. VI. p. 329. Id. Supp.

I. p. 265. - Pen. Britt. Zool. II. p. 5I9. Arct. Zool. II. p. 5 I б. 11. 436 . Ediv. av. t. 359. f. I.

Der Dumme Lumme, Bechst. Nat. D. IV. p. 574. Avis Funior: Uria Svarthag et Ringuin Brümn. Bor. p. 27. 11. IIO. III.

Colymbus Minor, Gmel. Syst. I. p. 585.

Lesser Guillemot, Pen. Britt. Zool. II. p. 520. n. 23.5. Arct. Zool. Suppl. p. 69. - Lath. Syn. VI. p. 332.

Blekingis: Silladopping.

\section{DESCRIPTIO.}

Longit. I6 - I7 poll. Rostrum (superne) I $\frac{7}{2}$ poll. ab angulo oris $2 \frac{\pi}{2}$ poll. A flexura al: $7 \frac{\pi}{2}$. Tibia $I \frac{\pi}{2}$ poil. Digit. med. $2 \frac{\tau}{8}$ poll. Cauda 2 poll.

Rostrum virescenti-njigrum. Iris brunnea, Pedes lutescenti-nigri membranis natatoriis nigris.

Caput, pars colli antice, totumque collum postice, Dorsum, uropygium, Alx et cauda nigra in cinereum vergentia; Pars inferior colli antici, Pectus, Abdomen, Crissum, Alarum et caudx inferiores tectrices apicesque Remigum secundariorum alba. Senior avi.

Femina Mare aliquaintulum minor.

Funior Avis: Pedes et basis Rostri lutescenti - fusca. Iris nigro - fusca.

Caput et Collum supra, Dorsum, ale et cauda sattrrate cinereo-fusca. Fascia ejusdem coloris pone oculos versus nucham ducta. Latera occipitis albo-ma- 
culata. Gula, jugulum, pectus, abdomen, crissum et apices remigum secundariorum pure alba. Hypochondria striis quibusdam transversis fuscis notantur.

\section{HIS T ORIA,}

Habitat in partibus borealibus marium, qux Scandinavian cingunt, tum in Mari Balthico tum occidentali. Autumno et hieme juxta oras Scandinavix meridionalioris quoque obvenit. Nidulatur in insulis et rupibus marinis tum intra tum extra circulum polarem sitis, ex. gr. in Insula Gottlandia \&c.

\section{URIA GRYLLE, Lath.}

DiAGN: Rostrum nigrum, pedes rubri, tectrices alarum albæ.

\section{SYNONYMA.}

Uria Grylle, Lath. Ind. II. p. 797. Retz Faun. p. I48. n. I08. Fabr. Fn. p. 92. n. 60. Ödm. Act. Acad.

Holm. I781. p. 228. Brünn. Bor. II3.

Uria minor nigra, Briss. Orn. 2. p. 379.

Colymbus Grylle, Lin. Syst. I. p. 220. Fin. Sv. 11. I4S.

Gmel. Syst. I. 2. p. 584. n. I.

Le petit Guillemot noir, Büff. Ois. IX. p. 354.

Guillemot à miroir blanc, Ternm. Man. p. 608. Blach Guillemot, Lath. Syn. VI. p. 332. Pen. Brit. Zool. II. p. 52I. Pen. ArEt. Zool. II. p. 5I6. r. 437. Edw. Av. t. 50.

Der schwarze Lumme, Bechst. Deutschl. IV. p. 586. Meyer Liv. u. Esthl. p. 223.

Avis junior: Uria Baltica et Grylloides Brünn. Bor. p. 28. n. II4. II5. II6. 
Colymbus Laćteolus, Gmel. Syst. I. 2. p. 583. n. I3.

Cepphus Lacteolus, Pall. Spic. V. p. 33.

Uria Lncteola, Lath. Ind. II. 798.

Uria Albida, Bechst. Nat. D. IV. p. 593.

Svecis: Grisla, Gresla; Gottl. Grylle. Norveg. Teiste, Teste. Scanis: Iste.

\section{DESCRIPTIO.}

Longit. I3 poll. Alæ extens 22 poll. Rostr. IT poll. $\Lambda$ flexurn alæ 7 poll. Tibia $I_{\frac{3}{8}}^{3}$ poll. Digit. med. $I \frac{\kappa}{8}$ poll.

Rostrum nigrum. Iris nigro-brunnea. Os intus et pedes rubri. Lingua semiteres, supra plana, acuta apice bifido.

Caput, collum et totum corpus nigrum, tectricibus alarum inferioribus et superiorum majoribus et mediis albis, unde maeula magna alarum alba in ala compo. sita oritur.

Femina aliquantum minor colore nigro minus saturate. Avis junior autumno: Rostrum nigrum, pedes nigri, antice sordide rubescentes. Os intus pallide rubrum. Iris nigro - hrunnea.

Caput supra nigrum. Dorsum nigrum pennis uro. pygii et scapularum albido fimbriatis. Gula, pcêtus et abdomen alba. Latera capitis, collum et hypochondria alba, nigro tenuissime et irregulariter undulata. Alæ saturate nigræ macula magna alba, nigro sæpe irrorata vel variegata.

\section{OBSERV.}

$\mathrm{Ab}$ hoc pulli colore sensim abit avis in colorem Senioris ance descriptum, quem tamen non ante quartum vel quintun ztaris annum perfe the adtingit. 


\section{H ISTORIA.}

Habitat ad insulas maris occidentalis et Baltici, in arcticis frequentius, in plagis meridionalioribus rarius astivans. Nidulatur tamen quotannis in insulis et rupibus qux ad oras Bahusix, Hallandix et Scanix sita sunt ex. gr. Hallands wäderö, et ad Gottlandiam \&c. In oris tamen scopulosis Norvegix longe frequentissima, ibique migratoria per hiemen abit. Ora 2 albida nigro-maculata, in fissura rupis cujusdam ponit. Vescitur pisciculis et crustaceis.

\section{URIA ALLE, Temminck.}

DiAGN: Supra saturate nigra, subtus remigibusque secundariis apice albis. Rostrum brevissimum.

\section{SYNONYMA.}

Uria Alle, (Guillensot Nain) Temminck Manuel d' Ornith. p. 6 II.

Uria Minor, Briss. Orin. 2. p. 378.

Alca Alle, Lin. Syst. I. p. 2 I I. Gmel. I. 2. p. 554.

Lath. Ind. II. p. 795. IO. Lin. Faun. n. I42.

Fabl. Faun, Groenl. p. 84. n. 54.

Little Auk, Pen. Brit. Zool. II. tab. 82. n. 233. (Non descript.)

Small black and zohite Diver, Edw. Av. t. 9I. Avis Funior: Alca Alle, Retz. Faun. Sr. p. I42, n. IO0. Brünn. bor. p. 26. n. I06.

Le Petit Guillemot femelle, Buff. PI. enl. 917. Little Auk, Pen. Brit. Zool. II. p. 5I7. (Descr.) Aret. Zool. II. p. 5I2. n. 429. Lath. Syn. V. p. 327. Peder Dricker, Gunn. Act. Nidros. I. p. 26I. t. 6. Der kleine Alk, Bechst. Nat. D. IV. p. 732. 
Nautis Svec., Grönlands Dufwa. Norucgis: Pederdricker.

\section{DESCRIPTIO.}

Longit. $8 \frac{x}{2}$ poll. Rostr. superne $\frac{T}{2}$ poll. Ala a flexura $4 \frac{6}{8}$; Tibia $\frac{6}{8}$ poll. Digit. med. I $\frac{2}{8}$ poll.

Rostrum nigrum. Iris nigro-fusca. Pedes fusco. rufescentes membranis nigricantibus.

Obs. Rostrum brevissimum; preterea omnes habet characteres ad Genus Uria pertinentes.

Caput, gula et jugulum, collumque supra, Dorsum, Alæ et Cauda saturate nigra, Remigibus secundariis apice albis, primoribus fuscis. Collum inferius antice, pectus, abdomen et crissum pure alba. Avis junior: Gula et Jugulum pure alba. Regio aurium et stria occipitalis alba, nigro quasi irrorata. "Palpe* bra superior alba. Pennæ scapulares striis quibusdam longitudinalibus albis, praterea seniori similia.

\section{HISTORIA.}

Incolit Maria maxime borealia, nec nisi hieme et quiden raro oras meridionalioris Suecix visitat, Vescitur crustaceis, insectis et vermibus marinis. Nidulatur in fissuris rupium præruptarum. 
TRIBUS POSTERIOR: Pedibus tetradaCtylis.

\section{SECTIO PRIOR.}

Digitis anticis anembrana integra junctis, po. stico libero.

\section{A. Restro edentulo: \\ C O L Y M B U S.}

Rostrum mediocre, rętum, subulatum, compresso - teres, acutissimum, maxillis striktis margine inflexis.

Nares laterales, perviæ, juxta basin rostri sitx, membrana plumosa semitecta.

Pedes mediocres, retrąti. Tibice compressissima.

Digiti antici longi palmari: posticus membrana lobiformi instructus; Ungvibus latis et obtusis.

Ala breves remige prima ceteris longiore.

Canda brevis rotundata.

CCVI. COLYMBUS GLACIALIS, Lir.

Dragn: Caput et collum nigra. Fascia gulæ et macula cervicis interrupte alba. Corpus supra nigrum maculis quadrangularibus albis (Senior). 
Diagn: Caput et collum supra cinereo-fusca. Dorsum fuscum marginibus pennarum cinereis. Collúm antice et corpus subtus alba. (Junior).

\section{SYNONYMA.}

Plongeon Imbrim, Tem. Man. d'Ornith. p. 597. Avis Sentior:

Colymbus Glacialis, Lin. Syst. I. p. 22I. n. 5. Gmel. Syst. I. 2. p. 588. Lath. Ind. II. p. 799. Fabric. Faun. Groenl. p. 97. n. 62.

Colymbus torquatus, Brünn. Bor. p. 4I. n. I34.

L'Imbrim ou Grand Plongeon, Buff. Ois. VIII. p. 258. t. 22, - Pl. Enl. 952.

Northern Diver, Pen. Brit. Zool. II. p. 523. t. 84. n. 237. Arct. Zool. II. p. 518. n. 439. Lath. Syn. VI. p. 337.

Hav-Hymber, Gunner. Act. Nidros. III. p. 125.

Eistaucher, Bechst. N. D. IV. p. 595. - Meisner u. Schinz Vögel der Schweiz p. 257.

Avis funior:

Colymbus Immer, Brïnn. 1. c. n. 129. Linn. 1. c. p. 222.

Imber Diver, Pen. Brit. Zool. II. p. 524. t. 84. 11. 238. Himbryne, Olafs. et Pov. Reis. IsI. I. p. 556. tab. 40, Imber Taucher, Bechst. N. D. IV. p. 62I.

Imber, Gunner. Act. Nidros. III. p. 240. tab. II. f. I. Svec. Bahusiens. et Norveg. Immer, Imber, Emmer, Ömmer. Hymber, Hav-Hymber.

\section{DESCRIPTIO.}

Longitudo Avis usque ad 30 poll. Rostrum superne fere 3 poll.; a sinu oris 4 poll.; basi $I \frac{T}{B}$ poll. latum, 
maxillis vix inflexis. A flexura ala 15 poll. Tibix $3 \frac{3}{3}$ poll. Digit. extim. $4 \frac{5}{8}$ poll.; med. 4 poll. intimus $3 \frac{x}{2}$ poll.

Rostrun nigrum, majus et basi crassius quam se. quentis Speciei. Maxilla superior porrecta, inferior sursum curvata. - Pedes extus nigricantes, intus et membrana albicantes.

Caput et collum nigra viridi-et coeruleo-nitentia. Linea juguli transversalis alba, et cervicis macula semilunaris alba, nigro striata. Dorsum, scapulares et alarum tectrices nigra, maculis albis in dorso et scapu. Iis quadrangularibus, in tectricibus alarum et uropygio fusco, minoribus, rotundatis. Pectus, abdomen et crissum alba, hypochondriis nigro striatis.

Avis Funior:

Mus. Upsal.

Rostrum supra griseo-fuscum; subtus albidum.

Caput, occiput et cervix cinereo-fusca. Dorsum, Alæ et Uropygium saturate fusca marginibus pennarum cinereis. Gula et jugulum totumque corpus subtus alba.

\section{HIS TORIA.}

Habitat xstate in lacubus plagæ areticæ, præsertim Finmarkix, ubi in insulis nidulatur. Ova 2, fuscescentia. Pullis exclusis mare petit, ubi excellenter urinando prædam ex piscibus, insectis aliisque animalculis marinis quærit. Hyeme interdum, sed 1:rius, oras Bahusiz visitat.

\section{COLYMBUS ARCTICUS, Lin.}

Dragn: Caput et Collum supra cinerea; gula et jugulum nigra fascia gula aliaque laterum colli 
interrupte alba. Corpus supra nigrum, maculis quadrangularibus albis. (Senior).

\section{SYNONYMA.}

Columbus Arćticus, Lin. Syst. Nat. I. p. 22I. Fn. Svec. p. 52. n. 150. Retz. Fn. p. I47. n. IO6. Gmel. Syst. I. p. 587. Lath. Ind. II. p. 800.

Petit Plongeon de la mer du Nord, Büff. Ois. VIII. p. $26 \mathrm{I}$. Plongeon Lumme, Tem. Man. d'Orn. p. 599.

Black throntcd Diver, Pen. Brit. Zool. II. p. 527. n. 24I. tab. 85. fig, infer. - Aret. Zool. II. p. 520. 21. 444. Lath, Syn. VI. p. 343. Edw. Av. III. tab. 146.

Der Polar Taucher, Bechst. Nat. D. IV. p. 600.

Schroarzkehliger Seetnucher, Meyer Liv- und Esthl. p. 225. Meisner u. Schinz Vögel der Schweiz p. 258. Funior Avis:

Colymbus ignotus, Rechst. N. D. Ima Edit. II, p. 782. Id. IIda Edit. IV. p. 625.

Svecis: ILom.

\section{DESCRIPTIO.}

Longit. $26 \cdot 28$ poll. Rostr. superne $2 \frac{6}{8}$; a sinubus oris $3 \frac{1}{2}$ poll. Basi $\frac{5}{8}$ poll. latum, multo magis quam antecedentis compressum et marginibus inflexis. A flexura alæ ad apicem I3 poll. Tibia 3 poll. Digit, extim. $4 \frac{3}{8} ;$ postic. $\frac{5}{8}$ poll.

Rostrum nigricans maxilla superiore subdecurvata, inferiore ad medium xquali altitudine, subtus sulco longitudinali exarata, dehine in apicem acutissimum decrescente. Iris fusca. Pedes extus fusci, intus es membrana albidis.

Nilssen Ornith. Pars II.

L. 
Caput et collum supra cinerea, qui color versus frontem et in lateribus capitis sensim in nigrum transit. Gula et jugulum nigra violaceo-nitentia, linea transversali Gulæ alba, nigro interrupta. Latera colli fascia longitudinali alba, nigro longitudinaliter striata. Corpus supra et latera nigra. Pennæ scapulares et latera interscapulii maculis quadrangularibus albis in ordinibus positis. Teetrices alarum maculis minoribus albis adspersis. Collum inferius album nigro striatum. Peetus et totum corpus suvtus alba.

Avis Funior: Pullus similis est juniori Col. Glaciali, in omnibus fere, excepta magnitudine. Sensim pennæ nigra in gula, jugulo et dorso erumpunt, qux magis magisque augentur. Anno secundo maculæ scapularum albrexplicantur et anno tertio peracto colorem manentem plerumque recepit avis.

\section{HIS T OR IA.}

Habitat in lacubus et fluviis per totam Sveciam vul. garis excepta Scania. Migrat instante byeme tumque in maribus nostris occurrit. Nidulatur in lacuum insulis vel ripis inter gramina et arundines. Ova duo fusca. Vescitur piscibus, ranis, insectis et plantis aquaticis.

CCVIII. COLYMBUS SEPTENTRIONALIS, Litr.

Dragn: Capitis et colli latera cinerea. Jugulum rufum. Cervix nigricans, albo striatus. (Senior). 


\section{SYNONYMA.}

Colymbus Septentrionalis, Lin. Syst. p. 220. Retz. Faun. p. 148. n. 107. Fabr. Faun. Groenl. p. 94. n. 6 [. Gmel. Syst. I. 2. p. 586. Lath. Ind. II. p. 80 I. n.

5. - Gunner. Act. Nidros. I. p. 244. t. 2. f. 2. Colymbus Lumme, Brünn. Orn. bor. p. 39. n. I32. Plongeon Cat-marin, Temm. Man. d'Orn. p. 602. Plougeon a gorge rouge, Buff. Ois. VIII. p. 264. Pl. enl. 308.

Red throated Diver, Pen. Brit. Zool. II. p. 526. 11. 240. tab. 85. fig. sup. - Aret. Zool. II. p. 520. n. 443. - Lath. Syn. VI. p. 344. Edw. Av. II. t. 97.

Ruth Kehliger Taucher, Bechst. N. D. IV. p. 609. Meyer Liv. u. Esthl. p. 22\%. Meisner und Schinz Vögel der Schweiz p. 259.

Avis Funior:

Colymbus Stellatus, Gmel. Syst. I. 2. p. 587. Lath. Ind. II. p. 8OI.

Plongeon Cat-marin et Petit Plongeon, Buff. Ois. VIII. p. 256. et 254. t. 2I. P1. enl. 992.

Speckled Diver, Pen. Brit. Zool. II. p. 525. Lath. Syr. VI. p. 34 I.

Gesprcukelte Taucher, Bechst. N. D. IV. p. 6I3.

OBSERV.

Colymbus Stristus Gmel. et Col. borealis Brün. ad hane Speciem pertinent.

\section{DESCR I P T IO.}

Longit. 22-24 poll. Rostr. superne $2 \frac{2}{8}$ poll. $A B$ angulo oris 3 poll. A flexura ala ad cjusdem apicem I I $\frac{\pi}{2}$ poll. Tibia 3 poll. Digit, extim. $3 \frac{1}{2}$ poll.

L 2 
Rostrum nigrum, rectum, compressum, maxillis margine inflexis, inferiore aliquantum sursum flexa. Iris fusco-aurantia. Pedes extus virescenti-nigri, intus cum membranâ virescenti - albi.

Gula, latera capitis et colli cinerea; pileus fuscus marginibus pennarum cinereis. Nucha, cervix et colli inferioris latera nigricantia, striis longitudinalibus albis. Jugulum macula longitudinali rufa. Corpus supra fusco-nigricans, in senioribus immaculatum, in junioribus adspersum maculis parvis albidis. Pectus et totum corpus subtus album.

Avis Funior habitu hyemali:

Caput et collum supra cinereo-fusca. Dorsum er alx cinereo-nigricantia marginibus pennarum tenuibus albidis. Gula, genx, pectus et abdomen alta; Latera colli, capitis inferiora et collum antice fusco quasi sordida. Fascis per anum fusca.

\section{HISTORIA.}

Habitat in lacubus et fluviis Svecix, in borealibus plagis frequentior. Autumno migrans extra oras marinas frequens obvenit. Nidulatur juxta aquas inter gramina et arundines. Ova duo fuseo-grisea maculis rarioribus nigris ornata. - Victus ut antecedentis. 
GENUS.

\section{$S$ T E R N A.}

Rostrum mediocre, subrectum, compressum, acutum.

Nores pone medium rostri, linares, pervia.

Pedes tenues, supra genua nudi.

Alce longæ, acuminatx.

Cauda forficata.

CCIX. STERNA CASPIA, Pall.

DiAGN: Rostrum robustum, coccineum. Pileus e\& pedes nigri. Corpus supra canescens, subtus et cauda fissa alba.

\section{SYNONYMA.}

Sterna Caspia, Pall. nov. Comm Petrop. XIV. p. 582. Sparrm. Mus. Carlss. fasc. III. t. 62. Ödmann N. Act. Acad. Holm. 1782. p. 230. Gmel. Syst. I. 2. p. 603 Lath. Ind. II. p. 803. Retz. Faun. p. I64. n. 126.

Sterna Megarhynchos, Meyer Taschenb. Deutschl. p. 457.

Hirondelle de mer Tschegrawa, Tem. Man. d'Ornith. p. 476.

Caspian Tern, Lath. Syn. VI, p. 350. 
Caspische Meesschrolhe, Bechst. N. D. IV. p. 675. Roslngis: Skränmåse.

\section{DESC RIP T IÓ.}

Longit. 20-2I poll. Rostr. $2 \frac{5}{8}$ poll. validum, $\left(\frac{7}{8}\right.$ poll. altum), compressum, maxillis acutissimis. A flexura alx ad apicem $15 \frac{x}{2}$ poll. Cauda $6 \frac{1}{2}$ poll. Tibia $I \frac{6}{8}$; Digit. med. I $\frac{5}{8}$ poll.

Rostrum coccineum. Iris fusco-flavescens. Pedes nigri.

Pileus et pars cervicis nigra. Dorsum, alæ et cauda supra pallide cana. Collum, pectus, abdomen et tectrices inferiores pure alba. Remiges primores pallide cinerex, latere interiore et apice griseo-fuscx.

Funior: Caput supra nigricans albo maculatum et irrora. tum. Dorsum et alæ cana maculis fuscis. Cauda parum fissa. Rectricibus canis, ante apicem album fusco - maculatis.

Mus, Thunb,

\section{HISTORIA.}

Habitat ad oras Balthicas Svecix medix: ex. gr. Roslagix \&c. rarior tamen avis. Nidulatur in arene litorali, vel in rupibus juxta litora sitis. Ova $2-3$ alba maculis nigris ornata. (Vide præterea ödm. 1.c.)

\section{STERNA HIRUNDO.}

Diagn: Rostrum et pedes rubri. Corpus supra canum pileo nigro; subtus cano-album. Remiges et Re Etrices extimæ extus nigricantes. Funior: Rostrum nigricans basi subtus luteo-ru= brum. Pedes aurantii. 


\section{SYNONYMA.}

Sterna Hirundo, Linn. Syst. 1. p. 227. Fn. Svee. n。 I58. Retz. Fn. Svec. p. I6́2. n. I24. (Obs. Descriptio posterior huic adjuncta ad Sternam nigram juniorem pertinet.) Lath. Ind. II. p. 807. Fabr. Groenl. p. 105. n. 69. Gmel. Syst. I. 2. p. 605. Hirondelle de Mer, Pièrre Garrin, Buff. Ois. VIII. p. 331. t. 27. Pl Enl. 987. Temm. Man. d'Orn. p. $48 \mathrm{I}$.

Grent Tirn, Pen. Brit. Zool. II. p. 545. t. 90: Arct. Zool. II. p. 524. Lath, Syn. VI. p. 361.

Gemeine Meerschrolbe, Bechst. N D. IV. p. 682. Mey. Liv. p. 229. Meisner u. Schinz Vögel Schweiz p. $26 \mathrm{I}$.

Svecis: Tärna.

\section{DESCRIPTIO.}

Longit. I4 poll. Rostr. a fronte I $\frac{7}{2}$. Tibia I poll. Digit. med. $\frac{\sigma}{8}$; Cauda fere 6 poll. A flexura alx ad apicem ejusdem $10 \frac{1}{2}$ poll.

Avis Senior:

Rostrum rubrum apice (plerumque) nigro. Pedes rubri. Iris rufa.

Pileus et pars cervicis nigra; latera capitis inferiora et colli, gula, jugulum, uropygium et crissum alba. Dorsum et alæ pulcherrime cana. Pectus album cano colore levissime illitum. Abdonen album. Remiges primores rachibus et margine interiore albis; prima nigro-cinerea, ceterx cano-cinerex; secundarix colore dorsi apice albo marginatæ. Cauda forficata alba, Rectricibus lateralibus 2-3. extus nigro-cinereis. Funior: Rostrum nigricans basi subtus luteo-rubrum. Pedes aurnntii. Frons sordide alba, vertex fronti 
concolor sed nigro-maculatus, stria per oculos et occio put nigra, albido irrorata. Dorsum medium et tectrices alarum medix cana, apicibus pennarum sordide luteo-albidis. Tectrices alarum minores nigricantes. Rectrices laterales latere interiore albx, exteriore nigrocinerex, ceteræ albx, cano tinctx. Tectrices caudx superiores, collum corpusque totum subtus album.

\section{H IS T O R I A.}

Inter congeneres maxime vulgaris Species. Habitat ad oras maritimas et in insulis usque intra circulum pola"em. In lacubus quoque fere omnibus Svecix meridionalis obvenit et nidulatur. Migrat mense Septembri et mature revertitur. Ova 3 - 4 fusco-olivacea, cinereo et nigro maculata, in arena litorali vel in ipsa rupe, sine strato, ponit. Vescitur pisciculis et insectis aquaticis.

\section{STERNA CANESCENS,}

\section{Meyer.}

Diagn: Rostrum tenue, nigrum. Pileus et pedes nigri. Corpus supra canescens, cauda forficata alba.

\section{SYNONYMA:}

Sterna Canescens, Meyer Taschenb. II. p. 458. Sterna Boysii, Lath. Ind. II. p. 806.

Sterna Stubberica, Otto Buff. Ois. XXXI. p. I04. cum firg. Bechst N. D. IV. p. 679.

Sandzpich Terne, Lath. Syn. VI. p. 356. Id. suppl. I. p. 266. 
Avis Funior: Sterna Nubilosa, Retz. Fn, p. I65. 11. I 27.

Sterna Striata, Lath. Ind, II. p. 807. Lath. Syn. VI. p. 358. tab. 98.

\section{DESCRIPTIO.}

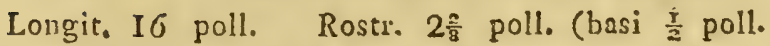
altum). A flexura alæ ad apicem II $\frac{T}{2}$ poll. Cauda $5 \frac{x}{2}$; Tibia $1 \frac{x}{8}$ poll. Digit. med. $1 \frac{x}{8}$ poll.

Rostrum compressum, strikum, marginibus utrin. que infexis, gracile, nigrum, apice pellucidum, lutescens. Pedes nigri.

Avis habitu cestivali:

Pileus niger pennis occipitis longioribus. Dorsum et alx pallide cana. Remiges cinerex, latere interiore albr. Ceterum caput, collum, corpus subtus et carta alba.

Mus. Thunb.

OBSERV. I.

Aven halitu hiemali, quam descripsit cel. Temminck 1. C. videre mihi non licuit, nec apud nos observari potest, $\mathrm{cum}$ ante hyemeen oras nostras selinquit.

\section{OBSERV. 2.}

Sterna Nubilosa, Sparrm. Mus. Carlss. tab. 63, sine dabio ut junior ad hane speciem pertinet; quam avem cum in Museo Academix Scient, Holmensi videre et describere mihi licuit; descriptionem heic adjungain:

Longit. I I $\frac{r}{2}$ poll. Rostr. a fronte I $\frac{3}{8}$ poll. A flexura alx ad apicem 10 poll. Cauda fere 5 poll. ejusque pennæ medix 3 poll. Alre extra caudam exten. duntur ad 2 poll. - Femors nuda $\frac{5}{8}$ poll. Tibia fere

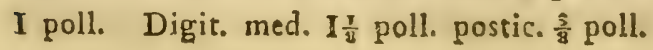


Rostrum strictum, parum decurvatum, nigrum, basi latius ( $\frac{1}{2}$ poll.), extus valde compressum, maxillis margine inflexis. Pedes fusci.

Frons et tota avis subtus alba. Fascia fuscescens inter rostrum et oculum. Caput, collum, dorsum, alx et cauda cinereo-fusca. Remiges primores nigræ, margine interiore pallide cinerex.

\section{OBSERV. 3.}

Quoad magnitudinem, formas et proportiones, colore tantum excepto, mirandum in modum similis est Sterne Alba (Mus. Carlss. 1. tab. II.) qua quoque in Museo eodern asservatur.

\section{H IST ORIA.}

Habitat rarius in insulis maris Balthici. Nidulatur in arena litorali vel in rupibus nudis. Ova $2-3$ albida, maculis nigricantibus picta. Victitat piscibus.

\section{STERNA NIGRA, Lin.}

Disgn: Rostrum nigrum. $P_{e d e s}$ brunnei. Coput nigrum. Crissum album. Corpus supra canum subtus nigro - cinereum.

\section{SYNON YMA.}

Sterna Nigra, Lin. Syst. I. p. 227. n. 3. Fn. Sv. 11. I59. Lath. Ind. II. p. 8 IO. Gmel. Syst. I. 2. p. 608. Retz. Fn. p. I64. n. I25.

Sterna fissipes, Linn. Syst. I. p. 228. n. 7. Gmel. Syst.

I. 2. p. 6IO. Lath. Ind. II. p. 810 .

Sterna obscura, Gmel. 1. e. p. 608. Latk. 1. c. p. 810. Hirondelle de mer à tête noire ou Gnchet, Buff. Ois. VIII. p. 342 . 
Guifette noire ou Epouvantail, Buff. Ois. VIII. p. 34I. Pl. enl. 333.

Black Tern, Lesser Sen Swallow et hrown Tern, Penu. Brit. Zool. II. p. 547. n. 256. Lnth. Syn. VI. p. 366. n. 22. A. n. 23.

Schroarze Meerschwallic, Bechst. N. D. IV. p. 692 et 697.

Funior autumno:

Sterna Nevia, Linn. Syst. I. p. 228. n. 5. Gmel. Syst.

I. 2. p. 609. 11. 5 .

Sterna Boysii, var. a. Lath. Ind. II. p. 806.

$L_{\boldsymbol{B}}$ Guiette, Buff. Pl. enl. 924.

OBSERV.

Huc pertinet Descriptio sub. Stern. Hirand. data in Retz. Fn. Sv. p. 163.

\section{DESCRIPTIO.}

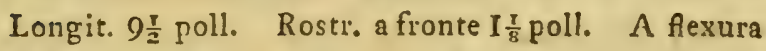
alx ad apicem fere 9 poll. Cauda $3 \frac{3}{8}$ poll. Tibia $\frac{\pi}{8}$; Digit. med. $\frac{7}{8}$; postic. $\frac{3}{14}$ poll.

Rostrum nigrum, valde compressum et acuminatum. Pedes rubri fusco tincti, membranâ natatoriâ excisâ, unde semipalmati appareant. Cauda bifida, alis 2 fere pollic, brevior.

\section{Senior astare:}

Caput et collum nigra, gula, collum antice, pectus et abdomen cinereo-nigra. Dorsum, alx et cauda coeruleo - cinerea. Remiges primores nigro-cinerez latere exteriore pallido, rachibus albis. Rectrices laterales albx versus apices cinerascentes. Crissum, tectrices caudx et alarum inferiores pure alba. 
Avis post autumnalem pennarum mu:ationem:

Pileus niger fascia frontali alba. Corpus totum cinereo - canum tectricibus caudæ inferioribus et gula albis juguloque albo maculato. Reetrices concolores. Pullus ante autumnalcm pennarum nutationem:

Caput supra et nucha nigra fronte sordide alba. Tota avis subtus a gula ad caudam lateraque colli alba, qui color totum fere collum cingit. Stria nigricans per oculos. Corpus supra cinereo-fuscum pennis scapuiaribus et tectricibus alarum ferruginescenti marginatis, Uropygium pallide cinereum. Cauda parum forficata Rectricibus apice obtusis, cinereis apice rufes. centi marginatis.

\section{H I S T OR I A.}

Habitat in paludibus et carectis passim frequens, non solum in Scaxía sed quoque in media Suecia. Ni. dulatur inter gramina. Ova 2.4 olivacea maculis fuscis et nigris adspersa. Vescitur insectis et vermi. bus aquaticis.

\section{STERNA MINUTA, Lin.}

Diagn: Rostrum et pedes lutei, Pileus et lora nigra, fronte superciliisque albis. Corpus supra pallide canum, subrus et cauda alba. Remiges tres extimæ extus nigricantes.

\section{SYNONYMA.}

Sierna Minuta, Lin. Syst. I. p. 228. Lath. Ind. II. p. s09.

La petite Hirondelle de mer, Buff. Ois. VIII. p. 337. Pl. enl, 996. Temm. Man. d'Ornith. p. 487. 
Lesser Tern, Pen. Brit. Zool. II. p. 546. n. 255. Lath. Syn. VI. p. 364.

Die kleine Meerschrvalhe, Bechst. N. D. IV. p. 690. Meisner u. Schinz Vügel Schweiz. p. 265.

\section{DESCRIPTIO.}

Longit. $8 \frac{x}{2} \cdot 9$ poll. Rostr. a fronte $I \frac{\frac{7}{5}}{5}$. A fexura alir 7 poll. Cauda $3 \frac{3}{8}$ poll. Tibia $\frac{6}{6}$ poll. Digit. med. $\frac{6}{8}$ poll.

Rostrum compressum, acuminatum, luteum apice nigro. Nares lineares pervix. Pedes sordide flavi unguibus acuris nigris. Cauda valde forficata.

Caput supra, nucha et lora nigra. Frons et supercilia alba. Dorsum et alx supra pallide cana. Remiges primores tres nigricantes vexillo interiore dimidiate albo, ceteræ colore dorsi, margine interiore albo. Tota avis subtus et cauda alba, Pęore canescente.

\section{HISTORIA.}

Nova hæc Faunx nostra incola, intex maxime vul. gares sui generis, saltem in Scania, est numeranda. Habitat in litoribus marinis et in campis adjacentibus arenosis Scanix et Hallandix (In Scanörsljung copiose). In Norvegia illam quoque observavi. Nidulatur in arena. Ova 2-3 pallide virescentia maculis majoribus minoribusque brunneis et cinereis ornata, mense Junio leguntur. Vescitur insectis et vermiculis maxinis. 
GENUS.

\section{A R U S.}

$\boldsymbol{R}_{\text {ostrum mediocre, compressum, cultratum, nu- }}$ dum, apice subadunco: maxilla inferiore pone apicem subius angularo - gibba, compressa.

Nares in medio rostri, sublineares, postice angustiores, pervire.

$p_{\text {edes }}$ graciliusculi femoribus seminudis. Ale longæ Remigibus $\mathrm{x}$ ma et $\cong \mathrm{da}$ fere æqualibus. Cauda brevis, æqualis.

\section{LARUS MARINUS, Linn。}

DraGn: Albus dorso et alis nigris; Remigibus apice albis. Rostrum luteum angulo rubro. Pedes albidi.

\section{SYNONYMA.}

Jarus Marinus, Limn. Syst. I. p. 225. Fn. Sv. n. I55.

Ödmann N. Act. Holm. I783. p. IOI Gmel.

Syst. I. 2. p. 598. Lath. Ind. II. p. 813 . n. 6.

Goeland à mantenu noir, Temm. Man. d'Ornith. p. 490.

Buff. Ois. VIII. p. 405. Pl. enl. 990.

Blackhacked Gull, Penn. Brit. Zool. II. p. 528. 11. 242.

Arct. Zool. II. p. 527. n. 45 I. Lath. Syn. VI. p. $37 \mathrm{r}$. 
Mantel Meve, Bechst. N. D. IV. p. 653. Meyer Liv. und Esthl. p. 230. Meisner u. Schinz 1. c. p. 266. Funior:

Larus Navius, Linn. Syst. I. p. 225. 11. 5. Gmel. Syst. I. 2. p. 598.

Goeland varié ou Grisard, Buff. Ois. VIII. p. 4I3. t. 33. Pl. enl. 266.

Wagel Gull, Penn. Brit. Zool. II. p. 536. tab. 8s. n. 247.

Svecis: Hafsmäse; Scanis: Hafnäka. Blekingis Homåke 1. Hålmäka,

\section{DESCR I P TIO.}

Longit. 30-3I poll. Alæ extensæ 5 ped. 8 poll. A flexura alæ ad apicem I ped. 8 poll. Rostrum a fronte fere 3 poll. A sinu oris 4 poll. Cauda 8 poll. Alx extra caudam extenduntur fere ad 2 poll. Femora nuda $I \frac{\pi}{2}$ poll. Tibia $3 \frac{2}{8}$ poll. Digit. med. $3 \frac{x}{2}$ poll. postic. $\frac{x}{2}$ poll. Fensina aliquantum minor est.

Rostrum pallide luteum apice albo: maxilla inferior pone apicem lateribus rubra. Margo palpebrarum aurantius. Iris luteo.grisea. Pedes albidi unguibus corneis.

Avis senior habitu estivali:

Caput, collum, corpus subtus et cauda nivea; dorso, scapularibus et alis nigris cinereo tinctis. Remex. prima apice longo alto, secunda quoque apice albo, sed ante apicem macula vexilli interioris nigra; tertia ante apicem album nigra; quarta ante apicem album macula nigra, antequan minor alba. Remiges secundarix et pennx scapulares apice albæ. Margo alartum albus. 
Avis senior habitu hiemali:

Simillima jàm descriptæa, a qua tantuın dignoscitur striis longitudinalibus fuscis in vertice nucha et lateribus capitis.

Avis junior primo anno:

Rostrum nigrum. Pedes pallide fusci.

Caput, collum et corpus subtus albida (sordide alba) striis longitudinalibus fuscis in capite et collo, maer. lisque transversis fuscis in pectore, abdomine et crisso. Dorsum, scapula et teetrices alarum fusca pennis albido-rufescenti terminatis et transverse fasciatis. Remiges primores nigricantes apicibus vix albidis. Rectrices nigricantes versus basin albo marmoratis, apicibus albis. Albedo in lateralibus magis pravalet.

\section{Secundo anno:}

Maculæ fusca capitis, colli et inferioris corporis sen. sim evanescunt, ita ut pennx harum partium magis in pure album vergant. Rostrum nigrum apice et basi luteo-albidum. - Post hoc tempus partes corporis su. periores sensin deponunt colorem variegatum et induunt coloren nigricantem, et quarto xtatis anno avis coloribus absolutis induitur.

\section{HIS T ORIA.}

Habitat in utroque mari ad oras Svecix et Norvegix, et in insulis eas adjacentibus. In insulis plagre borealis imprimis frequens. Lacus et flumina nunquam visitat. Vißtus ejus sunt imprimis pisces, cadavera in unda natantia et ovula piscium. Interdum quoque pullos aliarum avium devorat. Nidulatur mense Majo in scopulis marinis tum intra tum extra circulum polarem. Ova 3.4 saturate viridi-olivacea, maculis majoribus et minoribus nigris ornata. Plumis et pen- 
nis utilis est avis. Caro vero nunquam, quond sciam, in Svecia comeditur. In Norvegir vero borealis insulis pulli hujus et sequentis speciei ab incolis capiuntur, et tamquam apud nos Anseres, farciuntur, fars. tique comeduntur.

\section{LARUS GLAUCUS, Limn.}

DIAGN: Albus, dorso et alis canis, Remigibus apice albis. Rostrum luteum angulo rubro. Pedes pallidi.

\section{SYNONYMA.}

Larus Glaucus, Fabr. Fn. Groenl. p. I00. n. 64. Öd. mann N. A\&t. Holm. 1783. p. 97. Lath. Ind. II. p. 814 . Gmel. Syst. I. 2. p. 600. Retz. Fn. p. I56. n. II6. Brim. Orn. bor. p. 44. Goelnard à mantenu blen, Temm. Man. d'Orn. p. 493.

Buff. Ois, VIII. p. 406. t. 32. Pl. enl. 253.

Glancous Gull, Lath.Syn. VI. p. 374. Penn. Aret. Zool.

II. p. 532.

Herring Gull, Pen. Br. Zool. II. p. 533. n. 246. Arct.

Zool. II. p. 527. Lath. Syn. VI. p. 372. n. 3.

Weissgraue Meve, Bechst. N. D. IV. p. б62.

Svecis: Gråtrut, Gråmåse. Blekingis: Slagmåka, Win. termåka.

\section{DESCR IP TIO.}

Longit. 25 poll. Nlx extensix 4 yed. 9 poll. ad 5 ped. A flexura alx ad apicem $I 7 \frac{x}{2}$ poll. Rostrum 8 fronte $2 \frac{\pi}{8}$ poll. A sinu oris $3 \frac{T}{2}$ poil. Cauda $7 \frac{\pi}{2}$ poll. Alre extra caudam extenduntur ad 2 poll. Tibin 3 poll. Digit. ned. $2 \frac{6}{8}$ poll. Nilsson Ornith. Pars II. 


\section{O BSERV. I.}

Femina in omnibus aliquantum minor.

\section{O BSERV: 2.}

Hre Specics in omnibus atatibus a pracedente dignoscitur sostro et tibiis minoribus præcipue gracilioribus, nec tam ut in L. Marino robustis.

Rostrum pallide flavum angulo maxillæ inferioris rubro. Margo palpebrarum miniatus. Iris pallide lutea. Pedes pallide grisei unguibus nigro-fuscis. Avis senior habitu estivali:

Caput, collum, corpus subtus et cauda nivea immaculata, dorso, scapularibus et alis canis (coerulescenti-cinereis). Remiges primores I- 6 ante apicen nigrx, apice ipso albr; secundarix et scapulares canx albo terminatx.

Avis senior habitu hiemali:

Similis jam descripta, a qua tantum differt: striis longitudinalibus fuscis in vertice, nuche et lateribus cnpitis.

Avis junior primo anno: (Mense Aug.)

Rostrum nigrum basi tantum pallide griseum. Iris griseo-fusca. Pedes pallide grisei.

Caput, collum et corpus subtus grisea maculis fuscis freque ntibus nebulosa et variegata. Dorsum et alæ variegata e fusco, griseo et rufescente. Remiges primores fusca apice vix albx. Rectrices fuscr versus busin variegatze fusco et albido, lutescenti terminatr. Avis secundo atatis anno (Mense Aug. occisa).

Rostrum pallide griseum (1. Intescens) verșus apicem fuscum, macula utrinque lutea, apice ipso griseo. Pedes pallide grisei unguibus fuscis.

Caput, collum et tota subtus alba maculis et striis fuscis in superiore cepitis et colli porte atque ad latera 
pectoris. Dorsum et seapulx cana, inspersis pennis sordide griseis, fusco et albido transverse maculatis. Tectrices alarum fuscx marginibus et fasciis pallide griseis s. albidis. Remiges primores nigricantes, apice albidx et extima ante apiccm macula magna alba; secundarix fuscx albo marginatx et terminatx. Uropygium et tectrices caudx alba fasciis transversis fuscis. Reetrices versus apicem nigricantes, versus basin albo marmoratx, qux albedo in lateralibus prævalet.

Varietas. Tota pallide grisen, fuscescenti nebulosa ct variegata,

Mus, Lund.

\section{HIS T ORIA.}

Habitat in insulis utriusque maris, neque tamen nisi hiemali tempore juxta litora Scanix a me visa. Lacus quoque et flumina interdum visitat. Habeo specimen in flumine prope Lund occisum. In insulis tamen et rupibus maris Norvegici usque intra circulum polarem multo frequentius occurrit. Vietus ut pracedentis speciei. Nidulatur in rupibus nudis. Ova $2-3$ colore variant, jam saturate olivacea, jatn coerulescentia maculis semper nigris et cinereis picta. Pulli farcti ab insulanis Norvegicis comeduntur.

\section{LARUS FUSCUS, Iinn.}

DiAgn: Albus dorso et alis coerulescenti - nigris. Remiges primores nigræ, extimis duabus macula ante apicem ovali alba. Rostrum luteum angulo rubro. Pedes flavi.

\section{SYNONYMA.}

Larus Fuscus, Limn. Syst. I. p. 225. Fn. Sv. N. I54. Retz. Fn. p. 151. n. II8. Ödm, 1. c. p. I05. Gmel. M 2 
Syst. I. 2. p. 599. Lath. Ind. Orn. II. p. 8 IJ $^{\text {g. }}$ 11. 8 .

Larus Flavipes, Meyer Vögel Liv. u. Esthl. p. 23 I.

Taschenb. Deutsch. II. p. 469. cum tab. Herrings Meve, Bechst. N.:D. IV. p. 6.58 . Svecis: Sillmåse, Sillmaikn.

\section{DESCRIP TIO.}

Longit. 22 poll. A flexura alx ad apicem It poll. Rostrum a fronte 2 poll. A sinu oris 3 poll. Cauda $6 \frac{\frac{5}{8}}{3}$ poll. Tibia $2 \frac{3}{8}$ poll. Digit. med. $2 \frac{2}{8}$ poll.

Femina aliquantum minor.

Rostrum citrinum, angulo maxillæ inferioris rubro. Iris et margines palpebrarum flava.: Pedes flavi. Alr extra caudam longius progrediuntur.

Senior habitu estivali:

Caput, collum, corpus subtus et cauda cum tectlicibus nivea immaculata. Dorsum, scapulares et alre nigra coerulescenti tincta. Remiges primores saturate nigræ apicibus tenuiter albis, prima (et interdum quoque secunda) ante apicem maeula longa alba: secunda. rix et scapulares apicibus late albis.

Senior habitu hiemali:

Similis jam descriptr, a qua tantum differt: striis longitudinalibus fuscis in vertice, nucha et lateribus capitis.

Funior prino anno: Rostrum nigrum basi fusco. Pedes sordide ochracei. Remiges nigræ immaculatx. Præterea tam similes sunt pullis Lari Marini, ut nihil fere nisi magnitudine dignoseantur.

\section{HISTORIA.}

i Habitat juxta oras maris Balthici et occidentalis; in borealibus tamen plagis peninsula multo frequantior 
quam in meridionalioribus. Nidulatur in rupibus marinis vel in arena litorali. Ova 3 griseo - fusca ma. culis nigris ornata.

\section{LARUS EBURNEUS, Gmel.}

DiaGN: Totus albus, rostro pallide luteo, basi coerulescente, pedibus nigris.

\section{S Y NONYMA.}

Lowus Eburneus, Gmel. Syst. I. 2. p. 596. Lath. Ind. II. p. 8 I6. n. I6.

Larus niveus, Mart. Spitzb. t. L. f. A. Phipps Reise. Larus candidus, Fabr. Groenl. p. I03. n. 67. Monette Elanche ou Sénateur, Temm. Man. d'Ornith.p. 498. Buff. Ois. VIII. p. 422. Pl, enl. 994. Ivory Gull, Lath. Syn. VI. p. 377.

\section{DESCRIPTIO.}

Longit $17 \frac{x}{2}-18$ poll. A flexura alæ ad apicem $14 \frac{T}{2}$ poll. Rostr. a fronts I $\frac{x}{2}$ poll. A sinu oris 2 poll. Tibia $1 \frac{x}{2}$ poll. Digit. med. $I \frac{6}{8}$ poll. postic. $\frac{3}{8}$ poll.

Rostrum robustum et minus quain in Laro cano com. pressum, basi coerulescens, apice pallide luteum. Pedes nigri.

Avis senior habitu astivali:

Tota avis pulcherrime alba immaculata.

"Pulli maculati sunt maculis oblongis nigris præsertim in dorso alisque. Rostrum illorum etiam nigricat. Fabric. 1 c.

\section{H I S T OR I A.}

Habitat in oceano maxime boreali juxta Spitzbergam et Groenlandiam. Hieme meridionaliores plagas petens 
oras Norvegix, Hallandix \&c. interdum visitat; unde ut opinor, rupes Bahusienses quoque salutat, quamvis nondum ibidem ab ornithologo observatus fuerit. (Specimen descriptum in Norvegia occisum est). Oeconomia et propagatio adhuc plane incognitæ sunt.

\section{LARUS CANUS, Limn.}

Diagn: Albus dorso et alis canis. Remiges extima dur macula ante apicem alba. Rostrum luteum. Pedes Anvi.

\section{SYNONYMA.}

Serior habitu astivali:

Larus canus, Lin. Fn. Svec. p. 54. n. I53. Ödmann 1. c. p. I09. Retz, Fn. p. I58. 11. I 19. Gmel. Syst. I. 2. p. 596.

Common Gull, Pen. Brit. Zool. II. p. 538. n. 249. Latl. Syn. VI. p. 378.

Senior habitu hiemali:

Larus Cynnorhynchus, Mey. Taschenb. II. p. 480. n. 5.

Mouette a pieds bleis, Buff. Ois. VIII. p. 428. P1. enl. 977.

Funior avis:

Larus hybernus, Gmel. I. 2. p. 596.

Larus procellosus, Bechst. N. D. IV. p. 647. (junior). In Mouette d'Hiver, Buff. Ois. VIII. p. 437.

Winter Gull, Pen. Brit. Zool, II. p. 537. Lath. Syı. VI. p. 384 .

Svecis: Fiskmåse, Fiskmåkn. 


\section{DESCRIPTIO.}

Longitudo 17 poll. Alæ extensæ 3 ped. 2.3 poll. Rostrum a fronte $\frac{3}{8}$ poll. A sinu oris $2 \frac{7}{8}$ poll. A flexura alæ ad apicen $13 \frac{x}{2}$ poll. Cauda $5 \frac{T}{2}$ poll.; Alis complicatis 3 pollices brevior. Tibia $I \frac{7}{8}$ poll. Digit. med. I $\frac{5}{8}$, postic. $\frac{2}{8}$ poll.

Rostrum virescenti-luteum. Os intus aurantium. Anguli oris et margines palpebrarum cinabarini. Iris fusco-grisea. pedes viridi-flari, unguibus fuscis.

Avis senior habitu astivali:

Caput, collum, corpus subtus, uropygium et cauda nivea, inmaculata. Dorsum, scapulares et ala cana (coerulescenti - cinerea). Remiges primores nigra, basi cinereæ, primæ duæ macula magna alba ante apicem nigrum; ceteræ apice albæ: secundarix apicibus late albis.

\section{Avis senior habitu hiemali:}

Similis jan descriptæ, a qua tantum differt: striis longitudinalibus fuscis in vertice et nucha; orbitis antice nigricantibus.

Avis junior primo snno: Rostrum nigrum. Orbitz ni. gricantes. Pedes lutescentes.

Capur, collum et tota avis supra fuseo-grisea, pennis dorsi et alarum lutescenti-alho marginatis. Frons, collum antice et avis subtus alba griseo-nebulosa, prie sertim in collo et lateribus pectoris. Remiges primores nigricantes, immaculatie. Rectrices canescenti-albr, fascia ante apicem lata nigro - fusca, apicibus albo terminatis.

\section{OBSERV.}

Post primam mutationem pennarum pennas unicolores cane in dorso hinc inde erumpunt. Caput albuin fusco-striatum. 
Tum in capite et collo, tum in partibus corporis inferioribus color albus magis magisque angetur. Rostrum basi lutescens apice nigro.

\section{H IS T ORIA.}

Ex hoc genere maxime apud nos vulgaris Species. Non solum maria Scandinavian alluentia frequens incolit, sed quoque lacus et fumina visitat. Mare procellâ agitatum relinquit et interdum, maximis gregibus socista, prata depressa visitat. Nidulatur inter gramen in insulis vel maris l. lacuum tum in Scania tum in Norvegia maxime boreali. Ova 3 saturate viridia, nigro-maculata. Vescitur piscibus, testaceis et vermibus.

\section{LARUS TRIDACTYLUS, Lim.}

DiagN: Albus dorso et alis canis; Remigibus extimis apice nigris. Digitus posticus verruciformis, muticus.

\section{SYNONYMA.}

Larus Tridactylns, Lath. Ind. II. p. 8I\%. 11. II.

Larus Rissa, Brünn. Orn. n. I40. Lin. Syst. I. p. 224.

n. 1. Olavs. et Pov. Isl, Reise I. p. 356. tab. 23. (Senior).

Kittiwake, Pen. Brit. Zool. II. p. 539. n. 250. Lath. Syn. VI. p. 393.

Mouette tridactyle, Temm. Man. p. 502.

Serior habitu hiemali:

Larus tridactylus, Bechst. Nat. D. IV. p. 628.

$\boldsymbol{L}_{a}$ Mouette cendrée, Briss. OMn. VI. p. '175. n. 8. t. I6. f. I. 
Funior:

Larus Tridactyluis, Lin. Syst. I. p. 224. n. 2. Fn. SF. p. 55. n. 157. Gmel. Syst. I. 2. p. 595.

Moutte cendrée, tachetée, Buff. Ois. VIII. p. 424. PI. enl. 387.

Tarrock Gull, Pen. Erit. Zool. II. p. 540. n. 25 I. Aret. Zool. II. p. 533. - Id. p. 529. n. 456. Lath. Syn. VI. p. 3922 et var. A. Id. Suppl. V. I. p. 268.

\section{DESCRIPTIO.}

Longit. I5 - I5 $\frac{1}{2}$ poll. Rostr. a fronte $1 \frac{3}{8}$ poll. A sinu oris 2 poll. A flexura alx I2 poll. Cauda 5 poll. Tibia $1 \frac{3}{8}$ poll. Digit. med, $1 \frac{7}{8}$ poll.

Rostrum acutissimum, aliquantum decurvatum, angulo maxillx inferioris vix conspicuo. Femora sem:nuda. Tibix breves, verruca ungue destituta pro digito postico.

Senior habitu astivali:

Rostrum virescenti-luteum. Os intus et margines palpebrarum rubra. Iris fusca. Pedes olivaceo - fusci.

Caput, collum, corpus subtus et cauda nivea, immaculata. Dorsum, scapulares et alr canx. Remiges primores quatuor nigro-terminatæ, quarum tres apice tenui albo notatx sunt, quinta maculam majorem alban habet. Extima extus longitudinaliter nigra.

Senior habitu hiemali:

Vertex, occiput, nucha et colli latera cana immaculata, striis minutis nigris ante oculos. Frons, orbita, coilum antice, corpus subtus, uropygiun et cand alba immaculata. Cetera ut in prrcedenti.

Avis junior: Primo anno: Rostrum nigrum. Iris et margines palpebrarum nigrientia. 
Caput, collum et corpus subtus albida maculâ nigrâ ante oculos, aliaque majore nigrescente prope occiput. Cervix macula magna semilunari nigra. Dorsum et alx saturate cana, pennis nigricanti terminatis. Angulus alæ et tectrices ejus minores nigri. Scapulares et remiges secundariæ nigro maculatæ. Remiges primores nigrre. Cauda alba fascia prope apicem nigra, albo terminata.

Post autumnalem pennarum mutationem:

Frons, genu, gula, collum antice et corpus subtus alba immaculata. Orbita antice pilis nigris adspersa. Vertex, cervix, dorsum et alx cana, macula obsoleta nigricans ad latera occipitis, alisque majori interrupta basin cervicis cingit. Dorsum et scapulares immaculata. Tectrices alarum minores nigræ; unde fascia lon. gitudinalis nigra in nla composita oritur. Remiges primores 4 nigra, margine interiore canæ. 5-6. albo canescentes macula nigra ante apicem: secundarix albocanescentes. Cauda alba, fascia juxta apicem nigra; pennis lateralibus albis immaculatis.

\section{HIS T ORIA.}

Habitat juxta oras scopulosas Bahusir et Norvegix, prasertim tamen in regionibus arcticis. Nunquam, quantum equidem scio, i: mari Balthico occurrit. Sinus maris et insulas interiores frequentius quam exteriores incolic. Vescitur piscibus et insectis marinis. Nidulatur in rupibus. Ova 3 virescentia maculis fuscis.

CCXX. LARUS RIDIBUNDUS, Linn. Diagn: Caput nigro - fuscum. Dorsam canum. Corpus subus et cauda alba, rostro pedibusque rubris. Sen, astate. 


\section{OBSER V.}

Dignoscitur Species omnibus anni termporibus:

Remigibus primoribus albis, apice et marginibus nigris.

\section{SYNONYMA.}

Larus ridibundus, Lin. Syst. I. p. 225. n. 9. Gmel. Syst. I. 2. p. 60I. - Lath. Ind. II. p. 8 I I. n. 2. Retz. Fn. p. 159. 11. 120.

Larus atricilloides, Gmel. Syst. I. 2. p. 60I. 11. 19. Lath. Ind. II. p. 8 I 3. n. 3.

Moutte Rieuse, Temm. Man. d'Ornith. p. 504. Buff. Ois. VIII. p. 433.

Black hended Gull, Pen. Brit. Zool. II. p. 54 I. n. 252 Lath. Syn. VI. p. 380. Schroarzköpfige Meve, Bechst. N. D. IV. p. 635.

Lach Meve, Meyer Liv. u. Esthl. p. 234.

\section{OBSER V.}

Lavus Atricilla, Retz. Fn. Sv. p. 160. no 121. est a Lare yidibundo distineta Species, qux in maribus australibus obvenir, nec, ut opinor, Faunce Suecic unquam adnumerari potest. Vide Temin. 1. c. p. 507. not.

Senior habitie hiemali:

Larus Cinerarius, Lin. Syst. I. p. 224. n. 4. Gmel. Syst. I. 2. p. 597.

Inrus procellosus, Bechst. N. D. IV: p. 647. (senior, junior enim est Larus canus).

La petite Mouette cendrée, Briss. Orn. VI. p. I78. n. 9. t. I7, f. I. Buff. Ois. VIII. p. 430. Pl. enl. 969. Red legged Gull, Lath. Syn. VI. p. 38 I. n. 10. Funior:

Larus erythropus, Gmel. Syst. I. 2. p. 597. Larus canescens, Bechst. N. D. IV. p. 649. 
Red legged Gull, Pen. Arç. Zool. II. p. 533. - Varier.

Lath. Syn. VI. p. 38I. n. IO. A.

Brown hended Gull, Lath. Syn. VI. p. 383.

\section{DESCR IPT IO.}

Longit. I5 poll. Rostrum superne $1 \frac{3}{8}$ poll. A sinu oris 2. poll. A flexura alx $I 2 \frac{\pi}{2}$. Cauda $4 \frac{x}{2}$ poll. Tibia $1 \frac{7}{8}$ poll. Digit. med. $1 \frac{1}{2}$ poll.

Rostrun et pedes rubri. Iris fusca.

Senior habitu cstivali:

Caput totum et pars colli superior saturate fusca. Oculi circulo albo inclusi. Basis colli, corpusque totum subtus et cauda alba, colore roseo tincta. Dorsum, scapulæ et alarum tedrices cana. Margo alarum exterior albus. Remiges primores alba, apice et margine interiore nigris; extimx quoque extus ab apice longitudinaliter nigræ.

Senior habitu hiemali:

Caput, collum, totum corpus subtus et cauda alba: macula nigra ante oculos, aliaque major ad aures. Cctera ut snteced.

Funior avis: primo anno ante autumnalem pennarum mutationem:

Rostrum nigricans basi et pedibus lutescentibus.

Caput et occipur fusca macula majori alba pone oculos. Corpus subtus et collare alba, rufesenti tincta. Dorsum, scapulares et minores tectrices fusca margunibus pennarum lutescentibus. Margo alarum inferior et uropygium alba. Cauda alba fascia ante apicem albidum nigra. Remiges albx, apice et margine utroque ab apice nigrx; extima per totam longitudinem extus nigra. Post autumnalem penuarum mutationen: Frons, gula, collum et corpus subtus alba. 
Orbita antice nigra. Macula auris fusca. Dorsum ct *hrum tętrices fusca marginibus pennarum lutescentibus, pennis canis unicoloribus, immixtis. Tectrices alarum plurimr canx. Remiges et rectrices ut in ave nuper descripta.

\section{H I S T OR I A.}

Habitat in mari ad oras prasertim Scanenses. Lacus quoque et fumina incolit, sed minus frequens. Verno tempore interdum agros nuper aratos vermium legendorum causa visitat. Vescitur insectis, vermibus et pisciculis. Nidulatur in graminosis locis juxta litora maris vel fuminum. Ova 3 saturate olivacea maculis majoribus fuscis adspersa.

\section{LARUS MINUTUS, Pall.}

Diagn: Albus capire nigro, dorso cano, rostro rubro, pedibus coccineis. Remiges apice albæ.

\section{SYNONYMA.}

Larus minutus, Pall. Itin. III. p. 702. 17. 35. Gnel. Syst. I. 2. p. 595. Lath. Ind. II. p. 813. n. 5. Retz. Fn. Sv. p. 278. n. 27 I.

Little Gull, Lath. Syn. VI. p. 39 I.

OBSERV.

Excellentiss. Comes Wachtmeister primus est Ornithologus, qui hanc Speciem ut Suecanaun detexit, vide Retz. Fn. Sv., postea vero pluribus locis inventa est.

\section{DESCR IP TIO.}

Longit. II poll. Rostrum $\frac{7}{8}$ poll. debile tuberculo parum exserto. A flexura ala ad apicem 9 poll. Cauda $3 \frac{\mathrm{T}}{2}$ poll. Tibia $\frac{\mathrm{T}}{8}$ poll. Digit. med. I $\frac{\hat{\mathrm{g}}}{\mathrm{B}}$ poll. 
Senior habitu castivali:

Rostrum saturate rubrum. Iris fusca. Pedes coc. cinei.

Caput totum et superior pars colli nigra; ceterum collum, corpus subtus et cauda alba, immaculata. Dorsum, scapulæ et alæ cana. Remiges apice albæ; primores latere exteriore dorso concolores; interiores obscuriores.

Junior mense Fulio occisus:

Rostrum supra et apice nigro - fuscum, ceterum luteo-rubrum. Pedes lutescentes. Vertex et oceiput nigra. Frons, gula et latcra colli totaque avis subtus alba. Dorsum et scapulares canæ apicibus pennartum fuscescentibus. Tectrices alarum minimæ nigricantes, cereræ et remiges secundariæ canx, apice albx. Remiges primores cinerex margine interiore albæ, apice saturatiores, extima extus nigra. Rectrices extima longiores, extus nigricantes, intus albx, ceteræalbæ.

\section{HISTORIA.}

Habitat in inșulis maris Balthici, Gottlandia et Oe. landia, ubi in palndibus quotamnis nidulatur. Occud. rit quoque sed rarius juxta litora australiora Scaniz. Hieme abit. Ova nondum vidi. 
GENUS.

\section{E S T R I S, Illig.}

Rostrum mediocre, teres, rectum, cera cornea ad nares vestitum; maxilla superiore apice adunco, inferiore pone apicem compressum, canaliculatum, gibba.

Nares ante medium rostrum, marginales, sublineares, postice angustiores, pervir.

$P_{\text {edes }}$ graciles, femoribus seminudis; unguibus curvis, acutis.

Ala mediocres remige prima ceteris longiore.

Canda rectricibus duabus mediis longioribus.

OBSERV.

Genus a Laris distinctum est, sive mores sive formam rostti respicias. Quoad hanc medium tener inter Laros et Procellarias, quos igitur conjungit; quoad vero illos, singulare omnino est.

\section{LESTRIS PARASITICUS.}

DiAGN: Rostrum et pedes nigri Redricibns duabus mediis longissimis. Mas supra niger, collo, pectore et abdomine albis. Femina tota nigro. fusca, subtus pallidior. 
- Pullus: fuscus marginibus pennarum lutes scentibus, cauda subæquali.

\section{DESCR IP TIO.}

Longitudo $a b$ apice rostri ad apices rectricum inter. mediarum 20 poll. Rostrum superne $I_{\frac{3}{8}}$ poll. A sinu oris $I \frac{7}{8}$ poll. Alx extensx 2 ped. 5 poll. A Alexura alx ad ejusdem apicem $13 \frac{\pi}{2}$ poll. Cauda $8 \frac{\pi}{2}$ poll. Tibia $I \frac{6}{8}$ poll. Digit. med. $I \frac{5}{8}$ poll.

Rostrum nigrum in coeruleum vergens. Iris fusca. Pedes nigri.

Mas senior habitu astivali:

Caput supra a rostro, per oculos ad nuchan, saturate nigrum. Dorsum, scapulx, uropygium, alx et canda nigra, cinereo - fusco levissime tincta. Crissum et tectrices caudx inferiores cinereo-nigra. Gula, Iatera capitis inferiora, collare latum, collum antice et totum corpus subtus alba; gulâ, lateribus capitis col. loque antice tinctura lutescenti sordidis. Remiges nigr: basi et rachibus albis, lateribus interioribus in rufo-cinereum vergentibus. Rectrices basi albx, ceterum nigrx, I. I-4. 4 subæquales apice rotundato; 5. 5. aliquantum longiores et acuminatx. 6.6 . longissimx, acuminatissimx.

Femina scrior habitu astivali:

Caput supra saturate fuscum; regio oculorum nigre. Dorsum et alarum tectrices nigro-fusca, unicoloria, rufo vix tincta. Gula, latera capitis, totum collum et corpus subtus griseo-fusça, rufo tineta. Remiges et rectrices ut in antecedenti.

Avis junior ante autumnalem pennarum mutationem: (Medio Mens. Augusti). 
Longitudo circa I4-I5 poll. Rostrum pallide plumbeum, apice fuscum. Pedes pallide coerulescen. tes, digitis et membrana natatoria basi coerulescentialbis, dehinc cum unguibus nigris.

Caput, collum et corpus subtus cinereo-fusca marginibus pennarum rufescentibus, aliquantum latioribus. Remiges primores nigrx, basi, latere interiore juxta basin et rachibus albis. Cauda alis brevior, fere xqualis retricibus mediis parun longioribus. Rectrices apice nigræe in medio in nigro-cinereum transeuntes, a brsi ad tertiam partem albx. Tectrices inferiores fusca fasciis transversis albidis.

Obs. I. Post prinam mutationem pennarum autumnalem, strix pennarum marginales rufescentes aut omnes aut ad partem evanuerunt, et avis tum Lestri crepidato Temminckii simillima fit, quacum, ni fallor, omnino identica est.

Obs. 2. Non dubito, quin ha omnes e sexu vel xtate ortæ varietates sub variis nominibus specificis in scriptis Ornithologorum obveniant. Eas vero ad unam eandernque speciem pertinere co certius adfirmare audeo, quo sub itinere Norvegico presertim ad oras Helgolandire et Saltenses hanc speciem diligenter observare millies mihi occasio fuerit. Mares et femi. nas unâ semper vatorem (qui nido illorum ex impro. vișo adpropinquabat) adgredientes, et pullos tum pusillos et lanatos, tum volatui aptos defendentes, pius quam semel fixi pullosque, quos nutriebant, cepi. Illi etian piscatores, qui rupes et insulas oceani Norvegici usque intra circulun polarem incolunt, nullam aliam avem $T y v$ - Foe quam descriptam Speciem agnoscunt; adfirmant vero aliam huic sub. similem avem, parum tamen majorem, ad oras Finnmarkix obvenire. Hanc Finnmarkikan avem pro Nilssan Ornith. Pars II. 
Cataracta Skua Brünnichii habeo (quamris descriptio Brünnichii præter magnitudinem potius ad juniorem quam seniorem avem pertinere mihi videatur). Sed ingenue confiteor me nondum, neque in natura, neque in Museis vidisse avem hujus generis, qux non ad hanc speciem pertineret; quare omittere quam erronee adferre Synonyma satius duxi. Neque tamen ne. gare possum, me de vera differentia trium ex quatuor illis speciebus, quas enumeravit celeberr. Temminck, valde dubitare, et dubitaturum, usque dum accuratissimo Auctori placuerit, distinctiores characteres specificos proponere. Longitudo enim Tibix (Tarsi) variat pro xtate (in pullis minor), variat ali. quantum pro individuis; Cauda quoque xtate crescit, et in pullis semper brevior est: qux tamen observationes scrutatorem Naturæ tam excellentem fugere non potuerint. - Ego vero fere credo, suum Lestrem parasiticuns p. 5 I2 esse marem et juniorem, L. pomarinum p. 5 I 2 esse juniorem avem post autumnalem pennarum mutationem, et L. crepidatum juniorem (p. 5I6) esse pullum. Nam sinc dubin, tamquam in Larorum gienere, plures prætereunt anni, antequam colores perfecti sunt.

Ut certa, quorum tamen plurima ad Mares tantum pertinent, hæc adferre licet

\section{SYNONYMA.}

Larus parasiticus, Lin. Fn. Sv. p. 55. 11. I56. Lath. Ind. II. p. 8I9. (it.)

Cataracta Parasitica, Brünn. Orn. p. 37. n. 12\%. (Mas senior). Retz. Fn. Sv, p. I60. 11. 122. (Mas). Coprotheres, Brünn. 1. c. n. I28. (femina senior). Cataracta Cepphus, Brünn. 1. c. p. 36. n. 126. (junior ante primam pennarum mutationem). 
Arctic Gull, Penn. Brit.Zool. II. p. 533. n. 245. (Descr. Maris et feminæ bona) tah. 87. Lath. Syn. VI. p. 389. t. 99.

Aretic bird, Fdw. Av. t. I48. (Mas senior) tab. 149. (Junior).

Stcrcoraine parasite, Temm. Man. d'Orn. p. 5 I2.

Tyv-Foen, Gunner. Act. Nidr. III. IO3. (Femina est cum Skua Hojeri confusa, a qua tamen, ut opinor; distincta est species).

Svecis: Swartlasse, Struntjager. Norvegis: Joen, Le. verjoen, Tyvjoen, Geaath etc.

\section{HISTOR I A.}

Habitat in rupibus et insulis oceani Norvegici et maris Balthici superioris. Nidulatur in tesquis et uliginosis insularum. Ova 2.3 saturate olivacea maculis fuscis. Vescitur piscibus, quos tamen raro ipse piscrtur, sed plerumque $\mathrm{ab}$ aliis avibus, prasertim a Lavo tridactylo, L. cono et Sterna Hirundine prædatur. In aëre has aves persequitur, donee prædam e gutture emittant, quam tunc in ipso aëre, mirabili velocitate, antequam aquam attigerit, arripit. Piscatorum quoque cymbas supervolitat et piscium viscera, quæ in mare ejicit piscator, statim arripit. - Pullos mira strenuitate defendit; non solum aves ex improviso transvolitantes adgreditur, verum homines quoque transeuutes, alis et rostro petit. Pullum etiam volatui aptum, ta. men ad tempus quoddam, donec ipse prædari didicerit, prada adportata alit. 


\section{GENUS. \\ PRO CELLARIA.}

Rostram mediocre basi subdepressum, apice compressum; maxilla superiore apice adunca, inferiore apice decurvata, canaliculata.

Nares cylindro supra rostrum, a basi ad medium, decumbente, truncato.

$P$ edes mediocres 1 . graciles femoribus seminudis, ungue postico sessili (absque digito).

Alce longx, Remige prima ceteris longiore.

CCXXIII. PROCELLARIA PELAGICA, Linn.

DIAGN: Nigra uropygio albo.

\section{SYMONYMA.}

Procellaria pelagica, Lin. Syst. I. p. 212. Fn. Sv. I43. Retz. Fn. p. I43. in IOI. Lath. Ind. II. p. 826. Gmel. Syst. I. F. 59 I. Briss. Orn. VI. p. I40. 1n I. t. I3. f. I.

Petrel pigmé, Temm. Man. d'Orn. p. 5 I9.

L'oiseau de Tempète, Buff. Ois. IX. p. 327.

Stormy Petrel, Pen. Brit. Zool. II. p. 553. 11. 259. Aret.

Zool. II. p. 536. Latk. Syn. Suppl. I. p. 269. Edw. Av. t. 90 . 
PALMIPEDES. PROCE LLARIA. 187

Geschäckter Stinnivogel, Bechst. N. D. IV. 2. p. 704. Ǩléinster Sturnuogel, Mícisn. u. Schinz Vögel der Schweiz p. 82 I.

Svecis: Stormwâdersfogel.

\section{DESCRIPTIO.}

I.ongit. $6 \frac{x}{2}$ poll. Rostrum $\frac{5}{8}$ poll. A. flexura ala ad apicem 6 poll. Cauda $3 \frac{2}{8}$ poll. Tibia $\frac{7}{8}$ poll. Digit. med. I. poll.

Rostrum et pedes nigri. Alx compositx canda $\frac{3}{4}$ pollices longiores. Cauda xqualis.

Tota avis fuliginoso-nigra, uropygio albo. Remiges primores et Rectrices saturate nigr:x. Tcetrices alarum fusco-grisex. Pennae scapulares et Remiges secundarir apicibus albicantes.

\section{HIS T ORIA.}

Habitat in Oceano boreali, raro nee nisi hieme oras occidentales Svecix visitat. Super undas currit, quare nomen a nautis Avis Sti Petri recepit. Instante procella naves petit, ut contra vim ventorum præsidium habeat. Vesciturinsectis et vermibus marinis, quæ in superficie maris natant. An in Scandinavix maxime borealis insulis nidificet nee ne, pro certo dicere non possum.

CCXXIV. PROCELLARIA GLACIA. LIS, Linz.

Diagn: Alba, dorso et alis canis, Remigibus griseofuscis. 


\section{SYNONYMA.}

Procellavia glacialis, Lin. Syst. I. p. 2 I3. Fn. Suec. I44.

Retz. Fn. p. I43. n. I02. Gmel. Syst. I. 2. p. 562.

Lath. Ind. II. p. 823.

Petrel fumar, Temm. Man. d'Ornith. p. 518. Buff.

Ois. IX. p. 325. t. 22. Pl. enl. 59.

Fultnar, Pen. Brit. Zool. II. p 549. t. 91. Arct. Zool.

II. p. 534. n. 46 I. Lath. Syn. VI. p. 403."

Havhest, Gunner. Acta Nidros. I. p. 182.

\section{DESCR I P TIO.}

Longitudo 16-I7 poll. Rostrum fiavum, superne subaurantium. Iris et pedes lutei.

Caput, collum, uropygium, cauda et totum corpus subtus alba. Dorsum, scapulares, alarum tectrices et remiges secundariae coerulescenti cinerea (cana). Re. miges primores extus pallide griseo-fuscr, intus albr. Cauda angulato-rotundata.

\section{HIS T ORIA.}

Habitat in ocenno boreali. Continentem terram et insulas eam adjacentes nunquam vel rarissime visitat. In insulis vero plagæ arcticæ (ex. gr. Nordlandix et Finnmarkix) a terra remotissimis ex piscatorum indiciis quotannis nidulatur. Ovatio adhue mihi incognita. Vescitur cadaveribus Cetaceorum aliisque animalibus marinis in superficie maris natantibus. 
B) Rostro dentato s. lameiloso.

GENUS.

\section{C $\quad \mathrm{Y}, \mathrm{G} N \mathrm{~N} \quad \mathrm{U}$.}

Rostrmm usque ad oculos nudum semicylindricum, basi altius quam latius, marginibus lamellosum, apice ungue obtuso, lato. Nares ovales in medio rostri sitr. Collum longum. Dorsum convexum.

\section{CYGNUS MELANORHYN. 。 CHUS, Mey.}

DiAGN: Albus capite et nucha lutescente. Pedes et rostrum nigra, cerâ luteâ.

\section{SYNONYMA.}

Cygnus Mclanorhynchus, Meyer Vögel Liv. u. Esth1. ₹. 240. Meyers u. Wolfs Taschenb. p. 498.

Cygnus Musicus, Bechst. N. D. IV. p. 830. Anas Cygnus, Gmel. Syst. I. p. 50I. Lath. Ind. II. p. 833. Retz. Fn. p. 109. n. 62.

Le Cygne snuvage, Buff. Ois. IX. p. 3. Temm. Man. p. 522.

Whistling or Wild Swan, Pen. Brit. Zool. II. p. 562. Arct. Zool. II. p. 54I. Edw. Av. III. t. I50. Lath. Syn. VI. p. 433. Id. Suppl, I. p. 272.

Svecis: Wild Swan. 


\section{DESCR IPTIO.}

Longit. 4 ped. $6-9$ poll.

Rostrum enode, nigrum cerâ basin et regionem ocu. lorum cingente, flava. Iris fusca; Pedes nigri.

Tota avis pure alba, exceptis capite et nucha, lute. scenti colore tinctis.

\section{HIS T ORIA.}

Habitat rarius et forte non nisi hyemali tempore in mari juxte oras Sveciæ meridionalioris. IEstatem in fluviis et lacubus Lapponia degere refertur. Pro corto scio, Cygnos interdum, sed rarius, in mari extra oras Nordlandix et Finnmarkix Norvegice visos esse, qui, ut opinor, ad hanc speciem pertinent. Olim in lacu Por. sion, jam campo subsicco, prope Mnlmogiam Scanix, habitasse, et quotannis pullos exclusisse dicitur. Ni. dulatur in graminosis juxta aquam. Ova $5-7$ vire. scenti-olıvacea. Vescitur herbis et insectis aquaticis.

\section{CYGNUS GIBBUS, Bechst.}

Diagn: Albus rostro luteo-rubro, cera gibbo, ungue et marginibus nigris. Pedes rufescentinigri.

\section{SYNONYMA.}

Cygnus Gibhus, Bechst. Nat. D.IV. p. 815. Meyer Liv. u. Esthl. p. 24 I.

Anas Olor, Ginel. Syst. t. 2 p. 50 I. Lath. Ind. II. p. 834. Retz. Fn. p. I09. n. 63.

Le Cygne, Buff. Ois. IX. p. 3. t. I. Pl. enl. 913.

Cygne tuberculé on donestique, Temm. Man. d'On. p. 523. 
Tame Swan, Pen. Brit. Zool. II. p. 564. Edw. Av. t. 150. (caput.) Pen. Arct. Zool. II. p. 543. Lath. Syn. VI. p: 436.

Höcker Schivan, Bechst. N. D. IV. p. 8 I5.

Svecis: Tam Svan.

\section{DESCRIPTIÓ.}

Longitudo 4 ped. 6 poll.

Frons juxta basin rostri gibbo magno, rotundo, carneo iustrueta. Rostrum luteo-rubrum, marginibus maxillarum, narium, ungue, cerâ et gibbo frontali nigris. Iris fusca. Pedes nigri, rufescenti tineta. Praterea tota avis pure alba.

Fensina minor gilibo minori, collo graciliori.

\section{H IS T OR I A.}

Habitat in mari oras Svecix meridionalis cingente. In lacubus quoque sed rarius obvenit. Tempore autumnali innumeranda copia in mari extra oras Scanix occurrit lıæc Species, quam venandi jus Gubernatori Provincix reservatum est. In hoc venatu, qui quotannis instituitur, uno sæpe die usque ad centum Cygni, vel occiduntur vel vivi capiuntur. Nullibi, quantum equidem scio, in Svecia ferus nidulatur, sed cicur in plurimis Magnatum prædiis vivit ibique se propagat. Ova 5.6 virescenti - albida. 
GENUS.

A. N A S.

Rostrum depresso-semicylindricum, antice quam basi latius, marginibus lamellosis, apice ungue lato, obtúso.

Nares ovales prope dorsum rostri, et (plerisque) juxta basin sitæ.

Pedes breves; Tibia digito medio aliquantum brevior.

Collum brevins.

Dorsum depressum.

Digitus posticus membrana lobiformi instruęus.

I) Speculo nullo:

CCXXVII. ANAS NIGRA, Linn.

DIAGN: Rostrum nigrum, medio superne flavum. Mas: Rostrum basi tuberculo osseo linea flava bipartito instructum. Corpus totum atrum.

Femina: Tuberculo rostri destituta. Corpus supra nigro-fuscum, subrus pallide griseum.

\section{SYNONYMA.}

Anas Nigra, Lin. Faun. Sv. p. 39. n. IIO. Reczii Faun. p. II3. n. 67. Syst. Nat. I. p. 196. n. 7. Gmel. 
Syst. I. 2. p. 508. Lath. Ind. Orn. II. p. 848. n. 43 :

Lo Macreuse, Buff. Ois. IX. p. 234 t. I6. Pl. enl. 978. Canard Macrense, Temm. Man. d'Orn, p. 554.

Scoter, Pen. Br. Zool. II. p. 584. n. 273. Aret. Zool. II. p. 556. n. 484 . Lath. Syn. VI. p. 480 n. 36. Vers. Germ. Bechst. VI. f. 4 I4.

Trauen-Ente, Bechst. Nat. D. IV. p. 963. Meyer Vö. gel Liv. u. Esthl. p. 242.

Obs., 'Sine dubio huc pertinet ut avis junior:

Anas Cinerea, S. G. Gmelin, Reise II. p. I84. Tab. I8. Anas Cinerascens, Bechst. N. D.W. p. 1025.

\section{DESCR IP TIO.}

Mas: Long. I8 poll. Rostr. 2 poll. A flexura ale ad apicem 6 poll.

Cauda 3 poll. Tibia I $\frac{\pi}{2}$ poll. Digit. med. fere 3 poll. postic. $\frac{6}{8}$ poll.

Rostrum depressum, pone apicem latum, apice ob. tuso ungue vix conspicto, nigrum medio superne flavum; Caruncula baseos hieme olivacea, æstate rubra, per striam flavam longitudinaliter bifida. Utraque max. illa dentibus lamellosis instrueta. Nares pone meditun rostri, ovales aurantix. Iris brunnea. Margo palpebra. ram luteus. Pedes sordide griseo-fusci, membranâ natatoria nigra. Digitus posticus elevatus, membrana natatoria instructus. Cauda cuneiformis Rectricibus 16.

Corpus totum atrum capite et collo purpurascentinitidis. Remiges latere interiore rubescenti-albida. Tectrices alarum inferiores fuscr.

Femina: Aliquantum minor. 
Rostrum nigrum basi altius', sulco instructum, câruncula tamen destitutum; macula in mediolutea. Pedes sordide viridi = lutei, membranâ nigrâ.

Corpus obscure fuscum, capite supra saturatiore. Collum antice sordide albidum, maculis obsoletis fuscis. Pectus et abdomen pallide grisea fusco maculata. Mas Funior hieme:

Supra fusca pennis nigris in pileo et scapulis insertis. Gula albida. Jugulum, latera colli et capitis pallide grisea obsolete fusco maculata, collo iuferiore et humeris undulis obsoletis cinereis. Pectus et abdomen albida undulis obsoletis cinereis. Pectus et abdomen albida undulis crebris fuscescentibus.

\section{HIS TORIA.}

Estivat in terris borealibus, ex. gr. Lapponia, Sibiria. Autumno et per hyemem in mari juxta oras nostras et in lacubus, rarius tamen, obvenit. Vescitur testaceis, insectis et plantis marinis. Nidus et ova incognita,

\section{ANAS MOLLISSIMA.}

DiAGn: Rostrum semicylindricum, cera postice acute bifida, rugosa, Mas: Supra albus pileo arro postice lineâ alba bipartito; subtus et postice niger. Femina: Testacea maculis transversis e: undulis nigris.

\section{SYNONYMA.}

Ants Mollissima, Lin。 Syst. I. p. 198. Lin. Fn. Sv. p. 41. 1. II7. Retz, Fn. p. I18, n. 74. Gmel. Syst. 
I. 2. p. 5 I4. Lath. Ind. II. p. 845. n. 35. Brünn. Orn. Bor. n. 57-61 et 64-65. Id. Monogr. Hafnix 1783.

Oie a Duvet ou Eider, Buff. Ois, IX. p. I03. t. 6. Pl. enl. 208 (fem ) 209 (mas.).

Canard Eider, Temm. Man. d'Ornithol. p. 549. (cum Anate spectabili confusa).

Grent black and white Duck, Edw. Av. t. 98, mas. et fem.

Eider or cuthber Duck, Penn. Brit. Zool. II. p. 58I. t. 95. Aret. Zool. II. p. 553. 12. 480. Lath. Syn. VI. p. 470. n. 29. Suppl. p. 274.

Die Eidcrgaus, Bechst. N. D. IV. p. 926.

Eiderente, Meyer Liv. u. Esthl. p. 243.

Siecis: Eiderfögrel, Eidergâs. fem: Ảda.

\section{DESCR IP T I O:}

Longit. 24.26 poll. Rostrum ab apice ad angulum frontis 3 poll. A flexura alæ ad apicem $12 \frac{r}{2}$ poll Tibia 2 poll. Digit. med. $3 \frac{2}{8}$ p. postic. $\frac{7}{8}$ p.

Feinina 24 poll. alx extensæ 3 ped.

Rostrum conico-semicylindricum, fronte aliquantum altius quam latum et versus apicem sensim decrescens ${ }^{*}$ ), apice supra convexo, rotundato. Caput majusculum ovatum, antice attenuatum. Cutis nuda, qux rostrum a basi ad nares obtegit, rugosa et sordide viridis, ad. latera frontis utrinque in angulum acutum adscendir. Rostrum dehinc pallidius. Oculi parvisub vertice siti. Iris brunnea. Pedes sordide griseo-virides membrana. natatoria nigra et ungues nigri.

*) Quare potius fortasse ad Auseris quam ad An?tis genus nu. ineranda est $\Lambda$ vis. 
Mis senior:

Albus pileo atro, postice lineâ alba bipartitn. Occiput et latera nuchæ pallide viridia. Pectus rubescenti tinctum. Dorsum posticum et uropygium nigra. Pectus postice, abdomen, crissum, hypochondria et femora atra. Remiges primores nigricantes, intimæ albæ. Rectrices I6 nigrx.

Femina seniov:

Testacea seu luteo-rufa capite pallidiore punctis et maculis minutis longitudinalibus nigris crebre adsperso; corpore supra saturatiore, striis et undulis transversis nigris, subtus grisea undulis nigro-fuscis. Remiges et Rectrices saturate fusca.

Obs. Remiges secundarix et Teetrices alarum proximx apicibus pallent, neque tamen unquam eas tam pallidas vidi, ut fascias binas alarum formarent, quod in Anate spectabili obtinet.

Avis annua - An femina?

Nigricans marginibus penuarum in dorso, scapulis et alarum tectricibus rufis. Fascia supra oculos ad nucham grisea striis et punctis albidis et nigris. Latera capitis inferiora rufescentia striis nigris. Pectus et ab. domen fusca undulis tenuibus albidis adspersa. Criscum, hypochondria, latera uropygii et tectrices caudæ nigra fasciis et undulis transversis rufis et griseis. Remiges nigra, intimæ juxta apicem extus rufo marginatx.

Mas biennis? Pectus et anterior pars colli albescit. Tectrices alarum minores et Remiges intimx, altr, nigro nebulosæ. Abdomen undulis tenuibus fuscis et albis. - Vide praterea Sparrm. Museum Carls. I. tab. $\sigma$.

Mas triennis? Caput et collum nigricantia pennis albis hine inde inspersis et ad latera nuche in serie positis. 
Gula pennis plurimis albis. Superior pars pectoris alba nigro superne inquinato. Dorsum album, antice nigro maculatum. Scapulares nigrx albis imınixtis. Tectrices alarum minores et medir remigesque intimre albx. Peetus inferius, abdomen, hypochondria, crissum, tectrices caudre et uropygium medium nigra immaculata. - Brünn. n. 60. Exemplar descriptum ad finem M. Maji captum est.

\section{ORSER V.}

Quarto ætatis' anno Mas induitur colore primum descripto, quem postea continuo retinet.

\section{HISTORIA.}

Habitat hæc Species copiosissine in mari scopuloso secundum oras Norvegie, præcipue intra circulnu arcticum. Obvenit quoque et nidulatur, quamvis minus numerose in maris Balthici quibusdam insulis et scopulis extra Scanix et Blekingix litora sitis. In. trat etiam hieme fretum Oeresund, ubi non raro in retibus piscatorum capitur. - Nidulatur in insulis et scopulis parum elevatis. Ova 5.6 virescentia. (Historiam præterea fuse dedit cel. Brünnich 1. c)

\section{OBSERV.}

In Faun. Groenl. p. 69, refertur avem esse monogamam, et marem in propinquitate feminæ incubantis juxta litus illam quasi custodire. Hoc mex in Norvegia facta experientix repugnat, et ni fallor, mas feminam, rempore amoris perafo, deserit altumque mare petic, nec prius ad suos redit, quam pulli ad volandum sunt apti. Saltem ego per totam æstatem innumeras vidi feminas, neque unum marem ante medium mensis Augusti observabam. Post hoc tempus sensim in adspeatum veniebant.

Præterea referebant piscatores, qui ultimas insulas frẹ̣nentaverant, mares hujus speciei extra has insulas in alto muri xsta- 
tem degere. Hanc consvetudinem, conjugem ova patientem et excludentem deserendi, cum plurimis, quas novimus, congene. ribus communem habet.

\section{ANAS GLACIALIS, Lin.}

Diagn: Rostrum breve nigrum fascia (plerisque) rubra. Pedes plumbei. Speculum nullum. Mas: Niger et albus, rectricibus intermediis longissimis. Femina: Supra nigro-fusca, subtus alba, capite et collo albis, vertice et macula subauriculari fuscis.

\section{SYNONYMA.}

Anus hiennalis, Lin. Fn. Sv. (Mas habitu hiemali). Syst. Nat. I. p. 202. 11. 29. - Fabric. Grönl. p. 7I. 11. 45. (Mas habitu hiemsli et fem. habitu xstivali). - Brünn. Orn. Bor. p. I7. n. 75. (Mas habitu hiemali) n. 76. (Mas habitu astivali). Anas glacialis, Lin. Syst. I. p. 203. n. 30. (Videtur esse mas tempore mutationis pennarum). Al-Foglen, Ödm. Act. Holm. 1783. p. 3 I3. Longtailed Ducle, Pen. Brit. Zool. II. p. 599. n. 233. (tab. 98. Marem habitu æstivali reprasentat; Descriptio ad marem deplumantem pertinere videtur) tab. 99. (fem.) - Edw. Av. tom. VI. tab. 280. Mas tempore deplumationis Id. tom. III. tab. 156. (Mas habitu æstivali, figura accuratissima). Connard de Michon, Temm. Man. d'Ornith. p. 558. Kanmbak, Lepechin Reise 3 B. p. 222. (Descr, bona) t. 2. habitu astivali.

Troförer, Ström Aça Nidros. V. p. 539. tab. VIII. fig. I. (Mas habitu xstivali. Descr. bona). 
Suecis: Ahlfogel; Winter-Al, Sommar-Al. Ad Ystad Scanic: Mas Gaddes, Femina: Ahla,

\section{DESCRIPTIO.}

Longit. 20.2I poll. (propter rectrices longissimas). Rostrum I $\frac{x}{8}$ poll. A flexura alæ ad apicem 9 poll. Cauda 8 poll. Tibia I $\frac{2}{8}$ poll. Digit. med. $2 \frac{\pi}{8}$ poll. postic. $\frac{x}{2}$ poll.

Rostrum breve, parvun, apice ungue incurvo obtuso, nigrum fascia in medio superne semipollicari coccinea, inferne angustiore luteo-alba. Dentes pectiniformes (compressi) infra marginem maxillæ superioris dependentes. Nares oblongo-ovales. Iris rufo. lutea. Pedes rubescenti-cinerei membrana natatoria ot unguibus nigris.

\section{Mas senior habitu hiemali:}

Caput et collum alba laterihus capitis antice pallide rubescenti - cinereis, lateribus colli maculâ ovali nigra, rufo inferne terminata. Inferior pars colli et pectus nigra, qux nigredo conjungitur cum nigredine dorsi. Dorsum, alæe et cauda nigra. Penr:x scapulares elongatx, acut:x, e cano slbx, unde in scapulis oritur - macula magna alba, cano pulcherrime tincta. Remiges primores nigrex latere interiore cinereo-fusc: secundarix rufescenti - nigra. Teetrices inferiores fusco-uigricantes. Inferior pars pectoris, abdomen, hypochondria et crissum alba axillis canis. Cauda supra nigra, subtus alba. Reetrices 14, quarum I. I. 2. 2. albx; 3. 4. 5. cinereo-nigrx marginibus late albis; ceterre nigrx acuminatæ, prasertim intermedix longissima.

Nilssolz Ornitho Pars 11 .

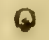


Femina senior habitu hiemali:

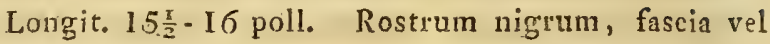
nulla vel pallidius aurantia, basi subtus plumbeum. Pedes antice plumbei, postice cum membrana natatoria nigri.

Caput et collum alba vertice et macula colli utrinque nigro-fuscis. Dorsum et pennæ scapulares nigræ cinerascenti-rufo late marginata. Dorsum posticum fusco-nigrum rufo obsolete maculatum. Remiges fuscr, latere interiore cinerex. Peatus cinerascens fusco et rufo immixtum, postice in album abdominis colorem sensim transiens. Cauda cuneata, rectricibus mediis fuscis, lateralibus sensim canescentibus.

Mas habitu cestivali:

Caput et collum nigra area oculorum et genis rubescenti-cinereis, postice albis, lateribus colli et pectore nigro in griseum transeuntibus. Dorsum et scapulares elongatr, acutr, nigra marginibus latis ferrugineis. Uropygium et tectrices caudx et alarum superne migra immaculata. Abdomen, hypochondria, latera uropygii et tectrices caudæ inferiores alba. Cauda exacte ut in mare primo descr.

Femina habitu restivali:

Caput supra et macula colli utrinque nigro-fusca, praterea caput et collum albida. Dorsum et uropygium nigricantia, immaculata. Pennæ scapulares et tectrices alarum nigro-fuscæ, marginibus qvasi dilutis, rufescentibus. Pectus griseo-fuscum, inferne sensim pallescit. Abdomen et crissum album. Rectrices cinereo - fuscr, apicibus albis.

Mas tempore deplumationis vernalis:

Latera capitis anterius cinerea. Ceterum caput et collum nigra, vertice, nucha et tænia cervicali albis. Gula et basis colli prasertim superne pennis albis du- 
dum immixtis, maculata. Peetus nigricans. Dorsum et uropygium nigra immaculata. Abdomen et crissum alba. Cauda ut in anteced. Scapula pennis albis, inter quas nigrx ferrugineo marginatæ intermixta sunt.

\section{O BSERV.}

Pennæ scapularmm seniores alba, et recentiores nigri ferrugineo fimbriatx cum aliis multis demonstrant, vestem hiemalem magis alban deponere et astivali magis obscura se induere avem jam incepisse. Demonstrant etiam habitum illum obscuriorem non solum ad pullos pertinere, ut plerique recentiores Ornithologi opinantur, sed a senioribus quoque tempore æstivali recipi, Cum hac sententia congruunt etiam omnes fere, quibus avem xstate in regionibus areticis observare contigit, neque unam vidi maris descriptionem vel iconem in plagis ar cticis xstate factam, qui habitum illum albidum representaret. At vero vix credere possumus ex. gr. clar. Lepechin, qui avem in regionibus mari glaciali contiguis rastate vidit, non nisi juniores invenisse; neque marem illum mense Junio occisum et a Rev. Ströra 1. c. descriptum pro pullo habeamus, cetera ut tacean. Ipse quoque mense Julio in sinu Ranenfjorden Norvegia marem et feminam hujus specici offendi.

Feminam supra descriptam plumbo fixi; Marem, descripto similem, perdidi,

\section{HISTORIA.}

Habitat æstate in plagis maxime borealibus Europz, Asix et Americx, et quidem in lacubus et fluviis oceano arctico contiguis. "Mense Junio ova ponit 5 figura et magnitudine Gallinæ junioris, alba cum tinctura pallide coerulescenti." (Fabric, 1. c.) Mensibus Octobri et Novembri oras Scanix visitat et per totam hiemem in mari gregatim occurrit. Voce sonora vel e longinquo dignoscitur. Mense Aprili et Majo abir.

० 2 
2) Speculo albo:

\section{ANAS SPECTABILIS, Linn.}

DiagN: Mas: Rostrum semicylindricum gibbo magno, superne pennis nigris bipartito ornatum. Caput supra canum. Remiges secundarix falcatæ. Femina: Brunnea maculis et siriis fuscis adspersa. Fascia alarum transversa, duplici, alba.

\section{SYNONYMA.}

Anas Spectabilis, Linn. Fn. Sv. p. 39. n. II2. Systo Nat. I. p. 195. Mus. Adolphi Fred. II. p. 25. Gmel. Syst. I. 2. p. 507. Fabr. Groenl. p. 63. Mus. Carls. II. t. 39. (Mas variet.) t. 40 (Femina). Grey-hended Duck, Edw. Av. III. tab. I54. (mas.) Hav-Orre, Leem Lappl. 274. t. 98. (mas.)

Dẹn pukkelnebbede Edderfugl, Fabric. Nat. Hist. Selsk. Skr. II. 2. p. 56. t. 9. (femina; figura bona).

\section{OBSER V.}

Singulare ommino est, hanc avem quamvis optinz ejus ef descriptiones et icones jam extent, a recentissimis tamen Ornttho. logis Temwiuck et Cuvier cum antecedenti Specie fuisse confusam.

\section{DESCRIPTIO.}

Longit. $22 \cdot 23$ poll. Rostr. a sinu oris $2 \frac{\pi}{2}$ poll. Mas senior:

Rostrum rubro-nigricans, semicylindricum, versus apicem angustius, basi supra gibbo maximo, compresso, flavescenti-rubro, superne longitudinaliter diviso et ad margines ornato pennis atris. Pedes rubri. 
Caput supira pallide cinereum. Latera capitis inferius albide-viridia. Gula alba macula atra postice bipartita. Dorsum anticum albo-cinereum; posticum et humeri nigro-fusca. Latera uropygii alba. Pectus superius album, flavo tinctum, inferius et abdomen nigra. Alæ nigro-fusce macula magna alba, Remiges secundarix falcatx.

Femina:

Mare aliquantum mino:, gibbum baseos rostri nee tam eminentem habet nec colorarum, sed carinam pennaceam latiorem, qux ceram rugosam dividit. Ro. strum et pedes obscuriores.

Tota avis ex brunneo et nigro maculata. Caput et collum brunnea striis longitudinalibus et maculis parvis obsoletis fuscis adspersa. Dorsum et alarum tectrices fusea marginibus pennarum pallidis, unde pallide undulata appareant. Abdomen subfuscum. Remiges fuscx, secundarix et tectrices proximz apicibus albæ; unde fascix binx, obliqux, albx in ala composita oriuntur.

\section{HIS TORIA.}

Habitat rarius in superioribus maris Balthici partibus. Hyeme interdum extra Upplandix oras descen. dit, unde inter aves, qux a piscatoribus Holmiz venditantur, non nunquam obvenit. - Oceanus tamen arcticus proprium est hujus: speciei domicilium, ubi fre. quentius in plagis maxime borealibus obvenit. Oras Finnmarkix sxpius visitat, rarissime vero in oris Helgelandix obvenit, et plagas meridionaliores vix unquam salutat. Oeconomia et vivendi ratio eadem esse refertur atque Anntis mollissime: 


\section{ANAS FUSCA, Linn.}

Diagn: Macula pone oculos striaque alarum albis. Mas ater. Femina nigro-fusca.

\section{SYNONYMA.}

Anas fusca, Lin. Syst. Nat. I. p. 196. Lin. Fn. Sv. p. 39. n. 109. Retz. Fn. p. I I2. 11. 66. Acta Nidros. I. Tab. IV. Lath. Ind. II. p. 848. Gmel. Syst. I. p. 507.

La double Macreuse, Buff. Ois. IX. p. 242. PI. enl. 11. 956. (mas senior.) Temm. Man. d'Orn. p. 552. $V_{\text {elvet }}$ Duck, Pen. Brit. Zool. II. p. 583. t. 96. Arct. Zool. II. p. 555. Lath. Syn. VI. p. 482. - Id. Suppl. I. p. 274.

Sanmet-Ente, Bechst. N. D. IV. I. p. 9.54. Anas Fuligula, Bechst. 1. c. p. 962.

Swarta, Lin. Itin. Gottl. p. 215-271. - Act. Acad. Scient. Holm. 1785. p. 391.

Söe-Orre, Gunner. Act. Nidros. I. p. 259. Tab. 4. fig. 5.

\section{DESCRIPTIO.}

Long. 2 I poll. Rostr. a sinu oris $2 \frac{\mathrm{T}}{2}$ poll. a fronte $I \frac{6}{8}$ poll. basi latum $I$ poll. pone apicem latum $I \frac{\frac{2}{8}}{8}$ poll. A flexura ali ad apicem II poll. Cauda 3 poll. Tibia $1 \frac{7}{8}$ poll. Digit, med, $3 \frac{2}{8}$ poll. postic. $\frac{7}{8}$ poll.

Rostrum magnum, latum, valde depressum, aurantium, gibbo, naribus et marginibus nigris; ungue obtuso luteo-rubro. Iris rubra. Pedes rubri membranâ natatoria nigrá. 
Corpus totum holosericeo-atrum macula parvá pone oculos er striâ in alis obliqua alba. Remiges primores nigre, secundarix et apices tectricum proximarum albr. Fomina: Rostrum luteo-fuscum gibbo carens. Pedes sordide rubri membrana nigra. Corpus supra nigrofuscuin gibbo carens. Pedes sordide rubri membrana nigra. Corpus supra nigro-fuscum, subtus griseoalbidum, fusco maculatum. In genis macula et in alis stria alba.

\section{HIS T ORIA.}

Habitat sstate in tota fere Scandinavia ex. gr. in Oelạndia, Gottlandia, Wermdö et in Norvegia usque ultra circulum arcticum, ubi per æstatem in sinubus interioribus quan in insulis et. alto mari frequentius occurrit. Mense Junio in terra sub frutice ova 8-10 alba ponit. Reliquo tempore maria frequentat.

\section{ANAS FULIGULA, Linn.}

Diagn: Nigricans abdomine et speculo albis. Crisra occipitis dependens. Mas capite et collopurpureo nitentibus.

\section{SYNONYMA.}

Anns fulizula, Lin. Syst. I. p. 207. Lin. Faun. Sv. p. 47. n. 132. Retz. Faun, p. I33. n. 91. Gmel. Sy'st.

I. 2. p. 543. Lath. Ind. II. p. 869.

The tufted Duck, Pen. Brit. Zool. II. p. 585. Arct. Zool.

II. p. 573. Lath. Syn. VI. p. 540, 11. 79.

Le Morillon, Buff. Ois. IX. p. 227. 23 I. t. 15. PI. enl. IOOI.

Reitzer. Ente, Bechst. N. D. IV. p. 997. Meyer Vüg. Liv. p. 247. 
Funior:

Anas Scandiaca, Gmel. Syst. I. 2. p. 520. Lath. Ind. If. p. 859.

Lappmark Duck, Lath. Syn. VI. p. 5 I.5.

Anas latirostra, Briinn. Orn. bor. p. 2I. n. 9 I.

Anas Skoorra, Müll. Prodr. Zool. Dan. p. I6. n. I30.

\section{DESCR IPTIO.}

Longit. 15-16 poll. Rostr. I $\frac{6}{3}$ p. A flexura alz ad apicem 8 poll. Cauda 2 poll. Tibia $I \frac{1}{2} p$. Digit. med. $2 \frac{1}{2}$ p. postic. $\frac{6}{8}$ poll.

Mas senior:

Rostrum pallide plumbeum ungue nigro. Iris fulva. Pedes plumbei membrana natatoria nigra.

Caput, collum et pectus ad medium nigra nitore violaceo 1. purpureo in capite et superiore collo. Occiput crista dependente bipollicari. Dorsum anticum, sca. pulares et tectrices alarum nigra viridi-nitentia punctis albidis minutissimis irrorata. Uropygium, crissum et tectrices caudæ nigra. Pectus postice et abdomen alba. Hypochondria grisea, albo tenuissime undulara. Remiges primores nigræ intus rersus basin cinereæ; se. cundarix albre apicibus nigris, unde speculum album nigro cinctum; intimx nigra splendore viridi. Caude fusco - nigra.

Femina Senior:

Rostrum et pedes obscuriores. Iris pallide lutea. Occiput cristâ breviore. Caput, collum et anterius pectus sordide rufo-fusca. Alæ fuscæ lineâ obliquâ alba, Abdomen album ferrugineo intermixto.

Avis junior post priman pennarum mutationem:

Rostrum fusco-plumbeum. Pedes plumbei membra. na nigra. Iris citrina. Occiput cristâ vix perspicua. 
Caput, collum et anterins pectus rufo-fusca. Ceterum peĉus et abdomenalba. Crissum griseum. Dorsum et alæ fųsco - nigricantia striâ alari alba.

\section{H I S T O R I A.}

Estivat in Lapponia et aliis regionibus areticis, ubi nidulatur, sed ovatio incognita est. Mense Oetobri meridionaliores terras petens in lacubus et fuviis Scanix obvenit. Vescitur piscibus, insectis et herbis aquaticis.

\section{CCXXXIII, ANAS CLANGULA, Liz.}

DIAGN: Rostrum breve, altum, nigricans. Caput tumidum. Iris flava, Mas: Caput atro-viridi nitens macula subrotunda alba ante oculos. Collum et corpus subtus alba. Dorsum nigrum. Femina: Caput rufum, pectus cinerascens. Ab. domen album. Dorsum fuscum.

\section{SYNONYMA.}

Anas Clangula, Lin. Syst. Nat. I. p. 201. n. 23. Faun. Sv. p. 43. 11. 122. Retz. Faun. Sv. p. 121. 11. 78. Gmel. Syst. I. p. 523. Lath. Ind. II. p. 867. n. 87. Briss. Orn. VI. p. 416. n. 27. t. 37. f. 2.

Le Garrot, Buff. Ois. IX. p. 222, Pl. enl. 802. (Mas). Golden-eye, Pen. Be, Zool. II. p. 587. n. 276. Arct. Zool. II. p. 557. n. 486. Lath. Syn. VI. p. 535. n. 76. Vers. Germ. Bechst. VI. p.

Die Schell-Ente, Eechst. Nat. D. IV. p. 985. tab. 37. (fenı.) Meyer Vögel Liv, u. Esthl. p. 249. 
Funiar avis:

Anas Glaucion, Lin. Faun. Sv. p. 43. n. I23. Retz.

Faun. p. I22. n. 79. Lath. Ind. 1I. p. 868. Arct.

Zool. II. p. 573.

Morillon, Pen. Brit. Zool. II. p. 588. n. $27 \%$.

Die Spatel-Ente, Bechst. 1. c. p. 1004.

Venitoribus Scamens. Dopping.

\section{DESCRIPTIO.}

Longit. I8 $\frac{\pi}{2}-19$ poll. Alæ extensæ 2 ped. 7 poll. Rostr. I $\frac{x}{2}$ poll. A flexura alæ ad apicem 9 poll. Cauda $3 \frac{2}{8}$ p. Tibia $1 \frac{3}{8}$. Digit. med. $2 \frac{6}{8}$, postic. $\frac{6}{8}$ poll.

Rostrum nigrum, breve, compresso-convexum et versus frontem elevatum. Nares in medio rostro sitæ, oblongo-ovales. Iris fulva, Pedes aurantii membranâ natatorin nigra.

Caput cum superiore colli parte tegitur pennis tumidis nigris atro-viridi nitentibus. Pone sinum oris macula subrotunda alba. Pars inferior colli, pectus, abdomen et hypochondria alba (nivea). Dorsum, uropygium et minimæ alarum tectrices nigra. Pennæ femorum longæ, acutx, albx, nigro marginatæ. Inter femora per ạnum fascia lata nigricans marginibus pennarum albis. Scapulares longae, acuminatæ, falcatx, albx, marginibus nigris. Tectrices alarum medizalbr; majores albæ basi nigræ. Remiges primores fusco. nigræ, medix (15-2I) albx, unde macula magua alba (interdum striâ nigrâ bipartita) in ala oritur. Remiges intimæ nigræ. Teçtrices inferiores nigro-cinerex. Cauda rotundata (pennis I6) supra cinereo-nigra, subtus fusca.

Femina: minor, I6 poll, longa. Alx extensa 2 ped. 2 poll. 
Rostrum fuscum, apice superne lutescens. Iris flava. Pedes viridi. lutei membranâ nigricante.

Caput tumidum cum superiore colli parte fusco-rufum. Collum medium pallide cinerascens. Pectus su. perius et latera cinerea marginibus pennarum albidis. Dorsum anterius, scapulares et minores teetrices fusca marginibus pennarum cinereis. Dorsum posticum cum uropygio nigricans, unicolor. Pectus inferius, abdomen et medix tectrices alba. Remiges primores fusconigra, medix albx, et tectrices majores albæ apice nigrx, unde in ala clausa macula magna alla striâ nigrâ bipartita oritu:. Per anum fascia cinerascens. Avis junior: Matri fere similis est.

\section{HISTORIA.}

Festivat in Svecia septentrionaliori, Norrlandia et Lapplandia. In arboribus cavis ova ponit 10 - I 2 alba. Hieme instante australiores plages petit et in mari Scaniam alluente aliquantum maturius quam Anas glacialis obvenit. Vescitur Insectis et vermibus aquaticis.

\section{ANAS MARILA, Lim.}

DIAGN : Caput et collum atra. Dorsum album lineis nigris transversim undulatum. Speculum et corpus subtus alba, postice nigra.

\section{SYNONYMA.}

Anas Marila, Lin. Syst. I. p. 196. Lin. Fn. p. 39. n。 III. (Mas) Retz. Fn. Sv. p. II 3. n. 68. Gmel. Syst. I. 2. p. 509. Lath. Ind. II. p. 853. Anas Franata, Mus, Carls. II. t. 38. (femina). 
Scaup Duck, Pen. Brit. Zool. II. p. 586. t. 100. Arê. Zool. II. p. 5ó5. Lath. Syn. VI. p. 500. n. 49. Vers. Germ. VI. p. 432.

Millouinan, Buff. Ois. IX. p. 22I. Pl, enl. n. 1002. Kagolka, Lepechin Reise 3 B. p. 223. t. IO. Berg. Ente, Bechst. N. D. IV. p. IOI6.

\section{DESCRIPTIO.}

Longit. I7 - I8 poll. Rostr. 2 poll. A flexura alz ad apicem $9 \frac{\pi}{2}$ poll. Cauda $2 \frac{\mathrm{T}}{2}$ poll.

Rostrum plumbeum magnum, postice altum, antice latum ungue incurvo. Iris fulva. Pedes coeruleo-cinerei membrana nigricante.

Mas: Caput, collum et pars pectoris nigra copite colloque viridi nitentibus. Dorsum anticum et scapulares alba nigro transversim tenuissime undulata. Peetus posticum et abdomen alba. Uropygium, crissum et tectrices caudæ fusco-nigra. Remiges primores nigricantes latere interiore cinereo; medix albæ apicibus nigricantibus; intimæ nigricantes juxta apices albo irroratæ. Tectrices alarum nigro - fusce lineis transversis undulatis albis. Cauda nigricans cinereo tincta.

Femina: Caput, collum et hypochondria rufo-fusca circa rostri basin fascia luteo-alba punetis nigris. Crissum, dorsum et cauda fusca. Cetera ut anteced.

Mas junior: Caput et collum nigra. Basis rostri pennis albidis cincta. Corpus supra fuscum albo tenuissime undulatum et punctatum. Pectus superius sordide rufescens, inferius cum abdomine album. Cauda æqualis fusea.

\section{H I S T OR I A.}

Estivat in Lapponiæ aquosis, ubi nidulatur, sed ovatio incognita est. Autumno et hyeme australiores 
Scandinavix oras visitat. Vescitur piscibus, insegtis et herbis aquaticis.

3) Speculo cinereo.

\section{ANAS FERINA, Limn。}

DiAGN: Capite et collo rufis; corpore undulato fascia pectorali, crisso et uropygio nigris.

\section{SYNONYMA.}

Anns ferina, Lin. Syst. Nat. I. p. 203. Lin. Fr. Sv. n. I27. Retz. Fn. p. 126. n. 83. Gmel. Syst. I. p. 530. Lath. II. p. 862. n. 77 .

Anas rufa, Lin. Faun. Sv. p. 47. n. 134. Gmel. Syst. I. 2. p. 5 I5. Lath. Ind. II. p. 863.

Anns Ferruginen, Retz. Fn. p. 136.

Ferruginous Duck, Pen. Brit. Zool. II. p. 60I. n. 285.

t. 99? Vide Obs, sub Anat. Penelop.

Canard Milonin, Buff. Ois. IX. p. 216. Pl. enl. 803.

Tem. Man, d'Orn. p. 564.

Pochard or Red-headed Wigeon, Pen. Brit. Zool. II. p. 600. n. 284. Lath. Syn. VI. p. 523.

Die Tafel-Ente, Bechst. N. D. IV. p. 1028. Meyer Liv. u. Esthl. p. 249. Meisner u. Schinz Vögel der Schw. p. 296.

\section{DESCRIPTIO.}

Longit. I6-I7 poll. Rostrum 2 poll. longum, cinereum, basi supra bipartita. Iris aurantia. Pedes plumbei.

Mas Senior:

Caput et collum rufa, dorso antice et pectore nigrofuscis. Dorsum, alnum teetrices minores et hypon 
chondria alba undulis tenuissimis et numerosissimis fuscis pulcherrime ornata. Abdomen albidum undulis obsoletis. Uropygium et crissum nigra. Remiges primores cinerex latere exteriore et apice fuscx; secun. daria cinerex apice albx. Speculum cinereum postice et inferne nigro cinctum.

Femina senior: Minor; Caput et collum ferruginea. Pectus.fuscum undulis rufescentibus. Crissum nigricans rufescenti tinctum.

Pullus: Caput et collum rufo-fusca. Corpus supra nigro-fuscum, subtus fuscum marginibus pennarum griseis.

\section{HISTORIA.}

Habitat in fluviis et lacubus Svecix septentrionalis. In Scania rarus obvenit. Nidulatur in juncetis. Ova 10. 12 virescenti -alba.

4) Speculo violaceo.

CCXXXVI. ANAS HISTRIONICA,

\section{Liwn.}

DIAGN: Rostrum parvum, compressum, ungue incurvo, Nares juxta basin approximatæ. Mas: Caput et collum violaceo-nigra fascia ad latera verticis ferruginen; maculis, linea collari et fascia pettorali albis. Femina: Supra fusca subrus albida fusco maculata, genis et macula pone oculos albidis.

\section{SYNONYMA.}

Anas histrionica, Lin. Syst. I. p. 304. 13, 35. Retz. Fn. Sv. p. 129. 11, 87. Brünn. Bor, p. 19, n. 84 . 
Gmel. Syst. I. p. 534, Lath. Ind, II. p. 849, Sparrm. Swensk Ornithol. (figura bona).

Canard ì Collier, ou Histrion, Temm. Man. p. 573. Buff. Ois, IX. p. 250. P1. enl. 798.

Harlequin Duck, Lath. Syn. VI. p. 485. Edw. to 99. Arct. Zool. II. p. 560.

Die Kragenente, Bechst. N. D. IV. p. 1037.

Straum Önd, Mohr. Isl. Nat. Hist. p. 24. t. I. Mas et fem.

Feminn:

Anas Minuta, Lin. Syst. I. p. 204. Brunn. Bor, n. 86. Lath. Ind. II. p. 849.

La Sarcelle brune et blanche, Pl. enl. 799.

Litle brown and zohite Duck, Edw. Av. to I5\%。

\section{DESCRIPTIO.}

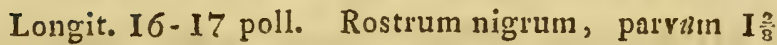
poll., compressum, fere anserirum, dentibus pectiniformibus juxta basin sub margine maxillæ superioris dependentibus, ungue incurvo. Pedes coeruleo-nigricantes.

Mns :

Genæ albx dimittunt striam utrinque ad latera fa. scix nigra, qux a fronte per verticem ad occiput longitudinaliter se extendit. Fascia ferruginea utrinque ad latera verticis. Aurium macula alba. Præterea latera capitis, gula et jugulum violaceo-nigra. Circa jugulum et latera colli fascia alba. Pectus, humeri et cervix saturate coerulei. Pectus, utrinque fascia se. milunari alba, nigro marginata cinctum. Dorsum fuscum; maculie in ala albx, speculo nigro-violaceo. 
Hypochondria foruginea. Tectrices caudx superiores nigrx. Crissum nigrum, macula utrinque alba. Cauda nigra acuminata.

Femina: Minor. Magnopere differt. Tota supra sarurate fusca, subtus albida, fusco maculata. Gula, frons, oculorum et aurium regio albida. Hypochondria rufa.

Varietas: Alba fusco hine inde maculata. E Groenlandia adductam olim mihi communicavit clar. Worm. skiold.

\section{HIS TORIA.}

Habitat hæc species in plagis terræ maxime borealibus, nec nisi rarissime et quidem hyeme oras nostras visitat. Nidulatur mense Junio. Ova 8 albida.

CCXXXVII. ANAS STELLERI, Pall.

Diagn: Mas: Albus, orbitis, gula, jugulo, collari et dorso nigris; macula nuchæ viridi, pec. rore rufo. Femina: Testacea fusco undulata; linea alarum duplici alba.

\section{SYNONYMA.}

Alas Stelleri, Pall. Spic. Zool. VI. p. 35. tab. 5. Retz. Fn. Sv. p. 133. 11. 92.

Anas dispar, Sparrm. Mus. Carls, fasc. I. to 7. (Mas) et t. 8. (fem.) Lath. Ind. II. 866.

Western) Duck, Pen. Arct. Zool. II. p. 564. t. 23. (Mas).

Stellers And, Swensk Zoologi. Tom. II. fasc. II. t. 62. 


\section{DESCRIPTIO.}

Magnitudo Anatis glacialis. Rostrum $\mathrm{I} \frac{6}{8}$ poll. A flexura alæ ad ejusdem apicem 9 poll. Cauda $3 \frac{r}{2}$ poll. Tibia $I \frac{\pi}{2}$ poll. Digit. med. $2 \frac{3}{3} ;$ Digit. postic. $\frac{\sigma}{8}$ poll.

Rostrum fere anserinum virescenti - nigrum lamellis pectiniformibus extus conspicuis. Pedes nigro-virides.

Mas: Caput album macula utrinque inter rostrum et oculum viridi; circa oculos macula nigra, macula nucha transversali virid: utrinque nigro terminata. Gula, jugulum et annulus circa collum inferius atra, hic fulgore viridi nitens. Dorsum nigrum. Pennæe scapulares longx, acuminatx, falcatæ latere exteriori atro-coeruleo nitentes, interiori alba. Tectrices om. nes alarum albx. Remiges primores et alula notha fuscx; secundarix fuscx apice albx; interiores latere interiori albx, exteriori atro-coerulex nitentes. Pectus et hypochondria alba sordide rufo-tincta; pectore in medio saturatiore, lineis transversis fuscis obsolete undulato; medium abdomen, crissum et cauda acuta nigro - fusca.

Femina: Fusca maculis et lineis transversis testaceis ubique adspersa. Abdomen, crissum et cauda nigrofusca, immaculata. Macula slarum coerulea, lineâ utrinque albâ.

\section{HISTORIA.}

Ter quaterse solumnodo hre species in Svecia, quantum scimus, observata fuit, seilicet in fuvio quodam Ostrogothix, in mari extra oras ejusdem provin. cix, et in oris insulæ Gottlandix. IEstatem in Asia maxime boreali degit; an quoque in Finnmarkia niduletur, nondum constat.

Nilsson Ornith. Pars 11 . 
Digitus posicus lolio membranaceo destitutus.

$$
\text { I) Speculo albo. }
$$

\section{ANAS STREPERA,}

$$
\text { Linn. }
$$

DiAGN: Rostrum aquale nigrum lamellis exseris. Pedes aurantii. Interscapulium, scapula et petus lineis arcuatis albis et nigris.

\section{SYNONYMA.}

Anas strepera, Lath. Ind. II. p. 859. 11. 69. Gmel. Syst. I. 2- p. 520.

Canard chipean ou ridenne, Temm. Man. p. 539. Buff. Ois. IX. p. 187. t. 12. (fem.) Pl. enl. 9.58. (Mas). Godwallor Grey, Lath. Syn. VI. p. 515. Pen. Brit. Zool. II. p. 603. n. 288. - Arct. Zool. II. p. 575. L.

Schnatterente, Bechst. N. D. IV. p. 1096. Meyer Taschenb. II. p. 533. Naum. Vögel Deutschl. III. B. p. 279. tab. 46. a. (Mas) tab. 45. fig. 65. (fem.)

\section{O BSER V.}

Linnei neque Faunam Svecia neigue Systema Natura heic citare potui. Utroque enim loco speculum ita delineatur, ut descriptio minime in hanc speciem, sed probius in An. Acutann quadret.

\section{DESC R IPTIŌ.}

Longit. I8 - I9 poll.

Rostrum nigrum, xquale, lamellis exsertis pectiniformibus. Iris brunnea. Pedes aurantii membrana natatoria nigra. 


\section{Mas senior:}

Vertex fuscus. Caput preterea et collum rufescen. tia maculis et punctis fuscis crebre adspersa. Gula albida immaculata. Pars inferior colli, superior pec. toris et dorsi nigro-fusca lineis semicircularibus albis pulcherrime adspersa. Dorsum posticum fuscum marginibus rufis. Hypochondria et femora fusca undulis crebris albidis. Abdomen album. Remiges primores fusco-cinerex, medix albæ (unde speculum album oritur), intimx longx, acutæ, cinerex apicibus pallidis. Tectrices proximæ nigrx, minores rufx. Cauda rotundara Rectricibus cinereis margine albis. Tectrices caudx superiores et inferiores nigræx.

Mus. Thunb.

Femina: Rostrum fuscum. Iris et pedes ut in Mare. Dorsum nigro-fuscum marginibus pennarum rufis. Peetus fusco-rufescens nigro-maculatum. Teetrices caudx superiores et inferiores griser.

\section{HIS T ORIA。}

Habitat in lacubus et paludibus, rarius tamen apud nos*). Nidificat in juncetis. Ova $8-9$ cinereo-viriscentia. Vescitur piscibus, insectis et plantis aquaticis.

\section{2) Speculo viridi.}

\section{ANAS ACUTA.}

DiaGN : Rostrum æquale et pedes cinerei. Speculum rufescenti, albo et nigro cinctum.

*) Vivan nonhum vidi; e Musøis descripsi. 
Mis: Caput et gula rufo-fusca, cervice linea utrine que alba in jugulum descendente. Dorsum lineis undulatis nigris er albis. Cauda acuminata, elongara.

Femina: Supra nigro-fusca striis undulatis rufescentibus vel albidis; subtus rufescenti-albida maculis fuscis.

\section{SINONYMA.}

Anas ncuta, Lin. Syst. 1. p. 202. Lin. Fn. 11. I26. Retz. F1. p. 123. n. 81. Gmel. Syst. I. 2. p. 528. n. 28. Lath. Ind. II, p. 864.

Anas Alandica, Sparrm. Mus. Carls. 3. t. 60. Retz Faun. p. I3I. ( $\mathrm{fem}$ ).

Anas Sparrmanni, Lath. Ind. II. p. 876. (fem).

Pintail, Pen. Brit. Zool. II. p. 598. Aret. Zool. II. p. 566. Lath. Syn. VI. p. 526. 11. 72. Vers. Germ. VI. 453.

Pilet ou Canard à longue queue, Buff. Ois. IX. p. 199. t. I3. Pl. enl. p. 954.

Spiess-Eute, Bechst. Nat. D. IV. p. III7. Meyer 1. c。 $25 \mathrm{I}$.

Scazis: Stjertand. Venator. Scan, Wi -anl.

\section{DESCRIPTIO.}

Longit. 2. ped. Rost. 2옹 poll. A flexura als sd apicem $10 \frac{\mathrm{T}}{2}$ poll. Cauda $7 \frac{\mathrm{r}}{2} \cdot 8$ poll. Tibia $I_{\frac{s}{8}}$. Digit. med. $2 \frac{2}{8}$ poll.

Rostrum longum, plumbeum, æquale; ungue ob. tuso dentibus pectiniformibus in medio rostri vix ex. sertis. Iris brunnea: Pedes cinerei. 
Caput et juguium rufo-fusca vertice maculis nigricantibus. Cervix nigra, ad cujus latus stria utrinque alba decurrit et cum collo antice albo se conjungit. Corpus subtus albam. Dorsum anterius, humeri, latera pectoris et hypochondria lineis transversis undu. latis nigris et albis. Tectrices candx superiores et scapulares longx, acuminatx nigre marginibus albis vel testaceo-albidis. Dorsum posticum fuscum undulis transversim linearibus albis. Crissum nigrum lateribus albis. Remiges primores griseo-fuscr, intimæ extus cinerex, intus nigrx. Speculum cupreo. viride antice rufescenti postice albo nigroque et $8 \mathrm{l}$ perne nigro-cinctum. Teetrices alarum superiores cinerex rufescenti tinctx. Rectrices dux intermedix nigrx longissimx angusta acuminatx, ceteræ cinerex marginibus albidis.

Femina: minor. Caput supra et cervix fusca marginibus pennarum rufescentibus. Collum sordide rufescenti-griseum maculis minutis fuscis. Dorsum, hy. pochondria et tectrices caudx superiores saturate fusca marginibus et lineis semilunaribus lutescenti - albis. Pectus et abdomen rufescenti - albida maculis fuscis. Remiges primores et intimæ fuscr. Speculum rufofuscum antice pallide rufescenti, postice nigra et alba linea marginatum. Cauda cuneata rectricibus acutis fuscis fasciis vel lineis undulatis pallide ferrugineis, marginibusque juxta apicem albis.

\section{O BS ERV.}

Quin huc pertineat Anas Almudica Spartm. 1. c. nullum dubium est. Exemplar enim, quod iconi ansam deưit, ipse examinavi. 


\section{HIS T OR IA.}

Habitat in septentrionslioris Scandinaviæ lacubus et fluviis. Raro in paludibus Scanix xstivat. Nidulatur in juncetis. Ova 8.9 coeruleo-virescentia. Hyeme abit. Mature redit.

\section{ANAS PENELOPE.}

DiagN: Pedes et rostrum parvum cinerea. Mas: Caput et collum rufa fronte lutescenti - albida. Speculum viride nigro marginatum. Dorsum nigro alboque tenuissime undulatum. Tectrices caudx inferiores nigrx. Femina: Caput et collum ferruginea nigro maculata; speculum cine. reum albo cinctum.

\section{SYNONYMA.}

Anas Pcnelope, Lin. Faun. Sv. p. 44. 1. I24. Retzii Faun. p. I23. n. 80. Syst. Nat. I. p. 202. 11. 27. Lath. Ind. II. p. 860. n. 7 I. Gmel. Syst. I. p. 527.

Anns fistularis, Briss. Orn. VI. p. 39I. no 21. to 35. f. 2 .

Canard Siffeur, Buff. Ois. IX. p. I69. t. IO. II. Vers. Germ. Otta XXXIII. p. 24I. cum tabula. Pl. enl. 825. (mas). Temm. Man. p. 542.

Wigeon, Pen. Br. Zool. II. p. GoI. n. 286. Arê. Zool. II. p. 574. R. Lath. Syn. VI. p. n。 63. Bechst. Nat. D. IV. p. 446. 11. 63.

Dic Pfeifente, Bechst. Nat. D. IV. p. II09. Meyer Vögel Liv. u. Esthl. p. 263. Huc pertinet: 
Anas ferruginea, Lindr. Mus. Grill. p. I3. n. 284. et forte

Anas rufa, Lin. Faun. Sv. 134. Cujus tamen icon inter pictas Rudbeckii aves jam desideratur. Diagnosis vero Linneana brevior est, quam ex qua quid certi concludatur.

I'cnntoribus Scnncns. Brunnacke.

\section{DESCRIPTIO.}

Longit. I8-I9 poll. Rostr. I $\frac{3}{8}$ poll. A flexurs alr ad apicem 10 poll. Cauda 3 poll. Tibix $1 \frac{5}{8}$, Digit. med. 2 poll. postic. $\frac{3}{8}$ poll.

Rostrum breve et tenue, depresso - semiteres basi altius, subangulatum, plumbeum, apice et subtus nigrum. Nares parve, ovales, marginatr. Iris fusca. Pedes cinereo-virides membrana nigricante.

Mas:

Caput et pars superior colli rufa maculis minutis viridi-nitentibus, Gula nigricans. Superior pars pectoris rufescenti-cinerea fusco maculata. Dorsum et scapulares undulis tenuissimis albis et fuscis ornata. Pars inferior pectoris et abdomen alba. Teetrices remigum secundariorum albx, unde macula magna alba in ala composita oritur, interdum cinerex marginibus albis; basis alre et tectrices rem. primorum saturate cinerex. Remiges primores cinereo-fusca, latere interiore pallidiores; secundarix nigre latere exteriore in medio cupreo colore nitente. Tertrices proxima albx apice nigrx, unde Speculum viride nitens nigro colore cingitur. Reniges intimx nigræx margine exteriore albo. Cauda acuta rectricibus cinereis albo marginatis. Tectrices candæ inferiores nigrx, superiores nigrtx albo marginatx.

Mus. Lund. 
Femina: Minor, : I6-I7 poll. long:

Rostrum et pedes sordide cinerea. Caput et collum pallide rufescentia maculis minutis rotundatis nigro. fuscis, præsertim superne confertis. Dorsum et scapulæ pennis fusco-nigricantibus rufo marginatis. Pectus superius rufun maculis transversis majoribus nigro fuscis. Ceterum pectus et abdomen alba. Crissum album maculis rufo-fuscis. Remiges cinereo-fuscæ. Tectrices alarum saturate cinerex in media ala fusco. maculacx. Speculum cinereum albo cinctum. Cauda acuta cinereo - fusca marginbus Rectricum albidis.

Mas Funior: Similis matri.

Mns mense Septcmbri: Dorsum tegitur pennis aliis e fusco et albo tenuissime undulatis, aliis nigro-fuscis stria transversa et marginibus rufis. Scapulares longa, superiores nigro-fusea stria simplici vel duplici et marginibus rufis, jnferiores cinerex albo tenuissime undulatæ. Pectus superne griseo-rufum maculis transversis nigris. Hypochondria rufa. Pectus et abdomen alba rufescenti tincta. Crissum album maculis nigris. Speculum viride.

\section{HISTORIA.}

Habitat xstate in aquis dulcibus regionum septentrio. nslium. Vere et autumno maria nostra turmatim visitat, ita ut mense Septembri inter omnes Anatum spe. cies in mari juxta littora Scanix maxime sit vulgaris. Vere in septenrrionale domicilium redit pars plurima. Manent in Scania nonnullæ et se propagant. Mense Aprili ova 9. alba, sordide virescentia in tumuli fossula juxta paludem s. aliam aquam ponit. 


\section{ANAS BOSCHAS.}

DiAGN: Rostrum luteo-viride. Pedes aurantii, Speculum violaceum utrinque fasciâ nigriâ a fascia alba distinctum. IVas: rectricibus intermediis recurvatis, capite et collo obscure viridinitentibus, collo inferne annulo albo cincto. Femina: supra nigro-fusca marginibus rufis et albidis; subtus rufescenti-alba, fusco maculata.

\section{SY NONYMA.}

Anas Boschas, Lin. Faun. Sv. p. 46. n. I3I. Retz. Faun. p. I31. n, 90. Lin. Syst. Nat. I. p. 205. n. 40. Gmel Syst. Nat. I. p. 538. Lath. Ind. II. p. 850, n. 49. Fabr. Fra. Groenl, p. 75. n. 47. Anas fera, Briss. Orn. VI. p. 3 I8. n. 4. Mallard or Wild Duck, Pen. Britt. Zool. p. 50I.n. 279. t. 97. Arct. Zool. II. p. 563. n. 494. Lath. Syn. VI. p. 489. n. 43. Vers. Germ. Bechst. VI. p. 424.

Canard sauvage, Buff. Ois. IX. p. II5. t. 7. 8.-Vers.

Germ, Otto XXXIII. p. 149. - Pl. enl. n. 776. (m:s) 777. (fem.) - Temm. Man. p. 538.

Gemeine Ente; Bechst. Nat. D. IV. p. I046. Stockente, Martzcnte, Meyer Vögel Liv. u. Esthl. p. 252.

Anas Platyriynchos, Lin. Faun. Sv. p. 42. n. I20. Retz. Faun. p. 189. 11. 76. Syst. Nat, XII. p. 200. (ut varietas Anatis Clypeate allata). Fauna Gottl. Billbergii. N. A\&t. Acad. Scient. 1809.

Anas fluviatilis ruffia, rostro subviridi, pedibus minia. ceis (fem.) Rudb, pict. fol. 23 I. 
Anas fluviatilis ruffa, rostro superius viresccnte inferins Ravescente, pedibus miniaceis, (mas.) Rudb. fol. 250. Est junior avis.

Svecis: Gräsand, Stockand.

\section{OBSERV.}

Hæ Rudbeckii icones ansam dederunt Annti Platyrhyncho in Fuun. Sv. quam Auctor postea in Systemate Nature edit. XIIma curn Anate clypeata conjunxit. Sed cum descriptio in Fauna Sv., tum icones jam allatre ostendunt aven ad Anatem Boscham pertinere.

\section{DESCR IP TIO.}

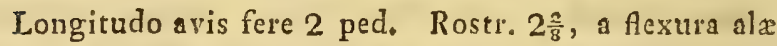
ad apicem $10 \frac{\mathrm{r}}{2}$ poll. Cauda $3 \frac{2}{8}$ poll. Tibi: $1 \frac{6}{8}$ poll. Digit. med. $2 \frac{1}{8}$; Digit. postic. $\frac{x}{2}$ poll.

Rostrum luteo-viride ungue parvo nigro, fere æquale, reetum antice depressum, parum latius, postice altius basi supra bipartita. Nares parver ovales. Iris brunnca. Pedes aurantii.

Mas: Caput et collum obscure viridi-nitentia *), fronte obscure castaneo, nucha rubro tincta. Collum inferne annulo albo cinctum. Superior pars pectoris castanea nitens. Ceterum pectus, abdomen et hypochondria alba undulis minutissimis et frequentissimis fuscis pul. cherrime ornata, presertim in hypochondriis conspicuis. Dorsum fuscum, antice rufescenti-griseo tinctum. Scapulares alhidre margine exteriore rufescen. res, undecunque undulis minutissimis fuscis confertis onatx. Tectrices alarum minores cinerex, fusco

*) Pro varia diredione luminis cupreo-viridi s, violaceo fulgore splendentia. 
inquinatx, majores apice albo, fascia nigra terminato. Remiges primores cinereo-fuscr; secundarix in medio extus violacer nitore viridi vel purpureo, pone apicem album fascia nigra; unde speculum violaceum viridi 1. purpureo nitens, superne fascia nigra, antice et postise extra fasciam nigram stria alba inclusum. Tec. trices caudx superiores et inferiores nigra. Cauda acuminata. Rectrices exteriores incana marginibus albis, medix binx, ternx 1. quaternx nigrx, recurvatæ.

Femina: Rostrum sordide olivaceum. Pedes aurantii,

Caput et collum supra nigro - fusca striis minutis angustis rufo-albis. Latera colli et capitis albida fusco longitudinaliter minute striata. Supra oculos linen albida et alia per oculos fusca. Gula alba luteo inqui21ata. Dorsum et scapulares nigro-fusca maiginibus pennartum rufis et albidis. Uropygium fuscum marginibus et fascia in medio pennarum albo-rufis. Pectus pallide rufescens maculis fuscis. Abdomen et crissum sordide alba fusco maculata. Tectrices alaruin minores et remiges primores cinereo-fuscr. Speculum violaceum purpureo et coeruleo nitens utrinque fascia alba extra fasciam nigram marginatum. Rectrices acuminatæ, rufescenti-albæ maculis irregularibus nigro - fuscis.

Variet. A) Anas Platyrhynchos Lin. Faun. Sv. n. I20. macula alari purpurea utrinque nigra albaque, pectore rufescente. Cet, descript. vide in Faun. 1. c. et icones (unde orta est) inter Rudbeckii aves pictas fol. 23I. et 250.

Voriet. B) Femina: Rostrum pallide olivaceum. Pedes luteo-rubri. Caput et collum suprn nigro - fusca striis albidis. Linea alba supra oculos et alia fusca per oculos. Dorsum et scapule nigro o fusca marginibus pen. 
narum latioribus albis 1. rufescenti-albis Uropygium pallide rufescens maculis nigro-fuscis rotundato-cordatis. Alæ ut in femina descripta. Latera capitis, collum et tota avis subtus alba, pectore et crisso levissime rufo tinctis. Rectrices albæ intermediis binis medio fuscis, marginibus albo variegatis. Tectrices superiores laterales albx.

Mus. Thųnb,

OBSERV.

Hæc est Anas Platyrhynchos Faunæ Gottlandice 1, c.

Mas habitu autumnali: Pileus niger viridi nitens. Genæ et gula nigra viridi-nitentia maculis albidis adspersa. Latera capitis, colli et jugulum sordide albida maculis minutis linearibus fuscis adspersa. Cervix fusca. Macula alba semilunaris circa collum. Dorsum fuscum. Scapula undulatæ. Collum inferne castaneum. Subtus præterea tenuissime undulata e fusco et albido. Caudx tectrices superiores et inferiores nigra. Cauda caret rectricibus recurvatis.

\section{H IS T OR I A.}

Habitat in omnibus fere Scandinaviz plagis et se propagat tum in Scania tum in Lapponia. Hieme cum gelu obteguntur lacus, scaturigines amnium visitat. Vescitur testaceis, piscibus, insectis, plantis aquaticis, cerealibus, \&c., Nidulatur in graminosis et juncetis, et interdum in arboribus juxta lacus et fluvios. Ova IO.I4 alba coeruleo-virescentia.

\section{ANAS TADORNA, Linn.}

DIAGN : Caput et pars colli atro-viridia. Corpus album fascia petoris et dorsi anterioris ferru- 
ginea. Scapulæ macula magna. Remiges et apex caudx corpusque subtus longitudinaliter nigra. Rostrum sanguineum. Pedes incarnati.

\section{SYNONYMA.}

Anas Tadorna, Lirn. Fn. Sv. II3. Retz. Fn. p. IIO. Gmel. Syst. I. p. 506. Lath. Ind. II. p. 854. n. 56. Lin. Itin. Scan. 2I2. Id, itin. Gottl, p. 2 I4. Tadorne, Buff. Ois. IX. p. 205. t. I4. P1. enl. 53 (Mas). Temm. Man. d'Orn. p. 536.

Shieldrake, Pen. Brit. Zool. II. p. 589. Lath. Syn, VI. p. 504. Id. Suppl. I. p. 275.

Brandente, Bechst. N. D. IV. p. 976.

Svecis: Grafgås, Brandgåk; Gottl. Jugås.

\section{DESCRIPTIO.}

Longit, avis 2 ped. I $\frac{T}{2}$ poll. Alæ extensæ 3 ped. 8 poll. Rostr. a sinu oris $2 \frac{\pi}{2}$ p., basi latum $\frac{x}{26}$ poll., pone apicem lat. $\frac{1}{x} \frac{5}{6}$ poll. A flexura alæ ad apicem $13^{\frac{r}{2}}$ poll. Cauda $4 \frac{\pi}{2}$ poll. Tibia $2 \frac{3}{8}$ poll. Digit. med. $2 \frac{5}{8}$, postic. $\frac{6}{8}$ poll.

Rostrum sanguineum, subrecurvatum, versus apicem latius, depressum, rotundatum, ungue nigro obtuso, incurvato; basi compressum, altius quam latius, superne carbuncula magna carnosa sangrinea, antice compressa ornatum. Nares ovales marginibus nigris. Maxilla superior lamelloso-dentata, dentibus pone medium infra marginem exsertis. Maxilla inferior apice truncata nigra lateribus lamellosis. Pedes pallide carnei femoribus supra genua nudis, unguibus fuscis. Digitus posticus compressus nulla tamen inembrana instructus. Cauda parum rotundata alas subxquans. 
Caput totum et colli pars superior atra viridi et vio. laceo nitentia. Pars inferior colli, dorsum, scapulares inferiores, uropygium, tectrices alarum super. et infer., latera pectoris et abdominis alba. Anteriora pec. toris et dorsi cingit fascia lata, tripollicaris, rufo.ferruginea. Scapulares superiore nigræ. Pectus et abdo. men in medio longitudinaliter nigra. Crissum pallide ferrugineo-fulvum. Remiges primores 10. nigræ, secundariz I I - 22 latere exteriore viridi-aurex, latere interiore nigræ, basi albx, sequentes $23-26$ latere exteriore saturate rufa stria juxta rachin nigra, interiore albæ, intimæ totæ albx. Tectrices proximæ rem. prim. nigræ et alula notha apice et latere exteriore nigra. Cauda alba rectricibus, exceptis extimis, apice nigris.

Femina: minor, caret carunculâ rostri. Caput et superior pars colli nigrescentia maculis albis in genis et in fronte. Frscia ferruginea circa pectus et interscapulium angustior, superne fusco irrorata. Nigredo abdominis interrupta et angusta.

Funior: Rostri apice fusco, præterea matri subsimilis.

\section{HISTORIA.}

Habitat xstate secundum litus marinum a Scania fere usque ad circulum polarem. Frequentius tamen in Scania, ubi advenit juxta finem mensis Aprilis et initio m. Maji, et unde abit mense Augusti Ova IO-I2 alba plerumque ponit in cuniculis horizontalibus, quos in arena fodit. Vescitur piscibus, testaceis, plantis marinis, Cicurata cerealibus delectatur. Caro insipida. 


\section{ANAS CLYPEATA, Linn。}

DIAGN : Rostrum longum spathulatum. Speculum nigro-viride. Tectrices alarum cyanex. Pe。 des aurantii.

\section{SYNONYMA}

Anns Clypeata, Lin. Fn. Sv. p. 42. n. I 19. Syst. Nat. I. p. 200. Retz. Fn. p. I18. n. 75. Gmel. Syst. I. 2. p. 518. Lath. Ird. II. p. 856. n. 60.

Anas rubens, Gmel. Syst. I. p. 519. Lath. 1. c. p. 857. Canard Souchet ou le Ronge, Buff. Ois. IX. p. 19I. P1. enl. 97 I. et 972. Tem. Man. d'Orn. p. 543.

Shovler, Penn. Britt. Zool. II. p. 596. Arct. Zool. II.

p. 557. Lath. Syn. VI. p. 50y.

Löffelente, Bechst. Nat. D. IV. p. IIOI. Meyer Liv. u. Esthl. p. 254. Meisner. u. Sch, Vög. der Schweitz. p. 302.

Venatoribus Scanensibus: Leffeland.

\section{DESCRIPTIO.}

Mas:

Longit. 18 poll. Alæ extensi $2 \frac{\gamma}{2}$ ped. Rostr. $2 \frac{1}{3}$ poll. A flexura ala ad apicem 9 poll. Tibia $I \frac{3}{8}$ poll. Digit. med. 2 poll.

Rostrum magnum spathulatum, extus dilatatem, apice rotundato, ungue ovato incurvo; supra nigrum margine dentibus a basi ultra medium longis exsertis, pectiniformibus ciliato; subtus subluteum. Pedes aurantii. Iris fulva.

Caput et superior pars colli atro-viridia, nitentia. Inferior pars colli et superior pectoris alba. Dorsum 
nigro fuscum. Pennæ scepulares longæ, laxæ, nigrre, extus albæ. Tedrices alarum minores pallide coerulex. Speculum saturate viride, albo cinctum. Abdo. men et hypochondria fusco-rufa.

Femina: Long. I7 poll.

Rostrum supra nigro - virescens, subtus pallide rubrum. Pedes aurantii membrana natatoria nigra. Caput supra, dorsum et scapulares nigro - fusca marginibus pennarum pallide rufescentibus. Caput præterea et collum pallide rufa striis et maculis minutis fuscis sparsa. Pectus et tota avis subtus pallide rufa maculis nigricantibus. Tectrices alarum pallide coerulei. Speculum saturate viride striâ antice alba.

\section{HIS T ORIA.}

Habitat passim in paludibus et lacubus Svecix meo ridionalioris ex. gr. in Scania, Gottlandia \&c. Vescitur piscibus, vermibus, plantis et insectis aquaticis. Nidulatur in arundinetis et juncetis juxta lacus et paludes. Ova 7 - 14. luteo-viridia.

\section{ANAS QUERQUEDULA,}

\section{Linn.}

Diagn: Rostrum depresso-subdilatatum dentibus exsertis ungue ovato. Speculum parvum viridifuscum, parum nitens, fascia utrinque alba cinfum.

Was: Latera capitis et collum rufa, albido striata. Gula nigra. Teetrices alarum pallide cinerex. Scapulares falcata. 
Femina: Fascia albida supra et infra oculos, fuscaque per oculos. Genæ et collum alba fusco striata. Tectrices alarum griseo-fuscæ.

\section{SYNONYMA.}

Anas Querquedula, Lin. Fin. Sv. p. 45. n. I28. Syst. Nat. I. p. 203. 11. 32. Retz. Fn. p. I27. 11, 84. Lath. Ind, II. p. 872. 11. 99. Gmel. Syst. t. 2. p. 531.

Cnnard Sarcelle d'ète, Temm. Man. p. 54.5. Buff. Ois. IX. p. 260. et 268. Pl. enl. 946. (Mas).

Gargancy, Pen. Brit. Zool. II. p. 604. n. 289. t. IOI.

(Mas et fem) Arct. Zool. Il. p. 576. O. Lath. Syn. VI. p. 550. 11. 87.

Die Kräckente, Bechst. N. D. IV. p. II35. Mey. Liv. u. Esthl. p. 255. Meisner u. Schinz die Vögel der Schweitz p. 303.

Venatoribus Scanens.: Kräckand, Årta.

\section{OBSERV.}

Qurnam esset avis Anas Circia Lin. inter Ornithologos sspe dissensio fuit, donec nuper fere inter omnes convenit, eandem esse Marem seniorem Anatis Querquedula. Quibus vero rationibus in hanc opinionem incidere potuerint, ego sane videre non possum. Speculi cnim color, qui in hoc genere nota maxime characteristica est, primo quoque adspectu, longe aliam indicas speciem. Neque enim $A$. querquedula habet: "maculam alarum coeruleam supra infraque nigram, ante et post album" ut Anas Circia Fauna Suec. neque "Speculum nigro virens supra infraque nigrum, margine antico posticoque album" ut. A. Circia Syst. Nat. Quænam igitur sint Linneanx aves curâ eruere conabor. Imo In folio 224. operis Rudbeckiani cxstat Figura, qua sine ullo dubio et exsistentiam ct nonen dedit Anati Circic Lin. Syst. Nat. p. 204. n. 34. - Synonyma a Rudbeckio ipso in schedula allata Nilsson Ornith. Pars II. 
hec sunt: "Atias Circin Gesn. p. 106. Aldr. L. 19. p. 209. Will. p. 291. Ray. p. 148." - Eandem exacte Synonymiam in Syst. Nat. 1. c. invenis. Hac vero icon Rudbeckiann egregie reprasentat Marem junioren Anatis Crecce, exale talem qualem infra describam. 2do Anas Circia Lin. Nn. n. 1;0. eadem est ac Rudbeckii Anas fusca media, rosiro et pedibus cinereis, que in fol. 242. Operis citati depicta est, et sine dubio Linneus nullibi vidit guem nisi in hoc opcte. - Rudbeckins ipse eam ab $A$. Circia (fol. 224) distinctan esse suspicatur, quare in schedula juxta posita interrogat: An Anas Circia major, vel etiame Mas? Hanc iconem ita olim descripsi: Rostrum breve cinereum apice nigricante. Caput et collum sordide rufescentia. Dorsum nigricans marginihus pennarum obsolete rufescentibus. Corpus subtus album. Cauda et Remiges saturate fusca. Teetrices alarum minores cincreo-fusca marginibus pallidis. Speculum viride antice et postice fascia obliqua nigra, extra quas linex albæ. Quin avis heic descripta sit Anas Circia Lin. Fn. Sv. nullum omnino et dubium. Quænam vero sit species, jam absolute determinare non possum. Ad Querquedulam sane non pertinet. Nonne potius Más junior Atzatis Peizelopes?

\section{DESCRIPTIO.}

Longit. I 5 poll. Rostrum is poll. A flexura alx ad apicem $7 \frac{\pi}{2}$ poll. Cauda $2 \frac{\pi}{2}$ poll. Tibia $I_{\frac{3}{8}} \mathrm{p}$. Di. git. med. $I \frac{5}{8}$ p. postic. $\frac{T}{2}$ poll.

Rostrum nigricans, basi coarctatum, antice dep:essum, subdilatatum ungue nigro ovato, obtuso, dentibus peetiniformibus pone medium exsertis*). Ocu. li sub vertice siti. Iris pallide brumnea. Pedes cine. rei.

Pileus et nucha nigra rufescenti (interdum) immixto striis frontis albidis. Fascia alba supra oculos utrun.

* Jac rostri formâ in omnibus anni tempestatibus optime dignoscitui ab An. Crecca. 
que ad latera cervicis ducta. Gula nîgrı. Caput præxterea et collum fusco-rufa striis minutis albis crebre sparsa. Inferior pars colli et pectus pallide ferruginea lineis semicircularibus nigro-fuscis. Dorsum fuscum marginibus pennarum griseis. Scapulares longæ, accuminatx, deorsum folcatæ, atro-virides, stria juxta rachin alba. Tectrices alarum pallide coerulescenti-cinerer, majores apice latius albæ. Speculum cinereo. virite fascia alba antice et postice cinctum. Abdomen album rufescenti tinctum. Hypochondria alba lineis undulatis nigris. Crissum lineis transversis et teetrices caudx inferiores maculis fuscis. Mas senior.

Femina: Minor. Rostrum et pedes grisea, Iris fusca. Pileus nigro fuscus rufo immixto. Fascia albida supra et infra oculos aliaque fusca per oculos, prrsertim po. stice conspicus. Ge:x et latera colli albida striis minutis fuscis sparsa. Gula alba. Dorsum et scapulares nigro-fusca marginibus pennarum rufescentibus et al. bidis. Pectus superius et hypochondria sordide sibs rufescenti tinda maculis fuscis. Ceterum subtus sor. dide alba, crisso maculis fuscis. Tectrices alarum griseo-fuscr, quarum proximæ, ut etiam remiges secundarix apice albæ sunt, unde speculum viridi-fuscum, nitore destitutum utring̨ue stria alba cingitur.

Mas junior: Feminæ seniori subsimilis differt magnitudine aliquantum majori fascia albida supra oculos ver. sus nucham ducta; tectricibus alarum fusco-einereis.

\section{H I S T OR I A.}

Estivat in paludibus, amnibus et lacubus, præsertim ubi ripæ graminose sunt vel juncetis cinctr. In Scania vulgaris. Vescitur plantis aquaticis, testaceis et insectis. Mense Novembri abit, vere redeunte redit. Ova 10-12 lutescenti-alha. 


\section{ANAS CRECCA, Lin.}

DIAGN: Rostrum angustum semireres, dentibus infra marginem vix conspicuis, ungue sublineari. Speculum magnum viride superne et inferne nigro, antice et postice albo cinctum.

\section{SYNONYMA.}

Anas Crecca, Lin. Fn. Sv. p. 46. n. 129. Retz. Fn. p. 127. 11. 85. Gmel. Syst. I. 2. p. 532. Lath. Ind. II. p. 872 .

La petite Sarcelle, Buff. Ois. IX. p. 265. t. I7 et I8. Pl. enl. 947. (mas).

Common Teal, Pen. Brit. Zool. II. p. 606. n. 290. Arê. Zool. II. p. 577. Lath. Syn. VI. p. 55 I.

Canard Sarcelle d'hiver, Temm. Man. p. 547. Krieckente, Bechst. N. D. IV. p. II 43 Meisn. et Schinz

Vög. Schw. p. 304. Meyer Liv. u. Esthl. p. 256. Svecis: Krickand.

\section{DESCRIPTIO.}

Longit. I4-I5 poll. Rostrum $I \frac{\pi}{2}$ poll. A flexure alx ad apicem 7 poll. Tibia $I \frac{2}{3}$; Digit. med. I $\frac{5}{3}$ p. postic. $\frac{3}{8}$ poll.

Rostrum nigrum, angustum, æquale, semiteres, ungue angusto lanceolato, dentibus infra marginem maxille haud conspicuis. Iris brunnea. Pedes cinerei membrana nigricante.

Caput et collum rufa. Gula nigra. Fascia lata atroviridis utrinque per oculos ad latera nucha ducta in cervice jungitur. Pars inferior colli, dorsum, scapulares intimx et hypochondria lineis undulatis albis et nigris ornata. Peetus rufescenti-album maculis parvis 
rotundatis (pisiformibus) fuscis. $\Lambda$ bdomen album, tec. trices alarum cinereo-fuscr, proximr apicibus late albis, vel rufo-albis. Remiges primores fusca. Speculun magnuın viride nitens cingitur superne fascia longitudinali inferne macula nigra, antice fascia et postice stria alba. Tectrices caudx inferiores nigra macula utrinque magna rufescente; superiores nigra marginibus pennarum albidis. Cauda acuminata. Mas senior.

Femina: Minor. Rostrum nigricans subtus rubro nebuIosum. Caput et corpus supra nigro - fusca marginibus pennarum rufis. Gula alba Corpus subtus album. Speculum dimidiato viride et nigrum, antice et postice stria alba.

Mas junior ante primam pennarum mutationem:

Feminæ subsimilis. Caput supra, dorsum et scapulares saturate fusca marginibus pennarum rufis. La. tera capitis et collum antice pallida maculis minutis fuscis sparsa. Palpebra inferior alba. Gula albida immaculata. Pectus superius albidum rufo tinctum maculis latis nigris ornatum. Ceterum pectus et aldo. men alba. Crissum rufescenti-album maculis nigris. Alæ exacte ut in mare seniori.

\section{OBSERV.}

Jam mense Augusto et Septembri incipiunt pennx ex albo et nigro variegase inter scapulares erumpere.

\section{HIS T ORIA.}

Habitat in amnibus et lacubus, in superiore Svecia magis quam in Scnnia vulgaris. Ova 12. rufescenti. alba, obsolete fusco maculata. Victus ut praced. 
GENUS.

\section{A N S E R.}

Rostram breve, conico - cylindricam basi altius quam Jatum, versus apicem sensim angustius, apice rotundato, maxilla utraque dentibus conicis in. strueta.

Nares oblongo - ovales, in medio rostro siræ.

$P_{e d e s}$ crassiusculi; Tibia cum digito medio subrqualis.

Digitus posticus membrana lobiformi destitutus. Collum longitudine mediocri.

Dorsum convexum.

CCXLVI. ANSER CINEREUS, Mey.

Diagn: Griseus, subrus albidus. Dorsum antice fuscum pennis griseo marginatis, postice cinereum. Rostrum aurantium ungue albo. Pedes pallide carnei. Alæ complicatæ caudâ breviores.

\section{SYNONYMA:}

Anser Cinereus, Mey. Liv. u. Esthl. p. 257. Bechst. Nat. D. IV. p. 842 .

Anas Anser ferus, Lath. Ind. II. p. 841. n. 26. Temm. Man. d'Ornith. p. 526. 
Grey Leg Goose, Pan. Brit. Zool. II. p. 570. n. 266. (Descr. bona) Lath. Syn. VI. p. 459. n. 2 I.

\section{OBSERV.}

Hanc Auscris speciem non novit linné. Avis enimquam sub nomine Auttis Auseris in Fauna Svecica descripsit, er quam piæsertim in Lapponia habitantem refert, minime ad hanc sed ad inscquentem Speciem pertinet. Hæc vera Anseris domestici stirps mihi et forte Fauna nostre diu adhuc plane incognita fuisset, nisi specimina et historiam ejus benigne mihi communicasser Excellentiss. Comes Trolle Wachtaeistea, cujus ingenio et favori erga me et studium Ornitholog. piurima deber Omichologia Parriæ.

\section{DESCR I P TIO.}

Longit. 2 ped. $9-10$ poll. Rostrum $2 \frac{T}{2}$ poll. A flexura alæ ad apicem 16 poll. Cauda 6 poil. Tilia $3 \frac{\frac{2}{3}}{3}$ poll. Digit. med. $3 \frac{x}{2}$ poll. postic. $1 \frac{1}{5}$ poll.

Rostrum totum luteo-aurantium, ungue albescente, robustius, pone apicem altius (minus depressum) ungueque majore, convexiori quam in sequenti Specie. Margines palpebrarum lutei. Iris saturate fusca. Pedes carneo-lutei. Alx compositie apicem cande non adtingunt.

Caput et collum supra griseo-fusca, marginibus pennarum pallide grisescentibus. Dorsum anticum, scapulares et tectrices alarum majores et medi:e fusca, marginibus pennarum tenuibus griseo-albescentibus. Tectrices minimx et margo alarun exterior pallide cinerea. Remiges basi pallide cinerex apice nigra. Dorsum mediun et uropygiun cinereum. Collum antice et pectus pallide grisea undulis obioletis fuscescentibus. Abdomen, crissum et tectrices coudæ inferiores pure alba. Interdum tamen sunt pectus et ab. domen maculata pennis hinc inde nigro-fuscis. Hy- 
pochondria cinereo-fusca griseo-albido marginata. Latera uropygii et te?trices caudæ superiores proximæalba. Rectrices cinereo - fuscr apice et marginibus albis, qua albedo ita augetur, ut laterales pennze totr sint albx.

\section{HIS T OR I A.}

Migratoria hxc avis juxta finem mensis Aprilis ad oras australiores Scanix et Blekingix, minus tamen frequens, advenit. Heic in paludibus a mari haud longe remotis nuptias celebrat, (ex gr. juxta Ahus Sconire, juxta Ysanne Blekingix etc.). Coitus tempore peracto mares feminas deserunt, et in mare adjacens abeunt, ubi extra oras scopulosas gregatim obveniunt. Femina interim remanens ova ponit 5.6 sordide virescentia. Medio mensis Junii matres pullos in mare conducunt, ubi postea gregatim simul eum maribus occurrunt usque ad initium mensis Augusti. Post hoc vero tempus oris nostris valedicunt plagas meridionaliores petentes.

CCXLVII. ANSER SEGETUM, Bechst.

Diagn: Caput et collum saturate fusca. Dorsum antice fuscum pennis griseo marginatis, postice nigricans, Rostrum nigrum fascia ante medium aurantia. Pedes aurantii, ale complicatæ caudam excedentes.

\section{SYNONYMA.}

Anser Segetum, Bechst. N. D. IV. p. 882. Meyer Liv. u. Esthl. p. 258.

Anas Segetum, Gmel. Syst. I. 2. p. 5 I2. Lath. Ind. II. g. 842, n. 28. Temm. Man. d’Oril. p. 527. 
Anas Anser, Lin. Fn. Sv. p. 40. 11. I14. Syst. Nat. I. p. 197.

Benn Goose, Pen, Brit. Zool. II. p. 575. n. 267. tab. 94. Lath Syn. VI. p. 464. Pen. Arct. Zool, II. p. 546. n. 472 .

Svecis: Wildgảs, Grågås.

\section{DESCR IP T'IO.}

Longit. 2 ped. $6-7$ poll. Alx extens 5 ped. Ro. str. $2 \frac{3}{8}$ poll. A flexura ala ad apicem 18 poll. Cauda 6 poll. Tibia $3 \frac{x}{2}$ poll. Digit, med. 3 poll. postic. $\frac{7}{\varepsilon}$ poll.

Rostrum (minus quam pracedentis, robustum et pone apicem magis depressum) basi et apice nigrum, fasciâ ante nares aurantiâ, in mandibula inferiore angustiore et minus perspicua. Hxc fascia interdum usque pone nares juxta margines maxillæ superioris producitur. Pedes aurantii unguibus nigro - coerulescentibus. Margines palpebrarum griseo.nigricantes. Iris saturate fusca. Alx complicatx apicem caudx excedunt.

Caput totum et collum supra saturate griseo - fuscum unicolor; Collum antice et versus pectus magis in ci. nereum inclinans, rufescenti interdum tinctum. Dorsum anticum, scapulares et tectrices alarum cinereo. fusca marginibus pennarum rufescentibus vel albidiss Dorsum medium et uropygiun nigricans, unicolor. Remiges intimx cum teetricibus nigro-fusce marginibus et apicibus albis; primores et medir nigra rachibus albis; extimx 3.5 cum tectricibus cinereo tinetr. Pectus et hypochondria pallide rufo-grisea marginibus sordide albidis 1. rufescentibus. Abulomen, crissun et tectrices caudre inferiores alba. Latera uropygii et tectrices caudæ superiores proxinn albr. Rectrices cinereo-fuscre, apice et marginibus apicis albr. 
Avis Funior: Maculis 2.3 albis supra basin rostri sxpissime notatur. "Margine frontis albo" Lin. Fn. Margines pennarum in hac magis rufescentes, in seniore magis albida.

\section{H I S T O R I A.}

E suo genere maxime vulgaris apud nos Species. Plagas tamen Svecix australiores vere tantum et autumno in maximis plerumque gregibus adsocista transmigrat, neque apud nos unquam xstatem degit vel se propagat. Habitat vero xstate in regionibus peninsulæ nostræ maxime horęalibus, scil. in insulis juxta oras Nordlan. dix et Finmmarckix sitis, tanque in his plagis copiosa est hac agris consitis nociva avis, ut inter agriculturæ incommoda haud contemnenda illam non sine caussa numerent incolæ. Vere enim rediens agros consitos sxpe devastat; autumno migrans segetem maturescentem adit, et per totam xstatem, mares presertim, agros in continenti terra haud longe a mari remotos frequentat. Nidulatur heic in insulis, nunquain in continenti terra, (quia ut incolx putant, Vulpen timet). Ova ponit 10-12 alba, immaculats.

\section{ANSER LEUCOPSIS, Bechst.}

Diagn: Frons, genæ, tempora et gula alba, Collum nigrum. Corpus supra canum maculis nigricantibus, subtus album. Rostrum et pedes nigra.

\section{S YNON YMA.}

Anser leacopsis, Bechst. N. D. IV. p. $92 \mathrm{I}$.

Anas leucopsis, Temm. Man. d'Oṛith. D. 530. 
Aias Erythropus Mas, Lin. Fn. Sv. p. 4I. n. II G. Retz.

Faun. Sv. p. Il 6. n, 72. Lath. Ind. II. p. 843.

(Obs. At Anas Erythropus Lin. Syst. I. p. 197. n.

II. ad insequentem Speciem pertinet).

Anas Canadensis, Retz. Fn. Sv. p. 277. n. 270.

The Bernacle, Pen. Brit. Zool. II. p. 577. 11. 269. Arc?.

Zool. II. p. 552. n. 479. Lath. Syn. VI. p. 466.

Die Mittlere Kaszarka, Lepechin Reise 3 B. p. 22 I. tab. 9.

\section{DES C R IP TIO.}

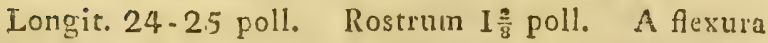
alra ad apicem 14 poll. Cauda 4 poll. Tibia $2 \frac{r}{2}$ p. Dig. med. $2 \frac{2}{8}$ poll.; postic. $\frac{x}{2}$ poll.

Fenine: maribus aliquantum minores sunt.

Rostrun parvum nigrum. Iris nigro-fusca. Pedes nigri.

Frons, latera capitis et gula alba. Stria nigra inter rostrum et oculos. Vertex, occiput et collum nigra. Dorsum et alx supra pallide coerulescenti - cineren (cana) apicibus pennarum nigricantibus, albido plerumque marginatis. Uropygium et cauda nigra. Remiges cinerex apicibus nigris. Pectus, alıdomen, crissum et tectrices caudx inferiores alba immaculata.

funior avis: Pedes nigro-fusci. Frons et genx nigro punctatr. Pennæ et alarum rufescenti - marginatx.

\section{HISTORIA.}

Fistivant in paludibus et lacubus Lapponix. Autumno merjdiem petentes, in magnis gregibus sociati interdum in litoribus Scaniax occurrunt. Haud timidi sunt, illisque quam congeneribus facilius insidiantu: venatores. Oeconomia eorum nondum ben: in patria boreali explorata est. 
CCXLIX. ANSER ALBIFRONS, Bechst.

Diagn: Frons alba. Collum cinereo - fuscum. Corpus supra fuscum pennis griseo marginatis, subtus albidum, pectore et abdomine pennis (rotis) nigris adspersis. Rostrum pallide incarnatun ungue albido. Pedes crocei.

\section{SYNON YMA.}

Aisser nlbifrons, Bechst. N. D. IV. p. 898. Meyer Liv. u. Esthl. p. 259.

Anas albifronts, Gmel. Syst. I. 2. p. 509. Lath. Ind. II.

p. 842. 11. 27. Retz. Tn. p. II6. n. 7 I. Brünn, Bor. n. 54.

Anas Erythropus femina, Lin. Fn. Sv. p. 4I. n. I I 6. L'oie ricuse, Buff. Ois. IX. p. 8 I. Otto XXXIII. p. 22. cuin fig. Tem. Man. p. 529.

Laughing Goose, Edw. Av. t. I53.

White - fronted Goose, Pen. Br. Zool. II. p. 576. n. 268. Arct. Zool. II. p. 548. 11. 476. Lath. Syn. VI. p. 463. 11. 22.

\section{DESCRIPTIO.}

Senior: Longit. 27.28 poll. Alr extensre 4 ped. 9-I2 poll. Rostrum II $\frac{x}{2}$. A flexura alx ad apicem I7 p. Cauda 5 poli. Tibia $2 \frac{6}{8}$ poll. Digit. med. $2 \frac{6}{8}$ poll.

Rostrum pallide incarnatum *) ungue albo. Margines palpebrarum et Iris fusca. Pedes crocei unguibus albidis.

*) Rostrum aurantium (quale ab Ornithologis describitur) in seniozi ave sung̨uam observavi. 
Frons alba, linea nigra cincta; prrterea caput et collum strintum cinereo-fusca, rufo tincta, summis pennarum apicibus pallidis. Dorsum, scapulares et majores alarum tectrices fusca, apicibus griseo-rufescenti marginatis. Dorsum posticum nigricans. Collum antice et pectus pallide grisea, pennis albido terminatiș. Abdomen medium, crissum et teĉrices caudx alba. Pectus et abdomen pennas habet plures paucioresve nigras, inspersas. (Unde macula majores transversales nigras has partes ornant). Remiges primores cinerex, apice nigræx, secundariæ nigræx, basi tantum cinerex, juxta apicem alho marginatx. Teetricesalnum minores, item ac proximx Rem. primorum cinerea, his apice albis, Cauda cinereo fusca, apice alba.

\section{O BSERV.}

Verosimillina est opinio Celeb. Temminuckii, hanc speciem per æstatem pectore et abdomine plane nigris ornatam esse. Habeo specimen quoddam, ab Excell. Comite Trolle Wachtmeister verno tempore missum, cujus macula peetorales et abdominalesnigra tain magnæx sunt, ut hinc inde confluant et fascias fere transversales forment, spatiumque haram partium longe maximam occupent.

\section{H IS TOR I A}

hujus avis nondum bene cognita est. Fistivat in Asiz et Europæ maxime borealis tesquis uliginosis et lacubus. Vere et autumno Scaniam transmigrat, et in mari paludibusque mari contiguis præsertim mens. Septembri et Octobri gregaria obvenit.

\section{O BSER V.}

In Miseo Academico Lundensi servatur specimen Anseris cujusdam Scandinavici, de quo mihi adhuc dubium est, an illad pro juniori Ansere albifronte habeam, an ut distinatam speciem describam. Est vero hæe ejus Descriptio: 


\section{DESCRIPTIO.}

Longit, 2 ped. Rostrum I $\frac{5}{8}$ poll. A fiexura ala ad apicem 15 poll. Cauda $4 \frac{6}{8}$ poll. Tibia $2 \frac{7}{2}$ poll. Digit, med. $2 \frac{6}{8}$ poll. posticus $\frac{5}{3}$ poll. Alre complicatæ caudâ breviores sunt.

Rostrum aurartium ungue et marginibus fusco-nigtis, Pedes crocei, membrana natatoria obscuriore.

Caput et colium fnsco-cinerea, rufescenti leviter tincta; vertice, fronte et genis saturatius fuscis. Mentuin fuscum, macula parva alba. Interscapuliuın, penne scapulares et tectrices alarum medix fusca marginibus pennarum griseo.rufescentibus, Dorsum posticum nigrum, Remiges primores cum te?tricibus cinerex, ąice nigræ; secundariæ nig!æ albo tenuissime marginate. Tcet)ices proximr pallide cinerez, apice albæ. Tęrices minores saturatius cinereæ. Pe@tus album, maculis parvis fere pisiformibus (sed marginibus irregularibus) fuscis adspersum. Abdomen, crissum et teetrices caudx inferiores superioresque alba, immaculata, Hypochondria fusca, pennis griseo-rufescenti marginatis. Femura cinereo-fusca, Cauda fusca, rectricibus apice et marginibus albis.

Si distincta esset species, his charndteribus a ceteris dignosceretur:

DraGN: Caput et collum rufescenti-grisea, vertice, fronte et genis saturatioribus. Corpus supra fuscum, marginibus pennarun griseis, subtus album; pectore maculis pisiformibus adsperso. Rostrum aurantium, ungue et marginibus fuscis. Pcdes crocei.

Specimen descriptum, juxta litus marinum, mense Octobri, ad Arup Scaniæ, sciopeto occisum fuit et ab Excellentiss. Comite Trolle Wachtrateister mecum benignissime communicatum.

Distinetam Anseris specien primo illam esse putavi; sed post accuratissimam ejus, quantum fieri potuit, cum 
Ansere Albifronte comparationem, non nisi pullum hujus speciei, ance primam pennarum mutationem, esse suspicor; præsertim cum venator mihi narravit se avem inter tres vel quatuor Anseres albifrontes fixisse. E pelle tamen siccata, calvariaque orbata, nihil certi concludere audeo; comparationem enim partium solidarun non nisi incompletam instituere potui. Quare determinationem magis absolutam in postermm differre, quam Systema novo nomine temere onerare, satius duxi.

\section{ANSER TORQATUS, Bechst.}

DIAGN : Caput et collum nigra, maculâ transversali alba utrinque ad latera colli. Corpus cinereofuscum crisso et tectricibus caudæ albis.

\section{SYNONYMA}

Anser Torquatus, Bechst. N. D. IV. p. 9 I I. Meyer Liv. u. Esthl. p. 260.

Anas Bernicla, Lin. Fn. p. 40. 1. 11 5. Retz. Fn. p. II7. n. 73. Lin. Syst. I. p. 198. n. I3. Lath. Ind, II. p. 844. Gmel. Syst. I. 2. p. 513.

The Brent Goose, Pcn. Brit. Zool. II. p. 579. n. 270. Aret. Zool. II. p. 55 I. n. 478. Lath. Syn. VI. p. 467. 12. 27.

I6 Cravant, Buff. Ois. IX. p. 87. Otto XXXIII. p. 53. cum fig.

Oic Cravant, Temm. Man. d'Ornith. p. 53 I.

Svecis: Prutgås; Blckingis: Taflacka.

\section{DESCRIPTIO.}

Longit. 22 poll. -2 ped. Rostrum I $\frac{1}{2}$ poll. A flexura alx ad apicem II poll. Cauda 4 poll. Tibia $2 \frac{r}{2}$ poll. Digit, med. $2 \frac{6}{5}$ poll. postic. $\frac{T}{2}$ poll. 
Rostrum nigrum, parvum (majus tamen quam Anseris Lencopsis) ungue rotundato, fere semigloboso. $\mathrm{Pe}$. des rufo-nigri.

Caput totum et collum usque ad sternum nigra. In latcribus colli macula utrinque transversalis alba, interdum antice juncta, unde torques oritur. Dorsum, sca. pula et tectrices alarum fusco-grisea, pennis rufescenti marginatis. Pectus, hypochondria et abdomen saturate cinerea albido marginatis. Uropygium medium cinereo-fuscum. Crissum, latera uropygii et tectrices caudx alba. Remiges nigræ, secundaria apice albr. Rectrices nigræ lateralibus apice albis.

\section{HIST OR IA.}

Habitat ærtate in paludibus et lacubus regionis arcticx. Vere et autumno plagas Scandinavix meridiona. liores transmigrans in oris Scanire et præsertim Blekingix gregatim et copiose occurrit. Caro sapida zstimatur.

\section{ANSER RUFICOLLIS, Pall.}

Diagn: Collum rufum capite, cervice, dorso et pectore nigris. Macula inter rostrum et oculum alba aliaque rufa albo marginara in temporibus et colli lateribus. Abdomen, crissum et tętrices caudx alba.

\section{SYNONYMA.}

Anser Ruficollis, Pallas Spicil. Zool. T. I. Fasc. VI. p. 2I. tab. IV. Gmel. Itin. II. p. I79. to I4. Bechst. N. D. IV. p. 9 I 6 . 
Anas Ruficollis, Retz. Fn. Sv. p. II4. Lath, Ind. II. p. 841 . Gmel. I. 2. p. 5 I I.

Anas torquata, Gmel. Syst. I. 2. p. 540. 11. 70.

Diekleine Kraszarka, Lepechin Reis. 2 B. p. 183.t. 5. Redbreasted Goose, Arct.Zool. p. 57I. C. Lath. Syn. VI. p. 455.

\section{DESCR I P TIO.}

Longit. 20 poll. Rostr. I poll. A flexura alæ ad apicem 14 poll. Cauda $3 \frac{6}{3}$ p. Tibia $2 \frac{1}{8}$ p. Digit. med. 2 poll. postic. brevis $\frac{\frac{2}{5}}{\bar{s}}$ elevatus.

Rostrum nigro-fuscum, breve, basi latius apicem versus angustius*), ungue obtuso, Nares oblongoovales. Pedes nigri.

Caput et cervix nigra macnla magna ovali alba inter rostrum et oculos. A temporibus in latera colli descendit macula ovalis rufa albo marginata. Collum præterca ad sternum usque rufum, a pectore et dorso linea alba distinctum. Pectus, dorsuus et scapulares nigra. Abdomen, crissum, latera uropygii et tectrices caudx alba. Hypochondria nigra, albo transversim maculata. Alæ nigr:e tectricibus griceo-albo marginatis. Rectrices XVI. nigræ, apice albx.

\section{H IS T OR I A.}

Incolit terras boreales Asix. Rarius Europam septertrionalem habitare videtur, cum versus meridiem nigrans rarissime Scandinaviam visitat. Non nisi semel apud nos inventa scil. initio Octobris I793, cum juxta Lund Scanire viva in fossula caperetur. Vide Retzii Faun, 1. c.

*) Vere anserinum.

Nilssone Ornith, Pars 11. 
GENUS.

M E R G U S.

Rastrum subulato-cylindricum, tenue, ręum, basi crassum, apice aduncum, maxilla utraque denticulata, denticulis retrorsum vergentibus. Neres parvæ, ovales, laterales, in medio rostro sitæ.

Pedes breves, digito postico membranx alæformi.

CCLII. MERGUSMERGANSER, Lin.

DraGn: Speculum alarum album sti ia transversali nulla. Crista brevis. Corpus subius album lu. teo tinctum.

Mas: Caput et superior colli pars nigra; inferior alba, lutescens. Dorsum antice nigrum.

Femina: Capur et superior colli pars rufa; inferior et pectus albida. Dorsum cinereum.

\section{SYNONYMA.}

Mergus Merganser, Ödmann Aata Holm. I785. p. $30 \%$. Temm. Man. d'Orn. p. 575. Meyer Liv, u. Esthl. p. 26I. Retz. Fn. p. I34.

Le Harle, Buff. Ois. VIII. p. 267. tab. 23. Pl. enl. $95 \mathrm{I}$.

Goosnuder, Pen. Brit. Zool, II. p. 556. n. 260. 
Taucher.Gaus, Bechst. N. D. IV. p. 78 I.

Fenina et junior avis:

Mergus Castor, Lin. Syst. I. p. 209. Gmel. Syst. I. 2. p. 545. Lath. Ind. II. p. 829.

Mergius vulbricapillus, Gmel.1. c. p. 545.

Havle Femelle, Buff. Ois VIII. p. 236. Pl. enl. 953.

Svecis: Skrake, Kôrfögel; Wrakfügel. Blckinges: Gulskräcka.

\section{DESCRIPTIÓ.}

Mas scnior: Longit. 28.29 poll. Alæ extensæ 3 ped. 6 poll. Rostrum a fronte $2 \frac{\frac{l}{2}}{2}$ p. A flexura alx $I \frac{r}{2}$ poll. Cauda $4 \frac{1}{2}$ poll. Tibia 2 poll. Digit. med. $2 \frac{6}{8}$ poll. postic. $\frac{5}{8} \mathrm{p}$.

Rostrum saturate sangvincum, supra longitudinaliter et ungulo fuscis. Os intus pallide luteum. Iris rufo-brunnea l. rubra. Pedes rubri. Caput cristatum crista brevi, tumida.

Caput totum et collum usque ad medium atra, viridi et violaceo nitentia. Ceterum collum, pectus, abdo. men et crissum alba luteo tincta. Dorsum antice et scapulares interiores nigra. Scapulares exteriores et tectrices alarum albx. Flexura alx nigricans. Dorsum medium et uropygium cinerea (interdum albo tenuissime undulata). Cauda cinerea. Remiges primores nigrx, medix, albx, intimx albx, margine exteriori nigræ.

Femina senior: 27.28 poll. Alx extensa 3 ped. 3 poll.

Rostrum supra fuscum, subtus sangvineum. Iris fusca. Pedes extus carnei, intus luteo-rubri. Crista longa, angustior.

Caput et superior pars colli rufa. Gula alba. Inferior pars colli, pectus et hypochondria cinerascenti-

R 2 
albida. Abdomen album luteo tinctum. Corpus to. tum supra cinereum speculo alarum albo, fascia transversali destituto.

Mas junior tempore deplumationis: Caput et superior pars colli rufa pennis nigris immixtis. Gula alba nigro maculata. Collum inferius album pennis cinereis rarius inspersis. Dorsum anterius et scapulares interiores nigræ, pennis cinereis immixtis. Scapulares exteriores et tectrices alarum albæ. Ala ad flexuram nigricans marginibus pennarum cinereis. Pectus, abdomen et crissum alba luteo tincta. Cetera ut in seniore.

\section{HIS TOR IA.}

Habitat frequens tum in lacubus et fluvis tum in mari inter rupes et insulas. Nidulatur in omnibus Suecix provinciis (Scania forte excepta) inter lapides, sub fruticibus et præsertim in arboribus cavis. Ova $12-15$ albida. Vescitur piscibus ct amphibiis. Hieme præ. sertim a piscatoribus in mari figitur.

CCLIV. MERGUS SERRATOR, Limn.

DIAGN: Speculum alarum album, stria transversali nigra vel grisea; Crista dependens. Corpus subtus album.

IIas: Caput et superior pars colli nigra; inferior rufescens nigro=maculata, collari albo. Dorsum nigrum.

Fenina: Capur et Collum rufescentia. Dorsum fuscum marginibus pennarum cinereis.

\section{SYNONYMA.}

Mergus Serrator, Retz. Fn. p. I36. 11. 94. Lath. Ind. II. p. 829. n. 4. Temm. Man, d'Ornith, p. 579. 
Mergus Merganser, Mas Lin. Fn. Sv. p. 48. n. I35. Harle Huppé, Buff. Ois. VIII. p. 273. Pl. enl. 207. Redbrcasted Merganser, Pen. Brit. Zool. II. p. 558. n. 26 I. Lath. Syn. VI. p. 423. Edw. Av. t. 95. Langscinabliger Sïger, Bechst. N. D. IV. p. 795. Meyer Liv. u. Esthl. 262.

Mas junior:

Mergus Serratus, Lin. Fn. Sv. p. 48. n. 136. Gmel Syst. I. p. 546. n. 3. var. a. - Mergus niger, ibid, y. Lath. Syn. VI. p. 426. B.

Gottlandis: Ard, Pracka. Blekingis: Stenskråcka. Norvegis: Siil And.

\section{DESCRIP TIO.}

Longit. $22 \cdot 23$ poll. Rostrum a fronte $2 \frac{\mathrm{T}}{2} \mathrm{p} \cdot \mathrm{A}$ flexura alx ad apicem 10 poll. Cauda 3 poll. Tibia $\mathbf{I} \frac{6}{8} \mathrm{p}$. Digit. med. $2 \frac{6}{8} \mathrm{p}$; ; postic. $\frac{6}{8}$ poll.

Rostrum et iris rubra. Pedes aurantii. Crista longa postice dependens.

Caput et superior colli pars nigra, viridi nitentia. Collare album. Collum inferius antice rufescens maculis 1. striis nigris. Dorsum, scapulares et pectoris latera ante flexuram alarum nigra. Juxta alæ flexuram utrinque maculæ quædam majores albæ nigro marginatx sunt. Speculum alarum album striis transversis duabus nigris. Pectus et abdomen alba. Uropygium et hypochondria lineis undulatis albis et fuscis. Remiges primores nigrx, secundariæ albæ basi nigrx.

Fenina senior: Longit. 2 I poll. Alæ extensæ $2 \frac{r}{2}$ poll. Rostrum supra fuscum, subtus sangvineum. Iris rubra. Pedes extus rubri, intus luteo-rubri inembrana natatoria virescente. 
Caput, crista et latera superiora colli rufescentia. Dorsum, scapulares et teêrices alarum minores saturnte fusca marginibus pennarum cinereis. Gula alba. Collum antice album undulis obsoletis albidis et rufescentibus. Pectus, abdomen et crissum alba. Remiges primores et tectrices proximæ nigro - fuscæ; secundariæ medietate exteriore albæ, unde macula alba in ala oritur. Uropygium ${ }_{j}$ et cauda fusco-cinerea.

\section{H IS T OR I A.}

Habitat frequens iisdem locis atque antecedens. Nidulatur inter lapides in rupibus marinis. Ova I2. 15 coerulescenti - alba.

\section{MERGUS ALBELLUS, Linn。}

Diagn: Rostrum et pedes cinerei. Crista tumida. Alæ variegata.

Mas: Albus occipire, macuiâ ad sinum oris et dorso nigris.

Finina: Pileo et nucha rufis. Gula et jugulo albis. Dorso fusco - cinereo.

\section{SYNONYMA.}

Mergus Albellus, Lin. Fn. Sv. p. 48. n. I37. Syst. Nat. I. p. 269. Lath, Ind. II. p. 83 I. 11. 6. Gmel. Syst. I. 2. p. 547. Retz. Fin. p. 138. n. 95.

Le petit Harle huppé ou la Rette, Buff. Ois. VIII. p. 275. Pl. enl. 449. (Mas) 450. (Fem). Harle Piette, Temm. Mnn. d'Ornith. p. $58 \mathrm{I}$. Smew or White Nun, Pen, Brit. Zool. II. p. 559. n. 262. Lath. Syn. VI, p. 428. Id. Suppl. I. p. 27 I. 
Weisser Sïger, Bechst. N. D. IV. p. 804. Meyer Liv. u. Esthl. p. 263.

Femina et junior avis:

Mergus minutus, Lin. Fn。 p. 49. n. I38. Syst. I p. 209. Lath. Ind. II. p. 832. Brunn. Bor. n. 98. Red headed Sillerv, Pen. Brit. Zool. II. p. 500. 11. 263. Lath. Syn. VI. p. 429.

\section{DESCRIPTIO.}

Longit. I $5 \frac{x}{2}-16$ poll. Rostrum a sinu oris $I \frac{2}{3}$ poll.

Rostrum et pedes coerulescenti-cinerei inembrana natatoria nigra. Iris brunnes. Crista tumida.

Latera capitis ad rostri hasin maculâ rotundâ nigrno Ad basin lateralem colli utrinque linea tiansversali nigra. Ceterum caput cum crista, collum et corpus subtus alba. Latera corporis alba fusco tenuissime et pulcherrime undulata. Dorsum nigrum. Alæ nigræ maculâ magna longitudinali alba. Cauda cinerea. Mas senior.

Fenhina senior: Long. I5 poll. Caput supra ct superior. pars cervicis saturate rufa. Gula et pars juguli pure alba. Inferior par's cervicis, dorsum, tectrices alarum et hypochondria fusco-cinerea. Alæx nigræ maculâ et fasciis binis albis.

Mas junior: Frons et genx fusca. Pileus et cervix ex fusco et albo varius. Dorsum et cauda cinereo-fusea. Hypochondria fusco-cinerea, pennis albis, fusco undulatis immixtis. Pectus et abdomen alba.

\section{H ISTORIA.}

Halitat in regionibus patrix maxime borealibus. Instante hieme interdum sed rarissime occurrit in Scanire fluviis et lacubus vel in mari. Vescitur pisci. bus. Ova 8.12 albida parere dicitur. 


\title{
SECTIO POSTERIOR.
}

Digitis omnibus membrana jundtis.

\author{
GENUS.
}

C A $\quad$ R $\quad B \quad O$.

Rostrum mediocre vel longum, ręum, subcylindricum, compressum, supra teres, edentulum, maxilla inferiore apice crassiore, superiore ungue exserto, dependente.

Orbita, Gens et Gula nuda.

Nares rima obliterata juxta basin rostri.

Carda rotundata.

Pedes breves, robusi, digitis omnibus membrana integra junetis.

\section{CARBO CORMORANUS,}

\section{Meyer.}

DingN: Nigricans, collari, macalis femorum et striis colli albis. Cauda rotundata rectricibus quatuordecim.

\section{SYNONYMA.}

Carbo Cormoranus, Meyer Liv. u. Esthl. p. 265. Temm. Manuel d'Ornith, p. 587. 
Pelecanus Carbo, Lin. Fn. Sv, p. 5I. n. I45. Gmel。 Syst. I. 2. p. 573. Lath, Ind. II. p. 880.

Le Cormoron, Buff. Ois. VII. p. 310. to 26. Pl. enl. 927.

Corvorant, Pen. Brit. Zool. II. p. 608. Lath. Syn. VI. p. 593.

Dor Schwarze Peliknu, Bechst. N. D. IV. 750. Suecis: Hafs,tjïder. Alkrok. Norvergis: Sknrv.

\section{DESCRIPTIO.}

Longit. 27-30 poll. Rostrum a fronte 3 poll. A sinu oris $4 \frac{\frac{7}{8}}{\mathrm{~s}}$ poll. A fiesura alix ad apicem $\mathrm{I} 4$ poll.

Rostrum cinereo-nigricans. Regio oculorum nuda viridi-lutca marginibus palpebrorum rubris. Gula nuda favcscens. Pedes nigri. Cauda rotundata, rectricibus quatuordecim.

Senior habitu cestivali:

Occiput cristatum. Caput et superior pars colli nigra lineis longitudinalibus albis. Sub gula collare album ad latcra capitis se extendens. Femora pennis longis pure albis. Ceterum collum, cauda et corpus subtus nigra viridi nitentia. Dorsum et alæ supra pennis in medio griseis colore xneo nitentibus, nigro marginatis.

\section{Senior habitu hienali:}

Caput lave. Gula et latera capitis inferiora albida. Caput supra, collum, pelus et totum corpus subtus nigra viridi nitentia. Strie ninutx albe in collo. Als et cauda nigra. Dorsum ut in anteced.

Avis junior: Caput supia nigricans. Collum et superior pars pçoris fuliginosa, gulâ sordide albâ. Pars inferior pectoris et abdomen alba, fusco obsolete ma. 
culata. Dorsum anterius, scapulares et tectrices ala. rum in medio grisea subnitontia, marginibus nigris cineta.

\section{HISTORIA.}

Hobitat in maribus Scandinaviam alluentibus, presertim ad oras et insulas Norvegiz septentrionalis. Vescitur piscibus. Nidulatur in scopulorum rimis. Ova 3.4 sordide virescenti-alba, oblonga, extremitate urraque æquali.

\section{CARBO GRACULUS, Mey.}

Diagn: Nigricans, Gula nigua, maculis femorum et striis colli albis. Cauda cuneiformis rectricibus auodecim.

\section{SYNONYMA.}

Carbo Graculus, Meyer Taschenb. II. p. 578. Temm. Man. d'Ornith. p. 589.

Pelecrnus Graculus, Lath. Ind. II. p. 887. n. I5. Gmel. Syst. I. 2. p. 574.

Pelecnnus Capensis, Sparrm. Mus. Carls. III. t. 61 . Shag, Pen. Brit. Zool. If. p. 6IO. n. 292. Lath. Syn. VI. p. 598.

Le petit Cormoran on Nigaud, Buff. Ois. VIII. p. 319. Krähen Pelikan, Bechst. N. D. IV. p. 762.

Norveg: Sinaa Skarv.

\section{DESCRIPTIO.}

Longit. 23-24 poll.

Rostrum cinereo-rufescens, supra nigricans. Regio oculorum et gula nuda rufescenti-lutea. Iris rufo. 
fusca. Pedes nigri. Cauda cuneiformis rectricibus duodecin.

Senior habitu astivali:

Occiput cristatum. Caput et superior pars colli nigra lineis longitudinalibus albis. Gula nigra. Fe. mora pennis longis pure albis. Ccterum collum et corpus subtus viridi-nigricantia. Dorsum ct alx supra pennis in medio saturate cinereis nigro late marginatis. Senior halitu hiemali:

Caput lixve. Caput, gula, collum, dorsum et corpus subtus virescenti-nigra. Stric minutæ albr in collo.

\section{HISTORIA.}

Habitat iisdem fere locis ac antecedens. Tempore hiemali rarius tamen oras nostras frequentat. Vescitur piscibus. In rupium rimis et in arboribus nidulatur. Ova $2-3$ albida. 
GENUS.

S. U L $\left.A^{*}\right)$.

Rostrum longum, rectum, basi crassum, dehine decrescens, apice acuminato, deflexo, marginibus incisuris serratis.

Facies et gula nuda. Nares lineares, occultr, juxta basin. Pedes breves, robusti, digitis omnibusmembrana integra junctis; ungue digiti medii serrato. Canda cuneiformis rectricibus duodecim.

\section{SULA ALBA, Mey.}

Diagn: Alba, remigibus nigris, facie coerulea. Funior: Fusca punctis triangularibus albis adspersa.

\section{SYNONYMA.}

Sula alba, Meyer Tashenb. Ir. p. 582.

Pelecanus Eassanus, Lin. Syst. I. p. 217. 11. 5. Fn. Sv. p. 52. n. I47. Retz, Fn, p. I46. n. 105. Gmel. Syst. I. 2. p. 577. Lath. Ind. II. p. 89 I.

*) Ad hoc Genus pertinet prxter mox describerdan speciem, (quam Sulam borealem nominarem), Pclecanus Carolinus Lin. Pel, Fiber Lin, et pl. 
Fon blanc on Le Basnn, Temm. Man. p. 593. Buff. Ois. VIII. p. 376. Pl. enl. 278.

Thie Gannet, Pen. Brit. Zool. II. p. 612, tab. 103. Lath. Syn. VI. p. 608.

Der Bassanische Pelikan, Bechst. N. D. IV. p. 765. Funior:

Pelecanus maculatus, Gmel. Syst. I. 2. p. 579.

Le Fou tacheté, Buff. Ois. VIII. p. 375. Pl. enl. 286. Suecis et Norvegis: Sula, Havsule.

\section{DESCR IP TIO.}

Longit. 2 ped. 7 poll. -3 ped.

Rostrum basi cocruleo-cinereum, apice album. Orbita nuda coerulescens. Facies et gula nuda coeruleo-nigricantia. Iris lutea. Pedes antice virides, postice et membrana natatoria nigra. Ungues albi.

Vertex et occiput lutea, ceterum caput et corpus totum alba remigibus alulaque notha nigris.

Funior; Caput et collum cinereo-fusca maculis minutis triangularibus adspersa. Dorsum cinereo-fuscum maculis triangularibus albis rarioribus et majoribus. Corpus subtus albidum maculis minutis fuscis. Remiges et rectrices nigricantes.

\section{HIS TORIA.}

Habitat in oceano Scandinavix maxime borealis, nea misi raro et hieme rigida oras Norvegix et Svecia occidentalis visitat. Nidulatur in insulis a terra maxime remotis. Ovum unum album. 


\section{SUPPLEMENTUM}

ET EMENDATIONES IN PARTEM PRIOREM ORNITHOLOGIF SVECICE.

$P_{\text {ag. 44. (Fulco peregrimus). HISTORIA: In pla- }}$ gis Svecix australioribus rarius obvenit, neque heic nisi uno loco nidulantem adhuc offendi, scil. in rupe prærupta Blekingix, Walhall (unde nomen ei Walkök a vulgo imponitur) ubi quotannis domicilium habet et se propagat. Advenit post medium mens. Martii, vel ad initium $M$. Aprilis ct abit mens. Novembri. In rupibus Svecix borealis et Norvegix haud rara est. In insula Lowanncn (vide Orn. Sv. II. p. 140.) ubi Alca Arctica infensissimus est, in rimis rupium nidificantem quoque invenimus.

Pog, 51. (Falco Tinnunculus) Habitat frequens, tum in austratioribus tum in borealibus peninsula plagis. Heic in preruptis montibus nidulatur. - In Scaniam post initia mens. $\Lambda$ prilis advenit.

Pagr. 65. lin. IO. Strix Uralensis Hoc Synonymum deleatur; distinctam enim adtinet speciem, que in boreali quoque Scandinavia observata est. Hujus igitur Diagnosin adjungam.

\section{STRIXMACROURA, Nutt.}

DiAgin: Supra fusco-cinerca maculis majoribus, ovalibus, albis; subtus albida maculis longirudinalibus fuscis. Cauda longa, cuneiformis, fusca, 
reniis septem transversalibus, albidis. Longit. $2 x-22$ poll. Cauda $10 \frac{\pi}{2}$ poll.

\section{SYNONYMA.}

Strix macroura, Mey. Vögel Liv. u. Esthi. p. 29. Choutte de l'Oural, Temm. Man. d'Ornith. p. 56.

\section{HABITAT}

in vastis Svecir borealis sylvis. In Lofoden Norvegix a Cl. Boie fuit visa.

Pag. 66. Strix Ulula Lin. HISTORIA. adde: In sylvis vastis Norrlandix, ex. gr. Herjedalix. Dalecarlix \&c. frequens occurrit. Eandem exacte regionem xstare occupat, ac Corvus infousius. In Scania non nisi hieme et quidem raro obvenit.

Pag. 80. Corvus clericus, Huic similem Coronem, macula gulari alba, sed aliquantum minori, juxta Arup Scanix, medio mens. Junii 1817 , fuit occisus. Junior erat avis.

Pag. 85. Corvus Monedula. Domicilium ejus ma. xime boreale in peninsuln Scandinavica est Stichlerstad, ultra Nidrosiam, ubi in templo quotannis nidulatur.

Pag. 89. Corvus infuustus. HISTORIA: Habitat passim frequens in pinetis regionum Svecire et Norvegix borealium. Circa Holmiam et Christianiam rarissime ob. venit, sed in Dalekarlia, Herjedalia et Westrobothnia, in Guldbrandsdalia, Gulldalia \&c. frequens occurrit. Quoad consuetudines ad Lanitm glandarium proxime acce. dit. Sicut ille Tetraones aliasque aves a Norrlandis autumno laqueis captas interdum plane devorat. Vescitur ccterum baccis, insectis, mammalibus minoribus et aviculis. Qva nondum vidimus; mature vero nidulatur; vidimus enim d. 20. mens. Junii 7 - 8 pullos; jam volatui ap. tos, qui parentes, a quibus nutriebantur, comitabantur. 


\section{Pag. 102. adde: SECTIO II. \\ Pedibus gressoriis. \\ GENUS. \\ $\begin{array}{llllll}M & E & R & O & P & S\end{array}$}

Rostrman mediocre, acutum, parum curvatum, compressum, supra subtusque carinatum.

Nores laterales, parvæ, juxta basin rostri sitæ. $p_{e d e s}$ breves, digitis tribus anticis, quorum extimus cum medio usque ad ulrimam articulationem juncus est; postico libero, basi lato.

\section{MEROPS APIASTER, LIN.}

DisgN. Dorsum ferrugineum, corpus subius virescenti-coeruleum. Gula fulva. Fascia per oculos nigra. Kectrices media bina longiores. Longit. II. poll.

Mas et fernina hujus speciei, sub æstate anni I 86 haud procul ab Ystad Scanix visi sunt, alterque sexus plumbo occisus fuit.

\section{SECTIO III.}

\section{Pedibus scansoriis, doc.}

Pag. I05. PICUS CANICEPS, mihi, lege: PICUS CANUS, Temm.

Diagn, Viridis, capite, collo et corpore subtus cinereis; fascia frontali (in mare) eoscinea; זx. 
nia nigra inter nares et oculos, alieque tenuiore ad larera juguli.

Pag. 138. Fringilla Montifringilla. Innumerandos greges, versus septemtrionem dimigrantes, in Norvegia meridionali, dieb. 8-I2 mens. Maji. observavimus. Iam initio mens. Iunii in sylvis subalpinis trans montes Dovrefield sitis, et postea per totam rstatem in sylvis septemtrionis per paria habitantes vidinus. Quoad consuetudines cum fringilla calibe maxime conveniunt. 'Interdum in fagetis Scanix hybernant et glandes fageas devastant.

Pag. I66. Emberiza Hortulana. In Norvegia quoque usque ad Dovrefield passim frequens occurrit.

Pag. I70. Emberiza Schoeniclus. In Scandinavix borealis pratis depressis et fruticosis passim frequens occurrit. Post medium Septembrem australiores terras pea tens saliceta Scaniæ visitat.

Pag. 177. Turdus Iliacus. Habitat frequens in superioris Svecix et Norvegir sylvaticis humidioribus. Nidulatur in fruticosis. Ova 6 coeruleo-virescentia, nigro maculata, mense Iunio invenimus.

Pag. 189. Muscicapa Grisols. Occurrit æstnte in Scandinavia usque ad circulum arcticum.

Pag. 192. Passim in borealibus etiam regionibus usque' ad circulum arcticum.

Pag. 205. Sylvia hortensis. Hane speciem in sylvis Svecix superioris et Norvegix frondosis, multis locis observavimus usque ad circulum polarem.

Pag. 220. Sylvin Hippolais, in Norvegix quoque sylvis usque ultra Nidrosiam occurrit. 


\section{SYLVIA RUFA, Lath.}

Diagn. Supra saturate grisea, superciliis flavescen. sibus; subtus sordide alba, flavo immixto. Pedes et rostrum nigro-fusca. Alarum tectrices subtus straminex;

\section{SYNONYMA.}

Sylvia abietiza, Nilss. Act. Holm. I819. p. II5. Tab. V. (figura, quoad rostrum ct pedes, mala). Sylvia rufa, Lath. Ind. II. p. 516. Bec-fin veloce, Tem. Man. d'Ornith。 p. 125. Weiden Sänger, Bechst. N. D. III. p. 649.

\section{DESCRIPTIONEM}

vide Acta Holm. 1. c.

\section{HISTORIA.}

Habitat in pinetis Norvegix et, sine dnbio etiam, Svecir borealis, haud rara. Cantans in summo abietis cacumine plerumque sedit. Cantus, quo facile etiam eminus dignoscitur, singularis magis quam amoenus, his fere syllabis exprimi potest: kipp, kipp, kipp, - kjapp, kjapp, kjapp, - teer, teer. - Vescitur Insectis eorumque larvis. Vide cet. Act. Holm. l. c.

Pag. 244. Anthus pratensis. Obvenit in omnibus Scandinaviz regionibus usque intra circulum polarem. Alpes boreales quoque adscendit et in earum lateribus humidiorihus inter gramina nidulatur.

Pag. 247. Anthus rupestris, in rupibus litoris Scandinavici a Scania et Blekingia usque ad Helgolandiam et Salten Norvegix obvenit.

Pag. 267. Sturnus vulgaris. In Seandinavia longe sersus septemtrionem adscendit. In regionibus quoque arcticis illum hine inde habitantem invenimus. 


\section{I n d e x}

nominum Latinorum \& Svecicorum.

Accipiter alaudarius I. 50. - Korschun I. 20. Ahla II. 199. Ahlfogel II, 199. Alauda Arborea I. 258.

- Arvensis I. 255.

- Campestris I. 248.

- cristatella I. 258.

- minor I. 251.

- nemorosa I. 258.

- novalium I. 248.

- obscura I. 245,

- petrosa I. 245 .

- pratensis I 242. 245.

- trivialis I. $25 \mathrm{I}$. Alca Alle II, 146.

- Arctica II. 139.

- Balthica Ir. 136.

- Impennis II. 137. 138 .

- minor II. 136.

- Pica II. 136.

- Torda II. 135.

- unisulcata II. 136.

Alfic II. 26.

Alkrok II. 255.

Allika I. 84.

Altita I. 273.

Alvargrim II. 7 . Ampelis Garrulus I. 185 . Anas Acuta II. 217. 218.

- Alandica II. 218.

- Albifions II. 242 .
Anas Anser II. 239.

- Anser ferus II. 236.

- Bernicla II. 245.

- Boschas II. 223.

- Canadensis II. 24I.

- cinerascens II. 193.

- Cinerea II. 193.

- Circia II. p. $23 \mathrm{I}$.

- Clangula II, 207.

- Clypeata II. 229.

- Crecca II. 234.

- Cygnus II. 189.

- dispar II, 214.

- Erythropus femina II. 242.

- Erythropus mas II. 24 I.

- fera II. 223.

- Ferina II. 211.

- Ferruginea II. $22 \%, 21 \%$.

- fistularis II. 220.

- Franatĭ II. 209.

- Fusca II. 204.

- Fuligula II. 204. 205.

- Glacialis II. 198.

- Glaucion-II. 208.

- hiemalis II. 198.

- Histrionica II. 212.

- larirostra II. 206.

- leucopsis II. 240.

- Marila II. 209.

- Minuta II. 262.

- Mollissima 1I. 194.

- Nigra II. 192.

- Olor II. 190. 
Anas Penelope II. 220.

- platyrhynchos II. 223.

- Qverquedula II. 230.23I.

- Rubens Iit. I 29.

- Rufa II. 211 . 22I,

- Ruficollis II. 247.

- Scandiacaj II. 206.

- Segetum II. 238.

- Skoorra II. 206.

- Spartmanni II. 218.

- Spectabilis II. 202.

- Stelleri II. $21 \mathrm{~A}$.

- Strepera II. 216.

- Tadorna II. 226. 227.

- torquata II. 247.

Anser Albifrons II. 242.

- Cinereus II. 236.

- Leucopsis II. 240.

- Ruficollis II. 246.

- Segetum II. 238.

- Torquatus II. 245.

Anthus Aquaticus I. 245.

- arboreus I. 25I.

- Campestris I. 248.

- Pratensis I. 241. 242.

- Rupestris I, 245.

Aquila aurea I. 2.

- Mogilnilk I. \%.

- navia I. 7 .

- nigra I, 2

Ard II. $25 \mathrm{I}$.

Ardea Ciconia II. 3 I 。

- Cinerea II. 36. $37^{\circ}$

- Grus II. 34.

- Major II. 37.

- Minuta II. 38.

- Nigra II. 33.

- Stellaris II. 3940.

Asilus I. 228 .
Astur I. 340

Augustisnåppa II. 52.

Backswala I. 285 .

Beckasin Dubbel II. 103.

- Enkel II. 65. 104.

- Halfenkel II, 108.

Bergfink I。 136 .

Berguf I. 53.

Bjuggbit II. II3.

Bjorkdrosscl I. 178 .

Blåfot I. 39.

Blåkråka I. ()2.

Blåmes I. 270 .

Bofink I. 138.

Brandgås II. $22 \%$ "

Branduggla I. $\sigma_{3}$.

Brockfogel II. 7 .

Brunnacke II. 22 I.

Brushane II. 72。

Busklus I. 230.

Busksqwătta I. 195.

Calendula I. 230.

Calidris Grisea II. 22. $86^{\circ}$

Caprimulgus Europæus I 288 .

Carduelis lulensis I, I 36.

Carbo Cormoranus II. 254 .

- Graculus II. 256.

Caryocatactes guttatus I. 90.

Cataracta Ceppus II. 184.

- Parasitica II. 184.

Cepphus Lacteolus II. 145.

Certhia Familiaris I. 98.

Charadrius Albifrons II. 17.

- Alexandrinus II. 17\%

- Apricarius II, 6.

- Auratus II. 6.

- Calidris II. $2 a_{\text {。 }}$ 
Charadritus Campestris II. 6. , - Curonicus II. 15.

- Fluviatilis II. 15.

- Fulvipes II. 15.

- Hiaticula II. 13.

- Litroralis II. 17.

- Morinellus II. 10.

- Minor II. 15.

- pluvialis II. 7 .

- Sibericus II. 10.

- tataricus II. 10.

Ciconia Alba II. 3 r.

- Fusca II. 33.

- Nigra II. 33.

Cinclus II. 94 .

Cinclus Aqvaticus I. 26I, 262 .

- torqvatus II. 90.

Colunba Domestica I. 295.296.

- livia I. 296.

- Oenas I. 293.

- Palumbus T. 291.

- rupicola I. 296.

- Saxatilis I. 296.

Colymbus Arcticus II, 150.151 .

- auritus II. 129.

- Cristatus II, I26.

- Glacialis II. I48.

- Grylle II. 144.

- ignotus II. I5I.

- Immer II. 149.

- Lacteolus II. 145.

- Lumm: III, I53.

- MinorII. 130.131.143.

- obscurus IL 130 .

- Parotis II. I 27.

- Rubricollis II, I27.

- Septentrionalis II, 152. 153.

- Stellatus II. 153.
Colymbus subcristatus If. 127.

- torqvatus II. I49.

- Troile II. II 42 .

- Urinator II. $12 \sigma_{0}$

Coprotheres II. 184.

Coracias Garrula I, 92.

- Galbula I. 94.

- Oriolus I. 94.

Cornix cinerea I. 83 .

- Gesneri I. 80.

Corvus Coryocatactes I. 90.

- Corax I. 78.

- Cornix 1. 82.83.

- Corone I. 79.

- Frugilegus I. 81.

- Glandarius I. 76.

... Infaustus I. 87.

- Monedula I. 84.

- Fica I. 85.

- Sibericus I. 87.

Crucirostra Abietina. I. I22. 123.

- Pinetorum I. 120.

I2I.

Cuculus Canotus I. I16.

- Hepaticus I, 119.

- Rufus I. I18. 'I19.

Curruca arundinacea I. 219.

- atricapilla I, 203.

- garrula I, 208.

- minur I. 204

- sepiaria I. 217.

Cyanecula I, 216.

- Gibraltariensis I. 2 r6.

Cygnus Gibbus II. 190.

- Melanorhynchus II, 189.

- musicus II. 189.

Cynchramus I, 162. 
Dendrofalco I, 46 .

Domherre I. 129.

Dopping II. 20.8 .

Eiderfogel II. 195.

Eidergås II. 195 .

Emberiza Calandia I. 162.

- Citrinella I. 166.

- Alva I. 166.

- hortulana I, I64.

- lapponica I. 157.

- Meelbyensis I. I65.

- Miliaria I. 162.

- Nivalis I. I60.

- Schoeniclus. I. 168.

Emmer II. I 49.

Entita I. 278 .

Eurynorhynchus Griseus II. 29.

Falco Esalon I. 48.

- Aruginosus I. 19. 20.

- albicaudus I. 11.

- albicilla I. 10. II

- albidus I. 28.

- Apivorus I. 3 I.

- Ater I. 25.

- Buteo I. 28.

- brunneus I. 50.

- Candicans I. 39.

- Chrysaëtos I. 1.2.

- Communis I. 43.

- Communis leucoceplialus

$$
\text { I. } 9 .
$$

- cyaneus I. 22.

- fasciatus I. 50.

- fulvus I. 2 .

- Fuscus I. 39.

- Gallinarius I. 34.
Falco Gentilis I. 34,42 .

- Gyrfalco I. 38. 39.

- Halietus I. 16.

- hinnuiarius I. 1 I.

- Hudsonius I, 23.

- Islandicus I. 39.

- Islandus I. 39.

- Lagopus I. 8.

- Lanarius 1.44.

- Levicocephalús I. II.

- Lithofalco I. 48.

- Longipes I. 18.

- Macrourus I. 22.

- Maculatus I. 7.

- Marginatus I. 34。

- Melanxtus I. 2, 1 I.

- Milvus I. 25. .

- Mogilnik I. 7.

- Nævins I. 7.

- Niger I. 2.

- Nisus I. 36. 37\%

- Ossifragus I. 14.15.

- Palumbarius I. 33.34 。

- pennatus I. 9 .

- Peregrinus I. 42.

- Pygargus I. 22.

- Rufus I. 20.

- Ruscicolis I- 39,

- S. Johannis I. 9 .

- Sclavonicus I. 9.

- Strigiceps I. 2 I.

- Subbuteo I. 45.

- Tinnunculus I. 49.

- Torquatus I, 22.

- Umbrinus I. 39.

- Variegatus I. 28.

- Versicolor I. 28.

Fisk-Kjuse I. 16.

Fiskmaka II, 172 . 
Fiskmåse II. 172.

Fjållripa I. 309.

Fjůlstar I. 174 .

Fogelkong I. 230 .

Fringilla Calcarata I, 158.

- Cannabina I, I44.

- Carduelis I. I5I.

- Coelebs I. 138.

- Domestica I. 140.

- Erythrina I. 155

- flammea I. 156.

- Flavirostris I. 146. 147.

- Lapponica I. 158.

- Linaria I. 149.

- Linota I. $344^{\circ}$

- Lulensis I. 136.

- Montana I. 142.158.

- Montifringilla I. 135 . 136.

- Nivalis I. 134.

- Obscura I. 156.

- purpurea I. 156.

- rosea I. 156.

- Spinus I. 153.

Fulica Ethiops II, 123.

- Aterrima II. I22.

- Atra II. I2 2.

- Chloropus II. 116.

Fältlärrka I. 248 .

Fältsparf I. I43.

\section{Gaddes 1I. 199.}

Gallinago Minor II. 108. Gallinula Chloropus II, I16.

- Crex II. Itz.

- major II. I16.

- Porzana II. 1.I4.
Geeath II. 185 .

Glada I, 26.

Glotris. Chloropus II. 57.

- Semipalmata II. 55.

Grafgàs II, 227 .

Gresla II, 145.

Grisla II, 145.

Grus Cinerea II, 34 .

Gryll II, 13.

Grylle II, 145.

Grågås II. 239.

Grảmåse II. 167.

Griifiska I. 149.

Gråsparf I, I 40 .

Gråspink I, 140.

Gråtrut II- $16 \%$.

Gräsand II. 224.

Gröngōling 103.

Gröning I I3.1.

Grőndlandsk Papegoja II. 139.

Grönlands Dufwa II. 147.

Grönsiska I. 158.

Grönspett I. 103.

Gulskräka II. 249.

Gulsparf I. 167.

Gultrast I. 94.

Guärla I. 237.

Gyllenrenna I. 103.

Gylling I. 940:

Gärdsmyg I, 132 :

Gülk I. 116 .

Gölityta 1. 114.

Haclsspett I. 109.

Hrematopus Ostralegus II, r9.

Hafmäka II, 165.

Hafsmäse II, 165. 
Hafstjädel Ir. 255.

Hafsörn I. 1.!.

Harfang I. 56.

Haruggla I. 6r.

Hav-Hymber II, 149.

Havsule II. 259.

Hirundo Apus I. 235. 286.

- Riparia I. $2 \$ 4$.

- Rustica I. 280.28 I.

- Urbica I. 283.

Hjärpe I. 305.

Homå ka II. 165.

Hornuf I. 53.

Hornuggia I, 54 .

Horsgök II. 104.

Husfink I. 140.

Hussivala I. 283 。

Hutti II. 7 .

Hymber II. 149.

Hålmäka II. 165.

Hårsnäppa II. 108.

Häger II. 37.

Härrfogel I. 96 .

Hämpling I. 144 .

Hönstjuf I 20 .

Imber II. 149.

Inmer II. 149.

Ioen II. $185^{\circ}$

Tordswala I. 285 .

Iris I 149.

Iste II. I 45 .

Jugrï II. $22 \%$.

Jynx Torquilla I. II 4 \%

Järnsparf I. 238.

II aja 1. $84 .{ }^{9}$

Kattuggla I, $6 \mathrm{I}$,
Keron I 309 .

TKlera I 174 .

Klädra I. 174 .

Kolerast I. 179.

Kongsfogel I. 230.

Kornknarr II. 113 .

Kornlärka I. 163.

Kornskrika I. 76 .

Korp I. 78 .

Korsnäbb Mindre I. 123.

- Större I. 121 .

Korșkäraré I. 123.

Kourpa II. 45.

Kramsfogel I. 174 .

- Dubbel I. 172 .

Krickand II, 234 .

Kräka I. 83.

Kräckand II. $23 \mathrm{X}$

Körfogel. II, 249.

Laduswala I. $28 \mathrm{I}$.

Lafskrika I. 87.

Lanđöry I. 3.

Lanius Biarmícus I. 273.

- caudatus I. 273.

- Collurio I. 72 .

- Excubitor I. 74.

- Garrulus I. 185.

- Glandarius I. 75 .

Lappskata I. 87.

Lappsparf I. 158.

Larus atricilloiles IT, $17 \%$.

- candidus II. 17 I.

- Canescens II. 177.

- Canus II. 172.

- Cinerarius II. 177.

- Cyanornynchus II. 172.

- Eburneus II. I7I.

- erythropus II, I77. 
Latus Flavipes II. 170.

- Fuscus II. 169.

- Glaucus II. 167.

- Hybernus II. 172.

- Marinus Ir. 164.

- Minutus II. $17 \%$.

- Nævins II. 165.

- niveus II. 171.

- parasiticus II. 184.

- procellosus II. 172.177.

- Ridibundus II. 176, 177 .

- Rissa II. 174.

- Tridactylus II, 174. 175.

Leffeland II. 229 .

Lestris Parasiticus II, $18 \mathrm{r}$.

Leverjoen II. 185.

Limosa fusca II. 59.

- Grisea II, $5 \%$.

- Melanura II. 49.

- Rufa II. 51. 52.

Liungspole II. 7 .

Ljunguipa II. 7 .

Lom II. I 5 .

Loxia Cardinalis I. I55.

- Chloris I. I3i.

- Coccothraustes I. 127 .

- Curvirostra I. 122.

- Enucleator I. 125 .

- Erythræa I. 155.

- erythrina I. 155.

- Flaningo I. 129.

- Pyrrhula I, 129.

Lunda II. 139.

Lundfogel II. 139 .

Lunne II. I39.

Lyngspole II. 7•.

Lảngnäbba II. 45.

Lärka I. 256.

Lärlkfalk I, 46 .
Marspitt II. 20.

Mergus Albellus II. 252.

- Castor II. 249.

- Merganser II. 248.

- merganser Mas II, $25 \mathrm{I}$.

- Minutus II. 253.

- niger II. $25 \mathrm{I}$.

- rubricapillus II. 249.

- Serrator II. 250.

- Serratus II. 251 .

Meshatt I. 275.

Morinella Collaris II. 81 . Morkulla II. IOI.

Motacilla Acredula I. 228 .

- alba I. 234.

- Arundinacea I. 22I.

- Atricapilla I. 202."

- Boarula I. 237.

- Curruca I. 208.

- Erithacus I. 212.

- Ficedula I. 187. 190.

- Flava I. 237.

- Gibraltariensis I. 215.

- Hippolais I. 204. 219.

- Luscinia I. 199.

- Modularis I. 217.

- Phoenicurus I. 2I2。

- Regulus I. 230.

- Rubecula I. 210.

- Ruberra I. 194.

- Salicaria I. 204. 219.

- Schocnobirnus I. 223.

- Scolopacina I.98.

- Svecica I. 216.

- Sylvia I. 206. 208.

- Titys I. 213.

- Trochilus I. 228.

- Troglodytes I, 2310 
Motacilla Oenanthe I. 197.

Mule II. 136.

Muscicapa Atricapilla I. 190.

- Grisola I, 187.

Muswåg I. 28.

Myrpyta II. 7 .

$N_{\text {attskräfwa I. } 289 .}$

Notrskürra I. 289.

Nattwaka I. 126.

Norrquint 1. 136.

Numenius Arqvata II. 44.

- ferrugineus II. $9 \%$.

- Minor II. 47.

- Phropus II. 46.4\%

- Viridis II. 42.

Nückergal I. 200.

Nötkraka I. go,

Nötpacka I. 100.

Nötskrika I. 76 .

Nötwäcka I. 100 .

Odens Swala II. 33 .

Olycksfogel I. 87 .

Oriolus Galbuia I. 94 .

Orre II. 301.

Orriane I. 301.

Orthöna I. зоI.

Ortygumetra II. II 2 .

Otis Tarda II. 1. 2 .

- Tetrax II. 3 .

Oäringsfogel I. 96 .

$P_{\text {arus Ater I. } 276 .}$

- Caudatus I. 273.

- Coeruleus I. 270.

- Cristatus I. 275.

- Cyanus I. 27 .
- Kujusciok I, 272.

- Major. I. 268.

- Palustris I. 277. 278.

- Sæbyensis I. 271 .

Pederdricker II, 147.

Pelecanus Bassanus II. 258.

- Capensis II. $25 \%$

- Graculus II. 256.

- Maculatus II. $259^{\circ}$

Pelikan II. 28.

Perdix Cinerea I. $3 \mathbf{I r}$.

- Coturnix I. 313.

Phalaropus Cinereus II, 130.

- fuscus II. 120.

- hyperboreus II. 120.

- lobatus II. 120.

- Rufus II. IIg.

Picus Canus I. 105.

- Leucunotus I. 107.

- Major I. 108.

- Martius I. I02.

- Medius I. Iro.

- Minar I. III.

-- Norvegícus I, 105.

- Tridactylus I. 112.

- Viridis I. 103.

Piplärka I. 252.

Platalea Leucorudia II, 27.

Fodiceps Auritus II. 128. 129.

- Cristatus II. 125.

- Minor II. I3I.

- Obscurus II. 130.

- Rubricollis II. $12 \%$.

- subcristatus II. 127.

Pomeransfogel II. 10.

Popp I. 96.

Pracka II. $25 \mathrm{I}$.

Procellaria Glacialis II. 187. 188. 
Procellaria Pelagica II: 186. Prutgås II. 245.

Qvidbonde II. 47.

Quidfogel I. 28.

$\mathrm{R}_{\text {allus aqvaticus }}$ II. 110.114 .

- Crex II, 112.

- Porzana II, II4.

Rapphöns I. 311 ,

Rauck I. 80.

Ravn I. 78.

Recurvirostra Avocetta II. 25. Rehusak I. 307.

Ringdufwa I. 292.

Ringswala I. $2: 6$.

Roka I. $8 \mathrm{I}$.

Rotgel I. 210 .

Råka I. 8 r.

Rödbena II. 62.

Rödbrösta I. 2 !O。

Rödbuk I. 2I3.

Rödstjert I. 213.

Rödtjuxa I. 87.

Rödwinge I. 176 .

Rördrom II. 40.

Sandrulling II, 13.

Saxicola Oenanthe I. $197^{\circ}$

- Rubetra T. 194.

"Scolopax Egocephala II. 5 I. Arquata II. 44.

- Calidris II. 63.

- Fusca II. 59. 60.

- Gallinago II. 104.

- Gallinula II. 107.108.

- Glottis II. 57.

- Lapponica II, 5I.
Scolopax Limosa Ir. 49.

- Major II. 102.

- media Ir. 102.

- paludosa I1. roz.

- Paykullii II. 106.

- Phropus II. 46.

- Rusticola II, I00.

- Semipalmata II. 55.

- subarqvara, II. 93.

- Totanus II. 60.62.

Seidreifwer II. II3.

Sidenswans I. 185.

Siil And II. 251 .

Silladopping, II, 143 .

Sillmåka 1I. 170.

Sillmåse Ir. 170.

Sitra Europza I. 100.

Sjutut I, 19?.

Sjöhök I, 16.

Sjöørre II. 123.

Skarv II: 255.

Skata I. 85.

Skogsdufiwa I. 294.

Skogsknett I, 220.

Skogstipa I. 307.

Skogsskata I 76.

Skrake II. 249 .

skränmise II. 156.

Skärfäcka II. 26 .

Slaghök I. 34 .

Slagmåka II. 167,

Smaa Skarv II. 256.

Simåspof II. 47.

Smátrapp II. 4.

Snöripa I. 307.

Srioskata I. 178.

Snöfparf I. 160.

Sommar - Al 1I. 199. 
Sommarguling I. 94. Spansk kräka I. 92. Sparfhög I. 37. Sparflung I. 229. Sparfugla I, 70. Spíllkråka I. 102。 Spof II. 45 . Stare I, 264 . Steglitza I. 15 IStellers And II. 214. Stenknäcls I. 127. Stenskräcka II. 251 . Stensquatta I. 197. Sterna Boisii II. 16r.

- Canescens II. 158.

- Caspia II. 1155.

- fissipes II. I60.

- Hirundo II. 156. 157.

- Megarhynchos II. 155.

- Minuta II. 162.

- Navia II. 161.

- Nigra II. 160.

- Nubilosa II. 159.

- obscura II. 160.

- stubberica II. 158.

Stjertand II. 218 .

Stockand II. 224 .

Stork II. 32.

Swart II. 33.

Stormwädersfogel II. 187 .

Storspof II. 45.

Stortrapp II. 2.

Strandpipare II. I3.

Strandsittare II, 69.

Strandskate II. 20.

Strandskjura II, 20.

Strandswala I, 285.

Strix Accipitrina I. 63.

- Aluco I, 60, 62.
Strix aretica I.63.

- Brachyoros I. 6;.

- Brachyura I. 62.

- Bubo I, 52.

- Flammea I. 62.

- Funerea 1. 65. 66.

- Hudsonia I. 65.

- Lapponica I. 58.

- Liturata I. 59.

- macroura II. 360.

- Nivea I. 56.

- noctua I. 66. 68.

- Nudipes I. 68.

- Nyctea I. 56.

- Otus I. 54 .

- Passerina I. 69.70.

- Scandiaca I. 56.

- Stridula I. 6062.

- Tengmalmi T. 66.

- Ulula I. 63.64.65.

- Uralensis I. 65.

Struntjägar: II. 185 .

Strömstare I. 262.

Sturnus Cinclus I, $26 \mathrm{r}$.

- Vulgaris I. 264.

Sula II. :259.

Sula Alba II. 258.

Swan Tam II. I9I.

- Wild II. 189.

Swansines I. 273.

Swarthätta I. 203.

Swartlasse II. 185.

Swenska I. 126.

Svensk Papgoja I. 126.

Sylvia Arundinacea I. $22 \mathrm{I}$,

- Atricapilla I. 202. 203.

- Cinerea I. 206.

- Curruca I. 208.

- Erithacus $\mathrm{I}, 2 \mathrm{I} \approx$. 
Sylvia Hippolais I. 289.

- Hortensis I 204.

- Luscinia I. 199.

- Modularis I. 217.

- Nisoria I. 20 r.

- Oenanthe I. 1.97.

- Passerina I. 204.

- Phrnicurus I. 212.

- Regulus I. 230.

- Rubecula I, 210.

- Rubetra I. 194.

- rufa II. 262.

- Salicaria I. 223.

- Schoenobxnus I. 223.

- Sibilatrix. I. 225.226.

- Svecica I. 216.

- Sylvicola T. 226.

- Titlys I. 215.

- Trochilus T. 228.

- Troglodytes I. $23 \mathrm{I}$.

Sånglärka I. 256.

Sådesärla I. 235.

Säfsparf I. 169.

Säfstigàre I. 224 .

Iaflacka II. 245.

Taigoxe I, 268 .

Talltrast I. I 74 .

Tantalus Falcinelius II, 42 .

Teiste II. 145.

Teste Ir. 145.

Tetrao Alpinus I. 31 r.

- Bonasia I. 305.

- Coturnix I. 316.

- hybridus I. 303.

- Lagopus I. 307. 311 ,

- Mutus I. 3 II.

- Perdix I 314.
Tetrao Rupestris I. 31 r.

- Subalyinus I, 307.

- Tetrix I. 300.

- Urogallus I. $297^{\circ}$

Tillkråka I. 102.

Tjäder I. 298.

Tofstitta I. 275.

Tofsmyssa I, 275 .

Tolk II. 82.

Tomlinge I. 278.

Tord II, 136.

Tordinule II. 136 .

Toppmes I. 275.

Toppuipa II. $7^{6}$.

Tornfalk I. 50,

Totanus Calidris II. 62.

- Chloropus II. 57.

- Cinereus II. 72.

- Fistulans II. 57.

- Fuscus 59.60.

- Glareoia II. 66.

- Glotris II. 57.

- Gregarius II. 52.

- Hypoleucos II, 68.

- Leucocephalus II. 52.

- Maculatus II. 60.

- natans II. 60.

- Ocropus II. 64.

- Pugnax II. 7 I.

- Ruber II. $6 \mathbf{2}$.

- Rufus II. 51 .

- Strintus II, 62 .

Trana II. 35 .

Trapp II. 2 .

Tringa Alpina II, 90.

- Arenaria II, 22.

- Arra II.60.

- Canurus II. 6g. 
Tringa Cinclus II. 94 *

- Cinerea II. 86.

- Ferruginea II. 84. 93,

- fulicaria 1I. 119.

- Gambetta II. 62.

- Glareola II, 64.66.

- Gregaria II. 52.

- Grenovicensis II, 72 .

- Grisea 11. 86.

- Helvetica 1I 78.

- hyperborea II. 119. 120.

- Hypoleucos II. 69.

- Interpres II. 81.

- Islandica II. 84.93.

- Litrorea II. 64.66. .

- lobata II. 120.

- Maritima II, 87. 88.

- Minuta II. 98.

- Ocropus II. 64.

- Pugnax II. 7I.

- pusilla II. 96. 98.

- Rufescens II. 72.

- Squatarola II. 78.

- Striata II. 62.88.

- Subarquata II. 92.

- Temminkii II. 96.

- Vanellus II. 75.

- Varia II. 78.

- Variabilis II, gr.

- Variegata II. 72.

Trädkrypare I, 98.

Trädlärka I. 258 。

Trädsparf I. 143 .

Tullare II, 45.

Tummelilla I. 232 .

Tummeliten I. 232 。

Turdus Iliacus I, 175, 176。

- major I. 172 .

- Memla I. I79.
Turdus Musicus I. 173: 174.

- Pilaris I. 177 .

- Roseus I. 182.

- Torquatus I. $18 \mathrm{I}$.

- Viscivorus I, 171.172。

Turemule II. 136.

Tyvjoen Ir. 185.

Tarna II. 157 ,

Törnskata I. 73 .

$U_{\text {f. }} I_{053 .}$

Upupa Epops I. 96.

Uria Albida II. I 45.

- Alle IT, 146 .

- Baltica \& Grylloides II. 144.

- Grylle II. 144.

- Lacreola II. 145.

- Lomvia II. 142.

- minor II 146 .

- Swartbag \& Ringuia If. 143.

- Troile II. $x+2$.

Wacktel I. 3 I 4 .

Wacktelkung II, II3.

Vanellus Cristatus II, 75.76 。

- griseus II. 78.

- Helveticus II. 78.

- Melanogaster II. 77 .

Warfogel I. 74 .

Wattenhöns II. 123.

Wattenstare I. 26:.

Wedknarr I. rog.

Wi-And II. II8.

Wilddu fwa I, 294 .

Wildgàs II. 239.

Windspole II. 45 . 
Winter - A1 II. 199.

Winterhämpling I. 149,

Wintermåka II. 167 .

Wipa II. $7^{6 .}$

Wrakfogel II, 249.

$\AA_{\text {da II. } 195 .}$

Ảkerhöns I. 3II, II, 7 .

Áta II, 23 s.
$\ddot{A}_{\text {nglärkn I. } 242 \text {. }}$

Ängsnärpa II, 113.

Ärla I. 235.

Onmer II, 149.

Örn I. 3 . 
TYIS HARTV. FRID, POPP. 


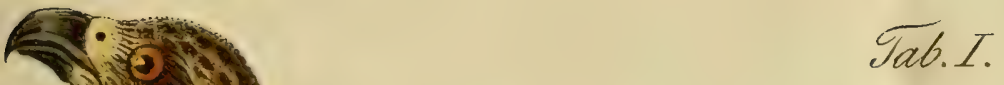

(it)

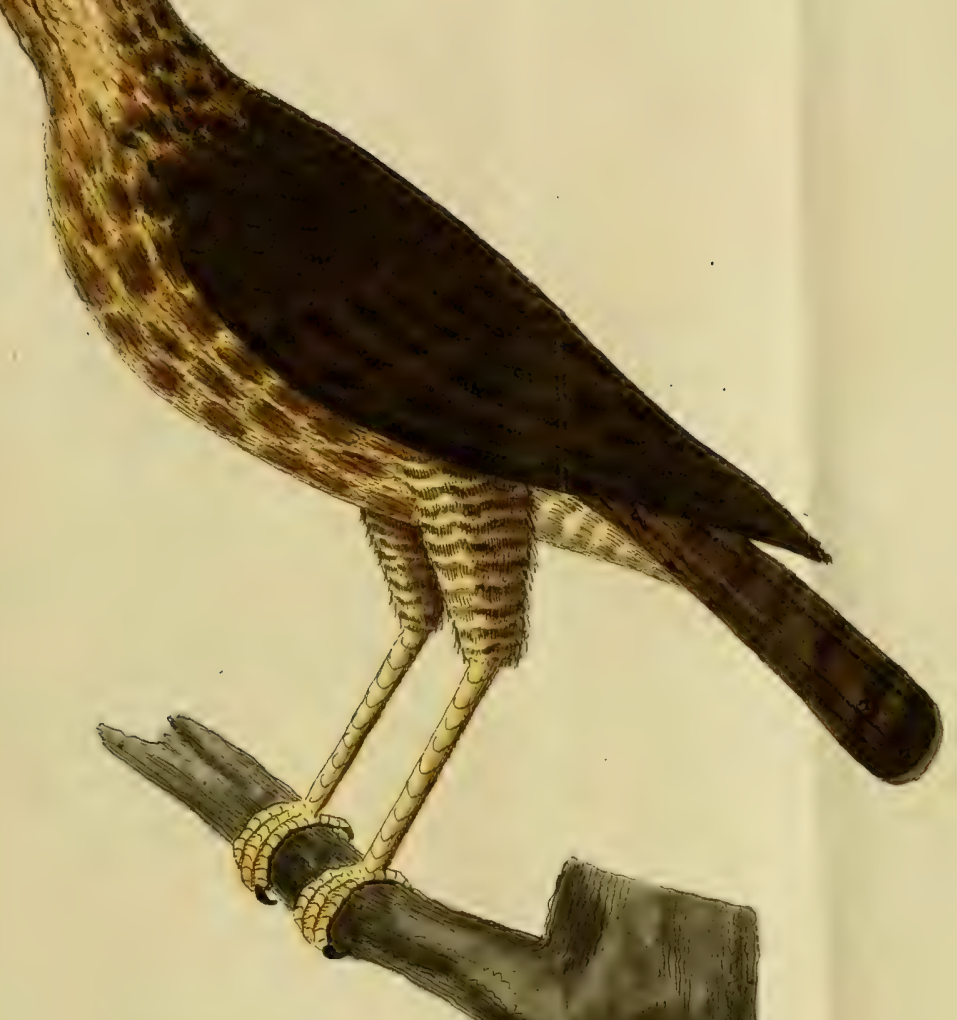

$\begin{array}{llllll}1 & 2 & 3 & 4 & 5 & 6 \\ 1 & 1 & 1 & 1 & 1 & 1\end{array}$
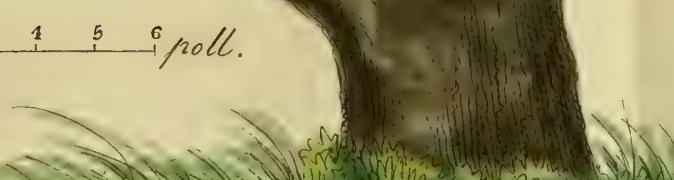

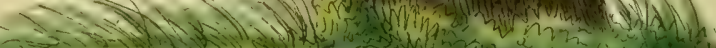

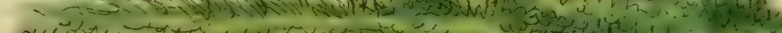

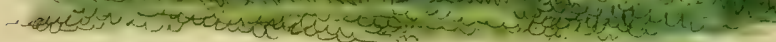





\section{Tab.II.}

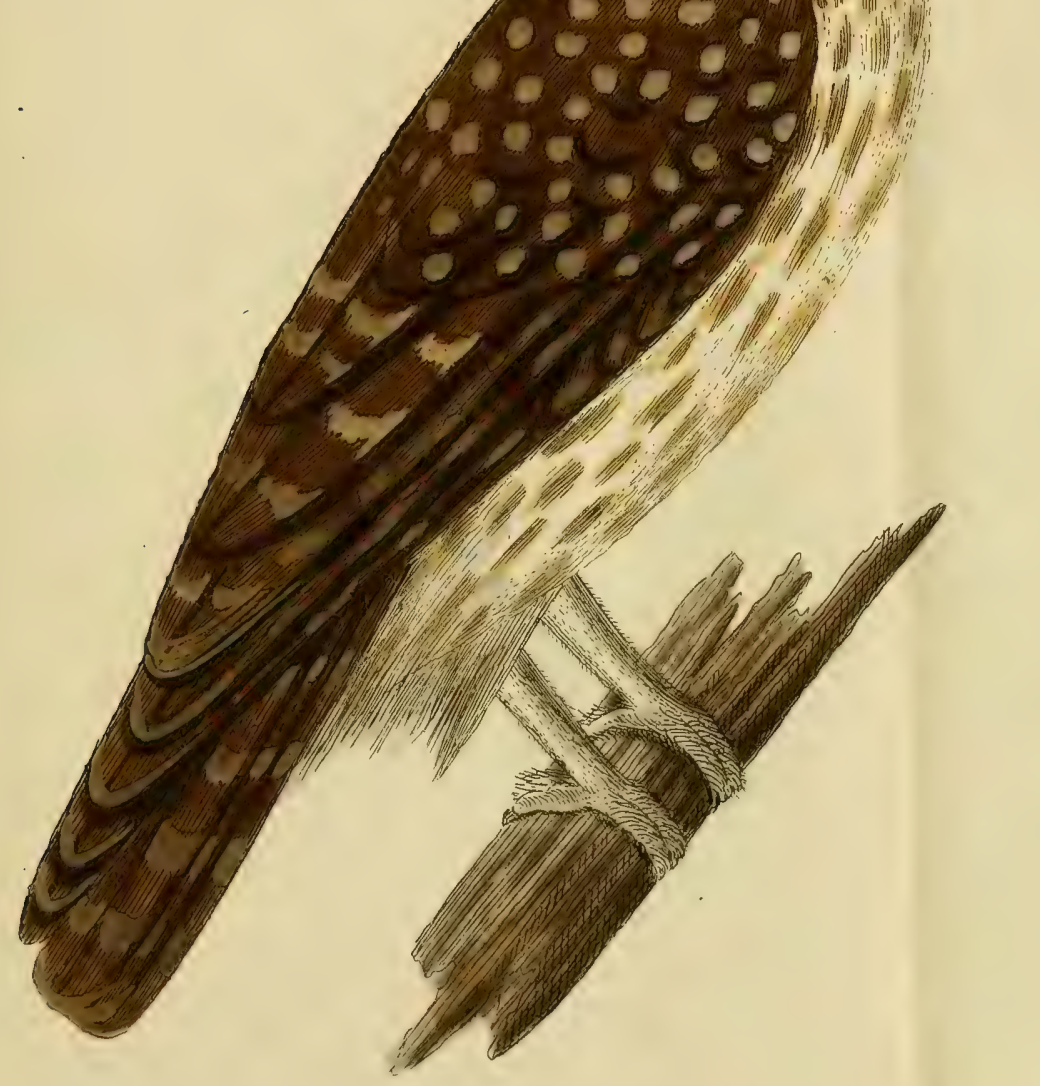





\section{Tab.III.}

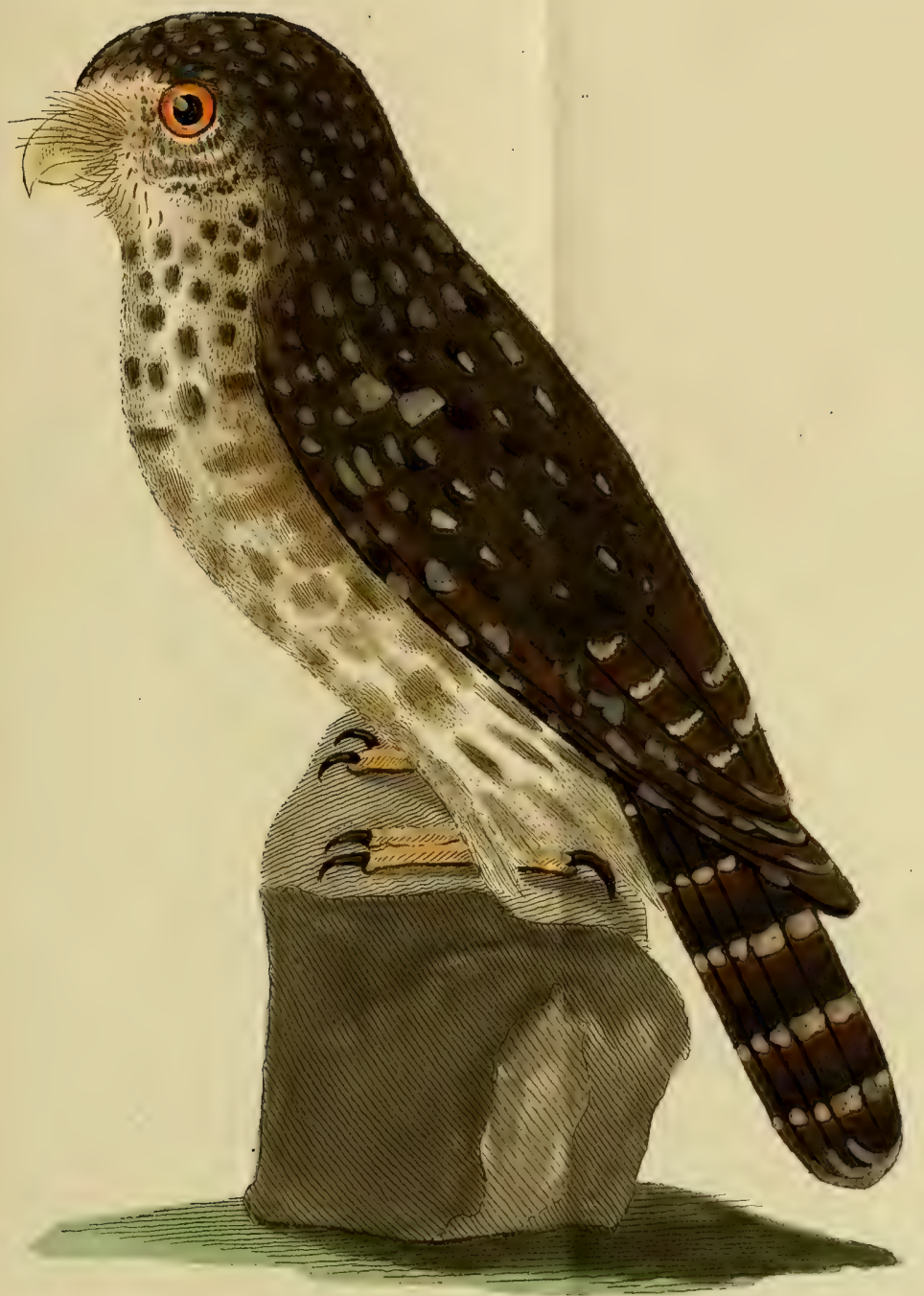



Tab. IV.

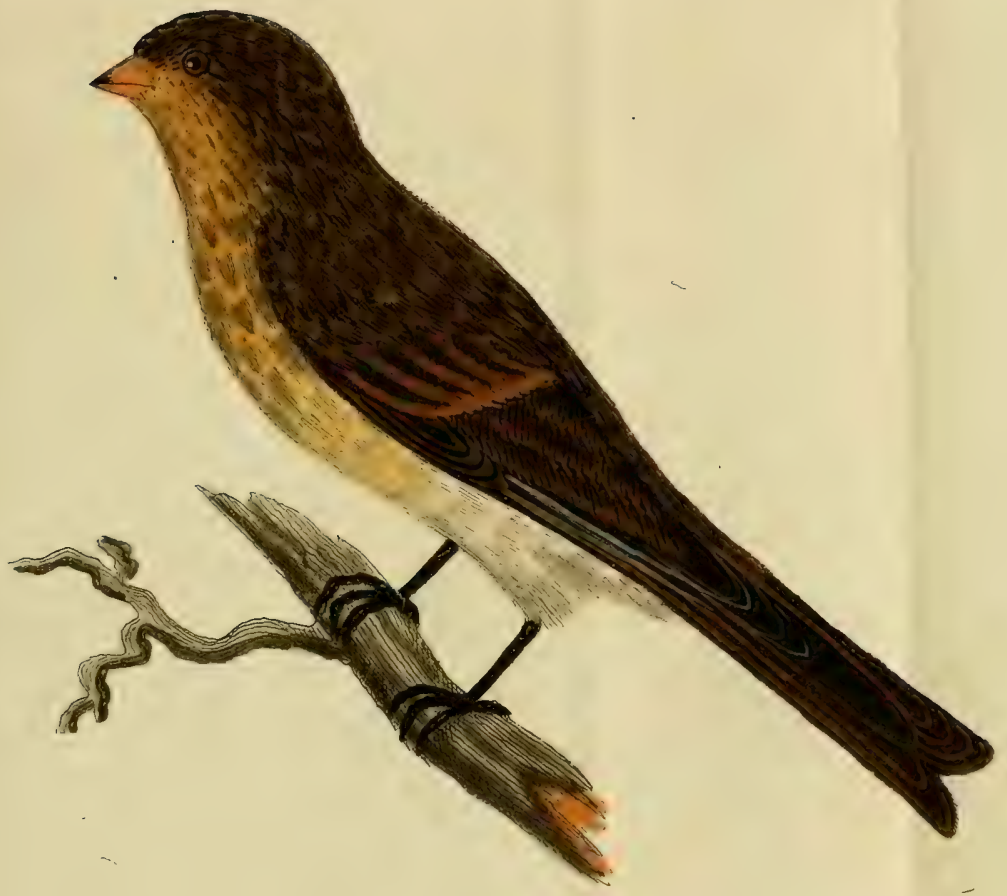



Tab.V.

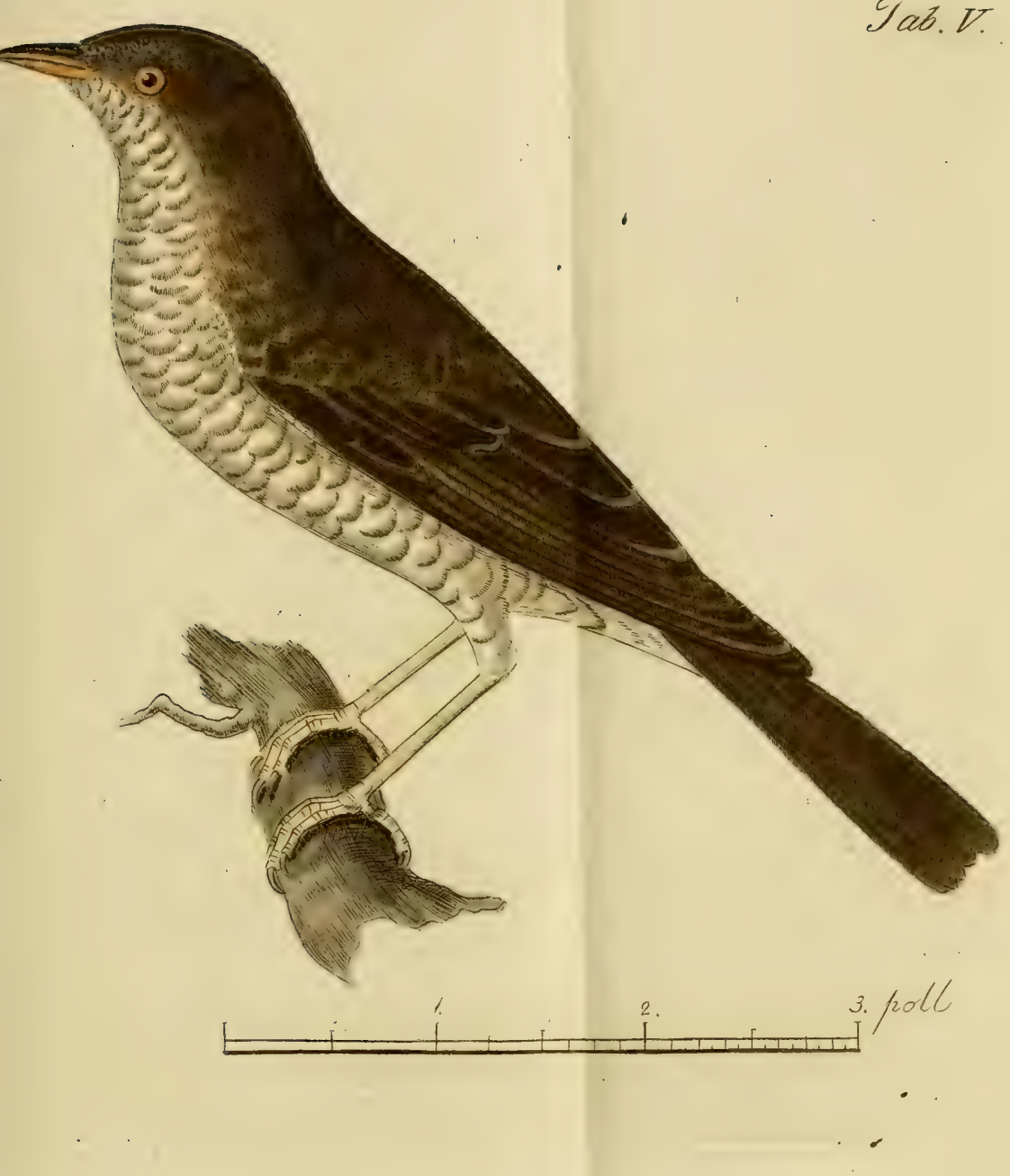



Tab.VI.

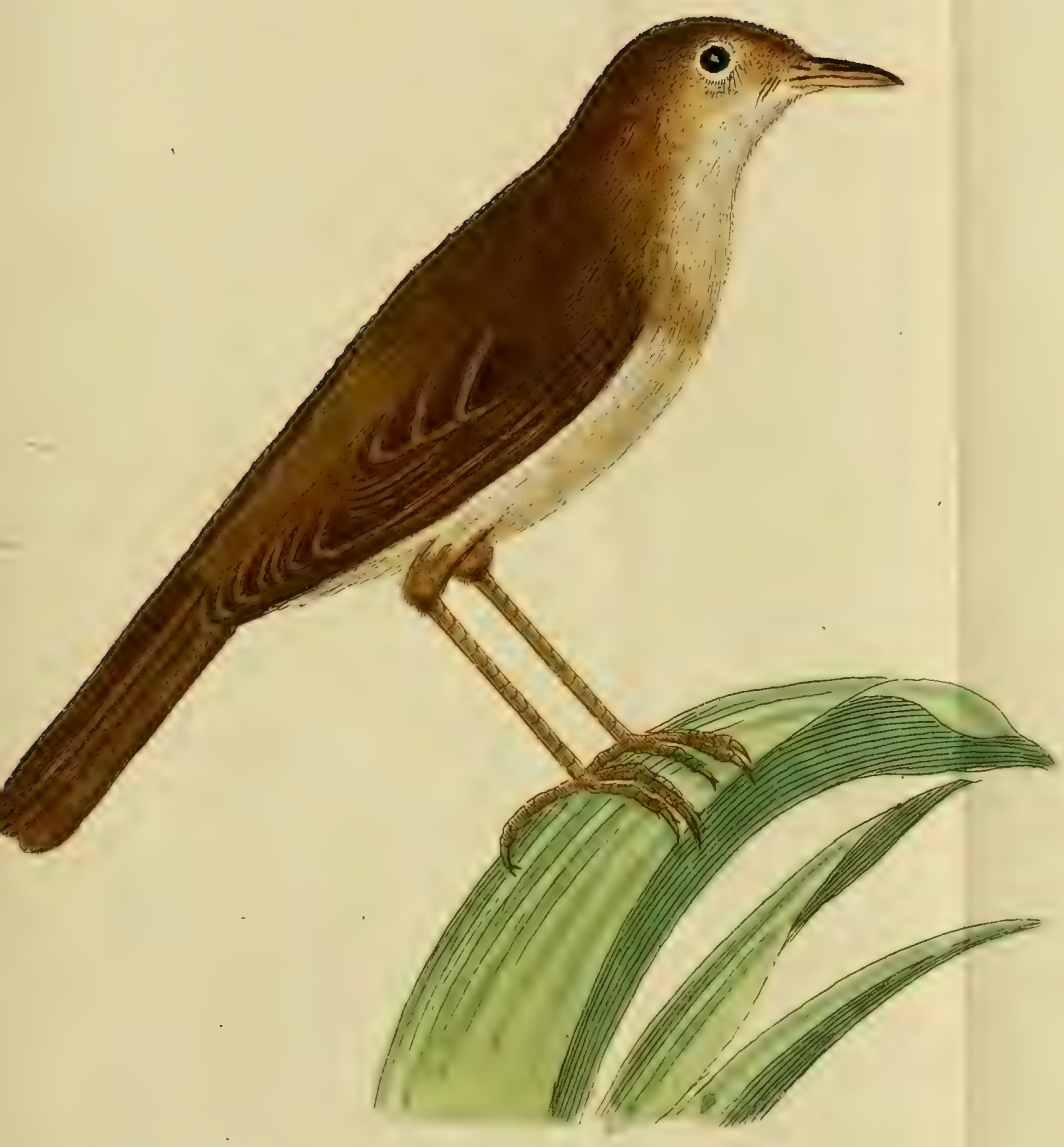




Tab. VIII. 

Tab.IX Fig.1.

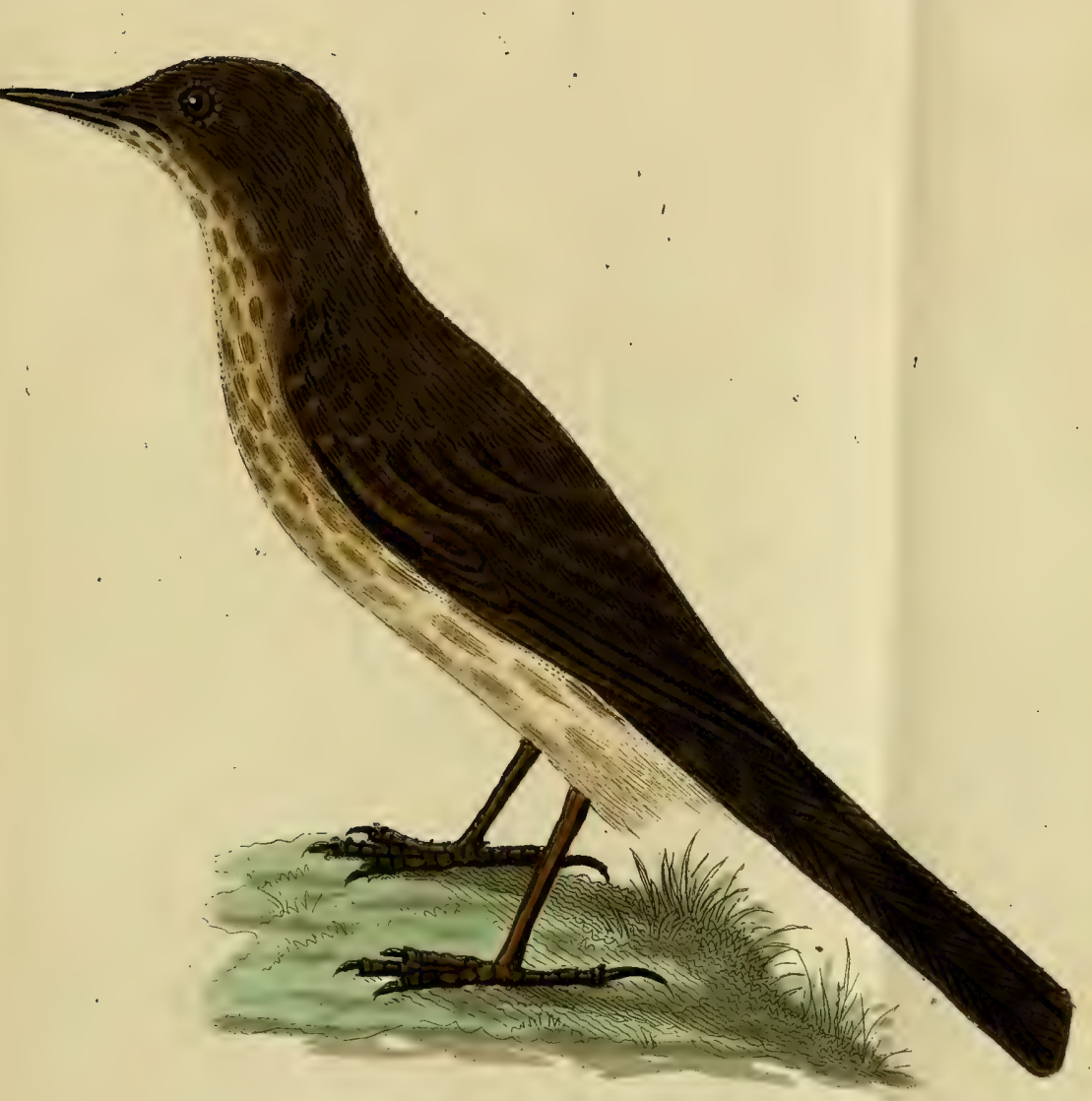





$$
1
$$



Tal..

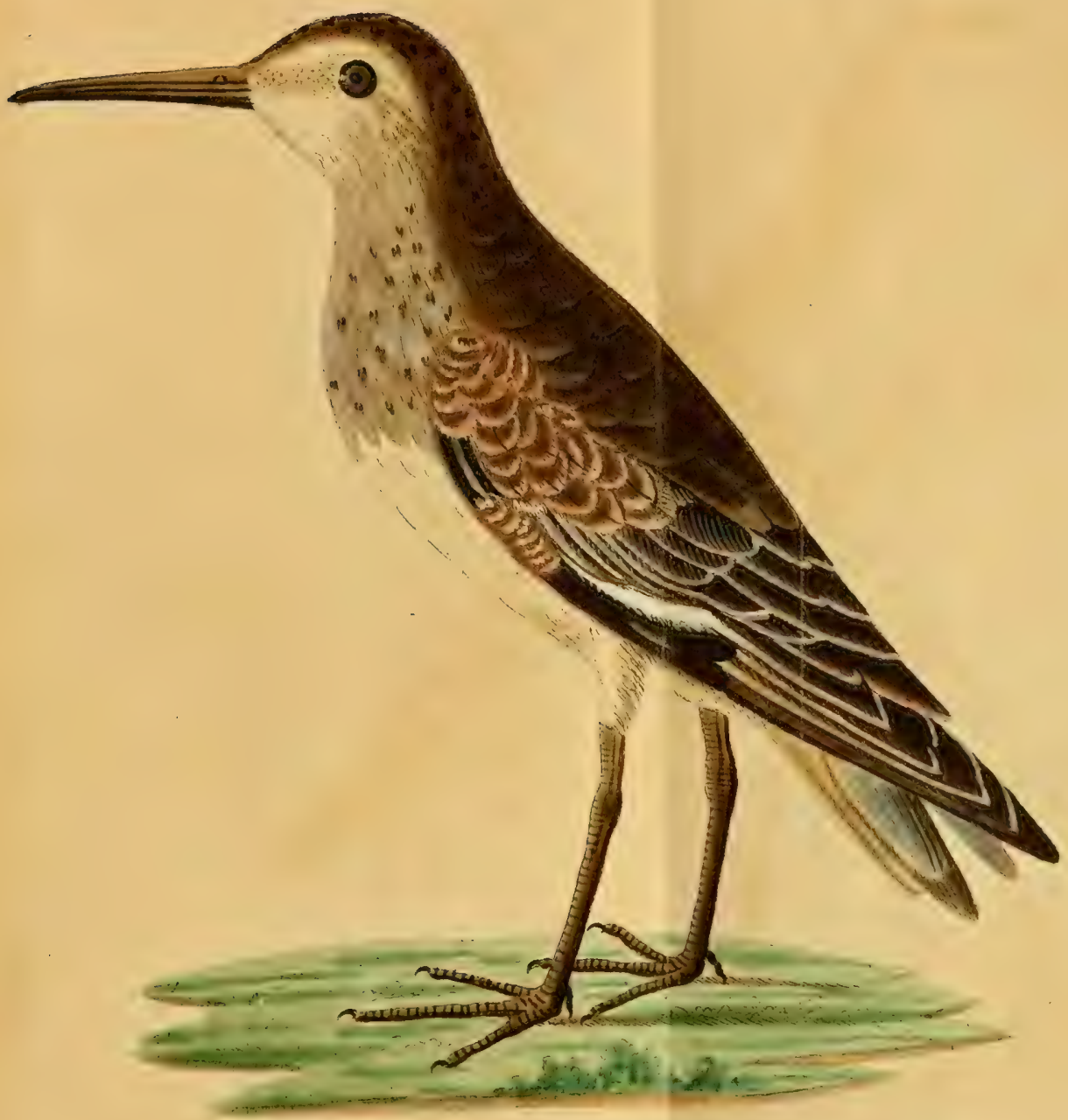



बत्व. IT. 


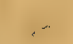


3. 4011 



SMITHSONIAN INSTITUTION LIBAARIES 